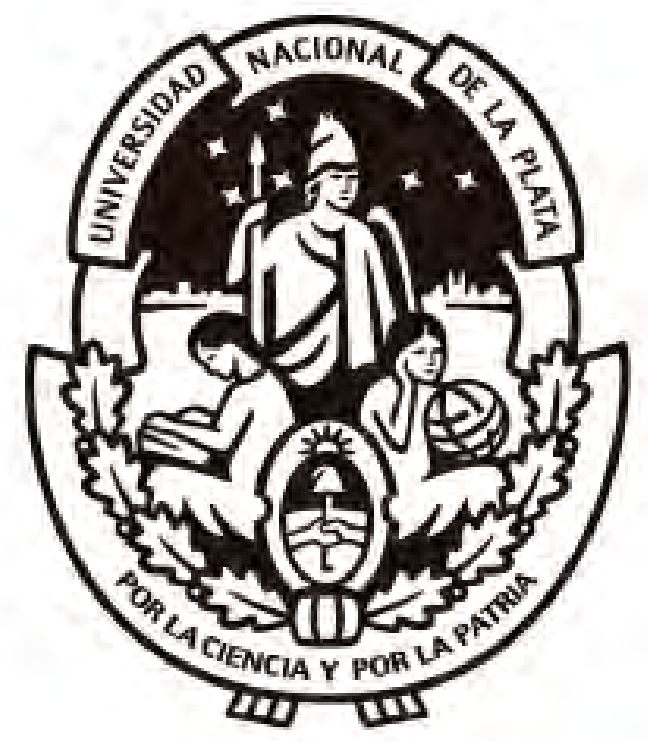

Universidad Nacional de La Plata

Facultad de Ciencias Astronómicas y Geofísicas

Tesis para obtener el grado académico de Doctor en Astronomía

Estudio Quemodinámico en Galaxias BCD

Lic. Federico Campuzano Castro

Director: Dr. Guillermo F. Hägele

Co-Director: Dr. Guillermo Bosch

La Plata, Argentina

- MARZO DE 2021 - 



\section{Prefacio}

Esta Tesis es presentada como parte de los requisitos para obtener el grado académico de Doctor en Astronomía de la Universidad Nacional de La Plata. La misma contiene los resultados de las investigaciones desarrolladas bajo la dirección de los Doctores Guillermo F. Hägele y Guillermo Bosch. Además se trabajó en colaboración con la Dra. Mónica Cardaci y la Dra. Verónica Firpo, investigadora y personal del Observatorio Gemini, y otros colegas durante los años 2014 y 2020.

Federico Campuzano Castro.

e-mail: fedecampu@fcaglp.unlp.edu.ar

La Plata, enero de 2021. 



\section{Resumen}

Uno de los desafíos de la astrofísica moderna en el estudio de galaxias es comprender las conexiones que subyacen entre el gas ionizado, los estados evolutivos y componentes químicos que lo constituyen. En particular, es el estudio de espectroscopía de alta resolución en el rango óptico el que nos permite analizar y medir numerosas líneas de emisión, a través de las cuales se pueden obtener resultados muy importantes por medio de cocientes de sus flujos. Las galaxias enanas cumplen un rol protagónico en el estudio y evolución de galaxias por considerarse a estas "pilares fundamentales" en la evolución de las galaxias. Comprender la naturaleza de los gases expuestos a los procesos físicos y químicos generados por los campos de radiación de los cúmulos jóvenes y sus estrellas masivas es importante para conocer cómo son los estadíos de evolución del gas ionizado.

En esta Tesis nos hemos abocado a investigar, por un lado, la complejidad de las múltiples componentes cinemáticas detectadas en los perfiles de las líneas espectrales de brotes intensos de formación estelar. Y por otro lado, las propiedades físicas y químicas del gas ionizado como densidades y temperaturas electrónicas, las abundancias químicas y iónicas y los campo de radiación al que este gas se ve expuesto. Este análisis completo y combinado es denominado Quimiodinámica.

En el Capítulo 1 hacemos una descripción general de las Regiones Hit y de las Galaxias BCDs (de sigla en inglés: Blue Compact Dwarf). Como nuestro trabajo se realizó a partir de espectroscopía de alta resolución, se hace una descripción de los espectros, así como también de las líneas espectrales y las propiedades físicas y químicas determinadas a partir de ellas.

En el Capítulo 2 se describen las observaciones y los procesos de reducción y calibración de la muestra de Regiones HiI estudiadas en esta Tesis. Las distintas reducciones y los detalles que han surgido al calibrar los espectros. Estos representan los primeros resultados de nuestro trabajo.

En el Capítulo 3 se define la cinemática de dichas regiones. Hemos desarrollado un programa en Python para avanzar en el estudio de las múltiples componentes cinemáticas presentes en los perfiles de las líneas de emisión. Este programa, apodado Kinematics, está basado en el código LMFit (https://lmfit.github.io/lmfit-py/) y nos permitirá validar las soluciones planteadas a través de la relación $\triangle \mathrm{AC}$ definida por el criterio estadístico de Akaike. Los ajustes de múltiples gaussianas, permitiréstimar la velocidad radial ( $\mathrm{v}_{\text {rad }}$ ), la dispersión de velocidades $(\sigma)$ y el flujo asociado a cada gaussiana. La metodoloía utilizada tiene sus orígenes en la Tesis Doctoral de la Doctora Verónica Firpo, entre otros trabajo desarrollados por el grupo de trabajo y colaboradores. Se presentan las soluciones cinemáticas y los ajustes correspondientes para cada región. En el Capítulo 4 se calculan las propiedades físicas del gas ionizado a partir de los resultados obtenidos para la cinemática. Nuestro análisis depende de manera directa de la detección de líneas intensas y de las líneas más débiles y sensibles a las temperaturas, para estimar de manera precisa, a través del método directo, los parámetros físicos. Para los casos en que fue necesario, se utilizaron modelos de fotoionización para la estimación de abundancias químicas, así como también hemos utilizado métodos empíricos y 
semiempíricos para la determinación y comparación de resultados. A través de los parámetros de ionización $\eta$ y $\eta$ ' del gas, se pudo dar una medida aproximada de las temperaturas de los campos de radiación ionizantes de los brotes intensos de formación estelar.

A lo largo de este proceso de realización de la Tesis, se ha participado en la elaboración de cinco propuestas de observación, donde se han obtenido tiempo en dos de ellas, y se espera trabajar y resolver la quimiodinámica de una muestra de BCDs obtenidas en distintos turnos de observación. En el Capítulo 5 presentamos los resultados y conclusiones de la Tesis, y también se presentan los candidatos a analizar y estudiar próximamente. 


\section{Originalidad}

Las ideas, desarrollos y resultados obtenidos en este trabajo son originales del autor y de los directores de la Tesis, salvo cuando se hace explícita mención. La mayor parte de los análisis, metodologías y resultados fueron presentados previamente en actas de congresos. Parte de la metodología fue publicada en Bosch et al. (2019). En este período de trabajo se han presentado solicitudes de tiempo de telescopio de diversos instrumentos, indicando la importancia del trabajo y las pulicaciones con los resultados obtenidos se encuentran en desarrollo. Para realizar los ajustes de la cinemática se ideó un código en Python, con el objetivo de optimizar tiempos en el análisis y metodología, en comparación con la herramienta utilizada anteriormente como IRAF (Image Reduction and Analysis Facility, distribuido por NOAO, operado por AURA, Inc., bajo contrato con NSF). Luego, para el estudio de las propiedades físicas del gas y la determinación de las abundancias químicas iónicas y totales, se ha utilizado el programa desarrollado por Guillermo Hägele que ha sido utilizado en numerosos trabajos Hägele et al. (2006, 2008, 2009, 2012) y está demostrado que funciona correctamente. Además, en esta Tesis, la cual se desprende de una serie de trabajos realizados por el grupo del trabajo del cual formo parte (Hägele et al., 2007, 2009, 2010; Firpo et al., 2011; Hägele et al., 2012; Amorín et al., 2012a; Hägele et al., 2013; Bosch et al., 2019), nos proponemos resolver la cinemática compleja de regiones de formación estelar violenta pertenecientes a galaxias compactas azules. Los estudios de múltiples componentes cinemáticas identificadas en las líneas de emisión, sumado al análisis de las propiedades físicas del gas ionizado, completa un estudio quimiodinámico. Este procedimiento pudo realizarse únicamente para un objeto en la literatura (Hägele et al., 2012), previo al desarrollo de esta Tesis. 

A toda persona que defienda la Universidad Pública, que luche por el sistema científico nacional, y que reconozca un valor cultural en este escrito.

A aquellos que valoran, desde la humildad y el respeto, el trabajo de un becario y su esfuerzo. A mis amigas y amigos, por su compañía y apoyo incondicional. A mi familia, Pau y Milo. 

A mi Madre y a mi abuela Norma.

Gracias por el amor de por vida.

Este escrito es producto de que siempre creyeron en mí.

Estarán por siempre en mi corazón. 



\section{Agradecimientos}

Esta Tesis es el resultado de varios años de trabajo y de estudio dentro de la Facultad de Ciencias Astronómicas y Geofísicas de La Plata. Fue la educación pública y de calidad que me abrió la puerta hacia un sistema público de investigación, en el cual pude desarrollar mi actividad con la financiación del CONICET; y estoy sumamente agradecido por ello.

En primer lugar quiero y debo agradecer a la sociedad y al país que apoya y sostiene un sistema científico y a la Universidad Pública. Este apoyo nos permite a los y las estudiantes de doctorado desarrollar nuestras investigaciones. A pesar de que durante los años 2016 y 2019 los medios hegemónicos buscaron menospreciar el trabajo de los investigadores del CONICET, agrediendo y ninguneando las actividades de muchos colegas, el apoyo de gran parte de la sociedad fue muy valioso y fundamental para sostener muchas actividades. Es por esto, que quiero destacar y agradecer a toda la gente que apoya y que apoyó el trabajo de las/os becarias/os y científicas/os argentinas/os.

A mis directores, Los Guilles, por hacer posible que pueda obtener una beca para hacer el doctorado y darme la oportunidad de vivir estos años de mi vida en el sistema científico. Se han complementado para enseñarme críticamente el trabajo de un científico. Gracias por permitirme participar en distintos proyectos de observación que me dejaron tener vivencias y experiencias muy importantes en mi formación como profesional y como persona.

A Verónica Firpo, por haber sido la colega y colaboradora principal en este trabajo. Gracias por ayudarme a crecer en muchos aspectos. Por ser un ejemplo de sacrificio y esfuerzo. Compartí los más grandes momentos durante este tiempo, desde los principios en la Secretaría de Extensión hasta la última visita al observatorio. Gracias por todo, pero en particular por haber creído en mí y por acompañarme en el trabajo de una manera única.

A Pi-Jey-Pessi que (quizás sin saberlo) fue la companía y compañera más justa y necesaria que pude haber tenido en este proceso. Fuiste la mejor compañía que pude tener y realmente espero que en algún futuro podamos hacer ciencia juntos.

A Eze Passaro, por la compañía y apoyo en un momento muy especial y particular de mi carrera.

A Lilia Bassino, que depositó su confianza en mí y me abrió la puerta a relaciones nuevas. Ya te lo he dicho personalmente, pero gracias nuevamente por tu confianza, fue uno de los gestos más grandes que recibí en estos años.

A Caro, por todo. Solo vos sabes lo importante que sos para mí. Tu apoyo y compañía (a la distancia!!) fue crucial y fundamental para poder cerrar esto.

A la Furia Mariani, gracias amigo. Sos de las personas que más aprecio y en este último tiempo me diste la fuerza necesaria para confiar en mí.

A Keiko, por su 1.495m de amistad. Al Gran Francisco Reguera, por todo. La compañía, la amistad y el apoyo en todo momento y circunstancia. Al Tin, al Tomi, a Mau, a Pe, al Fefu, a Iri, a Manu, al Santi, al Juls, a Tiaris, a Yani y al Juancho. Son mi familia y desde hace muchos años que alegran los días y la vida en La Plata. Al Lea y Joni, mis más grandes compañeros del corazón que siempre estuvieron y que son lo más grande que hay. 
Al Fola, por las charlas y momentos compartidos.

A mis amigas y amigos que me acompañan desde los inicios de la carrera y a todes les que fueron surgiendo a lo largo de este camino.

A mis compañeros de la cátedra de Física I, con los cuales hemos dado clase desde hace varios años y llevamos todo un curso durante pandemia aprendiendo muchos valores sobre el trabajo y la dedicación. A la UNLP y a la UNaB por ser sostenes y soportes laborales cuando los caminos de la Tesis estaban obstruídos y sentía que no había avances.

A Ani, Marce, Lea, Santi y Joaco, por su compañía y apoyo.

A la Facultad y a toda la gente que trabaja en ella. El obser es como mi segunda casa.

A quienes luchan por hacer público y accesible el conocimiento científico.

Y por último, y en especial, a Pau y a Milo. Las personas que me han acompañado y bancado en todo este proceso. Este trabajo es fruto de su apoyo y aliento.

Gracias. 


\section{Índice general}

$\begin{array}{ll}\text { Prefacio } & \text { iii }\end{array}$

Resumen $\quad$ v

Originalidad vii

Agradecimientos $\quad$ xiii

1. Introducción 1

1.1. Aspectos generales de las Regiones Hir . . . . . . . . . . . . . . . . . 1

1.2. Galaxias Compactas Azules . . . . . . . . . . . . . . . 4

1.3. Espectros de emisión en nebulosas gaseosas . . . . . . . . . . . . . . 9

1.3.1. Equilibrio de fotoionización . . . . . . . . . . . . . . . . 10

1.3.2. Líneas de recombinación . . . . . . . . . . . . . . . . . . . . . 13

1.3.3. Líneas prohibidas o de excitación colisional . . . . . . . . . . . . . 14

1.4. Abundancias quimicas y parámetros físicos . . . . . . . . . . . . . 16

1.4.1. Abundancias químicas . . . . . . . . . . . . . . . . . . . 16

1.4.2. Densidad . . . . . . . . . . . . . . . . . . 17

1.4.3. Temperatura . . . . . . . . . . . . . . . . . . 19

1.4.4. Corrección por enrojecimiento y extinción . . . . . . . . . . . . . . . . 22

1.5. Técnicas observacionales utilizadas . . . . . . . . . . . . . . 23

1.5.0.1. Espectroscopía . . . . . . . . . . . . . . . 24

1.6. Objetivos del Trabajo . . . . . . . . . . . . . . . . . . 31

2. Observaciones y reducción de datos 35

2.1. Importancia y tipo de observaciones . . . . . . . . . . . . . . . . . . 35

2.2. Descripción de las galaxias observadas . . . . . . . . . . . . . . 36

2.2.1. II Zwicky $33 \ldots \ldots \ldots \ldots \ldots \ldots \ldots$

2.2.2. Markarian $600 \ldots \ldots \ldots \ldots \ldots$

2.3. Observaciones Espectroscópicas . . . . . . . . . . . . . . . . . . . . 39

2.4. Proceso de reducción y calibración de las observaciones . . . . . . . . . . . . 42

2.4.1. Extracción del espectro y calibración en longitud de onda . . . . . . . 42

2.5. Extracción de los espectros de ciencia . . . . . . . . . . . . . 46

2.5.1. Calibración en flujo . . . . . . . . . . . . . . . . 51

2.6. Análisis de las regiones . . . . . . . . . . . . . . . . . 55

2.6.1. Extracción a partir de líneas intensas . . . . . . . . . . . . . 55

2.6.2. Problemas y soluciones a limitaciones instrumentales . . . . . . . . . 56

2.6.3. Análisis de las componentes espaciales . . . . . . . . . . . . 58

2.7. Conclusión . . . . . . . . . . . . . . . . . . . . . . 59 
3. Análisis y resultados obtenidos a partir de las observaciones

3.1. Estudio de la cinemática a través de espectroscopía de alta resolución en galaxias BCD . . . . . . . . . . . . . . . . 67

3.2. Metodología del análisis cinemático . . . . . . . . . . . . . . . . . . 69

3.2.1. Ajuste de múltiples gaussianas . . . . . . . . . . . . . . . . 69

3.2.2. Tarea de ajuste para las componentes: Kinematics . . . . . . . . . . 69

3.2.3. Dispersión de velocidades, $\sigma \ldots \ldots \ldots$. . . . . . . . . . 74

3.2.4. Flujo y errores en su determinación . . . . . . . . . . . . 74

3.3. Análisis de la cinemática de las regiones . . . . . . . . . . . . . . . . . . . . . 75

3.3.1. II Zw 33 . . . . . . . . . . . . . . . . . . . . . . 76

3.3.2. IIZw 33 Nudo A . . . . . . . . . . . . . . . 76

3.3.3. IIZw 33 Nudo B . . . . . . . . . . . . . . . . . . 81

3.3.4. IIZw 33 Nudo $\mathrm{C}$. . . . . . . . . . . . . . . . 87

3.3.5. Conclusiones de la Cinemática para II Zw 33 . . . . . . . . . . . . 92

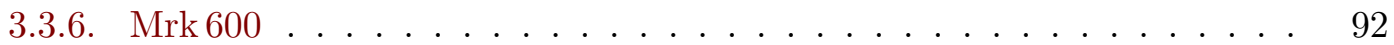

3.3.7. Mrk 600 Nudo A . . . . . . . . . . . . . . . 92

3.3.8. Mrk 600 Nudo B . . . . . . . . . . . . . . . . . 97

3.3.9. Conclusiones de la Cinemática para Mrk600 . . . . . . . . . . . 103

3.3.10. Diagramas Diagnósticos: BPT . . . . . . . . . . . . . 103

3.4. Conclusión ......................... 109

4. Determinación de las abundancias químicas y las propiedades físicas del gas ionizado111

4.1. Densidades y Temperaturas Electrónicas . . . . . . . . . . . . . . . . . 111

4.1.1. Método Directo . . . . . . . . . . . . . . . . . . . 115

4.1.2. Relación entre las temperaturas electrónicas . . . . . . . . . . . . 116

4.1.3. Métodos Empíricos para la determinación de temperaturas . . . . . 119

4.2. Abundancias Químicas . . . . . . . . . . . . . . . . . . 119

4.2.1. Helio . . . . . . . . . . . . . . . . 120

4.2.2. Abundancias iónicas y totales a partir de líneas prohibidas . . . . . 121

4.3. Discusión de las observaciones Echelle . . . . . . . . . . . . . . . . . . . . . 124

4.3.1. Comparación de los resultados determinados entre nudos . . . . . . 146

4.4. Abundancias químicas estimadas a partir de calibradores empíricos . . . . . . 153

4.4.1. Discusión de los resultados determinados a partir de los parámetros empíricos para II Zw 33 . . . . . . . . . . . . . . . 162

4.4.2. Discusión de los resultados determinados a partir de los parámetros empíricos para Mrk600 . . . . . . . . . . . . . . . 164

4.5. Estructura de Ionización . . . . . . . . . . . . . . . . . . . . . . 166

4.6. Resumen ............................ 177

5. Resumen, conclusiones y trabajo a futuro 18

5.1. Trabajo a Futuro . . . . . . . . . . . . . . . . . . . . . 187 


\section{Acrónimos}

Lista de acrónimos utilizados en esta tesis (notar que las siglas usualmente corresponden a las utilizadas en el idioma inglés):

- RHir: Región Hiı

- RHiıG: Región Hir Gigante

- BCDs: Galaxias Compactas Azules (Blue Compact Dwarf Galaxies)

- LCO: Observatorio Las Campanas (Las Campanas Observatory)

- II Zw 33: II Zwicky 33

- Mrk600: Markarian 600

- CR: Rayo cósmico (Cosmic Ray)

- S/N: señal a ruido

- $\mathrm{T}_{e}$ : Temperatura electrónica

- $\mathrm{T}_{e} f f:$ Temperatura efectiva

- $\mathrm{n}_{e}$ : Densidad electrónica

- $\eta$ : Parámetro de Blandura (Softness Parameter)

- U: Parámetro de Ionización

- LMFit: Non-Linear Least-Squares Minimization and Curve-Fitting for Python

- $\sigma_{i n t r}=$ Dispersión de velocidades intrínseca

- $\sigma_{o b s}=$ Dispersión de velocidades observacional

- $\sigma_{i n s t r}=$ Dispersión de velocidades instrumental

- $\sigma_{\text {term }}=$ Dispersión de velocidades térmica

- SNR: Remanente de supernova (Supernova Remnant)

- UL: Límite superior (Upper Limit)

- ICF: Factor de Corrección por Ionización (Ionization Correction Factor)

- WR: Wolf-Rayet 



\section{Índice de figuras}

1.1. Nebulosa del Corazón - Nebulosa de emisión . . . . . . . . . . . . . . . 2

1.2. Galaxias BCD . . . . . . . . . . . . . . . . . 7

1.3. Regiones Hir a escala . . . . . . . . . . . . . . . . . . . . . . . . 11

1.4. Diagramas Diagnóstico de densidades . . . . . . . . . . . . . . . 18

1.5. Diagramas Diagnósticos de Densidades y Temperaturas . . . . . . . . . . . . 20

1.6. Diagrama Diagnóstico Osterbrock . . . . . . . . . . . . . . . . . 21

1.7. Diagrama de Gotrian para el Orı . . . . . . . . . . . . . . . . . . . . . 21

1.8. Espectros Estelares y Nebulares . . . . . . . . . . . . . . . . . 25

1.9. Desplazamiento de las líneas debido al corrimiento al rojo . . . . . . . . 25

1.10. Espectrógrafos y sus elementos dispersores . . . . . . . . . . . . . . . 27

1.11. Espectro resultante . . . . . . . . . . . . . . . . . . 27

1.12. Superposición de los órdenes espectrales . . . . . . . . . . . . . . . . 29

1.13. Ángulo de posición de la rendija . . . . . . . . . . . . . . . . . 30

2.1. Imagen del continuo y contornos de emisión de $\mathrm{H} \alpha$ para II Zw $33 \quad \ldots$. . . . . 37

2.2. Imagen del continuo y contornos de emisión de $\mathrm{H} \alpha$ para Mrk $600 \quad \ldots$. . . . . . 39

2.3. Simulación en falso color de como se ajusta un espectro Echelle en el detector. 40

2.4. Regiones Observadas . . . . . . . . . . . . . . . . . . . . . . 42

2.5. Imágenes de calibración: Bias, Flat y lámpara . . . . . . . . . . . . . . . . 43

2.6. Imagen defectuosa . . . . . . . . . . . . . . . . . . . . 44

2.7. Imágenes del proceso de extracción de rayos cósmicos . . . . . . . . . . . . 47

2.8. Traza del espectro de la estrella estándar . . . . . . . . . . . . . . . . . . . . 48

2.9. Líneas del espectro con contaminación de otra fuente . . . . . . . . . . . . 49

2.10. Imágenes del doblete del $[\mathrm{OII}] 3726,29 \AA$ A para ambas galaxias . . . . . . . . . . 50

2.11. Líneas de la lámpara de comparación . . . . . . . . . . . . . . . 52

2.13. Ajuste del polinomio para la calibración en flujo de los espectros . . . . . . . 54

2.14. Ajuste en longitud de onda y flujo de la estrella estándar . . . . . . . . . . . 55

2.15. Ventanas de extracción del espectro en líneas de emisión . . . . . . . . . . 56

2.16. Perfíl de línea de emisión en el cross-over . . . . . . . . . . . . . . . . 57

2.17. Zona del cross-over para MIKE . . . . . . . . . . . . . . . . 58

2.18. Líneas de emisión con estructura espacial distinguible . . . . . . . . . . . . . 59

2.19. Extracción del espectro en el eje espacial . . . . . . . . . . . . . . . . 60

3.1. Criterio de Akaike para la determinación de componentes en el Nudo A de

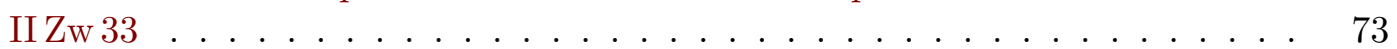

3.2. Ajustes de Múltiples Componentes Cinemáticas en II Zw 33 A . . . . . . . . . 80

3.3. Ajuste de la componente cinemática reflejada hacia el rojo . . . . . . . . . . . . 81

3.4. Determinación de las múltiples componentes en II Zw 33 B . . . . . . . . . 82

3.5. Ajustes de Múltiples Componentes Cinemáticas en II Zw 33 B . . . . . . . . 86 
3.6. Ajustes de múltiples gaussianas para realizados para líneas del doblete del oxígeno en $3729 \AA \ldots \ldots \ldots$. . . . . . . . . . . . . . . . 87

3.7. Determinación de las múltiples componentes en II Zw 33 C . . . . . . . . . 88

3.8. Ajustes de Múltiples Componentes Cinemáticas en II Zw 33 C . . . . . . . . . 89

3.9. Determinación de las múltiples componentes en Mrk600 A _ . . . . . . . 93

3.10. Ajustes de Múltiples Componentes Cinemáticas en Mrk600 A . . . . . . . . 98

3.11. Determinación de las múltiples componentes en Mrk600 B . . . . . . . . . . 99

3.12. Ajustes de Múltiples Componentes Cinemáticas en Mrk600 B . . . . . . . . . 100

3.13. Diagrama Diagnóstico $[\mathrm{OIII}] \lambda 5007 \AA / \mathrm{H} \beta$ vs. $[\mathrm{NiI}] \lambda 6584 \AA / \mathrm{H} \alpha$ para II Zw 33 . . 104

3.14. Diagrama Diagnóstico $[\mathrm{OIII}] \lambda 5007 \AA / \mathrm{H} \beta$ vs. $[\mathrm{SiI}](\lambda 6717 \AA+\lambda 6731 \AA) / \mathrm{H} \alpha$ para II Zw $33 \ldots \ldots \ldots \ldots \ldots \ldots \ldots \ldots \ldots \ldots$

3.15. Diagrama Diagnóstico $[\mathrm{OIII}] \lambda 5007 \AA / \mathrm{H} \beta$ vs. [OI] $] 26300 \AA / \mathrm{H} \alpha$ para II Zw $33 \ldots 106$

3.16. Diagrama Diagnóstico $[\mathrm{OIII}] \lambda 5007 \AA / \mathrm{H} \beta$ vs. $[\mathrm{NII}] \lambda 6584 \AA / \mathrm{H} \alpha$ para Mrk 600 . . 107

3.17. Diagrama Diagnóstico $[\mathrm{OIII}] \lambda 5007 \AA / \mathrm{H} \beta$ vs. $[\mathrm{SiI}](\lambda 6717 \AA+\lambda 6731 \AA) / \mathrm{H} \alpha$ para Mrk $600 \ldots \ldots \ldots \ldots \ldots \ldots$

4.1. Diagramas de niveles de transición . . . . . . . . . . . . . . . . . . . 112

4.2. Estructuras de Ionización de la RHi . . . . . . . . . . . . . . . . . . 117

4.3. Gráfico de las abundancias de N/O para II Zw $33 \ldots \ldots \ldots$

4.4. Gráfico de las abundancias de $\mathrm{S} / \mathrm{O}$ para II Zw 33 . . . . . . . . . . . . . . 139

4.5. Gráfico de las abundancias de $\mathrm{Ne} / \mathrm{O}$ para II Zw 33 . . . . . . . . . . . . . . 140

4.6. Gráfico de las abundancias de $\mathrm{Ar} / \mathrm{O}$ para II Zw $33 \ldots \ldots$. . . . . . . . . 141

4.7. Gráfico de las abundancias de N/O para Mrk600 . . . . . . . . . . . . . . . 148

4.8. Gráfico de las abundancias de S/O para Mrk600 . . . . . . . . . . . . . 149

4.9. Gráfico de las abundancias de Ne/O para Mrk600 . . . . . . . . . . . . 150

4.10. Gráfico de las abundancias de Ar/O para Mrk600 . . . . . . . . . . . . . 151

4.11. Estimación de las abundancias químicas a partir de Métodos Empíricos . . . 160

4.12. Estimación de las abundancias químicas a partir de Métodos Empíricos . . . 161

4.13. Parámetro de dureza del campo de radiación: $\eta$. . . . . . . . . . . . . 170

4.14. Parámetro de dureza del campo de radiación: $\eta$. . . . . . . . . . . . . 173

4.15. Parámetro de dureza del campo de radiación Observacional: $\eta$ ' . . . . . . . . 174

4.16. Parámetro de dureza del campo de radiación Observacional: $\eta^{\prime} \ldots$. . . . . . . 175

5.1. Soluciones cinemáticas preliminares para los tres nudos principales de formación estelar de la galaxias BCD Haro $11 \ldots$. . . . . . . . . . . . . .

5.2. Soluciones cinemáticas preliminares para tres nudos intensos de formación estelar de la galaxias BCD Tol2 . . . . . . . . . . . . . . . . . . . .

5.3. Solución cinemática preliminares para la región centraol de la galaxias BCD Tol0610-387. . . . . . . . . . . . . . . . . . . .

5.4. Soluciones cinemáticas preliminares para los dos nudos principales observados en la galaxia BCD SCHG 0341-407 . . . . . . . . . . . . . . . .

5.5. Solución cinemática preliminares para la región de formación observada en la galaxia BCD Haro 14. . . . . . . . . . . . . . . . . . . . . 196

5.6. Soluciones cinemáticas preliminares para la galaxia BCD NGC 1702 . . . . . 197

5.7. Calibraciones preliminares de los datos de Gemini Norte . . . . . . . . . . 198 


\section{Índice de tablas}

2.1. Características de ambos brazos del espectrógrafo MIKE . . . . . . . . . . . . 39

2.2. Esquema de las Observaciones realizadas . . . . . . . . . . . . . . . . . 41

3.1. Parámetros de los ajustes de las diferentes líneas, sus múltiples componentes y el perfíl Global del Nudo A de II Zw 33 . . . . . . . . . . . . . . . . 77

3.2. Parámetros de los ajustes de las diferentes líneas, sus múltiples componentes y el perfíl Global del Nudo B de IIZw 33 . . . . . . . . . . . . . . . 83

3.3. Parámetros de los ajustes de las diferentes líneas, sus múltiples componentes y el perfíl Global del Nudo C de II Zw 33 . . . . . . . . . . . . . . . .

3.4. Parámetros de los ajustes de las diferentes líneas, sus múltiples componentes y el perfíl Global del Nudo A de Mrk600 . . . . . . . . . . . . . . . . .

3.5. Parámetros de los ajustes de las diferentes líneas, sus múltiples componentes y el perfíl Global del Nudo B de Mrk600 . . . . . . . . . . . . . . . . . . . . 101

4.1. Cocientes utilizados para la determinación de las densidades y temperaturas electrónicas . . . . . . . . . . . . . . . . . . . . 114

4.2. Ecuaciones iónicas para las diferentes especies químicas . . . . . . . . . . 125

4.3. Intensidad de las líneas corregidas por enrojecimiento para el Nudo A de II Zw 33128

4.4. Resultados de las Propiedades Físicas del gas ionizado para II Zw 33 A . . . . 130

4.5. Intensidad de las líneas corregidas por enrojecimiento para el Nudo B de II Zw 33132

4.6. Resultados de las Propiedades Físicas del gas ionizado para II Zw 33 B . . . . 134

4.7. Intensidad de las líneas corregidas por enrojecimiento para el Nudo C de II Zw 33135

4.8. Resultados de las Propiedades Físicas del gas ionizado para II Zw 33 C . . . . 137

4.9. Intensidad de las líneas corregidas por enrojecimiento para el Nudo A de Mrk 600143

4.10. Resultados de las Propiedades Físicas del gas ionizado para Mrk 600 A . . . . 145

4.11. Intensidad de las líneas corregidas por enrojecimiento para el Nudo B de Mrk 600146

4.12. Resultados de las Propiedades Físicas del gas ionizado para Mrk 600 B . . . . 147

4.13. Parámetros Empíricos . . . . . . . . . . . . . . . . . . . 162

4.14. Cocientes para obtener $\log \eta \mathrm{y} \log \eta \ldots \ldots \ldots \ldots$. . . . . . . . . 168

4.15. Propiedades generales deducidas a partir de observaciones de las Galaxias . . 176 



\section{Capítulo 1}

\section{Introducción}

\subsection{Aspectos generales de las Regiones HII}

Las Regiones HiI son objetos que ocupan inmensas zonas del espacio, están bien definidas y se observan principalmente en el disco de galaxias espirales e irregulares, donde en muchos casos son tan brillantes que dominan la emisión de la galaxia a la que pertenecen. Su nombre se debe a que sus espectros se ven caracterizados por la emisión de intensas líneas provenientes de la recombinación del hidrógeno ionizado. El flujo de energía que ioniza el gas circundante proviene principalmente de las estrellas masivas ubicadas, en general, en el centro de las Regiones Hiı (RHiı, de ahora en más), lo que genera estas hermosas e imponentes nebulosas, con una gran variedad de formas y tamaños. A través de estas estructuras, es posible estimar densidades y composiciones químicas del medio, la cantidad y naturaleza de las estrellas ionizantes y comprender la dinámica de los vientos estelares, entre otros (Sargent \& Searle, 1970; Searle \& Sargent, 1972; Thuan \& Martin, 1981). En la Figura 1.1 se presenta La Nebulosa del Corazón (del inglés: The Heart Nebula. IC 1805, Sh2-190) que es una nebulosa de emisión y una región de formación estelar de alrededor de 60 pc de diámetro. Está ubicada dentro del Brazo Perseo de nuestra galaxia, a una distancia de $2.3 \mathrm{kpc}$ en la constelación norte de Casiopea. Ambas estructuras observables en el centro y en la región inferior derecha de la imagen son parte de un complejo conjunto de regiones de formación estelar que se encuentran en el borde de una gran nube molecular. Puede observarse una mezcla de gas interestelar brillante y nubes de polvo oscuro. La nebulosa parece relativamente desprovista de estrellas debido a la oscuridad del polvo y su eficacia para absober radiación en el óptico. Sin embargo, la formación de estas nebulosas está asociada a los intensos vientos irradiados por las estrellas masivas.

Las temperaturas de las estrellas que ionizan el medio interestelar abarcan un rango amplio desde los $30.000 \mathrm{k}$ a los $150.000 \mathrm{k}$, aproximadamente. El límite inferior es el valor a partir del cual las estrellas producen campos de radiación con energías suficientes para ionizar el medio interestelar. El límite superior es muy difícil de determinar con certeza. Entre otras cosas, esta es una de las causas por la que estos objetos de estudio son tan importantes, tanto para la comprensión de la evolución estelar como para el estudio del medio y material interestelar en que estas estrellas se encuentran embebidas (Osterbrock \& Ferland, 2006). Las estrellas excitatrices en las RHiI pueden ser de baja masa (masas estelares $<5 \mathrm{M}_{\odot}$ ) pero que afectan volúmenes relativamente pequeños de la nube. Las estrellas masivas $\left(\geq 10 \mathrm{M}_{\odot}\right)$ generan campos de radiación y vientos mucho más intensos que las de baja masa, son capaces de ionizar el material atómico de la nebulosa circundante y generan así las regiones ionizadas 


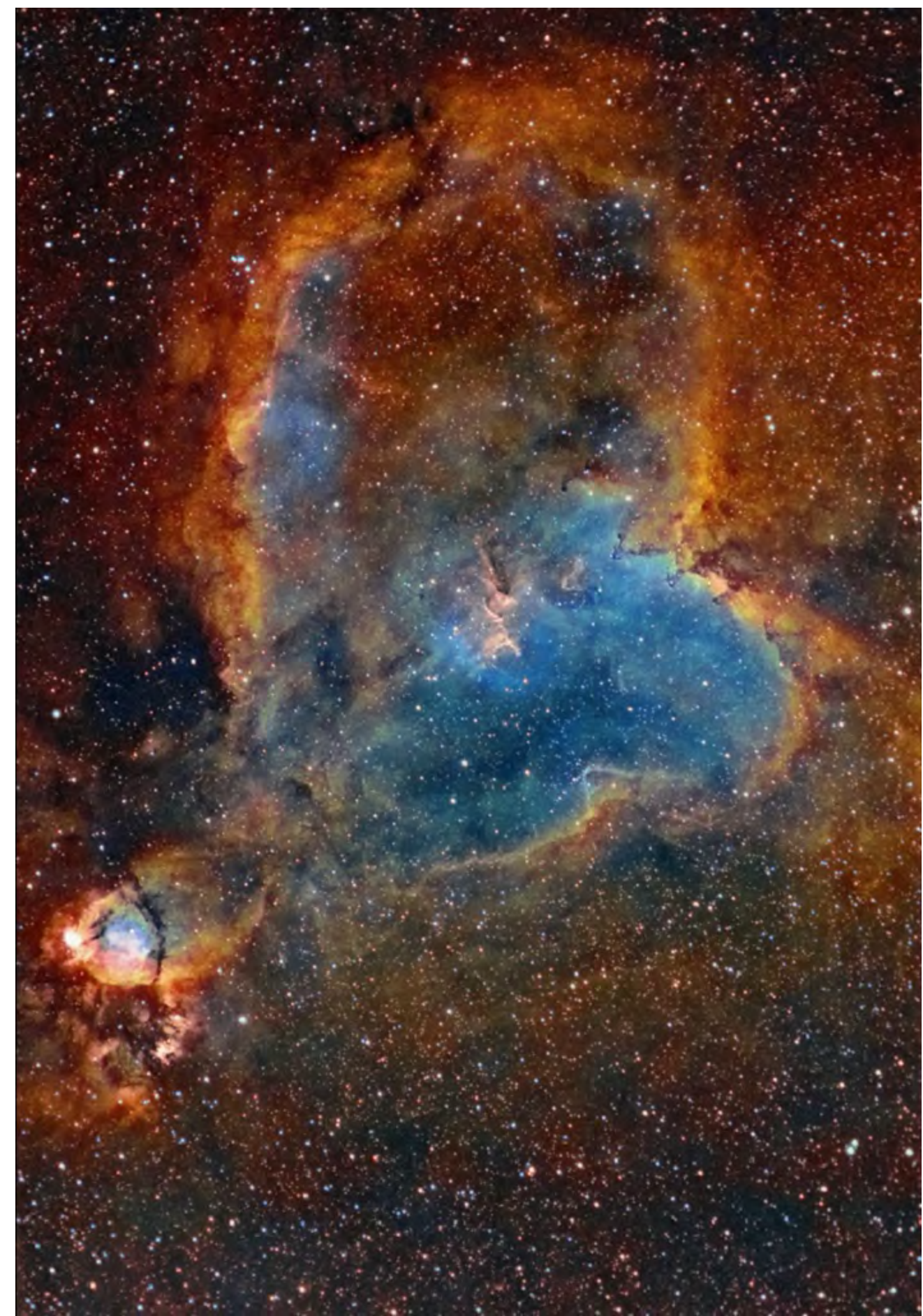

Figura 1.1. La Nebulosa del Corazón (del inglés: The Heart Nebula. IC 1805, Sh2-190). Nebulosa de emisión con varias estructuras en el centro y en la región inferior izquierda de la imagen con intensos brotes de formación estelar que se encuentran en el borde de una gran nube molecular. Fuente: Telescopio Espacial Hubble ${ }^{(\mathrm{i})}$. 
tan particulares y deslumbrantes que observamos en el Universo. Debido a los campos de radiación intensos que generan las estrellas masivas, un puñado de ellas son capaces de barrer el gas denso de las regiones de formación estelar y ionizar todo su material, exponiendo el cúmulo excitactriz recientemente formado.

Los rangos de temperaturas superficiales se extienden desde los $\mathrm{T}_{\text {eff }} 1 \times 10^{4} \mathrm{k}-5 \times 10^{4} \mathrm{k}$, luminosidades de $\approx 10^{4} \mathrm{~L}_{\odot}$ y masas entre 10 - $100 \mathrm{M}_{\odot}$. Los estudios sobre las estrellas masivas tuvieron una gran revolución a partir de las observaciones de los telescopios espaciales que permitieron observar la distribución de energía espectral que alcanza su máximo en longitudes de onda del ultravioleta, el cual es inaccesible utilizando los telescopios terrestres (ver Massey \& Olsen, 2003). La relación $\mathrm{N} \propto \mathrm{M}^{-2.35}$ dada por Salpeter (1955), indica que la cantidad de estrellas masivas que se esperan encontrar por intervalo de masas con respecto a las estrellas de tipo solar, es mucho menor. Los fuertes vientos de estas estrellas a lo largo de su evolución y hasta en la etapa final de supernova, enriquecen el medio interestelar (MIE) (Maeder, 1981; Abbott, 1982) y juegan un rol fundamental en él y en la evolución galáctica. Las estrellas más masivas conocidas en la actualidad son: Eta Carinae $\left(100-150 \mathrm{M}_{\odot}\right.$, van Genderen \& The, 1984), la estrella Pistol (100 - $150 \mathrm{M}_{\odot}$, Figer et al., 1998, de inglés "The Pistol Star") y LBV 1806-20 (130-200 $\mathrm{M}_{\odot}$, Figer et al., 2004).

Los espectros de las Regiones HiI, observados en el rango óptico, son característicos debido a que se detectan fuertes líneas en emisión correspondientes a diferentes elementos químicos, tanto en estados neutros como ionizados. Entre los más comúnes encontramos: el Hidrógeno y el Helio, el Nitrógeno, el Argón, el Neón, el Oxígeno, el Azufre, el Hierro y el Carbono. Las líneas de emisión observadas de dichas especies tienen su naturaleza en la desexcitación colisional y en la recombinación. Las transiciones prohibidas son críticas para la estimación de abundancias químicas, densidades y temperaturas del gas ionizado. Existen también líneas de recombinación débiles de, por ejemplo, Oxígeno y Carbono que también pueden ser utilizadas para la estimación de dichos parámetros. Para esto, es necesarios tener datos de una alta calidad, lo cual resulta muy costoso a nivel instrumental y de telescopio. Además, como las líneas en emisión aportan información muy valiosa sobre la cinemática del gas ionizado, se vuelven muy importantes en el análisis de la distribución espacial y velocidades de los iones emisores.

Dentro de las características del gas se estima que la temperatura electrónica representativa de estas regiones es $\sim 10^{4} \mathrm{k}$, y las densidades se encuentran en el rango de $10^{1}-10^{3}$ partículas por $\mathrm{cm}^{3}$, menores al límite de desexcitación colisional. Este valor indica densidades electrónicas bajas en comparación con regiones circumestelares o de nebulosas planetarias, que son varios órdenes de magnitud mayores.

Las RHir también se clasifican según sus estados evolutivos y/o tamaños. En nuestro trabajo nos centramos en las RHir Gigantes (RHiıG, de ahora en más) donde las líneas intensas espectrales presentan anchos supersónicos, y en las cuales pueden resolverse varias componentes (Bergvall \& Östlin, 2002). Este fenómeno se produce a partir de la ionización del gas a causa de los flujos de energía ultra-violeta de las estrellas masivas y jóvenes que barren con el material interestelar circundante (De Young \& Heckman, 1994).

En la Nube Mayor de Magallanes está la Región Gigante Hiı conocida como La Nebulosa de la Tarántula (o 30 Doradus), ha sido muy estudiada a lo largo de la historia. Particularmente, después de los años 60', esta RHII se ha transformado en centro de todo tipo de análisis por ser la más grande y activa región de formación estelar extragaláctica cercana, transformándose en un centro de estudios en todos los rangos de longitudes de onda para el análisis de poblaciones estelares, distribuciones de gas y polvo. A través de espectroscopía Fabry-Perot, Smith \& Weedman (1972) mapearon la emisión del [OIII] $\lambda 5007 \AA$ detectando grandes dispersiones de velocidades en las regiones más internas de la región, que asociaron 
a los fuertes vientos producidos por estrellas del tipo Wolf Rayet. El gas ionizado en dicha región fue estudiado por Chu \& Kennicutt (1994) y Melnick et al. (1999) en donde utilizaron espectroscopía de resolución baja-intermedia (long-slit) y espectroscopía de alta resolución (Echelle), respectivamente. La cinemática analizada por los primeros autores reveló un comportamiento complejo del gas ionizado, con diversas capas en expansión supersónicas que fueron asociadas a remanentes de supernovas. Por el lado del trabajo realizado por Melnick et al. (1999), la espectroscopía de alta resolución les permitió analizar los perfiles de las líneas de emisión de $\mathrm{H} \alpha$ en distintas subregiones de 30 Doradus. A través de los espectros de alta resolución del gas ionizado de las RHII, analizaron distintos mecanismos causantes de los ensanchamientos de las líneas espectrales. Los autores determinaron una componente ancha de muy baja intensidad que explicaba el comportamiento de las alas de las líneas de emisión de los perfiles integrados de 30 Doradus. Estos son solo algunos de los estudios que proporcionaron información útil sobre las dispersiones de velocidades supersónicas que le agregan la característica de Gigante a esta RHiı.

Por otro lado, los autores de Firpo et al. (2005) comprueba la naturaleza gigante de RHII en los brazos de las galaxias espirales NGC 2997 y NGC 7552 a partir de los ajustes de la cinemática con componentes supersónicas. Posteriormente, en los trabajos publicados por Firpo et al. (2010) y Firpo et al. (2011) (entre otros), se ampliaron los análisis para nuevas RHiı en las galaxias espirales NGC 6070 y NGC 7479 y en la galaxia Enana Compacta Azul Haro 15, en la que se estudiaron los brotes de formación estelar con mayor emisión en H $\alpha$.

\subsection{Galaxias Compactas Azules}

Las galaxias constituyen una pieza clave a la hora de analizar la distribución de materia del universo, a pequeña y gran escala. Dentro de las distinciones observacionales que podemos encontrar de manera directa, las galaxias presentan una gran variedad de formas que van desde las más simples (Esferoidales), a las más complejas estructuras y formas (Espirales Barradas), que permiten estimar el origen, formación y evolución estelar.

Para clasificar según su morfología a las galaxias, la Secuencia de Hubble es el sistema mayormente utilizado. Este esquema fue diseñado por Edwin Hubble (entre 1926 y 1936 Hubble, 1926, 1936) y describe una clasificación morfológica de las galaxias, dividiéndolas en tres tipos: Elípticas, Lenticulares y Espirales, basándose en la apariencia revelada en las placas fotográficas de la época. Hay una cuarta especie dentro de esta división conocidas como galaxias Irregulares, que muestran una estructura amorfa, grandes cantidades de polvo, gas y estrellas jóvenes. Este esquema no indica estados evolutivos de las galaxias, pero es una clasificación muy utilizada y útil para diferenciar a estos objetos.

En el marco de esta Tesis, vamos a enfocarnos en un tipo de galaxias enanas muy particulares debido a que sus espectros presentan características observacionales muy similares a Regiones Hir típicas. Dentro de la descripción morfológica, son enanas de baja masa con (al menos) un evento reciente de formación estelar violenta concentrada en unos pocos parsecs cercanos a sus núcleos, en donde los flujos provenientes de estas estrellas jóvenes y masivas son los que dominan la luz de esta subclase de galaxias enanas (Sargent \& Searle, 1970; Searle \& Sargent, 1972; Thuan \& Martin, 1981; Melnick et al., 1985b,a; Hägele et al., 2006, 2008).

Las galaxias enanas son las más numerosas en el universo (Marzke \& da Costa, 1997) y juegan un papel crucial en nuestra comprensión sobre la formación y evolución de las galaxias. Se considera una galaxia enana a aquella cuya magnitud absoluta en la banda $B$ es $\mathrm{M}_{B} \geq-18$ (ver por ejemplo Grebel et al., 2001), y son considerados como los "pilares fundamentales" en escenarios jerárquicos de la formación de galaxias (Mateo, 1998). Este es un análisis realizado desde un punto de vista cosmológico donde se predice que las primeras estrellas se formarían 
en subhalos de materia oscura, dando lugar a las primeras galaxias enanas, que más tarde se fusionarían y darían lugar a galaxias de mayor masa.

Las galaxias enanas de bajas masas son típicamente pobres en metales $(7.0 \leq 12+\log (\mathrm{O} / \mathrm{H})$ $\leq 8.6$ (Searle \& Sargent, 1972; Pagel, 1992; Izotov et al., 1994; Thuan \& Izotov, 1999; Kunth \& Östlin, 2000; Kniazev et al., 2004; Papaderos et al., 2008), indicando un estado poco evolucionado que las hace tener condiciones similares a galaxias a alto redshift (Amorín et al., 2017). El $95 \%$ de la población de galaxias que constituyen el Grupo Local, son galaxias enanas, y son utilizadas como base de estudio para revelar las propiedades físicas de las galaxias en el universo primitivo (Newton et al., 2018).

Además de su morfología, podemos separar (en una simplificación excesiva) a las enanas en dos tipos: enanas de tipo temprano ( $\mathrm{dE}, \mathrm{dSph}, \mathrm{dS} 0, \mathrm{cE}$ ) y de tipo irregular (dI, BCDs, etc). Las BCDs (Las Enanas Compactas Azules), del inglés: Blue Compact Dwarf, Enanas Elípticas (dEs, en inglés: dwarf Elliptical; ver Binggeli \& Popescu, 1995; Kunth \& Östlin, 2000, , entre otros), Enanas Irregulares (dIs, del inglés: dwarf irregular; ver Karachentsev et al., 2004). Las galaxias Elípticas y las Esferoidales presentan en general una distribución espacial esférica, bajo contenido de gas y son representativas de las galaxias enanas con poca (o prácticamente nula) actividad de formación estelar y bajo brillo. La población estelar que las compone es principalmente vieja, a pesar de que se observan estrellas tipo B, algo de gas y polvo. Su contenido de metales es bajo $\left(\frac{1}{30} \leq \frac{Z}{Z_{\odot}} \leq \frac{1}{3}\right)$ (ver Brinchmann \& Ellis, 2000; Grebel, 2000; Grebel et al., 2001; Amorín et al., 2009, y referencias en ellos). Estudios actuales sobre las propiedades físicas de este tipo de galaxias apuntan a que tuvieron que haber experimentado períodos largos de formación estelar para poder explicar sus poblaciones estelares. Generalmente se encuentran en cúmulos o grupos de galaxias, como ocurre con el Grupo Local, y la mayor parte de ellas poseen cúmulos globulares muy viejos, brillos superficiales $\mu_{V} \geq 21 \mathrm{mag} / \operatorname{arcseg}^{2}$, radio $\leq 4 \mathrm{kpc}$, Masas de $\mathrm{HI} \leq 10^{8} \mathrm{M}_{\odot}$. y masas totales $\leq$ $10^{9} \mathrm{M}_{\odot}$ (ver Amorín et al., 2009, y sus referencias en él).

Por el contrario, las galaxias dIs se caracterizan por un aspecto visual irregular, presentan bajo contenido de polvo y grandes depósitos de gas (Cairós et al., 2001). A las galaxias de esta clase no se las observa agrupadas entorno a galaxias masivas y en el caso del Grupo Local parecen estar distribuidas de forma aleatoria.

Son objetos de apariencia irregular, propia de galaxias tardías, con bastante cantidad de gas. Muestran evidencias de una formación estelar activa, cuya extensión espacial es del orden de los kpc y se estiman tasas de formación estelar (SFR, del ingl $\tilde{A} @$ Cs: star formation rate) moderadas (o bajas) (Kunth \& Östlin, 2000). Son sistemas con poco enriquecimiento químico, con un rango de metalicidades $\frac{1}{30} \leq \frac{Z}{Z_{\odot}} \leq \frac{1}{3}$, que aparentan haber experimentado una formación estelar aproximadamente continua a lo largo de un tiempo de Hubble $\left(\mathrm{t}_{H}\right.$ $=1 / \mathrm{H}_{0}$, donde $\mathrm{H}_{0}$ es la constante de Hubble) , y que por tanto habrían evolucionado relativamente despacio, especialmente aquellas de menor brillo (Bothun et al., 1991). Las primeras galaxias irregulares enanas en ser observadas fueron Las Nubes de Magallanes (MC, de su sigla en inglés Magellanic Clouds) ya que su cercanía a la Vía Láctea las hace observables a simple vista (Noeske et al., 1998; Martínez-Delgado et al., 2009). Las dI estudiadas en grupo local presentan características comunes: magnitud superficial $\mu_{V} \geq 23 \mathrm{mag} / \mathrm{arcseg}^{2}$, radios $\leq 5 \mathrm{kpc}$, masas de $\mathrm{Hi} \leq 10^{9} \mathrm{M}_{\odot}$ y masas totales $\leq 10^{10} \mathrm{M}_{\odot}($ Mateo, 1998; Grebel et al., 2001). En Tolstoy et al. (2009) se propone una relación evolutiva entre las BCDs, Id y las dEs, realizando ajustes entre las propiedades fotométricas tales como el perfil de brillo, color, y la estructura de estas galaxias enanas. Estos autores concluyen que existen relaciones entre los brillos centrales de estas galaxias y su estructura, lo que deja abierto el planteo de nuevas teorías sobre la evolución de las galaxias. 


\section{Introducción}

En esta Tesis nos centraremos en el estudio de las BCDs ${ }^{(i)}$. Estas galaxias no son de las más abundantes dentro de las enanas pero tienen ciertas propiedades típicas tales como que presentan grandes cantidades de gas (Zwicky \& Rudnicki, 1966; Gil de Paz et al., 2003b; Gil de Paz \& Madore, 2005), con fracciones de masa de Hi normalmente superiores al $30 \%$ de la masa total, su luminosidad está dominada por (al menos) un brote de formación estelar, con un aspecto muy similar a las RHIIG detectadas en galaxias masivas (ver por ejemplo, Hägele et al., 2006, y referencias en él). La gran mayoría está formando estrellas a un ritmo alto ( $>93 \%$, ver Gil de Paz et al., 2003a), que muestran una importante emisión de $\mathrm{H} \alpha$ $(E W \geq 20 \AA$ ). La tasa de formación estelar (SFR, del inglés star formation rate) derivada para estas galaxias alcanza hasta $10 \mathrm{M}_{\odot} \mathrm{yr}^{-1}$ (ver por ejemplo Fanelli et al., 1998; Gil de Paz et al., 2003a) y en algunos casos se registran valores mayores aún. Encontramos que las Green Peas (GPs Cardamone et al., 2009), galaxias que se encuentran a redshifts altos, presentan SFR mayores, pero estas tasas relativamente altas, combinado con altos contenidos de gas, implica que el consumo de gas se da en escalas de tiempo de $10^{9} \mathrm{yrs}$, que son mucho más cortas que la edad del universo.

Las BCDs son muy pobres en metales (Kunth \& Östlin, 2000; Hägele et al., 2006, 2008) y nos permiten estudiar la formación estelar y enriquecimiento químico en un entorno pobre en metales. Como tales, son importantes para nuestra comprensión de la formación y evolución de las galaxias. Además, en promedio presentan brillos superficiales $\mu_{V} \geq 19 \mathrm{mag} / \mathrm{arcseg}^{2}$ , radio $\leq 2 \mathrm{kpc}$, Masas $\mathrm{HI} \leq 5 \times 10^{9} \mathrm{M}_{\odot}$ y masas totales $\leq 10^{11} \mathrm{M}_{\odot}($ Gil de Paz \& Madore, 2005).

Las galaxias enanas con brotes de formación estelar intensos presentan espectros con líneas en emisión muy similares a los de las RHitG. Por lo tanto, es posible estudiar las propiedades de estas galaxias a través de su espectro mediante las mismas técnicas aplicadas para las mediciones en las RHıI, y así derivar por ejemplo las temperaturas, densidades electrónicas y composiciones químicas del gas interestelar de este tipo de galaxias. Este análisis lo detallaremos en las siguientes secciones al describir las propiedades físicas que estudiaremos a partir de las líneas de emisión intensas y aurorales (ver Hägele et al., 2006, 2008, 2012).

En ciertos casos es posible detectar en las galaxias BCDs poblaciones estelares de edades más avanzadas que tienen una distribución espacial más uniforme que las poblaciones de estrellas más jóvenes y brillantes asociadas a los grupos ionizantes. Estas poblaciones de estrellas más viejas producen un espectro característico de absorción que afecta, principalmente, a las líneas de emisión de recombinación de hidrógeno (ver discusión en Díaz, 1988; Guseva et al., 2006; Hägele et al., 2006), correspondientes a las series de Balmer y Paschen en el rango espectral que nosotros estudiamos.

Los estudios de las regiones de formación estelar presentes en estas galaxias han permitido indagar sobre los posibles mecanismos que desencadenan y activan a la formación estelar en ellas. Presentan índices de color $(B-V)$ que varían de 0 a 0.03 , lo que corresponde a estrellas de secuencia principal tipo $A$, indicando que esas galaxias están atravesando períodos de formación estelar abrupta con brotes muy marcados, resultando en colores más azules que otras galaxias ${ }^{(i i)}$.

En Hägele et al. (2011) se realiza un análisis espectral de distintas regiones de formación estelar en la galaxia BCD Haro 15 del cual se concluye que dicha galaxia tiene características más propias de las galaxias espirales. Con una magnitud absoluta entre -14 y -17 , con masas del orden de $10^{9} \mathrm{M}_{\odot}$ y con diámetros de unos pocos kpc, presentan una gran abundancia de gas y polvo con masas de $\mathrm{HI}$ del orden de $10^{8} \mathrm{M}_{\odot} \mathrm{y}$ de $\mathrm{HII} \approx 10^{6} \mathrm{M}_{\odot}$, representando alrededor

\footnotetext{
${ }^{(i)}$ En la literatura también pueden encontrarse como Galaxias HıI aunque no neceariamente todas las galaxias Hil son galaxias BCD, como se lo indica en Terlevich et al. (1999).

${ }^{(i i)}$ De ahí el término azul (de "blue" en inglés) en su nombre.
} 



Figura 1.2. Imágenes del SDSS (Sloan Digital Sky Survey) de una muestra de galaxias BCD presentadas en el artículo de James et al. (2015). 
del $20 \%$ de la masa total de la galaxia (Papaderos et al., 1999; Östlin et al., 2003; Gil de Paz et al., 2003b). Su aspecto denso, similar al de una estrella, determinado por las placas fotográficas de los años 60' les adjudicó el nombre de objetos compactos (Zwicky, 1965; Zwicky \& Rudnicki, 1966). En principio se especuló que las BCDs podían ser galaxias jóvenes (experimentando su primer episodio de formación estelar) debido a sus bajas metalicidades y a que la población estelar joven fue el único componente observado.

De las diversas teorías que existen sobre la formación de este tipo de galaxias, la mayormente aceptada es la que explica que las galaxias BCDs fueron formadas como consecuencia de interacciones y fusiones, y los resultados de una perturbación externa pueden explicar los espectros observados y la comprensión de estos objetos compactos. Pero el punto débil de esta teoría está en que se han encontrado muchas galaxias compactas aisladas, por lo que es difícil encontrar una fuente de perturbación externa que afecte la dinámica interna de la galaxia (Telles et al., 1997). De forma que, si es aislada, no tiene interacción y esta teoría no es del todo válida.

Una discusión abierta, que no tiene un acuerdo en la literatura aún, es establecer si el proceso de formación estelar que da origen a la actividad actual es producto de la actividad interna de la propia galaxia, como puede ser el colapso de nubes moleculares, vientos estelares, supernovas, etc; o si, por el contrario, es debido a un proceso externo como una posible interacción, colisión, o encuentro con alguna galaxia cercana.

Las BCDs se las encuentra tanto en el universo local como hacia corrimientos al rojo mayores $(0.001<\mathrm{z}<0.01$. Para mayor información ver Gil de Paz et al., 2003b; Gil de Paz \& Madore, 2005, y referencias en él) y son ellas las que nos permite realizar estudios sobre contenido de metales, poblaciones estelares y de los procesos de formación estelar en un entorno de bajas metalicidades, con una precisión que las galaxias con alto desplazamiento al rojo no nos permiten.

Las galaxias GPs que son similares a las de alto corrimiento al rojo ( $\mathrm{z} \sim 0.1-0.3$ ) y comparten una serie de propiedades con las BCDs (Amorín et al., 2017), que podemos relacionar resultados encontrados. Las GPs son Galaxias HiI y se consideran excelentes laboratorios para estudiar las propiedades físicas del gas ionizado y la dureza de los campos de radiación ionizantes en ambientes de baja metalicidad en zonas más distantes.

En líneas generales, el estudio de las galaxias enanas, y el de las BCDs en particular, es de interés debido a:

- Su baja metalicidad las convierte en excelentes laboratorios para estudiar la evolución química de galaxias (derivar abundancias, temperaturas y densidades electrónicas, etc) (Thuan \& Izotov, 1999).

- Permiten el análisis de procesos de formación estelar de estrellas masivas y de los procesos de "retroalimentación" del material interestelar a través de supernovas.

- Las explosiones de supernova y los vientos podrían marcar de manera drástica la evolución de las galaxias enanas debido a la energía mecánica que es transferida al material interestelar, que se calienta y puede cambiar las propiedades físicas y químicas del medio. Varios autores (Dekel \& Silk, 1986; De Young \& Gallagher, 1990; De Young \& Heckman, 1994, entre otros) proponen que las galaxias enanas con intensos episodios de formación estelar podrían estar sufriendo una pérdida sustancial de masa, conforme el medio interestelar es calentado y eyectado, dando lugar a supervientos galácticos. Este proceso, podría explicar el bajo contenido en metales y el bajo brillo superficial de las galaxias enanas. 
- Nos permiten realizar estudios sobre la Función Inicial de Masas (de sus siglas en inglés: IMF, Initial Mass Function), y sobre cómo ésta se puede ver alterada o modificada por los distintos procesos físico-químicos que se desarrollan a lo largo de la historia de formación estelar de la galaxia anfitriona. Sabemos que la función inicial de masas puede variar debido a:

- Procesos de colisión: pueden provocar variaciones en la función de masa aumentando la tasa de formación estelar.

- La baja metalicidad: la metalicidad actúa como agente enfriador, por lo que para metalicidades bajas, el enfriamiento es menos efectivo y puede variar favoreciendo el nacimiento de estrellas nuevas (Martínez-Delgado et al., 2009).

\subsection{Espectros de emisión en nebulosas gaseosas}

Si comparamos los espectros de nebulosas gaseosas con los estelares podemos encontrar una importante cantidad de diferencias en los niveles de energía del continuo, en los tipos y formas de líneas. Los espectros de estrellas presentan depresiones en su continuo a causa de que los fotones tienen que atravesar zonas de gas con una menor temperatura en su camino hacia el exterior de la atmósfera. Esto genera en los fotones una pérdida de energía y en consecuencia en los espectros encontramos líneas típicas en absorción. En algunas estrellas, como las $\mathrm{B}_{e}$, la existencia de una envoltura conduce a la presencia de líneas de emisión en el espectro (ver por ejemplo, Slettebak, 1979; Jaschek \& Egret, 1981; Zorec et al., 2004). Por el lado de las nebulosas gaseosas, en sus espectros dominan las líneas de emisión respecto al continuo, debido a distintas caracterísitas como la densidad, las abundancias, las temperaturas y los estados ionizados de los elementos que componen el gas. El mecanismo mediante el cual el gas se ve excitado es producto de la presencia cercana de una o varias estrellas calientes que irradian energía excitando los átomos de los elementos químicos existentes en su fase gaseosa, los cuales emiten radiación en ciertas frecuencias, que constituyen los espectros de emisión.

Las nebulosas gaseosas presentan líneas en emisión que corresponden a iones de distintos elementos químicos. Entre las más importantes dentro del rango óptico del espectro electromagnético podemos encontrar el doblete del [OIII] en $\lambda 4959 \AA$ y $\lambda 5007 \AA$, las cuales fueron las primeras líneas nebulares medidas y que dieron lugar a la discusión sobre el nuevo elemento: el Nebulio (ver por ejemplo, Nicholson, 1911). Estas líneas se observaban en las nebuosas pero no correspondían a ningún elemento conocido hasta ese momento, y cuya existencia planteaba inconsistencias relacionadas con el número atómico y el peso atómico del mismo. Posteriormente, Bowen $(1927,1928)$ determinaron que este doblete correspondía a transiciones prohibidas del [OIII]. En un átomo los electrones pueden encontrarse solo en ciertos niveles de energía (diferentes para cada átomo), y un electrón puede saltar a un nivel superior si absorbe la diferencia de energía o a un inferior si la emite. Pero estos saltos no pueden ser de cualquier manera, sino que los hace siguiendo las reglas de selección, que permite que se den unas transiciones y otras no. De esta manera, si una transición no cumple las reglas de selección definidas por el dipolo, se dice que es una transición prohibida. Por lo que a medida que se fueron descubriendo e interpretando las condiciones necesarias para poblar los niveles atómicos, resultó que el Nebulio no era otra cosa más que el oxígeno dos veces ionizado que no se encuentra en la Tierra, pero dadas las condiciones físicas del medio sí se puede encontrar en las nebulosas.

Hacia la zona más azul del espectro encontramos las líneas del doblete del [OII] en $3726 \AA$ y $3729 \AA$, las cuales se observan como una sola línea en los espectros de baja resolución. Estas líneas aportan información sobre las características del medio a partir de la cual se 
pueden estimar densidades electrónicas del gas ionizado, como describiremos en las siguientes secciones. Hacia la parte más roja del espectro óptico, es decir, más allá de los $\lambda 6500 \AA$, encontramos a las líneas del doblete del [Sir] en $6717 \AA$ y $6731 \AA$. Al igual que las líneas del doblete del [OII] en el azul, son muy importantes para la determinación de las densidades electrónicas.

Se encuentran también líneas de interés para estudios físicos y químicos de más especies, como las de [SIII] $\lambda \lambda$ 9069, 9532 mos las líneas de recombinación de la serie de Balmer del Hidrógeno: $\mathrm{H} \alpha \lambda 6563 \AA \mathrm{H} \beta \lambda 4861 \AA$ $\mathrm{H} \gamma \lambda 4340 \AA$ y $\mathrm{H} \delta \lambda 4100 \AA^{(\mathrm{i})}$. Estas son fundamentales en el estudio de la extinción causada por el polvo y del enrojecimiento en las observaciones en general. Otras líneas de recombinación de interés, y características de estas regiones, son las líneas del HeI, en particular $5876 \AA$, que son considerablemente más débiles que las líneas del hidrógeno. En las nebulosas con alto grado de ionización, es posible observar a la línea débil del HeII $\lambda 4686 \AA$, debido a que la fuente excitatríz con un flujo ionizante muy energético alcanza a ionizar al HeII.

Como objetos con espectros dominados por líneas en emisión podemos mencionar a las RHir típicas de los brazos de galaxias espirales (por ej. la Nebulosa de Orión), a RHir Gigantes observadas en otras galaxias (por ejemplo, 30 Doradus en la Nube Menor de Magallanes), las Galaxias Hir, las galaxias con altas SFR (Starbursts Galaxies), así como a nebulosas planetarias. La diferencia más notoria con estas últimas, es que la fuente central es una enana blanca con $\mathrm{T}_{\text {eff }} \approx 100.000 \mathrm{k}$ (estrella o sistema binario) y la dureza del campo de radiación es mayor que en las antes mencionadas, proporcionada por fotones duros del UV (ver por ejemplo, Schönberner \& Blöcker, 1996). Como la ionización es más alta, pueden encontrarse átomos ionizados de otros elementos que en las RHII no encontramos habitualmente, como [FeVII], [Nev], distintos iones del carbono, líneas de recombinación del O, entre otros (ver por ejemplo, Peimbert, 1978; Aller \& Czyzak, 1983). Otra característica central que diferencia a las regiones de gas son las densidades electrónicas, que en las nebulosas planetarias pueden llegar a ser varios órdenes de magnitud mayor que en RHII (Osterbrock \& Ferland, 2006). En la Figura 1.3 se muestra la diferencia de tamaños entre una region Hiı galáctica y una RHıI gigantes extragalácticas (van der Werf \& Snijders, 2006). Nuestra galaxia no presenta RHiIG observadas y en otras galaxias uno puede encontrar RHiıG y RHi clásicas también.

Los espectros de nebulosas presentan un continuo débil formado por las componentes debido a la reflexión y componentes atómicas neutras y ionizadas. La primera consiste en la luz estelar dispersada por el polvo, de manera que dependiendo de las cantidades de polvo la intensidad del continuo varía. En el rango espectral óptico del espectro electromagnético el continuo atómico se emite fundamentalmente por transiciones libre-ligado (Osterbrock \& Ferland, 2006), en el continuo de Paschen del Hi a longitudes de onda menores a $8245 \AA$, en el extremo rojo, y el continuo de Balmer a $\lambda<3648 \AA$, en el rango UV. Por otro lado, en el espectro IR, el continuo nebular corresponde en gran parte a radiación térmica emitida por el polvo, que se calienta al absorber la radiación estelar, y reemite la radiación a longitudes de onda más largas.

\subsubsection{Equilibrio de fotoionización}

Para poder interpretar los espectros observados de nebulosas fotoionizadas, es necesario comprender algunos de los procesos físicos que tienen lugar en ellas. Como mencionamos previamente, la radiación UV de las estrellas en las nebulosas de emisión proveen el campo de radiación que calienta, excita e ioniza al medio. La energía de la fuente excitatríz (estrellas o cúmulos estelares) es transferida al medio y a la nebulosa a través de los fotones UVs que

\footnotetext{
${ }^{(i)}$ Las longitudes de onda indicadas son para los iones en reposo. Ver García-Rojas et al. (2004).
} 


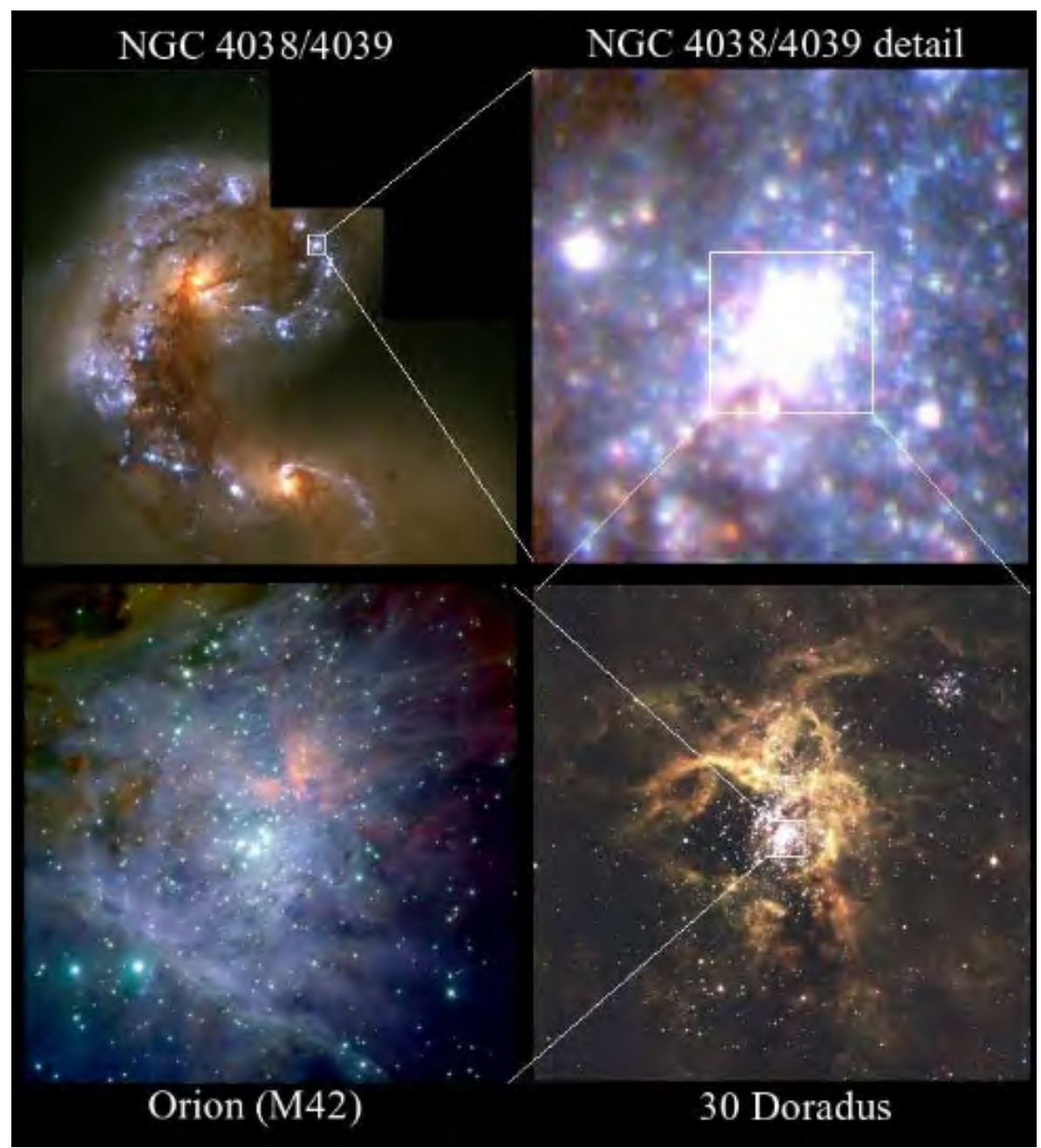

Figura 1.3. Montaje que muestra los tamaños relativos de RHiı. La Nebulosa de Orión presenta una extensión de 2 pc y percenece a la Vía Láctea; 30 Doradus $\approx 380$ pc perteneciente a una de las galaxias satélite de la Vía Láctea, la Nube Mayor de Magallanes; para una galaxia con formación estelar intensa, NCG 4038 en la imagen, una región como 30 Dorados corresponde a una de los brotes intensos de sus brazos. Es decir que en las galaxias starburst están compuestas por una gran cantidad de regiones gigantes HiI entre otras RHıI clásicas (imagen extraída de van der Werf \& Snijders, 2006). 
fotoionizan a los átomos de distintos elementos presentes en el medio en que las estrellas están embebidas. El hidrógeno es, por mucho, el elemento más abundante del cosmos. Compone en un $90 \%$ al medio interestelar, y su energía de ionización es relativamente baja $(13.6 \mathrm{eV}$, ver Osterbrock \& Ferland, 2006) de manera que los fotones energéticos de las estrellas se encargan de transferir su energía al gas excitando y ionizando al $\mathrm{H}$ neutro presente. Este es uno de los principales mecanismos de inyección de energía a las nebulosas, que en un diagrama diagnóstico, como por ejemplo los BPT (Baldwin et al., 1981; Kewley et al., 2001; Kauffmann et al., 2003; Kewley et al., 2006) se clasifican como objeto tipo HiI.

En las nebulosas gaseosas fotoionizadas el flujo de fotones ionizantes emitidos por la fuente determina la tasa de fotoionización para unas dadas condiciones de densidad, temperatura y abundancias químicas. Si suponemos una nebulosa de $\mathrm{H}$ puro, solo la radiación con $\nu>\nu_{0}$ (se define a $\nu_{0}$ la frecuencia umbral de fotoionización del $\mathrm{H}$ ) es efectiva a la hora de fotoionizar el $\mathrm{H}$ desde el nivel fundamental. En condiciones estacionarias, el número de fotoionizaciones alcanza el equilibrio con las recombinaciones, y lo podemos representar para cada punto de la nebulosa de la siguiente manera:

$$
\mathrm{n}\left(\mathrm{H}^{0}\right) \int_{\nu_{0}}^{\infty} \frac{4 \pi \mathrm{J}_{\nu}}{\mathrm{h} \nu} \mathrm{a}_{\nu}\left(\mathrm{H}^{0}\right) d_{\nu}=\mathrm{n}_{p} \mathrm{n}_{e} \alpha\left(\mathrm{H}^{0}, \mathrm{~T}_{\mathrm{e}}\right),
$$

en donde $\mathrm{J}_{\nu}$ es la intensidad media de la radiación a una frecuencia dada en cada punto, $\mathrm{a}_{\nu}$ es la sección eficaz de fotoionización del $\mathrm{H}$ para fotones con energía h $\nu$ superior al umbral de ionización, $\mathrm{n}\left(\mathrm{H}^{0}\right), \mathrm{n}_{p}$ y $\mathrm{n}_{e}$ son las densidades de $\mathrm{H}^{0}$, protones $\left(\mathrm{H}^{+}\right)$y electrones $\left(\mathrm{e}^{-}\right), \mathrm{y}$ $\alpha\left(\mathrm{H}^{0}, \mathrm{~T}_{e}\right)$ es el coeficiente de recombinación a una dada temperatura electrónica $\mathrm{T}_{e}$. La parte izquierda de la ecuación indica la tasa de fotoionizaciones por unidad de volumen y tiempo (en unidades de $\mathrm{cm}^{-3} \mathrm{~s}^{-1}$ ), mientras que la derecha describe la tasa de recombinaciones por unidad de volumen y tiempo.

Si consideramos una situación más real, en donde además del H, tenemos He y elementos pesados (como O, N, S, Ar, Ne, etc.) la tasa de ionizaciones depende de la presencia de estos otros elementos que compiten con el $\mathrm{H}$ en la absorción de los fotones ionizantes. Para refinar el cálculo del equilibrio de fotoionización resulta fundamental tener en cuenta otros procesos como los efectos del campo de radiación difuso (propio de la emisión del gas ionizado), la recombinación dielectrónica o las reacciones de transferencia de carga (ver Osterbrock \& Ferland, 2006). En un caso ideal, en el que no existiera acoplamiento en las estructuras de ionización, la expresión de la ecuación de equilibrio de fotoionización para dos estados sucesivos de ionización $(+\mathrm{i}$ y $(+\mathrm{i}+1))$ de un elemento cualquiera, $\mathrm{X}$, se puede aproximar por:

$$
\left(\mathrm{X}^{+i}\right) \int_{\nu_{i}}^{\infty} \frac{4 \pi \mathrm{J}_{\nu}}{\mathrm{h} \nu} \mathrm{a}_{\nu}\left(\mathrm{X}^{+i}\right) \mathrm{d}_{\nu}=\mathrm{n}\left(\mathrm{X}^{+i+1}\right) \mathrm{n}_{e} \alpha_{\mathrm{G}}\left(\mathrm{X}^{+i}, \mathrm{~T}_{\mathrm{e}}\right)
$$

donde $\mathrm{n}\left(\mathrm{X}^{+i+1}\right)$ es la densidad (proporcional a la abundancia) del estado de ionización $\mathrm{i}+1$ de dicho elemento $\mathrm{X} ; \mathrm{a}_{\nu}\left(\mathrm{X}^{+i}\right)$ es la sección eficaz de fotoionización desde el nivel fundamental del ión $\mathrm{X}^{+i}$, con energía umbral de fotoionización $\mathrm{h} \nu_{i}$; y $\alpha_{G}\left(\mathrm{X}^{+i}, \mathrm{~T}_{e}\right)$ es el coeficiente de recombinación del nivel fundamental de $\mathrm{X}^{+i+1}$ a todos los niveles de $\mathrm{X}^{+i}$. De esta manera podemos determinar el equilibrio de ionización para cada punto, teniendo en cuenta: i) las ecuaciones mencionadas y ii) el número total de iones de todos los estados de ionización:

$$
\sum_{i=0}^{\infty} \mathrm{X}^{+i}=\mathrm{n}(\mathrm{X}) .
$$

Como comentamos previamente, la interacción del flujo de radiación ionizante con el gas de una nebulosa origina un espectro de líneas de emisión, donde las relaciones de las líneas están definidas por la composición química del gas, la forma del continuo ionizante y la 
distribución geométrica del gas. Estos factores son determinantes para la estudiar el grado de ionización del gas de la nebulosa y temperaturas del campo de radiación de las fuentes excitatrices (Hägele et al., 2006, 2008). Podemos definir en general la intensidad de una línea de emisión observada como:

$$
\mathrm{I}_{\lambda}=\int \mathrm{j}_{\lambda} \mathrm{ds}=\int \mathrm{n}_{i} \mathrm{n}_{e} \epsilon_{\lambda}\left(\mathrm{T}_{e}\right) \mathrm{ds}
$$

donde $\epsilon_{\lambda}\left(\mathrm{T}_{e}\right)$ es el coeficiente de emision de la línea a una dada $\mathrm{T}_{e}, \mathrm{n}_{i} \mathrm{y} \mathrm{n}_{e}$ son la densidad del ión responsable de la emisión y la densidad electrónica, respectivamente.

Las líneas observadas las vamos a clasificar en dos tipos dependiendo de su origen: líneas permitidas (que son de recombinación o de fluorescencia) y líneas prohibidas (originadas por excitación colisional).

\subsubsection{Líneas de recombinación}

Son el resultado de que los iones recapturen a los electrones termalizados. El grado de ionización en cada punto de la nebulosa viene dado por el equilibrio entre las fotoionizaciones y las recombinaciones (ver ec. 1.2). En este proceso de recombinación los electrones son recapturados a niveles excitados dando lugar a un átomo excitado. Dicho átomo decae a niveles cada vez más bajos mediante transiciones radiativas finalizando eventualmente en el nivel fundamental, generando en cada uno de estos decaimientos radiativos la emisión de una línea.

Se define emisividad de una línea de recombinación (LR) a la magnitud que depende de la diferencia de energía entre los niveles involucrados en la transición, y del producto entre la densidad electrónica y la densidad del ión que al recombinarse va a dar origen a la línea. La expresión de la emisividad viene dada por:

$$
\mathrm{j}_{\lambda}=\mathrm{j}_{n n^{\prime}}=\frac{\mathrm{h} \nu_{n n^{\prime}}}{4 \pi} \mathrm{n}\left(\mathrm{X}^{+p+1}\right) \mathrm{n}_{e} \alpha_{n L, n^{\prime} L^{\prime}}^{e f f}
$$

donde el coeficiente de recombinación efectiva, $\alpha$, indica la posibilidad de que un electrón del nivel $\mathrm{nL}$ decaiga en cascada hasta el nivel n' $\mathrm{L}$ ' teniendo en cuenta todos los caminos posibles. Finalmente, la emisividad de una LR se puede expresar en función de la población de los niveles $\mathrm{nL}$ y sus posibilidades de transiciones $\mathrm{A}_{n L, n^{\prime} L^{\prime}}$ de un nivel a otro:

$$
\mathrm{j}_{\lambda}=\mathrm{j}_{n n^{\prime}}=\frac{\mathrm{h} \nu_{n n^{\prime}}}{4 \pi} \mathrm{N}\left({ }_{n L}\right) \mathrm{A}_{n L, n^{\prime} L^{\prime}}
$$

(ver Osterbrock \& Ferland, 2006). Si trabajamos en equilibrio termodinámico la población de los niveles se determina resolviendo la ecuación de Boltzmann y así es posible determinar la emisividad de una forma sencilla.

Para el caso de nebulosas fotoionizadas, encontramos que si tomamos una densidad característica de $\mathrm{n}_{e}<10^{3} \mathrm{~cm}^{-3}$, en su mayoría, los niveles atómicos no estarán en equilibrio termodinámico. Para estos casos hay que resolver los decaimientos en cascada que permiten determinar las poblaciones de todos los niveles en simultáneo (a través de una matriz compleja). En estos procesos se emiten fotones que dan origen a líneas de recombinación de $\mathrm{H}$, por ejemplo de Paschen y Balmer. Estas series de hidrógeno neutro son observadas en todas las nebulosas con gas fotoionizado. También se detectan líneas colisionales y de recombinación (más difíciles) deotros elementos como el $\mathrm{S}, \mathrm{O}, \mathrm{N}$ y C, entre los más significativos. 


\subsubsection{Líneas prohibidas o de excitación colisional}

Las colisiones entre electrones libres y los iones pueden excitar los niveles de energía más bajos de dichos iones, principalmente los de elementos pesados, más allá del Helio. La nebulosa genera un espectro con líneas conocidas como prohibidas, que como mencionamos anteriormente se las denomina así porque no siguen las reglas de selección de la mecánica cuántica para la radiación de dipolo eléctrico, y que son muy difícil de simular en condiciones de laboratorio. Las transiciones radiativas desde los niveles metaestables hacia subniveles inferiores tienen una probabilidad de transición muy pequeña en ambientes de bajas densidades $\left(\mathrm{n}_{e}<10^{3} \mathrm{~cm}^{-3}\right)$.

La emisividad de una línea excitada colisionalmente se puede determinar conociendo la población del nivel de partida, por lo que hay que tener en cuenta tanto los procesos que pueblan como los que los despueblan. Este balance entre los diferentes procesos que pueden modificar el estado energético de un sistema (átomo, molécula o ión) se debe tanto a los procesos radiativos como a los procesos colisionales. Se necesita resolver la ecuación de Equilibrio Estadístico para obtener la población de los niveles. Esta viene dada por la expresión:

$$
\mathrm{N}_{j} \mathrm{n}_{e} \mathrm{q}_{j i}+\mathrm{N}_{j} \mathrm{~A}_{j i}+\mathrm{N}_{j} \mathrm{~B}_{j i} I_{\nu}=\mathrm{N}_{i} \mathrm{n}_{e} \mathrm{q}_{i j}+\mathrm{N}_{i} \mathrm{~A}_{i j}+\mathrm{N}_{i} \mathrm{~B}_{i j} \mathrm{I}_{\nu}
$$

donde $\mathrm{j}>\mathrm{i}$ y en el término de la izquierda aparecen recopiladas las transiciones que provocan desexcitaciones y del derecho las que provocan excitaciones. Tenemos que $\mathrm{N}_{i}$ es la población

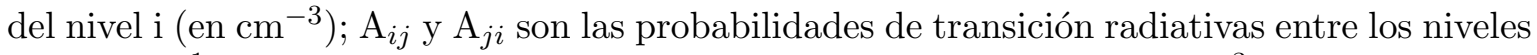
i y j $\left(\right.$ en s$\left.^{-1}\right)$; y $\mathrm{n}_{e}$ corresponde es la densidad electrónica del gas $\left(\mathrm{en}^{-3}\right)$, donde $\mathrm{q}_{j i}$ y $\mathrm{q}_{i j}$ corresponden a los coeficientes de desexcitación y excitación colisional respectivamente (en unidades de $\mathrm{cm}^{3} \mathrm{~s}^{-1}$ ) y la probabilidad de las transiciones dependen del campo de radiación incidente $\mathrm{I}_{\nu}$ y del coeficiente de absorción de Einstein $\mathrm{B}_{i j}$ (en unidades de $\mathrm{erg}^{-1} \mathrm{~cm}^{2} \mathrm{~s} \mathrm{~Hz}$ ).

Para entender estos mecanismos es común recurrir a la aproximación de un átomo con solo dos niveles energéticos 1 y 2 , siendo n1 y n2 las poblaciones del estado inferior y superior, respectivamente.

- Transiciones Radiativas: cuando la transición entre los niveles involucra un fotón de energía: $\Delta \mathrm{E}=\mathrm{E}_{2}-\mathrm{E}_{1}$. Se describen tres tipos:

- Desexcitacion espontánea de un electrón desde el nivel 2 al 1 emitiendo un fotón con energía $E_{2}-E_{1}$. La probabilidad de transición por unidad de tiempo se define como el coeficiente de Einstein de emisión espontánea, $\mathrm{A}_{21}$ ( $\mathrm{o}_{\mathrm{A}}$ ) (en unidades de $\mathrm{s}^{-1}$ ), cuyo inverso es el tiempo de vida de la partícula en el nivel superior. $\mathrm{Su}$ contribución es el término: $\mathrm{N}_{j i} \mathrm{~A}_{j i}=\mathrm{N}_{i j} \mathrm{~A}_{i j}$.

- Absorción de un fotón de energía $\mathrm{E}_{2}-\mathrm{E}_{1}$ que provoca la excitación de un electrón del nivel 1 al 2. La probabilidad de la transición depende del campo de radiación incidente $\left(\mathrm{I}_{n u}\right)$, siendo la constante de proporcionalidad el coeficiente de Einstein de absorción, $\mathrm{B}_{12}\left(\mathrm{erg}^{-1} \mathrm{~cm}^{2}\right.$ seg Hz), y su contribución es: $\mathrm{N}_{i} \mathrm{~B}_{i j} \mathrm{I}_{\nu}$.

- Emisión estimulada o desexcitación de un electrón del nivel 2 al 1 inducida por un fotón incidente y acompañada de la emisión de otro fotón. Se considera una absorción inversa con probabilidad dada por el coeficiente de Einstein de emisión estimulada, $\mathrm{B}_{21}$. Su contribución corresponde al: $\mathrm{N}_{j} \mathrm{~B}_{j i} \mathrm{I}_{\nu}$.

- Transiciones colisionales: el choque del átomo con una partícula externa provoca una excitación del nivel 1 al 2 (el sistema gana parte de la energía cinética de la partícula) o una desexcitación del nivel 2 al 1 (el sistema cede energía a la partícula). La probabilidad 
de transición colisional se define como: $\mathrm{C}_{12}=\mathrm{n}_{e} \cdot \mathrm{q}_{12}$, para el caso de excitaciones y $\mathrm{C}_{21}$ para las desexitaciones. Su constribución corresponde a: $\mathrm{N}_{j} \mathrm{n}_{e} \mathrm{q}_{j i}$ y $\mathrm{N}_{i} \mathrm{n}_{e} \mathrm{q}_{i j}$.

Si reescribimos la ecuación 1.6:

$$
\mathrm{N}_{2} \mathrm{n}_{e} \mathrm{q}_{21}+\mathrm{N}_{2} \mathrm{~A}_{21}+\mathrm{N}_{2} \mathrm{~B}_{21} \mathrm{I}_{\nu}=\mathrm{N}_{1} \mathrm{n}_{e} \mathrm{q}_{12}+\mathrm{N}_{1} \mathrm{~A}_{12}+\mathrm{N}_{1} \mathrm{~B}_{12} \mathrm{I}_{\nu},
$$

vemos que del lado derecho nos quedan los procesos debido a: emisión inducida, estimulada y desexcitación colisional, y del lado izquierdo: absorción y excitación colisional. Para una RHiı típica en donde el medio está muy diluído, se puede considerar que las colisiones se producen solo con electrones, y además el proceso más importante es la emisión espontánea y podemos considerar $\mathrm{B}_{12} \approx \mathrm{B}_{12} \approx 0$. Suponiendo esto, la expresión de la 1.7 queda simplificada a:

$$
\mathrm{N}_{2} \mathrm{n}_{e} \mathrm{q}_{21}+\mathrm{N}_{2} \mathrm{~A}_{21}=\mathrm{N}_{1} \mathrm{n}_{e} \mathrm{q}_{12}+\mathrm{N}_{1} \mathrm{~A}_{12} .
$$

Para analizar que tipos de transiciones son las que dominan, se estudia la densidad crítica definida como: $\mathrm{n}_{\text {crit }}=\mathrm{A}_{21} / \mathrm{q}_{21}$. Si reescribimos la expresión 1.8 como:

$$
\frac{\mathrm{N}_{2}}{\mathrm{~N}_{1}}=\frac{\mathrm{n}_{e} \mathrm{q}_{12}}{\mathrm{n}_{e} \mathrm{q}_{21}+\mathrm{A}_{21}}=\frac{\mathrm{n}_{e} \mathrm{q}_{12} / \mathrm{A}_{21}}{\mathrm{n}_{e}+\mathrm{n}_{\text {crit }}},
$$

podemos obtener 2 situaciones límites en función de la densidad electrónica del medio:

- Si la densidad del medio es alta $\left(\mathrm{n}_{e}>\mathrm{n}_{\text {crit }}\right)$ deducimos que el cociente $\mathrm{n}_{2} / \mathrm{n}_{1}$ tiende a la razón de los coeficientes de Einstein $\left(\mathrm{q}_{12} / \mathrm{q}_{21}\right)$ y las transiciones colisionales dominan por sobre las radiativas. Este es el caso conocido como una región termalizada dado que la temperatura de excitación coincide con la temperatura cinética del electrón.

- Si la densidad del medio es baja $\left(\mathrm{n}_{e}<<\mathrm{n}_{\text {crit }}\right)$ se obtiene: $\frac{\mathrm{q}_{12} \cdot q_{21}}{n_{\text {crit }}}$. Esto indica que los niveles dependen del coeficiente $\mathrm{A}_{21}$ y que dominan las transiciones radiativas.

Las transiciones que dominan en las RHII son las radiativas, caracterizadas por dos procesos para regiones de bajas densidades en los que un electrón en estado excitado puede regresar al nivel fundamental:

I - Líneas permitidas: producidas por interacciones dipolares eléctricas con valores de $\mathrm{A}_{21}$ altos, de manera que el tiempo de vida en el estado superior es corto.

II - Líneas prohibidas: producidas por interacciones cuadrupolares eléctricas y dipolares magnéticas en que los tiempos de vida $\mathrm{A}_{21}$ son mucho más altos $(\approx 400$ años) y generan las líneas prohibidas.

Observar líneas prohibidas en emisión de distintos elementos como [OII] y [OIII], [NII], [SII] y [SIII], es muy importante en el contexto de las RHir. A pesar de que la abundancia relativa de dichos iones es mucho menor que la del abundancia del $\mathrm{H}$ o del $\mathrm{He}$, las condiciones físicas extremas alcanzadas en el gas ionizado permiten la exhibición de estos metales. Las colisiones entre iones y electrones termalizados excitan los niveles de baja energía de los iones, lo cual produce las transiciones de electrones hacia niveles superiores. Las transiciones radiativas de estos niveles excitados tienen una probabilidad de transición muy pequeña, pero en las bajas densidades electrónicas de las RHII, la desexcitación colisional es muy poco probable y 
prácticamente cada excitación entre niveles electrónicos viene acompañada de la emisión de un fotón.

Los espectros con líneas prohibidas, generados en las nebulosas gaseosas, son muy difÃciles de reproducir en el laboratorio por causa de las bajas densidades electrónicas necesarias para crear estos fenómenos. A continuación veremos cómo se pueden estimar parámetros físicos del gas ionizado por medio de relaciones entre cocientes de líneas intensas y líneas débiles, cocientes de líneas emitidas desde niveles con potenciales de ionización similares. Pero antes me parece importante mencionar que la ecuación del Equilibrio Estadístico (E.E.) puede explicarse con un modelo sencillo de dos niveles, pero como depende de los iones y la mayoría tiene más niveles que dan lugar a un mayor número de transiciones radiativas y colisionales, hay que resolver la ecuación. de E.E. generalizada (1.6), a la que se le agrega la Ec. de Cierre $\left(\mathrm{N}=\sum_{j}^{n} \mathrm{~N}_{j}\right)$, donde $\mathrm{N}$ representa el número total de iones, obteniendo la población de cada nivel. Este es un complejo sistema de ecuaciones que suele resolverse adoptando distintas aproximaciones (ver Osterbrock \& Ferland, 2006). Sin embargo, teniendo en cuenta que en el análisis de nebulosas gaseosas la mayoría de los iones presentan una estructura sencilla de cinco niveles de baja energía, es posible utilizar una aproximación que considara únicamente esos cinco niveles para resolver las ecuaciones de E.E.

Un código muy utilizado es IONIC (ver Pagel, 1992) del software para reducción de imágenes y datos astronómicos IRAF que utiliza el átomo de 5 niveles como aproximación. El modelo de átomo de 5 niveles es válido para átomos ligeros. La mayoría de los iones que intervienen en los mecanismos de enfriamiento de una nebulosa, y cuyas líneas son observables (por ejemplo, $\mathrm{O}^{++}, \mathrm{N}^{+}$), presentan configuraciones electrónicas en el estado fundamental $\mathrm{p} 2$, p3 o p4, las cuales contienen 5 niveles de baja energía. Five-level (Robertis, 1986, FIVEL), que fue el programa que dio lugar al nebular en IRAF, supone que estos 5 niveles son los físicamente relevante para el problema del cálculo del espectro de emisión observado, dado que los niveles más altos de estos iones no han sido poblados significativamente por colisiones u otros mecanismos (por ejemplo, fluorescencia) (ver Robertis, 1986; Shaw \& Dufour, 1995).

\subsection{Abundancias quimicas y parámetros físicos}

Hemos indicado los procesos de formación de las líneas y veremos las importancia de ciertas líneas en la determinación de las condiciones físicas del gas ionizado. Como la emisividad de las líneas tiene una dependencia con la densidad y la temperatura electrónica, al determinar cocientes entre líneas estos serán funciones de ambos parámetros. Las línea tienen dependencias distintas con las densidades y temperaturas electrónicas, tal que para cocientes de líneas en donde la dependencia entre densidades o temperaturas sea similar, este será prácticamente independiente de esa y sensible a la otra. En la siguiente sección describiremos muy brevemente los distintos métodos usados habitualmente para determinar las condiciones físicas de las regiones fotoionizadas que son importantes para nuestro análisis.

\subsubsection{Abundancias químicas}

Dado que la estimación de los parámetros físicos y las abundancias químicas es uno de los objetivos de esta Tesis, explicar los lineamientos generales y las propiedades físicas utilizadas es conveniente para una mejor interpretación del tema.

Las determinaciones de las abundancias químicas parten del hecho de que los tipos de líneas que hemos visto, tanto las de recombinación como las prohibidas, son ópticamente delgadas. Por lo tanto, el flujo que podemos medir de ellas es directamente proporcional a la 
abundancia del ión que la produce. Bajo esta hipótesis es que se pueden calcular abundancias por medio de las razones de intensidades de estas líneas. Para las líneas de recombinación (H y He) tenemos la ecuación en función de los coeficientes $\alpha$ de recombinación:

$$
\frac{\mathrm{n}\left(\mathrm{He}^{+}\right)}{\mathrm{n}\left(\mathrm{H}^{+}\right)}=\left(\frac{\mathrm{I}\left(\lambda, \mathrm{He}^{+}\right)}{\mathrm{I}(\mathrm{H} \beta)}\right) \frac{\alpha(\mathrm{H} \beta)}{\alpha(\lambda, \mathrm{He})} \frac{4861}{\lambda} .
$$

Este es el método más confiable para la determinación de abundancias en RHiı, ya que dichos coeficientes dependen débilmente de la temperatura, y además son muy similares, por lo que esta dependencia se cancela.

Por otro lado, para el resto de iones no se encontrarán líneas de recombinación en la parte óptica del espectro sino que únicamente se cuenta con las líneas colisionales. El análisis del cociente de flujos de dichas líneas viene dado por:

$$
\frac{\mathrm{n}\left(\mathrm{X}^{i}\right)}{\mathrm{n}\left(\mathrm{Y}^{j}\right)}=\left(\frac{\mathrm{I}\left(\lambda_{1} i, \mathrm{Y}^{i}\right)}{\mathrm{I}\left(\lambda_{2}, \mathrm{Y}^{i}\right)}\right) \frac{\mathrm{n}_{e} \mathrm{c}(H \beta)}{\mathrm{c}\left(\lambda, X^{i}\right)} \frac{4861}{\lambda} .
$$

En este caso hay una dependencia de los coeficientes de excitación colisional "c", que dependen a su vez de la temperatura, pero el comportamiento es equivalente al del coeficiente $\alpha$ en las líneas de recombinación, por lo que su dependencia es baja. Debido a esto, la manera más común de determinar la abundancia de una línea de excitación colisional (LEC) es comparando la intensidad de la línea correspondiente con una línea de recombinación de hidrógeno (que por default se considera $\mathrm{H} \beta$ ).

\subsubsection{Densidad}

La densidad electrónica de un gas excitado por fotoionización puede calcularse a partir de las líneas intensas en emisión observadas. No puede utilizarse cualquier línea, sino aquellas que procedan de niveles de energía de un mismo ión con potenciales de excitación similares, de manera que la intensidad de estas líneas dependerá del cociente de los coeficientes de las fuerzas de colisión de dichos niveles. Por lo tanto, las líneas reflejan los efectos de la desexcitación colisional. Si los dos niveles tienen diferentes probabilidades de transición o presentan ritmos de desexcitación colisional diferentes, el cociente de la intensidad de sus líneas espectrales (así como la población relativa de ambos), dependerá de la densidad de los electrones presentes en el medio (Osterbrock \& Ferland, 2006). 

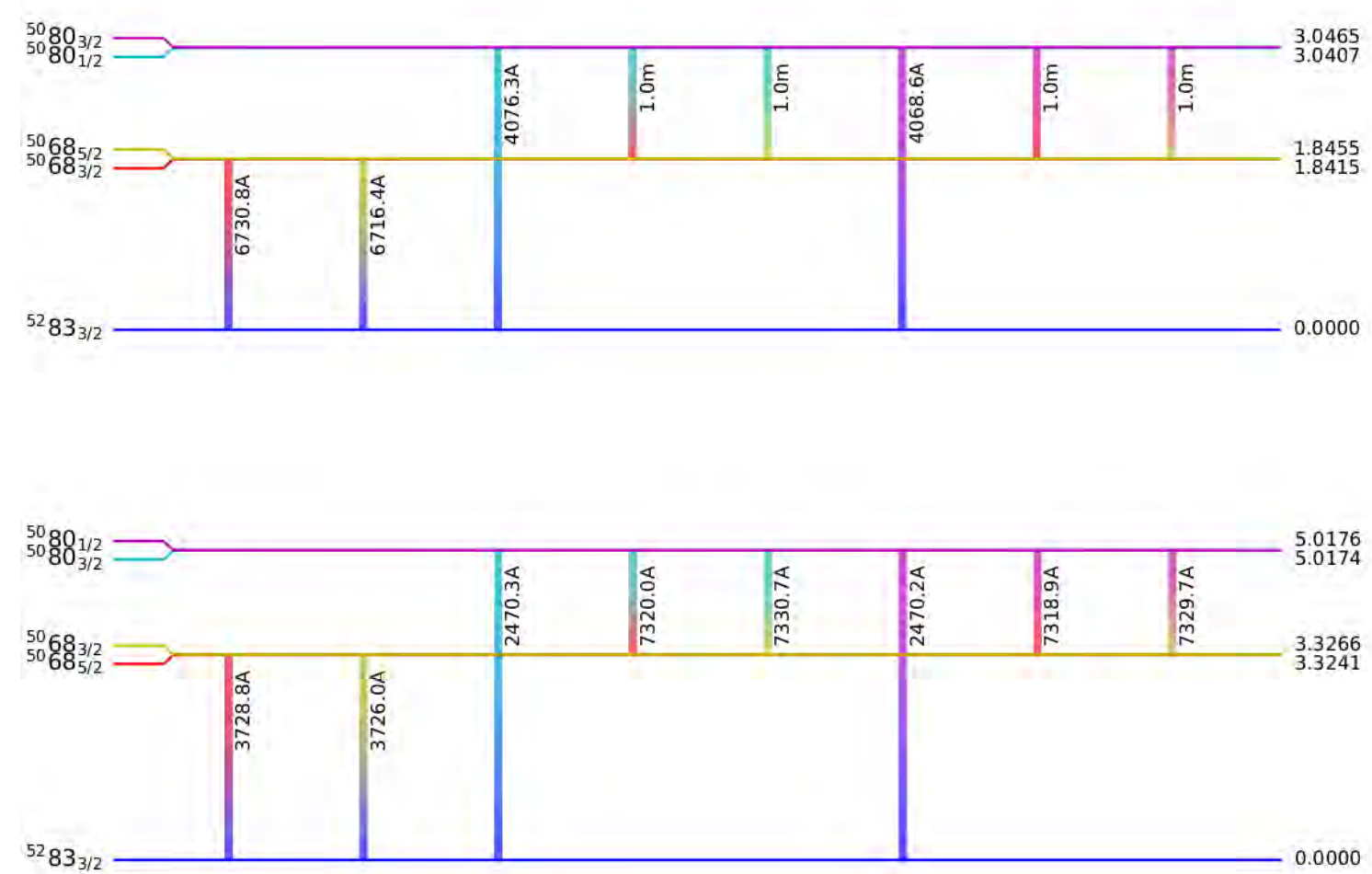

Figura 1.4. Diagrama Gotrian de los iones [OII] y [SII]. En los paneles puede observarse como el par de líneas para la determinación de densidades tiene orígenes en niveles con energías muy similares que decaen al nivel fundamental. A partir de los cocientes de dichas líneas, es posible determinar la densidad del medio nebular (Osterbrock \& Ferland, 2006). Imágen generada utilizando el software $\mathrm{PyNeb}^{(\mathrm{i})}$. 
En el rango óptico del espectro electromagnético los principales cocientes de líneas utilizados, siguiendo la física de nebulosas desarrollada por Osterbrock \& Ferland (2006), son [SII] $\lambda 6716 \AA / \lambda 6731 \AA$ y [OII] $\lambda 3729 \AA / \lambda 3726 \AA$. Estos cocientes de líneas son válidos para un régimen de densidades bajas e intermedias de $\mathrm{n}_{e}=10^{1}-10^{4} \mathrm{~cm}^{-3}$.

Cuando estamos en el límite de baja densidad (menores a 100 partículas por $\mathrm{cm}^{-3}$ ) las desexcitaciones colisionales se hacen despreciables y el cociente entre las intensidades coincide con los pesos estadísticos de los niveles de los que parten las líneas, dado que ambos niveles tienen energías de excitación muy similares. Por el contrario, en el límite de alta densidad (densidades superiores a la densidad crítica, $\mathrm{n}_{c} \sim 500-2000$ part $/ \mathrm{cm}^{3}$ ) las poblaciones de los niveles son dominadas por las excitaciones y desexcitaciones colisionales y se distribuyen según la ecuación de Maxwell-Bolzmann. Así vemos que el cociente es muy sensible a la densidad y poco dependiente de la temperatura.

Los cocientes de las intensidades de las líneas de [SII] y del [OII] en función de la densidad electrónica son muy sensibles dentro de los rangos de densidades bajas e intermedias, que son los valores típicos estimados para las RHir. En la Figura 1.6 podemos ver la variación de los cocientes con respecto a la densidad para una temperatura de $10.000 \mathrm{k}$, típica de estas regiones. Los valores estimados de densidades a partir de cocientes de líneas pertenecen espacialmente a las zonas en las que se encuentran los iones de los elementos correspondientes y son un valor medio representativo de la nebulosa.

Además de los mencionados, hay cocientes de líneas de otros elementos que pueden ser utilizados para estimar densidades como por ejemplo: [Cl III] $\lambda 5517 \AA / \lambda 5537 \AA$. Si bien este cociente es sensible a altas densidades electrónicas y es característico en nebulosas planetarias, fue detectado con SOAR ${ }^{(i i)}$ en RHII bajo ciertas condiciones particulares. Y por otro lado, el [Ar IV] $\lambda 4711 \AA / \lambda 4741 \AA$ detectado en regiones HII y un buen indicador de densidades electrónicas para regiones de densidades medias. Sin embargo, estas líneas son intrísecamente débiles y esto es una complicación para su detección, como por ejemplo las líneas del Argón ([Ar IV $] \lambda 4741 \AA$ ).

\subsubsection{Temperatura}

La estimación de la temperatura electrónica resulta fundamental para la determinación de las abundancias químicas. Los métodos que se utilizan para el cálculo de una temperatura representativa del gas ionizado en RHII son el método directo, métodos empíricos y semiempíricos y temperaturas derivadas a partir de modelos de fotoionización (Hägele et al., 2006, 2008, 2012). El método más preciso es el primero, que se basa en la medida directa de las líneas intensas y de las sensibles a la temperatura, conocidas como aurorales. A través de los cocientes de intensidades de dichas líneas, se puede estimar la $\mathrm{T}_{e}$ para la región donde el ion correspondiente se encuentre. La complicación que presenta este método es que se requiere de una alta calidad espectral para poder observar y medir las líneas aurorales, debido a que estas son intrínsecamente débiles. Es importante mencionar que si las metalicidades del gas son altas, las temperaturas son menores y por lo tanto las líneas se hacen más débiles. Hace falta un equilibrio entre metalicidad y temperatura para poder medir este tipo de líneas débiles (ver Pettini \& Pagel, 2004; Osterbrock \& Ferland, 2006; Hägele et al., 2006, 2008).

En las nebulosas, se suele indicar a la $\mathrm{T}_{e}[\mathrm{OIII}]$ como la representativa de la región, debido a que la línea auroral del $[\mathrm{O} I I]] \lambda 4363 \AA$ es la que presenta una probabilidad mayor de detección frente a los demás elementos. Por lo tanto, para determinar la $\mathrm{T}_{e}$ se utilizan los cocientes de intensidades de LEC de [OIII] $\lambda \lambda 4959,5007 \AA / \lambda 4363 \AA$. En la Figura 1.7 podemos ver como

\footnotetext{
${ }^{(i i)}$ El telescopio Southern Astrophysical Research (SOAR) es un telescopio con una apertura de 4.1 metros, diseñado para trabajar desde el azul en $320 \mathrm{~nm}$ hasta el infrarrojo cercano $\approx 800 \mathrm{~nm}$ (http://opcc.cl/soar/).
} 

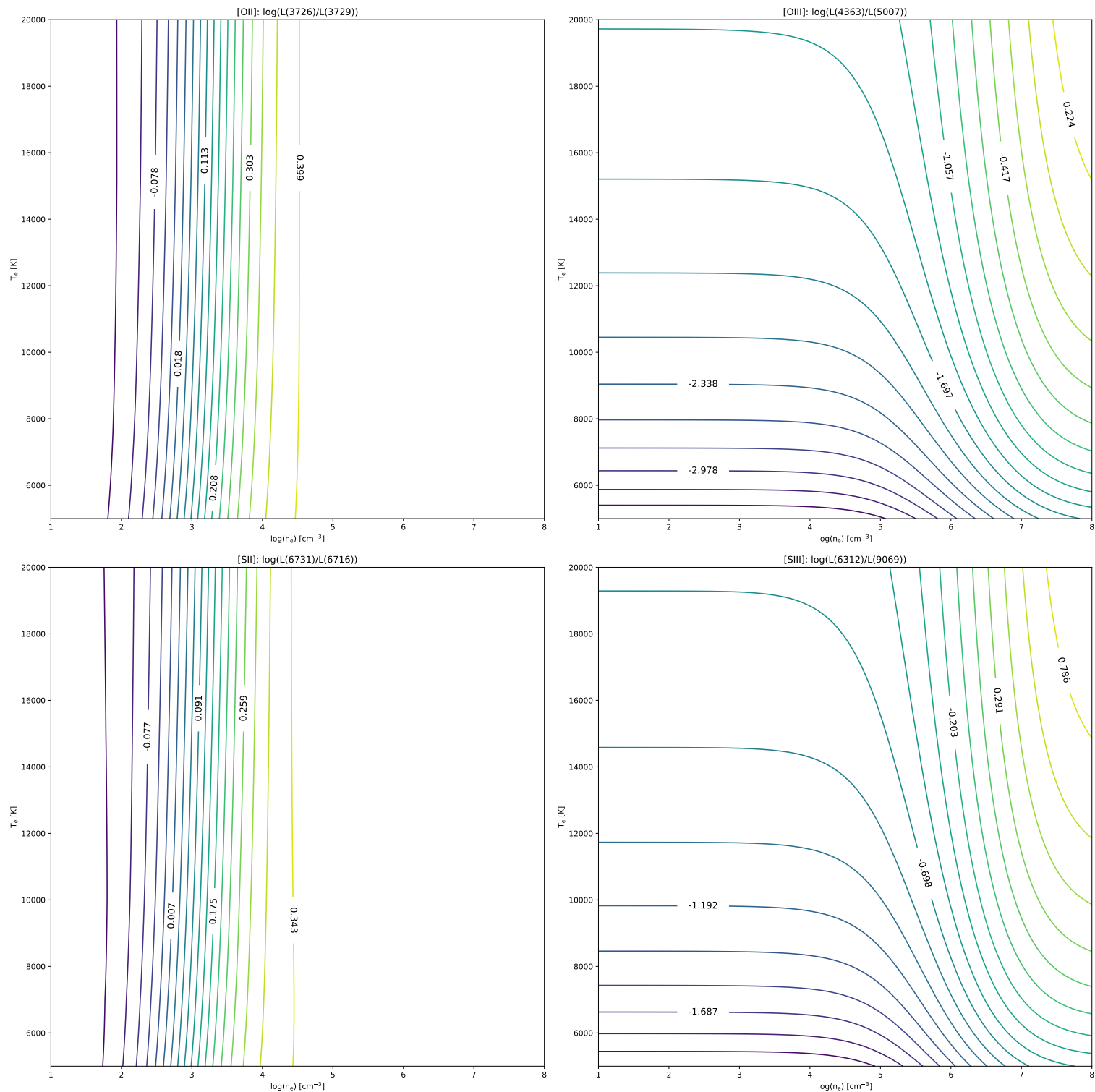

Figura 1.5. Diagramas diagnósticos fundamentales para interpretar cómo se comportan los cocientes de líneas con el objetivo de determinar los valores de densidad y temperatura. De arriba a abajo y de izquierda a derecha, $\log (\mathrm{L}(\lambda 3726 \AA) / \mathrm{L}(\lambda 3729 \AA))$ un excelente estimador de la densidad electrónica para un amplio rango de temperaturas; $\log (\mathrm{L}(\lambda 4363 \AA) / \mathrm{L}(\lambda 5007 \AA))$ un estimador de temperaturas electrónicas para densidades bajas, mientras que para un régimen de alta densidad, puede funcionar como estimador de densidad electrónica; $\log (\mathrm{L}(\lambda 6731 \AA) / \mathrm{L}(\lambda 6716 \AA))$ un excelente estimador de la densidad densidad electrónica para un amplio rango de temperaturas $\log (\mathrm{L}(\lambda 6312 \AA) / \mathrm{L}(\lambda 9060 \AA))$ un estimador de temperaturas electrónicas para densidades bajas, mientras que para un régimen de alta densidad, puede funcionar como estimador de densidad electrónica. 


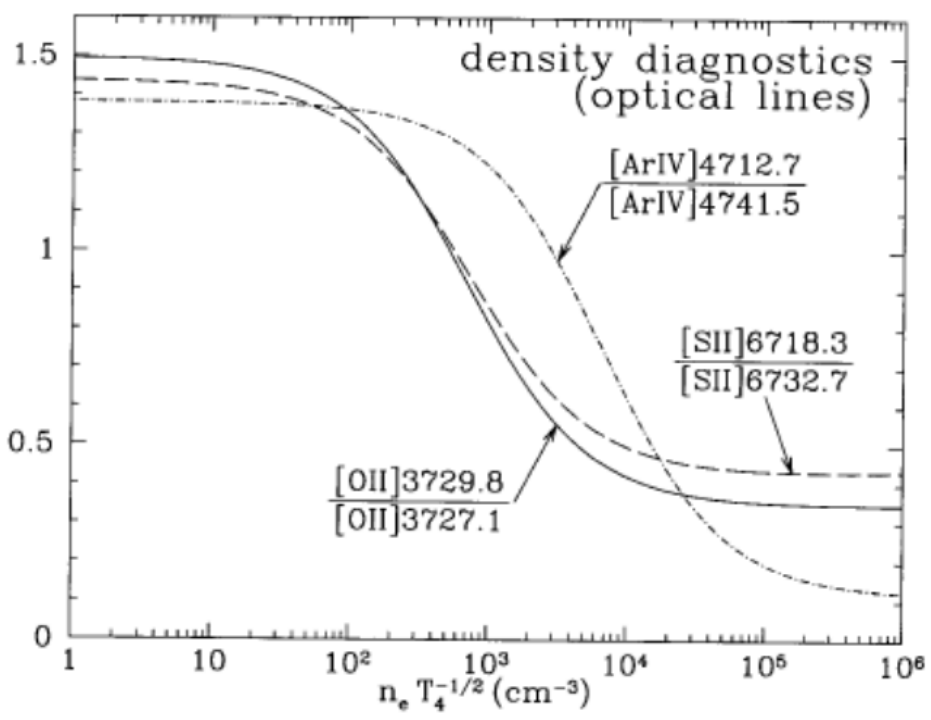

Figura 1.6. Cocientes de intensidades de líneas y su relación con las densidades electrónicas bajas e intermedias. Según la relación, los cocientes pueden toman valores entre $\sim 1.5$ y $\sim 0.4$, lo que se traduce en densidades bajas, cuando la razón estimada es mayor a 1, y altas, cuando el cociente es menor a 1. El límite inferior, a densidades altas, corresponde a densidades críticas donde se produce la desexcitación colisional de los electrones. Imagen extraída de la página web de la Universidad de Meryland (www.astro.umd.edu/ `bolatto/ teaching/ASTR670_Spring17/Graphics_HIIRegions.pdf).

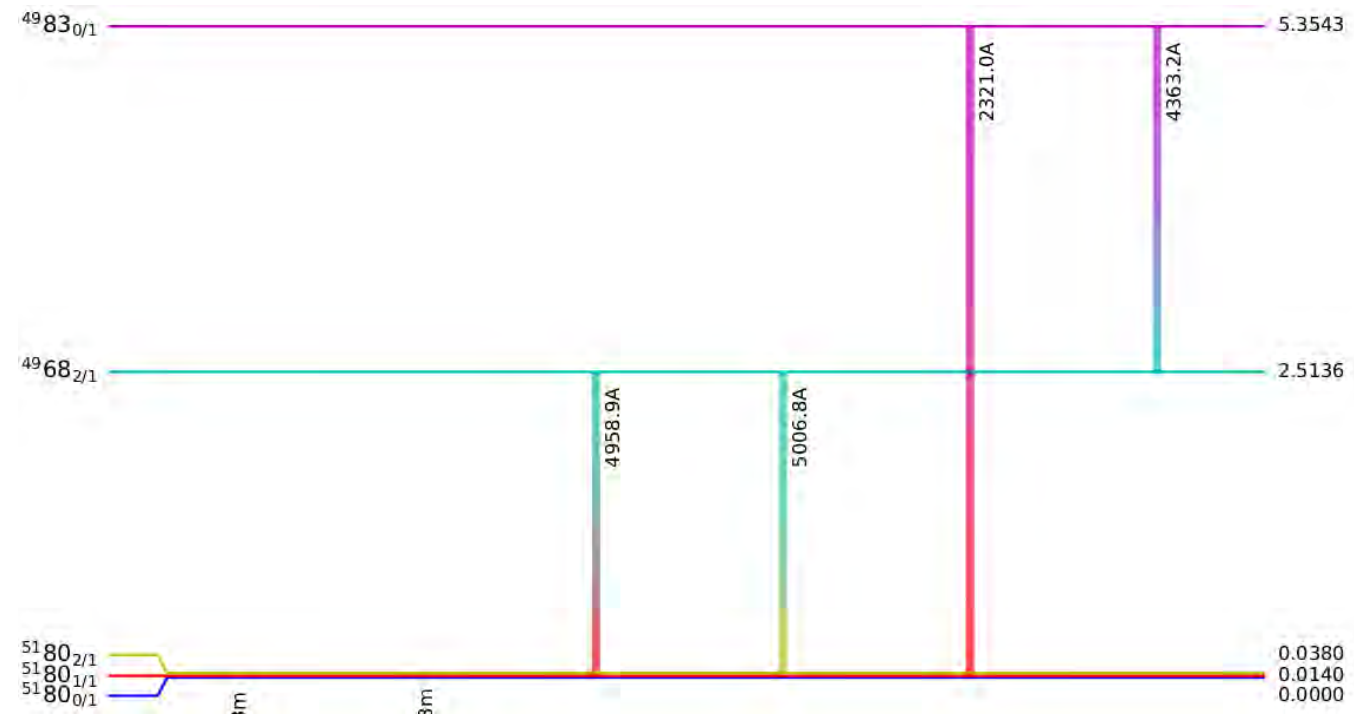

Figura 1.7. Transiciones electrónicas entre distintos niveles de energía del [OIII]. Se puede ver que la línea $4363 \AA$ proviene de un nivel superior, distinto a las líneas $4959 \AA$ y $5007 \AA$. A partir de la intensidad de la línea débil, 4363Å, y de la razón con las líneas intensas, se puede estimar la temperatura que genera que una cierta cantidad de electrones se exciten al nivel superior. Imágen generada utilizando el software PyNeb. 
ejemplos algunas de las líneas referentes en nuestro análisis y sus correspondientes niveles de energías, con potenciales de excitación muy diferentes. Se puede ver que la línea $4363 \AA$ proviene de un nivel superior, distinto a las líneas $4959 \AA$ y $5007 \AA$. Entonces, a partir de la intensidad de la línea débil, $4363 \AA$, y de la razón con las líneas intensas, se puede estimar la temperatura que genera que una cierta cantidad de electrones se exciten al nivel superior. Esto lo convierte en un buen indicador de la temperatura. La línea [OIII] $\lambda 4363 \AA$ tendrá una mayor probabilidad de observarse con una buena $\mathrm{S} / \mathrm{N}$ (señal-a-ruido, del inglés: signal-to-noise) para metalicidades $12+\log (\mathrm{O} / \mathrm{H})<7.8$, mientras que para las mayores a $12+\log (\mathrm{O} / \mathrm{H})>8.3$ será muy difícil de detectar (Pettini \& Pagel, 2004), a causa de que a metalicidades mayores el enfriamiento de la nebulosa es más eficiente y las líneas débiles resulta difícil observarlas.

En el rango espectral óptico y el IR cercano, desde $\lambda 3800 \AA$ hasta $\lambda 9500 \AA$, se pueden estimar las temperaturas electrónicas de otros iones de manera directa si se dispone de las líneas aurorales correspondientes. Por ejemplo: [SiI] $\lambda 4068 \AA$, [NII] $\lambda 5755 \AA$, [OII] $\lambda \lambda 7319$ y $7330 \AA$, $[\mathrm{SIII}] \lambda 6312 \AA^{\text {(iii) }}$.

\subsubsection{Corrección por enrojecimiento y extinción}

El polvo que existe en la línea de visión entre el objeto emisor y el observador, enrojese la luz emitida por este. Los fotones en las longitudes de onda más cortas se dispersan más que las largas, es fundamental tener en cuenta este efecto y corregirlo para obtener medidas de la intensidad real que emite la región. Se define la extinción para una dada longitud de onda, $A_{\lambda}$, como la diferencia entre la magnitud aparente observada y la magnitud intrínseca (sin efecto del polvo):

$$
\mathrm{A}_{\lambda}=\mathrm{m}_{\lambda}-\mathrm{m}_{\lambda}^{0} .
$$

Calculando el exceso de color $\mathrm{E}(\mathrm{B}-\mathrm{V})$, se mide la extinción en diferentes longitudes de onda permitiendo derivar el enrojecimiento que sufre la fuente.

La extinción y el exceso de color los podemos relacionar mediante una ley de extinción:

$$
\mathrm{X}_{\lambda}=\frac{\mathrm{A}_{\lambda}}{\mathrm{E}(\mathrm{B}-\mathrm{V})}=\mathrm{R}_{V} \frac{\mathrm{A}_{\lambda}}{\mathrm{A}_{V}},
$$

en donde $\mathrm{R}_{V}$ es la extinción total en la banda $\mathrm{V}$. Son muchas las leyes de extinción que han proporcionado una solución a los problemas introducidos por el polvo (Whitford, 1958) en el óptico, Seaton (1979) en el óptico y UV, Howarth \& Wilson (1983) en el IR cercano y Calzetti et al. (1989); Calzetti (2000) en el UV/óptico/NIR. En este trabajo utilizamos la parametrización definida por Cardelli et al. (1989), que define:

$$
\mathrm{X}_{\lambda}=\mathrm{a}_{\lambda} \mathrm{R}_{V}+\mathrm{b}_{\lambda},
$$

donde consideramos $\mathrm{R}_{V}=3.1$, y en donde los polinomios a $\mathrm{y} \mathrm{b}$ en función de $\lambda$ están en unidades de $\mu m^{-1}$. El flujo que medimos como observadores, $F(\lambda)$, es la intensidad emitida por el objeto, $\mathrm{I}(\lambda)$, que está atenuada debido a la extinción:

$$
\mathrm{F}(\lambda)=\mathrm{I}(\lambda) 10^{0.4 \mathrm{~A}_{\lambda}} .
$$

La extinción no depende del objeto en sí, sino que depende de la cantidad de polvo entre el emisor y el observador y la ley de extinción utilizada. Esta relación se presenta como:

$$
\mathrm{A}_{\lambda}=2.5[\mathrm{f}(\lambda)+1] \cdot \mathrm{c}(\mathrm{H} \beta),
$$

\footnotetext{
${ }^{(i i i)}$ La longitud de onda indicada, $\lambda$, corresponde a las longitudes de onda en reposo, $\lambda_{0}$, por lo que hay que tener en cuenta el corrimiento al rojo de las regiones cuando se las observa. Ver Hägele et al. (2008, 2011, 2012).
} 
donde $\mathrm{f}$ es la función de enrojecimiento y $\mathrm{c}(\mathrm{H} \beta)$ es la constante de enrojecimiento. Esta es un indicador del apantallamiento que sufre la luz que recibimos del gas emisor por acción del polvo. En nuestro trabajo lo hemos estimado a partir de las cuatro líneas de recombinación del hidrógeno más intensas de la serie de decremento de Balmer $(\mathrm{H} \alpha, \mathrm{H} \beta, \mathrm{H} \gamma$ y $\mathrm{H} \delta)$, relativas a $\mathrm{H}(\beta)$ a partir de un ajuste de mínimos cuadrados en donde la pendiente determinada es el coeficiente buscado (Hägele et al., 2006, 2008, 2013). Las intensidades y los cocientes teóricos son prácticamente constantes (Osterbrock \& Ferland, 2006) y han sido determinados por Storey \& Hummer (1995) para ciertos valores de densidad y temperatura electrónica.

A partir del coeficiente de enrojecimiento es posible:

- corregir por enrojecimiento a los cocientes relativos de todas las líneas:

$$
\mathrm{I}(\lambda) / \mathrm{I}(\mathrm{H} \beta)=\mathrm{F}(\lambda) / \mathrm{F}(\mathrm{H} \beta) 10^{\mathrm{c}(\mathrm{H} \beta) \mathrm{f}(\lambda)} .
$$

- estimar un valor para la extinción $\left(\mathrm{A}_{V}\right)$ y para el exceso de color $(\mathrm{E}(\mathrm{B}-\mathrm{V}))$. Por lo tanto, para corregir a la luz de estos efectos se debe corregir por efecto Doppler (debido a que la fuente no esta en reposo), y por corriemiento al rojo cosmológico si la fuente está a una distancia considerable.

\subsection{Técnicas observacionales utilizadas}

La astronomía suele dividirse en dos grandes áreas de trabajo: el área observacional y el área teórica. Ambas están vincualadas y sus desarrollos se guían y evolucionan, conjuntamente, con sus respectivos avances. Los resultados observacionales ayudan a confirmar los modelos teóricos y estos, a su vez, brindan interpretaciones a las observaciones, impulsando nuevos desafíos en desarrollos científicos y tecnológicos. Se pueden mencionar un sin fin de hitos alcanzados desde las primeras observaciones des espectro solar por Newton o las diversas observaciones realizadas por Galileo, que dieron inicio a una historia que continuó creciendo y nutriéndose, hasta llegar a una era en la que es natural hablar de satélites, telescopios gigantes, estaciones y misiones espaciales, radiotelescopios y grandes observatorios.

Comprender la luz que recibimos de los astros es un trabajo muy complejo que ha propuesto desafíos en la construcción de instrumentales de distintos tipos (cámaras, monturas, diseños, entre otros) y con diversos objetivos (espectrógrafos, rangos espectrales de detección, filtros y bandas angostas de observación, entre otras). Uno de los punto claves está en desarrollar instrumentos que logren recolectar la luz que recibimos de los astros de la manera más "limpia" y efectiva posible. Telescopios con espejos de grandes áreas colectoras de luz, detectores e instrumentos en general con grandes eficiencias cuánticas, hacen posible que una mayor cantidad de fotones puedan ser detectados, para analizarlos y posteriormente procesarlos.

En los próximos años presenciaremos un gran avance en el área del desarrollo instrumental, con telescopios de $30 \mathrm{~m}$ que ya estan en construcción y telescopios de $8 \mathrm{~m}$ que van a mapear el cielo con una profundidad y detalle superior a lo que tenemos hoy. Esto se traduce en que en una única noche, se van a generar más datos de los que tenemos hasta hoy en día. Observatorios como Gemini, que desde hace 20 años observan los cielos Sur y Norte desde sus telescopios en Chile y Hawaii, estudian ambos hemisferios, complementando las observaciones de todo el cielo; El Observatorio Las Campanas y La Silla, en Chile, que cuentan con más de un telescopio y disponen de una gran variedad de instrumental de distintas características; radiotelescopios que trabajan día y noche observando la contraparte de los astros en longitudes de onda de radio en el espectro milimétrico están hoy en día llegando a resoluciones angulares de la más alta calidad con ALMA (de su sigla en inglés Atacama Large Millimiter/submillimeter). 
Estos son algunos de los proyectos astronómicos que funcionan hoy en día en busca de respuestas y de estudios del cosmos, y una de las motivaciones de este trabajo es continuar con los estudios a través de la técnica observacional de la espectroscopía, que nos permite estudiar la luz es distintos rangos de longitudes de onda.

\subsubsection{Espectroscopía}

La espectroscopía es una técnica que analiza la distribución de la radiación electromagnética en función de la longitud de onda, y resulta fundamental para conocer la naturaleza de los astros a partir de la luz que recibimos de ellos. A esta distribución es la que comúnmente denominamos "espectro" y para obtenerlo utilizamos instrumentos conocidos como espectrógrafos, que son los encargados de realizar la descomposición de la luz a través de dispersores como los prismas o redes de difracción. Esta técnica es mucho más exigente, en cuanto a la cantidad de tiempo de observación empleado, que la fotometría, ya que es equivalente a una fotometría en banda estrecha en múltiples bandas.

Dicha técnica tiene sus inicios en 1666, con el mismísimo Isaac Newton. Él se encontraba trabajando en la construcción del primer telescopio refractor cuando logró comprender que los colores que se producían cuando la luz blanca atravesaba un prisma eran una propiedad intrínseca de la luz, y no una consecuencia de algún fenómeno producido por el vidrio. Al observar el espectro solar identificó un arco iris de color y varias líneas características oscuras, las cuales fueron descritas por primera vez recién en 1817 por el astrónomo alemán Joseph von Fraunhofer, quien le dio una identidad al espectro solar.

Este episodio dio origen a un campo que tuvo su máximo desarrollo en el siglo XIX conduciendo a un detallado entendimiento de la forma en que los átomos y moléculas pueden absorber y emitir radiación, desarrollado por G. Kirchoff y R. Bunsen y postulado en sus tres leyes:

- Una fuente (sólida incandecente o un gas denso y caliente) emite un espectro continuo de luz en todas las longitudes de onda.

- Líneas espectrales: regiones pequeñas donde el continuo se interrumpe y vuelve a recuperarse. Si la línea representa una disminución de intensidad respecto al nivel del continuo, se la llama línea de absorción. Y sí se ve un aumento se la llama línea de emisión (ver Figura 1.8).

- Bandas espectrales: iguales que las líneas, pero tienen mucha mayor ancho.

Si se conoce el corrimiento al rojo de una fuente y/o las características del medio interestelar se pueden identificar las líneas medidas en los espectros.

Entre alguno de los logros más importantes de esta técnica podemos mencionar a la clasificación de las estrellas según las características observadas en sus espectros. El astrónomo italiano Angelo Secchi a mediados de 1800 realizó una clasificación de las estrellas según sus tipos espectrales. Para ello se basó en el número e intensidad de las líneas de absorción de sus espectros. Otras características fundamentales que podemos estimar a partir del análisis espectral son los campos de velocidades y los desplazamientos al rojo (o al azul) que ocurren cuando la radiación electromagnética emitida (o reflejada) por un objeto es desplazada hacia longitudes de onda más largas (o más cortas) en el espectro electromagnético (ver Figura $1.9)$. 
Figura 1.8. Espectros de distintas clases de objetos. Los espectros estelares con líneas de absorción y los espectros nebulares caraceterísticos por su emisión pobre de continuo pero con líneas intensas en emisión. Imagen extraída de la pagína wed de la Universidad de Virginia (http://faculty.virginia.edu/skrutskie/ASTR1210/notes/kirch.html).
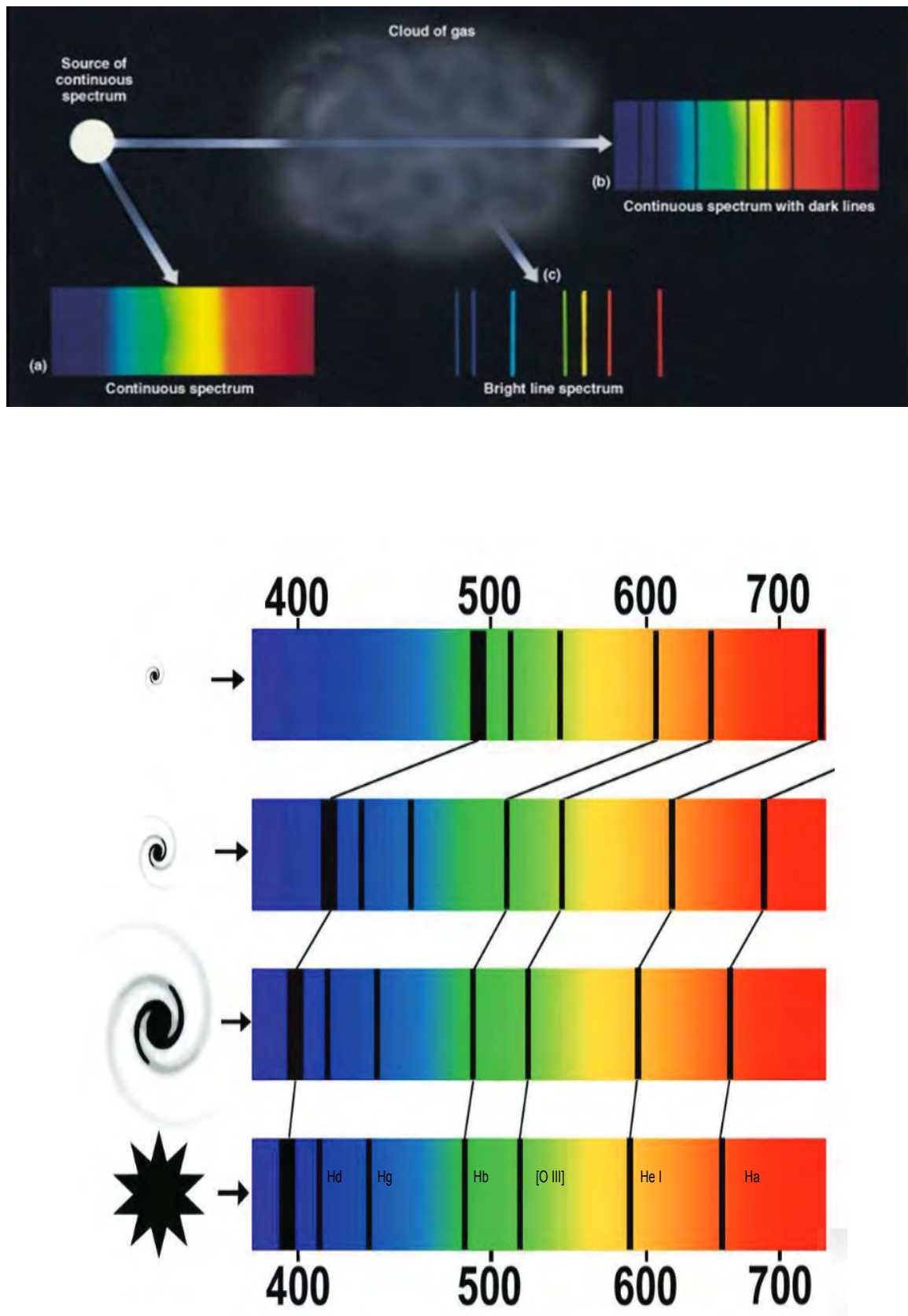

Figura 1.9. La expansión del Universo y el corrimiento al rojo lo podemos determinar a partir de las líneas espectrales y cuánto se alejan estas de la longitud de onda en reposo $\lambda_{0}$. En la Figura se indical las líneas de excitación colisional del [OIII], las líneas de recombinación de la serie de Balmer de Hidrógeno y la línea de recombinación de HeI, en el reposo, correspondiente a la referencia estelar. A medida que se van observando objetos más lejanos, espectralmente se identifica el corrimiento de cada una de las líneas correspondientes. El efecto se nota mirando de arriba hacia abajo. Imagen adaptada de la cátedra de astrofísica de la Universidad de La Laguna: http://astronomia.net/cosmologia/FAQ2.htm. 


\section{Introducción}

El corrimiento al rojo de las galaxias es consecuencia de su alejamiento, el cual fue descubierto en la segunda década del siglo XX por el astrónomo norteamericano Edwin Hubble. El carácter no estacionario de todo el sistema de galaxias en el Universo había sido previamente demostrado de manera teórica por A. Fridman (uno de los fundadores de la cosmología moderna). Según la ley definida por la teoría de Fridman y demostrada por Hubble con sus observaciones, las galaxias se alejan a velocidades $v$ proporcionales a las distancias $(d)$ hasta ellas, de modo que cuanto más alejadas están mayor es el valor de su velocidad observada. Para las galaxias próximas se demuestra que: $\mathrm{v}=\mathrm{H} \cdot \mathrm{d}$, donde $H$ es la constante de Hubble (coeficiente de proporcionalidad) que se determina a partir de observaciones. Esta ley es consistente con el principio cosmológico y muestra que no hay observadores privilegiados en el Universo. A causa del corrimiento al rojo, el alejamiento de las galaxias provoca el desplazamiento de sus líneas espectrales hacia el rojo del espectro. La dependencia del corrimiento al rojo, $z$, de la velocidad de alejamiento, $v$, se expresa mediante la siguiente formula: $\mathrm{z}=\mathrm{v} / \mathrm{c}$, siendo $c$ la velocidad de la luz. Si en esta fórmula introducimos la ley de Hubble, obtenemos la expresión básica utilizada para la estimación de distancias a galaxias y cúmulos estelares, dada por: $\mathrm{z}=\mathrm{H} \cdot \mathrm{d} / \mathrm{c}$. Además, esta técnica ha permitido el análisis de distintos parámetros como la presión de radiación, la gravedad, los campos magnéticos, etc.

Hay varios aspectos y clasificaciones posibles en espectroscopía, dependiendo de las maneras en que quieran llevarse adelante las investigaciones. Para la realización de este trabajo, nos enfocamos explícitamente en la espectroscopía de alta dispersión. Dentro de los instrumentos que permiten obtener espectros de alta resolución y un amplio rango espectral a la vez, se encuentran los espectros Echelle, que son los que trabajaremos en esta Tesis. Hay a su vez otros tipos de espectrógrafos de alta resolución, que no detallaremos en esta breve introducción.

Existen dos principios ópticos fundamentales que permiten dispersar la luz: la refracción diferencial y la interferencia. El primero da lugar a los espectrógrafos de prisma y el segundo a los basados en redes de difracción. Existen también elementos dispersores híbridos, que son la combinación de al menos un elemento de cada uno (ver la fuente "Astronomical optics"). Este tipo de instrumento puede tener varias configuraciones aunque normalmente consta de los siguientes elementos básicos (ver Figura 1.10):

- Ranura: se encuentra en el plano focal del telescopio y permite aislar la zona de la imagen (objeto) que se desea observar.

- Colimador: convierte el frente de luz en paralelo. Es decir, para que llegue al dispersor en la dirección óptima.

- Dispersores: prismas o redes de difracción.

- Cámara o detector: registra la imágen que luego generará el espectrógrafo.

El elemento dispersor es quien separa el haz incidente de manera que cada haz tiene un ángulo de salida, $\theta$, diferente. De aquí es que se define a la dispersión angular como la relación entre el ángulo de separación por intervalo de longitud de onda: $d \theta / d \lambda$. Además, queda definida la dispersión lineal en el plano del detector (plano focal) como la cantidad $d \lambda / d x$, y se suele expresar en unidades de $\AA /$ píxel o $\AA / \mathrm{mm}$. Esta es la cantidad que surge debido a que el espectrógrafo debe enfocar diferentes haces de luz incidentes con la misma $\lambda$ en un mismo valor de "x" (ver Figura 1.11).

El poder de resolución del espectrógrafo indica la capacidad de un instrumento para distinguir dos longitudes de onda separadas por $\Delta \lambda$, y viene dado por la relación: $R=\lambda / \Delta \lambda$. $\mathrm{Al}$ ir aumentando el valor de $\mathrm{R}$ se pueden obtener espectros con mayores resoluciones que 

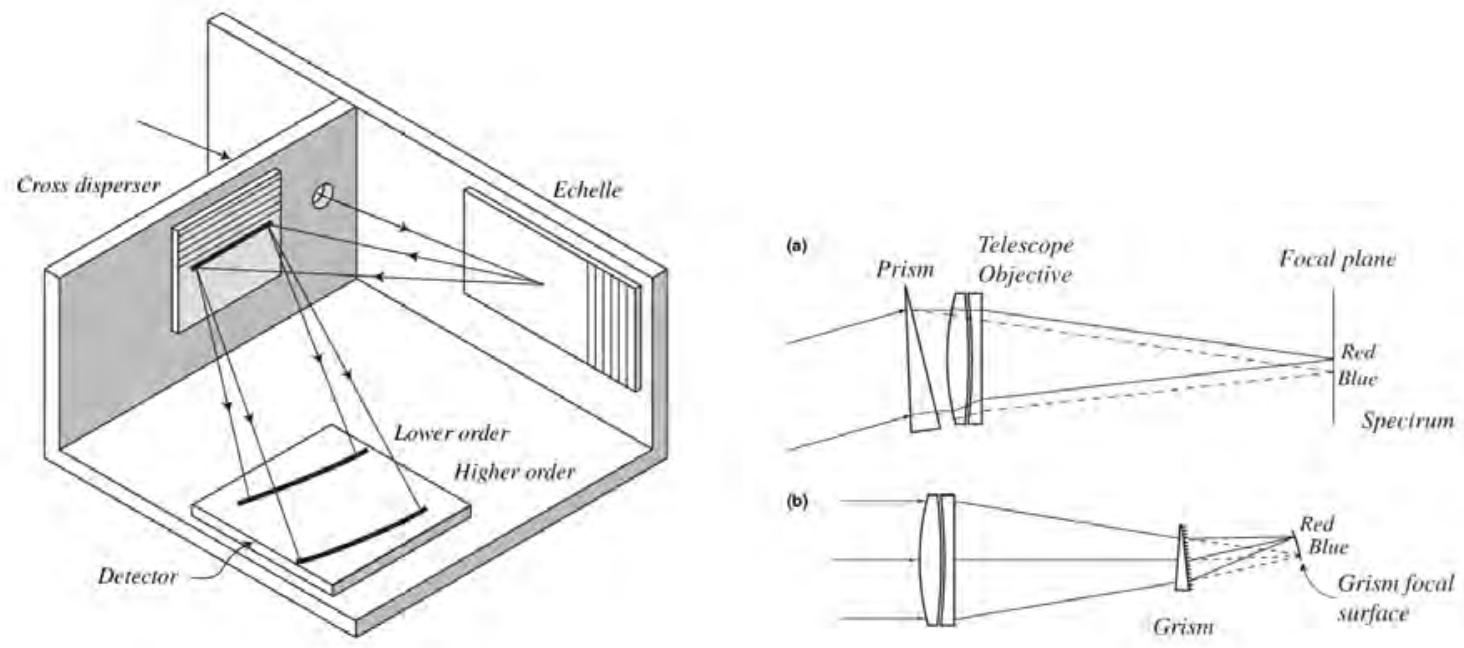

Figura 1.10. Panel izquierdo: Se muestran únicamente dos órdenes espectrales generados en el plano focal del detector para una major claridad. Panel derecho: dos modelos de espectrógrafo según sus elementos dispersones. El superior es el caso de un prisma, que en los telescopios Schmidt se montaría justo en frente de la placa correctora. En el caso inferior una red que dispersa la luz en longitud de onda hacia el plano focal. Las imágenes fueron extraída de "Astronomical optics" (Schroeder et al. 2000).

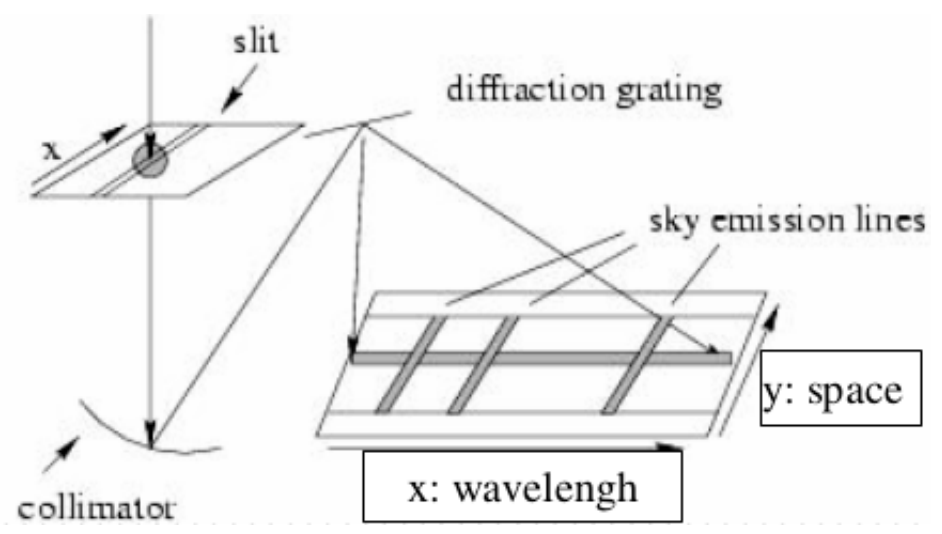

Figura 1.11. Representación gráfica del espectro resultante tras la observación de una fuente a través de una rendija (imagen extraída de "Astronomical optics"). 
permiten observar las líneas con suficiente detalle, dando lugar a estudio de componentes cinemáticas, perfiles de líneas y mecanismos de ensanchamientos de líneas. Esto depende también de los fenómenos y tipos de objetos observados, por ejemplo los AGNs (o galaxias en general) presentan anchos de líneas varios órdenes de magnitud mayor que las RHiI medidas observadas en las BCDs, y dobletes de líneas como [Ör] $\lambda \lambda 3726 \AA$, $3729 \AA y$ [Sir] $\lambda \lambda 6717 \AA$, $6731 \AA$, serían imposibles de separar aunque se disponga de una alta resolución espectral. Es decir, la dispersión de velocidades hace que las líneas tengan un ancho natural grande, y entonces por más que R sea alto, no se pueden "resolver" dichas 1 ineas. En los casos de las Galaxias BCDs, con una resolución adecuada, es posible separar los perfiles de las líneas de dichos dobletes. Por lo tanto, lo que permitirá que se separen o no las líneas es el R del espectrógrafo, y la condición de movimeinto del gas.

Junto a la resolución espectral, se define el poder resolvente como el valor de $\delta \lambda$ que viene dado por el seeing (o visibilidad) o el tamaño de la ranura (el menor de los dos) y la dispersión del instrumento utilizado. La resolución espectral es uno de los aspectos más importantes y fundamentales que se tienen en cuenta en los proyectos observacionales. Para estudios de alta resolución espectroscópica normalmente se utiliza la dispersión cruzada o Espectroscopía Echelle. A través de la red Echelle y del elemento dispersor, se separa el espectro de la luz en distintos órdenes, y en el detector se difracta el haz de luz en una cantidad definida de órdenes.

Continuando, la red de difracción (elemento dispersor del espectrógrafo) presenta propiedades que vienen dadas por la ecuación que describe la diferencia del camino de la luz como:

$$
m \cdot \lambda=\sigma(\operatorname{sen} \alpha+\operatorname{sen} \theta),
$$

donde $\sigma$ es distancia entre líneas de la red, $m$ corresponde al número entero de los órdenes, $\alpha$ es el ángulo de incidencia respecto a la normal, $\theta$ el ángulo de difracción respecto a la normal. Esta es la expresión que gobierna el comportamiento óptico de una red de difracción, y se debe a que para que se produzca una interferencia constructiva la diferencia del recorrido entre dos rayos de luz debe ser un número entero de longitudes de onda (ver Figura $1.12^{(\mathrm{i})}$ ). Esta ecuación de red indica que para un dado valor de $\sigma$ y $\alpha$, el ángulo de difracción $\theta$ depende de la longitud de onda $\lambda$. De esta manera, se producen espectros de la fuente de entrada para cada valor de $m$. Es decir que cada espectro es un orden de difracción (para una información más detallada, ver Schroeder et al. 2000).

Hay dos problemas que pueden afectar a las observaciones debido a la superposición de órdenes entre sí (como se indica en el panel izquierdo de la Figura 1.12) y son la aparición de líneas del segundo orden en alguno de los órdenes elevados y la problemática de que alguna línea de interés se vea afectada por las líneas de segundo orden. Este efecto de la superposición de órdenes no deseados puede eliminarse colocando un filtro antes de la red. Las redes de difracción sencillas tienen el problema de que casi toda la luz va a parar al orden cero, que es donde menos interesa. Para evitar esto la mayoría de las redes son diseñadas con forma de serrucho (o dientes de sierra), con un cierto ángulo entre los "dientes", lo que genera que el máximo de luz caiga en el orden deseado (a una dada longitud de onda). Es lo que se conoce como ángulo de blaze (ver imagen superior de la Figura 1.12), y para las redes indica donde se produce la máxima intensidad. Esto viene dado por:

$$
m \cdot \lambda=2 \sigma \operatorname{sen}(\epsilon) \cos (\alpha-\epsilon),
$$

siendo $\epsilon$ el ángulo el ángulo del braze (Chromey, 2010). Los ángulos $\alpha, \beta, \theta$ y $\epsilon$ cumplen con las siguientes relaciones:

\footnotetext{
${ }^{(i)}$ https://web.williams.edu/Astronomy/Course-Pages/211/assignment.html
} 

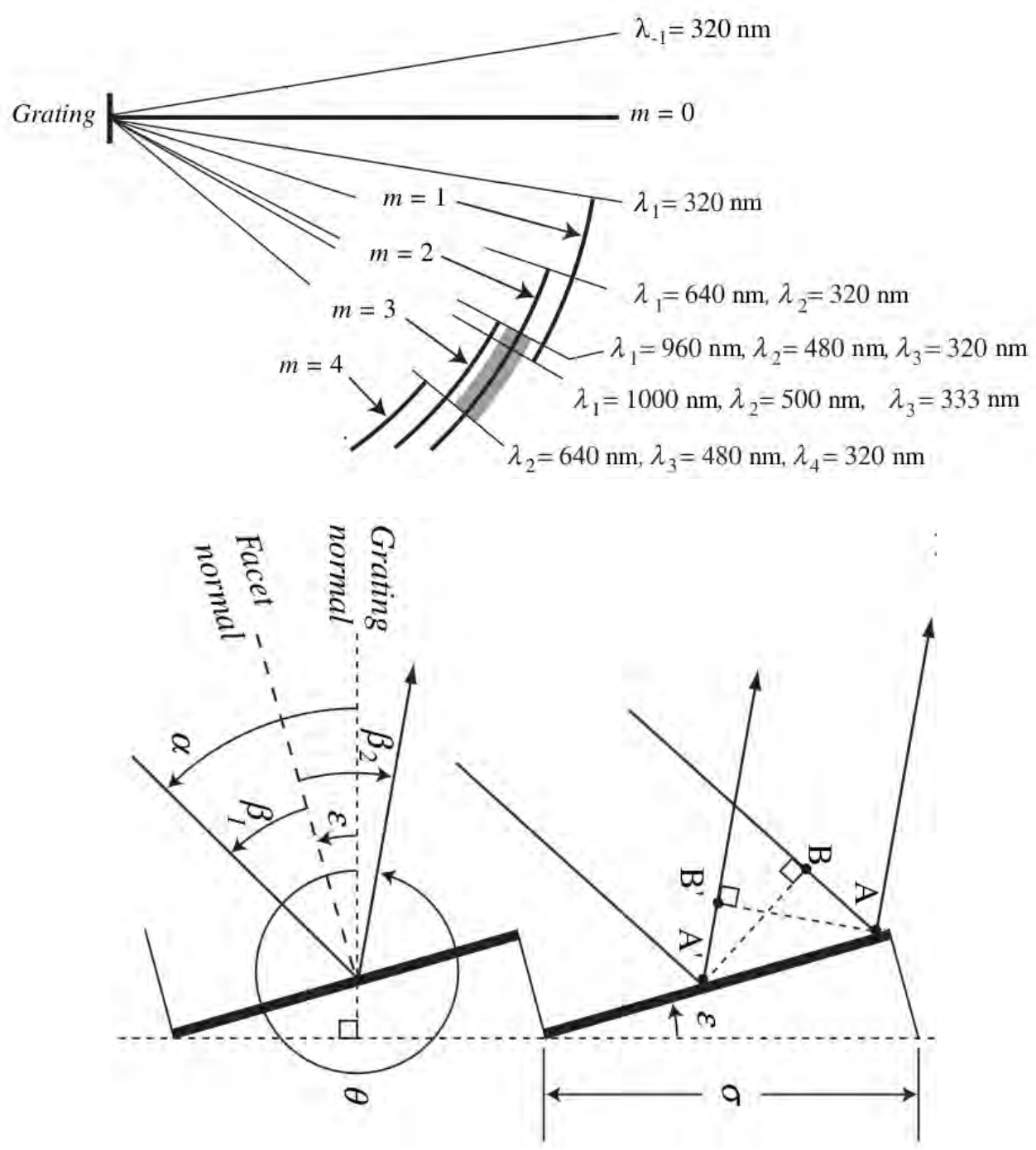

Figura 1.12. Superposición angular de los órdenes de la rendija. En la imagen superior se muestran las posiciones de los bordes del azul (tomado en $3200 \AA$ ) de órdenes -1 a 4 . El arco gris oscuro indica el rango espectral libre del segundo orden, suponiendo que $\mathrm{k}_{m} a x=6400 \AA$. La imagen inferior indica los ángulos del haz incidente y los correspondientes al blaze. Las imágenes fueron extraídas de "Astronomical optics". 


\section{(a)}

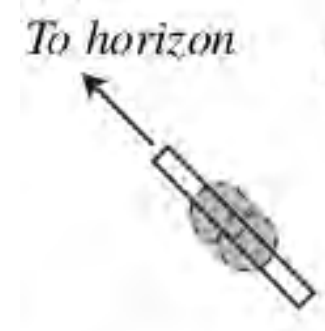

(b)

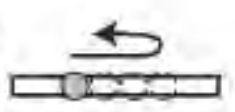

\section{To horizon}

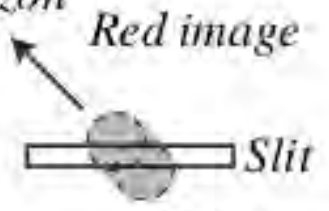

Blue image
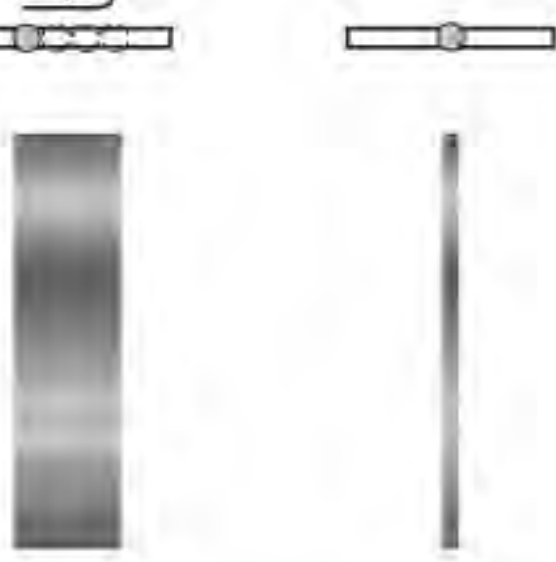

Figura 1.13. La imagen muestra la fuente desplazándose espacialmente en un intervalo de tiempo, y la importancia de colocar la rendija con el ángulo de posición correcto para no perder luz a lo largo de la observación. Imágenes extraídas de "Astronomical optics".

$$
\alpha=\beta+\epsilon, \theta=2 \pi+\epsilon-\beta ; \mathrm{y} \alpha=2 \epsilon,
$$

para que los rayos difractados estén en fase y se tengan las condiciones para una interferencia constructiva (ver imagen inferior de la Figura 1.12).

Luego, a partir de la expresión derivada de la ecuación de la red:

$$
d \theta / d \lambda=m / \sigma \cos \theta
$$

Entonces, para incrementar la dispersión angular es necesario:

- incrementar la cantidad de líneas por milímetro de la red (disminuir $\sigma$ ),

- trabajar con espectros de órdenes elevados, y de esta manera evitar los efectos de segundo orden.

Otro concepto importante a tener en cuenta de las observaciones espectroscópicas es el ángulo de posición que se elijen para las ranuras a la hora de realizar las observaciones. Se debe definir el ángulo paraláctico, el cual depende de la declinación $(\delta)$ y del ángulo horario $(t)$, que es la posición que recorre la ranura a lo largo del circulo vertical para evitar pédida de luz por efectos de la refracción diferencial producida por la atmósfera (ver Figura 1.13). 


\subsection{Objetivos del Trabajo}

El estudio de las Regiones HiI es fundamental para comprender la composición y la evolución química en el Universo, especialmente en ambientes extragalácticos. Resulta fundamental conocer si los métodos tradicionales de determinación de abundancias en nebulosas ionizadas son confiables o no. Además, es posible estudiar varios aspectos de los procesos de formación estelar a partir de la comprensión del gas ionizado que rodea a las estrellas masivas y jóvenes que se forman en sucesivos episodios en las nubes moleculares gigantes. La presente Tesis se enmarca en la astrofísica observacional sobre el estudio del gas ionizado de las Regiones HiI pertenecientes a Galaxias BCD. A través del espectro de líneas de emisión, se buscará estimar y analizar la cinemática y las propiedades físicas del gas nebular que rodea a las regiones de formación estelar. Estudiaremos, en un primer lugar, la cinemática del gas en estas Regiones Hir. Los perfiles de las líneas de emisión en estos objetos muestran evidencias de cinemáticas complejas a través de múltiples componentes de gas ionizado detectadas en dichas líneas. En un segundo lugar, se determinarán metalicidades y abundancias químicas y las propiedades físicas del gas ionizado. Esto es importante para una mejor comprensión de estos ambientes. El estudio de las propiedades del gas emisor, el cual es una consecuencia directa de la interacción del gas con los campos de energía ionizante que irradian los cúmulos estelares jóvenes (o fuentes excitatrices), permiten modelar y estudiar diversos procesos de la formación y evolución estelar. Las abundancias químicas son un aspecto determinante y para su estimación es necesario un análisis riguroso y cuidadoso de las líneas de emisión y de los errores asociados a las observaciones, mediciones y ajustes.

El objetivo fundamental de esta Tesis es completar el estudio de la quimiodinámica de una muestra de Regiones HiI pertenecientes a BCDs. La quiniodinámica fue definida por Esteban \& Vilchez (1992) donde combinaron, por primera vez, una alta resolución espectral y espacial en el rango óptico del espectro, pudiendo desarrollar un análisis de las condiciones físicas y químicas para cada componente identificada a distinta velocidad del gas ionizado y así llevar a cabo la determinación de abundancias con buena precisión utilizando el método directo para las distintas componentes cinemáticas del gas perteneciente a regiones con formación estelar en galaxias BCDs. Para lograrlo se requiere de una alta calidad de los datos (espectros con alta señal-a-ruido) y un tratamiento realista y cuidadoso de los errores, así como un análisis cinemático complejo. El análisis quimiodinámico se ha podido realizar muy pocas veces hasta el momento, incluyendo una única determinación para regiones His gigantes extragalácticas con formación estelar violenta en la galaxia Haro 15 (para más información del trabajo, ver Hägele et al., 2012). Con este trabajo buscamos ampliar la muestra de los objetos de estudio y perfeccionar la técnica de medición y calibración de la cinemática.

Presentaremos un análisis detallado de datos espectroscópicos de cinco Regiones Hiı pertenecientes a las galaxias: II Zwicky 33 y Markarian 600. Los datos han sido adquiridos con el espectrógrafo Echelle MIKE (The Magellan Inamori Kyocera Echelle) del telescopio Clay de Magallanes de 6.5m (Megallan Telescope), en el Observatorio de las Campanas (LCO), Chile, durante dos campañas de observación. En cada una de las regiones se ha cubierto un amplio rango de longitud de onda utilizando el doble brazo $(3500 \AA-9400 \AA)$, con altas resoluciones espectrales $(\mathrm{R}>30.000)$. Hemos detectado y medido cientos de líneas de emisión, las cuales corresponden a distintas especies químicas y son de origen colisional o de recombinación. Esta líneas serán fundamentales en el desarrollo de esta Tesis, en la cual abordaremos distintos temas relacionados a la dinámica, la física y la química del gas ionizado. Por lo tanto, la información que las líneas de emisión nos brinden resultarán fundamentales para cumplir con nuestros objetivos. 
Objetivos específicos:

- Reducción y calibración de las imágenes: Haciendo uso de los métodos clásicos de reducción de espectros (por ejemplo, Firpo, 2011) se procesarán las imágenes y se calibrarán los espectros en longitud de onda y flujo. Se trabajará haciendo uso de las observaciones de las lámparas de calibración y de las estrellas estándard de flujo, para realizar las respectivas calibraciones.

- Para la reducción final de los espectros, se harán pruebas y verificaciones de los ajustes utilizando la estrella estándard de flujo y se comprobará que los ajustes sean consistentes.

- Análisis de la cinemática interna del gas: continuado con las metodologías de estudio adoptadas en las Tesis del Dr. G. Hägele (Hagele, 2009) y de la Dra. V. Firpo (Firpo, 2011) se buscará determinar las múltiples componentes cinemáticas presentes en los perfiles de las líneas de emisión. Se ha propuesto una mejora en la metodología de trabajo, desarrollando un programa en Python para realizar el análisis de los ajustes de gaussianas, reemplazando al ngauss de IRAF. El programa apodado Kinematics está basado en el código LMFit ${ }^{(i)}$ para el ajuste de funciones a los perfiles de líneas. Para validar los ajustes de múltiples gaussianas, se introduce el criterio estadístico de Akaike.

- Determinación de las propiedades físicas del gas ionizado: Se continuará con la metodología de análisis desarrollada por el grupo de investigación en la serie de trabajos Hägele et al. (2006), Hägele et al. (2008), Hägele et al. (2009), Hägele et al. (2011) y Firpo (2011). Se buscará determinar las condiciones físicas del gas ionizado a partir del análisis de las líneas de emisión, tanto en las componentes individuales como para la medida global. Se estimarán temperaturas electrónicas para distintos iones: $\mathrm{T}_{e}[\mathrm{OIII}]$, $\mathrm{T}_{e}[\mathrm{OII}], \mathrm{T}_{e}[\mathrm{SII}], \mathrm{T}_{e}[\mathrm{SIII}]$ y $\mathrm{T}_{e}[\mathrm{NII}]$, utilizando el método directo, en los casos en que este fuera posible, a partir de relaciones entre las líneas intensas y las débiles aurorales. Para los casos en que no fuera posible estimar las líneas aurorales, se utilizarán relaciones empíricas entre temperaturas o se utilizará como temperatura inicial la temperatura típica para este tipo de regiones de $10^{4} \mathrm{k}$ para $\mathrm{T}_{e}[\mathrm{OIII}]$, la cual suele indicarse como la temperatura representativa de la nebulosa. Dependiendo de la señal-a-ruido (de ahora en más, $\mathrm{S} / \mathrm{N}$ del inglés signal-to-noise) espectros se buscará determinar las abundancias iónicas y total de varios elementos como $\mathrm{O}, \mathrm{S}, \mathrm{N}, \mathrm{Ne}$, Ar y He, a partir de líneas de excitación colisional y de recombinación.

- A patir de las líneas de recombinación más intensas de la Serie de Balmer ( $\mathrm{H} \alpha, \mathrm{H} \beta, \mathrm{H} \gamma$ y $\mathrm{H} \delta)$, se buscará calcular la constante de enrojecimiento $(\mathrm{c}(\mathrm{H} \beta))$ para nuestras regiones. Esto lo realizaremos a través de un ajuste lineal que utiliza los cuatro cocientes para mejorar la pendiente del ajuste.

- Estudiaremos distintos diagramas diagnósticos para las relaciones de abundancias relativas al oxígeno de los elementos mas abundantes como: S/O, N/O, Ne/O y Ar/O. Estas relaciones nos permitirán validar y comprender las abundancias relativas para las RHiI observadas en esta muestra con observaciones trabajos previos que encontramos en la literatura.

- Con la determinación de las magnitudes físicas mencionadas, buscaremos estimar los parámetros de ionización $\eta$ y $\eta$ ' para dar una medida aproximada de las temperaturas de los campos de radiación ionizantes de nuestros brotes intensos de formación estelar.

(i)https://lmfit.github.io/lmfit-py/ 
- En los casos que fuera posible, se calcularán las abundancias totales de oxígeno a partir de la calibración de parámetros empíricos. Propondremos distintos calibradores empíricos para la determinación tanto de las temperaturas electrónicas como de las abundancias químicas de nuestras regiones, a partir de la información obtenida de las componentes cinemáticas del gas.

Estos enfoques nos permitirán concretamente:

- Determinar componentes cinemáticas en los perfiles complejos de líneas de emisión.

- Definir componentes de distintas naturalezas.

- Establecer criterios para indicar cuales son los modelos correctos planteados para la solución de la cinemática (Bosch et al., 2019).

- Estimar densidades y temperaturas electrónicas para las regiones dadas.

- Determinar las propiedades físicas del gas y de abundancias químicas precisas a partir del método directo.

- Realizar un tratamiento detallado y cuidadoso de los errores.

- Obtener abundancias de distintos elementos químicos como: O, S, N, Ne, Ar y He, presentes en el gas ionizado.

- Determinar estructuras de ionización y abundancias químicas utilizando métodos empíricos y semiempíricos

- Completar un estudio Quimiodinámico para nuevas galaxias y presentar datos novedosos. 



\section{Capítulo 2}

\section{Observaciones y reducción de datos}

En este Capítulo presentamos la descripción detallada de las observaciones que se llevaron a cabo para la realización de este trabajo, así como de los pasos seguidos para extraer los espectros unidimensionales calibrados en longitud de onda y flujo, listos para ser analizados.

\subsection{Importancia y tipo de observaciones}

El desarrollo experimental del presente trabajo se ha basado en imágenes de espectros de alta resolución espectral de cinco regiones Hir gigantes pertenecientes a dos galaxias BCD, obtenidas con el espectrógrafo Echelle "Magellan Inamori Kyocera Echelle" (de ahora en adelante $\mathrm{MIKE}^{(\mathrm{i})}$ ) en dos campañas de observación en el telescopio Clay de los Magallanes, en el Observatorio Las Campanas, Chile. Dichas campañas tuvieron lugar en los meses de noviembre del 2005 y en enero del 2006, y fueron llevadas a cabo por las Doctoras Verónica Firpo y Nidia Morrell.

La idea de medir líneas de recombinación del H y He y prohibidas débiles de [OII], [OIII], [SII], [SIII] y [NII] utilizando un instrumento de alta calidad como MIKE junto al gran telescopio de 6.5m Magallanes, surgió a raíz de la publicación de Firpo et al. (2005). A partir de un estudio espectroscópico detallado de un conjunto de Regiones HiI Gigantes en galaxias espirales medidas con el telescopio de 2.5m Irene DuPont y con el Clay 6.5m de Magallanes, donde se lograron medir líneas de emisión de varios iones de distintos elementos químicos. Luego, en el trabajo realizado por Firpo et al. (2011) se logró llevar a cabo un estudio completo y detallado de cinemática usando las líneas más intensas y en el de Hägele et al. (2012) se realizó por primera vez un estudio completo y detallado de las propiedades físicas del gas ionizado para distintas componentes cinemáticas medidas en los perfiles de las líneas de emisión más intensas de los espectros de la galaxia Haro 15, utilizando imágenes también del telescopio DuPont. Este fue un trabajo pionero que concretó un estudió quimiodinámico detallado para cuatro nudos de emisión intensos de dicha galaxia, que mostró resultados muy interesantes sobre la cinemática de los nudos de formación estelar intensa. En esta Tesis se busca ampliar la muestra de regiones de formación estelar para estudiar los comportamientos químicos y la cinemática del gas de dichas regiones pertenecientes a este tipo de galaxias para comprender de manera más clara propiedades como las densidades electrónicas, las abundancias químicas y las relaciones entre los distintos elementos químicos, con la profundidad que espectros de alta resolución brindan.

\footnotetext{
${ }^{(i)}$ Para más información detallada sobre el espectrógrafo: Bernstein et al. (2003). En la página web del Observatorio Las Campanas (https://www.lco.cl) se encuentra toda la información técnica y observacional del espectrógrafo.
} 
Como hemos comentado en el Capítulo 1 Sección 1.5, para observar líneas prohibidas débiles se necesita de tiempo de telescopio y de instrumentos adecuados. Los resultados encontrados en Haro 15 dieron nuevas perspectivas sobre el análisis de múltiples componentes cinemáticas en los perfiles de líneas de regiones de formación estelar intensas en galaxias, para ser estudiadas con instrumentos de mayor envergadura. Esto posibilitaría la obtención de imágenes más profundas de los brotes de formación estelar, que faciliten el análisis de múltiples componentes cinemáticas de líneas débiles con mejor relación señal-a-ruido. Esta combinación de alta resolución espectral y estudio de la cinemática compleja, busca completar un análisis quimiodinámico de las regiones. Los resultados obtenidos en Haro 15 demostraron que con observaciones profundas pueden obtenerse resultados novedosos, que aportan nueva información para los estudios de evolución de galaxias, formación estelar y campos de radiación.

En el análisis desarrollado en el trabajo de esta Tesis hemos podido medir líneas de recombinación de Helio y de las series de Balmer del Hidrógeno, líneas prohibidas débiles aurorales (además obviamente de las líneas colisionales fuertes) que empleamos para la estimación de las temperaturas. Por su parte, se midió el doblete del azufre ([SII] $\lambda \lambda 6717,6731 \AA$ ) para la determinación de densidades electrónicas. El rango espectral de las observaciones es algo crucial para llevar a cabo este trabajo, ya que se requieren de coberturas desde el extremo azul del espectro óptico para poder observar el doblete de las líneas de [Оіг] $\lambda \lambda 3726,3729 \AA$, hasta el extremo del IR cercano (más allá de los $9000 \AA$ ) para alcanzar a medir las líneas del $[\mathrm{SIII}] \lambda \lambda 9069 \AA 9 \AA^{2} 9532 \AA$, necesarias para el análisis de las abundancias químicas iónicas y totales de dichos elementos.

Las regiones más brillantes de las galaxias BCDs pertenecen a regiones HII gigantes que son el indicador de las regiones de formación estelar violenta. Además, los brotes de formación estelar seleccionados fueron escogidos según el flujo de emisión de $\mathrm{H} \alpha$ publicado, para garantizar la detección de algunas líneas débiles prohibidas, que se estima que son posibles de observar bajo ciertas condiciones y fundamentales para el desarrollo de nuestro trabajo. Siguiendo estos criterios se han analizado cinco regiones pertenecientes a dos galaxias BCD: II Zwicky 33 (tres regiones) y Markarian 600 (dos regiones). Dichos espectros fueron obtenidos en dos turnos de observación de cuatro noches en total, dos en noviembre del 2005 y dos en enero de 2006 (respectivamente), en los que se tomaron las imágenes de ciencia y de calibración de dichas regiones (más adelante se detallan en la tabla ver 2.2).

\subsection{Descripción de las galaxias observadas}

Las galaxias estudiadas en esta Tesis corresponden a dos objetos muy estudiados en la bibliografía desde los catálogos de los 60' de Zwicky (Zwicky, 1965; Zwicky \& Rudnicki, 1966), hasta trabajos recientes (Walter et al., 1997; Méndez et al., 1999; Cairós et al., 2001; Gil de Paz et al., 2003a; Gil de Paz \& Madore, 2005; Amorín et al., 2009) en los cuales se han llevado a cabo análisis fotométricos detallados de sus estructuras y sus características observacionales. Sin embargo, la quimiodinámica de estas galaxias no ha sido estudiada hasta la fecha. Nuestro trabajo resulta novedoso ya que se analizan múltiples componentes del gas ionizado de sus regiones de formación estelar más intensas.

\subsubsection{Zwicky 33}

La primera galaxia estudiada es IIZw 33 la cual aparece en los primeros catálogos de galaxias enanas de Zwicky (1965) y que fuera analizada en detalle por Walter et al. (1997) y por Cairós et al. (1998), quienes reportan la presencia de un gran número de estrellas 


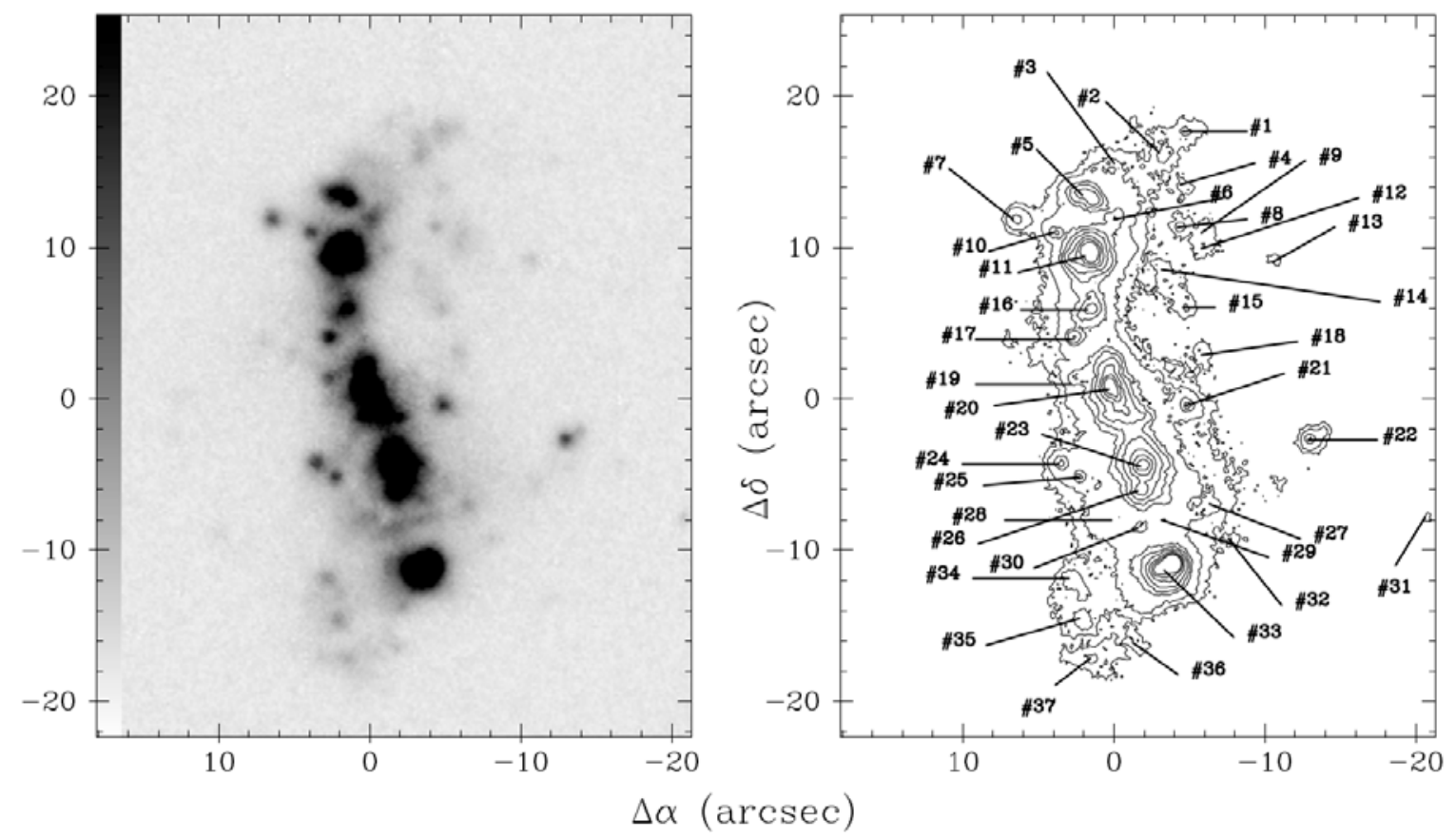

Figura 2.1. Representación en escala de grises (izquierda) y diagrama de contorno (derecha) de la imagen de $\mathrm{H} \alpha$ de II Zw 33. Las etiquetas en el diagrama de contorno refieren a los diferentes nudos de emisión de $\mathrm{H} \alpha$ donde están ocurriendo episodios de formación estelar. El norte está en la parte superior y el este a la izquierdaen la figura. La imagen es adoptada de Méndez et al. (1999).

Wolf Rayet (de ahora en adelante WR). En este último trabajo se determinaron los mapas de dispersión de velocidades con valores de hasta $24 \mathrm{~km} / \mathrm{s}$, lo que confirma actividad de formación de estrellas. Al observar la imagen en la banda de $\mathrm{H} \alpha$ de II Zw 33 publicada en dicho trabajo, podemos identificar estructuras complejas de diferentes nudos de formación estelar que han sido resueltos espacialmente (ver en la Figura 2.1 los contornos de emisión en $\mathrm{H} \alpha$ ). Se han determinado más de treinta nudos intensos distribuidos a lo largo de una barra central con dos elongaciones difusas en cada uno de sus extremos, que parecen seguir los cortos brazos espirales vistos en la imagen presentada por Walter et al. (1997). Por otro lado, Méndez et al. (1999) indican que los eventos de formación estelar más recientes se distribuyen en diferentes nudos a lo largo de la barra central, y que a su vez la formación estelar tendría sus comienzos en el centro de la galaxia. Posteriormente se desarrollaría en una barra compuesta por nudos intensos de formación estelar hacia las partes más externas de la galaxia, en dirección nortesur.

II Zw 33 es definida en este último trabajo como una galaxia tipo WR (dado que muestra características espectrales asociadas a la presencia de un alto número de estrellas WR, ver Walter et al., 1997) rica en gas, con una compañera de bajo brillo superficial cuya masa de Hi resulta similar a la de II Zw 33, mientras que sus propiedades ópticas son diferentes. La distancia estimada a II Zw 33 es $36.4 \mathrm{Mpc}$ calculada suponiendo una constante de Hubble $\mathrm{H}_{0}=75 \mathrm{~km} \mathrm{~s}^{-1} \mathrm{Mpc}^{-1}$, tomando como referencia las distancias de Virgo Infall (Amorín et al., 2009). En los datos publicados por Cairós et al. (2001) la $\mathrm{m}_{B}$ es 14.64 mag y el B-V estimado con fotometría integrada es de $0.68 \pm 0.04$, en acuerdo con el B- $\mathrm{V}=0.81$ estimado por Amorín et al. (2009), donde este exceso en B indicaría la presencia de población jóven. 


\subsubsection{Markarian 600}

Markarian 600 (en adelante Mrk 600; Markarian et al., 1989) es una galaxia compacta azul (BCD) iE según la clasificación del catálogo de Gil de Paz \& Madore (2005). Sin embargo, la forma alargada y la presencia de varias regiones más débiles más allá del cuerpo principal de la galaxia indican una morfología cometaria. La formación estelar activa en esta galaxia se concentra principalmente en las dos nudos de emisión $\mathrm{H} \alpha$, correspondientes a Regiones HiI Gigantes. Los nudos de emisión resueltos espacialmente son consistentes con las regiones de formación estelar asociadas a los brotes de estrellas jóvenes. La distribución de estas Regiones HiI puede ser el resultado de una interacción reciente dada la presencia de una compañera cercana solo observable en hidrógeno neutro (HI) (Taylor et al., 1993) como lo indica Noeske et al. (2005). Estudios realizados por Augarde et al. (1990) en base a espectros de ranura larga, indican una metalicdad de $1 / 8 \mathrm{Z}_{o}$. En dicho trabajo se identifican los dos nudos principales de gas ionizado por estrellas calientes con temperaturas efectivas del orden de $T_{e f} \approx 50.000 \mathrm{~K}$, que están inmersos en una envoltura roja muy tenue correspondiente a una población estelar vieja. Las observaciones pueden explicarse en términos de un período de formación estelar a un ritmo constante durante los últimos $1.5 \times 10^{8}$ años.

Estimaciones de las abundancias de oxígeno fueron realizadas por Guseva et al. (2011) donde obtuvieron $12+\log (\mathrm{O} / \mathrm{H})=7.88 \pm 0.03$. Los índices de color $\mathrm{B}-\mathrm{V}$ determinados en un estudio fotométrico por Cairós et al. (2001) indican un índice B-V global de 0.26, en acuerdo con $\mathrm{B}-\mathrm{V}=0.31$ calculado por Amorín et al. (2009). Del estudio de estos mapas, queda claro que las BCD generalmente tienen fuertes gradientes de color, con colores que se vuelven más rojos hacia las zonas más externas. Se estimó a partir del $\mathrm{F}(\mathrm{H} \alpha) \approx 3.3 \pm 0.2\left(10^{-13} \mathrm{erg} \mathrm{cm}^{-2} \mathrm{~s}^{-1}\right)$ una luminosidad de $\log (\mathrm{L}(\mathrm{H} \alpha)) \approx 39.95 \mathrm{erg} \mathrm{s}^{-1}$ y una tasa de formación estelar de $0.01 \mathrm{M}_{\odot} \mathrm{yr}^{-1}$. Las regiones del formación estelar se han resuelto en dos nudos compactos, mientras que en otras de las varias galaxias estudiadas por dichos autores las regiones más intensas con brotes de formación se distinguen claramente de la población subyacente de estrellas más viejas. Lagos et al. (2018) estudiaron los dos nudos principales (A y B) y regiones secundarias más débiles (ver Figura 2.2), utilizando observaciones de VIMOS-IFU y estimaron sus abundancias totales de oxígeno en $7.85 \pm 0.09$ y $7.83 \pm 0.01$, respectivamente.

La distancia determinada en Amorín et al. (2009) es de $\approx 12.81 \mathrm{Mpc}$, según la referencia de las distancias de Virgo Infall, siendo una galaxia relativamente cercana a la Vía Láctea en comparación con Haro $15(\approx 84.15 \mathrm{Mpc})$ o II Zw $33(\approx 36 \mathrm{Mpc})$. 


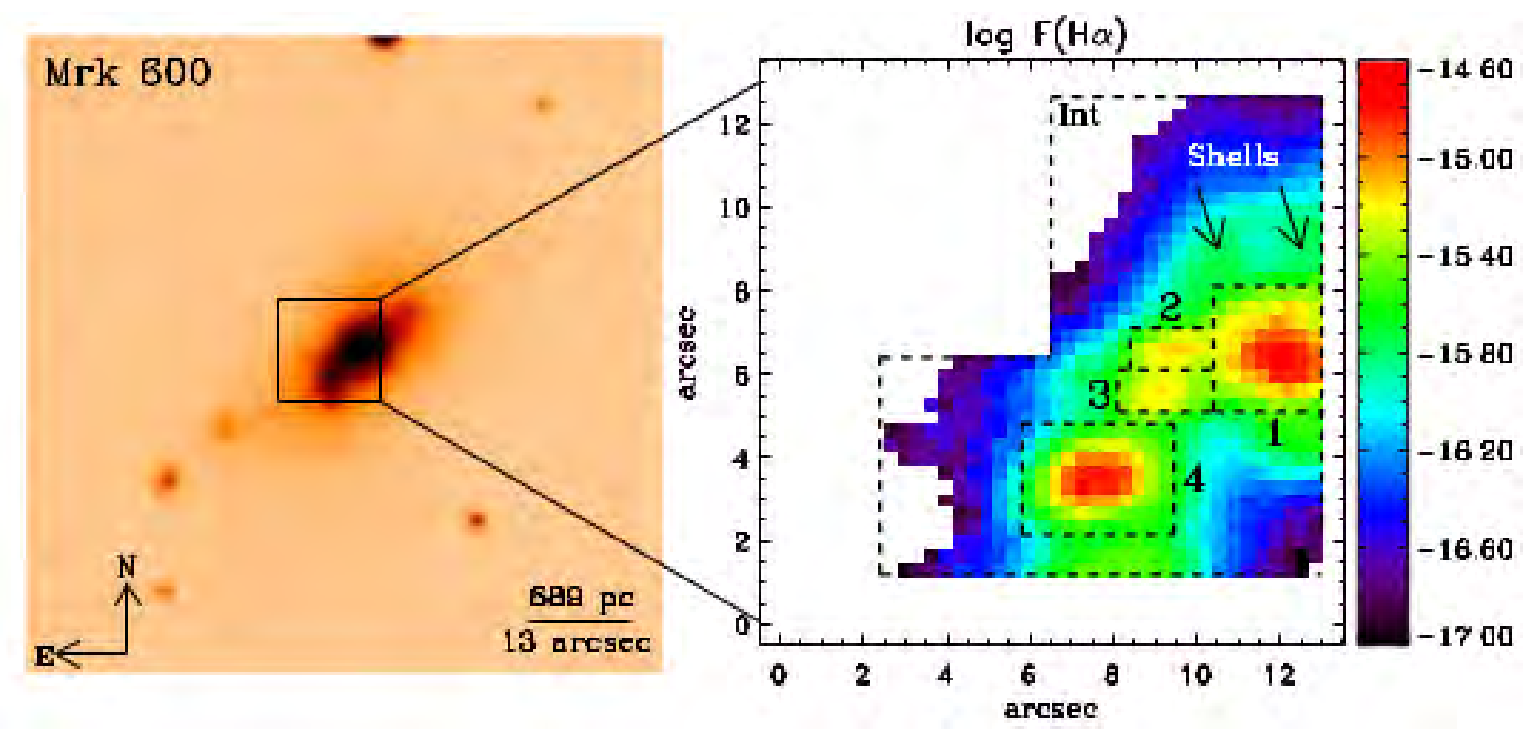

Figura 2.2. Izquierda: Representación de la banda $R$ adoptada de Gil de Paz et al. (2003b). Derecha: mapas de flujo de emisión $\mathrm{H} \alpha$, en escala logarítmica, para el campo de visión de VIMOS-IFU de $13^{\prime \prime} \times 13^{\prime \prime}$ (imagen adoptada de Lagos et al., 2018). Las regiones indicadas con lineas punteadas 1 y 3 , corresponden a los Nudos A y B, respectivamente. Estas son las regiones que abarcaremos en este trabajo.

\begin{tabular}{|l|c|c|}
\hline & Brazo Azul & Brazo Rojo \\
\hline Escala del CCD & $0.12^{\prime \prime} / \mathrm{pix}$ & $0.13^{\prime \prime} / \mathrm{pix}$ \\
\hline A $/$ pix & -0.02 & -0.05 \\
\hline Detector & $2048 \times 4096(15 \mu \mathrm{m}$ pix $)$ & $2048 \times 4096(15 \mu \mathrm{m} \mathrm{pix})$ \\
\hline Ganancia & $-0.47 \mathrm{e}^{-}$ & $-1.0 \mathrm{e}^{-}$ \\
\hline Ruido de Lectura & $-2 \mathrm{e}^{-} / \mathrm{pix}$ & $-3.5 \mathrm{e}^{-} / \mathrm{pix}$ \\
\hline Rangos de $\lambda$ & $3200-5100$ & $4900-9800$ \\
\hline Resolución $\left(0.35^{\prime \prime}\right.$ slit $)$ & 83.000 & 65.000 \\
\hline Resolución $\left(1.0^{\prime \prime}\right.$ slit $)$ & 28.000 & 22.000 \\
\hline
\end{tabular}

Tabla 2.1. Características de cada uno de los detectores. En la segunda columna lo referente al brazo azul y en la tercera al brazo rojo (Tabla extraída de http://www.lco.cl/?epkb_ post_type_1=the-mike-magellan-inamori-kyocera-echelle-users-guide).

\subsection{Observaciones Espectroscópicas}

MIKE es un espectrógrafo Echelle con dispersión cruzada de dos brazos (rojo y azul) que permite cubrir todo el rango espectral óptico en forma simultánea a través de detectores en dos brazos independientes. El haz de luz incidente es dividido por un dicróico que refleja/transmite la luz a partir de una longitud de onda crítica a cada uno de los brazos, los cuales a su vez disponen de su propio obturador y cámara CCD, por lo que se pueden usar simultáneamente con tiempos de exposición independientes. Esto permite realizar distintos estudios espectrales de una misma fuente. Las especificaciones básicas de cada brazo se enumeran en la Tabla 2.1.

Los parámetros que el observador puede elegir son la cobertura en longitud de onda, el ancho y el largo de la rendija. Para nuestras observaciones se escogieron dos rendijas de distintos anchos: $0.75^{\prime \prime}$ (segundos de arco) para II Zw 33 y 0.35" para Mrk 600. Ambas galaxias fueron observadas con rendijas de $5^{\prime \prime}$ de largo. El espectrógrafo ofrece una cobertura de longitud de onda completa del espectro óptico de aproximadamente $3300 \AA-5100 \AA$ en el brazo azul y de 


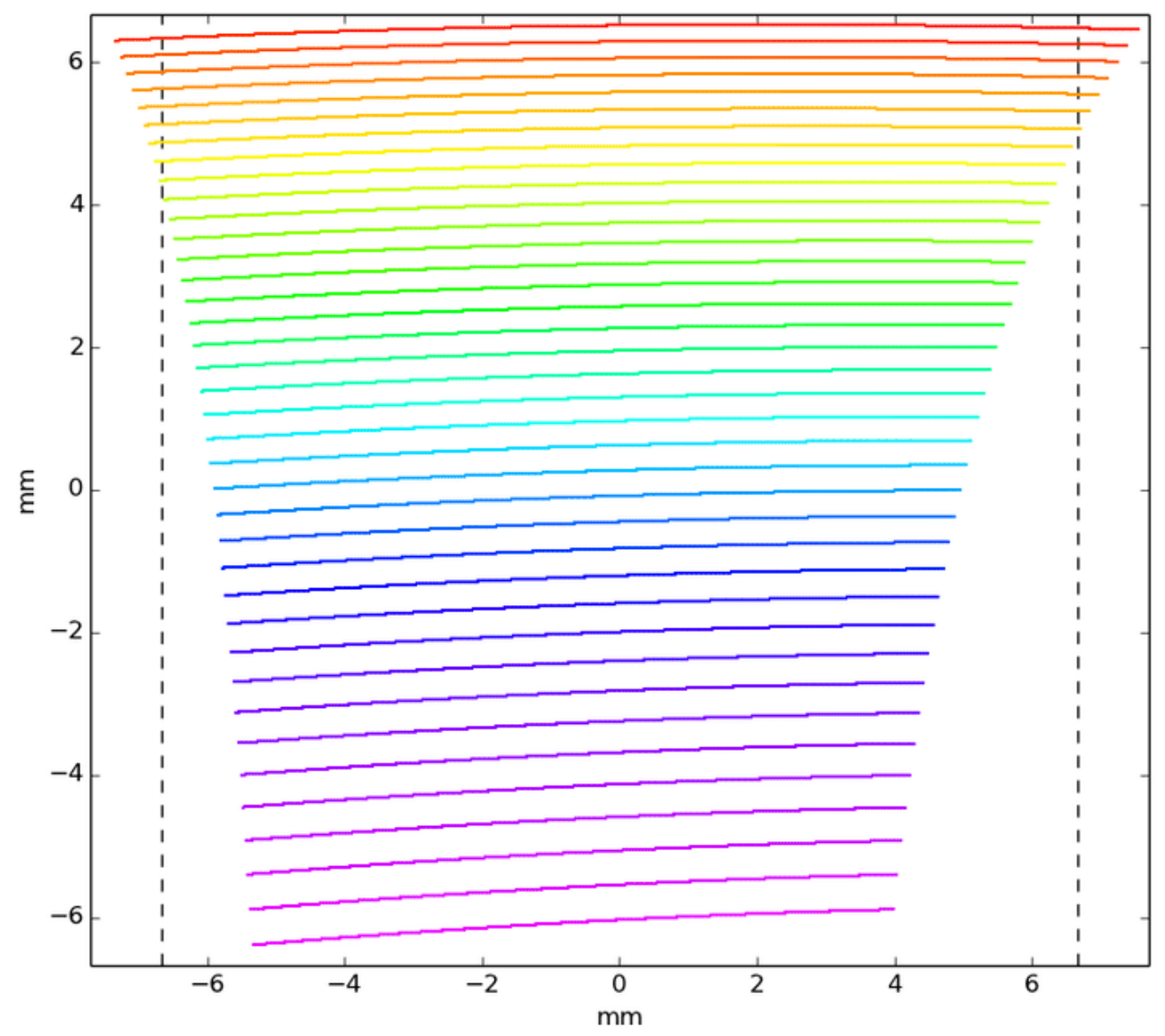

Figura 2.3. Cada línea corresponde con un orden espectral. Los órdenes del Echelle abarcan la mayor parte del rango espectral desde los $3500 \AA$ a $9500 \AA$. Las líneas verticales discontinuas indican con los bordes del detector CCD, lo que muestra que los órdenes más extremos al rojo se encuentran cortados ligeramente. Imagen extraída de: https ://www.researchgate. net/.


mediante la longitud de onda del dicróico en $4950 \AA$. Como observador, puede elegir que se ajuste el ángulo del dispersor cruzado de manera de seleccionar el rango espectral cubierto en cada brazo, y así alcanzar el extremo azul (hasta $3200 \AA$ ) y/o el rojo (hasta $10000 \AA$ ). Pero no es posible utilizar dicróicos, dispersores o prismas diferentes. Estos elementos son permanentes.

Para la realización de las observaciones, se usó la configuración estándar que dispone el espectrógrafo en ambos brazos, con lo que se cubrió una amplia región espectral: de $3350 \AA$ a $9400 \AA$. Cada brazo dispone de dos redes de dispersión cruzada trabajando en el primer orden espectral. Los rangos de longitud de onda han sido observados en su totalidad, con excepción de unos intervalos en los órdenes del extremo rojo a partir de los $6500 \AA$ hasta los $\approx 9400 \AA$, debido a que los órdenes más extremos al rojo se ven levemente truncados por los bordes del detector (ver Figura 2.3).

La rendija se situó con orientación este-oeste en las observaciones $\left(\mathrm{PA}=0^{\circ}\right)$, y se usó 
el corrector de dispersión atmosférica (Atmospheric Dispersion Corrector) con el objetivo de mantener la misma región observada dentro del área cubierta por la rendija a pesar de los cambios en los valores de la masa de aire, por los efectos atmosféricos mencionados en el capítulo anterior. Los anchos de las ranuras elegidos nos devuelven espectros con resoluciones $R \approx 30.000$ en el rojo y $R \approx 40.000$ en el azul, para $0.75^{\prime \prime} \times 5^{\prime \prime}$, y la máxima para la ranura de $0.35^{\prime \prime} \times 5^{\prime \prime}(R \approx 65.000$ para el brazo rojo y $\mathrm{R} \approx 83.000$ para el brazo azul) como lo indicamos en la Tabla 2.1. Dichos anchos fueron elegidos con el fin de obtener una buena relación señal-a-ruido (de ahora en más $\mathrm{S} / \mathrm{N}$ ) en nuestros espectros, manteniendo la resolución requerida para separar e identificar las líneas débiles necesarias para este proyecto, habiendo cambiado la ranura a la más pequeña en el segundo turno de observación para obtener una mejor resolución. Las condiciones atmosféricas de todas las noches fueron buenas, con seeing promedio de $\sim 0.6$.

Para el turno de observación en el que se observaron los nudos A, B y C de II Zw 33, se realizó una sola observación por nudo de 1200s, mientras que en en el segundo turno donde se observó a Mrk 600, se observaron los dos nudos principales, A y B, con 3 observaciones de 1200s cada una, con el fin de combinarlas y obtener una imagen resultante con mayor relación $\mathrm{S} / \mathrm{N}$. Además, combinar imágenes individuales nos permite eliminar los rayos cósmicos que contaminan a las observaciones. Realizar una sola observación de 60 minutos no es recomendable ya que el número de rayos cósmicos crece y la remoción por algoritmos de detección se hace imposible. Además, el seguimiento del telescopio en observaciones largas puede ser un riesgo. También se tomaron exposiciones más cortas, de dos minutos, para las estrellas estándar de flujo y para la lámpara de calibración se tomaron imágenes de cinco segundos. En la Figura 2.4 presentamos en ambos paneles las imágenes de las galaxias (extraídas del $\mathrm{NED}^{(\mathrm{i})}$ ) para indicar la ubicación de las Regiones Hit Gigantes estudiadas, y superpuestas las rendijas con sus respectivos tamaños y posiciones para cada una de las galaxias. En la Tabla 2.2 se muestra un esquema con las fechas de observación (columna 3), los tiempos de exposición (columna 4), el promedio de las masa de aire de las observaciones (columna 5), así como para las estrellas utilizadas para calibrar en flujo (columna 6).

\begin{tabular}{|l|c|c|c|c|c|}
\hline Obs & Nudos & Fecha & Tiempo de Exp. & Masa de aire & Estándar de flujo \\
\hline Echelle & II Zw 33 & & & & \\
& A B C & 2005 nov 21 & $1 \times 1200 \mathrm{~s}$ & 1.219 & GD108 \\
& $\begin{array}{c}\text { Mrk 600 } \\
\text { A B }\end{array}$ & 2006 ene 10 & $3 \times 1200 \mathrm{~s}$ & 1.215 & Feige110 \\
\hline
\end{tabular}

Tabla 2.2. Registro de las observaciones. En la segunda columna presentamos los nudos de las galaxias observados con la nomenclatura utilizada en este trabajo; las columnas 3 y 4 enumeran la fecha de observación y los tiempos totales de exposiciones, la columna 5 las masas de aire y la columna 6 indica las estrellas estándard para la calibración en flujo.

\footnotetext{
${ }^{(i)}$ https://ned.ipac.caltech.edu/
} 


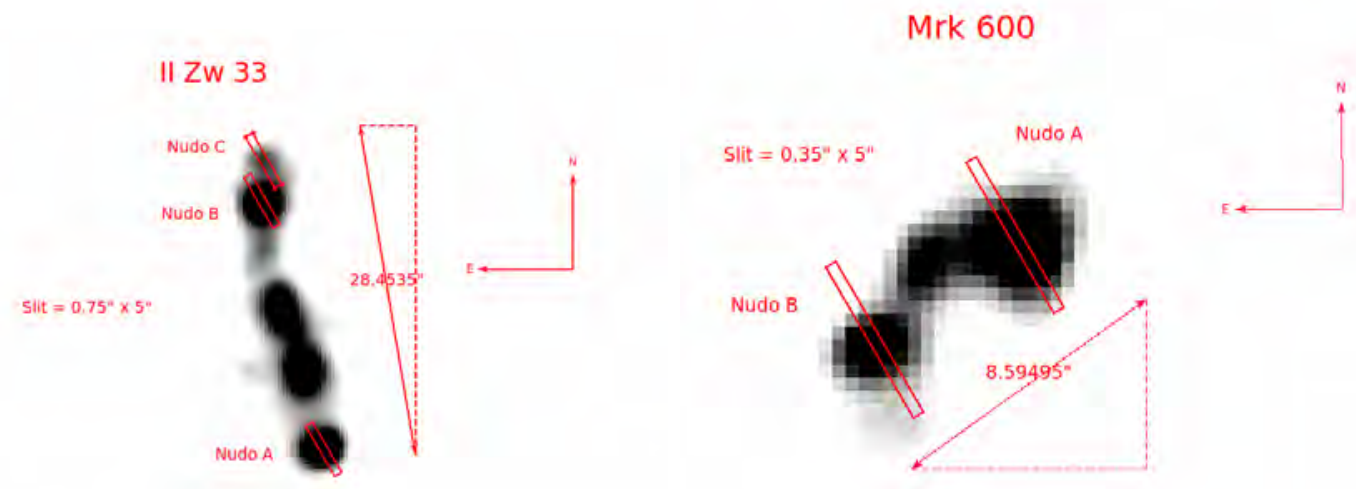

Figura 2.4. Panel izquierdo: Imagen de la banda angosta $\mathrm{H} \alpha$ de II $\mathrm{Zw} 33$. En rojo las tres rendijas correspondientes a los nudos de formación estelar observados. Panel derecho: Imagen de la banda angosta $\mathrm{H} \alpha$ de Mrk 600. En rojo las dos rendijas correspondientes a los brotes de formación estelar observados. Ambras imágenes fueron obtenidas con el telescopio Du Pont del Observatorio las Campanas (LCO).

\subsection{Proceso de reducción y calibración de las observaciones}

Uno de los ejes de este trabajo ha sido el análisis minucioso llevado a cabo para la correcta reducción y calibración de los datos espectrales. Cada una de las observaciones ha tenido distintos atributos que las ha distiguido y les ha dado una identidad especial. Debido a esto, hemos tenido que recurrir a exigentes procesos de reducción. Una correcta manipulación de los datos es fundamental y sumamente importante para que los espectros finales calibrados en longitud de onda y flujo estén en óptimas condiciones para poder ser estudiados. En este capítulo describimos los pasos de reducción más importantes y los distintos caminos que hemos elegido para obtener los espectros reducidos.

\subsubsection{Extracción del espectro y calibración en longitud de onda}

El procesamiento de los datos fue realizado siguiendo la forma tradicional a través del software para análisis y reducción de imágenes astronómicas IRAF (ver Firpo, 2011, y referencias en él). Los espectros de nuestras regiones presentan una gran cantidad de líneas de emisión intensas de diversos iones, que son fundamentales para plantear nuestro trabajo y para realizar la extracción del espectro, a partir de algunas líneas puntuales. La S/N de nuestras líneas en algunos casos es muy buena $\left(\mathrm{S} / \mathrm{N}_{H \alpha} \approx 400, \mathrm{~S} / \mathrm{N}_{[O i i i]} \lambda 5007 \AA \approx 400\right)$ y esto es uno de los puntos fundamentales para poder llevar adelante dicho trabajo, ya que nos permitirán estudiar el comportamiento cinemático del gas ionizado, el cual aplicaremos para descomponer los perfiles de las líneas prohibidas más débiles, como son las líneas aurorales. En esta sección indicamos la importancia de las líneas de emisión para llevar adelante la extracción del espectro y los distintos caminos que llevaron a optimizar la reducción hasta obtener espectros finales de buena calidad.

Cada conjunto de datos obtenidos en las noches de observaciones contienen todas las imágenes necesarias para poder realizar la correcta extracción y reducción de los datos. Están compuestos por imágenes de calibración: Flats, Bias y lámpara de comparación, e imágenes de ciencia, estrella estándar de flujo e imágenes correspondientes a la ciencia (en la Figura 2.5 se muestran ejemplos de imágenes de calibración).

Lo primero que hacemos es desplegar en el programa SAO Image DS9 las imágenes, para 


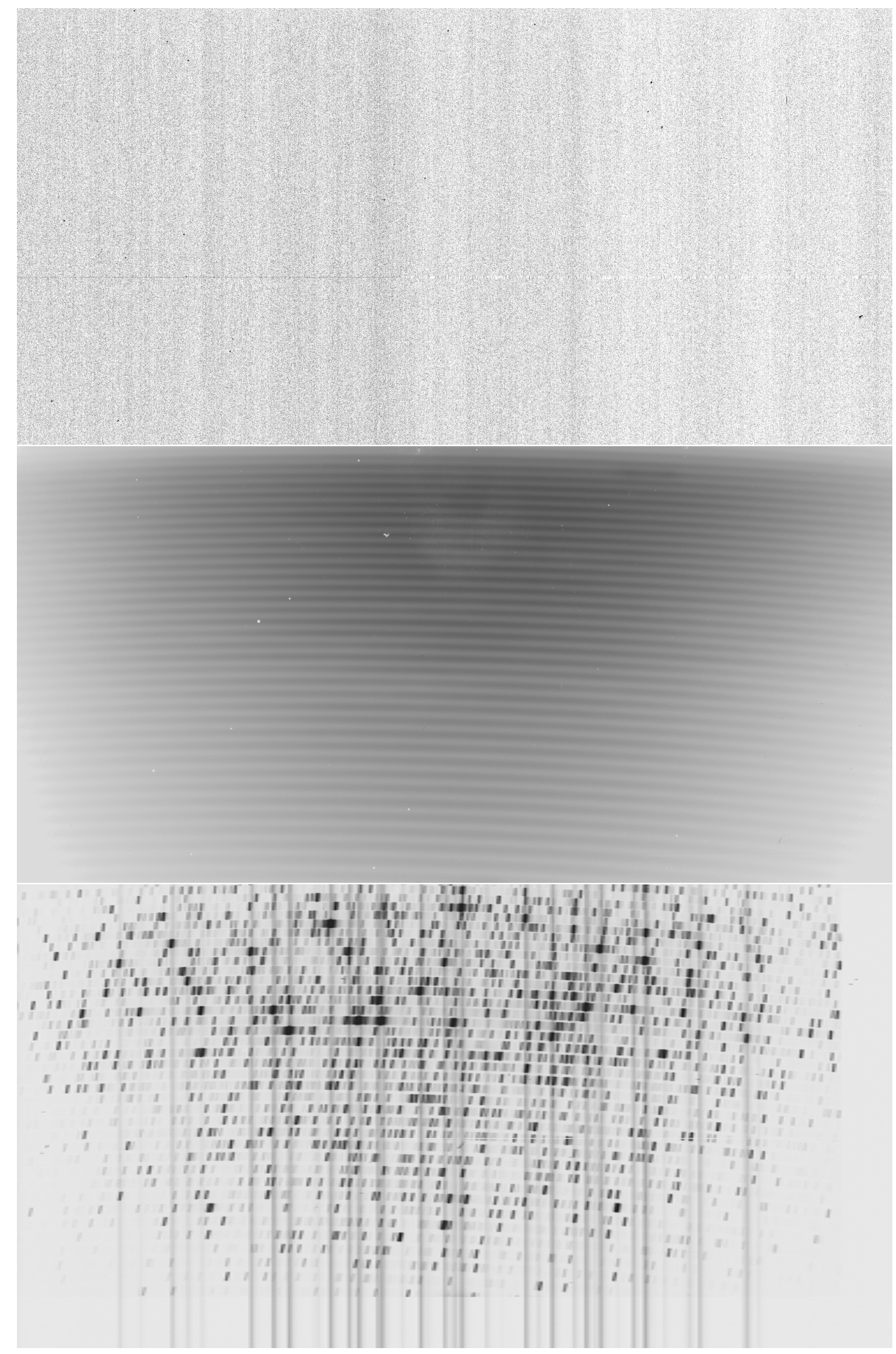

Figura 2.5. Ejemplo de bias (panel superior), b) flat field (panel central) y lámpara de calibración (panel inferior). 


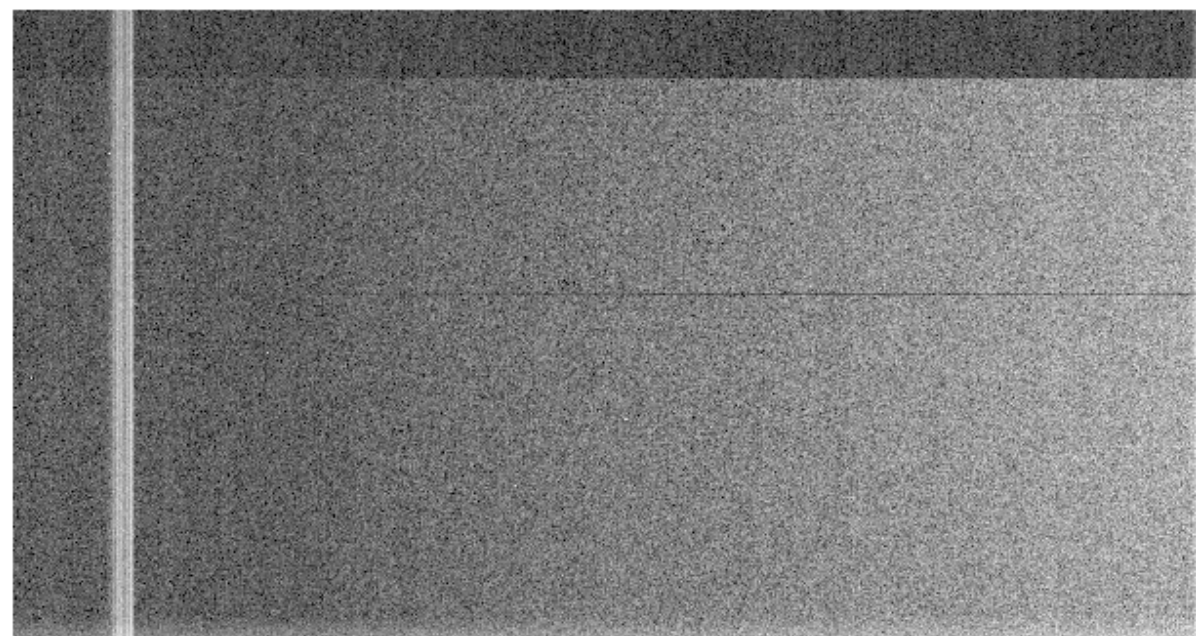

Figura 2.6. Imagen de un Bias que tuvo un problema durante el proceso de lectura y presenta varias columnas defectuosas. Estas imágenes no tienen que ser tenidos en cuenta para generar el master bias.

ver que aspecto presentan. Es decir, si tienen columnas malas, píxeles defectuosos o alguna otra particularidad (ver ejemplo de un bias defectuoso en la Figura 2.6) que nos que indique que deban ser descaratadas. De dicha manera se identifican una por una cada imagen y se las agrupa según su naturaleza: bias, flats, ciencia, estrella estándar; para procesarlas en grupos, según convenga.

Cada imagen FITS (del inglés Flexible Image Transport System, el cual es el formato de archivo utilizado comúnmente en astronomía) tiene toda la información referida a la observación, en un header. Este es fundamental para poder conocer parámetros necesarios en la reducción, como el tiempo de exposición, masa de aire, angulo de posición del slit, ángulo paraláctico, instrumento, etc. Para visualizarlo se utiliza la tarea imheader $l+$. Esto es muy útil para previamente clasificar las imágenes y saber a que tipo corresponde cada una. Entonces, una recomendación útil es agrupar en listas diferentes cada una de las imágenes, para llamarlas luego todas juntas, refiriéndolas al archivo correspondiente (por ejemplo, bias.dat y flat.dat).

Las imágenes presentan un sector conocido como overscan que es una zona que contiene información de la electrónica del detector. Es una zona del CCD que no se expone. En ella se registra el nivel de pedestal que se agrega a cada lectura, y que resulta variable imagen a imagen (porque depende de la temperatura y del tiempo). En el proceso de reducción se mide ese nivel de pedestal, y se resta a las imágenes. En el caso de los espectros de esta Tesis, hay que corregir por overscan a todas las imágenes. Hay instrumentos donde no se aplica esta correccion, ya que si es estable, estará en cada imagen (y al restar el bias, entonces se anula). Además, dentro de este primer paso, se realiza el trimming donde se recorta la imágen, descartando las columnas y filas más cercanas a los bordes del detector, que pueden tener algunos errores de lectura o cuentas que no son confiables a causa de los mismos efectos de borde, y y la zona del overscan que como dijimos no tiene datos. Entonces, se utiliza la tarea ccdproc disponible en los paquetes imred (imagen reduction), ccdred (ccd reduction), Echelle. Aquí se indican los rangos en píxeles con los que se va a trabajar, eligiendo las dimensiones del overscan y del trimming.

Una vez corregido por overscan, continuamos con la corrección de las imágenes. A continuación se detalla qué son los Bias y los Flats.

- BIAS: es una imagen tomada con el obturador cerrado y con tiempo de exposición 
cero. Contiene el offset de cada px́el en relación al nivel de pedestal que se registra en el overscan. Es diferente pixel a pixel, pero es fijo para cada pixel. Ese nivel de pedestal registra el ruido de lectura del amplificador (aleatorio) y el ruido electrónico de la cámara, por lo que la resta del BIAS permite remover estos efectos de la imagen.

- FLAT FIELD: es una imagen de una superficie uniformemente iluminada sobre la cual se realiza un ajuste que corrige de manera global las deficiencias cuánticas del telescopio e instrumento intruducidas en las imágenes. Para dicho ajuste suelen utilizarse polinomios de órdenes bajos para evitar los efectos en los residuos y defectos en los bordes. Al dividir a las imágenes de ciencia por el ajuste efectuado en los flats se corrigen las variaciones de la imagen.

En un espectro que se obtiene iluminando la ranura con una lampara blanca, y se mide como responde pixel a pixel a la longitud de onda que cae en cada píxel (que es distinta). Se ajusta con un polinomio de orden bajo que intenta corregir el flat por el color de la lamara. De esa forma, una vez que se corrige el flat por dicho polinomio (se lo normaliza por dicho polinomio), resulta una imagen que solo contiene la variación de sensibilidad píxel a píxel (que además depende de la longitud de onda).

Para corregir por Bias generamos una imagen "Superbias", combinando todos los bias y hacemos un promedio con la tarea zerocombine. Esta imagen resultante es utilizada para la resta sobre las imágenes de ciencia. La imagen bias (o de nivel cero) se genera a partir de un promedio de $\mathrm{n}_{\text {bias }}$ tomados con un tiempo de integración cero. El ruido de lectura en una imagen de ciencia al restar el Superbias es:

$$
\text { Ruidolectura }_{\text {lo }}=R d \cdot \sqrt{1 / n_{\text {bias }}},
$$

donde $\mathrm{Rd}$ es el ruido de lectura del CCD. Los bias se combinan para hacer una imagen promedio de las cuentas y restársela a las imágenes de los flats y de esta expresión se puede ver por qué es necesario tomar las imágenes del bias y promediarlas para reducir el ruido de lectura que se va generando por la resta de una imagen a otra. En esta tarea hay que prestar atención en modificar los ruidos de lecturas y ganancias correspondientes al detector de cada brazo de MIKE con los que fueran realizadas las observaciones, para cada reducción.

Luego, el ruido total es la suma de las siguientes contribuciones:

- ruido propio de la señal: toda señal proveniente de una fuente luminosa tiene un ruido inherente asociado. La naturaleza de la emisión en sí misma, el brillo observado de una estrella es el valor medio del número de fotones emitidos por unidad de tiempo y la dispersión de dichos valores es igual a la raíz cuadrada del valor medio. Es decir, ruido $\mathrm{S}=\sqrt{\operatorname{señal}}$ y en donde se define a $\mathrm{S}$ como el ruido poissoniano de la señal en sí.

- ruido de lectura. Este ruido es la contribución del CCD al leer la carga de fotones.

- ruido de fondo. Al observar objetos débiles esta señal aumenta y su contribución es mucho mayor que las otras fuentes.

Continuando con el procesamiento de los Flats, se lleva a cabo por intermedio de la tarea flatcombine, donde se genera un Superflat combinando todas las imágenes de los Flats, que son fundamentales para corregir la no uniformidad global y a gran escala del detector CCD. Para esto se necesitan imágenes de un campo suficientemente plano y con un nivel alto de cuentas para reducir el impacto del ruido de lectura contra el poissoniano, y que el S/N crezca con $\sqrt{S}$ (>10000 cuentas). La idea de la división por el ajuste de los flats es para corregir los bumps y falta de uniformidad que hay en los espectros. Con estos pasos estamos en condiciones de 
corregir a las imágenes de ciencia por ambos efectos, entonces en ccdproc indicamos como entrada a nuestra lista con las imágenes de ciencia (por ejemplo, @ciencia.dat) y corregimos por Bias y Flat. Como resultado obtenemos las imágenes corregidas por efectos propios de un detector tipo CCD.

\subsection{Extracción de los espectros de ciencia}

En el proceso de obtener el espectro final, hemos emprendido distintos análisis y estudios a lo largo de la tesis. En esta sección se detallan los pasos necesarios para obtener los espectros con la mayor calidad posible para los estudios llevados a cabo en esta Tesis.

Corregidas las imágenes por Bias y Flat, para la extracción del espectro se utiliza la tarea apall del paquete Echelle o imred. Pero antes, es necesario realizar la remoción de los rayos cósmicos de las imágenes. Los rayos cósmicos son partículas muy energéticas que generan un gran número de electrones libres al impactar en el semiconductor del CCD. La cantidad de rayos cósmicos presentes en una imagen es proporcional al tiempo de exposición con la que fue tomada la misma. Como nuestra ciencia requiere de tiempos de exposición prolongados para poder sumar la mayor cantidad de flujo proveniente de estas regiones de formación estelar, indefectiblemente se van a ver afectadas por los rayos cósmicos. Para los espectros de II Zw 33 se han empleado algortimos de limpieza que buscan rayos en una imagen individual. Otras formas requieren del empleo de múltiples imágenes, que en el caso de esta galaxia no disponemos.

Para su identificación y limpieza hemos utilizado dos programas de extracción distintos: el CosmicRay de IRAF, y el Detect and Remove Cosmic Rays ${ }^{(\mathrm{i})}$ (dcr). Hemos procedido a realizar primero una limpieza de los más intensos con el CosmicRay, y luego, una extracción más profunda con el dcr. Por medio de esta limpieza, se limpia al espectro de impurezas que pueden generar problemas en la determinación de propiedades a partir de las líneas de emisión (ver Figura 2.7; allí mostramos un espectro bidimensional, la máscara de identificación de los rayos cósmicos y la resta resultante a la imagen de ciencia).

Ambas tareas brindan una limpieza necesaria de los rayos sobre el espectro original 2$\mathrm{D}$, para luego continuar con la reducción sobre espectros con menores contaminantes que pueden alterar el ajuste o interpretación de las líneas particulares de interés, ya que vamos a medir emisiones. Las tareas funcionan correctamente y la identificación que realizan de rayos cósmicos es muy precisa, es decir líneas que no tienen anchos que puedan llegar a confundirse con líneas de emisión. Por el lado de las líneas de cielo, en nuestro análisis no nos hemos tomado el trabajo de restarlas ni de analizar esta sección del espectro debido a que las líneas de interés no se ven afectadas en estos corrimientos al rojo. Hay algunas líneas débiles que pueden verse afectadas pero en ese caso decidimos no hacer la resta del cielo solo por una línea débil que no era crucial para llevar adelante nuestro análisis.

Los espectros de esta clase de objetos estudiados en la presente Tesis se caracterizan por tener una muy baja emisión del continuo estelar, y por la presencia de líneas de emisión claramente identificables en las longitudes de onda correspondientes. Debido a esto, resulta imposible realizar una extracción de espectros de forma rutinaria sino que es necesario utilizar otros objetos como guía. Lo primero que hay que hacer es obtener una traza de un objeto con buena emisión de continuo para luego realizar la extracción del espectro de ciencia utilizando dicha traza. Para ello tomamos la estrella estándar de flujo para definir la traza (ver Figura 2.8), extraer el espectro y luego, tomar esta solución para la obtención de los espectros unidimensionales de ciencia sin calibrar en longitud de onda ni en flujo.

\footnotetext{
${ }^{(i)}$ http://users.camk.edu.pl/pych/DCR/
} 


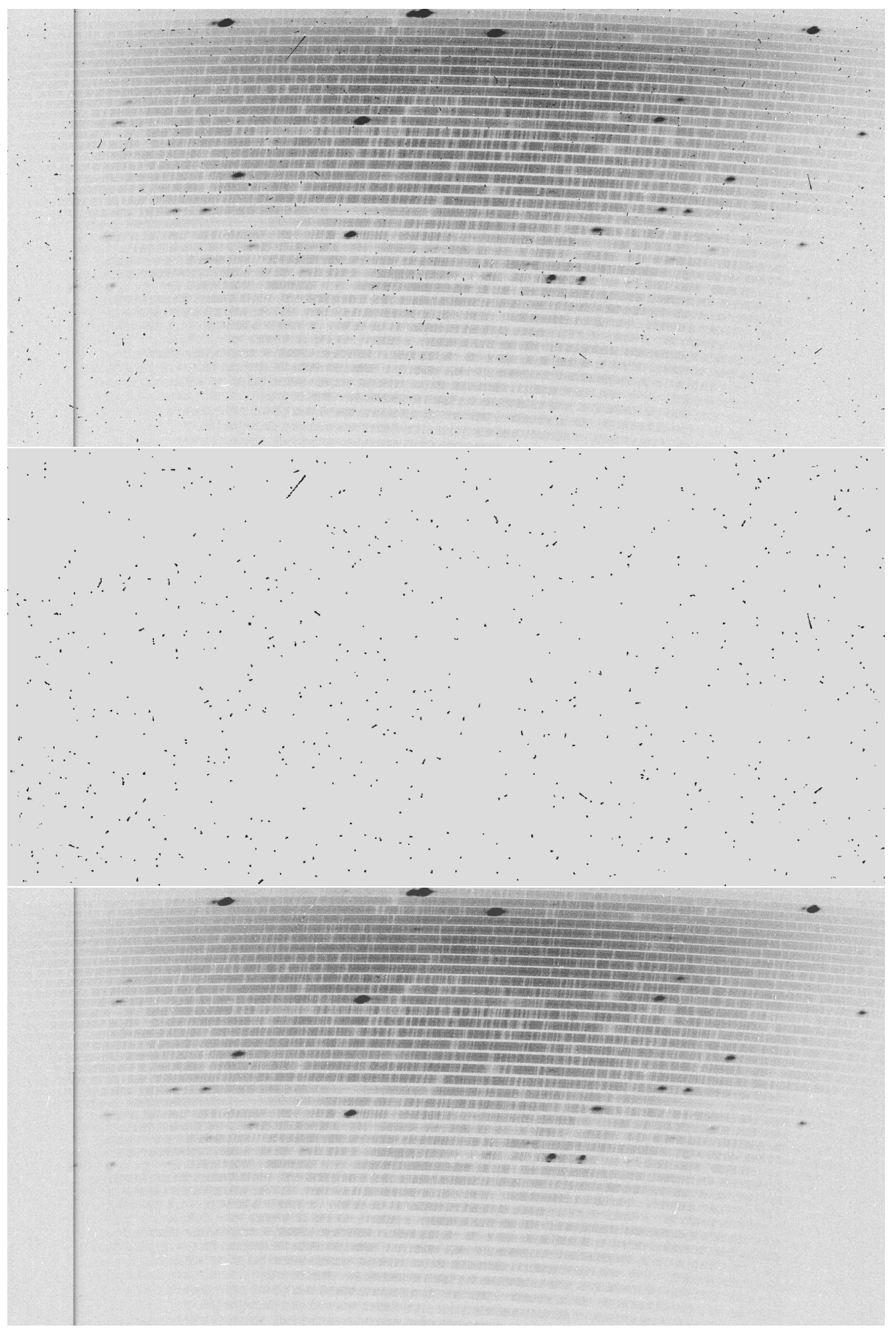

Figura 2.7. Imagen de un espectro Echelle del nudo A de Mrk600 donde se aprecia en la imagen superior el espectro con rayos cósmicos, en la central la máscara de identificación de rayos cósmicos y en la inferior la resta de los rayos al espectro de ciencia. La última imagen corresponde al espectro sobre el cual se realizó la extracción de los datos. 

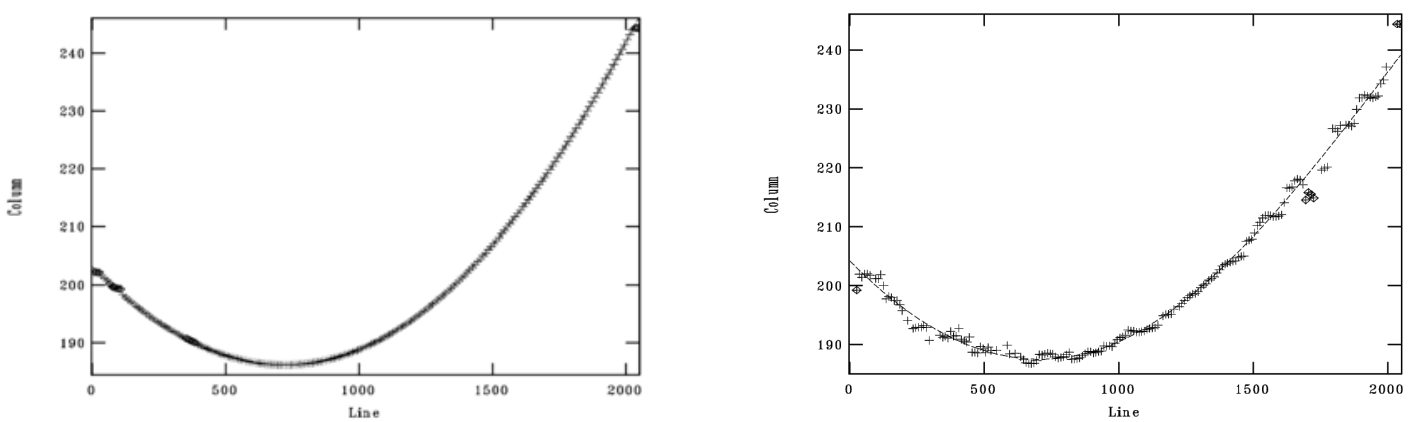

Figura 2.8. Traza de uno de los órdenes ajustados en la estrella estándar de flujo (izquierda) y copiado el ajuste para la imagen de ciencia con pobre nivel de continuo (derecha).

Con dicha traza, determinada con la estrella estándar, tomamos como referencia a las líneas más intensas, como $\mathrm{H} \alpha$, [OIII] $\lambda 5007 \AA$ y $\mathrm{H} \beta$, siguiendo los procedimientos publicados en la Tesis de la Dra. Verónica Firpo. Además extraemos el plano de varianza y no restamos el background, ya que en estos objetos difusos y extendidos, la resta del background puede afectar señal en las líneas débiles y esto sería un grave problema.

En los parámetros de extracción indicamos que se despliegue la ventana interactiva (interactive $=$ yes) y definimos la apertura sobre la cual vamos a extraer el espectro. Con el display abierto en el $D S 9$, se identifica la línea de referencia para realizar la extracción, definiendo la regiones de extracción en píxeles (ver Figura 2.9).

Tratándose de objetos extendidos, en muchos casos resueltos espacialmente, la definición del tamaño de la ventana de extracción fue objeto de varias pruebas hasta alcanzar el criterio óptimo en el cual definimos la región según la intensidad de la zona central hasta que esta decae a un $50 \%$. De esta manera, al tomar esa ventana nos aseguramos de no incluir en la extracción señales espurias. Resulta interesante mencionar que originalmente, en las primeras extracciones y calibraciones de los espectros, tomamos como ventana de extracción a todo el ancho del orden del Echelle (ver en el panel inferior de la Figura 2.9 la sección de color verde), para integrar toda la señal proveniente de la RHII.

Las líneas de emisión en el espectro 2-D tenían aspectos levemente distintos. En algunos casos, se puede observar cómo la línea parece tener contribución de zonas espacialmente separadas, que no necesariamente pertenecen al brote de formación estelar principal, sino que posiblemente pertenezca a una región muy cercana, la cual contamina el perfil compuesto final. Dado esto, decidimos rehacer las extracciones considerando únicamente como ventana de extracción la zona de intensidad máxima de la línea en el orden del Echelle. Esta decisión es el producto de diversas pruebas luego de las cuales privilegiamos minimizar las fuentes de contaminación sobre la recolección del mayor número de fotones y resignamos un poco de señal para mejorar la confiabilidad del resultado.

Estas ventanas tiene un ancho de entre 10 y 14 píxeles, dependiendo de las regiones observadas, y así omitimos cualquier contaminación debido a la presencia de regiones débiles cercanas a nuestra región. En la Figura 2.9 se presentan dos imágenes correspondiente a Mrk600 en la región azul del espectro, en las cuales se observa la contaminación en la línea por una emisión que no pertenece al nudo bajo estudio sino que proviene de una region distinta, espacialmente distinguible.

En el panel superior vemos las distintas líneas de emisión del UV del espectro en el ran-

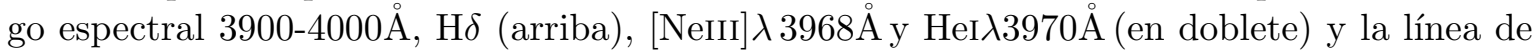
recombinación de H 8 (debajo a la izquierda), con la emisión del nudo central y la contaminación hacia el límite inferior del orden de la rendija. Y en el panel inferior podemos ver 


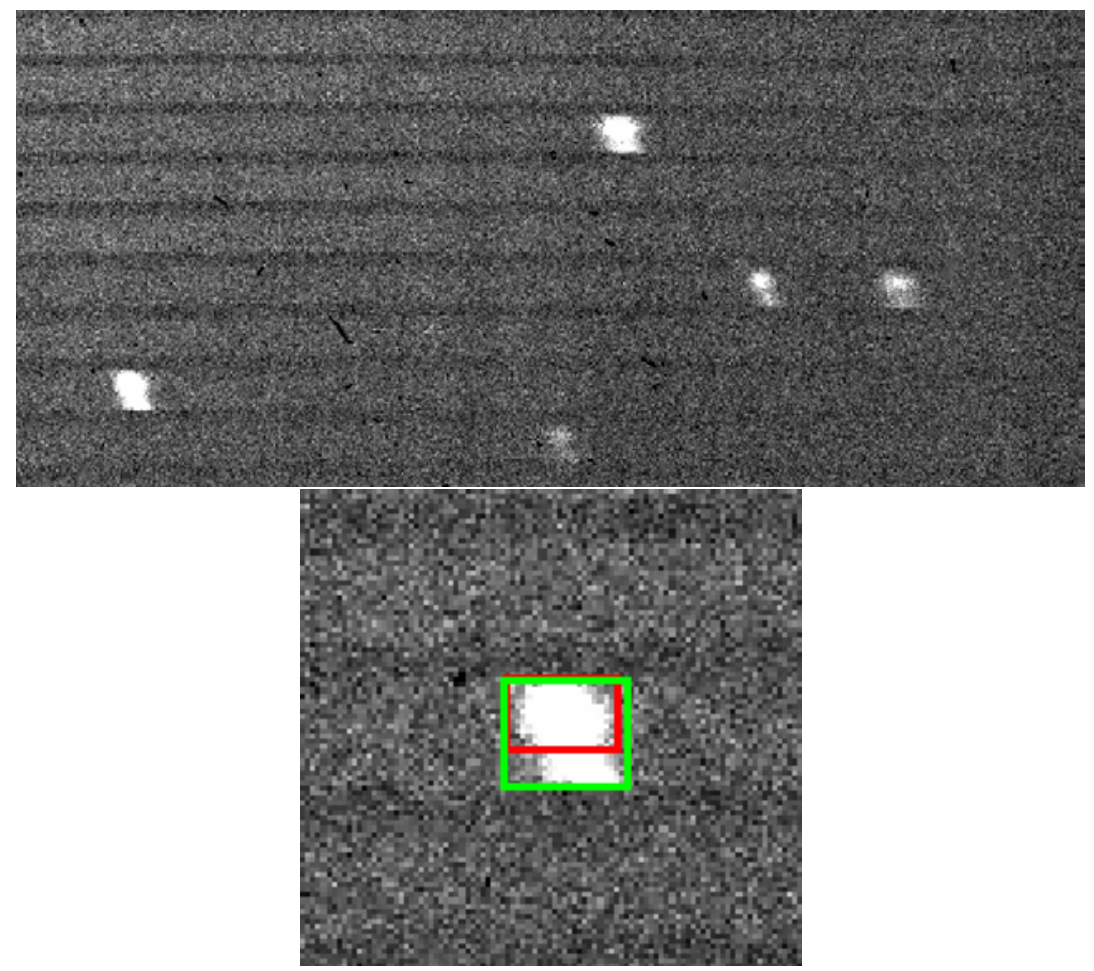

Figura 2.9. En el panel superior se identifican distintas líneas de emisión en el rango espectral entre $\approx 3800-4000 \AA$ : H $\delta$ (arriba), [NeIII] $3968 \AA$ y HeI $\lambda 3970 \AA$ (doblete central hacia la derecha) y H8 (debajo a la izquierda más intenso), con la emisión del nudo central y una emisión débil hacia el límite inferior de la ranura.

Por otra parte, en el panel inferior podemos ver dos casillas superpuestas sobre la línea de emisión $\mathrm{H} \delta$, una verde que corresponde al tamaño total del slit de 20 píxeles y otra roja, que corresponde a la ventana de extracción final que hemos considerado que es de $\approx 13$ píxeles.

dos cajas, una verde que corresponde al tamaño total del slit de 20 píxeles y otra roja, que corresponde a la ventana de extracción final que hemos considerado que es de 13 píxeles aproximadamente. Este trabajo nos permitió obtener finalmente unos espectros con bajos niveles de contaminación de flujo en sus líneas, para lo cual fue necesario separar de la zona de extracción a las componentes espacialmente resueltas así como también a regiones con poca señal que introducen ruido en las líneas (ver Figura 2.10).

La extracción de los espectros que resulta del apall está en cuentas vs. píxeles, y para la calibración final deben estar representados en flujo vs. longitud de onda. Por lo que el paso siguiente, es calibrar a los espectros en longitud de onda, tarea que se lleva a cabo con ecidentify. Como se indicó anteriormente, las observaciones tienen intercaladas los espectros de la lámpara de calibración, el cual se utiliza para hacer una óptima calibración en longitud de onda por medio de las líneas de emisión conocidas. En este caso, hemos trabajado con lámparas de Th-Ar (Tório y Argón) donde cada orden espectral contiene una cantidad de líneas que pueden ser identificadas a partir de su atlas respectivo (ver Figura 2.11), que puede encontrarse en la página de los observatorios como datos de archivo (https://www.lco.cl, en nuestro caso). Para una correcta calibración, es necesario identificar líneas a lo largo del orden (seis líneas por orden es un número suficiente), pero resulta fundamental, en caso de poderse, identificar líneas en los bordes de los órdenes que se encuentren en dos órdenes consecutivos debido a la superposición de estos en longitud de onda. Esto es un buen ejercicio 



Figura 2.10. Líneas de $[\mathrm{OII}] \lambda 3726,29 \AA$. Los paneles superiores corresponden al Nudo A de Mrk600. A la izquierda se presenta el espectro extraído con una ventana menor (13 pix). A la derecha la extracción del espectro con una ventana de igual tamaño total del orden (20 pix). Los paneles inferiores corresponden al Nudo C de II Zw 33. Ambas representan una extracción del espectro teniendo en cuenta distintas aperturas. 
para que el ajuste final de la solución tenga un buen acuerdo orden a orden.

Una vez identificadas las líneas en todos los órdenes, se ajustó una solución y se completó la calibración en longitud de onda. El ajuste de la solución será bueno o malo dependiendo del valor del rms (la desviación del valor cuadrático medio). El rms lo consideramos uno de los parámetros más importantes a tener en cuenta, y lo determinamos a partir de la distribución de los residuos (ver Figura 2.12a). Si la desviación media de la solución es baja, se está ajustando un polinomio que tiene un error relativo bajo punto a punto y en donde además la distribución de los residuos presenta una forma uniforme. Esto garantiza una solución confiable en longitud de onda. Para la identificación de líneas de la lámpara que nos facilite la buena calibración en longitud de onda primero se suelen tomar parámetros generales, y luego se aproxima la función de ajuste con un polinomio de orden 5 con un número total de líneas de una baja cantidad (unas 300), un margen de separación mínima, un umbral mínimo de identificación, y un match=1. Luego de una primera identificación de líneas en los bordes y en los centros de cada órden, se procede a hacer un primer ajuste, y luego de este se comienzan a variar los parámetros de identificación y así se va iterando hasta mejorar la solución. Este proceso es fundamental para obtener ajustes con dispersiones bajas. En los paneles de la Figura 2.12a se pueden ver tres ajustes distintos donde se fueron mejorando los valores de los parámetros de identificación hasta obtener un rms del ajuste muy bueno.

Este ajuste puede ser aplicado como solución inicial a otras lámparas de calibración tomadas con la misma configuración y luego las utilizamos como referencia para las imágenes de ciencia, por medio de la tarea refspec, donde asignamos a cada imagen de ciencia su respectiva lámpara de referencia. Una vez que se tiene el archivo de calibración asociado a las imágenes, por medio de la tarea dispcor se aplica la corrección por dispersión. De esta manera, quedan calibrados los espectros en longitud de onda.

\subsubsection{Calibración en flujo}

Para medir flujos emitidos por cada línea de interés y así derivar condiciones físicas del gas ionizado debemos transformar los espectros de cuentas a flujo. Para ello se utiliza a la estrella estándar tomada en los turnos de observación junto a las imágenes de ciencia. En este procedimiento, es necesario disponer del archivo calibrado de la estándar en longitud de onda, para hacer la calibración en flujo.

Se ha utilizado la estrella estándar de flujo GD 108, descargada de la página ${ }^{(i i)}$ de la ESO (European South Observatory), donde hay una amplia base de datos de distintos objetos observados con el espectrógrafo UVES (Ultraviolet and Visual Echelle Spectrograph) y pueden descargarse los espectros calibrados de varias de las estrellas estándar de flujo. Se encuentran archivos con distintas resoluciones y con distintos pasos (p. e. $0.1 \AA_{p x^{-1}}^{-1} 1.2 \mathrm{pp}^{-1}, 3 \mathrm{ppx}^{-1}$ ) que pueden servir para tener calibraciones en flujo más precisas, dependiendo de la ciencia que se lleve adelante.

Los pasos a seguir para la calibración en flujo utilizando IRAF, se deben realizar utilizandon tres tareas: standard, sensfunction y calibrate. En la primera, se selecciona el espectro de nuestra estrella estándar calibrado en longitud de onda, y luego se hace un "match" para identificar intervalos del espectro medido en cuentas. Se puede optar por ver en modo interactivo el espectro orden a orden y la superposición de este con la tabla de la estándar calibrada y eliminar por ejemplo intervalos de puntos que corresponden a regiones del espectro con bajo nivel de cuentas, los cuales están dominados por ruido de lectura del detector.

Haciendo esto orden a orden se genera una tabla de valores de cuentas y sus correspondientes flujos (o magnitudes) que usaremos en una segunda etapa (sensfunction) para el ajuste

\footnotetext{
${ }^{(\text {ii })}$ https://www.eso.org/sci/facilities/paranal/instruments/uves.html
} 


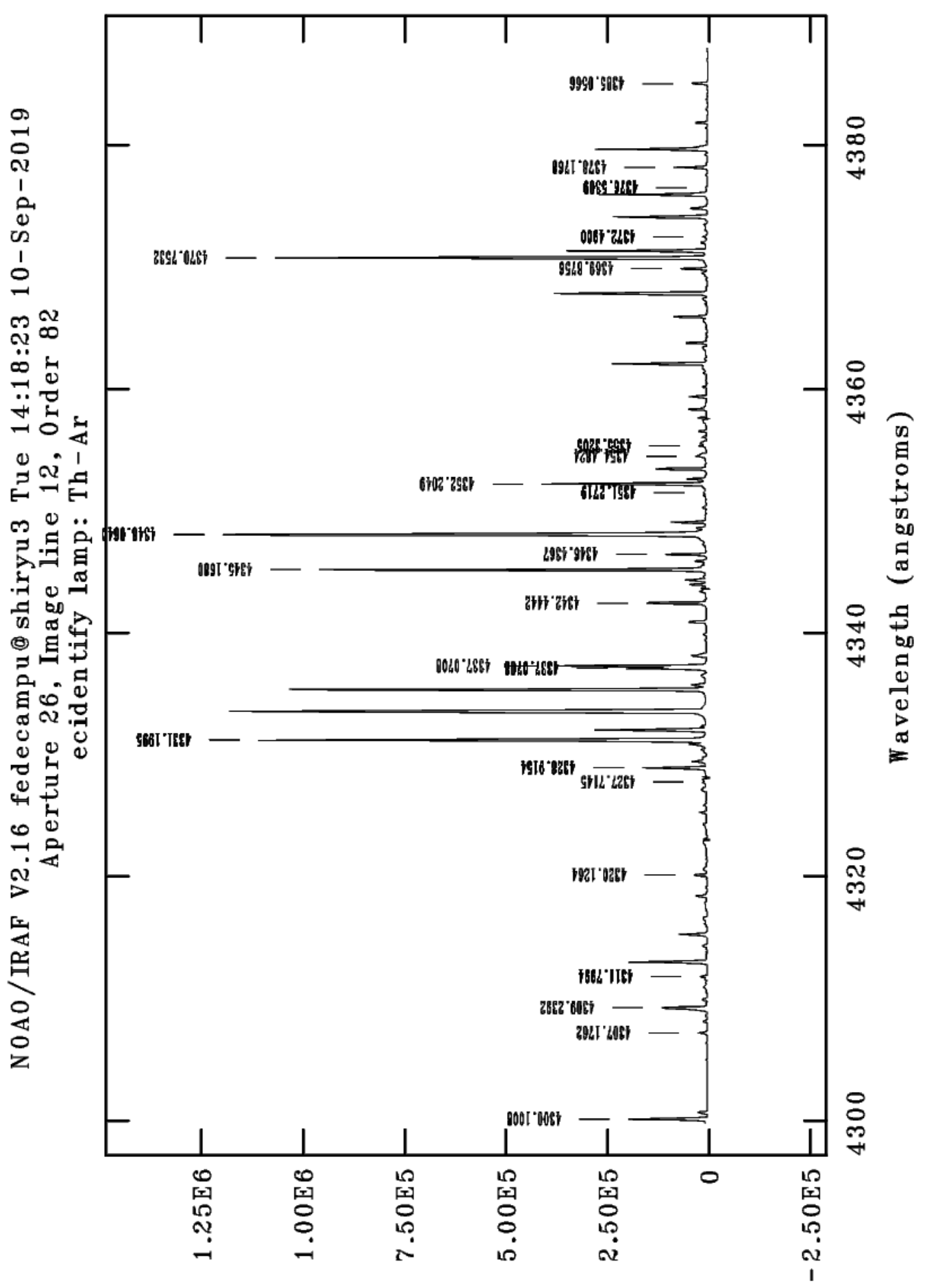

Figura 2.11. Un orden espectral de la lámpara de Th-Ar en donde se indentifican algunas de las líneas para la calibración en longitud de onda. 

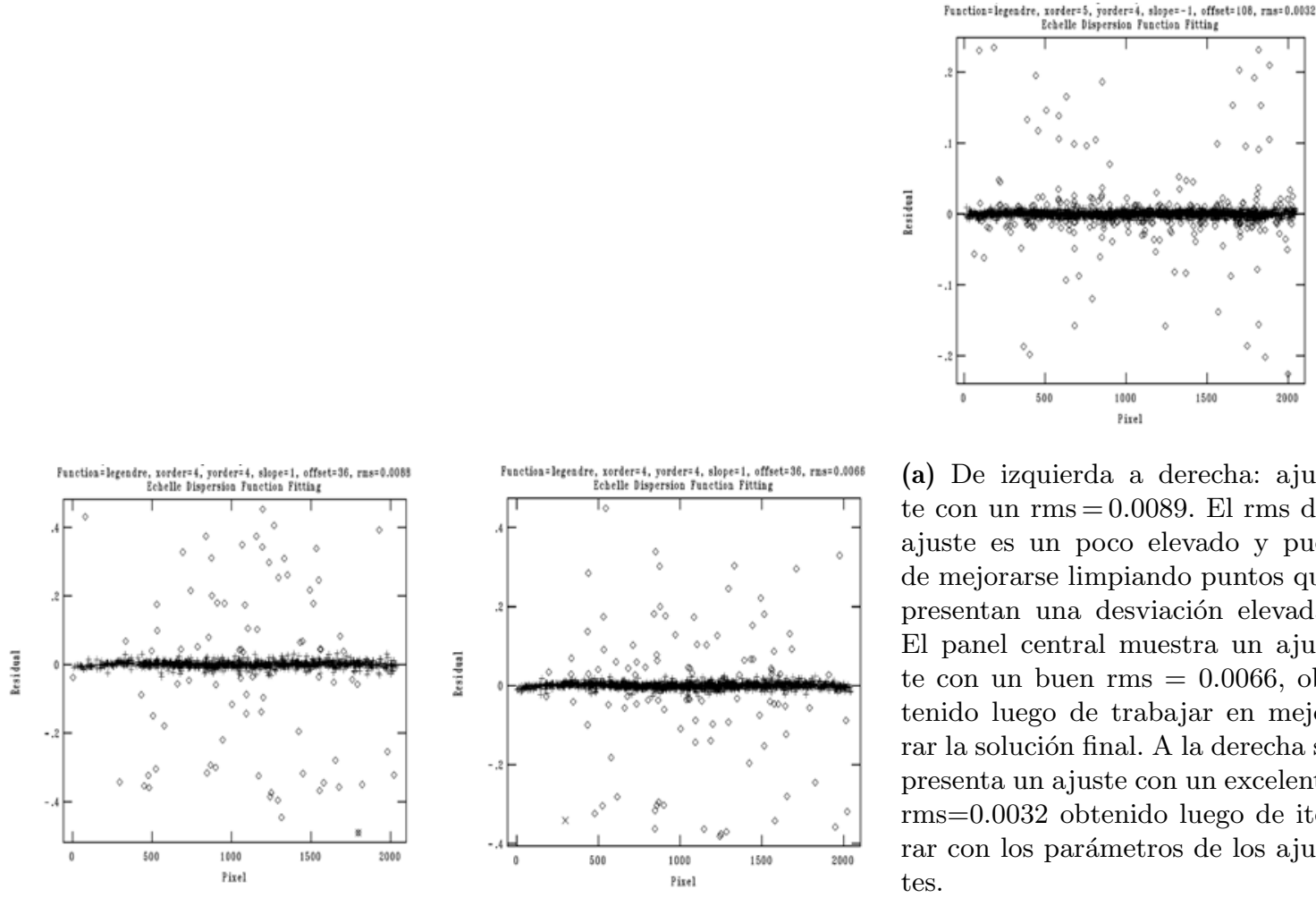

(a) De izquierda a derecha: ajuste con un rms $=0.0089$. El rms del ajuste es un poco elevado y puede mejorarse limpiando puntos que presentan una desviación elevada. El panel central muestra un ajuste con un buen rms $=0.0066$, obtenido luego de trabajar en mejorar la solución final. A la derecha se presenta un ajuste con un excelente $\mathrm{rms}=0.0032$ obtenido luego de iterar con los parámetros de los ajustes.

final a través del ajuste de un polinomio de orden adecuado, teniendo en cuenta un balance entre los puntos ajustados y el grado del orden utilizado. Por lo tanto, la validez estadística de reducir el rms injustificadamente. Si se toman órdenes altos el $\mathrm{rms}$ disminuye, pero esto trae consigo errores de los órdenes altos también, por lo que lo recomendable es minimizar y encontrar el mejor ajuste con polinomios de órdenes no muy elevados (por ejemplo, orden 8 o 9). Algunos ejemplos de dichos ajustes para la calibración final en flujo se pueden ver el los paneles de la Figura 2.13.

Resulta de interés mencionar que los puntos del "borde" de los órdenes son fundamentales para que los ajustes se comporten lo mejor posible en los extremos de cada orden y no conviene que se eliminen, aunque el rms mejore significativamente. Estos puntos ayudan a que se siga la traza de los órdenes y la calibración en flujo no se pierda. Por efectos del blaze de las redes Echelle que tienen una baja transmisión en los extremos de los órdenes, en general los intervalos en estas regiones tienen pocas cuentas, por lo que hay que prestar atención en no eliminarlos para que el orden se pueda trazar de la manera más completa posible. Finalmente, con la tabla generada de la tarea sensfunc con las cuentas y las magnitudes, se calibran los espectros a través de la tercera tarea calibrate. Se introduce el nombre del archivo a calibrar, la solución del polinomio obtenida en sensfunc y de esta manera queda calibrado en flujo nuestro espectro.

Un método eficiente para testear el ajuste realizado es calibrar el espectro de la estrella estándar de flujo consigo misma. Esto suele realizarse antes de calibrar los espectros de ciencia, para asegurarse de que el procedimiento es correcto y luego continuar con el análisis sobre los espectros de ciencia. Las calibraciones realizadas en longitud de onda y posteriormente en flujo para algunos de los órdenes del espectro se presentan la Figura 2.14.

Estos pasos mencionados son necesarios para una buena calibración en longitud de onda y posteriormente en flujo de los espectros. Cualquier error en la calibración en longitud de onda puede ser crucial en el análisis de la cinemática de las líneas e introduciría un error 


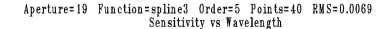

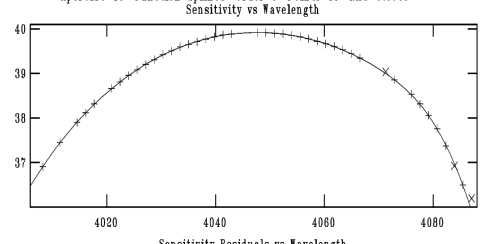

Sositivity Residuals vs Varelen

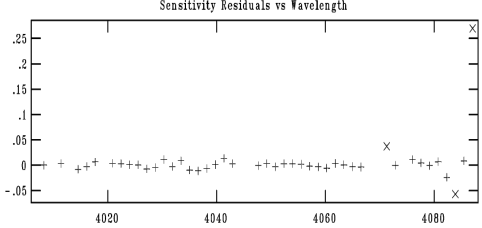

Aperture $=5$ Function $=$ spline3 Order $=5$ Points $=79$ RUS $=0.0098$

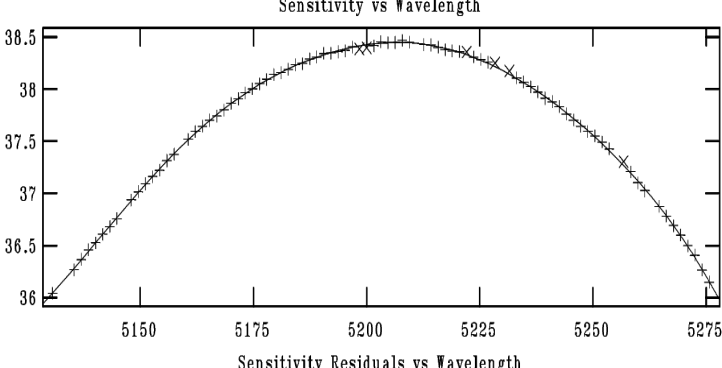

vity Residuals vs "avelength

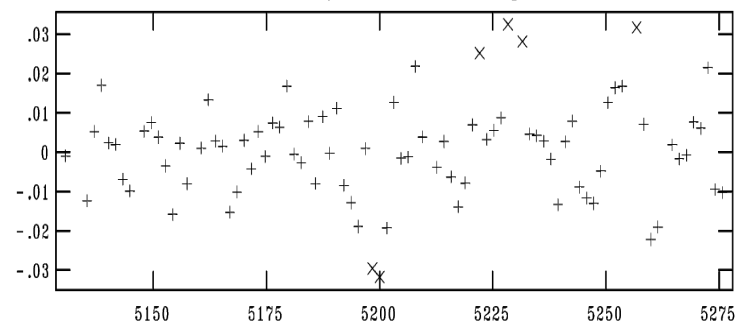

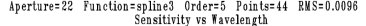

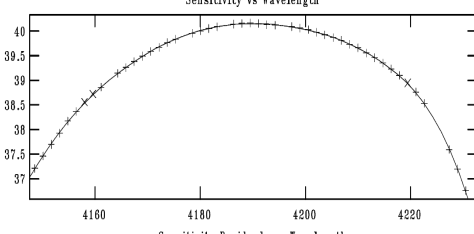

Sensitivity Residaals rs Iavele



Aperture $=14$ Function $=$ spline3 Order= 5 Points= 103 RMS $=0.009$

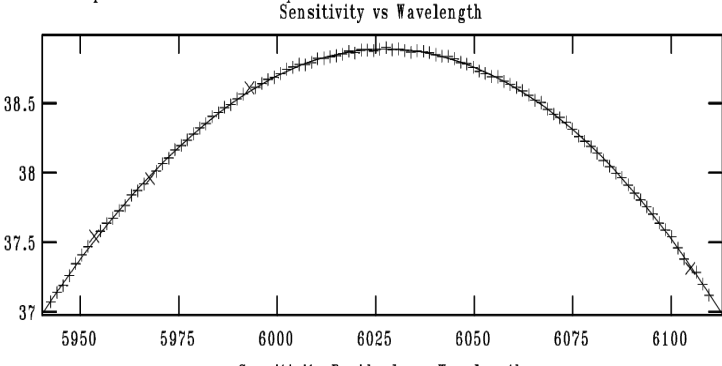

Sensitivity Residuals vs Iavelength

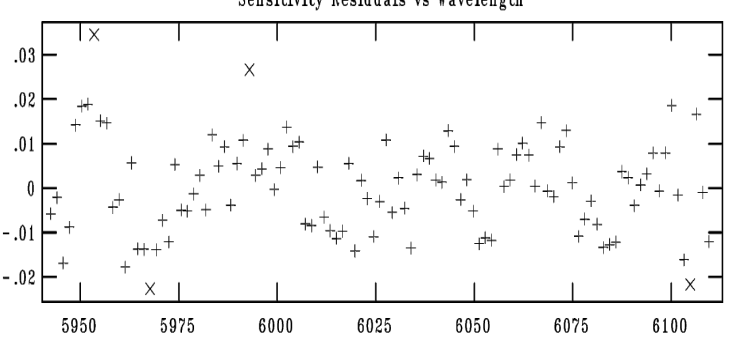

Figura 2.13. En los paneles se muestran distintos órdenes y los ajustes de los puntos identificados en cada uno de ellos. A partir de estos ajustes se conforma la función para la calibración en flujo de nuestros órdenes, orden por orden. En los tres paneles superiores tenemos de izquierda a derecha el ajuste de órdenes del brazo azul de MIKE, y en los paneles inferiores ajustes del brazo rojo de MIKE. Se pueden ver los rms bajos, necesarios para una calibración en flujo confiable. 

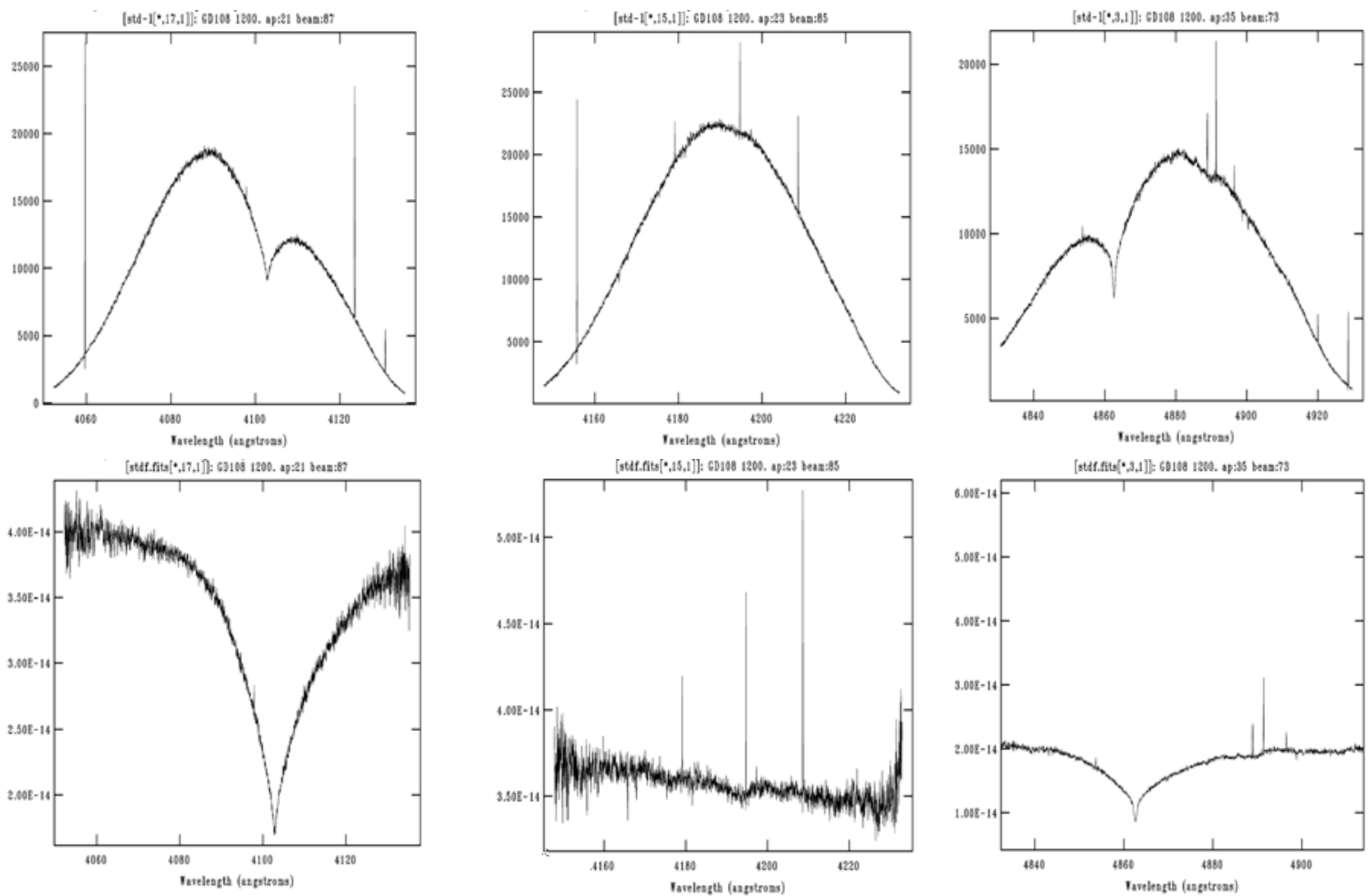

Figura 2.14. En los paneles se pueden ver tres órdenes del brazo azul de la estrella estándar GD 108. En los paneles superiores se encuentran los órdenes calibrados en longitud de onda y en los paneles inferiores calibrados en flujo.

en la calibración en flujo. Así, una mala calibración en flujo puede afectarnos de manera crítica en la determinación de los parámetros físicos de nuestras regiones, como la densidad y temperatura, y la determinación de abundancias químicas.

\subsection{Análisis de las regiones}

\subsubsection{Extracción a partir de líneas intensas}

$\mathrm{Al}$ disponer de un espectrógrafo como MIKE que trabaja con dos brazos que funcionan como instrumentos individuales e independientes, se han llevado a cabo reducciones y calibraciones para los dos detectores de maneras independientes. Para extraer los espectros de las imágenes 2-D, hemos elegido como referencia en cada brazo líneas intensas, como $\mathrm{H} \beta$ y $\mathrm{H} \gamma$ (en el azul), y $\mathrm{H} \alpha$ y [Sir] $\lambda 6716 \AA$ (en el rojo). El trabajo más complejo, que requirió de mucha precisión, consistió en tomar las mismas ventanas de extracción para ambos brazos, para cada proceso de extracción llevado a cabo en las regiones estudiadas. En resumen, para elegir la ventana de extracción a utilizar tenemos en cuenta:

- cuáles son los contornos de emisión más intensos, y cuando estos caen al $50 \%$ del máximo.

- al ajustar la ventana tomamos una sección de la imagen del objeto para garantizarnos aquella con mayor señal, y evitar contaminación o señales espurias.

- cuales son los contornos de emisión más intensos de las líneas, para corresponder esta sección a la ventana y extraer esa porción del espectro. Este procedimiento se ha reali- 


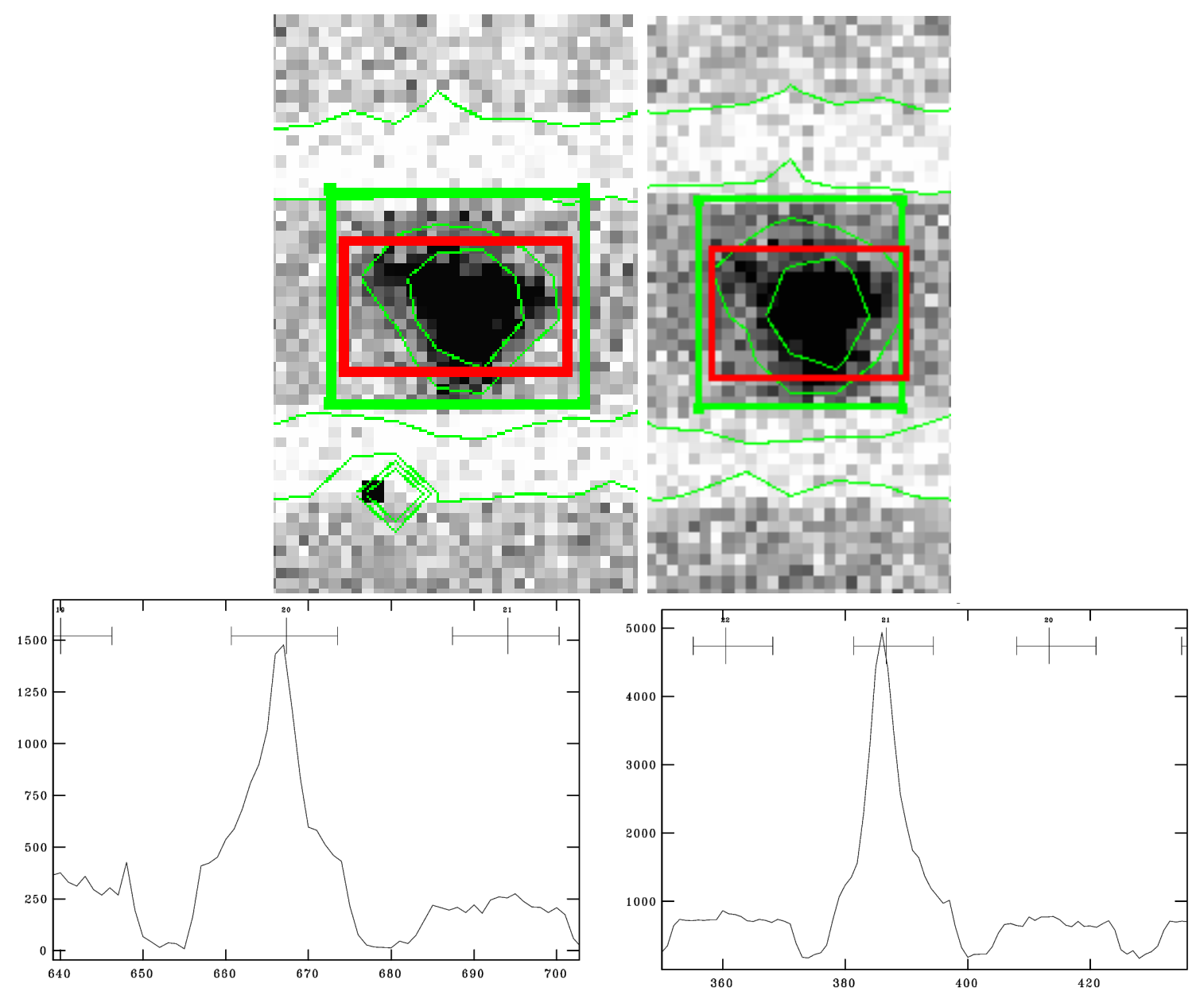

Figura 2.15. Paneles superiores: líneas de emisión con los contornos más intensos superpuestos. A la izquierda $\mathrm{H} \delta$ y a la derecha $[\mathrm{SII}] \lambda 6716 \AA$, se han elegido porque son más ilustrativas para visualizar los contornos. En rojo la casilla que representa la ventana de extracción y en verde la casilla que representa el tamaño total del orden. Paneles inferiores: ventanas de extracción seleccionadas en los cortes espaciales, en donde se pueden ver tres aperturas separados por los espacios entre órdenes.

zado para todos los nudos estudiados, tomando distintas líneas de emisión. Siempre se han tomado como referencia a las líneas $\mathrm{H} \beta$ y $\mathrm{H} \alpha$, para el brazo rojo y el brazo azul, respectivamente.

En la Figura 2.15 se puede ver como ejemplo la extracción realizada en Mrk 600 A donde se tomaron las líneas de $\mathrm{H} \delta$ y $[\mathrm{SiI}] \lambda 6716 \AA$ como referencia porque son más ilustrativas para la visualización de los contornos a partir de los cuales se extrajeron los espectros. Para las líneas intensas, al modificar con los contrastes y los niveles de emisión, las visualizaciones no resultan del todo claras debido a que no se reconoce detalladamente el nivel de señal de los órdenes espectrales.

\subsubsection{Problemas y soluciones a limitaciones instrumentales}

En el proceso de análisis de los perfiles cinemáticos de las líneas nos encontramos con algunas inconsistencias en las componentes cinemáticas, particularmente en el doblete de las líneas del $[\mathrm{OIII}] \lambda \lambda 4959 \AA$ y $5007 \AA$, que nos llevó a estudiar qué podría generar tal efecto en las líneas. Dichas líneas se encuentran en los bordes de los detectores (en los últimos órdenes 


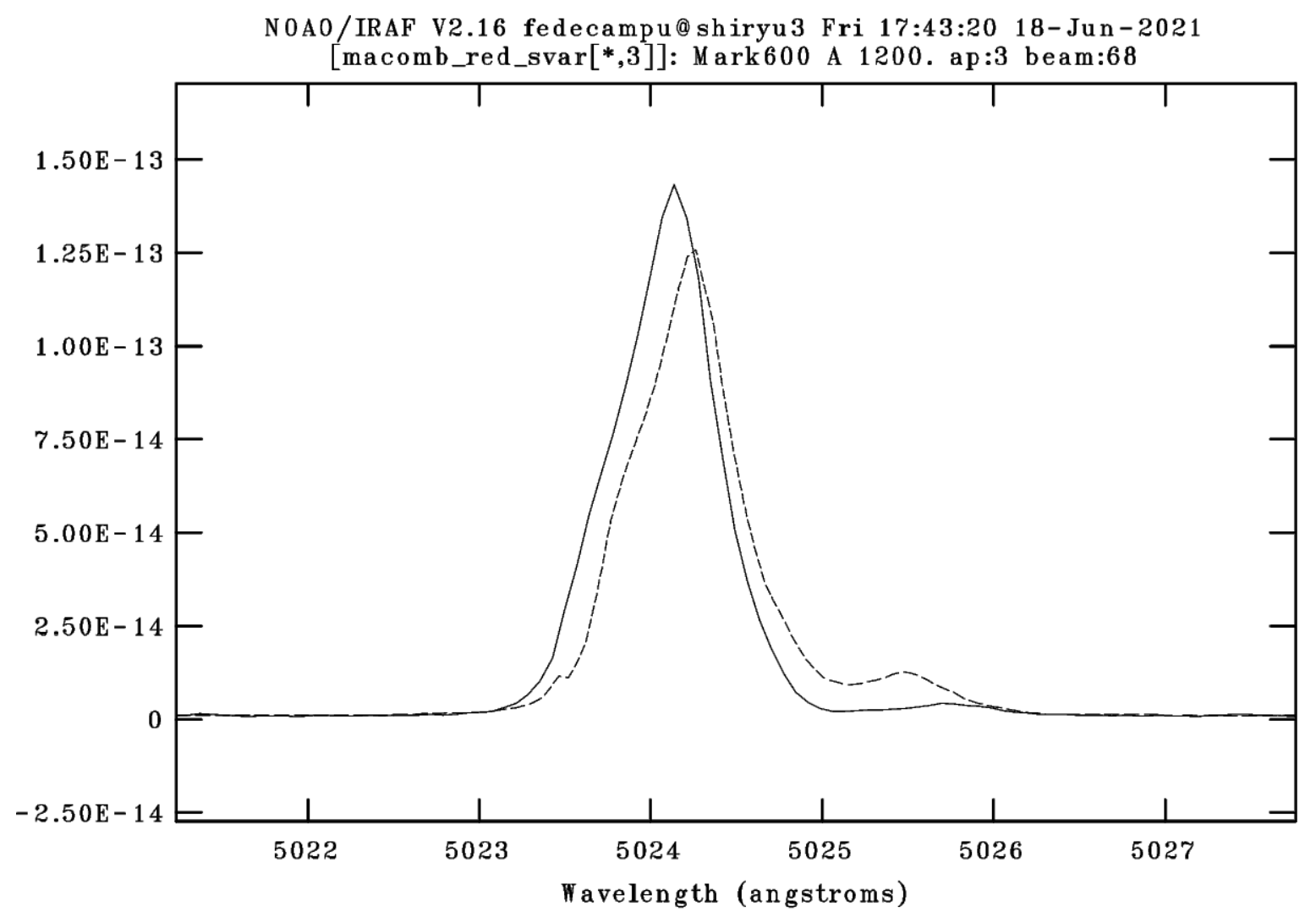

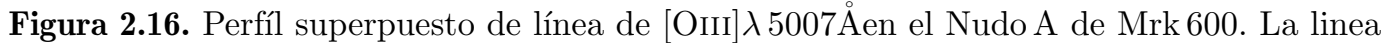
continua corresponde al brazo rojo y el perfil a líneas punteadas a la correspondiente al brazo azul.

del brazo azul y en los primeros del brazo rojo), y no coinciden con los perfiles de las demás líneas. Esta observación hizo que realicemos varias consultas con el personal del Observatorio Las Campanas para encontrar el supuesto error o problema.

Como se puede ver en la Figura 2.16 que es el perfíl de la línea del [OIII] $\lambda$ 5007Å, la misma línea presente en los dos brazos tiene una forma distinta, con una marcada componente hacia el rojo en el espectro extraído del brazo azul. Luego de discutir con expertos de $\mathrm{LCO}^{(\mathrm{i})}$ y de verificar las extracciones, encontramos que el poblema se explica debido a la presencia de un espectro fantasma generado por el dicróico justo en el rango espectral de la superposición de ambos brazos del espectrógrafo (cross-over) en los $\approx 5000 \AA$ (ver Figura 2.17). En conclusión, el espectrógrafo presenta el cruce de los rangos de detección de los brazos en la región espectral donde se encuentran las líneas nebulares de interés, como las líneas de $\mathrm{O}$, HeI e inclusive $\mathrm{H} \beta$, que puede verse afectada dependiendo de la velocidad radial del objeto.

Nuestros espectros presentan 37 órdenes en el brazo azul y 34 en el brazo rojo. Las líneas afectadas por el efecto del dicróico se hallan en los órdenes 36 y 37 del brazo azul y en el primero y en el segundo orden del brazo rojo. Este efecto se observa en todos nuestros espectros y hemos decidido trabajar con las líneas de [OIII] detectadas en el tercer orden del brazo rojo ya que el espectro fantasma producido por la dispersión del dicróico ya no se encuentra (o es despreciable en comparación al del 2do orden). Por el lado de la línea de $\mathrm{H} \beta$ (en $\lambda_{0} 4861 \AA$ ) hemos utilizado la observada en el brazo azul, ya que se encuentra en el orden

\footnotetext{
${ }^{(i)}$ Los contactos con el personal del observatorio ayudó a clarificar algunas indicaciones y especificaciones dadas en el manual, que no eran claras. Ver, http://www.lco.cl/Members/magins/mike-kb/mike-user-manual
} 


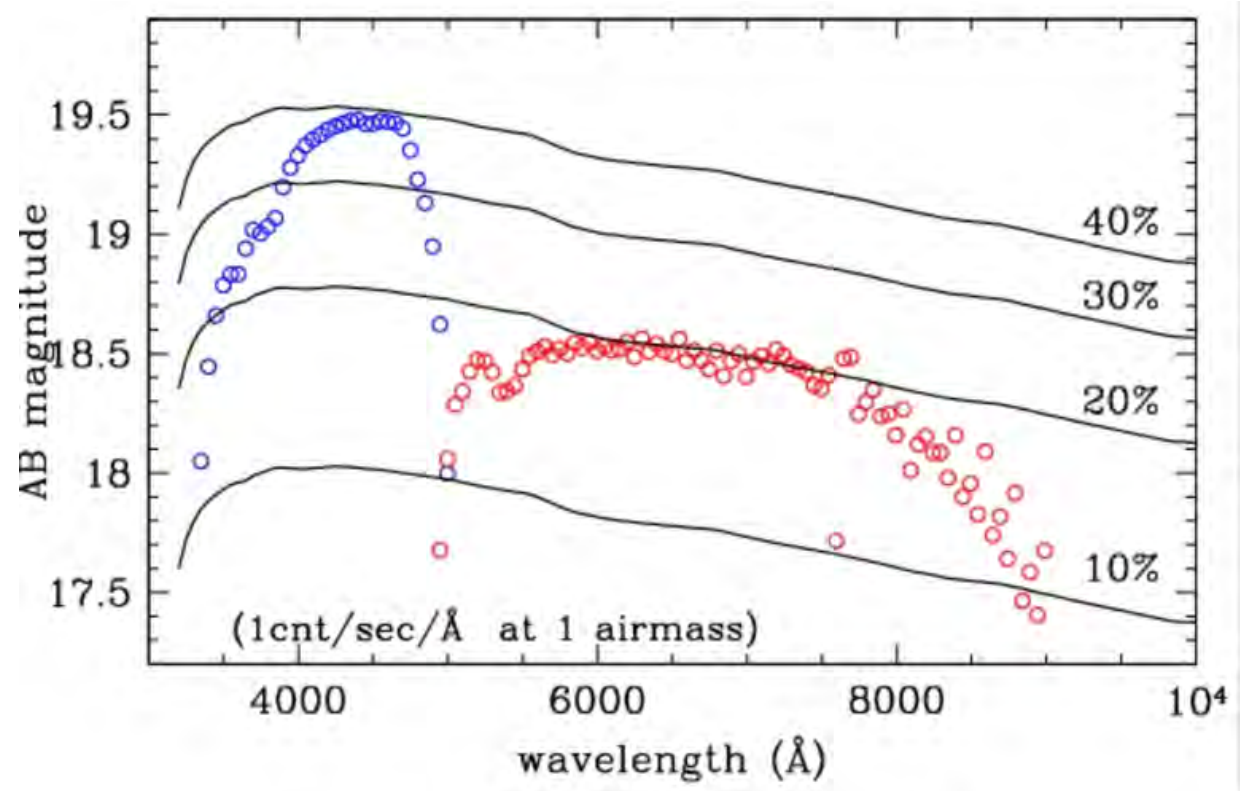

Figura 2.17. Gráfico extraído de la página web de LCO para estimar tiempos de integración con MIKE, que además muestra la zona del cross-over en los $\lambda 4950 \AA$. Los círculos azules corresponden a la eficiencia del detector azul mientras que los cículos rojos al brazo rojo. La zona en que ambos se encuentran es la zona del cross-over

35 mientras que en el brazo rojo se encuentra en el orden 1, donde el para este brazo el efecto es máximo.

\subsubsection{Análisis de las componentes espaciales}

Como parte del análisis llevado a cabo en el presente trabajo sobre los perfiles de las líneas de emisión, además de hacer el estudio espectral de las distintas componentes cinemáticas, hemos realizado un estudio de la variación de los perfiles espaciales en cada línea. Para ello hemos buscando componentes que se puedan separar a lo largo de la ranura, con el fin de hacer un estudio lo más profundo posible de las líneas espectrales y de la región en que estas se generan. Las regiones de formación estelar estudiadas abarcan varios cientos de parsecs y dependiendo de la sensibilidad de los espectrógrafos es posible obtener una descomposición espacial en distintas regiones de emisión (ver por ej. Hägele et al., 2011).

Para ello, se han estudiado perfiles espaciales que fueron extraídos con la misma tarea utilizada para la extracción de los espectros pero modificando el eje, y así obtenemos un perfíl espacial a lo largo de la ranura. En algunos casos, dentro de un mismo orden se identifica una estructura y diferencias espaciales en las líneas (ver por ejemplo la Figura 2.18).

$\mathrm{Al}$ analizar la imagen extraída en la dirección espacial, se pueden ver diferencias en los centros de la líneas. Lamentablemente, la resolución espacial no es lo suficientemente alta para realizar un análisis detallado y con señal suficiente de las diferentes componentes espaciales encontradas, ya que la superposición y contaminación de cada componente espacial identificada es muy grande y los resultados encontrados presentan muchas incertezas (ver Figura 2.19). La discusión planteada en este Capítulo fue presentada en congresos nacionales e internacionales, y en formato de presentación oral y poster. Particularmente, el estudio de componentes espaciales fue parte de las presentaciones que el grupo de trabajo expuso en un congreso internacional. Se presentó un poster y posteriormente fue publicado en el procceding de la revista de dicha reunión (ver, Campuzano-Castro et al., 2018). 


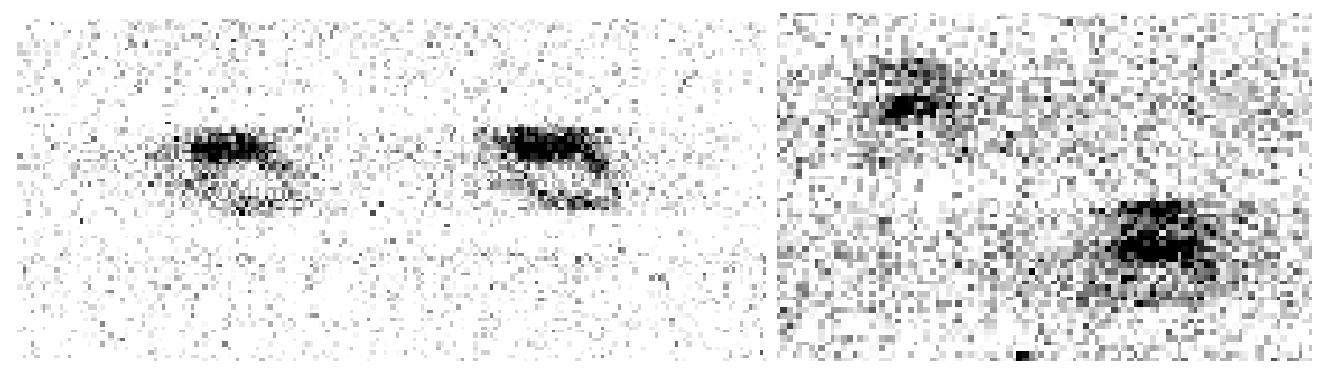

Figura 2.18. Líneas de emisión con estructura espacial en el brote de formación estelar II Zw 33 C. A la izquierda el doblete del [OII] $\lambda \lambda 3726 \AA, 3729 \AA$ y a la derecha $[\mathrm{NII}] \lambda 6584 \AA$ (superior) y $[\mathrm{SII}] \lambda 6716 \AA$ (inferior).

\subsection{Conclusión}

La reducción de datos astronómicos es un proceso que consta de varios pasos que conllevan cierto entendimiento y correlación entre sí. Es crucial que las calibraciones de las observaciones tengan la mejor calidad posible para que puedan ser analizadas, y que estas cuenten con el mínimo error asociado por la calibración de los datos. Es por esto que se ha analizado y se ha trabajado detalladamente en las observaciones para que estas presenten tanto la calibración en longitud de onda como la calibración en flujo lo más precisas posibles, habiendo tratado a los errores y a los ajustes con el mayor detalle y rigurosidad. Hemos medido numerosos espectros y hemos comparado observaciones para proceder con el estudio de los perfiles de las líneas de emisión. La calibración en longitud de onda llevada a cabo a través de las líneas de la lámpara y determinada para datos de una de las noches, la hemos utilizado como referencia para la calibración de otras lámparas de noches sucesivas, con errores $(\mathrm{rms}) \approx 0.0070 \AA \mathrm{px}^{-1}$.

La calibración en flujo se realizó tomando las estrellas estándar GD 108 y Feige 100, para las cuales se tomaron distintos archivos de calibración ${ }^{(i)}$, con distintas resoluciones $(0.1 \AA, 1 \AA$ y $3 \AA$ ), chequeando y comprobando distintas calibraciones hasta obtener un espectro calibrado en flujo de nuestra ciencia.

En los espectros calibrados encontramos algunas inconsistencias en los perfiles de las líneas de emisión presentes en el rango del cross-over del espectrógrafo, entre los $4950 \AA$ y los $5100 \AA ̊$ que nos llevó a investigar al respecto. Hemos entendido que debido al dicróico que dispersa la luz a ambos brazos del espectro, las líneas presentan un espectro "fantasma" hacia las alas que no es por la cinemática del gas, sino por un problema instrumental al dispersar la luz. Este patrón se hace muy importante en los órdenes que corresponden al cross-over, que son los últimos dos del brazo azul y los dos primeros del brazo rojo. Por suerte para nuestros datos, tanto las líneas del doblete del [OIII] como las de $\mathrm{H} \beta$ las tenemos presentes en ambos brazos, lo que nos permitió trabajar y analizar de igual manera a los perfiles de las líneas tomándolas del brazo rojo y descartando las del azul dado que se encuentran en el ante-último y último orden, respectivamente.

Así, hemos completado una extracción y calibración de las imágenes teniendo en cuenta la mayor cantidad de factores y efectos posibles con el afán de lograr los mejores espectros para el posterior análisis. En la siguiente sección se hace una descripción detallada de la cinemática de las regiones aquí mencionadas.

\footnotetext{
${ }^{(i)}$ Descargados de la página web del espectrógrafo UVES del Observatorio Europeo: https://www.eso.org
} 


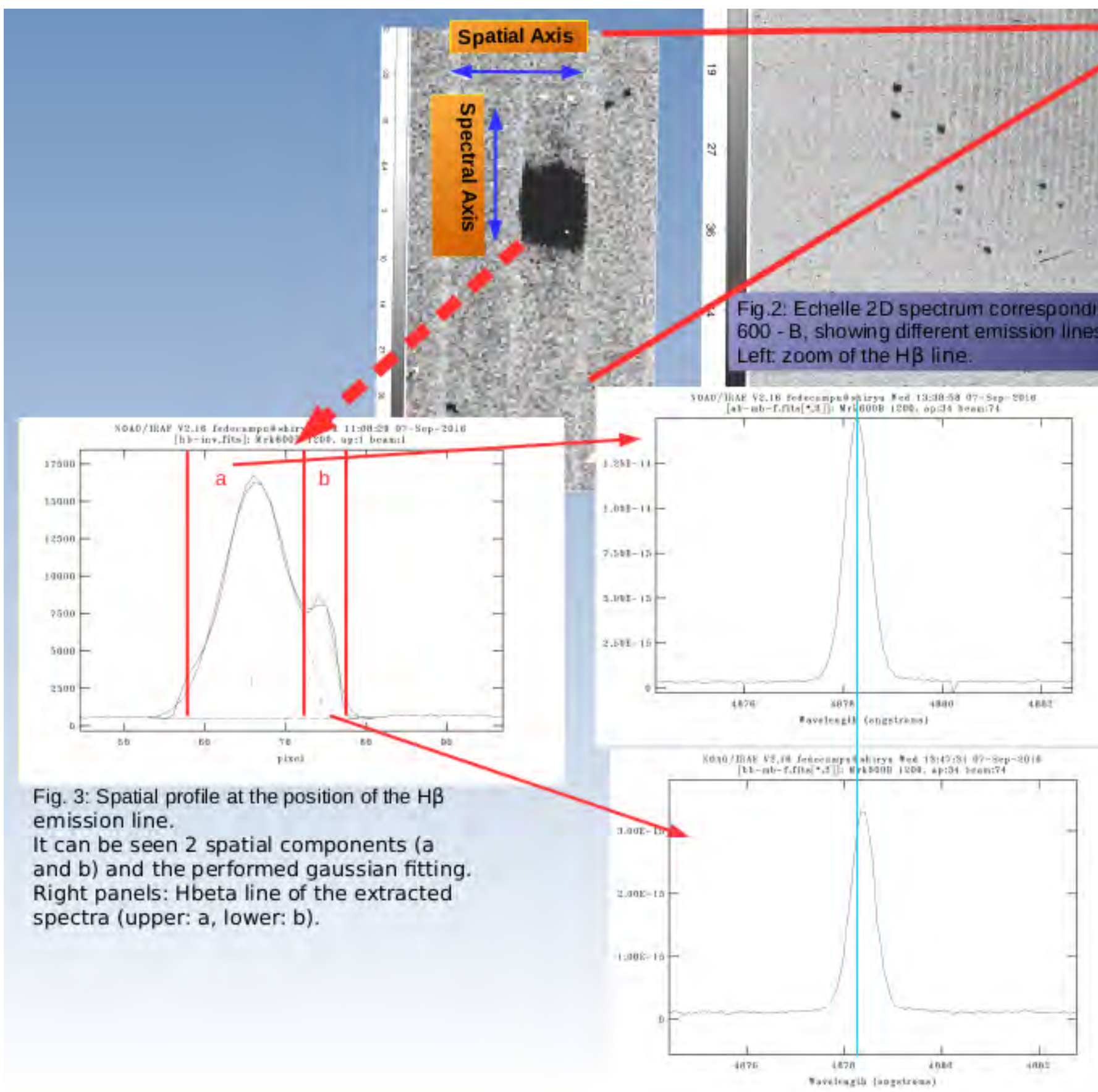

Figura 2.19. Extracción utilizando dos zonas distintas determinadas en $\mathrm{H} \beta$ en donde se puede apreciar un desplazamiento en longitud de onda. La imagen es un extracto del poster presentado en el Workshop Chemical Abundance in gaseous Nebulae, en Brasil 2016. 
Chemical Abundances in Gaseous Nebulae

AAA Workshop Series 10, 2016

G. Hägele, M. Cardaci \& E. Pérez-Montero, eds.

Poster

\title{
Chemodynamics of Blue Compact Dwarf galaxies
}

\author{
F. Campuzano-Castro ${ }^{1,2}$, G. F. Hägele ${ }^{1,2}$, G. Bosch ${ }^{1,2}$, V. Firpo ${ }^{3,4}$, \\ N. Morrell ${ }^{5}$, M. Cardaci ${ }^{1,2}$ \\ ${ }^{1}$ Facultad de Ciencias Astronómicas y Geofísicas, Universidad Nacional \\ de La Plata (FCAG-UNLP) \\ ${ }^{2}$ Instituto de Astrofísica de La Plata (IALP-CONICET) \\ ${ }^{3}$ Universidad de La Serena \\ ${ }^{4}$ Gemini Observatory, Chile \\ ${ }^{5}$ Las Campanas Observatory, Chile
}

\begin{abstract}
This work presents a brief summary of the analysis we are performing on the physical and chemical properties of the ionizing gas in star-forming regions belonging to two Blue Compact Dwarf galaxies (BCDs), using high resolution echelle spectra. Our aim is to perform a detailed study of the Chemodynamics on BCDs. To do that, we use our own Python code using the LMFIT (Non-Linear Least-Squares Minimization and Curve-Fitting for Python) package. We deconvolve the emission-line profiles fitting several gaussians to the different kinematical components to be able to estimate the properties and the nature of the ionized gas. Our next step is to use the kinematical information to perform the chemical abundance analysis and to infer the physical properties of the gas (the chemodynamical study) by using the methodology published in Hägele et al. $(2008,2012)$.
\end{abstract}

\section{Introduction}

Giant Extragalactic Hir Regions (GHIIRs) are extended objects, very luminous and located in the discs of spirals and in irregular galaxies. They are formed due to the presence of young and massive stars whose strong ultraviolet flux ionizes their surrounding gas. The observed emission line spectra of HiI galaxies and Blue Compact Dwarf galaxies (BCDs) are similar to those shown by Giant HII Regions (Sargent \& Searle, 1970; French, 1980), therefore, we are able to use similar analysis techniques to study the physical properties (electron densities and temperatures) and chemical abundances of the emitting gas of the star-forming regions (see e.g. Hägele et al., 2006) belonging to these low metallicity galaxies (Terlevich et al., 1991). BCDs present strong star formation easily identified through their intense $\mathrm{H} \alpha$ emission and narrow emission lines, low metallicity environments and complex star formation history. Due to these characteristics they are interesting objects to study metallicity effects in galaxies (Kunth \& Östlin, $2000)$. 

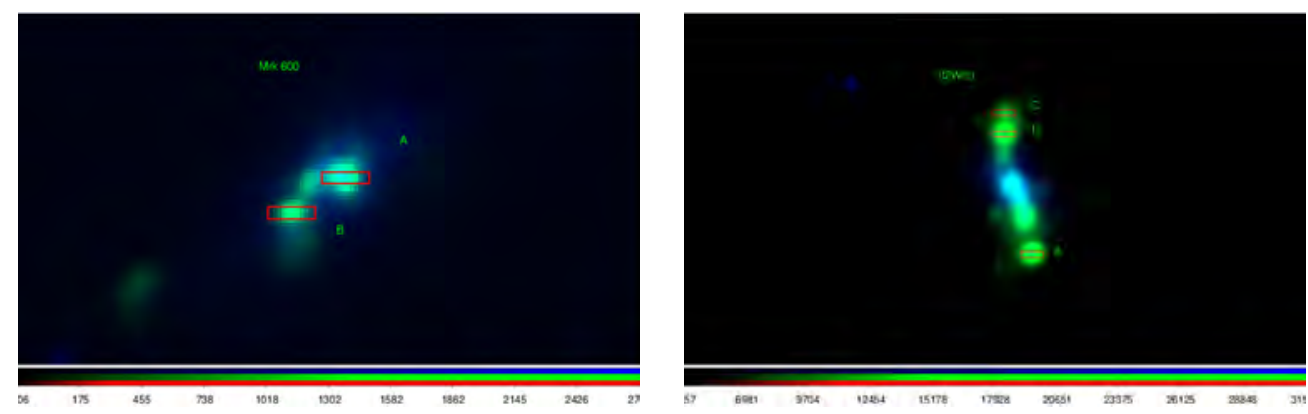

Figure 1. Composite images of Mrk600 (left panel) and IIZw 33 (right panel). Green and blue are $\mathrm{H} \alpha$ and $\mathrm{R}$ band, respectively, images acquired with the 1.8 telescope at Monte Palomar (images taken from NED). The echelle slits are overimposed.

Many BCDs that used to be considered compact objects, currently show a complex spatial structure thanks to the improvement and enlargement of telescopes and instruments (Hägele et al., 2011). For example, Haro 15, currently classified as a spiral galaxy, were included in BCDs catalogues (see discussion in Firpo et al., 2011; Hägele et al., 2012).

\section{Observations}

Our data were acquired in 2005 and 2006, using the high resolution echelle spectrograph mounted at the Clay Magellan Telescope $(6.5 \mathrm{~m})$ at Las Campanas Observatory (LCO), Chile. The spectrograph uses a dichroic to separate the light in 2 different spectral ranges. The $1 \times 4 \operatorname{arcsec}^{2}$ slit was used with a blue and red spectral resolutions of $\mathrm{R} 28000$ and $\mathrm{R} 22000$, respectively. The blue and red spectral ranges were $3300-5100 \AA$ and $4850-9300 \AA$, respectively. We used IRAF $^{1}$ routines in the usual manner to reduce the data. GD108 was used as standard star. We observed 5 star-forming knots: 2 belonging to Mrk 600 and 3 to IIZw 33 . Each studied star-forming region was also divided in sub-components accordingly to the spatial components identified in its spatial profile (see the procedure description in §3). In total we have observations of 11 regions: 4 in Mrk 600 (2 components for each knot, A and B; see left panel of Fig. 1) and 7 for IIZw 33 (3 sub-components for Knot A, and 2 for knots B and C; see right panel of Figs. 1).

\section{Results}

\section{Spatial components}

Analyzing the spatial profile around the $\mathrm{H} \beta$ emission line in the $2 \mathrm{D}$ echelle spectra we identified more than one different spatial components for each studied

\footnotetext{
${ }^{1}$ IRAF: the Image Reduction and Analysis Facility is distributed by the National Optical Astronomy Observatories, which is operated by the Association of Universities for Research in Astronomy, Inc. (AURA) under cooperative agreement with the National Science Foundation (NSF).
} 


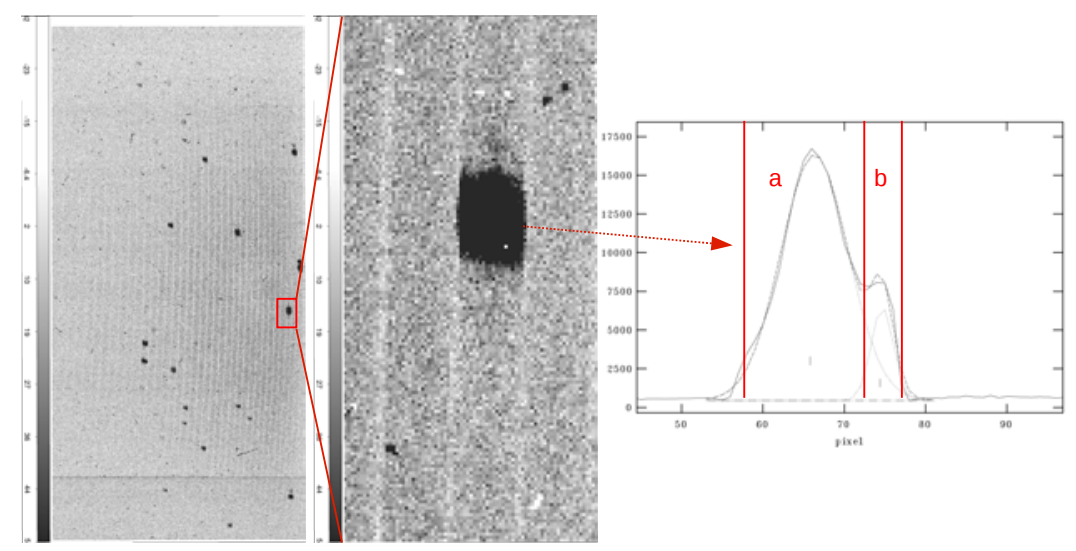

Figure 2. Left: 2D echelle spectrum corresponding to Mrk600-B, showing different emission lines and orders. Middle: zoom of the 2D echelle spectrum around the $\mathrm{H} \beta$ emission line. Right: spatial profile at the position of the $\mathrm{H} \beta$ emission line. It can be seen two spatial components and the performed spatial-Gaussian fitting.

star-forming region (see an example of the $2 \mathrm{D}$ echelle spectra together with the $\mathrm{H} \beta$ spatial profile and the performed fitting in Fig. 2). We performed an extraction for each of the identified spatial components. We are able to perform the chemodynamical study for each of these spatial components.

\section{Kinematical components}

Following the methodology proposed, developped and used in Hägele et al. (2007, 2009, 2010, 2012, 2013); Firpo et al. (2010, 2011) we deconvolve the emission line profiles in different kinematical components. We performed the analysis of the strong emission lines detected in our regions using our own Python code based on the use of the LMFIT (Non-Linear Least-Squares Minimization and Curve-Fitting for Python) package. We proposed a model composed by a linear function and "n" Gaussians or normal distributions, to fit the local continuum and the emission-line profile, respectively. We started our analysis modeling the strongest emission-lines: $\mathrm{H} \alpha$ and $[\mathrm{OIII}] \lambda 5007 \AA$. Then, we used these kinematical results, line positions (velocities) and widths, as the initial approximations for the other emission-lines with similar ionization state. For [OI], [OII], [SII] and $[\mathrm{NII}]$ emission-lines we used the solution found for the $\mathrm{H} \alpha$ recombination line. While for HeI, [SIII], [NeIII], [ArIII] and [ArIV], we used the solution found for the $[\mathrm{OIII}] \lambda 5007 \mathrm{~A}$ emission-line. It must be noted that for the very weak temperature sensitive auroral emission-lines: [ОІгі] $\lambda 4363 \AA$, [Онг] $\lambda \lambda 7319,7330 \AA$, $[\mathrm{NII}] \lambda 5755 \AA$, [SIII] $\lambda 6312 \AA$, and $[\mathrm{SII}] \lambda 4068 \AA$, we used the kinematical solution obtained for the strong emission-line of the same atomic ion since the kinematical solution must be the same (or very similar) and therefore we only varied their amplitudes (see a complete discussion about this point in Hägele et al. (2012). In Fig. 3 some examples of the results of the fittings to the emission-line profiles of Knot A of IIZw 33 are shown. 

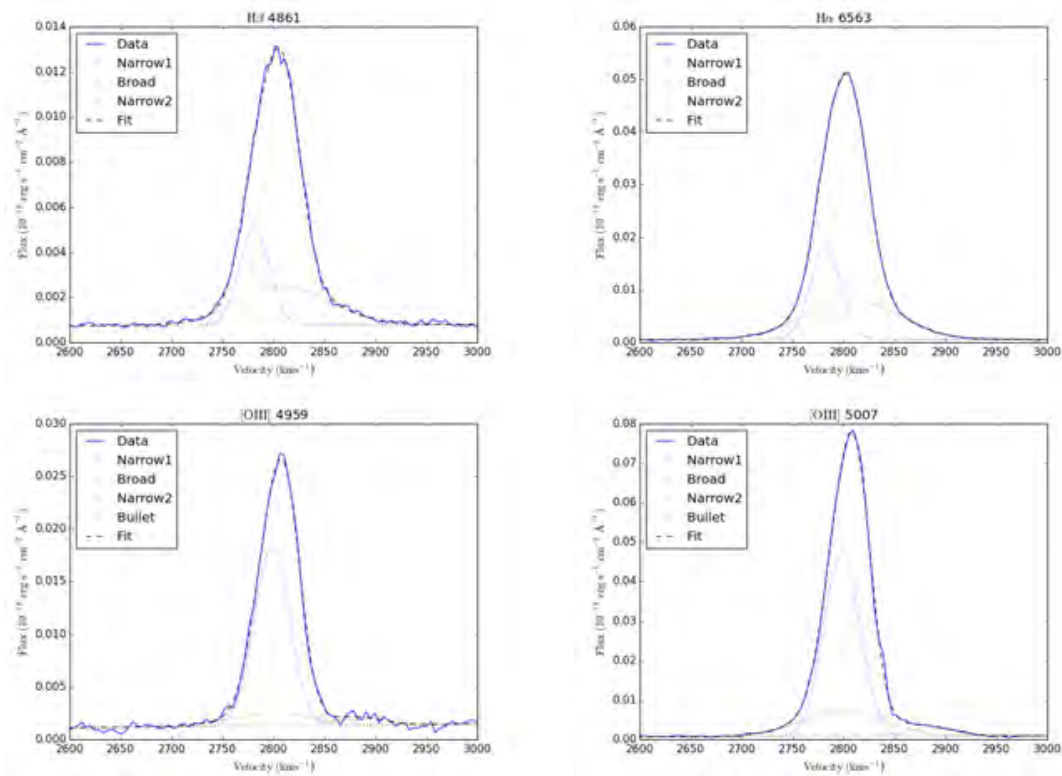

Figure 3. Some results of the fittings performed to the emission-line profiles of Knot A of IIZw 33.

\section{Conclusions}

We observed 11 star-forming knots belonging to 2 BCDs using high resolution echelle spectroscopy that will allow us to carry on a chemodynamical study of their physical conditions and chemical abundances. We used our own Python code base on the LMFIT package to perform the kinematical decomposition of the emission lines profiles. Our near future objectives are to derive for each kinematical component: (i) the reddening constant from the hydrogen recombination lines in all the regions; (ii) the electron density in the low excitation zone from the emission-line ratios of $[\mathrm{SII}] \lambda 6717 \AA / \lambda 6731 \AA$ and $[\mathrm{OII}] \lambda 3727 \AA / \lambda 3729 \AA$; (iii) the electron temperatures Te ([OOII]), Te ([OIII]), Te ([SII]), Te ([SIII]), and Te ([NII]) using the auroral emission lines present in the spectra of several of our regions and applying the direct method (see e.g. Hägele et al., 2008) or empirical relations and photo-ionization models (see e.g. Pérez-Montero \& Díaz, 2005; Hägele et al., 2006); (iv) ionic abundances of $\mathrm{He}+, \mathrm{O}+, \mathrm{O} 2+, \mathrm{S}+, \mathrm{S} 2+, \mathrm{N}+$, $\mathrm{Ne} 2+, \mathrm{Ar} 2+$ and Ar3+; (v) the total chemical abundances of He, O, S, N, Ne and Ar; (vi) the ionization degree of the nebular gas from the $\eta$ and $\eta$ ' parameters (Vílchez \& Pagel, 1988); and (vii) the relationship between the luminosities and velocity dispersion: L vs. $\sigma$ (see e.g. Bosch et al., 2002).

We also have observations of other 5 BCDs acquired using the echelle spectrograph mounted at the du Pont telescope at LCO, for which will continue our chemodynamical study.

Acknowledgments. This research has made use of the NASA/IPAC Extragalactic Database (NED) which is operated by the Jet Propulsion Laboratory, California Institute of Technology, under contract with the National Aeronautics and Space Administration. 


\section{References}

Bosch G., Terlevich E., Terlevich R., 2002, MNRAS, 329, 481

Firpo V., Bosch G., Hägele G. F., et al., 2011, MNRAS, 414, 3288

Firpo V., Bosch G., Hägele G. F., Morrell N., 2010, MNRAS, 406, 1094

French H. B., 1980, ApJ, 240, 41

Hägele G. F., Díaz A. I., Cardaci M. V., et al., 2007, $M N R A S$, 378, 163

Hägele G. F., Díaz A. I., Cardaci M. V., et al., 2009, MNRAS, 396, 2295

Hägele G. F., Díaz A. I., Cardaci M. V., et al., 2010, MNRAS, 402, 1005

Hägele G. F., Díaz A. I., Terlevich E., et al., 2008, MNRAS, 383, 209

Hägele G. F., Díaz A. I., Terlevich R., et al., 2013, MNRAS, 432, 810

Hägele G. F., Firpo V., Bosch G., et al., 2012, MNRAS, 422, 3475

Hägele G. F., García-Benito R., Pérez-Montero E., et al., 2011, MNRAS, 414, 272

Hägele G. F., Pérez-Montero E., Díaz A. I., et al., 2006, MNRAS, 372, 293

Kunth D., Östlin G., 2000, A\&A Rev., 10, 1

Pérez-Montero E., Díaz A. I., 2005, MNRAS, 361, 1063

Sargent W. L. W., Searle L., 1970, ApJL, 162, L155

Terlevich R., Melnick J., Masegosa J., et al., 1991, A\&AS, 91, 285

Vílchez J. M., Pagel B. E. J., 1988, MNRAS, 231, 257 


\section{Capítulo 3}

\section{Análisis y resultados obtenidos a partir de las observaciones}

En el Capítulo 2 Sección 2.2 se han definido las regiones observadas y en el presente capítulo detallaremos la metodología de análisis de los espectros calibrados. Haremos una descripción de los métodos empleados para medir las propiedades de las líneas como sus centros, anchos y sus flujos, necesarios para un estudio cinemático. Presentaremos los valores de las mediciones en tablas correspondientes y las gráficas de los ajustes realizados en las líneas espectrales de nuestra muestra de RHII, concluyendo con las discusiones acerca de la cinemática de los Nudos más intensos de estos objetos.

\subsection{Estudio de la cinemática a través de espectroscopía de alta reso- lución en galaxias BCD}

La espectroscopía de alta resolución nos permite descomponer los perfiles de las líneas espectrales y esto es fundamental para poder estudiar, describir y comprender la compleja cinemática observada en el gas ionizado. A través del análisis de estas líneas presentes en los espectros de las RHiI y RHIIG extragalácticas, producidas por la interacción de los flujos de energía de las estrellas jóvenes, que excitan el gas, inmersas en dichas regiones y el medio nebular es posible definir propiedades y obtener información sobre la naturalezas de este gas.

En el análisis cinemático de los perfiles de las líneas de emisión realizado por Firpo et al. (2011), se encontró que las regiones de formación estelar observadas en la galaxia BCD Haro 15 muestran ensanchamientos en las alas de las líneas. Esto es muy notorio en las líneas más intensas como $\mathrm{H} \alpha$ y $[\mathrm{OIII}] \lambda 5007 \AA$ y se observa además en otras líneas de emisión más débiles. Una RHiI es considerada una región gigante cuando los perfiles de sus líneas de emisión presentan anchos supersónicos. (definición introducida por Terlevich \& Melnick, 1981).

En la literatura, son pocos los trabajos que hacen un estudio cinemático de múltiples componentes sobre los perfiles de las líneas de emisión del gas ionizado. Clayton (1990) obtuvo perfiles de alta resolución de la línea de [OIII] $\lambda 5007 \AA$ en diferentes zonas de NGC3603, encontrando claras componentes de velocidad hacia el rojo en la mayoría de la extensión espacial cubierta. Este autor encontró desplazamientos en velocidad entre $30 \mathrm{~km} \mathrm{~s}^{-1}$ y $40 \mathrm{~km} \mathrm{~s}^{-1}$ con respecto a la componente principal. También indicó que, al igual que en muchas RHiıG, los movimientos del gas en NGC 3603 son muy complejos y pueden ser interpretados como el producto de varias estructuras diferentes que se están expandiendo con distintas velocidades de hasta $100 \mathrm{~km} \mathrm{~s}^{-1}$.

Posteriormente, en el trabajo de Firpo et al. (2005), el análisis detallado de la cinemática 
en RHII extragalácticas confirma la naturaleza gigante de estas regiones a partir de los valores de las dispersiones de velocidades, mayores a $25 \mathrm{~km} \mathrm{~s}^{-1}$. Estos ajustes fueron realizados con una sola componente angosta, en espectros obtenidos a partir de observaciones de alta resolución usando MIKE (instrumento descripto en el Capítulo 2 Sección 2.3). El estudio de componentes anchas es un trabajo que presenta una limitación dada por la baja $\mathrm{S} / \mathrm{N}$, que hace que muchas veces sea difícil ajustarla y consecuentemente estudiarla. Es por esto que vale la pena mencionar la importancia de este último trabajo en el resultado obtenido, que confirma que las RHir detectadas en galaxias muestran perfiles de líneas con dispersión de velocidades de carácter supersónico, lo que las define como gigantes.

En muchos casos, las componentes anchas pueden explicar las alas del perfil integrado (Díaz et al., 1987; Muñoz-Tunon et al., 1996; Terlevich et al., 1996; Melnick et al., 1999; Hägele et al., 2007, 2009, 2010; Firpo, 2011; Hägele et al., 2013), y por otro lado se apoya la idea de que estos estos perfiles tengan dos componentes además de la componente central o principal: un ala azul y otra roja (Chu \& Kennicutt, 1994; Relaño \& Beckman, 2005; Buckalew \& Kobulnicky, 2006; Rozas et al., 2006).

Por ejemplo, encontramos que Yang et al. (1996) al estudiar NGC604, la región más brillante de M33 y la segunda RHiıG más cercana, propone que las alas extendidas observadas en los espectros están asociadas a estructuras shells (cáscaras o capas) en expansión impulsadas por vientos estelares y remanentes de supernovas. Además se hace mención a que estas estructuras de cáscaras son muy similares a las estudiadas en 30 Dorados, en donde los autores de Melnick et al. (1999) identificaron una componente ancha en las líneas de recombinación en la zona central de las RGHır. También, Relaño \& Beckman (2005) indican que es muy dificultoso afirmar la existencia de una componente ancha de baja intensidad relativa y proponen el ajuste de dos componentes simétricas en las alas, reforzando la idea de shells en expansión. Westmoquette et al. (2007) asocian la componente ancha a las regiones de interacción entre el gas más caliente y más fríos del MI. Esta interacción entre las altas velocidades de los vientos de gas más caliente con las regiones de gas frío crean distintas capas con dispersión de velocidades altas. Amorín et al. (2012b) indica que las dispersiones de velocidades altas puede deberse a mecanismos o fenómenos que inyecten suficiente energía para impulsar el gas a grandes velocidades, como los choques (Tenorio-Tagle et al., 1997), la gravedad (Terlevich \& Melnick, 1981), por acreción de material (Elmegreen \& Burkert, 2010) o la retroalimentación de material de la formación de estrellas (Lehnert et al., 2009).

Estudios realizados por Hägele et al. (2007, 2009, 2010) muestran buenos ajustes en las líneas de emisión del gas ionizado en regiones circumnucleares de formación estelar combinando componentes angosta y una componente ancha para ajustar las alas de los perfiles. Firpo et al. (2010, 2011) confirmar este tipo de ajustes en Regiones HiI Gigantes pertenecientes a galaxias espirales y en regiones de formación estelar en la BCD Haro 15. Amorín et al. (2012b) y Bosch et al. (2019) (por ejemplo) hacen lo mismo para el análisis de la cinemática de regiones de formación estelar en galaxias Green Pea. En este trabajo buscamos una explicación a la existencia de alas en los perfiles de nuestras líneas y para ello hemos propuesto y analizado distintas soluciones.

Además, en los trabajos llevados adelante por miembros del grupo de investigación del cual formo parte, se muestra que los perfiles de las líneas de emisión no se pueden representar de manera fiel mediante una única función gaussiana, sino que, en general es necesario agregar una componente ancha para poder representar las alas de una manera completa. En muchos de los casos también se requieren más de una componente angosta para representar fielmente los perfiles complejos. Estos resultados fueron presentados en la Tesis de Dr. G. Hägele (Hagele, 2009) en donde se presenta la metodología que luego dio origen al trabajo realizado por Firpo (2011) en donde se estudiaron los Nudos de formación estelar más intensos de la Galaxia BCD 
Haro 15, y del cual se desprende a su vez el trabajo de esta Tesis. En esta serie de trabajos se probaron ajustes con otras funciones como Lorenzianas o perfiles de Voigt pero los ajustes que mejor representan al análisis de la cinemática son las funciones gaussianas.

\subsection{Metodología del análisis cinemático}

Como hemos mencionado anteriormente, el presente trabajo es una continuación del estudio previo realizado en la Tesis Doctoral de Verónica Firpo (Firpo, 2011), de la que hemos adaptado algunas de las técnicas y metodologías.

\subsubsection{Ajuste de múltiples gaussianas}

Al desplegar los espectros y visualizar los perfiles de las líneas de emisión, se observa que no presentan un comportamiento simétrico respecto al centroide del perfil. Uno de los objetivos es poder representar a las líneas observadas con funciones gaussianas, para identificar y estudiar de esta manera, y por medio de dichas funciones, a las distintas componentes de gas presentes en las líneas de emisión.

Debido a que los ajustes mediante una gaussiana devuelven residuos grandes en las alas y en los picos del perfil, es necesario proponer soluciones que contengan más de una componente gaussiana (ver por ejemplo Figura 3.1). Para poder comparar resultados e indicar si los ajustes son estadísticamente válidos se suele utilizar uno o varios criterios estadísticos. Por lo general, en la literatura encontramos comúnmente tres: $\chi^{2}$, Akaike o el criterio bayesiano. A continuación describimos el uso y el análisis en nuestra metodología.

Los ajustes iniciales los realizamos en las líneas más intensas de nuestros espectros con mayor S/N y luego copiamos la mejor solución encontrada a las demás líneas más débiles, a veces como aproximación inicial y otras veces como solución cinemática fija, dependiendo de la $\mathrm{S} / \mathrm{N}$ de las líneas. Dichas líneas de referencia son $\mathrm{H} \alpha$ y $[\mathrm{OIII}] \lambda 5007 \AA ̊$, que además de ser las líneas más intensas presentes en los espectros, corresponden a dos elementos asociados a regiones características con menor y mayor potencial de ionización, respectivamente.

Por lo dicho anteriormente y dado que uno de los ejes de análisis de esta Tesis fue la medición y determinación de parámetros de líneas de emisión, dichas líneas resultan fundamentales para llevar adelante nuestro trabajo. En consecuencia, hemos medido más de 200 líneas en la muestra de las RHII estudiadas, para las cuales calculamos los tres parámetros que se desprenden del ajuste de las múltiples componentes gaussianas:

- centro de la línea, en velocidad, v [ $\left.\mathrm{km} \mathrm{s}^{-1}\right]$

- dispersión de velocidades, $\sigma$ [ $\left.\mathrm{km} \mathrm{s}^{-1}\right]$

- flujos $\left[\mathrm{erg} \mathrm{s}^{-1} \mathrm{~cm}^{-2}\left(\mathrm{~km} \mathrm{~s}^{-1}\right)^{-1}\right]$.

El centro de la línea queda determinado por el centroide de la solución gaussiana de cada componente ajustada. Es necesario conocer la velocidad sistémica de la galaxia o de los nudos observados para calcular la velocidad de cada una de las componentes respecto a la velocidad central. Para ello hemos tomado las velocidades de trabajos publicados en la literatura , $2970 \mathrm{~km} / \mathrm{s}$ para II Zw 33 (Méndez et al., 1999) y $1016 \mathrm{~km} / \mathrm{s}$ para Mrk 600 (Lagos et al., 2018).

\subsubsection{Tarea de ajuste para las componentes: Kinematics}

En esta subsección describimos el desarrollo de una herramienta computacional para llevar adelante el proceso de ajuste de las líneas. Este programa nos permitió optimizar tiempos y 
llevar adelante el análisis de manera más efectiva. Originalmente, la metodología se planteó en Hägele et al. (2007), como se mencionó, y posteriormente, por ejemplo en Firpo (2011) se perfeccionó dicha metodología. La herramientas de ajustes de funciones utilizada fue el ngaussfit de IRAF, en donde se ajustaron gaussianas a los perfiles de líneas. Utilizando este tarea se han encontrado resultados novedosos y consistentes que han sido publicados en los trabajos mencionados. A través de dicha herramienta se realiza un ajuste gaussiano iterativo que permite seleccionar diferentes parámetros para ser ajustados, y posteriormente se guardan los valores ajustados de los parámetros y sus errores estadísticos. La herramienta necesita de valores iniciales, los cuales pueden ser proporcionados mediante tablas o a través de una ventana interactiva. El procedimiento llevado a cabo consiste en:

- Se define una primera componente gaussiana de modo de tener un ajuste global de la línea. Dependiendo del resultado, del indicador estadístico $\chi^{2}$ y de los residuos del ajuste con la observación, se procede a ajustar otra componente más, con el fin de mejorar la solución. Este proceso se repite hasta encontrar la mejor representación.

- Este procedimiento depende de los perfiles de las líneas, pero en muchas situaciones las alas son difíciles de ajustar. Si tenemos un perfil simétrico, elegir entre una componente ancha o 2 angostas para cada ala, dependiendo del trabajo y el objetivo.

- En aquellos casos en que la componente ancha no ajusta muy bien el perfil, entonces se procede a considerar dos componentes angostas: una corrida al azul y otra al rojo respecto a la gaussiana original.

- Los ajustes se hacen para las líneas más intensas y luego se toman los parámetros de las funciones gaussianas y se las copia en las línea más débiles, en algunos casos como aproximación inicial de su propio ajuste, y en otros se deja la solución cinemática fija dejando variar solamente la intensidad o amplitud de las líneas, dependiendo de la señal-a-ruido de las líneas. En muchas ocasiones esto resulta muy difícil de concretar debido a la baja señal de algunas líneas, por lo que la presencia de estas componentes se hace más clara en las líneas intensas. Esto no quiere decir que estas componentes no existan, sino que no pueden ser detectadas en líneas débiles.

- Es importante señalar que el indicador estadístico $\chi^{2}$ no da cuenta de que el ajuste sea físicamente coherente, sino que lo que nos indica es que matemáticamente la solución se acerca al mejor ajuste por sobre el dato observacional. Entonces, cuantas más componentes gaussianas se agreguen, el indicador $\chi^{2}$ mejorará. Esto no implica ninguna interpretación física sobre la solución, de manera que a aquella que contiene el menor número de componentes con un buen ajuste a ojo, será la mejor solución estimada.

Esta herramienta fue "durante muchos años" la forma de trabajo mediante la cual se llevaban a cabo estudios de componentes gaussianas en espectros. Una de sus desventajas es que no resulta práctica para hacer ajustes en varias líneas al mismo tiempo. En nuestro caso, realizar el ajuste de más de 200 líneas, trabajando una por una, fue una tarea que se tornó extremadamente ardua y poco práctica. Esto nos impulsó a que busquemos una herramienta computacional nueva, actual y con mayor y mejores aplicaciones, que pueda reducir el tiempo de análisis a través de la automatización de tareas y llevar a cabo el trabajo de una manera más práctica. Utilizando la tarea LMFit (Non-Linear Least-Squares Minimization and CurveFitting for Python, Newville et al., 2014) que proporciona una interfaz de alto nivel para la optimización no lineal y el ajuste de curvas, desarrollamos un programa para resolver nuestra problemática, y reemplazar al ngauss. A este programa lo llamamos Kinematics. 
A través de Kinematics hemos incorporado varias tareas para el análisis de la cinemática por medio del ajuste de la combinación de una función lineal y de funciones gaussianas, lo cual nos permite modelar de forma conjunta el continuo local y el perfil de las líneas. Por medio de un ajuste de mínimos cuadrados (el algoritmo de Levenberg-Marquardt) se busca minimizar la suma cuadrática de los residuos utilizando a su vez los métodos de Gauss-Newton y el método de descenso por gradiente entre el espectro y el ajuste (Newville et al., 2016).

Estas tareas se realizan de la siguiente manera:

- A cada línea se le define el rango espectral local en el cual se aplicará el ajuste de las funciones. Este rango espectral lo presentamos en velocidades y no en longitud de onda, dado que será más útil para el trabajo posterior, en los análisis de los parámetros de las gaussianas.

- Para el ajuste del perfil de la línea se definen funciones gaussianas y para el ajuste del continuo local se define una función lineal. Cada componente gaussiana presenta los tres parámetros mencionados anteriormente: centro, dispersión de velocidad y amplitud (área bajo la curva), mientras que para la función lineal para el ajuste del continuo se deben definir los dos parámetros, la de la pendiente y la ordenada al origen. Esta última es una función robusta que nos ayuda a fijar el cero de la base de las gaussianas.

- Con las funciones definidas, se propone como modelo de ajuste a la combinación de la función lineal más las componentes gaussianas. Como este método solo calcula mínimos locales la solución depende fuertemente de los parámetros iniciales. Para cada una de las componentes hay que indicar los valores iniciales de las variables, en donde es importante que los valores iniciales se encuentren cercanos al valor del mejor ajuste para que la solución converja en un número menor de procesos y evite caer en una minimización local que no sea la correcta. Esto se obtiene haciendo inicialmente algunas de prueba hasta encontrar un conjunto de valores para las condiciones iniciales que sean próximas a las correspondientes al mejor ajuste.

- Las principales diferencias entre LMFit y el ngauss surgen cuando indicamos la cantidad de funciones que vamos a ajustar en nuestro modelo, dado que este último presenta una limitación y reestricción en el ajuste de los parámetros de las funciones, siendo posible definirlos como libres o fijos únicamente. Mientras que en la nueva metodología uno puede indicar cotas a los parámetros de las funciones, lo que es ventajoso y de suma importancia para restringir mínimos en flujo y evitar que una minimización del ajuste presente líneas de absorción como una posibilidad. Por ello, en los modelos, se define como cota inferior de flujo (amplitud) un valor nulo, de manera de evitar falsos perfiles de absorción y permitiéndole al ajuste anular una componente si no hay señal que lo justifique.

- Para justificar el número de gaussianas de la mejor solución usamos las herramientas estadísticas disponibles del LMFit, las cuales se indican en los parámetros del análisis como los criterios estadísticos empleados (Fit Statistics), y estos son: reduced chi-square, Akaike info crit. y Bayesian info crit. En este trabajo utilizamos Akaike, el cual se basa en una comparación en el valor de los índices entre un ajuste con un número "n" de componentes Gaussianas y otro con " $\mathrm{n}+1$ " (para mayor detalle, ver Bosch et al., 2019). Cuando la diferencia entre los coeficientes es menor que 10, el criterio establece que la mejor solución es aquella con " $n$ " componentes. En la Figura 3.1 se pueden ver los ajustes para una y más componentes con los residuos de dichos ajustes en la línea de $\mathrm{H} \alpha$ del Nudo A de II Zw 33. En el ajuste de una componente queda clara la forma y que 
una sola componente no ajusta de manera correcta las alas. Si comparamos con ngauss, mientras $\chi^{2}$ mejora a medida que agregamos más componentes gaussianas, debido a que estadísticamente contamos con más parámetros para minimizar los errores, Akaike penaliza el agregado de gaussianas cuando el criterio estadístico dado por $\Delta \mathrm{AC} \leq 10$ se cumple (Jun-Jie et al., 2016). Para el caso del Nudo A de II Zw 33 los distintos ajustes indican un índice de Akaike de -263.002 para una componente, -531.409 para dos componentes, -669.427 para tres componentes y -674.350 para el ajuste con cuatro componentes. Debido a que el $\triangle \mathrm{AC}$ entre el ajuste con 3 y 4 componentes es menor que 10, nos quedamos con la solución con tres componentes. En Bosch et al. (2019) se presentan los resultados del ajuste de distintas componentes gaussianas y su mejor solución utilizando el criterio de Akaike, en el estudio de espectros con líneas de emisión intensas pertenecientes a una galaxias Green Pea.

- Al definir los rangos espectrales y el modelo de ajuste, puede ocurrir que los parámetros no converjan a un valor por problemas de borde en los ajustes. Esto puede deberse a que el ajuste se "choque" con un límite o porque el cambio de los parámetros libres no afecta al resultado. En estos casos se deben redefinir los rangos espectrales, ya sea agrandando el espacio de las velocidades en el eje de las abscisas y disponer así de más puntos de continuo para el ajuste, o también fijando los valores para la disminución de parámetros libres a ajustar, y chequear que la diferencia entre los parámetros ajustados menos el original sea $<10^{-8}$.

- Asumiendo como válido un escenario con dos zonas de ionización (Garnett, 1992b; López-Hernández et al., 2013), seguimos el procedimiento presentado en Hägele et al. (2012) y copiamos como aproximación inicial las soluciones calculadas para $\mathrm{H} \alpha$ y [OIII] $\lambda 5007 \AA$ (las líneas de emisión más intensa de nuestros espectros) a las demás líneas presentes. Dependiendo de los estados de ionización de los iones, los elementos de baja ionización que van a copiar la solución hallada para $\mathrm{H} \alpha$ son las líneas de recombinación de la serie de Balmer y las líneas de excitación colisional: [SII], [OII], [OI], [NII] y [ArIII]. Respecto a los elementos de alta ionización, los que adoptarán la solución calculada para el [OIII], serán las líneas de los iones de excitación colisional: [NeIII], [ArIv] y las de recombinación del He I y HeII.

- Para las líneas débiles, se propone tomar la solución hallada para las líneas intensas y copiarla dejando fijos los centros y la dispersión de velocidades, pero dejando libre la amplitud. Así, los flujos de las líneas más débiles pueden ajustarse. En este proceso puede suceder que las soluciones finales no se ajusten del todo al perfil de la línea, teniendo que definir nuevas condiciones en los parámetros para obtener una solución válida. En estos casos, se permite que dichos parámetros varíen \pm 3 veces los errores determinados para $\sigma$. Este procedimiento es necesario para obtener ajustes confiables y aceptables dentro de los errores, y así poder ajustar la cinemática a todos los conjuntos de líneas presentes y poder llevar adelante un análisis cinemático lo más completo posible.

- Una vez que se estudian todas las líneas, con el programa graficamos cada uno de los ajustes, con las componentes gaussianas definidas y superpuestas a los perfiles de línea para cada ion.

El desarrollo de este programa nos permitió mejorar y optimizar el método de análisis de múltiples componentes en los perfiles de líneas de emisión de nuestros espectros, donde hemos logrado realizar el ajuste de todas las líneas simultaneamente. Hemos verificado su utilidad 
Figura 3.1. Los ajustes gaussianos al perfil de línea de $\mathrm{H} \alpha$ para el Nudo A de II Zw 33 .

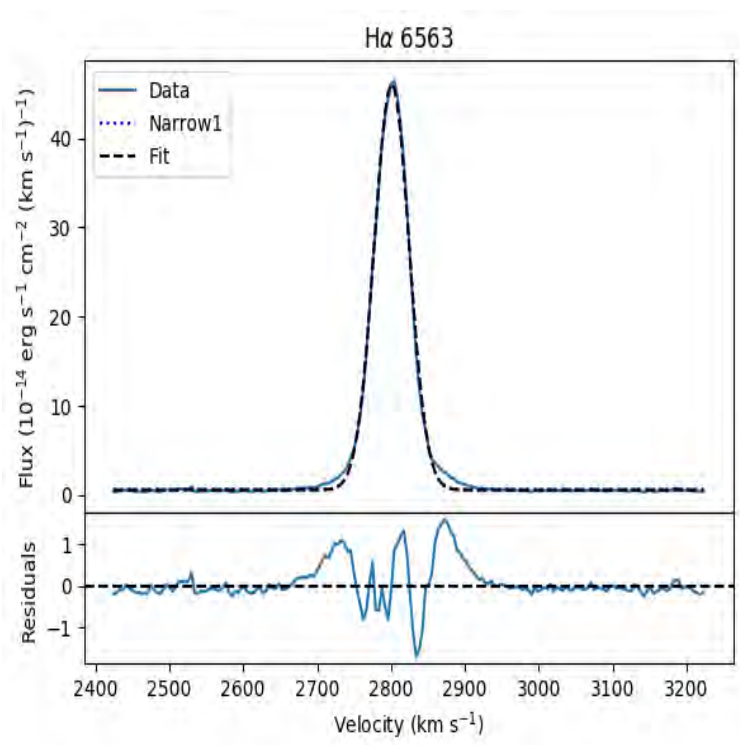

(a) Ajuste de una Gaussiana

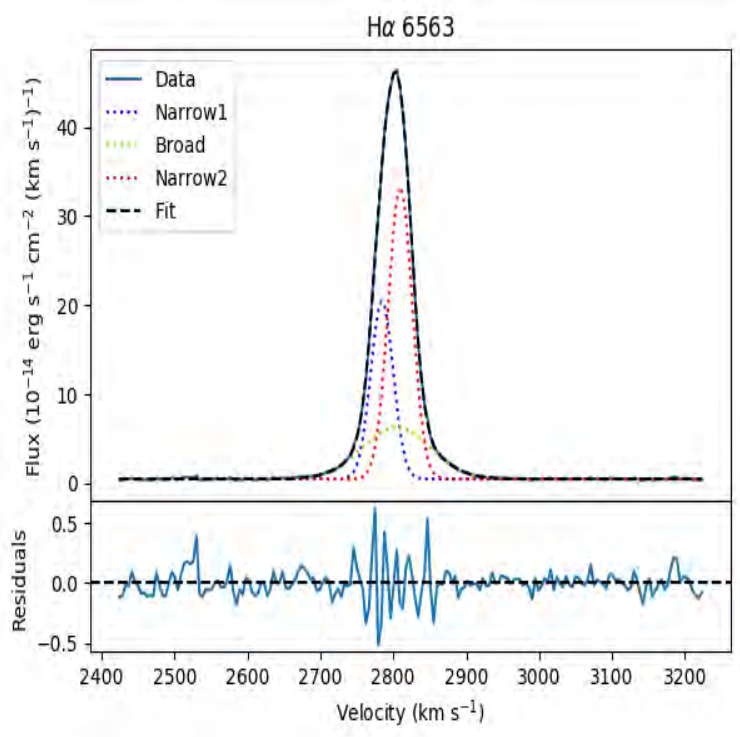

(c) Tres componentes: N1, N2 y ancha

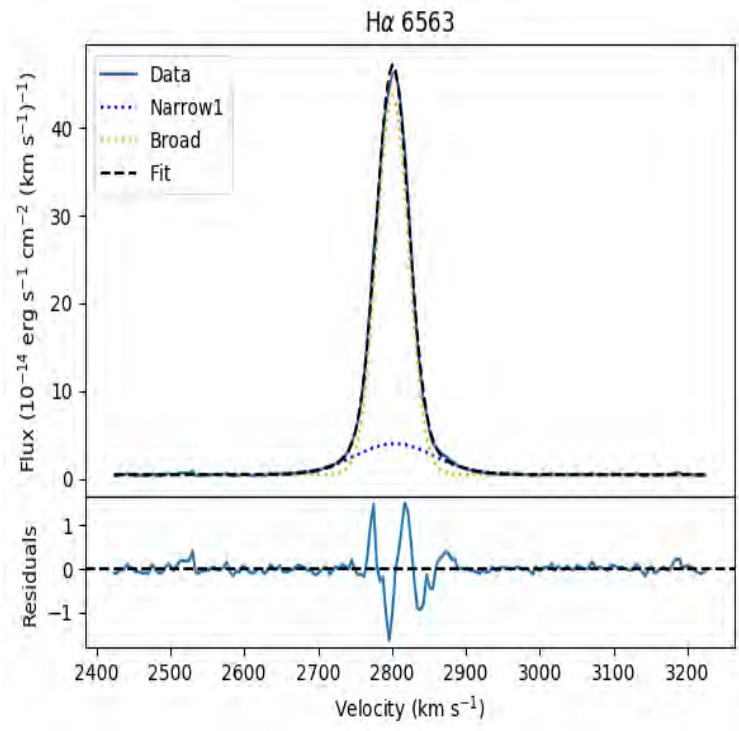

(b) Dos componentes gaussianas: N1 y ancha

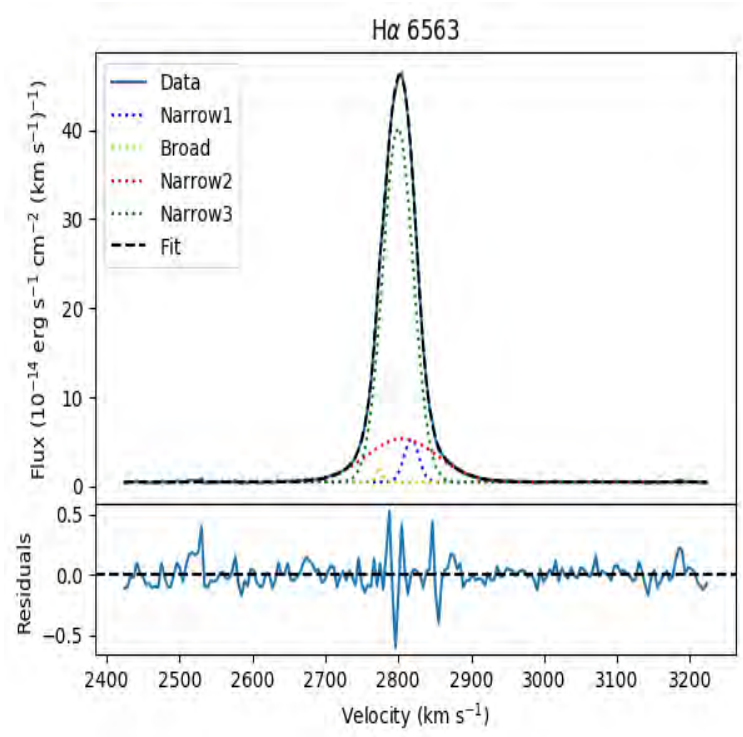

(d) Ajuste de cuatro componentes 
para el análisis de diversos métodos de espectroscopía: Echelle, IFU y long-slit (CampuzanoCastro et al., 2019; Bosch et al., 2019, y Firpo et al. en preparación); así como también en distintos tipos de regiones de formación estelar pertenecientes a galaxias BCDs, Green Peas y Regiones Circunnucleares de formación estelar, debido a que nos permite analizar la cinemática de toda clase de regiones nebulares.

Originalmente, el programa fue pensado como una herramienta para simplificar y agilizar el análisis cinemático, y así abordar y resolver un objetivo específico. Luego, observamos que tenía un potencial muy grande como herramienta de trabajo. Por lo tanto, comenzamos un trabajo más profundo y en detalle con la colaboración de un experto en programación, Daniel Muthukrishna, en ese momento pasante en Gemini Sur, y con una especialista en el uso del ngauss, la Dra. Verónica Firpo, Dra. en Astronomía y Staff de Gemini Sur. Desde el 2017 hemos trabajado en la generación y optimización del programa, para crear un programa que pueda ser publicable en sí mismo. De esta manera, hemos mantenido discusiones con ambos colegas con el fin de potenciar el trabajo y obtener un producto de primera calidad. Este trabajo fue presentado en distintas reuniones en formato poster y en contribución oral con el fin de mostrar su potencial y los resultados alcanzados en nuestro trabajo por medio de su uso. Además como un objetivo a corto plazo que se desprende de esta Tesis, es poder publicar el programa para un uso más genérico y abarcativo.

El trabajo realizado y los resultados obtenidos muestran el análisis robusto llevado adelante en las múltiples componentes para los perfiles de línea de emisión. A continuación, se presentan los resultados y la discusión de la cinemática calculada para nuestras Regiones HII.

\subsubsection{Dispersión de velocidades, $\sigma$}

Mediante el ajuste de funciones gaussianas a nuestros perfiles, tenemos como objetivo clasificar e identificar distintas componentes del gas ionizado. El ancho del perfil observado está afectado por el efecto térmico y por el efecto instrumental del espectrógrafo con el que se realizaron las observaciones. De manera que la dispersión de velocidades final, la intrínseca, viene dada por: $\sigma_{i n t r}=\sqrt{\sigma_{o b s}^{2}-\sigma_{\text {instr }}^{2}-\sigma_{\text {term }}^{2}}$, donde $\sigma_{\text {intr }}$ es dispersión de velocidades intrínsecas, $\sigma_{o b s}$ es es la dispersión observada, $\sigma_{i n s t r}$ el correspondiente a la dispersión introducida por el instrumento y $\sigma_{\text {term }}$ el correspondiente al térmico (consecuencia de la dispersión que se genera por la temperatura del gas). Este planteo es válido si se supone que la RHII observada tiene una temperatura cinética estimada por la distribución de Maxwell-Boltzmann de $\mathrm{T} \sim 10^{4} \mathrm{k}$. El ancho instrumental $\left(\sigma_{\text {instr }}\right)$ mínimo dado por el FWHM, se obtiene del perfil de las líneas de emisión (angostas) de la lámpara de comparación de Th-Ar. La dispersión de velocidades $(\sigma)$ a partir de los ajustes del ancho a media altura, abreviada FWHM (del inglés Full Width at Half Maximum) de las líneasa se puede determinar a través de la relación: $\sigma=$ $0.4249 \times$ FWHM. Además, para una temperatura cinética de $\mathrm{T}_{e}=10^{4} \mathrm{k}$ la velocidad del sonido en este medio es de $\sim 13 \mathrm{~km} / \mathrm{s}$, de manera que velocidades mayores a esta son consideradas velocidades supersónicas, una característica que precisamente define a las RHIIG como tales y las distingue de aglomeraciones de RHiı clásicas.

\subsubsection{Flujo y errores en su determinación}

La medición correcta (o más precisa posible) de los flujos de las líneas es uno de los puntos más importantes del trabajo, por lo que hemos realizado un análisis detallado y minucioso. En todos los casos hemos medido las intensidades integrando todo el flujo para cada una de las componentes y con la suma de ellas determinamos el flujo global. Los límites corresponden al rango espectral tomado y el continuo fue estimado con una función lineal, definida en el ajuste. El flujo queda definido por el ajuste del parámetro Amplitude, que en el código 
LMFit indica el área bajo la gaussiana. Debido a la dificultad de limpiar rayos cósmicos sobre los perfiles de líneas, no hemos considerado aquellas líneas que se encuentran solapadas con líneas de cielo y rayos cósmicos. Para los casos en que las líneas del cielo pueden ser resueltas en velocidad, no hubo problemas para realizar los ajustes de manera independiente y así quedarnos con la línea nebular de interés. Este efecto se presentó en los rangos espectrales más allá de los $6000 \AA$, donde líneas del [ArIII], HeI, [OI], [SII] y [SIII] han sido las más afectadas, y muchas veces difíciles de medir. Para el análisis de múltiples componentes, la presencia de una línea de cielo solapada al perfil hace prácticamente imposible llevar adelante el análisis del perfil compuesto, por la dificultad de ajustar correctamente las componentes. Los errores observacionales asociados al flujo han sido determinados teniendo en cuenta tres fuentes principales de incertidumbre: errores estadísticos en las medidas del flujo de las líneas, errores asociados a la corrección por extinción (indicado en la siguiente sección) y los rayos cósmicos y líneas de cielo.

\subsection{Análisis de la cinemática de las regiones}

Para el estudio de la cinemática en nuestras regiones a través de las líneas de emisión de los espectros calibrados en longitud de onda, $\lambda$, hemos acotado los rangos espectrales en torno a las líneas. Haciendo uso de los corrimientos al rojo de las galaxias, transformamos nuestro sistema al plano de Velocidades $\left[\mathrm{km} \mathrm{s}^{-1}\right]$ versus Flujo $\left[\operatorname{erg~s}^{-1} \mathrm{~cm}^{-2}(\mathrm{~km} / \mathrm{s})^{-1}\right.$, a través de la corrección de $\lambda$. Para Mrk 600 tomamos como redshift $\mathrm{z}=0.00336$ (de Vaucouleurs, 1991), y para II Zw 33 consideramos z $=0.00944$ (Haynes et al., 1997), los cuales hemos verificado tomando líneas intensas de nuestro espectro como las líneas de recombinación del hidrógeno, el doblete del $[\mathrm{SiI}] \lambda \lambda 6717,6731 \AA$ y el $[\mathrm{SIII}] \lambda 9069 \AA$. Hemos calculado su corrimiento respecto a la longitud de onda central adoptadas del trabajo de García-Rojas et al. (2005), encontrando buen acuerdo en los valores de z. Además, la velocidad sistémica de las regiones de II Zw 33 se han adaptado del estudio de las $\mathrm{V}_{\text {rad }}$ estimadas en Méndez et al. (1999) mediante un ajuste de una gaussiana para el perfil de $\mathrm{H} \alpha$, que proporciona una velocidad heliocéntrica sistémica promedio de $2790 \mathrm{~km} \mathrm{~s}^{-1}$. A su vez, esta velocidad sistémica está en acuerdo con las velocidades estimadas tomando los perfiles de líneas de Hi (ver Lauqué, 1973). Por la parte de Mrk 600, se ha tomado como referencia la velocidad sistémica determinada en Lagos et al. (2018) donde a través de un estudio realizado utilizando el instrumento VIMOS-IFU de VLT midieron un valor de $1016 \mathrm{~km} \mathrm{~s}^{-1}$.

En las Tablas 3.1, 3.2, 3.3, 3.4 y 3.5 se presentan las velocidades radiales para cada componente referidas a las velocidades sistémicas, las dispersiones de velocidades $\sigma_{\text {intr }}$, los flujos de cada componente y las componentes globales y las Medidas de Emisión (EM, de su sigla en inglés) para cada componente, donde la medida de emisión se define como el porcentaje de flujo de cada componente cinemática respecto al flujo total. En las medidas determinadas para las dispersiones de velocidades $\sigma_{\text {intr }}$ se pueden apreciar las velocidades supersónicas del gas en estas regiones. Para los ajustes hemos evaluado distintas componentes cinemáticas de distintos anchos. Se puede observar la presencia de una componente ancha y, en varios casos, más de una componente angosta. Estas suelen indicar una componente de gas hacia el azul y otra hacia el rojo del espectro electromagnético respectivamente, con diferencias en sus anchos de hasta $40 \mathrm{~km} \mathrm{~s}^{-1}$ asociada a distintas fuentes de gas ionizado. De esta manera, hemos planteado una metodología para explicar las alas del perfil integrado en cada una de las regiones. A continuación haremos una descripción detallada del estudio de la cinemática. 


\subsubsection{Zw 33}

Describiremos las soluciones cinemáticas determinadas para las tres regiones observadas. En el panel izquierdo de la Figura 2.4 de la Sección 2.4, indicamos las regiones observadas según la identificación de Nudo A, B y C. El primero y más intenso es el brote de formación estelar inferior, identificado como A. El del centro corresponde a la región B. Por último, el más débil es el Nudo C que está ubicado hacia el Norte en la imagen.

\subsubsection{Zw 33 Nudo A}

En su descripción recibe el nombre de Nudo A, ya que de las tres regiones observadas en II Zw 33, esta es la más luminosa, $\operatorname{con} \log (\mathrm{L}(\mathrm{H} \alpha)) \approx 40.35 \operatorname{erg~s}^{-1}$ (Méndez et al., 1999). Se han identificado y ajustado perfiles gaussianos a las líneas de recombinación más intensas de hidrógeno $(\mathrm{H} \alpha, \mathrm{H} \beta, \mathrm{H} \gamma$ y $\mathrm{H} \delta)$ y de helio (HeI $\lambda 5875, \lambda 6678, \lambda 4471$ y $\lambda 7065 \AA$ ), a las líneas intensas y a la línea auroral del [OIII] $\lambda 5007, \lambda 4959$ y $\lambda 4363 \AA$, respectivamente. las líneas del $[\mathrm{NII}] \lambda \lambda 6584,6548 \AA$, el doblete del $[\mathrm{SII}] \lambda \lambda 6716,6731 \AA$, así como también a líneas más débiles como el $[\mathrm{SiII}] \lambda$ 9069 $\AA$, [NeIII] $\lambda 3868 \AA$, [ArIv] $\lambda 7751 \AA$, entre las más importantes.

El mejor ajuste al perfil de $\mathrm{H} \alpha$ revela la presencia de dos componentes cinemáticas angostas, claramente separadas y distinguibles, a las que llamamos N1 y N2 (N indicando la abreviatura de narrow, del inglés: angosto), y una componente más ancha, que denominamos "Ancha", para el ajuste de las alas. Dicha solución cinemática fue determinada por medio de una comparación con modelos de ajustes con distinta cantidad de componentes gaussianas entre una y otra solución. Siguiendo lo indicado por el criterio de Akaike (AC, del inglés: Akaike Criterion), como mencionamos en la sección anterior, cuando se cumple que el $\Delta \mathrm{AC} \leq 10$, en la diferencia entre distintas soluciones, nos quedamos con la que tiene menos componentes. Veamos como son los ajustes y el valor del índice de Akaike para las líneas intensas de recombinación del hidrógeno según distintas componentes.

Para una componente (1c, de ahora en más) el índice Akaike $=-263.002$; dos componentes (2c, de ahora en más $)=-531.409$, tres componentes $(3 \mathrm{c}$, de ahora en más $)=-669.427$ y cuatro componentes (4c, de ahora en más $)=-674.350$. Dado que la diferencia entre el ajuste de tres y el consecutivo, de cuatro componentes, no es mayor que 10, según la relación $\Delta \mathrm{AC} \leq 10$ determinamos que la mejor solución es con tres componentes (ver ajustes correspondientes en la Figura 3.1). Los ajustes gaussianos de las líneas de los distintos elementos se muestran en la Figura 3.2.

Entre las componentes angostas, vemos que la N1 muestra un perfil ligeramente más angosto que la componente N2 ( $\sigma_{\text {intr }}: 6.3 \pm 1.7 \mathrm{~km} \mathrm{~s}^{-1}$ y $8.4 \pm 1.1 \mathrm{~km} \mathrm{~s}^{-1}$, respectivamente). Con respecto a la velocidad radial referidas a la velocidad sistémica, la componente N1 se encuentra hacia el azul $\left(V_{\text {rad }}:-5.5 \pm 2.1 \mathrm{~km} \mathrm{~s}^{-1}\right)$ y la componente $\mathrm{N} 2$ hacia el rojo ( $V_{\text {rad }}$ : $\left.19.6 \pm 1.5 \mathrm{~km} \mathrm{~s}^{-1}\right)$. Por su parte, la componente ancha presenta un $\sigma_{\text {intr }}=44.2 \pm 0.9 \mathrm{~km} \mathrm{~s}^{-1}$, mayor que las angostas y su velocidad resulta $V_{\text {rad }}=14.1 \pm 0.7 \mathrm{~km} \mathrm{~s}^{-1}$.

$\mathrm{Al}$ igual que la solución para $\mathrm{H} \alpha$, en el perfil de línea del [OIII] hemos ajustado tres componentes, dos angostas y una ancha. Los respectivos $\sigma_{\text {intr }}$ de las componentes resultan: $\sigma_{N 1}=9.8 \pm 2.8 \mathrm{~km} \mathrm{~s}^{-1}, \sigma_{N 2}=12.1 \pm 1.3 \mathrm{~km} \mathrm{~s}^{-1}$ y $\sigma_{B}=34.3 \pm 3.9 \mathrm{~km} \mathrm{~s}^{-1}$. Para los centros, en $\mathrm{N} 1=-2.8 \pm 4.9 \mathrm{~km} \mathrm{~s}^{-1}, \mathrm{~N} 2=21.2 \pm 2.3 \mathrm{~km} \mathrm{~s}^{-1}$ y Ancha $=6.5 \pm 4.1 \mathrm{~km} \mathrm{~s}^{-1}$. Dichas componentes muestran una gran coherencia tanto en las velocidades radiales individuales como en la dispersión de velocidades entre las diferentes líneas de emisión ajustadas a partir de los ajustes de las líneas más intensas. Además, hemos propuesto una componente con un $\sigma_{\text {intr }}$ de $\sim 100 \mathrm{~km} \mathrm{~s}^{-1}$ hacia el rojo para ajustar una componente (o imagen fantasma) que acompaña a la línea intensa de [OIII] $\lambda 5007 \AA$, debido a que está en los primeros órdenes del espectrógrafo, lo que genera esa pequeña componente, como se indicó y se explicó en 
el Capítulo 2. Esta componente no entra en la discusión de la cinemática ya que ha sido ajustada y analizada únicamente para garantizar un ajuste robusto de las componentes en la línea principal (ver Figura 3.3).

Vale la pena señalar que aunque el ajuste de las líneas del [OIII] y de $\mathrm{H} \alpha$ muestra resultados generales similares (dentro de los errores para N1 y a dos $\sigma$ para N2), las componentes N1 y N2 del [OIII] muestran un perfil más amplio (del órden de los $3 \mathrm{~km} \mathrm{~s}^{-1}$ ). Esto podría estar relacionado con un comportamiento cinemático diferente entre el gas altamente ionizado y el gas correspondiente a regiones más externas, lo cual debe confirmarse para otras líneas de alta excitación. Siguiendo el procedimiento descrito en Firpo et al. (2010), pudimos ajustar una componente ancha, con un $\sigma$ mayor que $25 \mathrm{~km} \mathrm{~s}^{-1}$ en las líneas más intensas, y luego trasladarla a las líneas más débiles. En $\mathrm{H} \alpha$ encontramos que esta línea ancha tiene una presencia más importante que en las otras líneas débiles, a causa de la $\mathrm{S} / \mathrm{N}$, por lo que muchas veces se hace dificultoso llevar el ajuste de esta componente ancha a las líneas más débiles. Es el caso de la línea auroral del [OIII] $\lambda 4363 \AA$, en que la señal es $\sim 133$ veces menor que $\lambda 5007 \AA$, la componente ancha no la pudimos ajustar. Y usando la misma solución cinemática que para [OIII $] \lambda 5007$ Åvemos que liberando las amplitudes de las dos componentes angosta obtenemos un muy buen ajuste (ver Figura 3.2). La Tabla 3.1 muestra los parámetros cinemáticos determinados a partir de los ajustes para las tres componentes gaussianas que se han ajustado y para el perfil global. Los flujos de cada componentes los presentamos como el porcentaje dado por la medida de emisión en relación con el flujo total de la línea, como indica el trabajo de Relaño \& Beckman (2005) y como se presenta en el análisis cinemático presentado en Firpo (2011). Los casos en que no se lista ningún error a los parámetros cinemáticos del ajuste es porque la solución cinemática fue copiada de la inicial (de H $\alpha$ o [OIII] $\lambda 5007 \AA$ ) sin dejar variar el centro ni $\sigma$, debido a que las variaciones del ajuste generan grandes cambios en la solución que no son cinemáticamente consistentes con la solución obtenida para las líneas intensas. En la Figura 3.2 se muestra el ajuste realizado con el LMFit de las múltiples componentes para algunas de las líneas de interés.

Tabla 3.1. Parámetros de los ajustes de las diferentes líneas de emisión para el Nudo A de II Zw 33. En la primer y segunda columna se identifica la longitud de onda en reposo, $\lambda_{0}$, y el ion correspondiente, la tercer columna indica el nombre de las componentes ajustadas para cada línea, la cuarta las velocidades radiales, $\mathrm{V}_{r}$, respecto a la Velocidad Sistémica: $\mathrm{V}_{\text {Sistem }}=2790 \mathrm{~km} / \mathrm{s}$, la quinta las dispersiones de velocidades, $\sigma$, la sexta columna indica los Flujos para cada componente y la última columna corresponde a las Medidas de Emisión de flujo de cada componente $\left(\mathrm{EM}_{f}\right)$.

\begin{tabular}{lllcccc}
\hline $\begin{array}{l}\lambda_{0} \\
{[\AA]}\end{array}$ & Ion & Comp. & $\begin{array}{c}\mathrm{v}_{\mathrm{r}} \\
{\left[\mathrm{km} \mathrm{s}^{-1}\right]}\end{array}$ & $\begin{array}{c}\sigma_{\text {int }} \\
{\left[\mathrm{km} \mathrm{s}^{-1}\right]}\end{array}$ & $\begin{array}{c}\text { Flujo } \\
{\left[10^{-17} \mathrm{erg} \mathrm{s}^{-1} \mathrm{~cm}^{-2}\right]}\end{array}$ & EM $_{\mathrm{f}}$ \\
\hline 6563 & $\mathrm{H} \alpha$ & Angosta 1 & $-5.5 \pm 2.1$ & $6.3 \pm 1.7$ & $769 \pm 6$ & 27.5 \\
& & Angosta 2 & $19.6 \pm 1.5$ & $8.4 \pm 1.1$ & $1348 \pm 6$ & 48.2 \\
& & Ancha & $14.1 \pm 0.7$ & $44.2 \pm 0.9$ & $679 \pm 10$ & 24.3 \\
& & Flujo Global & $2796 \pm 13$ & & & \\
4861 & $\mathrm{H} \beta$ & Angosta 1 & -5.5 & 6.3 & $169 \pm 4$ & 19.6 \\
& & Angosta 2 & 19.6 & 8.4 & $447 \pm 5$ & 51.8 \\
& & Ancha & 14.1 & 44.2 & $247 \pm 9$ & 28.6 \\
& & Flujo Global & $863 \pm 11$ & & & \\
4340 & $\mathrm{H} \gamma$ & Angosta 1 & -5.5 & 6.3 & $87 \pm 3$ & 18.3 \\
& & Angosta 2 & 19.6 & 8.4 & $222 \pm 4$ & 47 \\
& & Ancha & 14.1 & 44.2 & $166 \pm 7$ & 35
\end{tabular}


Flujo Global $\quad 474 \pm 8$

\begin{tabular}{|c|c|c|c|c|c|c|}
\hline \multirow[t]{4}{*}{4102} & $\mathrm{H} \delta$ & Angosta 1 & -5.5 & 6.3 & $46 \pm 3$ & 16.5 \\
\hline & & Angosta 2 & 19.6 & 8.4 & $133 \pm 3$ & 47.5 \\
\hline & & Ancha & 14.1 & 44.2 & $101 \pm 7$ & 36.0 \\
\hline & & Flujo Global & $281 \pm 8$ & & & \\
\hline \multirow[t]{4}{*}{5007} & [OIII] & Angosta 1 & $-2.8 \pm 4.9$ & $9.8 \pm 2.8$ & $788 \pm 23$ & 18.7 \\
\hline & & Angosta 2 & $21.2 \pm 2.3$ & $12.1 \pm 1.3$ & $2668 \pm 25$ & 60.34 \\
\hline & & Ancha & $6.5 \pm 4.1$ & $34.3 \pm 3.9$ & $913 \pm 47$ & 20.96 \\
\hline & & Flujo Global & $4372 \pm 58$ & & & \\
\hline \multirow[t]{4}{*}{4959} & [Öні] & Angosta 1 & -2.8 & 9.8 & $287 \pm 16$ & 21.3 \\
\hline & & Angosta 2 & 21.2 & 12.1 & $869 \pm 17$ & 64.6 \\
\hline & & Ancha & 6.5 & 34.3 & $190 \pm 34$ & 14.1 \\
\hline & & Flujo Global & $1346 \pm 42$ & & & \\
\hline \multirow[t]{4}{*}{4363} & [OІІІ] & Angosta 1 & -2.8 & 9.8 & $6 \pm 2$ & 19.0 \\
\hline & & Angosta 2 & 21.2 & 12.1 & $27 \pm 2$ & 81.0 \\
\hline & & Ancha & 6.5 & 34.3 & $0.00 \pm 3.314$ & 0.0 \\
\hline & & Flujo Global & $33 \pm 4$ & & & \\
\hline \multirow[t]{4}{*}{6584} & {$[\mathrm{NII}]$} & Angosta 1 & -2.7 & 6.3 & $37 \pm 2$ & 29.5 \\
\hline & & Angosta 2 & 19.6 & 8.5 & $44 \pm 2$ & 35.2 \\
\hline & & Ancha & 13.0 & 44.2 & $44 \pm 5$ & 35.3 \\
\hline & & Flujo Global & $125 \pm 6$ & & & \\
\hline \multirow[t]{4}{*}{6548} & {$[\mathrm{NII}]$} & Angosta 1 & -2.7 & 6.3 & $10 \pm 1$ & 22.8 \\
\hline & & Angosta 2 & 19.6 & 8.5 & $11 \pm 1$ & 24.1 \\
\hline & & Ancha & 13.0 & 44.2 & $23 \pm 3$ & 53.1 \\
\hline & & Flujo Global & $44 \pm 4$ & & & \\
\hline \multirow[t]{4}{*}{6717} & {$[\mathrm{SII}]$} & Angosta 1 & -2.7 & 6.3 & $31 \pm 2$ & 18.1 \\
\hline & & Angosta 2 & 19.6 & 8.5 & $42 \pm 3$ & 24.3 \\
\hline & & Ancha & 13.0 & 44.2 & $100 \pm 7$ & 57.6 \\
\hline & & Flujo Global & $173 \pm 8$ & & & \\
\hline \multirow[t]{4}{*}{6731} & {$[\mathrm{SII}]$} & Angosta 1 & -2.7 & 6.3 & $23 \pm 2$ & 19.4 \\
\hline & & Angosta 2 & 19.6 & 8.5 & $40 \pm 3$ & 34.1 \\
\hline & & Ancha & 13.0 & 44.2 & $55 \pm 7$ & 46.5 \\
\hline & & Flujo Global & $118 \pm 8$ & & & \\
\hline \multirow[t]{4}{*}{3729} & [OII] & Angosta 1 & -6.8 & 6.3 & $266 \pm 12$ & 21.4 \\
\hline & & Angosta 2 & 18.9 & 8.4 & $398 \pm 14$ & 32.0 \\
\hline & & Ancha & 13.3 & 44.2 & $581 \pm 44$ & 46.7 \\
\hline & & Flujo Global & $1246 \pm 48$ & & & \\
\hline
\end{tabular}




\begin{tabular}{|c|c|c|c|c|c|c|}
\hline \multirow[t]{4}{*}{3726} & \multirow[t]{4}{*}{ [Оні] } & Angosta 1 & -5.1 & 6.3 & $208 \pm 8$ & 22.9 \\
\hline & & Angosta 2 & 19.8 & 8.5 & $311 \pm 10$ & 34.3 \\
\hline & & Ancha & 10.6 & 44.1 & $389 \pm 32$ & 42.9 \\
\hline & & Flujo Global & $907 \pm 34$ & & & \\
\hline \multirow[t]{4}{*}{5876} & \multirow[t]{4}{*}{ HeI } & Angosta 1 & -2.8 & 9.8 & $28 \pm 7$ & 29.7 \\
\hline & & Angosta 2 & 21.2 & 12.1 & $63 \pm 8$ & 66.0 \\
\hline & & Ancha & 6.5 & 34.3 & $4 \pm 16$ & 4.3 \\
\hline & & Flujo Global & $95 \pm 19$ & & & \\
\hline \multirow[t]{4}{*}{4471} & \multirow[t]{4}{*}{ HeI } & Angosta 1 & 0.0 & 9.3 & $1.75 \pm 1.699$ & 5.5 \\
\hline & & Angosta 2 & 24.0 & 12.6 & $19.87 \pm 1.909$ & 62.1 \\
\hline & & Ancha & 9.3 & 35.3 & $10.38 \pm 4.763$ & 32.4 \\
\hline & & Flujo Global & $32 \pm 5$ & & & \\
\hline \multirow[t]{4}{*}{6678} & \multirow[t]{4}{*}{ HeI } & Angosta 1 & -2.8 & 9.8 & $9 \pm 2$ & 32.9 \\
\hline & & Angosta 2 & 21.2 & 12.1 & $17 \pm 3$ & 65.9 \\
\hline & & Ancha & 6.5 & 34.3 & 0.31 & 1.2 \\
\hline & & Flujo Global & $26 \pm 7$ & & & \\
\hline \multirow[t]{4}{*}{7065} & \multirow[t]{4}{*}{ HeI } & Angosta 1 & -2.8 & 9.8 & $6 \pm 1$ & 35.7 \\
\hline & & Angosta 2 & 21.2 & 12.1 & $10 \pm 2$ & 59.1 \\
\hline & & Ancha & 6.5 & 34.3 & 0.89 & 5.2 \\
\hline & & Flujo Global & $17 \pm 5$ & & & \\
\hline \multirow[t]{4}{*}{6300} & \multirow[t]{4}{*}{ [OI $]$} & Angosta 1 & -5.5 & 6.3 & $6 \pm 1$ & 17.9 \\
\hline & & Angosta 2 & $20.3 \pm 1.3$ & 8.4 & $14 \pm 2$ & 43.3 \\
\hline & & Ancha & 14.1 & 44.2 & $13 \pm 5$ & 38.8 \\
\hline & & Flujo Global & $32 \pm 5$ & & & \\
\hline \multirow[t]{4}{*}{9069} & \multirow[t]{4}{*}[\mathrm{SiII}]{} & Angosta 1 & -0.2 & 9.8 & $11 \pm 7$ & 6.6 \\
\hline & & Angosta 2 & 20.2 & 12.1 & $76 \pm 9$ & 46.2 \\
\hline & & Ancha & 9.3 & 34.3 & $77 \pm 16$ & 47.1 \\
\hline & & Flujo Global & $163 \pm 19$ & & & \\
\hline \multirow[t]{4}{*}{3868} & \multirow[t]{4}{*}{ [NeIII] } & Angosta 1 & -4.3 & 9.8 & $23 \pm 6$ & 5.3 \\
\hline & & Angosta 2 & 24.0 & 12.1 & $240 \pm 7$ & 54.5 \\
\hline & & Ancha & 9.3 & 34.3 & $178 \pm 17$ & 40.2 \\
\hline & & Flujo Global & $441 \pm 19$ & & & \\
\hline \multirow[t]{4}{*}{7136} & \multirow[t]{4}{*}{ [ArIII] } & Angosta 1 & -2.7 & 6.3 & $21 \pm 2$ & 26.3 \\
\hline & & Angosta 2 & 20.9 & 8.5 & $30 \pm 2$ & 37.0 \\
\hline & & Ancha & 16.9 & 44.2 & $29 \pm 6$ & 36.6 \\
\hline & & Flujo Global & $80 \pm 7$ & & & \\
\hline
\end{tabular}


Figura 3.2. Perfiles de las líneas de emisión más intensas del Nudo A de II Zw 33 con sus respectivas componentes cinemáticas superpuestas. Cada espectro está calibrado en flujo, y en el eje $\mathrm{x}$ encontramos las velocidades radiales respecto al centro de la línea. En el panel inferior se presenta el residuo entre el ajuste y la observación. Para aumentar los detalles en las bajas señales, aplicamos escala logarítmica en flujo. Desde arriba hacia abajo y de izquiera a derecha, encontramos a: $\mathrm{H} \alpha, \mathrm{H} \beta, \mathrm{H} \gamma, \mathrm{H} \delta,[\mathrm{OIII}] \lambda 5007 \AA$, [NII] $\lambda 6584 \AA$, [SII] $] 6717 \AA$, $[\mathrm{SII}] \lambda 6731 \AA$ y $[\mathrm{OIII}] \lambda 4363 \AA$.
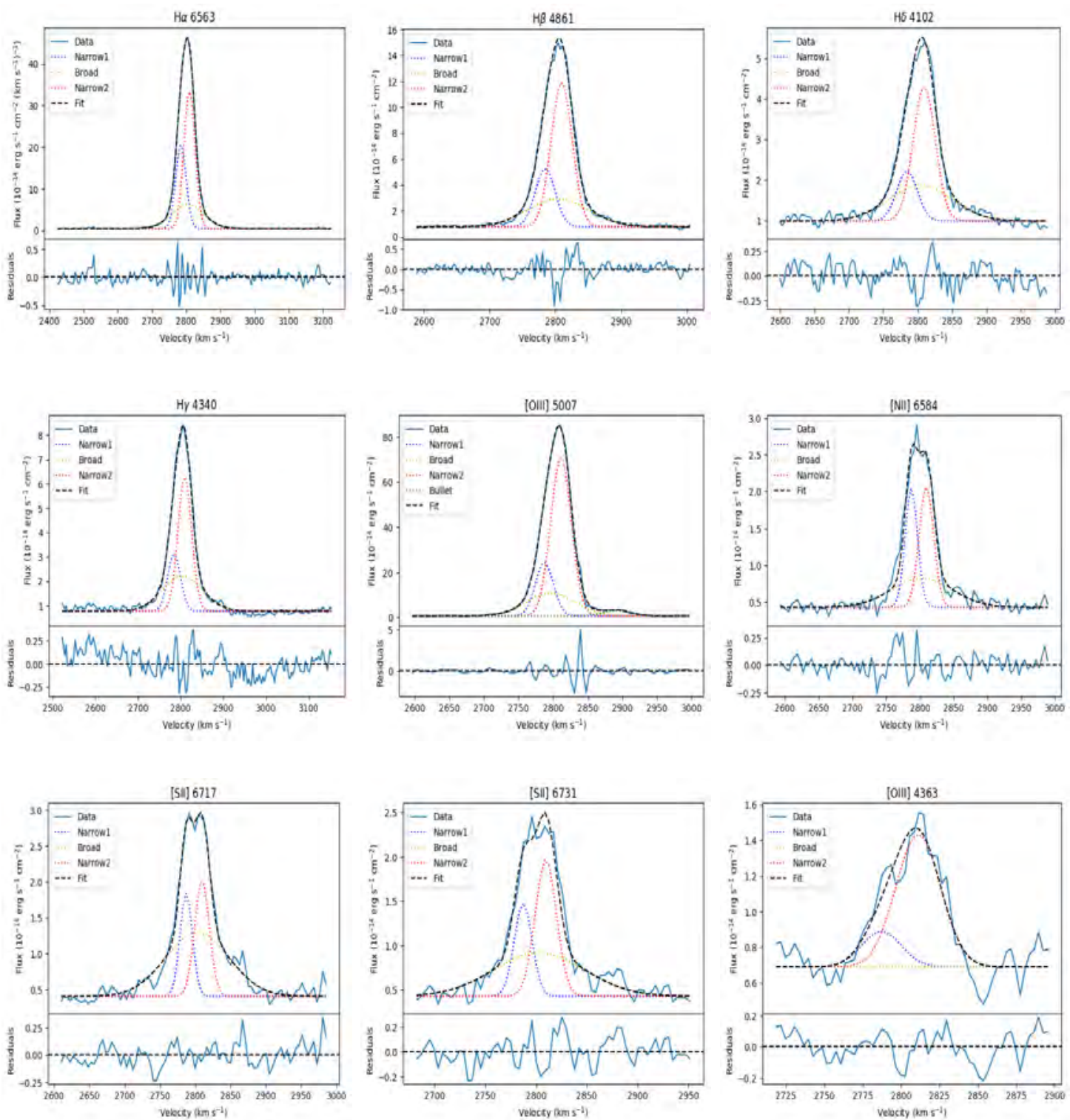
Figura 3.3. Ajuste de las múltimples componentes en la línea de [OIII] $\lambda 5007 \AA ̊$ en el Nudo A de Mrk 600. En el centroide de la línea se observan las múltiples componentes y hacia el rojo una componente fantasma en emisión de la línea. Los ajustes de las múltiples componentes cinemáticas se presentan azul, rojo, verde y negro y las componentes fijadas en el espectro fantasma generado por el dicróico (descripto en el capítulo anterior) se muestran en amarillo (Bullet 1 y Bullet 2).

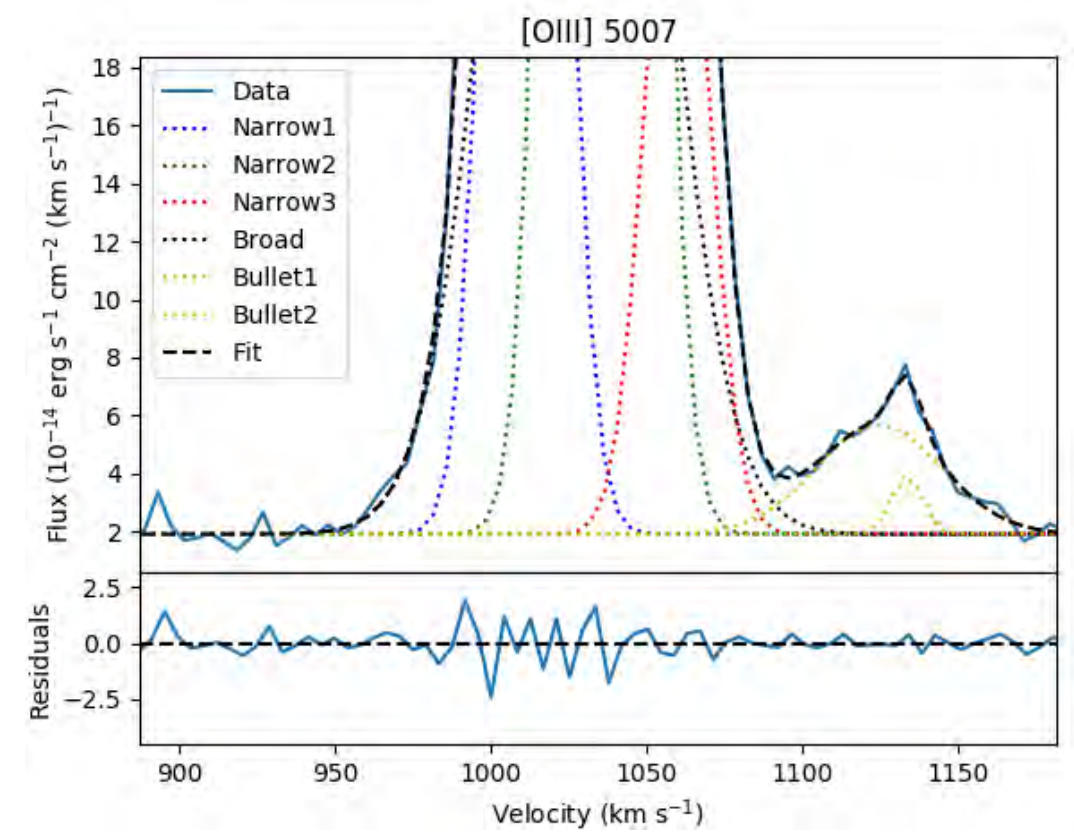

\subsubsection{Zw 33 Nudo B}

El espectro del Nudo B $\left(\log (\mathrm{L}(\mathrm{H} \alpha)) \approx 40.10 \mathrm{erg} \mathrm{s}^{-1}\right.$, Méndez et al., 1999)), al igual que el de la región A, está dominado por líneas de emisión correspondientes a gas ionizado en la nebulosa. Detectamos y medimos líneas débiles sensibles a la temperatura de dos iones: del [OIII] en $\lambda 4363 \AA$ y la correspondiente al [SIII] $\lambda 6312 \AA$. Identificamos y ajustamos múltiples componentes a las líneas de recombinación del H y del HeI, así como también a las líneas intensas del [OIII], al doblete del [OII] y del [SII], a las líneas débiles del [NII] y [SIII], [ArIII] y [NeIII] con suficiente S/N para ajustar las múltiples componentes en los perfiles de las líneas. El comportamiento cinemático medido para la región es complejo, donde el mejor ajuste presenta tres componentes: dos angostas y la restante, una ancha, para ajustar de manera efectiva las alas de los perfiles. Los ajustes determinados para $\mathrm{H} \alpha$ y el [OIII] muestran un gran acuerdo, por lo que se puede afirmar que existe un comportamiento cinemático compatible entre las distintas zonas de ionización propuestas para esta región.

A partir de los valores dados del índice de Akaike, se determinan las mejores soluciones del ajuste. En este caso, la mejor solución de tres componentes queda determinada al comparar las distintas soluciones entre dos, tres y cuatro componentes. Para el ajuste con 1c tenemos un índice $\mathrm{AC}=-137.25 ; 2 \mathrm{c}=-413.66 ; 3 \mathrm{c}=-538.65$ y $4 \mathrm{c}=-408.74$. Se determina que para la solución con 4 componentes el índice no mejora. Por lo tanto, la mejor solución encontrada es la de 3 componentes. Los distintos ajustes se muestran en los paneles de la Figura 3.4.

Para la componente ancha, se eliminaron los residuos de las alas de las líneas de emisión. Presenta un $\sigma_{\text {int }}$ de $42.1 \pm 1.1 \mathrm{~km} \mathrm{~s}^{-1}$ para $\mathrm{H} \alpha$ y de $37.5 \pm 1.6 \mathrm{~km} \mathrm{~s}^{-1}$ para [OIII] $\lambda 5007 \AA$. En los paneles de la Figura 3.5 se muestran no solo los ajustes de las múltiples componentes de algunas de las líneas de interés sino que además se presentan los residuos de los ajustes. Es 
Figura 3.4. Ajustes con distinta cantidad de gaussianas al perfil de la línea de $\mathrm{H} \alpha$ para el Nudo B de II Zw 33.

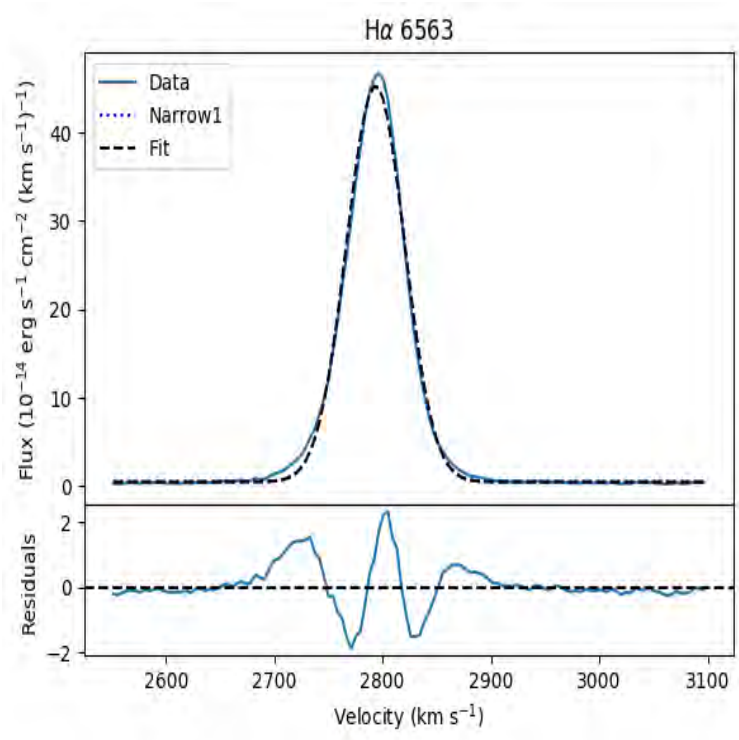

(a) Ajuste de una gaussiana.

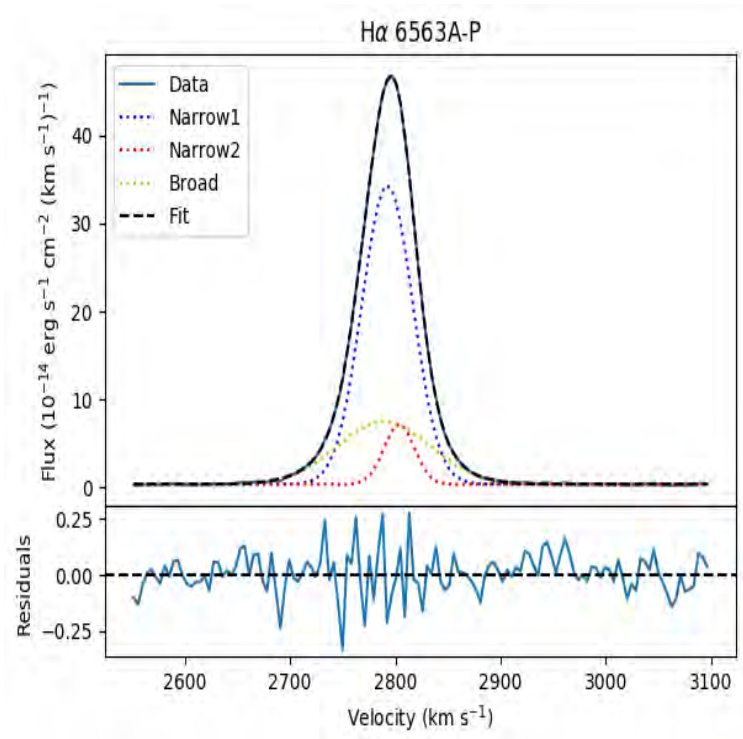

(c) Tres componentes: N1, N2 y ancha.

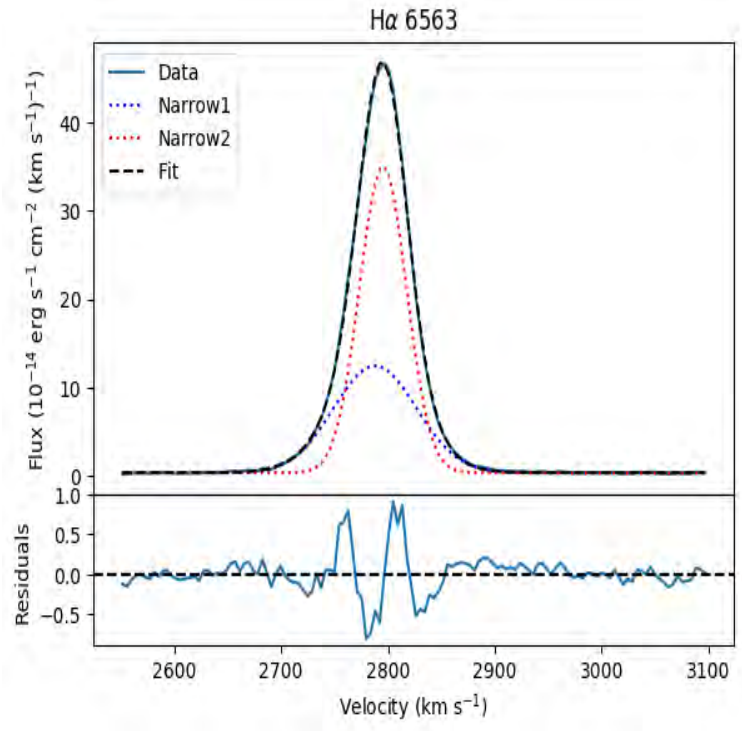

(b) Dos componentes gaussianas: N1 y ancha.

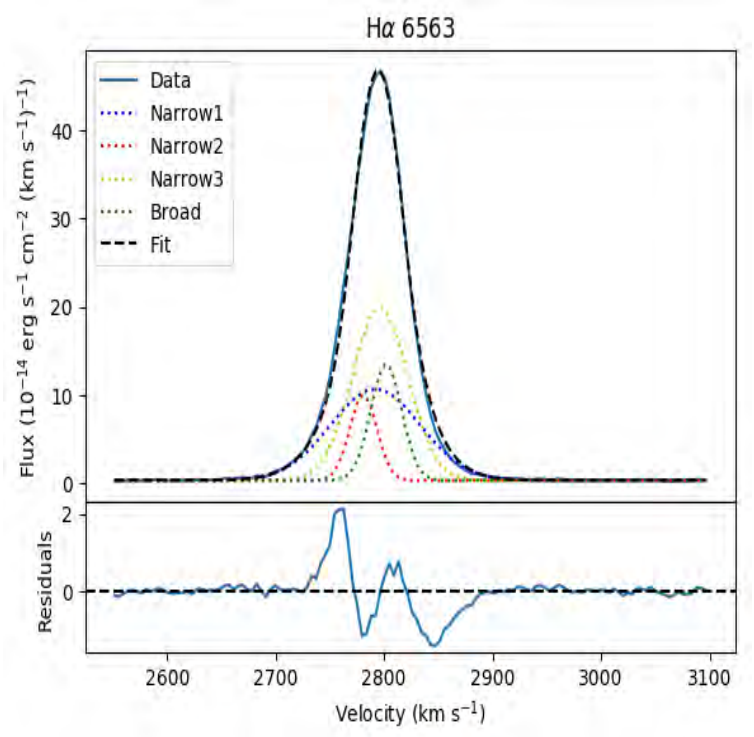

(d) Cuatro componentes. 
importante remarcar que el uso de la escala logarítmica para el eje de las ordenadas magnifica los residuos en las alas de perfil para dichas líneas. En la Tabla 3.2 se presentan las velocidades radiales (referidas a la Velocidad Sistémica $=2790 \mathrm{~km} \mathrm{~s}^{-1}$ ), las dispersiones de velocidades y los flujos de cada una de las componentes y del perfil global junto a los errores de los ajustes. Para los centros en $\mathrm{H} \alpha$ determinamos: $\mathrm{N} 1=2.5 \pm 0.3 \mathrm{~km} \mathrm{~s}^{-1}, \mathrm{~N} 2=13.8 \pm 0.8 \mathrm{~km} \mathrm{~s}^{-1}$, ancha $=-0.9 \pm 0.5 \mathrm{~km} \mathrm{~s}^{-1}$ y en [OIII] $\lambda 5007 \AA$ : $\mathrm{N} 1=6.4 \pm 0.7 \mathrm{~km} \mathrm{~s}^{-1}, \mathrm{~N} 2=13.9 \pm 1.3 \mathrm{~km} \mathrm{~s}^{-1}$ y para la ancha $=4.0 \pm 0.8 \mathrm{~km} \mathrm{~s}^{-1}$. Así mismo, los valores determinados $\sigma_{i n t}$, son $\sigma_{N 1}=$ $19.7 \pm 0.3 \mathrm{~km} \mathrm{~s}^{-1}, \sigma_{N 2}=3.3 \pm 0.8 \mathrm{~km} \mathrm{~s}^{-1}$ y $\sigma_{B}=42.2 \pm 1.1 \mathrm{~km} \mathrm{~s}^{-1} ; \sigma_{N 1}=18.1 \pm 0.9 \mathrm{~km} \mathrm{~s}^{-1}$, $\sigma_{N 2}=7.8 \pm 2.5 \mathrm{~km} \mathrm{~s}^{-1}$ y $\sigma_{B}=37.5 \pm 1.6 \mathrm{~km} \mathrm{~s}^{-1}$, para $\mathrm{H} \alpha$ y [OIII] respectivamente.

Por el lado de las líneas más débiles, se ha tomado la solución de las líneas intensas como valores iniciales de los parámetros de ajuste y en los casos en que fuera necesario y posible, se liberan los centros y $\sigma$ para que se "acomoden" mejor los perfiles. Este es el caso de las líneas del doblete del [OII] $\lambda \lambda 3726 \AA, 3729 \AA$, en donde hemos dejado que la solución cinemática varíe respecto a sus parámetros iniciales copiados de la solución cinemática de $\mathrm{H} \alpha$. En la Figura 3.6 se muestran los distintos ajustes para la línea de [OII]3729, sin dejar que varíen los parámetros cinemáticos de la aproximación inicial (derecha) y dejándolos variar dentro de una tolerancia (izquierda). Permitimos que varíen sus parámetros hasta que la solución converja y tenga valores confiables, siendo estos justamente los valores de los centros de la línea [OIII] $\lambda 5007 \AA$.

La componente ancha no se ha podido ajustar en las líneas aurorales débiles, debido a la baja $\mathrm{S} / \mathrm{N}$, pero sí se pudieron ajustar las dos componentes angostas. La relación entre el flujo de $[\mathrm{OIII}] \lambda 5007 \AA$ y $[\mathrm{OIII}] \lambda 4363 \AA$ es de $\sim 196$, mientras que entre $[\mathrm{SIII}] \lambda 9069 \AA$ y $[\mathrm{SIII}] \lambda 6312 \AA$ es de $\sim 11$ veces.

Tabla 3.2. Parámetros de los ajustes de las diferentes líneas de emisión para el Nudo B de II Zw 33. En la primer y segunda columna se identifica la longitud de onda en reposo, $\lambda_{0}$, y el ion correspondiente, la tercer columna indica el nombre de las componentes ajustadas para cada línea, la cuarta las velocidades radiales, $\mathrm{V}_{r}$, respecto a la Velocidad Sistémica: $\mathrm{V}_{\text {Sistem }}$ $=2790 \mathrm{~km} / \mathrm{s}$, la quinta las dispersiones de velocidades intrínseca, $\sigma_{i n t}$, la sexta columna indica los Flujos para cada componente y la última columna corresponde a las Medidas de Emisión de flujo (EM) correspondientes.

\begin{tabular}{lllcccc}
\hline $\begin{array}{l}\lambda_{0} \\
{[\AA]}\end{array}$ & Ion & Comp. & $\begin{array}{c}\mathrm{v}_{\mathrm{r}} \\
{\left[\mathrm{km} \mathrm{s}^{-1}\right]}\end{array}$ & $\begin{array}{c}\sigma_{\text {int }} \\
{\left[\mathrm{km} \mathrm{s}^{-1}\right]}\end{array}$ & $\begin{array}{c}\text { Flujo } \\
{\left[10^{-17} \mathrm{erg} \mathrm{s}^{-1} \mathrm{~cm}^{-2}\right]}\end{array}$ & EM $_{\mathrm{f}}$ \\
\hline 6563 & $\mathrm{H} \alpha$ & Angosta 1 & $2.5 \pm 0.3$ & $19.7 \pm 0.3$ & $2053 \pm 11$ & 66.4 \\
& & Angosta 2 & $13.8 \pm 0.8$ & $3.3 \pm 0.8$ & $246 \pm 6$ & 8.0 \\
& & Ancha & $-0.9 \pm 0.5$ & $42.2 \pm 1.1$ & $793 \pm 10$ & 25.6 \\
& & Flujo Global & $3092 \pm 16$ & & & \\
4861 & $\mathrm{H} \beta$ & Angosta 1 & 2.5 & 19.7 & $501 \pm 10$ & 59.2 \\
& & Angosta 2 & 13.8 & 3.3 & $89 \pm 5$ & 10.5 \\
& & Ancha & -0.9 & 42.2 & $257 \pm 10$ & 30.3 \\
& & Flujo Global & $847 \pm 15$ & & & \\
& & & & & & \\
4340 & $\mathrm{H} \gamma$ & Angosta 1 & 2.5 & 19.7 & $277 \pm 9$ & 60.8 \\
& & Angosta 2 & 13.8 & 3.3 & $29 \pm 4$ & 6.3 \\
& & Ancha & -0.9 & 42.2 & $149 \pm 8$ & 32.9 \\
& & Flujo Global & $455 \pm 13$ & & & \\
& & & & & & \\
4102 & $\mathrm{H} \delta$ & Angosta 1 & 2.5 & 19.7 & $155 \pm 7$ & 58.7 \\
& & Angosta 2 & 13.8 & 3.3 & $19 \pm 3$ & 7.4
\end{tabular}




\begin{tabular}{|c|c|c|c|c|c|c|}
\hline & & $\begin{array}{l}\text { Ancha } \\
\text { Flujo Global }\end{array}$ & $\begin{array}{c}-0.9 \\
265 \pm 10\end{array}$ & 42.2 & $90 \pm 7$ & 33.9 \\
\hline 5007 & [Öні] & $\begin{array}{l}\text { Angosta } 1 \\
\text { Angosta } 2 \\
\text { Ancha } \\
\text { Flujo Global }\end{array}$ & $\begin{array}{c}6.4 \pm 0.7 \\
13.9 \pm 1.3 \\
4.0 \pm 0.8 \\
3332 \pm 215\end{array}$ & $\begin{array}{c}18.1 \pm 0.9 \\
7.8 \pm 2.5 \\
37.5 \pm 1.6\end{array}$ & $\begin{array}{c}1853 \pm 127 \\
243 \pm 123 \\
1236 \pm 123\end{array}$ & $\begin{array}{c}55.0 \\
7.2 \\
37.7 \\
\end{array}$ \\
\hline 4959 & [Öн] & $\begin{array}{l}\text { Angosta } 1 \\
\text { Angosta } 2 \\
\text { Ancha } \\
\text { Flujo Global }\end{array}$ & $\begin{array}{c}4.7 \pm 1.6 \\
12.5 \pm 2.8 \\
6.8 \pm 2.9 \\
1021 \pm 81\end{array}$ & $\begin{array}{c}18.1 \\
7.8 \\
37.5\end{array}$ & $\begin{array}{c}572 \pm 60 \\
93 \pm 38 \\
356 \pm 40\end{array}$ & $\begin{array}{c}56.1 \\
9.1 \\
34.8\end{array}$ \\
\hline 4363 & [Öн] & $\begin{array}{l}\text { Angosta } 1 \\
\text { Angosta } 2 \\
\text { Flujo Global }\end{array}$ & $\begin{array}{c}6.4 \\
13.9 \\
17 \pm 4\end{array}$ & $\begin{array}{c}18.1 \\
7.8\end{array}$ & $\begin{array}{l}15 \pm 3 \\
2 \pm 0.5\end{array}$ & $\begin{array}{l}89.5 \\
10.5\end{array}$ \\
\hline 6584 & {$[\mathrm{NII}]$} & $\begin{array}{l}\text { Angosta } 1 \\
\text { Angosta } 2 \\
\text { Ancha } \\
\text { Flujo Global }\end{array}$ & $\begin{array}{c}2.5 \\
13.8 \\
-0.9 \\
191 \pm 10\end{array}$ & $\begin{array}{c}19.7 \\
3.3 \\
42.2\end{array}$ & $\begin{array}{l}87 \pm 7 \\
12 \pm 3 \\
92 \pm 8\end{array}$ & $\begin{array}{c}45.5 \\
6.2 \\
48.3\end{array}$ \\
\hline 6548 & {$[\mathrm{NII}]$} & $\begin{array}{l}\text { Angosta } 1 \\
\text { Angosta } 2 \\
\text { Ancha } \\
\text { Flujo Global }\end{array}$ & $\begin{array}{c}2.5 \\
13.8 \\
-0.9 \\
61 \pm 8\end{array}$ & $\begin{array}{c}19.7 \\
3.3 \\
42.2\end{array}$ & $\begin{array}{c}30 \pm 4 \\
4 \pm 1 \\
27 \pm 6\end{array}$ & $\begin{array}{c}48.8 \\
6.4 \\
44.8\end{array}$ \\
\hline 6717 & [SII] & $\begin{array}{l}\text { Angosta } 1 \\
\text { Angosta } 2 \\
\text { Ancha } \\
\text { Flujo Global }\end{array}$ & $\begin{array}{c}2.5 \\
13.8 \\
-0.9 \\
285 \pm 12\end{array}$ & $\begin{array}{c}19.7 \\
3.3 \\
42.2\end{array}$ & $\begin{array}{c}125 \pm 7 \\
11 \pm 3 \\
149 \pm 8\end{array}$ & $\begin{array}{c}44.0 \\
3.7 \\
52.2\end{array}$ \\
\hline 6731 & [SII] & $\begin{array}{l}\text { Angosta } 1 \\
\text { Angosta } 2 \\
\text { Ancha } \\
\text { Flujo Global }\end{array}$ & $\begin{array}{c}2.5 \\
13.8 \\
-0.9 \\
206 \pm 13\end{array}$ & $\begin{array}{c}19.7 \\
3.3 \\
42.2\end{array}$ & $\begin{array}{c}96 \pm 8 \\
7 \pm 2 \\
104 \pm 10\end{array}$ & $\begin{array}{c}46.5 \\
3.1 \\
50.4\end{array}$ \\
\hline 3729 & [OII] & $\begin{array}{l}\text { Angosta } 1 \\
\text { Angosta } 2 \\
\text { Ancha } \\
\text { Flujo Global }\end{array}$ & $\begin{array}{c}5.0 \pm 0.7 \\
19.5 \pm 0.4 \\
4.7 \pm 0.2 \\
1684 \pm 42\end{array}$ & $\begin{array}{c}19.7 \\
3.3 \\
42.2\end{array}$ & $\begin{aligned} 765 & \pm 24 \\
78 & \pm 8 \\
841 & \pm 34\end{aligned}$ & $\begin{array}{c}45.4 \\
4.7 \\
49.9\end{array}$ \\
\hline 3726 & [OII] & $\begin{array}{l}\text { Angosta } 1 \\
\text { Angosta } 2 \\
\text { Ancha } \\
\text { Flujo Global }\end{array}$ & $\begin{array}{c}5.0 \\
19.5 \\
4.7 \\
1146 \pm 43\end{array}$ & $\begin{array}{c}19.7 \\
3.3 \\
42.2\end{array}$ & $\begin{aligned} 488 & \pm 23 \\
70 & \pm 8 \\
587 & \pm 35\end{aligned}$ & $\begin{array}{c}42.6 \\
6.1 \\
51.2\end{array}$ \\
\hline 5876 & HeI & $\begin{array}{l}\text { Angosta } 1 \\
\text { Angosta } 2\end{array}$ & $\begin{array}{c}6.4 \\
13.9\end{array}$ & $\begin{array}{c}18.1 \\
7.8\end{array}$ & $\begin{array}{l}57 \pm 9 \\
12 \pm 5\end{array}$ & $\begin{array}{l}53.8 \\
11.4\end{array}$ \\
\hline
\end{tabular}




\begin{tabular}{|c|c|c|c|c|c|c|}
\hline & & $\begin{array}{l}\text { Ancha } \\
\text { Flujo Global }\end{array}$ & $\begin{array}{c}4.0 \\
107 \pm 14\end{array}$ & 37.5 & $37 \pm 9$ & 34.8 \\
\hline 4471 & HeI & $\begin{array}{l}\text { Angosta } 1 \\
\text { Angosta } 2 \\
\text { Ancha } \\
\text { Flujo Global }\end{array}$ & $\begin{array}{c}6.4 \\
13.9 \\
4.0 \\
31 \pm 6\end{array}$ & $\begin{array}{c}18.1 \\
7.8 \\
37.5\end{array}$ & $\begin{array}{c}16 \pm 4 \\
2 \pm 0.5 \\
13 \pm 4\end{array}$ & $\begin{array}{c}53.2 \\
5.1 \\
41.7\end{array}$ \\
\hline 6678 & HeI & $\begin{array}{l}\text { Angosta } 1 \\
\text { Angosta } 2 \\
\text { Ancha } \\
\text { Flujo Global }\end{array}$ & $\begin{array}{c}6.4 \\
13.9 \\
4.0 \\
35 \pm 7\end{array}$ & $\begin{array}{c}18.1 \\
7.8 \\
37.5\end{array}$ & $\begin{array}{c}16 \pm 5 \\
2 \pm 0.5 \\
18 \pm 4\end{array}$ & $\begin{array}{c}45.2 \\
5.3 \\
49.5\end{array}$ \\
\hline 7065 & HeI & $\begin{array}{l}\text { Angosta } 1 \\
\text { Angosta } 2 \\
\text { Ancha } \\
\text { Flujo Global }\end{array}$ & $\begin{array}{c}6.4 \\
13.9 \\
4.0 \\
20 \pm 5\end{array}$ & $\begin{array}{c}18.1 \\
7.8 \\
37.5\end{array}$ & $\begin{array}{c}17 \pm 3 \\
2 \pm 0.4 \\
1 \pm 0.35\end{array}$ & $\begin{array}{c}85.8 \\
7.9 \\
6.3\end{array}$ \\
\hline 6300 & [OI] & $\begin{array}{l}\text { Angosta } 1 \\
\text { Angosta } 2 \\
\text { Ancha } \\
\text { Flujo Global }\end{array}$ & $\begin{array}{c}2.5 \\
13.8 \\
-0.9 \\
47 \pm 9\end{array}$ & $\begin{array}{c}19.7 \\
3.3 \\
42.2\end{array}$ & $\begin{array}{c}38 \pm 5 \\
2 \\
7.39\end{array}$ & $\begin{array}{c}80.3 \\
4.0 \\
15.7\end{array}$ \\
\hline 6312 & [SIII] & $\begin{array}{l}\text { Angosta } 1 \\
\text { Angosta } 2 \\
\text { Ancha } \\
\text { Flujo Global }\end{array}$ & $\begin{array}{c}6.4 \\
30.7 \\
4.0 \\
17 \pm 6\end{array}$ & $\begin{array}{c}18.1 \\
7.8 \\
37.5\end{array}$ & $\begin{array}{l}13 \pm 3 \\
2 \pm 0.5 \\
2 \pm 0.4\end{array}$ & $\begin{array}{c}80.5 \\
6.7 \\
12.8\end{array}$ \\
\hline 9069 & [SIII] & $\begin{array}{l}\text { Angosta } 1 \\
\text { Angosta } 2 \\
\text { Ancha } \\
\text { Flujo Global }\end{array}$ & $\begin{array}{c}2.5 \\
1.8 \\
-0.9 \\
179 \pm 18\end{array}$ & $\begin{array}{c}19.7 \\
3.3 \\
42.2\end{array}$ & $\begin{array}{c}98 \pm 12 \\
13 \pm 4 \\
69 \pm 12\end{array}$ & $\begin{array}{c}54.4 \\
7.0 \\
38.5\end{array}$ \\
\hline 3868 & [NeIII] & $\begin{array}{l}\text { Angosta } 1 \\
\text { Angosta } 2 \\
\text { Ancha } \\
\text { Flujo Global }\end{array}$ & $\begin{array}{c}6.4 \\
13.9 \\
-4.4 \pm 1.7 \\
296 \pm 18\end{array}$ & $\begin{array}{c}18.1 \\
7.8 \\
37.3 \pm 5.1\end{array}$ & $\begin{aligned} 145 & \pm 14 \\
10 & \pm 4 \\
142 & \pm 10\end{aligned}$ & $\begin{array}{c}48.8 \\
3.4 \\
47.9\end{array}$ \\
\hline 7136 & [ArIII] & $\begin{array}{l}\text { Angosta } 1 \\
\text { Angosta } 2 \\
\text { Ancha } \\
\text { Flujo Global }\end{array}$ & $\begin{array}{c}2.5 \\
13.8 \\
-0.9 \\
78 \pm 7\end{array}$ & $\begin{array}{c}19.7 \\
3.3 \\
42.2\end{array}$ & $\begin{array}{c}59 \pm 4 \\
4 \pm 1 \\
15 \pm 5\end{array}$ & $\begin{array}{c}75.2 \\
5.1 \\
19.6\end{array}$ \\
\hline 7751 & [ArIII] & $\begin{array}{l}\text { Angosta } 1 \\
\text { Angosta } 2 \\
\text { Ancha } \\
\text { Flujo Global }\end{array}$ & $\begin{array}{c}2.5 \\
13.8 \\
-0.9 \\
22 \pm 5\end{array}$ & $\begin{array}{c}19.7 \\
3.3 \\
42.2\end{array}$ & $\begin{array}{c}6 \pm 2 \\
3 \pm 0.5 \\
13 \pm 4\end{array}$ & $\begin{array}{l}28.8 \\
12.5 \\
58.6\end{array}$ \\
\hline
\end{tabular}


Figura 3.5. Perfiles de las líneas de emisión más intensas del Nudo B de II Zw 33 con sus respectivas descomposiciones cinemáticas superpuestas. Los espectros están calibrado, en el eje $\mathrm{x}$ tenemos las velocidades radiales respecto al centro de la línea. En el panel inferior se presenta el del residuo entre el ajuste y la observación. Para aumentar los detalles en las bajas señales, aplicamos escala logarítmica en flujo. De arriba hacia abajo y de izquiera a derecha,



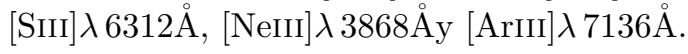
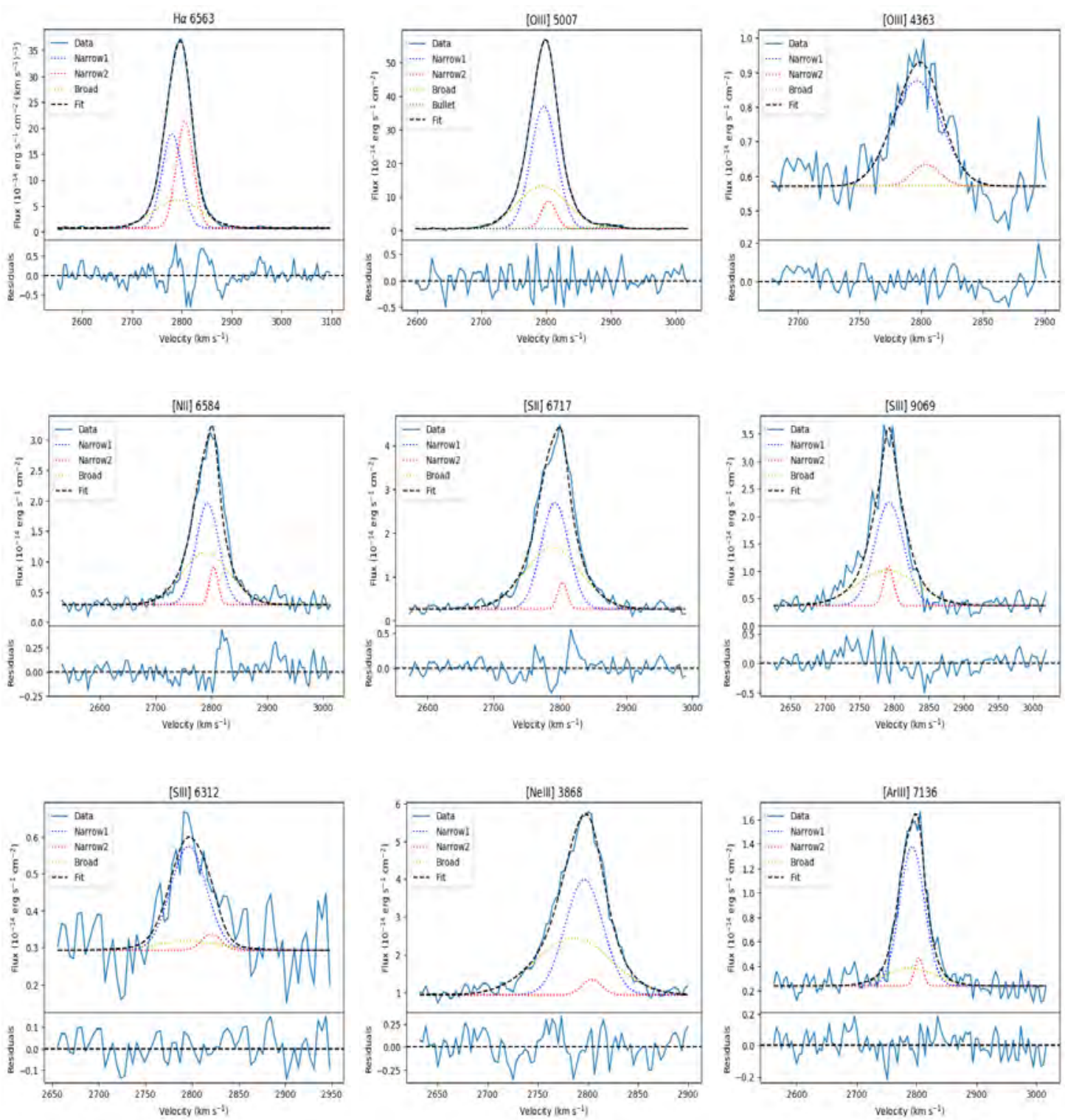
Figura 3.6. Ajustes de múltiples gaussianas para realizados para líneas de $[\mathrm{OII}] \lambda 3729 \AA$. A la derecha, la solución copiando los parámetros de $\mathrm{H} \alpha$ sin permitir que estos varíen y la izquierda la solución permitiendo que los parámetros varíen según la tolerancia definida por los errores del ajuste en centro y $\sigma$
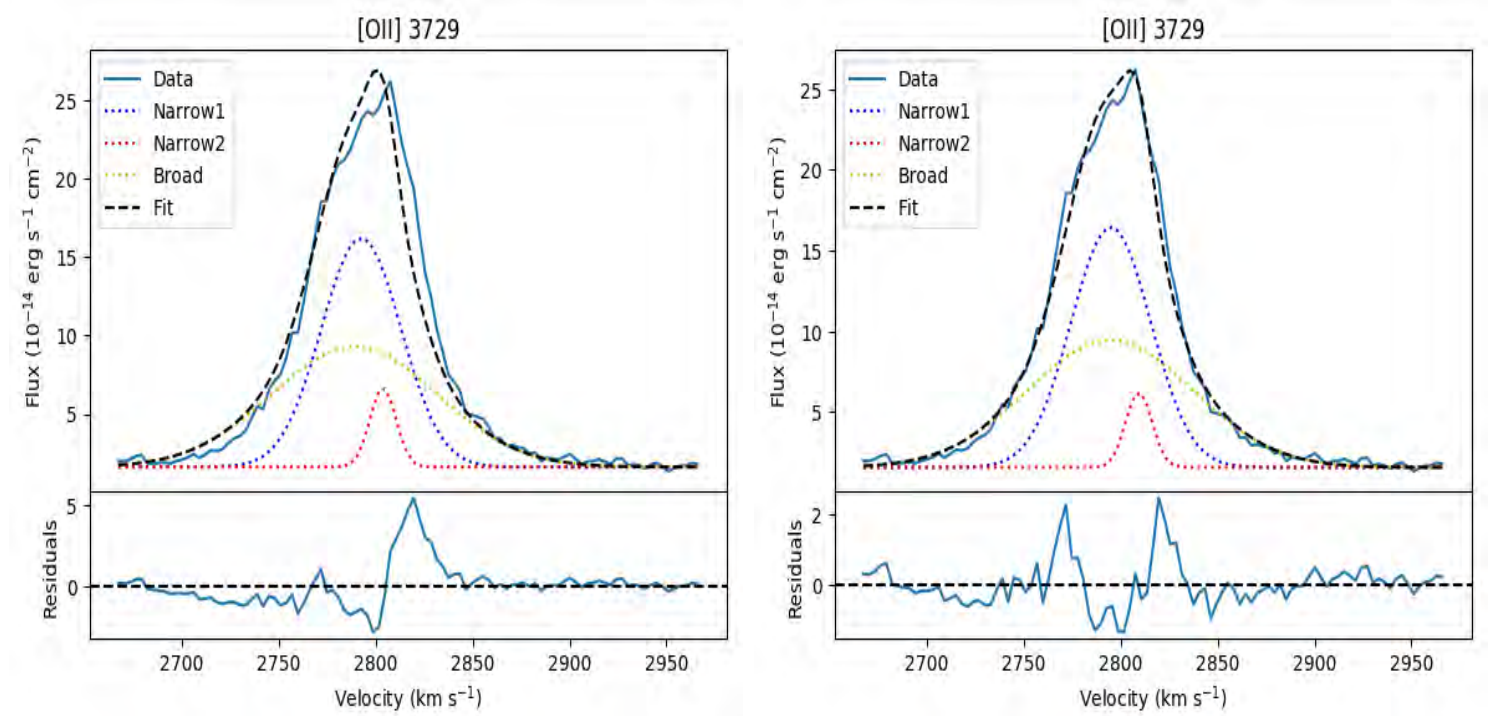

\subsubsection{Zw 33 Nudo C}

De los tres Nudos de II Zw 33, este nudo es el más débil $\left(\log (\mathrm{L}(\mathrm{H} \alpha)) \approx 39.50 \mathrm{erg} \mathrm{s}^{-1}\right.$ Méndez et al., 1999). A pesar de ello, pudimos identificar y ajustar los perfiles de las líneas más intensas del H, HeI, [SII], [NII] y [OII], entre otras, pero no ha sido posible ajustar las líneas aurorales débiles. El mejor ajuste muestra una solución con un total de tres componentes, dos de ellas angostas, junto a una componente ancha subyacente, tal como vinimos ajustando y observando en las regiones anteriores.

A partir de los ajustes y de los índices de Akaike, se determinó la mejor solución con 3 componentes gaussianas. Los índice de Akaike para $1 \mathrm{c}=-393.104 ; 2 \mathrm{c}=-421.156 ; 3 \mathrm{c}=$ -559.978 y $4 \mathrm{c}=-486.202$, donde se observa que el índice no mejora en la solución con 4 componentes. Por lo tanto, la relación del $\Delta \mathrm{AC} \leq 10$ se cumple para 3 componentes. Los distintos ajustes para $\mathrm{H} \alpha$ se muestran en la Figura 3.7.

Las soluciones de los centros y $\sigma_{\text {int }}\left(\right.$ en unidades de $\mathrm{km} \mathrm{s}^{-1}$ ) calculadas son: $\mathrm{N} 1=6.6 \pm 0.8$, $\mathrm{N} 2=40.5 \pm 2.2$ y Ancha $=6.1 \pm 1.8$ y $\sigma_{N 1}=12.8 \pm 0.8, \sigma_{N 2}=2.4 \pm 0.8$ y $\sigma_{B}=46.3 \pm 2.7 ; \mathrm{N} 1$ $=7.4 \pm 1.2, \mathrm{~N} 2=37.6 \pm 2.8$ y Ancha $=5.1 \pm 4.1$ y $\sigma_{N 1}=13.4 \pm 1.2, \sigma_{N 2}=8.2 \pm 2.2, \sigma_{B}=$ $39.4 \pm 5.7$, para $\mathrm{H} \alpha$ y [OIII] respectivamente.

En los paneles de la Figura 3.8 mostramos los ajustes de algunas de las líneas más importantes y en la Tabla 3.3 presentamos los parámetros de la cinemática correspondientes a II Zw $33 \mathrm{C}$.

Un resultado importante que hemos obtenido del análisis de esta región es que existe una probable contaminación en las líneas espectrales (como se indicó en el Capítulo 2) posiblemente debido a la contribución de un brote de formación estelar cercano. De la Figura 2.1, y considerando la posición en que fue colocado el slit, si el Nudo C corresponde al nudo \#5, posiblemente la contaminación provenga del nudo \#6, identificado hacia el sur-oste de la imagen. Este fue uno de los motivos por los cuales, para realizar los análisis de la cinemática de dichas regiones, hemos decidido restringir al máximo la zona de extracción del espectro. 
Figura 3.7. Ajustes con distintas cantidades de gaussianas para el perfil de la línea de $\mathrm{Ho}$ del Nudo C de II Zw 33.

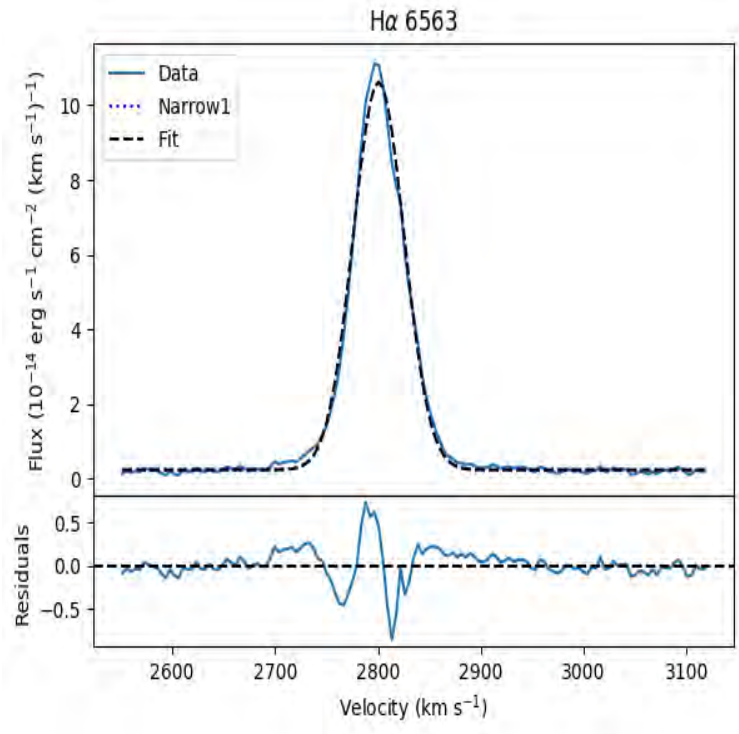

(a) Ajuste de una gaussiana.

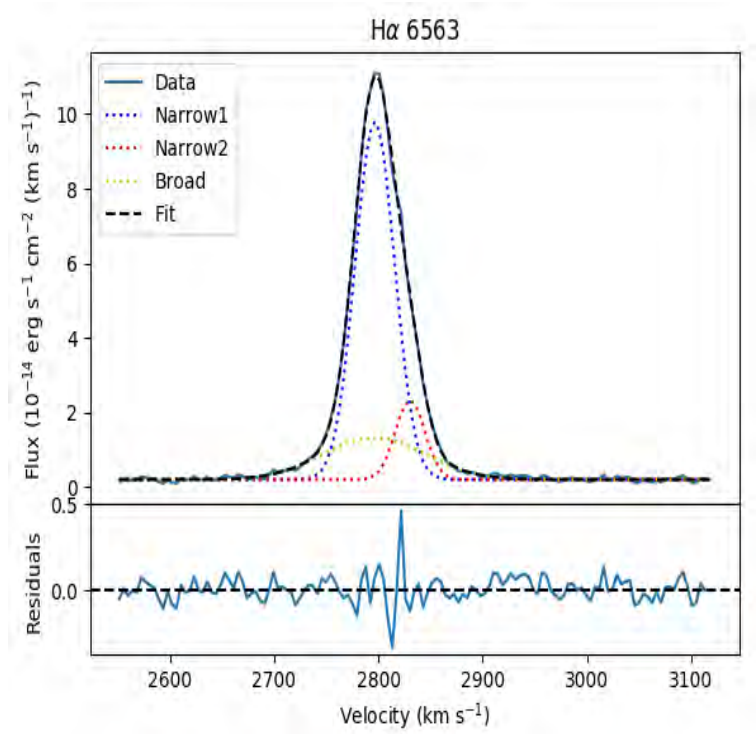

(c) Tres componentes: N1, N2 y ancha.

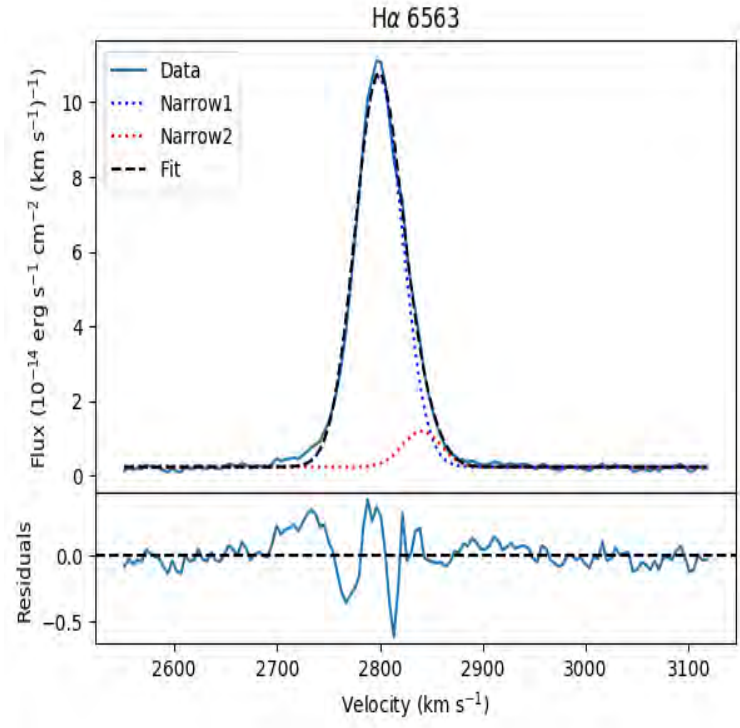

(b) Dos componentes gaussianas: N1 y ancha.



(d) Cuatro componentes. 
Figura 3.8. Perfiles de las líneas de emisión más intensas del nudo $\mathrm{C}$ de IIZw 33 con sus respectivas descomposiciones cinemáticas superpuestas. Los espectros están calibrado, en el eje $\mathrm{x}$ tenemos las velocidades radiales respecto al centro de la línea. En el panel inferior se presenta el del residuo entre el ajuste y la observación. Para aumentar los detalles en las bajas señales, aplicamos escala logarítmica en flujo. Desde arriba hacia abajo y de izquiera a derecha, encontramos a: $\mathrm{H} \alpha, \mathrm{H} \beta, \mathrm{H} \gamma, \mathrm{H} \delta,[\mathrm{OIII}] \lambda 5007 \AA$, [OII] $\lambda 3729 \AA$, [OII] $\lambda 3726 \AA$, $[\mathrm{SiI}] \lambda 6717 \AA$, [NII] $\lambda 6584 \AA$ y $[\mathrm{NeIII}] \lambda 3868 \AA$.
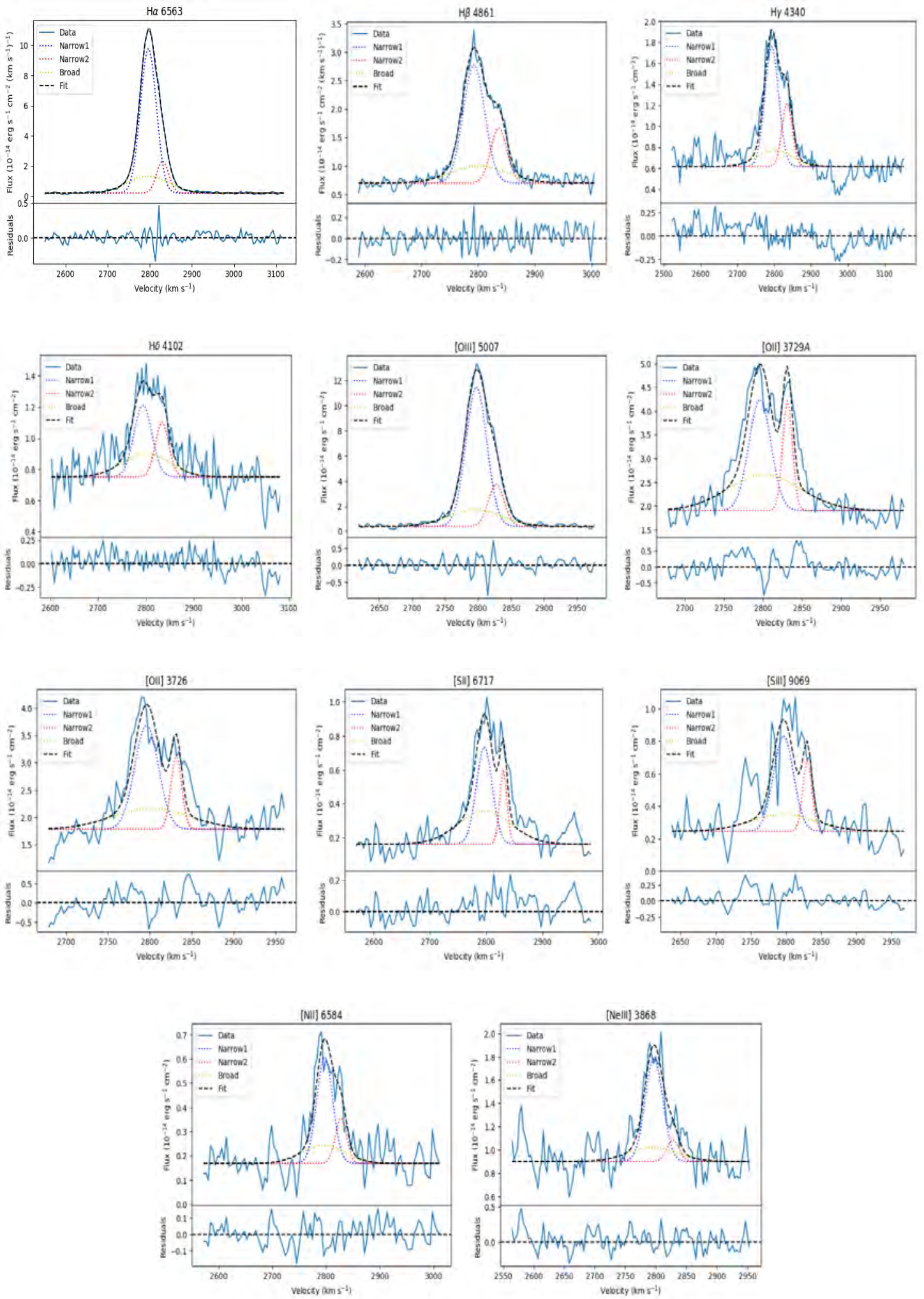
Tabla 3.3. Parámetros de los ajustes de las diferentes líneas de emisión del Nudo C de II Zw 33. En la primer y segunda columna se identifica la longitud de onda en reposo, $\lambda_{0}$, y el ion correspondiente, la tercer columna indica el nombre de las componentes ajustadas para cada línea, la cuarta las velocidades radiales, $\mathrm{V}_{r}$, respecto a la Velocidad Sistémica: $\mathrm{V}_{\text {Sistem }}=2790 \mathrm{~km} / \mathrm{s}$, la quinta las dispersiones de velocidades, $\sigma$, la sexta columna indica los Flujos para cada componente y la última columna corresponde a las Medidas de Emisión de flujo de cada componente $\left(\mathrm{EM}_{f}\right)$.

\begin{tabular}{|c|c|c|c|c|c|c|}
\hline $\begin{array}{l}\lambda_{0} \\
{[\AA]} \\
\end{array}$ & Ion & Comp. & $\begin{array}{c}\mathrm{V}_{\mathrm{r}} \\
{\left[\mathrm{km} \mathrm{s}^{-1}\right]}\end{array}$ & $\begin{array}{c}\sigma_{\text {int }} \\
{\left[\mathrm{km} \mathrm{s}^{-1}\right]}\end{array}$ & $\begin{array}{c}\text { Flujo } \\
{\left[10^{-17} \mathrm{erg} \mathrm{s}^{-1} \mathrm{~cm}^{-2}\right]}\end{array}$ & $\mathrm{EM}_{\mathrm{f}}$ \\
\hline \multirow[t]{4}{*}{6563} & $\mathrm{H} \alpha$ & Angosta 1 & $6.6 \pm 0.8$ & $12.8 \pm 0.8$ & $455 \pm 3$ & 68.2 \\
\hline & & Angosta 2 & $40.5 \pm 2.2$ & $2.4 \pm 0.8$ & $76 \pm 2$ & 11.3 \\
\hline & & Ancha & $6.1 \pm 1.8$ & $46.3 \pm 2.7$ & $137 \pm 5$ & 20.5 \\
\hline & & Flujo Global & $667 \pm 7$ & & & \\
\hline \multirow[t]{4}{*}{4861} & $\mathrm{H} \beta$ & Angosta 1 & 3.8 & 12.8 & $91 \pm 3$ & 53.1 \\
\hline & & Angosta 2 & 43.3 & 2.3 & $30 \pm 2$ & 17.8 \\
\hline & & Ancha & 8.9 & 46.3 & $50 \pm 6$ & 29.2 \\
\hline & & Flujo Global & $171 \pm 7$ & & & \\
\hline \multirow[t]{4}{*}{4340} & $\mathrm{H} \gamma$ & Angosta 1 & $3.3 \pm 1.5$ & 12.8 & $54 \pm 4$ & 57.3 \\
\hline & & Angosta 2 & $44.9 \pm 1.9$ & 2.3 & $21 \pm 2$ & 22.6 \\
\hline & & Ancha & $10.5 \pm 5.5$ & 46.3 & $19 \pm 34$ & 20.2 \\
\hline & & Flujo Global & $93 \pm 34$ & & & \\
\hline \multirow[t]{4}{*}{4102} & $\mathrm{H} \delta$ & Angosta 1 & $3.9 \pm 3.2$ & 12.8 & $22 \pm 3$ & 42.4 \\
\hline & & Angosta 2 & $43.8 \pm 3.0$ & 2.3 & $13 \pm 2$ & 23.9 \\
\hline & & Ancha & $10.6 \pm 4.5$ & 46.3 & $18 \pm 8$ & 33.8 \\
\hline & & Flujo Global & $52 \pm 9$ & & & \\
\hline \multirow[t]{4}{*}{5007} & [OIII] & Angosta 1 & $7.4 \pm 1.2$ & $13.4 \pm 1.2$ & $440 \pm 8$ & 65.7 \\
\hline & & Angosta 2 & $37.6 \pm 2.8$ & $8.2 \pm 2.2$ & $99 \pm 5$ & 14.8 \\
\hline & & Ancha & $5.1 \pm 4.1$ & $39.4 \pm 5.7$ & $131 \pm 12$ & 19.5 \\
\hline & & Flujo Global & $669 \pm 15$ & & & \\
\hline \multirow[t]{4}{*}{4959} & [OIII] & Angosta 1 & 7.4 & 13.4 & $140 \pm 10$ & 67.0 \\
\hline & & Angosta 2 & 37.6 & 8.2 & $31 \pm 6$ & 14.8 \\
\hline & & Ancha & 5.1 & 39.4 & $38 \pm 16$ & 18.2 \\
\hline & & Flujo Global & $210 \pm 20$ & & & \\
\hline \multirow[t]{4}{*}{6584} & {$[\mathrm{NII}]$} & Angosta 1 & 7.4 & 13.4 & $16 \pm 2$ & 57.0 \\
\hline & & Angosta 2 & 37.6 & 8.2 & $5 \pm 1$ & 17.2 \\
\hline & & Ancha & 5.1 & 39.4 & 7.43 & 25.9 \\
\hline & & Flujo Global & $28 \pm 7$ & & & \\
\hline \multirow[t]{4}{*}{6548} & {$[\mathrm{NII}]$} & Angosta 1 & 7.4 & 13.4 & $3 \pm 1$ & 27.4 \\
\hline & & Angosta 2 & 37.6 & 8.2 & 1.0 & 9.9 \\
\hline & & Ancha & 5.1 & 39.4 & 7 & 62.7 \\
\hline & & Flujo Global & $11 \pm 3$ & & & \\
\hline
\end{tabular}




\begin{tabular}{|c|c|c|c|c|c|c|}
\hline \multirow[t]{4}{*}{6717} & {$[\mathrm{SII}]$} & Angosta 1 & $6.6 \pm 1.8$ & 12.8 & $20 \pm 2$ & 40.2 \\
\hline & & Angosta 2 & $40.5 \pm 1.4$ & 2.4 & $7.11 \pm 1$ & 14.1 \\
\hline & & Ancha & $7.1 \pm 12.7$ & 46.3 & $23.00 \pm 1$ & 45.7 \\
\hline & & Flujo Global & $50 \pm 2$ & & & \\
\hline \multirow[t]{4}{*}{6731} & {$[\mathrm{SII}]$} & Angosta 1 & 6.6 & 12.8 & $15 \pm 2$ & 39.9 \\
\hline & & Angosta 2 & 40.5 & 2.4 & $4 \pm 1$ & 10.5 \\
\hline & & Ancha & 7.1 & 46.3 & $18 \pm 3$ & 49.6 \\
\hline & & Flujo Global & $37 \pm 3$ & & & \\
\hline \multirow[t]{4}{*}{3729} & {$[\mathrm{OII}]$} & Angosta 1 & $6.2 \pm 1.3$ & $12.8 \pm 1.4$ & $87 \pm 8$ & 45.1 \\
\hline & & Angosta 2 & $41.5 \pm 0.8$ & $2.4 \pm 5.8$ & $39 \pm 4$ & 20.1 \\
\hline & & Ancha & $11.8 \pm 3.5$ & $46.3 \pm 15.4$ & $67 \pm 13$ & 34.9 \\
\hline & & Flujo Global & $193 \pm 15$ & & & \\
\hline \multirow[t]{4}{*}{3726} & [OII $]$ & Angosta 1 & 6.2 & $12.8 \pm 20.6$ & $64 \pm 22$ & 49.7 \\
\hline & & Angosta 2 & 41.5 & $2.4 \pm 4.3$ & $20 \pm 6$ & 16.0 \\
\hline & & Ancha & 11.8 & $46.4 \pm 36.4$ & 44 & 34.3 \\
\hline & & Flujo Global & $128 \pm 13$ & & & \\
\hline \multirow[t]{4}{*}{5876} & HeI & Angosta 1 & 7.4 & 13.4 & $12 \pm 3$ & 54.6 \\
\hline & & Angosta 2 & 37.6 & 8.2 & $4 \pm 1$ & 19.8 \\
\hline & & Ancha & 5.1 & 39.4 & $5 \pm 1$ & 25.6 \\
\hline & & Flujo Global & $22 \pm 6$ & & & \\
\hline \multirow[t]{4}{*}{6678} & HeI & Angosta 1 & 7.4 & 13.4 & $5 \pm 1$ & 53.7 \\
\hline & & Angosta 2 & 37.6 & 8.2 & $1 \pm 0.35$ & 8.3 \\
\hline & & Ancha & 5.1 & 39.4 & $4 \pm 1$ & 38.0 \\
\hline & & Flujo Global & $10 \pm 3$ & & & \\
\hline \multirow[t]{3}{*}{6300} & {$[\mathrm{OI}]$} & Angosta 1 & 6.6 & 12.8 & $8 \pm 3$ & 76.8 \\
\hline & & Angosta 2 & 40.5 & 2.4 & $2 \pm 0.8$ & 23.0 \\
\hline & & Flujo Global & $10 \pm 3$ & & & \\
\hline \multirow[t]{4}{*}{9069} & [SIII $]$ & Angosta 1 & 6.6 & 12.8 & $20 \pm 3$ & 52.1 \\
\hline & & Angosta 2 & 40.5 & 2.4 & $7 \pm 2$ & 18.0 \\
\hline & & Ancha & 6.1 & 46.3 & $12 \pm 0.5$ & 29.9 \\
\hline & & Flujo Global & $40 \pm 4$ & & & \\
\hline \multirow[t]{4}{*}{3868} & {$[\mathrm{NeIII}]$} & Angosta 1 & $7.0 \pm 1.8$ & 13.4 & $32 \pm 4$ & 65.9 \\
\hline & & Angosta 2 & $37.9 \pm 6.1$ & 8.2 & $5 \pm 3$ & 9.5 \\
\hline & & Ancha & $4.8 \pm 20.7$ & 39.4 & $12 \pm 8$ & 24.7 \\
\hline & & Flujo Global & 65.9 & $49 \pm 9$ & & \\
\hline \multirow[t]{3}{*}{7136} & [ArIII] & Angosta 1 & $9.4 \pm 1.9$ & $12.8 \pm 1.1$ & $13 \pm 1$ & 87.6 \\
\hline & & Angosta 2 & $43.3 \pm 3.5$ & $2.4 \pm 32.0$ & $2 \pm 0.8$ & 12.4 \\
\hline & & Flujo Global & $15 \pm 2$ & & & \\
\hline
\end{tabular}




\subsubsection{Conclusiones de la Cinemática para II Zw 33}

Hemos podido medir una importante cantidad de líneas en las tres regiones de la galaxia II Zw 33. De todos los ajustes planteados, encontramos valores de $\mathrm{V}_{r}$ y de dispersión de velocidades levemente distintos unos de otros, pero lo importante de esto es poder notar las diferencias cinemáticas entre cada una de las regiones. Si bien en las tres regiones analizadas las soluciones cinemáticas planteadas presentan la misma descripción de dos componentes angostas y una ancha, los valores de los parámetros entre ellos varían significativamente.

\subsubsection{Mrk 600}

Describiremos las soluciones cinemáticas determinadas para las dos regiones principales y más intensas observadas en esta galaxia. En el panel derecho de la Figura 2.4 de la Sección 2.4, indicamos cuales son las regiones observadas, las que hemos identificado como Nudos A y B. El primero, y más intenso, es el brote de formación estelar superior derecho, identificado con la letra A. Hacia el sector inferior izquierdo indicamos a la región B.

\subsubsection{Mrk 600 Nudo A}

Como describimos en el capítulo anterior, la galaxia BCD Mrk 600 presenta una estructura compacta con dos regiones intensas centrales, las cuales hemos observado y denominado A y B según su luminosidad, $\log (\mathrm{L}(\mathrm{H} \alpha)) \approx 39.21 \mathrm{erg} \mathrm{s}^{-1} \mathrm{y} \approx 39.16 \mathrm{erg} \mathrm{s}^{-1} \mathrm{~cm}^{-2}$, respectivamente. La región A presenta una cinemática sumamente compleja, con cuatro componentes que describen el perfil de las líneas. Se han medido una gran cantidad de líneas de emisión tanto de recombinación como colisionales, intensas y débiles. El espectro tiene una gran calidad, con un continuo poco ruidoso, de manera que los perfiles de las líneas son bien claros, facilitando los ajustes de múltiples componentes.

A partir de los valores obtenidos de los índices de Akaike para ajustes con distinta cantidad de componentes, determinamos la mejor solución. Los índices de Akaike respectivos son: 1c $=-115.601 ; 2 \mathrm{c}=-194.281 ; 3 \mathrm{c}=-349.632 ; 4 \mathrm{c}=-410.190$ y $5 \mathrm{c}=-251.440$. De esta manera $\mathrm{y}$ según el criterio aplicado, la mejor solución en donde se cumple $\Delta \mathrm{AC} \leq 10$ corresponde a las cuatro componentes. Los distintos ajustes se muestran en la Figura 3.9.

La buena $\mathrm{S} / \mathrm{N}$ de las líneas de $\mathrm{H} \alpha$ y $[\mathrm{OIII}] \lambda 5007 \AA$ resultó muy importante para poder llevar adelante el ajuste de tres componentes angostas, (N1, N2 y N3, enumeradas en orden creciente de velocidades radiales respecto a la velocidad central de la región) y una componente ancha que nos permite ajustar las alas. De las tres componentes angostas, las que se ubican hacia el rojo y el azul tienen una menor amplitud que la angosta central. De ellas, la N1 es la más intensa, indicando un mayor flujo en la componente angosta con velocidad hacia el azul. Ambas líneas intensas muestran un interesante comportamiento en donde [OIII] $\lambda 5007 \AA ̊ 2.5 \times \mathrm{H} \alpha$. En esta región, es la línea del [OIII] la que domina el espectro como la línea más intensa. Las soluciones determinadas para cada componente cinemática presentan diferencias en sus velocidades radiales para $\mathrm{H} \alpha$ y [OIII], aunque al tener en cuenta los errores, son soluciones compatibles entre sí $(\mathrm{N} 1=-9.1 \pm 5.4, \mathrm{~N} 2=16.8 \pm 1.1, \mathrm{~N} 3=44.3 \pm 4.9$, ancha= 9.1 1.4 , para $\mathrm{H} \alpha ; \mathrm{N} 1=-3.1 \pm 1.1, \mathrm{~N} 2=21.2 \pm 0.3, \mathrm{~N} 3=45.1 \pm 2.7$, ancha $=13.2 \pm 3.0$ para $[\mathrm{OIII}]$ ). En el caso de las dispersiones de velocidades intrínsecas encontramos que la componente N3 en el caso de $\mathrm{H} \alpha$ es muy angosta. Esto es así debido a que el $\sigma$ resultante obtenido mediante el $\sigma_{o b s}$ menos las contribuciones instrumentales y térmica de la línea, dan como resultado una línea muy estrecha con un ancho compatible con cero. Esta línea se mantiene en el ajuste porque se ve que existe y aunque le ponemos cero sabemos que en realidad es una línea muy estrecha proveniente de material que no se está moviendo a velocidades supersónicas. 
Figura 3.9. Ajustes con distintas cantidades de gaussianas para $\mathrm{H} \alpha$ en el Nudo A de Mrk 600 .

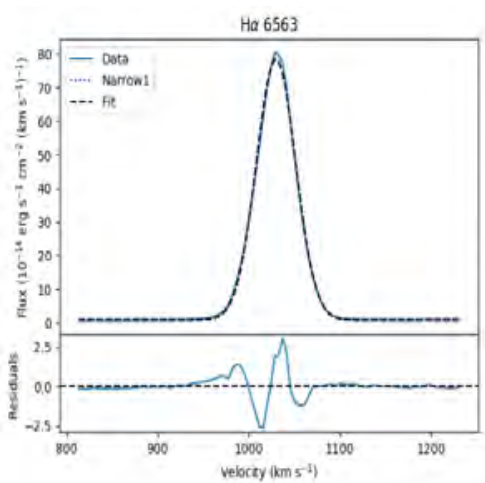

(a) Ajuste de una gaussiana

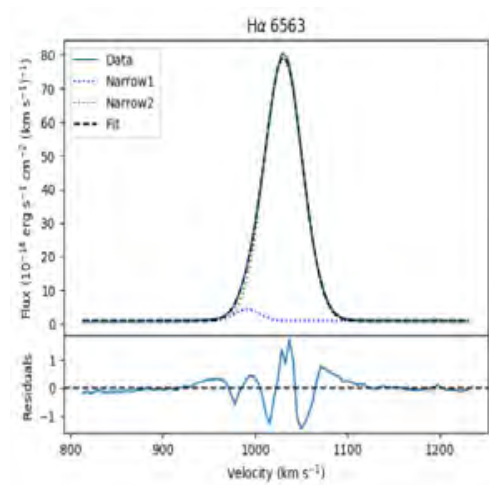

(b) dos componentes: N1 y N2

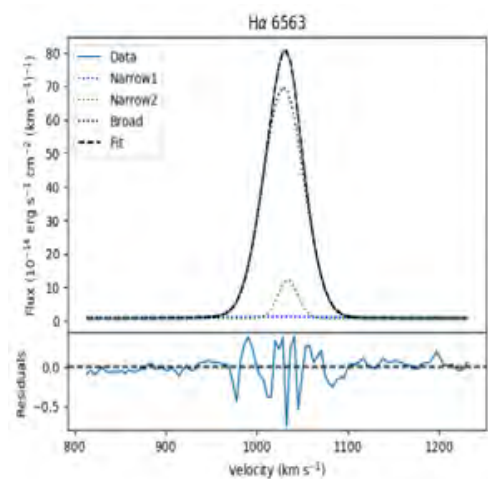

(c) N1, N2 y N3.

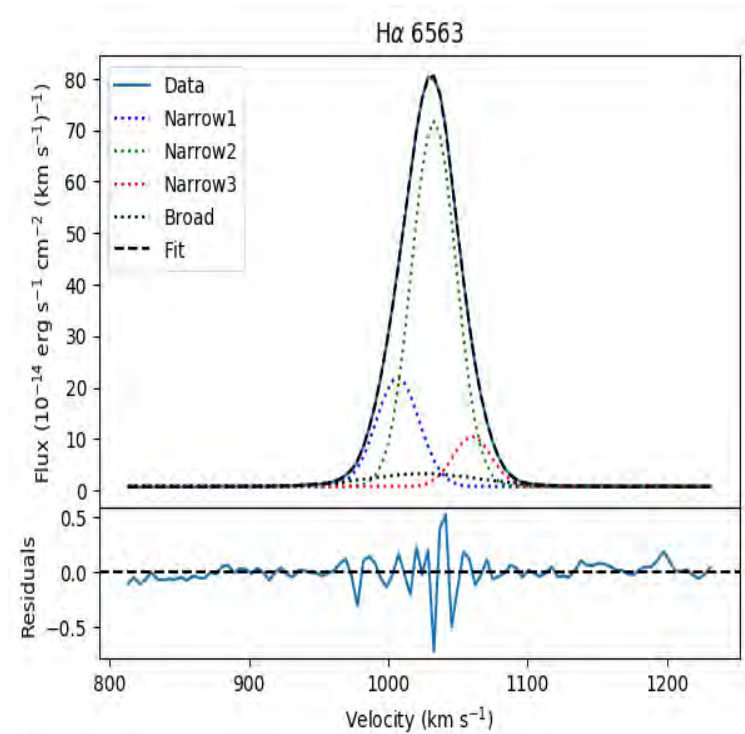

(d) Mejor ajuste con cuatro componentes

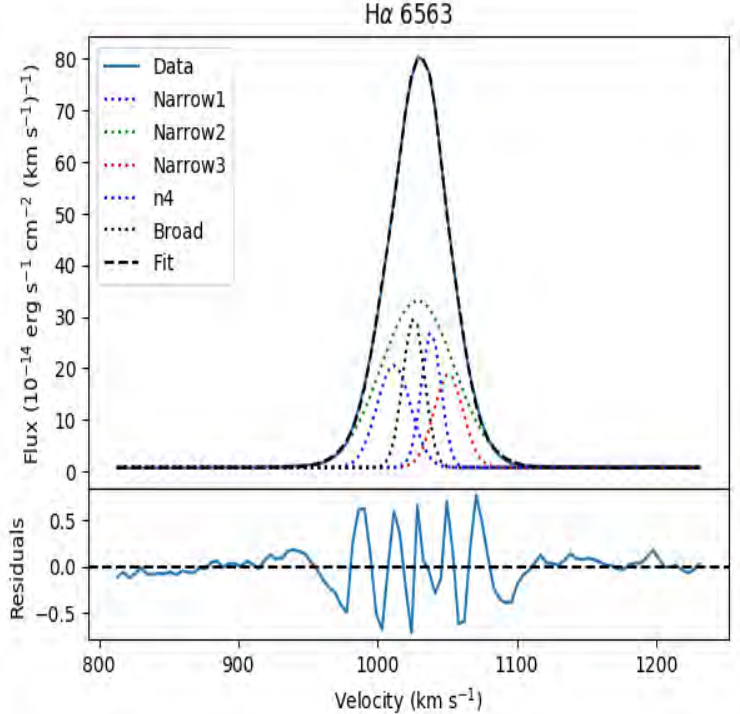

(e) Cinco componentes 
Por este motivo es que al $\sigma$ resultante se le asigna el valor nulo, haciendo referencia a que esta línea no tiene un comportamiento supersónico. Por lo tanto no refiere a una Región HiI Gigante. Los valores del $\sigma_{\text {intr }}$ determinados para nuestras componentes son N1 $=6.4 \pm 3.6$, $\mathrm{N} 2=7.5 \pm 3.6, \mathrm{~N} 3=0.0$, ancha $=38.2 \pm 3.4$, para $\mathrm{H} \alpha ; \mathrm{N} 1=7.4 \pm 1.2, \mathrm{~N} 2=8.6 \pm 0.7, \mathrm{~N} 3=7.1 \pm 2.2$ y ancha $=21.7 \pm 1.8$, para $[\mathrm{OIII}]$. La diferencia más apreciable es la de la componente ancha entre ambas soluciones.

Además, para las líneas del [SII], [NII] y [OII] se ha identificado que hacia el rojo del perfil las componentes resultan más intensas que su contraparte azul, mostrando un comportamiento levemente distinto a las líneas del [OIII] y H $\alpha$. En la Figura 3.10 mostramos los ajustes para algunas de las líneas de interés. Nos resulta muy importante presentar las componentes cinemática en las líneas débiles, en las que, a pesar de presentar una $\mathrm{S} / \mathrm{N}$ baja respecto a las líneas intensas, hemos podido ajustar las cuatro componentes, con un buen acuerdo en su mayoría. Dentro de las líneas prohibidas pudimos medir las líneas débiles aurorales de los iones del [OIII] y del [SIII], para las cuales calculamos su solución cinemática fijando los parámetros hallados en [OIII] de la velocidad radial y la dispersión de velocidades, permitiendo variar la amplitud únicamente. Es importante observar el excelente acuerdo conseguido para la solución de estas líneas, que nos permitirá analizar de manera directa las propiedades físicas del gas ionizado.

Los resultados de los ajustes de la cinemática pueden verse en la Tabla 3.4.

Tabla 3.4. Parámetros de los ajustes de las diferentes líneas de emisión del Nudo A de Mrk600. En la primera y segunda columna se identifica la longitud de onda en reposo, $\lambda_{0}$, y el ion correspondiente, la tercer columna indica el nombre de las componentes ajustadas para cada línea, la cuarta las velocidades radiales, $\mathrm{V}_{r}$, respecto a la Velocidad Sistémica: $\mathrm{V}_{\text {Sistem }}=1016 \mathrm{~km} / \mathrm{s}$, la quinta las dispersiones de velocidades intrínsecas, $\sigma_{\text {int }}$, la sexta columna indica los Flujos para cada componente y la última columna corresponde a las Medidas de Emisión de flujo cada componente $\left(\mathrm{EM}_{f}\right)$. El Flujo Global de cada línea lo indicamos en la fila al final de sus componentes respectivas.

\begin{tabular}{|c|c|c|c|c|c|c|}
\hline $\begin{array}{l}\lambda_{0} \\
{[\AA]}\end{array}$ & Ion & Comp. & $\begin{array}{c}\mathrm{V}_{\mathrm{r}} \\
{\left[\mathrm{km} \mathrm{s}^{-1}\right]}\end{array}$ & $\begin{array}{c}\sigma_{\mathrm{int}} \\
{\left[\mathrm{km} \mathrm{s}^{-1}\right]}\end{array}$ & $\begin{array}{c}\text { Flujo } \\
{\left[10^{-17} \mathrm{erg} \mathrm{s}^{-1} \mathrm{~cm}^{-2}\right]}\end{array}$ & $\mathrm{EM}_{\mathrm{f}}$ \\
\hline \multirow[t]{5}{*}{6563} & $\mathrm{H} \alpha$ & Angosta 1 & $-9.1 \pm 5.4$ & $6.4 \pm 3.6$ & $821 \pm 4$ & 19.4 \\
\hline & & Angosta 2 & $16.8 \pm 1.1$ & $7.5 \pm 3.4$ & $2828 \pm 4$ & 66.9 \\
\hline & & Angosta 3 & $44.3 \pm 4.9$ & 0.0 & $340 \pm 3$ & 8.0 \\
\hline & & Ancha & $9.1 \pm 1.4$ & $38.2 \pm 3.4$ & $240 \pm 9$ & 5.7 \\
\hline & & Flujo Global & $4230 \pm 12$ & & & \\
\hline \multirow[t]{5}{*}{4861} & $\mathrm{H} \beta$ & Angosta 1 & -9.1 & 6.4 & $331 \pm 6$ & 20.9 \\
\hline & & Angosta 2 & 16.8 & 7.5 & $965 \pm 5$ & 60.8 \\
\hline & & Angosta 3 & 44.3 & 0.0 & $163 \pm 4$ & 10.3 \\
\hline & & Ancha & 9.1 & 38.2 & $127 \pm 13$ & 8.0 \\
\hline & & Flujo Global & $1585 \pm 16$ & & & \\
\hline \multirow[t]{5}{*}{4341} & $\mathrm{H} \gamma$ & Angosta 1 & -9.1 & 6.4 & $139 \pm 7$ & 17.5 \\
\hline & & Angosta 2 & 16.8 & 7.5 & $512 \pm 6$ & 64.5 \\
\hline & & Angosta 3 & 44.3 & 0.0 & $90 \pm 5$ & 11.3 \\
\hline & & Ancha & 9.1 & 38.2 & $54 \pm 17$ & 6.7 \\
\hline & & Flujo Global & $794 \pm 20$ & & & \\
\hline
\end{tabular}




\begin{tabular}{|c|c|c|c|c|c|c|}
\hline 4102 & $\mathrm{H} \delta$ & Angosta 1 & -9.1 & 6.4 & $110 \pm 6$ & 24.9 \\
\hline & & Angosta 2 & 16.8 & 7.5 & $279 \pm 4$ & 63.4 \\
\hline & & Angosta 3 & 44.3 & 0.0 & $52 \pm 4$ & 11.8 \\
\hline & & Flujo Global & $441 \pm 10$ & & & \\
\hline 5007 & [OIII] & Angosta 1 & $-3.1 \pm 1.2$ & $7.4 \pm 1.1$ & $1676 \pm 42$ & 16.4 \\
\hline & & Angosta 2 & $21.2 \pm 1.2$ & $8.6 \pm 0.7$ & $4784 \pm 55$ & 47.2 \\
\hline & & Angosta 3 & $45.1 \pm 3.1$ & $7.1 \pm 2.3$ & $686 \pm 19$ & 8.7 \\
\hline & & Ancha & $13.2 \pm 0.8$ & $21.7 \pm 1.8$ & $2822 \pm 114$ & 27.8 \\
\hline & & Flujo Global & $9947 \pm 135$ & & & \\
\hline 4959 & [OIII] & Angosta 1 & $-2.1 \pm 1.5$ & $9.4 \pm 1.2$ & $673 \pm 25$ & 19.7 \\
\hline & & Angosta 2 & 21.2 & $8.2 \pm 1.1$ & $1598 \pm 22$ & 46.7 \\
\hline & & Angosta 3 & $44.0 \pm 1.9$ & $7.7 \pm 0.8$ & $289 \pm 11$ & 8.4 \\
\hline & & Ancha & $12.1 \pm 1.0$ & $24.0 \pm 1.3$ & $761 \pm 59$ & 24.7 \\
\hline & & Flujo Global & $3319 \pm 71$ & & & \\
\hline 4363 & [OIII] & Angosta 1 & -2.1 & 9.4 & $44.70 \pm 2.945$ & 19.8 \\
\hline & & Angosta 2 & 21.2 & 8.2 & $87 \pm 2$ & 38.7 \\
\hline & & Angosta 3 & 44.0 & 7.7 & $34 \pm 2$ & 14.9 \\
\hline & & Ancha & 12.1 & 24.0 & $60 \pm 0.5$ & 26.6 \\
\hline & & Flujo Global & $225 \pm 4$ & & & \\
\hline 6584 & {$[\mathrm{NII}]$} & Angosta 1 & -9.1 & 6.4 & $2.5 \pm 1$ & 5.8 \\
\hline & & Angosta 2 & 16.8 & 7.5 & $27 \pm 1$ & 65.8 \\
\hline & & Angosta 3 & 44.3 & 0.0 & $3 \pm 0.6$ & 6.6 \\
\hline & & Ancha & 9.1 & 38.2 & $9 \pm 6$ & 21.8 \\
\hline & & Flujo Global & $41 \pm 6$ & & & \\
\hline 6548 & {$[\mathrm{NII}]$} & Angosta 1 & -9.1 & 6.4 & $2 \pm 0.7$ & 17.2 \\
\hline & & Angosta 2 & 16.8 & 7.5 & $10 \pm 1$ & 77.7 \\
\hline & & Angosta 3 & 44.3 & 0.0 & 0.63 & 5.1 \\
\hline & & Flujo Global & $12 \pm 1$ & & & \\
\hline 6717 & [SII] & Angosta 1 & $-8.3 \pm 0.6$ & 6.4 & $8 \pm 2$ & 7.7 \\
\hline & & Angosta 2 & $16.3 \pm 0.7$ & 7.5 & $69 \pm 2$ & 65.9 \\
\hline & & Angosta 3 & $44.6 \pm 2.0$ & 0.0 & $8 \pm 1$ & 8.1 \\
\hline & & Ancha & $10.1 \pm 3.41$ & 38.2 & $19 \pm 6$ & 18.2 \\
\hline & & Flujo Global & $104 \pm 9$ & & & \\
\hline 6731 & [SII $]$ & Angosta 1 & -8.3 & 6.4 & $6 \pm 1$ & 8.9 \\
\hline & & Angosta 2 & 16.3 & 7.5 & $53 \pm 10$ & 71.7 \\
\hline & & Angosta 3 & 44.6 & 0.0 & $7 \pm 2$ & 9.9 \\
\hline & & Ancha & 10.1 & 38.2 & $7 \pm 2$ & 9.5 \\
\hline & & Flujo Global & $73 \pm 15$ & & & \\
\hline
\end{tabular}




\begin{tabular}{|c|c|c|c|c|c|c|}
\hline 3729 & [OII $]$ & Angosta 1 & $-1.6 \pm 3.4$ & 7.3 & $70 \pm 22$ & 9.2 \\
\hline & & Angosta 2 & $19.8 \pm 1.2$ & 8.6 & $505 \pm 28$ & 66.2 \\
\hline & & Angosta 3 & $47.2 \pm 0.7$ & 7.1 & $107 \pm 10$ & 14.1 \\
\hline & & Ancha & $11.1 \pm 1.3$ & 21.7 & $80 \pm 20$ & 10.4 \\
\hline & & Flujo Global & $762 \pm 79$ & & & \\
\hline 3726 & [OII] & Angosta 1 & -1.6 & 7.3 & $45 \pm 18$ & 10.6 \\
\hline & & Angosta 2 & 19.8 & 8.6 & $265 \pm 33$ & 61.7 \\
\hline & & Angosta 3 & 47.2 & 7.1 & $84 \pm 12$ & 19.5 \\
\hline & & Ancha & 11.1 & 21.7 & $36 \pm 14$ & 8.3 \\
\hline & & Flujo Global & $429 \pm 78$ & & & \\
\hline 5876 & HeI & Angosta 1 & -4.1 & 7.2 & $22 \pm 4$ & 11.8 \\
\hline & & Angosta 2 & 22.2 & 8.5 & $162 \pm 5$ & 86.1 \\
\hline & & Angosta 3 & 44.0 & 7.3 & $4 \pm 1$ & 2.1 \\
\hline & & Flujo Global & $188 \pm 8$ & & & \\
\hline 4471 & HeI & Angosta 1 & -4.1 & 7.2 & $21 \pm 2$ & 27.6 \\
\hline & & Angosta 2 & 22.2 & 8.5 & $41 \pm 2$ & 53.8 \\
\hline & & Angosta 3 & 44.0 & 7.3 & $14 \pm 2$ & 18.5 \\
\hline & & Flujo Global & $76 \pm 4$ & & & \\
\hline 6678 & HeI & Angosta 1 & -3.1 & 7.4 & $7 \pm 1$ & 16.2 \\
\hline & & Angosta 2 & 21.2 & 8.6 & $30 \pm 2$ & 70.6 \\
\hline & & Angosta 3 & 45.1 & 7.1 & $6 \pm 1$ & 13.2 \\
\hline & & Flujo Global & $42 \pm 3$ & & & \\
\hline 7065 & HeI & Angosta 1 & -3.1 & 7.4 & $19 \pm 1$ & 32.4 \\
\hline & & Angosta 2 & 21.2 & 8.6 & $29 \pm 1$ & 48.1 \\
\hline & & Angosta 3 & 45.1 & 7.1 & $12 \pm 2$ & 19.4 \\
\hline & & Flujo Global & $60 \pm 2$ & & & \\
\hline 3868 & [NeIII $]$ & Angosta 1 & -3.1 & 7.4 & $139 \pm 21$ & 13.7 \\
\hline & & Angosta 2 & 21.2 & 8.6 & $455 \pm 28$ & 45.0 \\
\hline & & Angosta 3 & 45.1 & 7.1 & $119 \pm 9$ & 11.7 \\
\hline & & Ancha & 13.2 & 21.7 & $299 \pm 59$ & 29.6 \\
\hline & & Flujo Global & $1012 \pm 70$ & & & \\
\hline 6312 & [SIII] & Angosta 1 & -3.1 & 7.4 & $3 \pm 1$ & 11.6 \\
\hline & & Angosta 2 & 21.2 & 8.6 & $14 \pm 5$ & 45.1 \\
\hline & & Angosta 3 & 45.1 & 7.1 & $5 \pm 1.5$ & 16.2 \\
\hline & & Ancha & 13.2 & 21.7 & $8 \pm 3$ & 27.2 \\
\hline & & Flujo Global & $30 \pm 12$ & & & \\
\hline 9069 & [SIII $]$ & Angosta 1 & -3.1 & 7.4 & $11 \pm 4$ & 10.2 \\
\hline & & Angosta 2 & 21.2 & 8.6 & $66 \pm 14$ & 63.0 \\
\hline & & Angosta 3 & 45.1 & 7.1 & $13 \pm 4$ & 12.1 \\
\hline & & Ancha & 13.2 & 21.7 & $15 \pm 3.5$ & 14.7 \\
\hline
\end{tabular}




\begin{tabular}{|c|c|c|c|c|c|c|}
\hline & & Flujo Global & $104 \pm 35$ & & & \\
\hline \multirow[t]{5}{*}{7136} & [ArIII] & Angosta 1 & $-7.1 \pm 2.4$ & 6.4 & $8 \pm 2$ & 8.3 \\
\hline & & Angosta 2 & $18.9 \pm 0.5$ & 7.5 & $54 \pm 3$ & 53.1 \\
\hline & & Angosta 3 & $42.1 \pm 1.2$ & 0.0 & $9 \pm 1.5$ & 8.6 \\
\hline & & Ancha & $11.1 \pm 4.9$ & 38.2 & $31 \pm 8$ & 30 \\
\hline & & Flujo Global & $102 \pm 8$ & & & \\
\hline \multirow[t]{4}{*}{7751} & [ArIII] & Angosta 1 & -3.1 & 7.4 & $3 \pm 1$ & 18.4 \\
\hline & & Angosta 2 & 21.2 & 8.6 & $13 \pm 1$ & 74.7 \\
\hline & & Angosta 3 & 45.1 & 7.1 & $1 \pm 0.4$ & 6.9 \\
\hline & & Flujo Global & $17 \pm 1$ & & & \\
\hline
\end{tabular}

\subsubsection{Mrk 600 Nudo B}

El espectro de la región B está dominado por líneas de emisión, siendo este el nudo en el que más líneas se han detectado. Además de medir las líneas más intensas detectadas en los nudos anteriores, se ha medido la línea débil auroral del $[\mathrm{SiI}] \lambda 4068 \AA$ y la línea de recombinación del HeII $\lambda 4686 \AA$, correspondiendo a una línea con un alto potencial de ionización, es decir que requiere de una fuente con un campo de radiación relativamente intenso o duro. Es interesante observar también que aunque la alta resolución espectral de las observaciones nos permite resolver los perfiles de las líneas espectrales con un muy buen detalle, fue posible resolver la cinemática con un modelo compuesto por únicamente dos componentes: una angosta y una ancha. Ambas componentes describen perfectamente la cinemática central y de las alas de las líneas con $\mathrm{V}_{r}$ y $\sigma_{\text {int }}$ con valores de $\mathrm{N} 1: \mathrm{Vr}=28.7 \pm 0.6 \mathrm{~km} \mathrm{~s}^{-1}$ y $\sigma=4.9 \pm 0.6 \mathrm{~km} \mathrm{~s}^{-1}$ y ancha: $\mathrm{Vr}=32.0 \pm 0.8 \mathrm{~km} \mathrm{~s}^{-1}$ y $\sigma_{\text {int }}=17.3 \pm 1.6$ para $\mathrm{H} \alpha$, y N1: $\mathrm{Vr}=31.8 \pm 0.1 \mathrm{~km} \mathrm{~s}^{-1} \mathrm{y}$ $\sigma=5.9 \pm 0.3 \mathrm{~km} \mathrm{~s}^{-1}$ y ancha: $\mathrm{Vr}=33.0 \pm 0.2 \mathrm{~km} \mathrm{~s}^{-1}$ y $\sigma=13.4 \pm 0.4$, para [OIII].

Una única componente gaussiana no ajustaba bien las alas como puede observarse en la Figura 3.11, por lo que se procedió a ajustar una componente ancha. La mejor solución de dos componentes fue determinada a partir de la determinación de los índices de Akaike para una, dos y tres componentes $(1 \mathrm{c}=-163.881 ; 2 \mathrm{c}=-250.536$ у $3 \mathrm{c}=-250.890)$. Vemos que el indicador no mejora entre la solución de dos componentes y la de tres, lo que nos indica que la mejor solución es la compuesta por la componente ancha y la angosta, de dos componentes.

Los resultados de los ajustes se pueden ver en la Figura 3.12, donde se presenta el análisis de las líneas más intensas y de las más débiles que se han podido ajustar. Para dichas líneas podemos encontrar las velocidades de los centros, las dispersiones de velocidad y sus errores correspondientes en la Tabla 3.1. Al tratarse de 2 componentes, hemos podido permitir que los parámetros de ajustes de las guassianas varíen libremente o hemos restringido algunos parámentros, permitiendo que se varíe únicamente los restantes. Hemos encontrado ajustes que varían levemente respecto a las soluciones iniciales, pero que están en acuerdo con las soluciones de las líneas más intensas, dentro de los errores. El caso de las líneas del [SiII], donde la componente N1 presenta un centro de línea ligeramente corrido al rojo respecto a las demás soluciones. Esto podría estar asociado al hecho de que se trata de las componentes del [SIII] en las que hemos determinado algunas particularidades por encontrarse hacia el extremo de los órdenes rojos. Además, la línea más intensa para este nudo, como en el caso A, es [OIII $] \lambda 5007 \AA$ siendo la intensidad un poco menos que el doble $\mathrm{H} \alpha$. 
Figura 3.10. Perfiles de las líneas de emisión más intensas del nudo A de Mrk 600 con sus respectivas componentes cinemáticas superpuestas. De arriba hacia abajo y de izquierda a derecha, se pueden identificar las líneas de emisión: $\mathrm{H} \alpha, \mathrm{H} \beta,[\mathrm{OIII}] \lambda 5007 \AA$, [OाII] $\lambda 4363 \AA$, $[\mathrm{SiI}] \lambda 6717 \AA, \quad[\mathrm{SiI}] \lambda 6731 \AA, \quad[\mathrm{OII}] \lambda 3729 \AA, \quad[\mathrm{OII}] \lambda 3726 \AA, \quad[\mathrm{SIII}] \lambda 6312 \AA, \quad[\mathrm{ArIII}] \lambda 7136 \AA$ $[$ NeIII] $] 3868 \AA$.
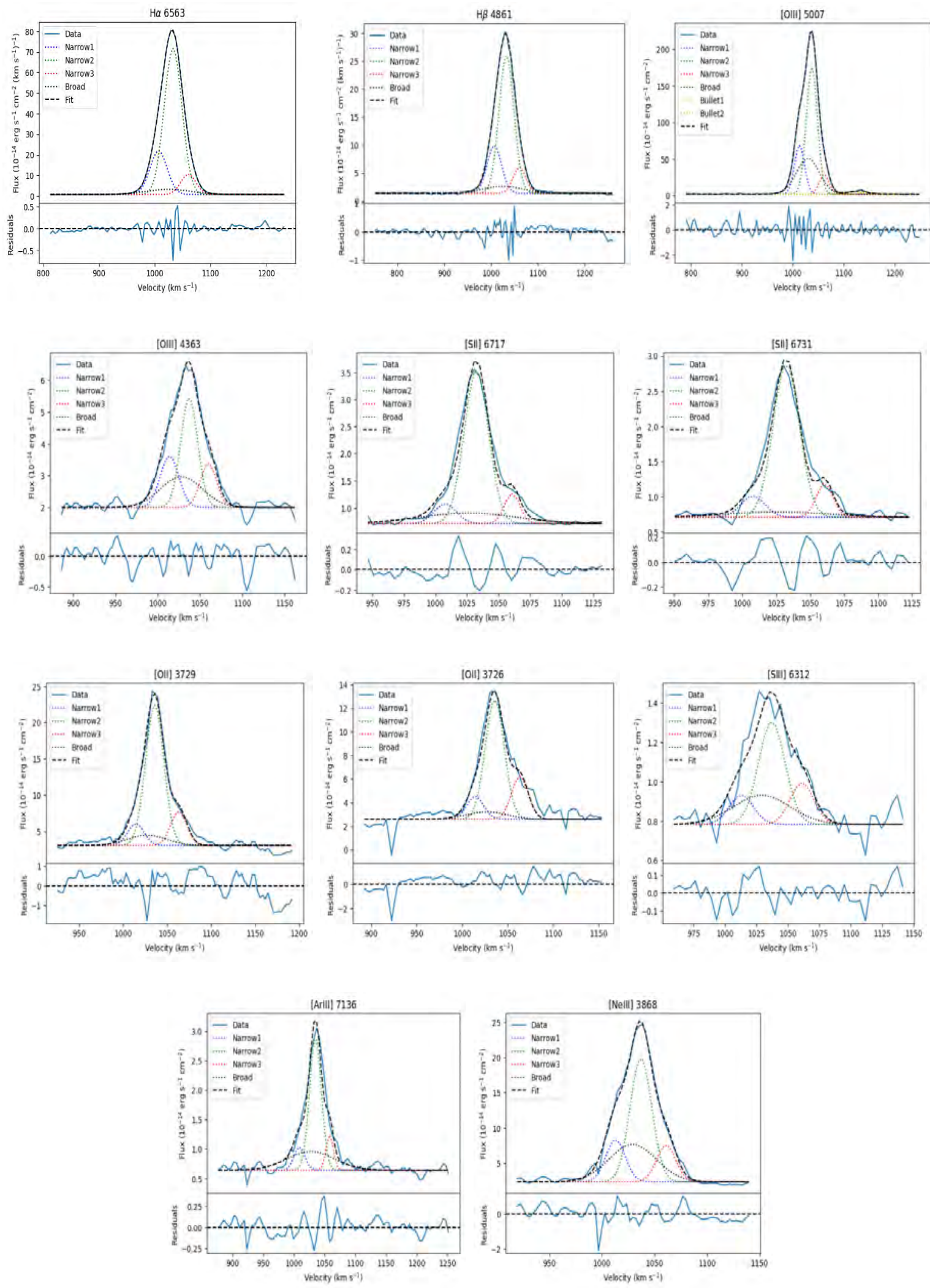
Figura 3.11. Ajustes con diferentes cantidades de gaussianas para $\mathrm{H} \alpha$ del Nudo B de Mrk 600.

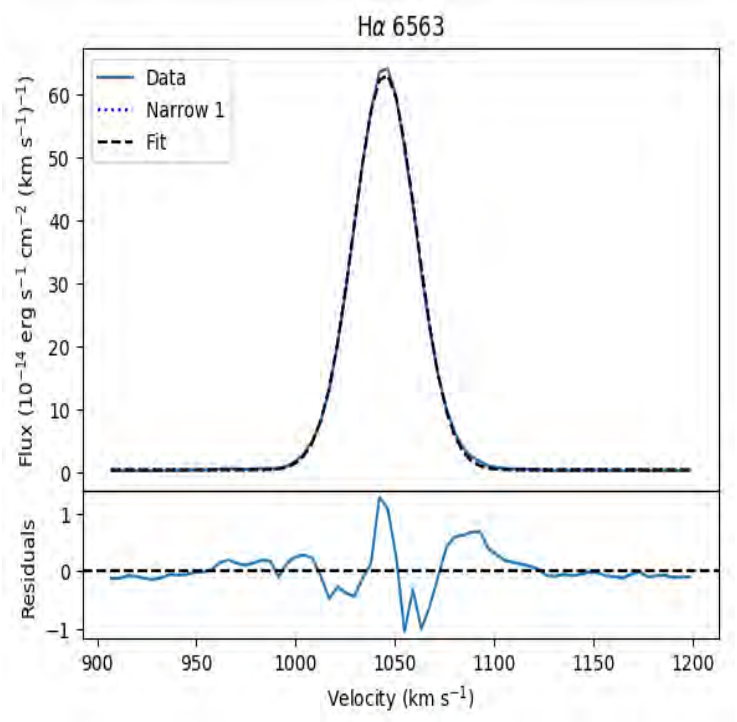

(a) Ajuste con una componente gaussiana.

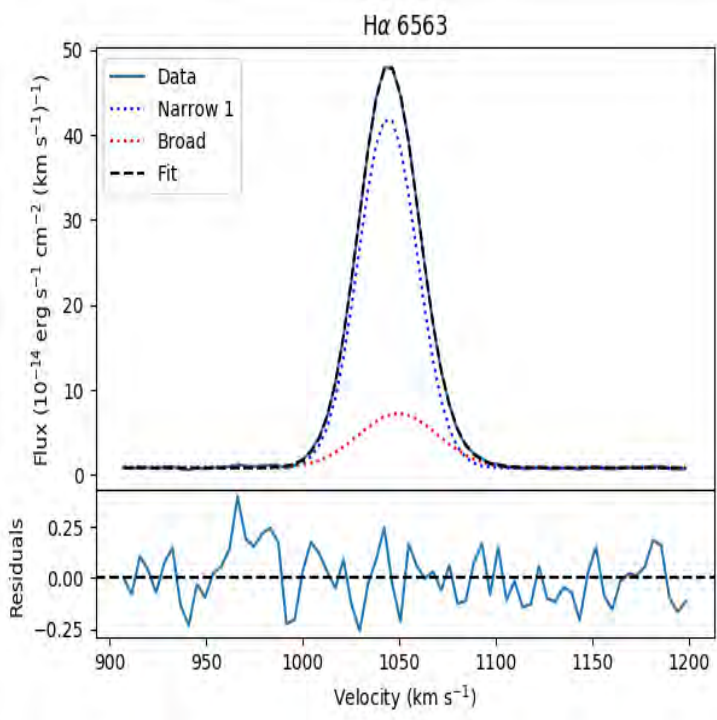

(b) Una componente ancha y una angosta.

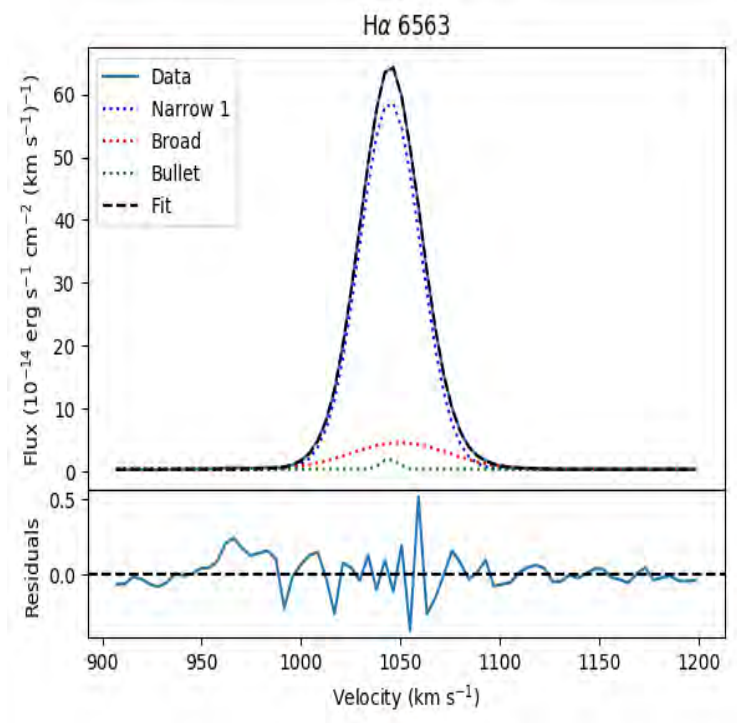

(c) Ajuste de tres componentes 
Figura 3.12. Algunas de las líneas intensas y débiles ajustadas con sus componentes anchas y angostas superpuestas. De arriba hacia abajo y de izquierda a derecha, se pueden identificar las líneas de emisión: $\mathrm{H} \alpha, \mathrm{H} \beta$, [Öні] $\lambda 5007 \AA$, [Öні] $\lambda 4363 \AA$, [SнI] $\lambda 6717 \AA$, [Sir] $\lambda 4068 \AA$, $[\mathrm{NII}] \lambda 6584 \AA$, [OII] $\lambda 3729 \AA$, [SIII] $\lambda 6312 \AA$, [NeIII] $\lambda 3868 \AA \mathrm{Ay}[$ ArIII] $\lambda 7136 \AA$.
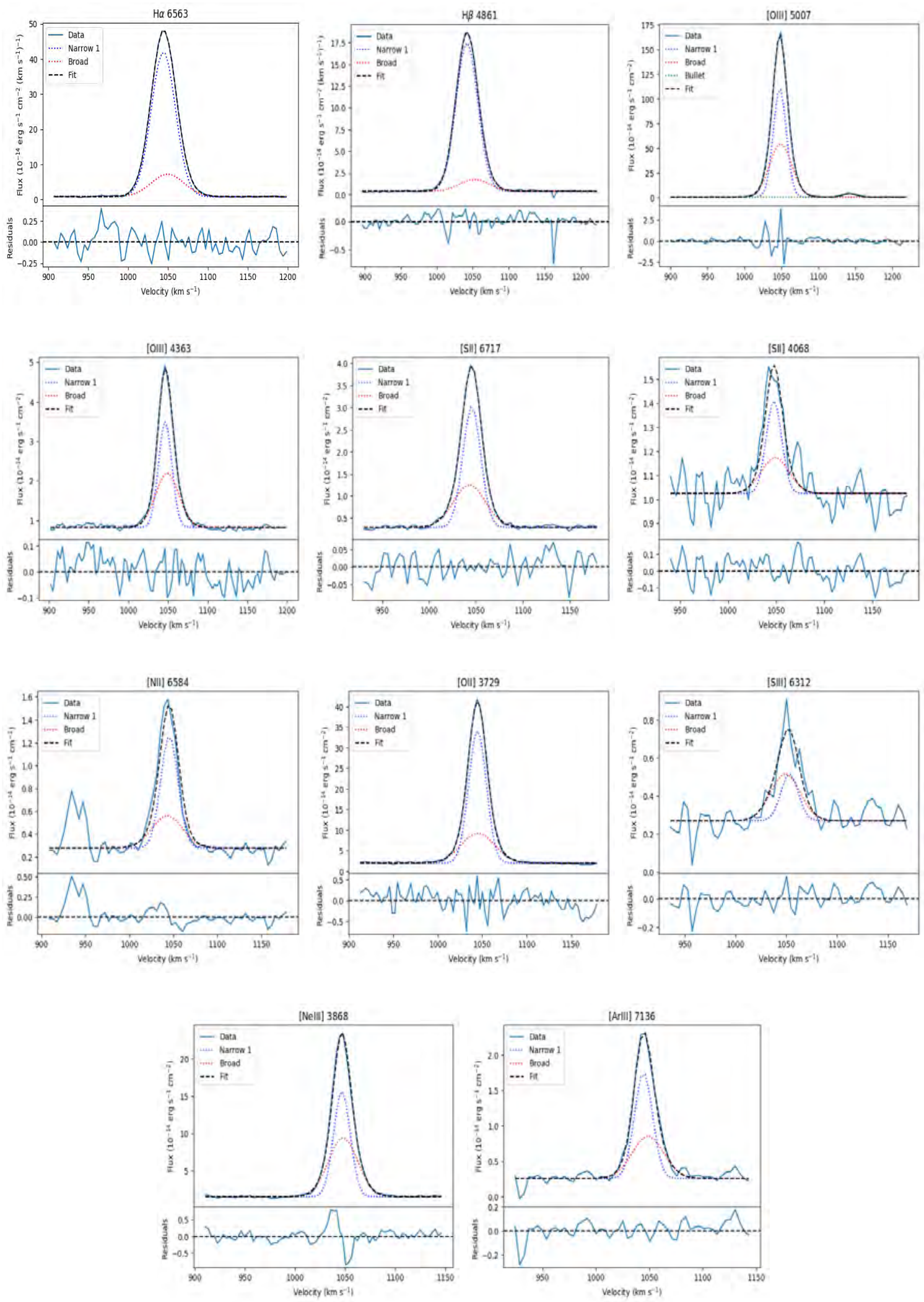
Tabla 3.5. Parámetros de los ajustes de las diferentes líneas de emisión del Nudo B de Mrk600. En la primer y segunda columna se identifica la longitud de onda en reposo, $\lambda_{0}$, y el ion correspondiente, la tercer columna indica el nombre de las componentes ajustadas para cada línea, la cuarta las velocidades radiales, $\mathrm{V}_{r}$, respecto a la Velocidad Sistémica: $\mathrm{V}_{\text {Sistem }}=1016 \mathrm{~km} / \mathrm{s}$, la quinta las dispersiones de velocidades, $\sigma$, la sexta columna indica los Flujos para cada componente y la última columna corresponde a las Medidas de Emisión de flujo cada componente (Med.Emi ${ }_{f}$ ).

\begin{tabular}{|c|c|c|c|c|c|c|}
\hline $\begin{array}{l}\lambda_{0} \\
{[\AA]}\end{array}$ & Ion & Comp. & $\begin{array}{c}\mathrm{V}_{\mathrm{r}} \\
{\left[\mathrm{km} \mathrm{s}^{-1}\right]}\end{array}$ & $\begin{array}{c}\sigma_{\mathrm{int}} \\
{\left[\mathrm{km} \mathrm{s}^{-1}\right]}\end{array}$ & $\begin{array}{c}\text { Flux } \\
{\left[10^{-17} \mathrm{erg} \mathrm{s}^{-1} \mathrm{~cm}^{-2}\right]}\end{array}$ & $\mathrm{EM}_{\mathrm{f}}$ \\
\hline \multirow[t]{3}{*}{6563} & $\mathrm{H} \alpha$ & Angosta 1 & $28.7 \pm 0.1$ & $4.9 \pm 0.6$ & $2077 \pm 11$ & 82.5 \\
\hline & & Ancha & $32.0 \pm 0.8$ & $17.3 \pm 1.6$ & $442 \pm 13$ & 17.5 \\
\hline & & Flujo Global & $2519 \pm 17$ & & & \\
\hline \multirow[t]{3}{*}{4861} & $\mathrm{H} \beta$ & Angosta 1 & $26.4 \pm 2.3$ & $4.8 \pm 1.3$ & $868 \pm 12$ & 84.5 \\
\hline & & Ancha & $31.2 \pm 0.8$ & $17.4 \pm 0.9$ & $159 \pm 14$ & 15.5 \\
\hline & & Flujo Global & $1028 \pm 18$ & & & \\
\hline \multirow[t]{3}{*}{4340} & $\mathrm{H} \gamma$ & Angosta 1 & $27.7 \pm 1.1$ & $3.2 \pm 0.4$ & $406 \pm 3$ & 75.5 \\
\hline & & Ancha & $29.8 \pm 1.2$ & $15.4 \pm 0.3$ & $132 \pm 4$ & 24.5 \\
\hline & & Flujo Global & $538 \pm 5$ & & & \\
\hline \multirow[t]{3}{*}{4102} & $\mathrm{H} \delta$ & Angosta 1 & 26.4 & 4.8 & $233.65 \pm 9.223$ & 72.9 \\
\hline & & Ancha & 31.2 & 17.4 & $86.81 \pm 11.178$ & 27.1 \\
\hline & & Flujo Global & $320 \pm 14$ & & & \\
\hline \multirow[t]{3}{*}{5007} & [OIII] & Angosta 1 & $31.8 \pm 0.1$ & $5.9 \pm 0.3$ & $2481 \pm 27$ & 54.45 \\
\hline & & Ancha & $33.0 \pm 0.2$ & $13.4 \pm 0.4$ & $2073 \pm 32$ & 45.55 \\
\hline & & Flujo Global & $4540 \pm 43$ & & & \\
\hline \multirow[t]{3}{*}{4959} & [OIII] & Angosta 1 & 31.8 & 5.9 & $876 \pm 42$ & 58.7 \\
\hline & & Ancha & 33.0 & 13.4 & $617 \pm 51$ & 41.3 \\
\hline & & Flujo Global & $1493 \pm 66$ & & & \\
\hline \multirow[t]{3}{*}{4363} & [OIII] & Angosta 1 & $30.4 \pm 0.3$ & $5.9 \pm 0.3$ & $55 \pm 1$ & 52.7 \\
\hline & & Ancha & $33.0 \pm 0.8$ & $13.4 \pm 1.3$ & $50 \pm 2$ & 47.3 \\
\hline & & Flujo Global & $106 \pm 2$ & & & \\
\hline \multirow[t]{3}{*}{6717} & {$[\mathrm{SII}]$} & Angosta 1 & $29.4 \pm 0.3$ & $7.4 \pm 0.7$ & $65 \pm 2$ & 61.4 \\
\hline & & Ancha & $26.9 \pm 1.0$ & $16.1 \pm 1.8$ & $41 \pm 2$ & 38.6 \\
\hline & & Flujo Global & $106 \pm 2$ & & & \\
\hline \multirow[t]{3}{*}{6731} & {$[\mathrm{SII}]$} & Angosta 1 & 29.4 & 7.4 & $43 \pm 3$ & 54.5 \\
\hline & & Ancha & 26.9 & 16.1 & $36 \pm 4$ & 45.5 \\
\hline & & Flujo Global & $80 \pm 5$ & & & \\
\hline \multirow[t]{2}{*}{6584} & {$[\mathrm{NII}]$} & Angosta 1 & 29.4 & 7.4 & $24 \pm 5$ & 66.4 \\
\hline & & Ancha & 26.9 & 16.1 & $12 \pm 6$ & 33.6 \\
\hline
\end{tabular}




\begin{tabular}{|c|c|c|c|c|c|c|}
\hline & & Flujo Global & $36 \pm 8$ & & & \\
\hline 6548 & {$[\mathrm{NII}]$} & $\begin{array}{l}\text { Angosta } 1 \\
\text { Ancha } \\
\text { Flujo Global }\end{array}$ & $\begin{array}{c}29.4 \\
26.9 \\
11 \pm 4\end{array}$ & $\begin{array}{c}7.4 \\
16.1\end{array}$ & $\begin{array}{c}5 \pm 2 \\
6 \pm 2.5\end{array}$ & $\begin{array}{l}47.3 \\
52.7\end{array}$ \\
\hline 4068 & {$[\mathrm{SII}]$} & $\begin{array}{l}\text { Angosta } 1 \\
\text { Ancha } \\
\text { Flujo Global }\end{array}$ & $\begin{array}{c}29.8 \\
33.0 \\
13 \pm 3\end{array}$ & $\begin{array}{c}5.9 \\
13.4\end{array}$ & $\begin{array}{l}8 \pm 2 \\
5 \pm 2\end{array}$ & $\begin{array}{l}58.8 \\
41.2\end{array}$ \\
\hline 3729 & [OII $]$ & $\begin{array}{l}\text { Angosta } 1 \\
\text { Ancha } \\
\text { Flujo Global }\end{array}$ & $\begin{array}{l}28.6 \pm 0.1 \\
29.2 \pm 0.5 \\
1141 \pm 14\end{array}$ & $\begin{array}{c}8.0 \pm 0.3 \\
18.0 \pm 1.2\end{array}$ & $\begin{array}{c}798 \pm 8 \\
343 \pm 11\end{array}$ & $\begin{array}{l}69.9 \\
30.1\end{array}$ \\
\hline 3726 & [OII $]$ & $\begin{array}{l}\text { Angosta } 1 \\
\text { Ancha } \\
\text { Flujo Global }\end{array}$ & $\begin{array}{c}28.6 \\
29.2 \\
823 \pm 12\end{array}$ & $\begin{array}{c}8.0 \\
18.0\end{array}$ & $\begin{array}{l}579 \pm 7.5 \\
245 \pm 10\end{array}$ & $\begin{array}{l}70.3 \\
29.7\end{array}$ \\
\hline 5876 & HeI & $\begin{array}{l}\text { Angosta } 1 \\
\text { Ancha } \\
\text { Flujo Global }\end{array}$ & $\begin{array}{c}31.8 \\
43.4 \pm 8.7 \\
110 \pm 20\end{array}$ & $\begin{array}{c}5.9 \\
13.4\end{array}$ & $\begin{array}{l}86 \pm 14 \\
24 \pm 14\end{array}$ & $\begin{array}{l}78.2 \\
21.8\end{array}$ \\
\hline 4471 & HeI & $\begin{array}{l}\text { Angosta } 1 \\
\text { Ancha } \\
\text { Flujo Global }\end{array}$ & $\begin{array}{c}31.8 \\
33.0 \\
43 \pm 3\end{array}$ & $\begin{array}{c}5.9 \\
13.4\end{array}$ & $\begin{array}{l}20 \pm 2 \\
22 \pm 3\end{array}$ & $\begin{array}{l}47.3 \\
52.7\end{array}$ \\
\hline 6678 & HeI & $\begin{array}{l}\text { Angosta } 1 \\
\text { Ancha } \\
\text { Flujo Global }\end{array}$ & $\begin{array}{c}29.6 \pm 1.3 \\
36.2 \\
25 \pm 3\end{array}$ & $\begin{array}{c}5.9 \\
13.4\end{array}$ & $\begin{array}{c}20 \pm 2 \\
6 \pm 2\end{array}$ & $\begin{array}{l}77.0 \\
23.0\end{array}$ \\
\hline 7065 & HeI & $\begin{array}{l}\text { Angosta } 1 \\
\text { Ancha } \\
\text { Flujo Global }\end{array}$ & $\begin{array}{c}27.6 \pm 4.2 \\
33.0 \\
22 \pm 5\end{array}$ & $\begin{array}{c}5.9 \\
13.4\end{array}$ & $\begin{array}{c}6 \pm 2.7 \\
17 \pm 3\end{array}$ & $\begin{array}{l}24.0 \\
76.0\end{array}$ \\
\hline 3868 & {$[\mathrm{NeIII}]$} & $\begin{array}{l}\text { Angosta } 1 \\
\text { Ancha } \\
\text { Flujo Global }\end{array}$ & $\begin{array}{c}30.7 \\
31.9 \pm 1.0 \\
579 \pm 10\end{array}$ & $\begin{array}{c}5.9 \\
13.4\end{array}$ & $\begin{array}{l}291 \pm 6 \\
288 \pm 8\end{array}$ & $\begin{array}{l}50.3 \\
49.7\end{array}$ \\
\hline 9069 & [SIII] & $\begin{array}{l}\text { Angosta } 1 \\
\text { Ancha } \\
\text { Flujo Global }\end{array}$ & $\begin{aligned} 37.1 & \pm 3.5 \\
33.0 & \pm 2.0 \\
61 & \pm 7\end{aligned}$ & $\begin{array}{c}6.1 \pm 1.5 \\
13.3 \pm 0.7\end{array}$ & $\begin{array}{l}25 \pm 4 \\
36 \pm 5\end{array}$ & $\begin{array}{l}40.6 \\
59.4\end{array}$ \\
\hline 6312 & [SIII] & $\begin{array}{l}\text { Angosta } 1 \\
\text { Ancha } \\
\text { Flujo Global }\end{array}$ & $\begin{array}{c}37.1 \\
33.0 \pm 3.0 \\
14 \pm 3\end{array}$ & $\begin{array}{c}6.1 \\
13.3\end{array}$ & $\begin{array}{l}5 \pm 2 \\
9 \pm 3\end{array}$ & $\begin{array}{l}37.1 \\
62.9\end{array}$ \\
\hline 7136 & {$[$ ArIII $]$} & $\begin{array}{l}\text { Angosta } 1 \\
\text { Ancha }\end{array}$ & $\begin{array}{l}28.5 \pm 1.1 \\
32.7 \pm 4.4\end{array}$ & $\begin{array}{c}6.0 \pm 3.7 \\
13.4 \pm 6.6\end{array}$ & $\begin{array}{l}32 \pm 3 \\
22 \pm 4\end{array}$ & $\begin{array}{l}59.0 \\
41.0\end{array}$ \\
\hline
\end{tabular}




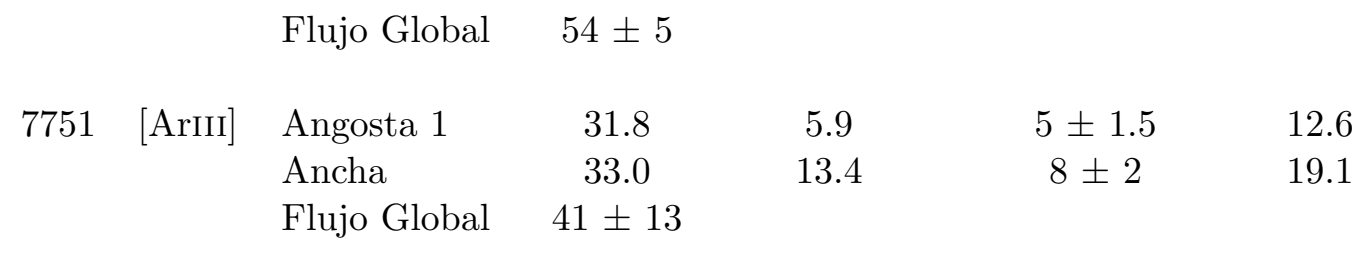

\subsubsection{Conclusiones de la Cinemática para Mrk 600}

Al igual que en las Regiones Hir Gigantes estudiadas en II Zw 33, hemos podido medir una importante cantidad de líneas en los dos nudos de Mrk600. De todos los ajustes planteados, se determinaron $\mathrm{V}_{r}$ y $\sigma$ para componentes anchas y angostas, con leves diferencias entre las soluciones determinadas para regiones de alta ionización mas cercanas al cúmulo ionizante y de más baja ionización. La diferencia encontrada entre la cinemática en ambas regiones de esta galaxia es sobresaliente, en donde hemos determinado una solución cinemática de múltiples componentes compuesta por tres componentes angostas y una componente ancha para el caso del Nudo A. Por otro lado, el Nudo B presentó un comportamiento cinemático posible de describir y analizar por medio de dos compontes únicamente.

\subsubsection{Diagramas Diagnósticos: BPT}

Con los resultados de la cinemática determinados para las componentes de gas ionizado sobre los perfiles de las líneas de emisión, utilizamos los diagramas diagnósticos BPT (Baldwin et al., 1981) para estudiar cuales son sus candidatos a fuente principal de ionización. En particular, usamos $[\mathrm{OIII}] \lambda 5007 \AA / \mathrm{H} \beta$ versus $[\mathrm{NII}] \lambda 6584 \AA / \mathrm{H} \alpha$ y $[\mathrm{OIII}] \lambda 5007 \AA / \mathrm{H} \beta$ versus $[\operatorname{Sir}](\lambda 6717 \AA+\lambda 6731 \AA) / \mathrm{H} \alpha$. Además, como fue posible medir la línea débil del $[\mathrm{OI}] \lambda 6300 \AA$, hemos utilizado el diagrama diagnóstico $[\mathrm{OIII}] \lambda 5007 \AA / \mathrm{H} \beta$ vs. [OI] $\lambda 6300 \AA / \mathrm{H} \alpha^{(\mathrm{i})}$. Este indicador es más difícil de estimar, dada la baja $\mathrm{S} / \mathrm{N}$ de las líneas del [OI], pero en este caso hemos podido medir en algunas componentes en las regiones de II Zw 33 (en algunos casos con errores grandes).

En las Figuras 3.13,3.14, 3.15 para II Zw 33 y en 3.16 y 3.17 para Mrk 600, presentamos los diagramas realizados para cada galaxia con las componentes de cada región. En los gráficos se indican las zonas asociadas a campos de ionización producidos por objetos tipo-Objetos HII, por Choques-AGN (del inglés: Active Galactic Nuclei) o por LINERs (del inglés: lowionization narrow emission-line region), según las relaciones dadas en Kewley et al. (2001); Kauffmann et al. (2003); Kewley et al. (2006).

El análisis de los diagramas nos indica un comportamiento similar y una muy buena concordancia entre la naturaleza de las fuentes ionizantes de las distintas componentes cinemáticas. En líneas generales, todas las componentes cinemáticas se ubican en las zonas de objetos tipo-HiI, exceptuando en algunos diagramas en donde algunas podrían indicar fuentes de ionización más energéticas asociandas a Choques o AGNs. Los anchos de las líneas de emisión en AGNs son significativamente mayores que los determinados en nuestros objetos y los diagramas BPT nos indican que las componentes que se ubican en zonas de cocientes vinculados con AGNs son las componentes anchas con velocidades de dispersión supersónicas. Si bien estos comportamientos analizados son resultado directos de los cocientes entre líneas, hay que complementarlo con otros análisis para determinar las fuentes ionizantes. En conclusión, los diagnósticos realizados sobre las componentes cinemáticas de las regiones estudiadas muestran un gran acuerdo en que corresponden a regiones tipo-HiI.

\footnotetext{
${ }^{(i)}$ para más información, ver: https://sites google.com/site/agndiagnostics/home/bpt.
} 
Figura 3.13. Diagrama diagnóstico $[\mathrm{OIII}] \lambda 5007 \AA \mathrm{A} / \mathrm{H} \beta$ vs. $[\mathrm{NII}] \lambda 6584 \AA \mathrm{A} / \mathrm{H} \alpha$ generado con el programa Kinematics para el análisis de la posible fuente ionizante para las componentes cinemáticas. Las curvas discontinuas en negro y rojo marcan el límite entre las distintas fuentes ionizantes Tipo-Objetos HiI, por Choques-AGNs o LINERs/Sy2, definidas por Kauffmann et al. (2003) y Kewley et al. (2001), respectivamente.

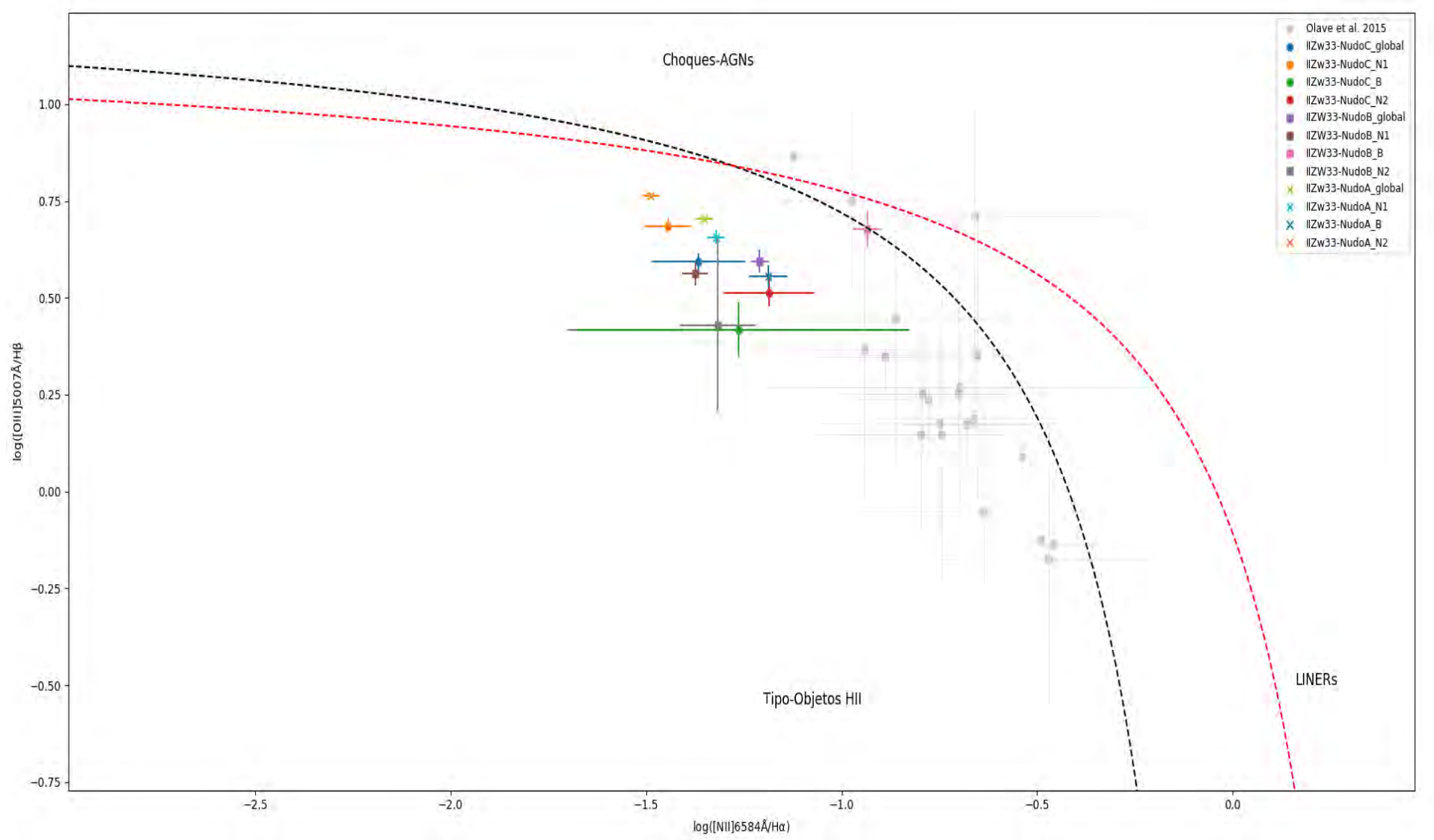


Figura 3.14. Diagrama diagnóstico $[\mathrm{OIII}] \lambda 5007 \AA / \mathrm{H} \beta$ vs. $[\mathrm{SII}](\lambda 6717 \AA+\lambda 6731 \AA) / \mathrm{H} \alpha$ generado con el programa Kinematics para el análisis de la posible fuente ionizante para cada componente de gas determinada. Las curvas discontinuas en negro y rojo marcan el límite entre las distintas fuentes ionizantes Tipo-Objetos HII, por Choques-AGNs o LINERs, definidas por Kewley et al. (2006).

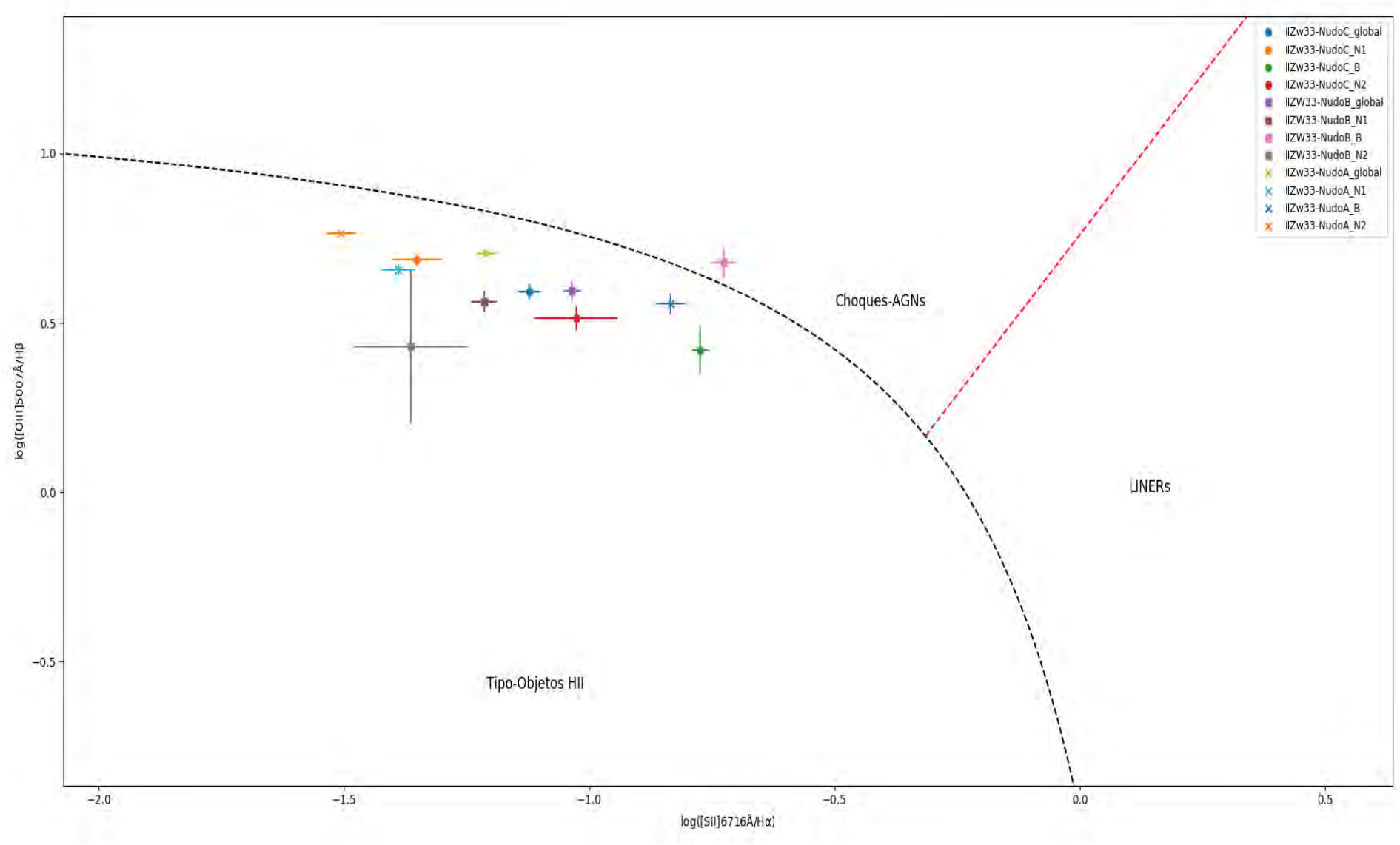


Figura 3.15. Diagrama diagnóstico $[\mathrm{OIII}] \lambda 5007 \AA / \mathrm{H} \beta$ vs. $[\mathrm{OI}] \lambda 6300 \AA / \mathrm{H} \alpha$ generado con el programa Kinematics para el análisis de la posible fuente ionizante para cada componente cinemática. Las curvas discontinuas en negro y rojo marcan el límite entre las distintas fuentes ionizantes Tipo-Objetos HII, por Choques-AGNs o LINERs, definidas por Kewley et al. (2006).




Figura 3.16. Diagrama diagnóstico $[\mathrm{OIII}] \lambda 5007 \AA / \mathrm{H} \beta$ vs. $[\mathrm{NII}] \lambda 6584 \AA$ $/ \mathrm{H} \alpha$ generado con el programa Kinematics para el análisis de la posible fuente ionizante para cada componente de gas determinada. Las curvas discontinuas en negro y rojo marcan el límite entre las distintas fuentes ionizantes Tipo-Objetos HII, por Choques-AGNs o LINERs/Sy2, definidas por Kauffmann et al. (2003) y Kewley et al. (2001), respectivamente.

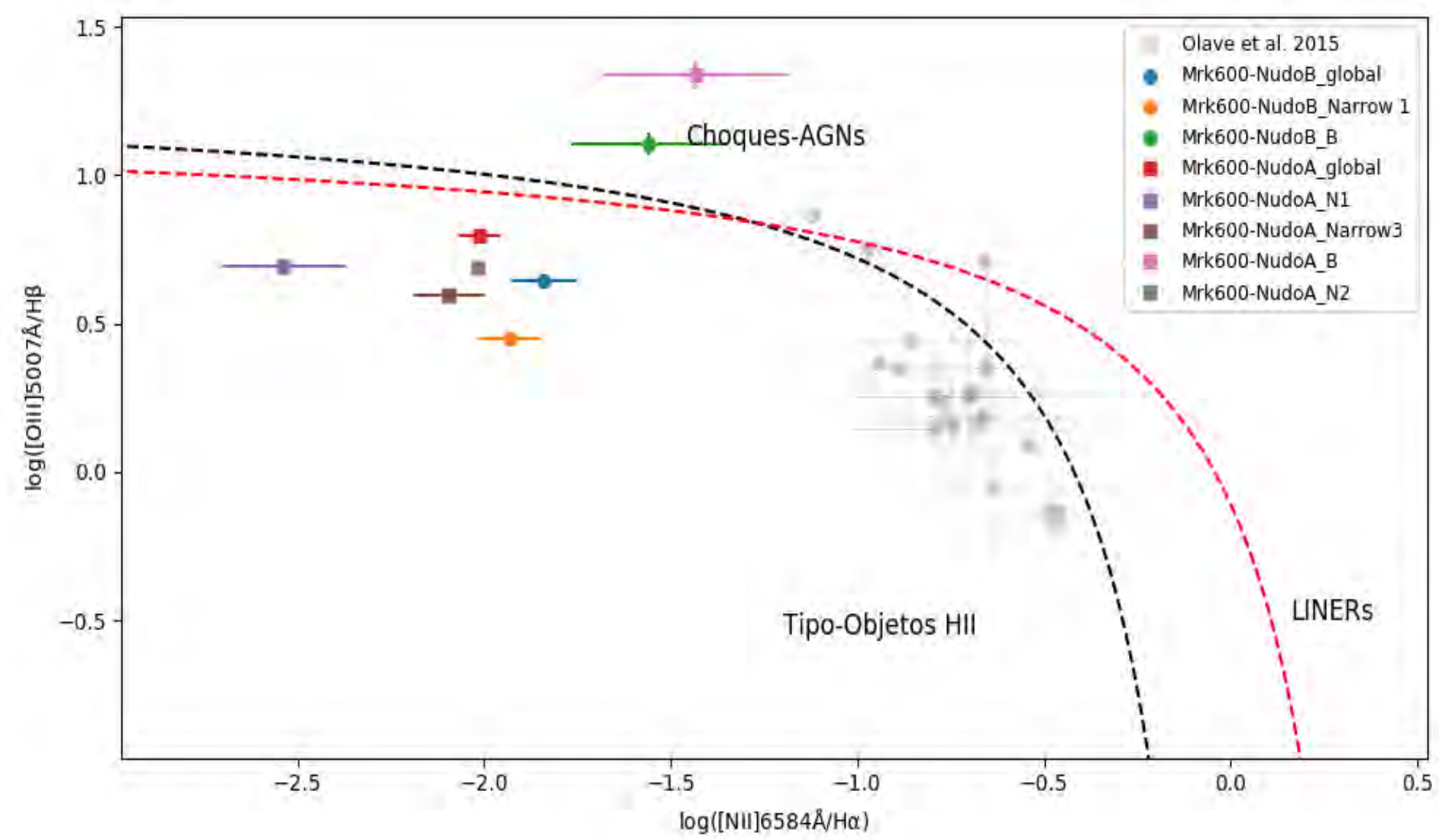


Figura 3.17. Diagrama diagnóstico $[\mathrm{OIII}] \lambda 5007 \AA / \mathrm{H} \beta$ vs. $[\mathrm{SiI}](\lambda 6717 \AA+\lambda 6731 \AA) / \mathrm{H} \alpha$ generado con el programa Kinematics para el análisis de la posible fuente ionizante para cada componente de gas determinada. Las curvas discontinuas en negro y rojo marcan el límite entre las distintas fuentes ionizantes Tipo-Objetos HII, por Choques-AGNs o LINERs, definidas por Kewley et al. (2006).

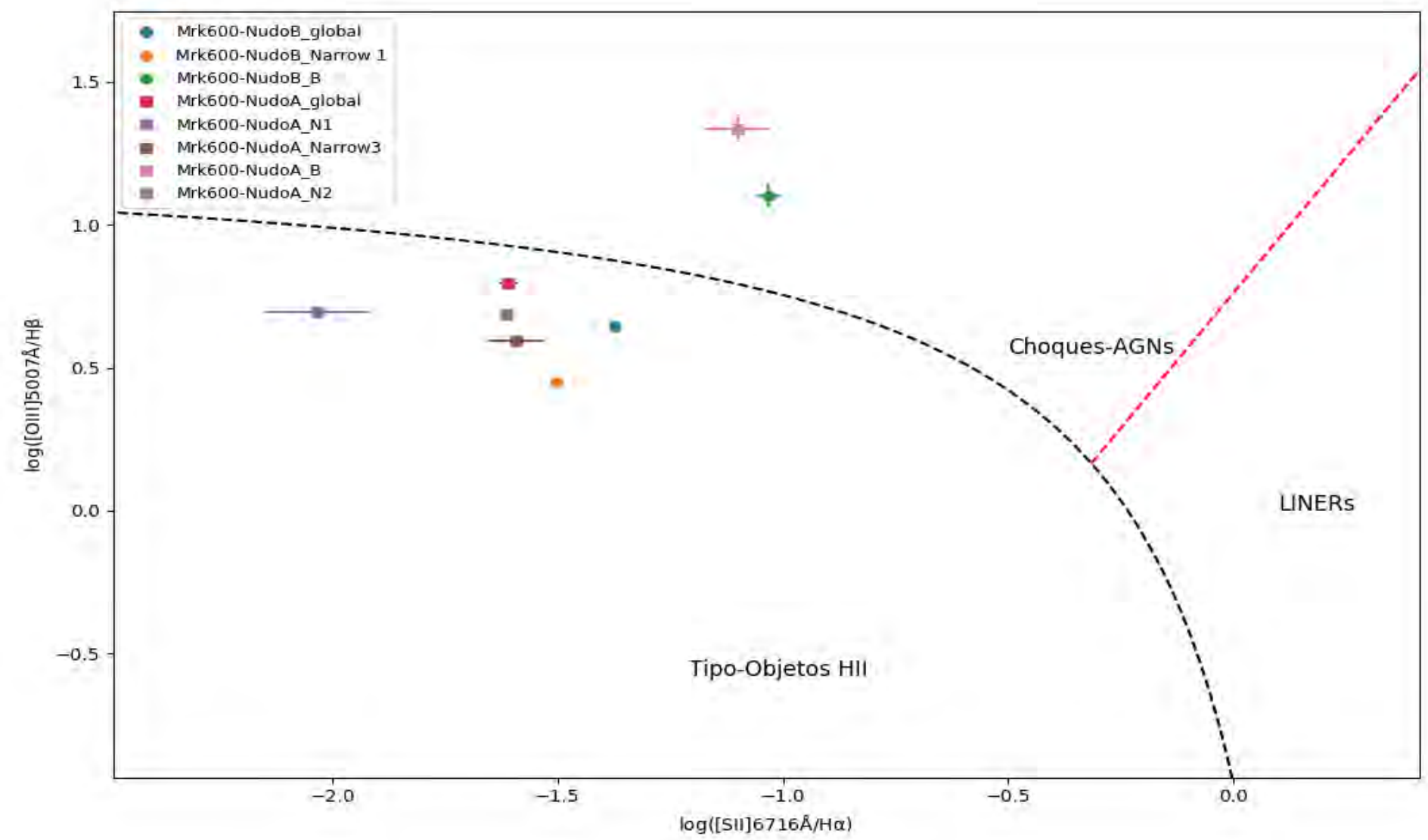




\subsection{Conclusión}

Se ha llevado a cabo un análisis detallado de la cinemática de nuestras regiones a través del ajuste de múltiples componentes gaussianas en los perfiles de las líneas de emisión intensas detectadas en nuestros espectros, haciendo uso de un programa en python desarrollado por el grupo y colaboradores, en el que utilizamos como herramienta de ajuste la tarea LMFit. Para la determinación de la cinemática, hemos seguido los pasos descriptos en los trabajos de Firpo et al. (2010); Firpo (2011) pero mejoramos y optimizamos el método de análisis creando un programa nuevo, más eficiente e interactivo para el estudio de los perfiles de líneas. Esto mejoró la técnica de ajuste y permitió el análisis de las regiones en simultáneo, así como también la generación de gráficos y tablas de una manera más metódica y eficiente. El trabajo en este programa requirió de mucho tiempo de pruebas y mejoras para hacerlo efectivo, robusto y confiable.

Descompusimos los perfiles de las líneas en múltiples componentes gaussianas, con anchos, flujos y centros directamente relacionados con las condiciones físicas del gas fotoionizado. Estas componentes gaussianas describen al gas ionizado a distintas velocidades, que pueden tener naturalezas distintas. Con este trabajo continuamos con las investigaciones realizadas por el grupo del cual he formado parte (Hägele et al., 2007, 2009, 2010; Firpo et al., 2011; Hägele et al., 2012; Amorín et al., 2012a; Hägele et al., 2013; Bosch et al., 2019), las cuales son las bases del mismo y nos dan un respaldo de muchos años de trabajo previo. Esto nos ayuda a comprender de una manera más completa la naturaleza de los perfiles de las líneas de emisión en este subtipo de galaxias. En este trabajo se presenta por primera vez un análisis detallado de la cinemática de las regiones de dichas galaxias.

El indicador estadístico de Akaike fue implementado como herramienta para validar las soluciones encontradas, dado que a partir de la condición que este plantea, podemos indicar si una solución tiene un sentido matemático acorde a nuestro plan de trabajo, penalizando los ajustes que tienen un mayor número de componentes que las estadísticamente válidas. De esta manera pudimos continuar con el estudio de la cinemática para las regiones con brotes de formación estelar violentas pertenecientes a galaxias BCD.

Hemos ajustado componentes anchas y múltiples componentes angostas, con dispersiones de velocidades supersónicas, que juntas nos permiten describir y encontrar una solución para los perfiles globales. Si tenemos en cuenta los trabajos previos realizados por los miembros del grupo de trabajo, podemos afirmar que los estudios de espectros de alta resolución de Regiones HiI nos permiten estudiar a partir de distintas componentes gaussianas, la cinemática compleja de dichas regiones. La determinación de gaussianas con $\sigma_{i n t}$ supersónicos nos permiten confirmar la naturaleza gigante de estas regiones, como ya fue planteado en trabajos anteriores del grupo.

Con los resultados determinados en esta sección, es posible continuar con el análsis de los flujos de las líneas y las relaciones de los cocientes entre ellas, para una determinación de las propiedades físicas del gas ionizado, tal como haremos en el siguiente Capítulo. 



\section{Capítulo 4}

\section{Determinación de las abundancias químicas y las propiedades físicas del gas ionizado}

Este capítulo presenta el análisis de los parámetros físicos de las regiones de gas ionizado de ambas galaxias analizadas, estudiando las subcomponentes cinemáticas y la global de cada región por separado. La presencia de gran cantidad de líneas de emisión en los espectros nos permite analizar las propiedades físicas y las abundancias químicas de dichas regiones a partir de los cocientes de sus líneas de emisión. La densidad, la temperatura, el enrojecimiento de las regiones, las abundancias químicas iónicas y totales, los parámetros de ionización, las tasas de formación estelar, son algunas de las propiedades que pueden determinarse a partir de los cocientes de las líneas. Siguiendo el trabajo de Hägele et al. (2012) llevamos a cabo un análisis detallado de las propiedades del gas ionizado para cada componente cinemática ajustada y descripta en el capítulo anterior, comparando las características estimadas para cada una de las componentes y de las regiones.

\subsection{Densidades y Temperaturas Electrónicas}

Como se ha descrito en el Capítulo 1 Sección 1.4, las densidades y temperaturas electrónicas son paráemtros físicos del gas en el medio nebular. Vimos que a través de líneas de emisión en el espectro óptico es posible estimar la densidad electrónica de un gas. Para ello, se comparan las intensidades de dos líneas del mismo ion, que emiten al caer a diferentes niveles con energía de excitación muy similares, de forma que las tasas de excitación relativas de los niveles dependan sólo del cociente de las fuerzas de la colisión. Si los dos niveles tienen diferentes probabilidades de transición o presentan ritmos de desexcitación colisional diferentes, el cociente de la intensidad de sus líneas dependerá de la densidad de los electrones presentes en el medio nebular.

En nuestro caso, los dobletes de líneas utilizados son: [OII] $\lambda 3729, \lambda 3726$ y $[\mathrm{SII}] \lambda 6717$, $\lambda 6731$.

Las líneas del [OII] se encuentran en el extremo azul del espectro por lo que son necesarios instrumentos que tengan buena eficiencia cuántica en esa región espectral. Otra opción es observar galaxias que presenten mayores corrimientos al rojo y así encontrar a este doblete desplazado hacia el rojo en un rango espectral donde el detector sea sensible. Por otro lado, las líneas del doblete del [SII] debido a su longitud de onda, de $\sim 6600 \AA$, no presentan el problema de la eficiencia cuántica, pero sí nos acercamos a las líneas de cielo que pueden ser 


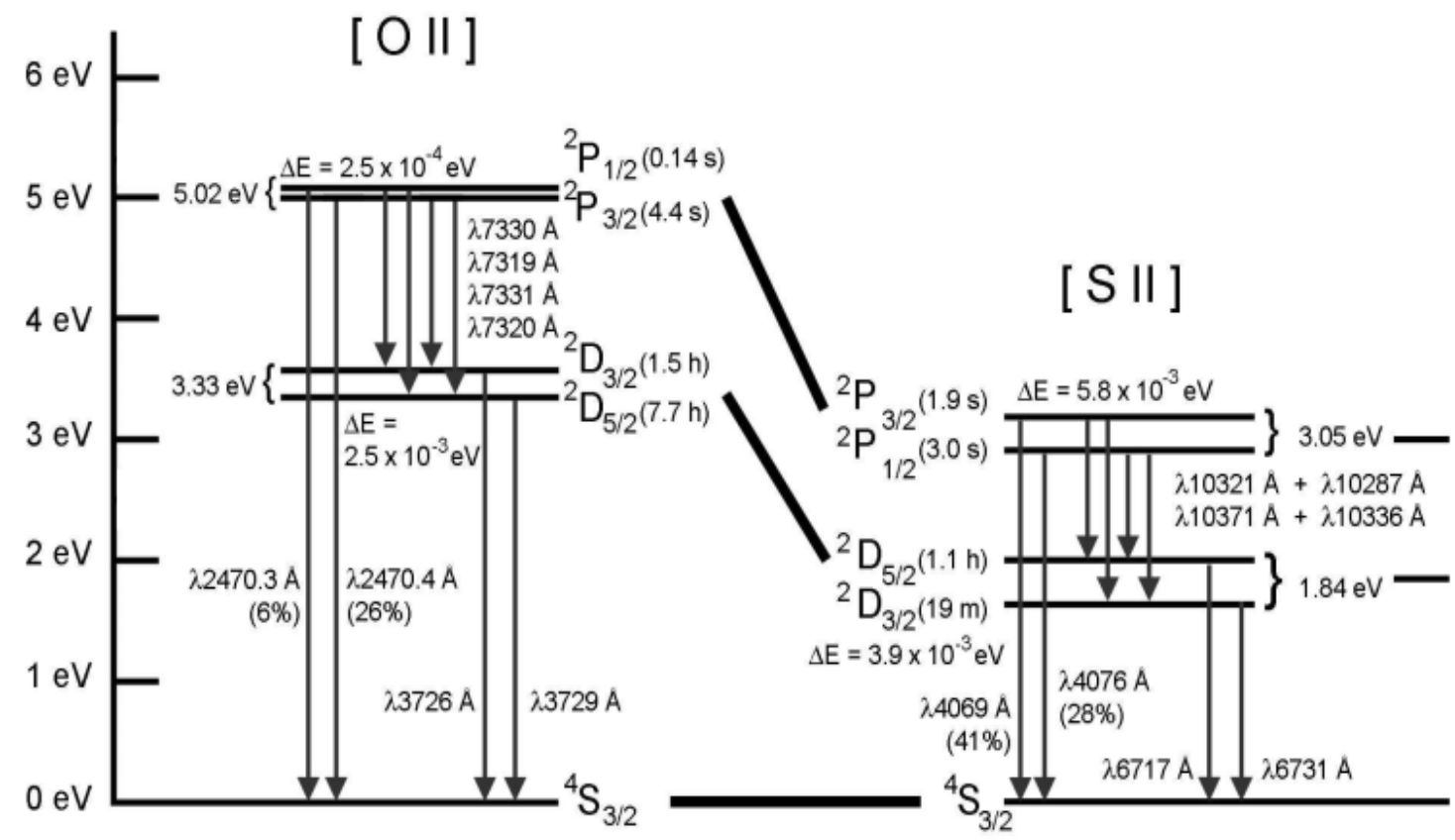

Figura 4.1. Diagramas de niveles de las transiciones de las líneas más brillantes de [OII] y [SII]. Fuente: Osterbrock \& Ferland (2006).

un gran problema si se superponen con dichas líneas. Pero lo que si hay que tener en cuenta es que a altos redshifts, las líneas del azufre, pueden verse afectadas por las líneas telúricas a partir de los $7000 \AA$. Por fortuna, en nuestro trabajo las observaciones con el espectrógrafo MIKE presentan un rango espectral que nos permite medir con una buena $\mathrm{S} / \mathrm{N}$ a las líneas del [OII] y también al doblete del [Sir].

Hemos calculado la densidad electrónica solamente a partir del cociente del doblete de [SII] $\lambda \lambda 6717 \AA / 6731 \AA ̊$ debido a que las densidades electrónicas estimadas a partir del doblete del [OII] en algunos casos presentan errores lo suficientemente grandes para no ser tenidos en cuenta.

En el límite de baja densidad, el cociente de flujos de las líneas se determina por las fuerzas de colisión de los niveles ${ }^{2} \mathrm{D}$, con un pequeño efecto debido a las cascadas provenientes del nivel ${ }^{2} \mathrm{P}$ (ver Figura 4.1), mientras que, en el caso de alta densidad electrónica, la intensidad relativa del doblete está dada por el cociente de las probabilidades de emisión radiativa, como lo vimos en el Capítulo 1 Sección 1.4. De esta manera, de la ecuación 1.9 se ve que a densidades bajas los términos en el denominador son mucho menores que los coeficiente de Einstein, $A_{21}$, ya que la intensidad de la línea es proporcional al cuadrado de la densidad.

Las estimaciones de los parámetros físicos derivados a partir de las líneas de emisión medidas en los espectros, fueron realizadas con el programa desarrollado por Hägele et al. (2006, 2008), que ha permitido los análisis desarrollados en los trabajos realizados por Hägele et al. $(2006,2008,2011,2012)$. Este programa se basa en el paquete temden del software de reducción astronómica IRAF, que proporciona una solución numérica que converge a la analítica a partir de la razón de los flujos de las líneas del doblete de azufre, tomando valores del cociente entre 0.46 - 1.421. Resulta interesante mencionar que en la literatura se encuentran cocientes que no están representados entre estos valores, debido a que en algunas situaciones estas cotas resultan ineficientes para explicar la densidad de una región, estimando valores del cocientes mayores a 1.421 que implicarían valores negativos de las densidades, lo 
cual es físicamente imposible (ver López-Hernández et al., 2013; Krabbe et al., 2014).

Presentamos a continuación la expresión utilizada para calcular las densidades a partir del cociente de las líneas de emisión de [SII].:

$$
n([\mathrm{SII}])=10^{3} \cdot \frac{R_{S 2} \cdot a_{0}(t)+a_{1}(t)}{R_{S 2} \cdot a_{2}(t)+a_{3}(t)},
$$

donde cada coeficiente $a_{i}$ corresponde a:

$$
\begin{gathered}
a_{0}(t)=2.21-1.3 / t_{e}-1.25 t_{e}+0.23 t_{e}^{2}, \\
a_{1}(t)=-3.35+1.94 / t_{e}+1.93 t_{e}-0.36 t_{e}^{2}, \\
a_{2}(t)=-4.33+2.33 / t_{e}+2.72 t_{e}-0.57 t_{e}^{2}, \\
a_{3}(t)=1.84-1 / t_{e}-1.14 t_{e}+0.24 t_{e}^{2} .
\end{gathered}
$$

El valor calculado para la densidad electrónica $[\mathrm{n}([\mathrm{SII}])]$ depende de la temperatura, $\mathrm{t}_{e}$, para la cual generalmente se adopta la temperatura electrónica del [OIII], donde $t_{e}=10^{-4} \mathrm{~T}_{e}$. En nuestro caso estimamos las temperaturas electrónicas para el [OIII] a través de un proceso iterativo, debido a que originalmente las líneas están sin corregir por enrojecimiento y la temperatura inicial estimada del [OIII] no es la correcta. En este proceso se utiliza la densidad, que no está afectada por enrojecimiento (ya que las líneas de [SII] están a unos pocos angstroms una de la otra) y la estimación de la temperatura del [OIII] obtenida a partir de las líneas enrojecidas. Se calcula una constante de enrojecimiento con dichos valores y se desenrojecen las líneas. Con estos nuevos valores de las intensidades podremos estimar una temperatura más confiable, y con esta temperatura y la densidad volvemos a calcular la constante de enrojecimiento volviendo a iterar hasta que converge en un valor aproximado. Cuando no se tiene algunas de las líneas necesarias para la estimación de la temperatura se usa una temperatura de 10.000k, que es representativa de las Regiones Hir y se estima un densidad para poder desenrojecer las líneas y estimar una temperatura más confiable de $\mathrm{T}_{e}([\mathrm{OIII}])$, repitiendo el proceso. Vale la pena aclarar que en algunos casos nos hemos encontrado que la constante de enrojecimiento calculada, $\mathrm{c}(\mathrm{H} \beta)$, presenta un valor negativo. Esto correspondería a un objeto blushift, es decir que a la región se estaría acercando a nosotros. Como es de esperarse que estas regiones estén apantalladas por el polvo, en las situaciones en que $\mathrm{c}(\mathrm{H} \beta)<0$, la reescribimos como $\mathrm{c}(\mathrm{H} \beta)=0$, y así suponemos un enrojecimiento nulo.

Las densidades derivadas para nuestros nudos, tanto en las medidas globales como para las componentes, pueden enmarcarse en los límites de las bajas densidades, excepto en unos pocos casos donde la densidad presenta errores grandes, provenientes de la propagación de los errores en los ajustes de las componentes en las líneas del azufre. Estos casos los discutiremos en detalle más adelante, cada uno en particular. Más allá de esto último, todos los valores resultan menores al crítico de desexcitación colisional en $\approx 2000 \mathrm{~cm}^{-3}$.

Una buena estimación de las temperaturas es fundamental para determinar las densidades de una manera más precisa. Suponer una temperatura de modelo introduce errores que luego se propagan en el análisis de las abundancias químicas de la nebulosa. Es posible estimar las temperaturas electrónicas de una manera directa a partir de cocientes de líneas provenientes del mismo ión que se generan en diferentes niveles y que tienen energías de excitación considerablemente diferentes (ver Figura 1.7 del Capítulo 1 Sección 1.4). Las excitaciones colisionales dependen de la temperatura del medio y por lo tanto, a través de las relaciones entre las poblaciones en cada nivel del ión es posible estimar la temperatura electrónica de una manera precisa, como se indica en Osterbrock \& Ferland (2006). En la tabla 4.1 se presentan los cocientes de líneas para la estimación de la temperatura electrónica según los distintos elementos químicos. 
Tabla 4.1. Cocientes comúnmente utilizados para la determinación de las densidades y temperaturas electrónicas en la nebulosa.

\begin{tabular}{|l|c|}
\hline \hline Cantidad Física & Cociente de líneas \\
\hline $\mathrm{n}_{e}[\mathrm{SII}]$ & $\mathrm{R}_{S 2}=\mathrm{I}(\lambda 6717 \AA) / \mathrm{I}(\lambda 6731 \AA)$ \\
$\mathrm{t}[\mathrm{OII}]$ & $\mathrm{R}_{O 2}=\mathrm{I}(\lambda \lambda 3726,3729 \AA) / \mathrm{I}(\lambda \lambda 7319,7330 \AA)$ \\
$\mathrm{t}[\mathrm{OIII}]$ & $\mathrm{R}_{O 3}=\mathrm{I}(\lambda \lambda 4959,5007 \AA) / \mathrm{I}(\lambda 4363 \AA)$ \\
$\mathrm{t}[\mathrm{SII}]$ & $\mathrm{R}_{S 2}^{\prime}=\mathrm{I}(\lambda \lambda 6717,6731 \AA) / \mathrm{I}(\lambda \lambda 4068,4076 \AA)$ \\
$\mathrm{t}[\mathrm{SIII}]$ & $\mathrm{R}_{S 3}=\mathrm{I}(\lambda \lambda 9069,9532 \AA) / \mathrm{I}(\lambda 6312 \AA)$ \\
$\mathrm{t}[\mathrm{NII}]$ & $\mathrm{R}_{N 2}=\mathrm{I}(\lambda \lambda 6548,6584 \AA) / \mathrm{I}(\lambda 5755 \AA)$ \\
\hline
\end{tabular}

Medir las líneas débiles aurorales es problemático debido a que las intensidades pueden ser varios órdenes de magnitud menores que las líneas más intensas y por lo tanto, la S/N no es lo suficientemente alta para aplicar el procedimiento descripto en el Capítulo 3.

La temperatura electrónica que se utiliza para caracterizar una temperatura global de la nebulosa es la $\mathrm{T}_{e}([\mathrm{OIII}])$, ya que generalmente las líneas de este ión son las más intensas en el espectro visible. También, existen otras temperaturas que resultan de interés en el rango observado, como la $\mathrm{T}_{e}([\mathrm{OII}]), \mathrm{T}_{e}([\mathrm{SII}]), \mathrm{T}_{e}([\mathrm{SIII}])$ y $\mathrm{T}_{e}([\mathrm{NII}])$, que se listan en la Tabla 4.1. La limitación que puede estar presente al pretender estimar la temperatura utilizando el cociente del azufre dos veces ionizado es que es necesario disponer de un espectro que cubra un amplio rango espectral para tener medidas de las líneas presentes en el extremo rojo del espectro óptico: [SIII] en $9069 \AA$ y $9532 \AA$. Esta zona del espectro cuenta con algunos factores como las líneas de cielo que pueden afectar a las líneas del azufre. Si además tenemos presente el corrimiento al rojo, que nos desplaza las líneas hacia longitudes de onda más largas, son varias las causas que pueden perjudicar o imposibilitar la medición de las líneas problemáticas del [SIII]. En nuestros casos hemos podido medir la línea de 9069 Án únicamente. La línea del [SIII] en $9532 \AA$, se encuentra fuera del rango espectral o justo en el último orden, donde los efectos de borde son muy grandes. Así es que hemos utilizado la relación teórica entre dichas líneas, dada por $\mathrm{I}(9069) \approx 2.44 \times \mathrm{I}(9532)$ (Osterbrock \& Ferland, 2006).

Para el [OII] encontramos la ventaja de que las líneas que se usan en este cociente se pueden medir bien en los espectros. Sin embargo, al estar muy separadas en longitud de onda la extinción interestelar es un factor importante a tener en cuenta y que hay que corregir, ya que afecta de manera diferente a las líneas. Como complicación en estos casos tenemos que las líneas de cielo pueden solaparse al doblete auroral en $\lambda 7319 \AA$ y $\lambda 7330 \AA$. Otra cuestión a tener en cuenta es la cobertura espectral en el ultravioleta, ya que en algunos casos en el extremo azul del espectro podemos tener restricciones para la determinación del doblete $\lambda \lambda 3726 \AA, 3729 \AA$. También debemos resaltar que todos los cocientes de líneas utilizados para determinar las temperaturas dependen de la densidad en cierta medida, especialmente $\mathrm{T}_{e}([\mathrm{OII}])$ y $\mathrm{T}_{e}([\mathrm{SII}])$ (Osterbrock \& Ferland, 2006). Debido a esto, las temperaturas electrónicas que se derivan comunmente son las de las líneas del [NII] y [OIII] ya que son los que llevan las determinaciones menos inciertas. Las $\mathrm{T}_{e}([\mathrm{OII}]), \mathrm{T}_{e}([\mathrm{SII}])$ y $\mathrm{T}_{e}([\mathrm{NII}])$ suelen usarse para caracterizar las zonas de menor grado de ionización, mientras que la $\mathrm{T}_{e}([\mathrm{OIII}])$ caracteriza las zonas de mayor grado de ionización. 


\subsubsection{Método Directo}

El método directo se basa en la determinación de las abundancias químicas iónicas y totales a través de la utilización de las densidades y temperaturas electrónicas, estimadas directamente de la observación y que caracterizan a los distintos iones. De esta manera se minimizan los errores introducidos por las suposiciones que dependen de modelos o determinaciones empíricas en los casos donde dichas densidades o temperaturas no pueden ser determinadas directamente de observables.

En varios casos fue posible medir las líneas aurorales del [OIII], [OII], [SIII] y [Sir]. Veamos ahora las expresiones utilizadas para la determinación de las temperaturas electrónicas en cada uno de los casos (ver Hägele et al., 2006, 2008).

- Oxígeno

OIII : Para el oxígeno dos veces ionizado utilizamos el cociente $\mathrm{R}_{O 3}$. Este cociente no depende de la densidad electrónica, por lo que es un excelente indicador de la temperatura a bajas densidades. La temperatura se determina de la siguiente expresión:

$$
\mathrm{t}_{e}([\mathrm{OIII}])=0.8254-0.0002415 \cdot \mathrm{R}_{O 3}+\frac{47.77}{\mathrm{R}_{O 3}},
$$

siendo $t_{e}$ la temperatura en unidades de $10^{4} \mathrm{k}$.

OII : En este caso la temperatura se determina a partir del cociente $\mathrm{R}_{O 2}$ indicado en la Tabla 4.1. Como mencionamos con anterioridad, el cuidado que hay que tener para estas líneas está relacionado a la contaminación del cielo y la recombinación en los $\lambda 7330 \AA$, para las líneas aurorales. Siguiendo el trabajo de Liu et al. (2001) la contribución por dichos efectos puede ser ajustada con $t$ en un rango de $0.5 \leq \mathrm{t} \leq 1$ a través de la siguiente expresión:

$$
\frac{\mathrm{I}_{R_{O 2}}(7319 \AA+7330 \AA)}{\mathrm{I}(\mathrm{H} \beta)}=9.36 \cdot t^{0.44} \cdot \frac{\mathrm{O}^{2+}}{\mathrm{H}^{+}} .
$$

Esta función es válida únicamente entre los $5.000 \mathrm{k}$ y los $10.000 \mathrm{k}$. De los cocientes mencionados para estimar temperaturas por el método directo, este es el que más fuertemente depende de la densidad. Como las líneas del doblete de $3726 \AA$ y $3729 \AA$ forman parte de esta relación, pueden utilizarse para la determinación de la densidad electrónica y por lo tanto, para la determinación de la temperatura electrónica. En los casos en que no se pueda contar con la densidad electrónica determinada a partir del cociente de las líneas del doblete del [OII], se toma la densidad electrónica determinada con el doblete del azufre, que en principio se suponen iguales. En nuestro caso, no ha sido posible medir el doblete de las líneas aurorales en $7319 \AA$ y $7330 \AA$. En los casos de no tener el doblete del UV para la determinación de la densidad, ya sea porque el rango cubierto no llega a tomarlas, porque la resolución no permite resolverla o porque la señal no es lo suficiente, suele utilizarse el valor de la densidad determinada a partir de las líneas del [SII]. Luego, se tiene que la relación para la estimación de la temperatura es:

$$
\mathrm{t}_{e}([\mathrm{OII}])=0.23+0.0017 \cdot \mathrm{R}_{O 2}+\frac{38.3}{\mathrm{R}_{O 2}}+\mathrm{f}_{1}\left(n_{e}\right),
$$

en donde $f_{1}$ es una función dependiente de la densidad electrónica. 
- Azufre

SIII : Disponer de un rango espectral amplio, incluyendo el IR cercano, más allá de $\operatorname{los} \lambda 9000 \AA$, es crucial para poder medir el doblete de líneas colisionales del $[\mathrm{SIII}] \lambda \lambda 9069 \AA, 9532 \AA$. La estimación directa de la temperatura de este ión se realiza utilizando la siguiente expresión que depende de los cocientes de las líneas de emisión:

$$
\mathrm{t}_{e}([\mathrm{SIII}])=\frac{\mathrm{R}_{S 3}+36.4}{1.8 \cdot \mathrm{R}_{S 3}-3.01} .
$$

SiI : Las líneas aurorales $4068 \AA$ y $4076 \AA$ presentan una relación teórica entre ellas dada por $\mathrm{I}(4068 \AA) \approx 3 \cdot \mathrm{I}(4076 \AA$ ) (ver Pérez-Montero et al., 2003) que nos permite estimar la temperatura por el método directo conociendo únicamente una de las dos. En nuestro caso hemos medido $4068 \AA$ en el brote B de Mrk 600 y hemos calculado la temperatura electrónica a través de:

$$
\mathrm{t}_{e}([\mathrm{SII}])=1.92-0.0375 \cdot R_{S 2}^{\prime}-\frac{14.5}{\mathrm{R}_{S 2}^{\prime}}+\frac{105.64}{\mathrm{R}_{S 2}^{\prime 2}}+\mathrm{f}_{2}\left(n_{e}\right)
$$

en donde $\mathrm{f}_{2}\left(\mathrm{n}_{e}\right)$ corresponde a una función de la densidad.

Por último, en el caso del nitrógeno la temperatura se puede estimar a través de los cocientes $\mathrm{R}_{N 2}$ (ver Tabla 4.1). En nuestros espectros contamos con una excelente señal para las líneas de emisión de [NII] en $6548 \AA$ y $6584 \AA$ pero no hemos detectado la línea auroral del $[\mathrm{NII}] \lambda 5755 \AA$ A . Debido a esto no se pueda estimar la temperatura del [NII] por el método directo, sino que se recurrieron a relaciones entre las distintas temperaturas a partir de relaciones teóricas, como se indica en Hägele et al. (2006, 2008).

En los casos en que no se pueden medir las líneas aurorales, no es posible estimar los parámetros físicos ni abundancias químicas a partir del método directo, como ocurre en el caso del Nudo C de II Zw 33, debido a la baja relación S/N de las líneas aurorales. Por otro lado, también sucede en regiones muy ricas en oxígeno, donde la línea auroral $\lambda 4363 \AA$ es muy débil y difícilmente detectable (Díaz et al., 2007). La elevada metalicidad produce un enfriamiento eficaz del gas a través de las líneas de los metales, principalemente del oxígeno, por lo que en estos casos la temperatura es muy baja. Para estas situaciones se utilizan las calibraciones empíricas tomando las medidas de las líneas de emisión más intensas para la determinación de las abundnacias del oxígeno. Esta alternativa, basada en modelos teóricos de fotoionización o relaciones empíricas (Stasińska, 2006; Díaz et al., 2007), nos permite estimar las temperaturas electrónicas en los casos en que no puedan determinarse con el método directo, a través de las relaciones entre distintos parámetros empíricos y las temperaturas, o entre distintas temperaturas (ver Díaz et al., 2007; Hägele et al., 2012). En los casos donde no podamos derivar las temperaturas electrónicas necesarias utilizaremos este tipo de relaciones, como explicaremos a continuación.

\subsubsection{Relación entre las temperaturas electrónicas}

Por lo mencionado anteriormente, la tarea de medir las líneas aurorales puede no ser posible por lo que las temperaturas electrónicas y las abundancias químicas no siempre se pueden calcular de manera directa. Es por esta razón que para los casos en que se logra medir algunas de las temperaturas electrónicas, es posible calcular por medio de distintas relaciones las correspondientes a los otros iones. 


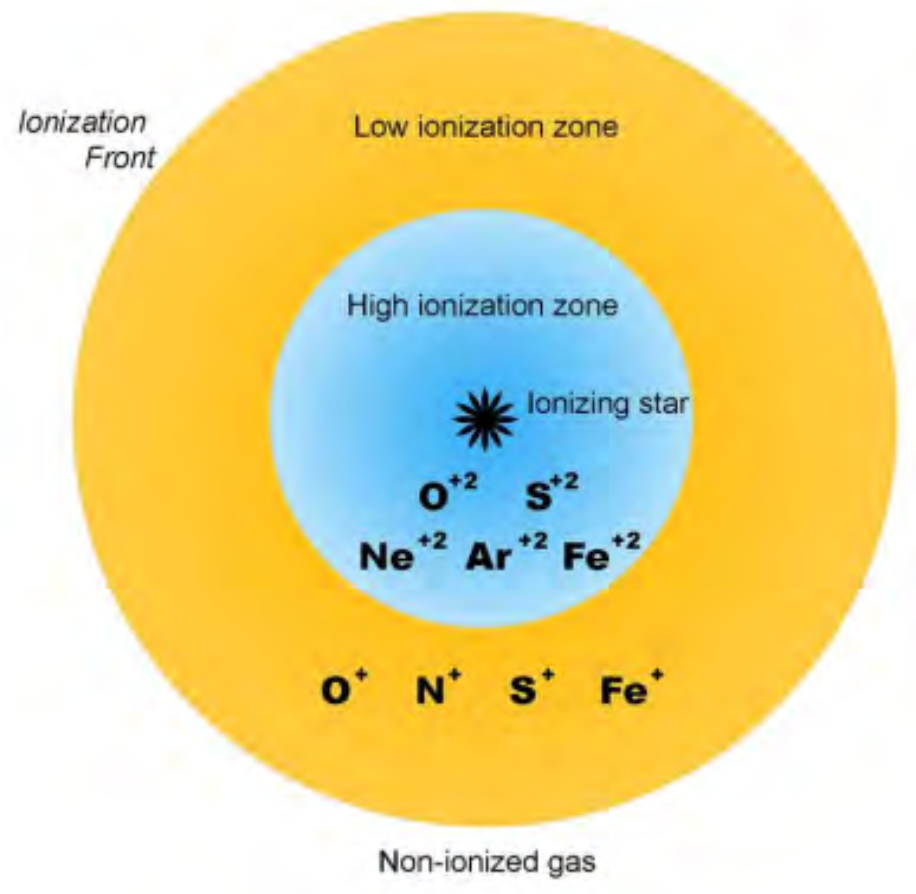

Figura 4.2. Iones que se originan en las distintas regiones de la nebulosa según la energía proveniente del campo de radiación de las fuentes excitatrices. Hacia el interior los iones que requieren mayores potenciales de ionización y hacia las capas más exteriores, los elementos que se ionizan con energías menores. Imágen adoptada de López-Sánchez et al. (2012).

Los estudios realizados por Peimbert (1967) demostraron la existencia de estructuras internas de ionización en regiones nebulares (ver Figura 4.2) lo que indica una variación espacial de las temperaturas electrónicas. Y debido a esto es importante para determinar las abundancias iónicas tener en cuenta las temperaturas de las zonas en las que los iones correspondientes se forman .

Una aproximación válida es considerar zonas con ionizaciones distintas dentro de la nebulosa, para poder plantear diferencias entre bajas y altas ionizaciones y la relación con los potenciales de ionización de los distintos iones y elementos químicos. Se plantean dos zonas, una de alta ionización asociada a las zonas más cercanas a las fuentes donde los campos de radiación ionizantes son más intensos, y otra más externa con flujos de energía más diluídos, caracterizados generalmente por las temperaturas del [OIII] y [OII], respectivamente (Pagel, 1992). En este trabajo hemos utilizado la metodología desarrollada por Hägele et al. (2008) (y modificada en Hägele et al., 2012) para derivar las distintas temperaturas a partir de las $\mathrm{t}_{e}([\mathrm{OIII}])$ determinada por el método directo. Se fijó el criterio de no indicar errores cuando las magnitudes determinadas son por modelos y/o relaciones empíricas, ya que en esos casos los valores son muy chicos y al expresar errores bajos puede parecer que es mejor hacer las estimaciones por modelos que de forma directa. Por lo tanto, como no es posible obtener una estimación realista de las incertidumbres asociadas a los modelos, en las tablas no se indicaron errores.

Es esperable que las temperaturas de $\mathrm{T}_{e}([\mathrm{OII}]), \mathrm{T}_{e}([\mathrm{SII}])$ y $\mathrm{T}_{e}([\mathrm{NII}])$ estén muy relacionadas entre sí ya que dichos iones se supone que se forman en la misma zona de ionización (baja) de la nebulosa. En la literatura se ha discutido que existen diferencias entre estas temperaturas electrónicas, de manera que se han utilizado distintas relaciones entre ellas cuando las líneas aurorales de estos iones no se han podido medir. En nuestro trabajo, la línea [NII] $\lambda 5755 \AA$ 
ha sido indetectable en los espectros, por lo que consideramos la aproximación con la temperatura del [SII]. Esta relación es sensible a la densidad y a la estructura de ionización de la nebulosa, de manera tal que el grado de incerteza que presenta no es despreciable y hay que considerarlo. A su vez, de distintos modelos de fotoionización (Pérez-Montero et al., 2003) resulta que la temperatura asociada al $\mathrm{S}^{+}$resulta ser menor que la calculada para los otros dos iones, lo que puede deberse a las fracciones de elementos de bajas excitaciones. Como las fracciones de azufre son mayores en la baja excitación que en las zonas de mayor ionización, esta mayor cantidad de elementos pesados produce una baja en la temperatura media estimada (Pérez-Montero et al., 2009, 2010).

Cuando alguna de las líneas aurorales de $\mathrm{S}^{+}$o de $\mathrm{O}^{+}$no puedan ser detectadas, se ha utilizado la relación entre las temperaturas electrónicas dada por Pérez-Montero et al. (2003):

$$
\mathrm{t}_{e}([\mathrm{SII}])=0.71 \cdot \mathrm{t}_{e}([\mathrm{OII}])+0.12 .
$$

Esta expresión lineal se ajustó para una densidad promedio $\mathrm{n}_{e}=10$ partículas $/ \mathrm{cm}^{3}$. La relación que resulta para densidades un orden de magnitud mayor $\left(\mathrm{n}_{e}=100\right)$ es:

$$
\mathrm{t}_{e}([\mathrm{SII}])=0.86 \cdot \mathrm{t}_{e}([\mathrm{OII}])+0.08 .
$$

Otras relaciones que hemos utilizado son las que obtenemos a partir del [OIII]. Las líneas aurorales del [OII] en muchos casos no son detectables, porque son muy débiles, o porque no tienen la suficiente señal para que podamos ajustar sus múltiples componentes. Pero, a partir de la $\mathrm{T}_{e}([\mathrm{OIII}])$ podemos estimar la $\mathrm{T}_{e}([\mathrm{OII}])$ a través de la relación dada por Pérez-Montero et al. (2003):

$$
t_{e}([\mathrm{OII}])=\frac{1.2+10.002 \cdot n_{e}+\frac{4.2}{n_{e}}}{t_{e}([\mathrm{OIII}])^{-1}+0.8+0.003 \cdot n_{e}+\frac{2.5}{n_{e}}}
$$

donde la determinación de $\mathrm{T}_{e}([\mathrm{OII}])$ depende explícitamente de la densidad electrónica. Esta expresión ha sido estudiada por Hägele et al. (2008) analizando el comportamiento de los ajustes de las curvas teóricas para los casos en que las temperaturas pudieron ser medidas por el método directo para testear su fiabilidad, y aunque las dispersiones eran considerables, no hay una tendencia clara de su relación con la densidad. En este trabajo, hemos utilizado esta relación para los casos en los que no pudieron detectarse las líneas aurorales del [OII].

Una de las relaciones más importantes que podemos encontrar en la literatura es aquella que vincula a las temperaturas del [OIII] con las del [SIII] dada por Hägele et al. (2008), la cual posteriormente en Binette et al. (2012) se buscó una interpretación a través de modelos. Los resultados presentados en Hägele et al. (2008) muestran que la $T_{e}([\mathrm{SIII}])$ es más alta que la $\mathrm{T}_{e}([\mathrm{OIII}])$ para los objetos menos metálicos y más baja para los más ricos en metales. Hemos utilizado la ecuación derivada por Hägele et al. (2006), la cual es una versión mejorada de la relación dada por Pérez-Montero \& Díaz (2005b) en la que se consideró un mayor número de observaciones para obtener una mejor estimación de las incertidumbre. De esta manera, la relación empírica que usaremos es:

$$
\mathrm{t}_{e}([\mathrm{SIII}])=(1.19 \pm 0.08) t_{e}([\mathrm{OIII}])-(0.32 \pm 0.10)
$$

Estas son las relaciones que se usan en este trabajo para estimar las temperaturas electrónicas cuando las líneas aurorales no pudieron medirse y por lo tanto, el cálculo no pudo hacerser mediante los cocientes de las líneas prohibidas. En los casos en que no sea posible medir ninguna línea auroral, puede suponerse una temperatura de $10.000 \mathrm{k}$, o pueden emplearse métodos empíricos utilizando las líneas más intensas, como explicaremos a continuación. 


\subsubsection{Métodos Empíricos para la determinación de temperaturas}

Cuando no fue posible medir las líneas aurorales, se utilizaron distintos métodos empíricos para determinar las temperaturas electrónicas de nuestras RHiI. Uno de estos métodos es el que nos permite estimar la temperatura del [SIII] a partir de relaciones entre las líneas intensas del oxígeno y del azufre, dadas por Díaz et al. (2007). Esta relación está basada en la combinación de los parámetros $\mathrm{O}_{23}$ (Pagel, 1979) y $\mathrm{S}_{23}$ (Vilchez \& Esteban, 1996), que son la suma de las líneas más intensas de los iones del oxígeno y del azufre ionizado respecto a $\mathrm{H} \beta$ :

$$
\begin{gathered}
\mathrm{O}_{23}=\frac{\mathrm{I}(3727,29 \AA)+\mathrm{I}(4959 \AA)+\mathrm{I}(5007 \AA)}{\mathrm{I}(\mathrm{H} \beta)}, \\
\mathrm{S}_{23}=\frac{\mathrm{I}(6717 \AA)+\mathrm{I}(6731 \AA)+\mathrm{I}(9069 \AA)+\mathrm{I}(9532 \AA)}{\mathrm{I}(\mathrm{H} \beta)}
\end{gathered}
$$

y que se conoce como $\mathrm{SO}_{23}$. La relación derivada por Díaz y colaboradores viene dada por la siguiente expresión:

$$
\mathrm{t}_{e}([\mathrm{SIII}])=0.596-0.283 \log \mathrm{SO}_{23}+0.199\left(\log \left(\mathrm{SO}_{23}\right)^{2}\right) .
$$

Dichos parámetros se utilizan para la determinación de las abundancias de oxígeno, que detallaremos en las siguientes secciones.

Si bien $\mathrm{SO}_{23}$ estaba pensada como un buen indicador de la temperatura para regiones con metalicidades altas, de la Figura 9 de Díaz et al. (2007) se deduce que además es un buen indicador para el extremo de la zona donde se encuentran las galaxias HiI, en donde la dispersión es baja. Dentro de los valores que toma el parámetros empírico en dicha zona de baja dispersión es donde encontramos a nuestras galaxias II Zw 33 y Mrk600. Con la temperatura del [SIII] estimada, hacemos uso de la relación empírica 4.1.2 para calcular $\mathrm{T}_{e}([\mathrm{OIII}])$, y a partir de esta, obtenemos por modelo a $\mathrm{T}_{e}([\mathrm{OII}])$, y luego, estimamos $\mathrm{T}_{e}([\mathrm{NII}])$ y $\mathrm{T}_{e}([\mathrm{SII}])$. Con estas temperaturas estimadas a partir de métodos semi-empíricos y junto a las líneas intensas es que podremos determinan las abundancias químicas.

Por último, para el caso único del Nudo C de II Zw 33 en el que no fue posible medir líneas aurorales ni algunas de las líneas intesas para el cálculo de tempearturas electrónicas a través de los métodos empíricos mencionados, se supone una temperatura electrónica para el [OIII] de 10.000k (se supone este valor como un promedio de la temperatura electrónica en este tipo de regiones, ver por ejemplo la discusión en Hägele et al., 2008). De esta manera, a partir de $\mathrm{T}_{e}([\mathrm{OIII}])$ y las relaciones que acabamos de mencionar, con la diferencia que usamos la ecuación 4.1.2 para derivar la temperatura de [SIII] a partir de la de [OIII], es que logramos estimar las demás temperaturas electrónicas.

\subsection{Abundancias Químicas}

A partir de las densidades y temperaturas calculadas, es posible determinar la abundancia química de varios elementos presentes en la nebulosa. A través de las abundancias iónicas de los elementos se pueden calcular las abundancias totales, utilizando distintas consideraciones como veremos a continuación, dependiendo de los elementos bajo análisis.

Para determinar la cantidad relativa de las distintas especies químicas respecto al hidrógeno, a partir de las líneas medidas, se utilizó la tarea ionic del paquete STSDAS de IRAF (como se describe en Hägele et al., 2008). Este paquete también se basa en la aproximación del átomo de cinco niveles en equilibrio estadístico, como hemos indicado previamente en el 
Capítulo 1). De esta manera se calculan las abundancias iónicas de un elemento respecto al hidrógeno resolviendo las ecuaciones del equilibrio estadístico para conocer las poblaciones relativas del nivel superior a la transición con la que se estiman las probabiliadades de transición, las diferencias de energías y luego las abundancias. Uno de los problemas que surgen al querer determinar las abundancias totales es que no se pueden medir las líneas de emisión de todos los estados de ionización de los elementos cuya abundancia química se quiere calcular. Una manera de sobrellevar esta dificultad es recurrir al factor de correción de ionización (ICF, de la sigla en inglés: ionization correction factor). Esta cantidad da cuenta de cuál es la contribución a la abundancia de las especies iónicas que no han sido observadas, y tienen que ser tenidos en cuenta para la determinación de las abundancias totales. Esta cantidad se calcula a partir de: $\mathrm{X} / \mathrm{H}=\mathrm{ICF}\left(\mathrm{X}^{+i}\right) \mathrm{X}^{+i} / \mathrm{H}^{+}$(Pérez-Montero et al., 2007; Hägele et al., 2008).

A continuación detallaremos las expresiones utilizadas para las determinaciones en cada especie atómica analizada, habiendo calculado previamente las densidades y temperaturas electrónicas, y posteriormente haremos el análisis de los resultados encontrados para cada una de las componentes cinemáticas en cada nudo estudiado.

\subsubsection{Helio}

Para el análisis de este elemento, necesitamos medir las líneas correspondientes al helio neutro, HeI, y una vez ionizado, HeII. Para estimar las abundancias iónicas del He, suelen utilizarse las líneas: para HeI $\lambda 4471 \AA$, $5876 \AA$, $6678 \AA$ y $7065 \AA$, y para el HeII $\lambda 4686 \AA$. Esta última, es una línea es muy difícil de detectar, en nuestro caso, por ser intrínsecamente débil y por encontrarse además en la zona del espectro del "bump" azul de Wolf-Rayet. A pesar de que las líneas del He se deben principalmente a la recombinación pura, estas pueden tener también una contribución por excitación colisional, produciendo un patrón de superposición, así como también puede sufrir la absorción producida por la población estelar subyacente (Olive \& Skillman, 2001, 2004).

Para un tratamiento consistente de los átomos de He, hemos tomado la temperatura electrónica del [OIII] como representativa de la zona en donde se originan las líneas de He. Esto es posible debido a que el potencial de ionización de los niveles que forman estas líneas son similares a las del [OIII] y por ende, las líneas se forman aproximadamente en la misma zona. Por lo tanto, utilizaremos la aproximación $\mathrm{T}_{e}(\mathrm{HeII}) \approx \mathrm{T}_{e}([\mathrm{OIII}])$, y la abundancia iónica del $\mathrm{He}$ una vez ionizado $\mathrm{He}^{+} / \mathrm{H}$ la estimamos siguiendo los trabajos de Olive \& Skillman (2001) donde utilizan las emisividades teóricas de $\mathrm{H} \beta$ estimadas por Benjamin et al. (1999):

$$
\begin{aligned}
\frac{\mathrm{E}(\mathrm{H} \beta)}{\mathrm{E}(4471)} & =2.00947 \cdot \mathrm{T}^{0.1259}, \\
\frac{\mathrm{E}(\mathrm{H} \beta)}{\mathrm{E}(5876)} & =0.7355 \cdot \mathrm{T}^{0.2298}, \\
\frac{\mathrm{E}(\mathrm{H} \beta)}{\mathrm{E}(6678)} & =2.5861 \cdot \mathrm{T}^{0.2475}, \\
\frac{\mathrm{E}(\mathrm{H} \beta)}{\mathrm{E}(7065)} & =4.3588 \cdot \mathrm{T}^{0.3456},
\end{aligned}
$$

y los factores de corrección colisional calculados por Kingdon \& Ferland (1995):

$$
\gamma(4471)=\left(6.95 \cdot \mathrm{T}^{0.15} \cdot e^{-4.545 / \mathrm{T}}+0.22 \cdot \mathrm{T}^{-0.55} \cdot e^{-4.884 / \mathrm{T}}+0.98 \cdot \mathrm{T}^{-0.45} \cdot e^{-4.901 / \mathrm{T}}\right) / \mathrm{D},
$$




$$
\begin{gathered}
\gamma(5876)=\left(6.78 \cdot \mathrm{T}^{0.07} \cdot e^{-3.776 / \mathrm{T}}+1.67 \cdot \mathrm{T}^{-0.15} \cdot e^{-4.545 / \mathrm{T}}+0.60 \cdot \mathrm{T}^{-0.34} \cdot e^{-4.901 / \mathrm{T}}\right) / \mathrm{D}, \\
\gamma(6678)=\left(3.15 \cdot \mathrm{T}^{-0.54} \cdot e^{-3.776 / \mathrm{T}}+0.51 \cdot \mathrm{T}^{-0.51} \cdot e^{-4.545 / \mathrm{T}}+0.20 \cdot \mathrm{T}^{-0.66} \cdot e^{-4.901 / \mathrm{T}}\right) / \mathrm{D}, \\
\gamma(7053)=\left(38.09 \cdot \mathrm{T}^{-1.09} \cdot e^{-3.364 / \mathrm{T}}+2.80 \cdot \mathrm{T}^{-1.06} \cdot e^{-3.699 / \mathrm{T}}\right) / \mathrm{D}
\end{gathered}
$$

donde $\mathrm{D}=1+3130 \cdot n^{-1} \cdot \mathrm{T}^{-0.50}$. Adoptamos como el valor de la abundacia iónica del HeI al promedio pesado con los errores de cada una de las estimaciones obtenidas con las distintas líneas de emisión medidas para este ión.

Para el helio dos veces ionizado, $\mathrm{He}^{+} / \mathrm{H}$, puede utilizarse la ecuación derivada por Kunth \& Sargent (1983):

$$
\frac{\mathrm{He}^{+}}{\mathrm{H}}=\mathrm{He}^{+}(4686)+\left(0.065+0.024 \cdot \mathrm{t}_{\mathrm{e}}-0.0052 \cdot \mathrm{t}_{\mathrm{e}}{ }^{2}\right) \frac{\mathrm{I}(\lambda 4686)}{\mathrm{I}(\mathrm{H} \beta)} .
$$

En los casos en que la línea de $[\mathrm{HeII}] \lambda 4686 \AA$ pueda ser medida, la estimación de la abundancia total quedará determinada por la suma directa de las dos abundancias iónicas:

$$
\frac{\mathrm{He}}{\mathrm{H}}=\frac{\mathrm{He}+\mathrm{He}^{+}}{\mathrm{H}} .
$$

Uno de los problemas en el cálculo de la abundancia total del helio, es la fracción de HeI no observable que representa la mayor fuente de error en la estimación de dicha abundancia (Storchi-Bergmann et al., 1994). Seg $\tilde{A}^{0}{ }^{\mathrm{n}}$ los autores, el factor de corrección se puede aproximar de una manera precisa para regiones HII ionizadas por estrellas muy calientes (con temperaturas efectivas $>40000 \mathrm{k}$ ). Una buena aproximación de la temperatura efectiva de las estrellas ionizantes de nuestros objetos se puede obtener a partir del parámetro de ionización $\eta$, que se define como la relación entre $\mathrm{O}^{+} / \mathrm{O}^{2+}$ y $\mathrm{S}^{+} / \mathrm{S}^{2+}$. En el caso de las galaxias HiI, estas se ubican en la zona correspondiente a valores grandes de estos parámetros, lo que equivale a altas temperaturas efectivas del campo de radiación, mayor a 40000k (ver discusión en Hägele et al., 2008).

En nuestro trabajo únicamente pudimos derivar la abundancia de $\mathrm{He}^{+} / \mathrm{H}$ para el Nudo B de Mrk 600, debido a que fue la única región en la que se pudo medir la línea en $4686 \AA$. Por esto, damos la abundancia total de $\mathrm{He} / \mathrm{H}$ solo para un objeto.

\subsubsection{Abundancias iónicas y totales a partir de líneas prohibidas}

Ahora vamos a describir en detalle el método y las ecuaciones utilizadas para el cálculo de las abundancias químicas iónicas y totales de las diferentes especies atómicas a través de las medidas de las líneas de emisión prohibidas, que serán fundamentales para describir las propiedades físicas del gas ionizado.

\section{- Oxígeno}

Las abundancias correspondientes a los iones $\mathrm{O}^{+}$y $\mathrm{O}^{2+}$ pueden ser derivadas de las líneas [OІІ] $\lambda \lambda 3727,29 \AA$ y [OІІІ $] \lambda \lambda 4959 \AA, 5007 \AA$, respectivamente, utilizando las siguientes ecuaciones (Hägele et al., 2008):

$$
\begin{gathered}
12+\log \left(\mathrm{O}^{+} / \mathrm{H}^{+}\right)=\log \left(\frac{\mathrm{I}(3727)}{\mathrm{I}(\mathrm{H} \beta)}\right)+5.992+\frac{1.583}{\mathrm{~T}_{e}}-0.681 \cdot \log \left(\mathrm{T}_{e}\right)+\log \left(1+0.00023 \cdot n_{e}\right) \\
12+\log \left(\mathrm{O}^{2+} / \mathrm{H}^{+}\right)=\log \left(\frac{\mathrm{I}(4959)+\mathrm{I}(5007)}{\mathrm{I}(\mathrm{H} \beta)}\right)+6.144+\frac{1.251}{\mathrm{~T}_{e}}-0.55 \log \left(\mathrm{T}_{e}\right)
\end{gathered}
$$


En estos casos $\mathrm{T}_{e}$ corresponde a la temperatura electrónica del ión en cuestión, $\mathrm{T}_{e}([\mathrm{OII}])$ para $O^{+}$y $\mathrm{T}_{e}([\mathrm{O} I \mathrm{I}])$ para $\mathrm{O}^{2+}$. Además, podemos ver que la abundancia del oxígeno una vez ionizado depende de la densidad electrónica.

La estimación de las temperaturas electrónicas del [OII] y del [OIII] permite una estimación más confiable de las abundancias totales del oxígeno. Dado que para las temperaturas típicas de las galaxias $\mathrm{HII}$ la mayor parte del oxígeno se encuentra en forma de $\mathrm{O}^{+}$ y $\mathrm{O}^{2+}$, es válido derivar las abudancias totales de oxígeno utilizando la aproximación:

$$
\frac{\mathrm{O}}{\mathrm{H}}=\frac{\mathrm{O}^{+}+\mathrm{O}^{2+}}{\mathrm{H}^{+}}
$$

- Azufre:

Para derivar la abundancia iónica del azufre una vez ionizado respecto al hidrógeno ionizado utilizaremos la temperatura electrónica $\mathrm{T}_{e}([\mathrm{SiI}])$ y los flujos de las líneas de emisión de [SII] $\lambda \lambda 6717,6731$ A(Hägele et al., 2008):

$12+\log \left(\mathrm{S}^{+} / \mathrm{H}^{+}\right)=\log \frac{\mathrm{I}(6717)+\mathrm{I}(6731)}{\mathrm{I}(\mathrm{H} \beta)}+5.423+\frac{0.929}{\mathrm{~T}_{e}}-0.28 \log \left(\mathrm{T}_{e}\right)+0.0001 \cdot n_{e}$,

donde puede verse que la abundancia del azufre una vez ionizado depende de la temperatura y de la densidad electrónica de [Sir].

De la misma manera, $\mathrm{S}^{2+} / \mathrm{H}^{+}$se puede obtener por medio de la temperatura electrónica $\mathrm{T}_{e}([\mathrm{SIII}])$ junto con las intensidades de las líneas de emisión de [SIII] $] \lambda 9069 \AA, 9532 \AA$ en el infrarrojo cercano:

$$
12+\log \left(\mathrm{S}^{2+} / \mathrm{H}^{+}\right)=\log \frac{\mathrm{I}(9069)+\mathrm{I}(9532)}{\mathrm{I}(\mathrm{H} \beta)}+5.8+\frac{0.771}{\mathrm{~T}_{e}}-0.22 \log \left(\mathrm{T}_{e}\right) .
$$

A diferencia de los elementos en los que la contribución a las abundancias totales de iones en estados mayores a dos veces ionizados resulta despreciable (del orden del $1 \%$, ver discusión en Firpo, 2011), en el azufre las contribuciones del [SIV] pueden resultar importantes, dependiendo de la temperatura de la nebulosa. Es por esto que no resulta conveniente utilizar la relación mencionada anteriormente para el caso del oxígeno, que estimaba la abundancia total como la suma de las dos abundancias iónicas observadas en nuestro rango espectral, sino que para el cálculo de la abundancia total del azufre se propone un factor de corrección por ionización (ICF, de la sigla en inglés: ionization correction factor) que tiene en cuenta la suma $\mathrm{S}^{+}+\mathrm{S}^{2+}$ de las abundancias iónicas ya estimadas para este elemento. Siguiendo la formulación publicada por Barker (1980), basada en los modelos de fotoionización de Stasińska (1978):

$$
\operatorname{ICF}\left(\mathrm{S}^{+}+\mathrm{S}^{2+}\right)=1-\left[1-\frac{\mathrm{O}^{+}}{\mathrm{O}^{+}+\mathrm{O}^{2+}}\right]^{\alpha-1 / \alpha},
$$

donde la expresión está en términos del cociente $\mathrm{O}^{+} /\left(\mathrm{O}^{+}+\mathrm{O}^{2+}\right)$, y $\alpha=2.5$ da el mejor ajuste a los pocos datos observacionales existentes de $\mathrm{S}^{3+}$ (Pérez-Montero et al., 2006). Aunque se suele utilizar la expresión de la fórmula de Barker como función de las fracciones iónicas $\mathrm{O}^{+} /\left(\mathrm{O}^{+}+\mathrm{O}^{2+}\right)$, es conveniente reescribirla en términos de $\mathrm{O}^{2+} /\left(\mathrm{O}^{+}+\mathrm{O}^{2+}\right)$ ya que los errores asociados a $\mathrm{O}^{2+}$ son considerablemente menores que los errores asociados a $\mathrm{O}^{+}$(Hägele et al., 2008). Luego, el ICF de $\mathrm{S}^{+}+\mathrm{S}^{2+}$ es: 


$$
\operatorname{ICF}\left(\mathrm{S}^{+}+\mathrm{S}^{2+}\right)=\left[1-\left(\frac{\mathrm{O}^{2+}}{\mathrm{O}^{+}+\mathrm{O}^{2+}}\right)^{2.5}\right]^{-0.4} .
$$

Ver también discusiń en Dors et al. (2016).

- Nitrógeno:

La abundancia iónica de nitrógeno $\mathrm{N}^{+} / \mathrm{H}^{+}$ha sido derivada de las intensidades de las líneas $[\mathrm{NII}] \lambda \lambda 6548,6584 \AA$ y la temperatura electrónica de [NII] se estima suponiendo que $\mathrm{T}_{e}([\mathrm{NII}])=\mathrm{T}_{e}([\mathrm{OII}])$ :

$$
12+\log \left(\mathrm{N}^{+} / \mathrm{H}^{+}\right)=\log \frac{\mathrm{I}(6548)+\mathrm{I}(6584)}{\mathrm{I}(\mathrm{H} \beta)}+6.273+\frac{1.558}{\mathrm{~T}_{e}}-0.504 \cdot \log \left(\mathrm{T}_{e}\right) .
$$

Luego, se deriva la abundancia total del nitrógeno utilizando la relación $\mathrm{N} / \mathrm{O}=\mathrm{N}^{+} / \mathrm{O}^{+}$ propuesta por Pagel (1992) y el cociente de N/H se determina utilizando la ecuación:

$$
\log \frac{\mathrm{N}}{\mathrm{H}}=\log \frac{N}{O}+\log \frac{O}{H} .
$$

- Neón:

El Ne se detecta en el extremo azul del espectro en las líneas [NeIII] $\lambda 3868 \AA$ y $\lambda 3968 \AA$ siendo la primera de ellas la más intensa y la más importante a la hora de trabajar. Para este ión asumimos que como las líneas del [NeIII] se forman en la zona de alta ionización la temperatura del OIII es representativa.

Clásicamente, la abudancia total de Ne se ha calculado asumiendo la relación dada por Peimbert \& Costero (1969):

$$
\frac{\mathrm{Ne}}{\mathrm{O}}=\frac{\mathrm{Ne}^{2+}}{\mathrm{O}^{2+}}
$$

Izotov et al. (2006a) notaron que esta suposición puede producir una sobreestimación del cociente $\mathrm{Ne} / \mathrm{O}$ para objetos con baja excitación, donde la transferencia de carga entre el $\mathrm{O}^{2+}$ y Hi comienza a ser importante. El ICF para el Ne puede ser calculado de acuerdo con la expresión dada por Pérez-Montero et al. (2007):

$$
\mathrm{ICF}\left(\mathrm{Ne}^{2+}\right)=0.142 \cdot x+0.753+\frac{0.171}{\mathrm{x}}
$$

donde $\mathrm{x}=\frac{\mathrm{O}^{2+}}{\left(\mathrm{O}^{+}+\mathrm{O}^{2+}\right)}$. Esta expresión ha sido derivada a partir de grillas de modelos de fotoionización construidas utilizando el código Cloudy (Ferland et al., 2008), tomando como fuentes de ionización las distribuciones espectrales de energía de estrellas O y B modeladas por Pauldrach et al. (2001). Ver también discusión en Dors et al. (2013).

- Argón:

En las regiones ionizadas podemos encontrar líneas de $\mathrm{Ar}^{2+}$ y $\mathrm{Ar}^{3+}$. La abundancia correspondiente al $\mathrm{Ar}^{2+}$ se puede calcular a partir de la medición de la línea de emisión $[$ ArIII $] \lambda 7136 \AA$, asumiendo que $\mathrm{T}_{e}([\mathrm{ArIII}]) \approx \mathrm{T}_{e}([\mathrm{SIII}])$ (Garnett, 1992b,a). Utilizando la expresión dada por Hägele et al. (2008), se estima: 


$$
12+\log \left(A r^{2+} / H^{+}\right)=\log \left(\frac{I(7137)}{I(H \beta)}\right)+6.157+\frac{0.808}{T_{e}}-0.508 \cdot \log \left(T_{e}\right) .
$$

Mientras que la abundancia iónica de $\mathrm{Ar}^{3+}$ se estima a partir de la línea de emisión de $[$ ArIv $] \lambda 4740 \AA$, bajo la suposición de que $\mathrm{T}_{e}([\operatorname{ArIv}]) \approx \mathrm{T}_{e}([\mathrm{OIII}])$ y utilizando la expresión dada por Hägele y colaboradores:

$$
12+\log \left(\mathrm{Ar}^{3+} / \mathrm{H}^{+}\right)=\log \left(\frac{I(4740)}{I(H \beta)}+5.705+\frac{1.246}{\mathrm{~T}_{e}}-0.156 \cdot \log \left(\mathrm{T}_{e}\right)\right.
$$

La abundancia total de Ar es la única que se calcula utilizando dos ICFs diferentes dependiendo de las abundancias iónicas previamente obtenidas. En ambos casos utilizaremos las ecuaciones derivadas por Pérez-Montero et al. (2007) de manera similar a la ecuación del ICF del Ne que utilizamos anteriormente. Solamente pudimos estimar la abundancia iónica de $\mathrm{Ar}^{2+}$, por lo que utilizamos la expresión del ICF dada por:

$$
\operatorname{ICF}\left(\operatorname{Ar}^{2+}\right)=0.507 \cdot(1-\mathrm{x})+0.749+\frac{0.064}{(1-\mathrm{x})}
$$

En el caso que podamos determinar ambas abundancias iónicas, $\mathrm{Ar}^{2+}$ y $\mathrm{Ar}^{3+}$, el ICF estará dado por:

$$
\mathrm{ICF}\left(\mathrm{Ar}^{2+}+\mathrm{Ar}^{3+}\right)=0.364 \cdot(1-\mathrm{x})+0.928+\frac{0.006}{(1-\mathrm{x})},
$$

donde para ambas ecuaciones: $\mathrm{x}=\mathrm{O}^{2+} /\left(\mathrm{O}^{+}+\mathrm{O}^{2+}\right)$.

En la Tabla 4.2 resumimos las ecuaciones utilizadas para el estudio de las abundancias iónicas, de los metales mencionados anteriormente.

Para nuestras regiones hemos podido determinar las abundancias químicas iónicas y totales del O, S, N, Ar y Ne, salvo en el caso del Nudo C de II Zw 33 para el cual la determinación de las abundancias de varios elementos químicos se vio afectada por la baja relación S/N y la imposibilidad de medir las líneas más importantes. Para los demás nudos correspondientes a Mrk 600 hemos podido medir con éxito abundancias iónicas y totales del O, S, N, Ne y Ar. Los resultados para cada una de nuestras regiones, sus factores de corrección de ionización (ICFs) y las abudancias totales con sus respectivos errores, se presentan en las Tablas 4.4, 4.6, 4.8, 4.10 y 4.12, para los nudos de II Zw 33 y Mrk600, respectivamente.

\subsection{Discusión de las observaciones Echelle}

En esta subsección se analizan los resultados obtenidos para las regiones estudiadas con lo datos Echelle de alta resolución espectral. Se presentan cada uno de los brotes por separado haciendo una descripción de sus componentes cinemáticas y de la solución global, indicando las propiedades físicas estudiadas como la temperatura y densidades electrónicas, las abundancias iónicas y totales. Luego, se presenta el análisis comparativo entre los brotes con los aspectos más relevantes de cada uno de ellos. Los flujos de las líneas de emisión de los nudos observados para ambas galaxias se listan en la Tablas 4.3, 4.5, 4.7, 4.9 y 4.11. En dichas Tablas se indican las línea de emisión corregidas por enrojecimiento, para cada componente cinemática y para el perfil global, junto a la constante de enrojecimiento y su error estimado del ajuste de mínimos cuadrados, y la intensidad de $\mathrm{H} \beta$ corregida por enrojecimiento. La 
Tabla 4.2. Ecuaciones iónicas para las diferentes especies químicas

\begin{tabular}{c|l}
\hline Iones $*$ & Expresiones \\
\hline $\mathrm{O}^{+}$ & $\log \frac{\mathrm{I}(3727)}{\mathrm{I}(\mathrm{H} \beta)}+5.992+\frac{1.583}{\mathrm{~T}_{e}}-0.681 \cdot \log \left(\mathrm{T}_{e}\right)+\log \left(1+2.3 \cdot n_{e}\right)$ \\
$\mathrm{O}^{2+}$ & $\log \frac{\mathrm{I}(4959)+\mathrm{I}(5007)}{\mathrm{I}(\mathrm{H} \beta)}+6.144+\frac{1.251}{\mathrm{~T}_{e}}-0.55 \cdot \log \left(\mathrm{T}_{e}\right)$ \\
$\mathrm{S}^{+}$ & $\log \frac{\mathrm{I}(6717)+\mathrm{I}(6731)}{\mathrm{I}(\mathrm{H} \beta)}+5.423+\frac{0.929}{\mathrm{~T}_{e}}-0.28 \cdot \log \left(\mathrm{T}_{e}\right)+n_{e}$ \\
$\mathrm{~S}^{2+}$ & $\log \frac{\mathrm{I}(9069)+\mathrm{I}(9532)}{\mathrm{I}(\mathrm{H} \beta)}+5.8+\frac{0.771}{\mathrm{~T}_{e}}-0.22 \cdot \log \left(\mathrm{T}_{e}\right)$ \\
$\mathrm{N}^{+}$ & $\log \frac{\mathrm{I}(6548)+\mathrm{I}(6584)}{\mathrm{I}(\mathrm{H} \beta)}+6.273+\frac{1.558}{\mathrm{~T}_{e}}-0.504 \cdot \log \left(\mathrm{T}_{e}\right)$ \\
$\mathrm{Ne}^{2+}$ & $\log \frac{\mathrm{I}(3868)}{\mathrm{I}(\mathrm{H} \beta)}+6.486+\frac{1.558}{\mathrm{~T}_{e}}-0.504 \cdot \log \left(\mathrm{T}_{e}\right)$ \\
$\mathrm{Ar}^{2+}$ & $\log \frac{\mathrm{I}(7137)}{\mathrm{I}(\mathrm{H} \beta)}+6.157+\frac{0.808}{\mathrm{~T}_{e}}-0.508 \cdot \log \left(\mathrm{T}_{e}\right)$ \\
$\mathrm{Ar}^{3+}$ & $\log \frac{\mathrm{I}(4740)}{\mathrm{I}(\mathrm{H} \beta)}+5.705+\frac{1.246}{\mathrm{~T}_{e}}-0.156 \cdot \log \left(\mathrm{T}_{e}\right)$ \\
\hline
\end{tabular}

donde $n_{e}$ corresponde a la densidad en $\mathrm{cm}^{-3}$ y $\mathrm{T}_{e}$ a la temperatura en $10^{4} \mathrm{k}$ * $12+\log \left(\mathrm{X} / \mathrm{H}^{+}\right)$

primer columna lista la longitud de onda y la identificación de las líneas medidas. La curva de enrojecimiento adoptada, $\mathrm{f}(\lambda)$, normalizada a $\mathrm{H} \beta$, se da en la columna dos. Las siguientes columnas muestran las intensidades de las líneas corregidas en enrojecimiento en relación con $\mathrm{H} \beta$ y sus errores correspondientes obtenidos como la propagación cuadrática, los errores de observación en los flujos de la línea de emisión y las incertidumbres de las constantes de enrojecimiento para cada componentes, respectivamente. Se ha adoptado en este trabajo no tener en cuenta los errores en las intensidades teóricas ya que son mucho más pequeños que los observacionales.

\section{- II Zw 33 Nudo A}

Para esta región se determinó un ajuste compuesto de tres gaussianas: dos componentes angostas (N1 y N2) y una componente ancha (B). Fue posible medir la línea auroral del [OIII] únicamente, mientras que las líneas débiles del [SIII] y de doblete del [OII] $\lambda \lambda 7319 \AA, 7330 \AA$ son líneas de baja $\mathrm{S} / \mathrm{N}$ que han sido ajustadas con grandes incertidumbres, por lo cual que se decidió no considerarlas en el análisis. Las temperaturas para las componentes N1 y N2 fueron determinadas a partir de las medidas directas de $5007 \AA$ y $4363 \AA$.

Por el lado de la componente ancha, los ajustes presentaban errores $>40 \%$, de manera tal que no la hemos considerado. Para esta componente tomamos la temperatura típica para estas regiones de $10^{4} \mathrm{k}$. Las temperaturas fueron estimadas siguiendo el procedimiento descripto en la Sección 4.1.2, a través de relaciones empíricas entre temperaturas o modelos cuando fuera necesario, donde pudimos determinar las temperturas de los iones [OII], [SII] y [NII].

Para las subcomponentes cinemáticas como para la componente global la densidad pudo ser analizada a través de las líneas del [SII] y estos valores se encuentran por debajo del límite de la densidad crítica por desexcitación colisional. En la medida global obtuvimos 
una densidad de 31 part $\mathrm{cm}^{-3}$. Para la componente ancha determinamos valores en el

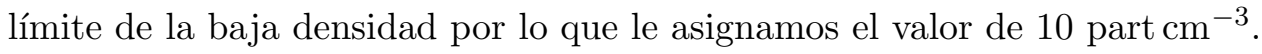

Por el lado de las componentes angostas, para ambas componente N1 y N2 se han

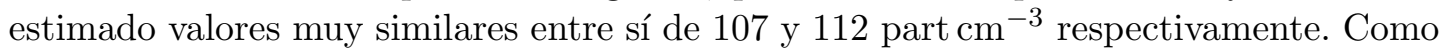
los errores de la N1 son muy grandes (mayores que el valor estimado de $\mathrm{n}_{e}$ ) se colocan ":" para indicar grandes errores. Hay que tener en cuenta que estos valores son estimaciones de la magnitud real, que presentan sus errores, y que en estas calibraciones todos los valores se ubican muy por debajo de los límites de densidades críticas, por lo que podemos afirmar con seguridad que todas las componentes corresponden a regiones de baja densidad. Los datos correspondientes a dichas densidades y temperaturas, así como también a las abundancias químicas iónicas y totales y los ICFs se presentan en la Tabla 4.4 .

Hemos calculado el valor de $\mathrm{He}^{+} / \mathrm{H}^{+}$a través de las líneas intensas de $4471 \AA$, $5876 \AA$, $6678 \AA$ y $7065 \AA$. En el perfil global obtuvimos $0.078 \pm 0.003$, pesado con los errores. Para las componentes N1 y N2 también se han utilizado dichas líneas pero la componente ancha únicamente la pudimos ajustar en $4471 \AA$ A. En comparación a los valores típicos que se estiman en Galaxias Hiı o RHir que fueron calculados por Hägele et al. (2008) encontramos un gran acuerdo. Para N1 estimamos un valor de: $0.075 \pm 0.031$, para N2 $=0.092 \pm 0.011$ y para la Global $=0.070 \pm 0.025$. Además, si tomamos el promedio del valor "adoptado" para las tres componentes indicadas, vemos que es aproximadamente el valor esperado de la medida global.

Los valores de las abundancias totales de oxígeno, el elemento más abundante en este tipo de regiones después del hidrógeno y el helio, presenta valores acordes a los determinados en la literatura para Regiones HII, donde en comparación con la abundancia solar (Allende Prieto et al., 2001, $12+\log (\mathrm{O} / \mathrm{H}) \odot=8.69)$ la medida global presenta un valor de $8.28 \pm 0.08,2.57$ veces menor que el valor solar. Para las distintas componentes cinemáticas obtuvimos abundancias totales de oxígeno de: $8.28 \pm 0.10$, para la N1, lo que corresponde a 2.57 veces menor que el valor solar; $8.28 \pm 0.09$ para la N2, igual a $\mathrm{N} 1$, y la ancha con $8.26 \pm 0.05$ representa 2.69 veces menor que la solar. Los valores determinados para las distintas componentes cinemáticas tienen un excelente acuerdo si tenemos en cuenta los errores, dado que la componente ancha es 0.02 dex menor que las componentes angostas. Los valores característicos de $12+\log (\mathrm{O} / \mathrm{H})$ que se encuentran en la literatura (Terlevich et al., 1991; Hägele et al., 2008; Amorín et al., 2009 , entre otros) abarcar el rango de $7.8<12+\log (\mathrm{O} / \mathrm{H})<8.3$. Por lo tanto, los valores calculados para nuestra región están en acuerdo con las abundancias para regiones de esta naturaleza.

Las abundancias estimadas para el azufre no resultan confiables, se alejan mucho de la media obtenida en la literatura para este tipo de objetos llegando a obtener abundancias mayores que para el oxígeno en varios casos. Esto puede estar emparentado con un problema en la $\mathrm{S} / \mathrm{N}$ de las líneas involucradas o por un problema mismo de la reducción de los datos hacia los órdenes en los extremos detector. Como los valores de las temperaturas, estimados a través de relaciones empíricas a partir de la temperatura electrónica del Oxígeno nos indican valores coherentes, estamos en presencia de un problema en la determinación de las abundancias. Por esto es que no seguiremos discutiendo estos valoresPara la abundancia total del nitrógeno, determinamos que los valores van de 6.27 a $6.42 \mathrm{dex}$, siendo estos rango esperables y en acuerdo con galaxias His si tenemos en cuenta la abundancia solar de nitrógeno (Holweger, 2001, 12+log $(\mathrm{N} / \mathrm{H}) \odot=7.93$ dex).

La calidad de los datos nos permitió ajustar múltiples componentes en las líneas débiles 
del [ArIII] y del [NeIII], por lo que pudimos estimar abundancias para ambos elementos. Para las abundancias iónicas del $\mathrm{Ar}^{2+} / \mathrm{H}^{+}$encontramos un excelente acuerdo entre las componentes y estas se encuentran dentro de los valores esperados para estas regiones. Por la parte del [NeIII] estimamos una abundancia total, teniendo en cuenta los $\mathrm{ICF}\left(\mathrm{Ne}^{+2}\right)$ de $7.95 \pm 0.4$ para la componente ancha, siendo la que presenta una mayor abundancia entre las componentes, pero con valores típicos dentro de los esperados. La componente N1 toma el menor valor de $7.15 \pm 0.33$, mientras que para la N2 y para la global son $7.57 \pm 0.08$ y $7.70 \pm 0.07$, respectivamente. En la Tabla 4.4 se presentan todos los valores determinados para las abundancias iónicas y totales de los elementos químicos medidos.

Cabe aclarar que las extinciones determinadas para las tres componentes cinemáticas tienen baja extinción, con $\mathrm{c}(\mathrm{H} \beta)$ de $0.02 \pm 0.01$ y $0.03 \pm 0.01$, pero para la componente $\mathrm{N} 1$ se detecta una extinción mayor $\mathrm{c}(\mathrm{H} \beta) \approx 0.48 \pm 0.02$, siendo esta una componente con mayor extinción y contribución del polvo.

Respecto a las abundancias del azufre relativas al oxígeno (S/O) encontramos valores elevados de azufre que se alejan considerablemente de lo esperado en todas las componentes salvo en una. Como mencionamos anteriormente, esto puede deberse a un error en los datos y los resultados obtenidos pueden no ser confiables. Para los demás cociente relativos al oxígeno analizados: $\mathrm{N} / \mathrm{O}, \mathrm{Ne} / \mathrm{O}$ y $\mathrm{Ar} / \mathrm{O}$ encontramos valores esperables para estas regiones y acordes con los publicados en la literatura (ver discusiones en: Amorín et al., 2010; Pérez-Montero et al., 2011b; Hägele et al., 2012).

En las Figuras 4.3, 4.4, 4.5 y 4.6 graficamos los cocientes para cada componente. Como puede apreciarse los cocientes presentan un acuerdo dentro de los errores. Si las diferencias no son muy evidentes (salvo para las componentes en el azufre, asociado a la impresición en la determinación de la temperatura electrónica), no se puede asegurar ni encontrar ninguna evidencia de evoluciones químicas diferentes entre componentes, sino que pequeñas diferencias entre ellos puede indicar que estos son distintas fases de un mismo gas. 
Tabla 4.3. Intensidad de las líneas corregidas por enrojecimiento para el Nudo A de II Zw 33 $[\mathrm{F}(\mathrm{H} \beta)=\mathrm{I}(\mathrm{H} \beta)=10.000]$.

\begin{tabular}{|c|c|c|c|c|c|c|c|c|c|}
\hline \multirow[b]{2}{*}{$\lambda(\AA)$} & \multicolumn{3}{|c|}{ Ancha } & \multicolumn{2}{|l|}{ N1 } & \multicolumn{2}{|l|}{$\mathrm{N} 2$} & \multicolumn{2}{|l|}{ Global } \\
\hline & $f(\lambda)$ & $I(\lambda)$ & $\begin{array}{c}\text { Error } \\
(\%)\end{array}$ & $I(\lambda)$ & $\begin{array}{c}\text { Error } \\
(\%)\end{array}$ & $I(\lambda)$ & $\begin{array}{c}\text { Error } \\
(\%)\end{array}$ & $\begin{array}{l}I(\lambda) \\
(\%)\end{array}$ & Error \\
\hline 3726 [OII] & 0.272 & $15749 \pm 1325$ & 8.41 & $16659 \pm 714$ & 4.29 & $7058 \pm 113$ & 1.60 & $10731 \pm 415$ & 3.87 \\
\hline $3727[\mathrm{OII}]^{b}$ & 0.271 & $39271 \pm 1476$ & 3.76 & $37952 \pm 1392$ & 3.67 & $16091 \pm 456$ & 2.83 & $25556 \pm 700$ & 2.74 \\
\hline 3729 [OII] & 0.271 & $23522 \pm 1854$ & 7.88 & $21286 \pm 988$ & 4.64 & $9032 \pm 326$ & 3.61 & $14789 \pm 578$ & 3.91 \\
\hline 3868 [NeIII] & 0.238 & $7206 \pm 684$ & 9.49 & $1805 \pm 468$ & 25.93 & $5437 \pm 163$ & 2.99 & $5204 \pm 228$ & 4.38 \\
\hline $4102 \mathrm{H} \delta$ & 0.188 & $4089 \pm 270$ & 6.60 & $3384 \pm 195$ & 5.78 & $3005 \pm 71$ & 2.37 & $3303 \pm 92$ & 2.79 \\
\hline $4340 \mathrm{H} \gamma$ & 0.142 & $6721 \pm 292$ & 4.34 & $6030 \pm 236$ & 3.91 & $5004 \pm 87$ & 1.75 & $5553 \pm 104$ & 1.87 \\
\hline 4363 [OIII] & 0.138 & - & - & $437 \pm 101$ & 23.20 & $608 \pm 38$ & 6.20 & $391 \pm 26$ & 6.72 \\
\hline $4471 \mathrm{HeI}$ & 0.106 & $421 \pm 149$ & 35.6 & $116 \pm 43$ & 37.21 & $448 \pm 43$ & 9.60 & $374 \pm 63$ & 16.91 \\
\hline $4861 \mathrm{H} \beta$ & 0.000 & $10000 \pm 366$ & 3.66 & $10000 \pm 241$ & 2.41 & $10000 \pm 106$ & 1.06 & $10000 \pm 127$ & 1.27 \\
\hline 4959 [Öні] & -0.024 & $7692 \pm 1389$ & 18.05 & $16541 \pm 940$ & 5.68 & $19416 \pm 387$ & 1.99 & $15615 \pm 482$ & 3.09 \\
\hline 5007 [ÖII] & -0.035 & $36923 \pm 7288$ & 19.74 & $43592 \pm 2704$ & 6.20 & $58058 \pm 10406$ & 17.92 & $52004 \pm 7974$ & 15.33 \\
\hline $5876 \mathrm{HeI}$ & -0.209 & - & - & $1318 \pm 333$ & 25.29 & $1427 \pm 279$ & 19.54 & $1082 \pm 219$ & 20.24 \\
\hline $6300[\mathrm{OI}]$ & -0.276 & $510 \pm 196$ & 38.47 & $252 \pm 59$ & 23.50 & $311 \pm 34$ & 10.80 & $369 \pm 60$ & 16.17 \\
\hline $6548[\mathrm{NII}]$ & -0.311 & $943 \pm 146$ & 15.45 & $417 \pm 53$ & 12.74 & $319 \pm 39$ & 12.37 & $457 \pm 40$ & 8.73 \\
\hline $6563 \mathrm{H} \alpha$ & -0.313 & $27490 \pm 397$ & 1.44 & $32095 \pm 232$ & 0.72 & $29704 \pm 137$ & 0.46 & $31674 \pm 146$ & 0.46 \\
\hline $6584[\mathrm{NII}]$ & -0.316 & $1781 \pm 205$ & 11.53 & $1527 \pm 84$ & 5.51 & $1346 \pm 57$ & 4.23 & $1414 \pm 66$ & 4.70 \\
\hline $6678 \mathrm{HeI}$ & -0.329 & - & - & $353 \pm 99$ & 27.96 & $378 \pm 57$ & 14.96 & $295 \pm 74$ & 25.19 \\
\hline 6717 [SII] & -0.334 & $3858 \pm 275$ & 7.12 & $1277 \pm 104$ & 8.13 & $943 \pm 62$ & 6.54 & $1954 \pm 88$ & 4.50 \\
\hline 6731 [SII] & -0.336 & $2008 \pm 285$ & 14.21 & $932 \pm 94$ & 10.08 & $842 \pm 64$ & 7.57 & $1333 \pm 91$ & 6.80 \\
\hline 7065 HeI & -0.377 & - & - & $238 \pm 59$ & 24.60 & $221 \pm 35$ & 15.77 & $194 \pm 52$ & 26.82 \\
\hline 7136 [ArIII] & -0.385 & $1186 \pm 242$ & 20.40 & $813 \pm 80$ & 9.89 & $649 \pm 51$ & 7.90 & $900 \pm 76$ & 8.42 \\
\hline 9069 [SIII] & -0.561 & $3101 \pm 644$ & 20.78 & $364 \pm 78$ & 21.44 & $1633 \pm 190$ & 11.64 & $1809 \pm 217$ & 12.01 \\
\hline $\mathrm{I}(\mathrm{H} \beta)\left(\mathrm{erg} \mathrm{seg}^{-1} \mathrm{~cm}^{-2}\right)$ & & $0.25 \times 10^{-}$ & & $0.17 \times 10^{-}$ & & $0.45 \times 10^{-}$ & & $0.86 \times 10^{-}$ & \\
\hline $\mathrm{c}(\mathrm{H} \beta)$ & & 0.00 & & $0.48 \pm 0$. & & $0.02 \pm 0$. & & $0.03 \pm 0$. & \\
\hline$-\mathrm{EW}(\mathrm{H} \beta)$ & & 8.49 & & 4.95 & & 4.25 & & 17.69 & \\
\hline
\end{tabular}




\section{- II Zw 33 Brote B}

En esta región se ajustaron tres gaussianas para representar el perfil global, fue posible medir las líneas aurorales del [OIII], [SIII], en $4363 \AA$ y $6312 \AA$, respectivamente. Al igual que en el caso del Nudo A, las líneas del doblete del [OII] en los $7225 \AA$ son muy débiles y no fue posible realizar un ajuste confiable para sus componentes. La medida global de la línea tampoco fue estimada por los errores mayores al $40 \%$. Las temperaturas para las componentes $\mathrm{N} 1$ y N2 fueron determinadas a partir de las líneas del [OIII], mientras que la componente ancha no pudo ser ajustada en las líneas aurorales, por lo que se utilizó la temperatura de modelo de $10^{4} \mathrm{k}$, y el procedimiento descripto anteriormente se pudieron determinar a través de relaciones teóricas las temperturas del [NII], [SII] y [OII]. Las densidades de las componentes y la global fueron estimadas a través de las líneas del [SII] obteniendo valores de densidad muy por debajo de la densidad crítica por desexcitación colisional. Para la medida global obtuvimos una densidad de $69 \mathrm{part}^{-3}$, para las

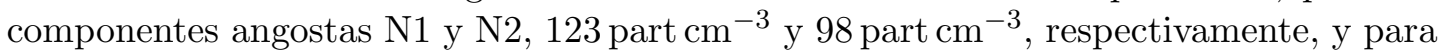
la ancha 75 part $\mathrm{cm}^{-3}$, indicando que todas las componentes presentan densidades bajas y típicas de las galaxias Hir. Para el HeI hemos calculado el valor de $\mathrm{He}^{+} / \mathrm{H}^{+}$a través de las líneas en $4471 \AA$, $5876 \AA$, $6678 \AA$ y $7065 \AA$, donde además resulta interesante indicar que en cada línea se pudieron ajustar todas las componentes cinemáticas determinadas en la solución de la línea intensa del [OIII]. Para el perfil global obtuvimos $0.083 \pm 0.006$, para las componentes N1, N2 $0.075 \pm 0.013$ y $0.062 \pm 0.022$, y la ancha, quien es la que presenta la mayor abundancia entre las componentes, $0.107 \pm 0.045$, también es la que presenta el error mayor en la medida. Los valores típicos que adoptan las galaxias Hir fueron discutidos en, por ejemplo, el trabajo realizado por Hägele et al. (2008) y en comparación a estos encontramos gran acuerdo con los estimados en este trabajo para este tipo de regiones.

Los valores de las abundancias totales de oxígeno presentan valores acordes a los determinados en la literatura para galaxias HII, en donde si se compara con la abundancia solar $(12+\log (\mathrm{O} / \mathrm{H}) \odot=8.69)$ nuestra medida global es de $8.43 \pm 0.06,1.82$ veces menor que el valor solar, coicidiendo dentro de los errores $( \pm 3 \sigma)$ con las componentes $\mathrm{N} 1 \mathrm{y}$ N2: $8.21 \pm 0.09$ (3.02 veces menor que la abundancia solar) y $8.13 \pm 0.06$ (3.63 veces menor que la abundancia solar), respectivamente. Para la componente ancha una posible explicación podría ser que debido a calculamos las temperaturas a través de modelos, el valor estimado de la abundancia presenta un valor y una incerteza mayor, y podría ser por esto que el valor se aleja levemente hacia un valor de abundancias mayores de $8.51 \pm 0.03$ (1.59 veces menor que la abundancia solar). Comparando estos valores totales de oxígeno calculado con los típicos de Galaxias Hir propuestos por Terlevich et al. (1991); Hägele et al. (2008); Amorín et al. (2009), encontramos un excelente acuerdo para nuestras componentes, con excepción de la componente ancha que presenta valores levemente mayores respecto a las demás.

Para el azufre hemos estimado que todas las componentes presentan abundancias menores que la solar $(12+\log (\mathrm{S} / \mathrm{H}) \odot=7.33$ dex, según Grevesse \& Sauval, 1998) (N1: $6.21 \pm 0.24 \mathrm{dex}, \mathrm{N} 2: 6.39 \pm 0.37 \mathrm{dex}$ y la componente global: $6.52 \pm 0.39 \mathrm{dex})$ aunque el valor obtenido para la componente ancha se aleja un poco hacia un valor mayor en relación a las medidas de las otras componentes (B: $7.08 \pm 0.27 \mathrm{dex}$ ). La abundancia total del nitrógeno con respecto a los valor solares (Holweger, 2001, 12+log $(\mathrm{N} / \mathrm{H}) \odot=$ $7.93 \mathrm{dex})$ es considerablemente menor para todas las regiones $(6.63 \mathrm{dex} \leq 12+\log (\mathrm{N} / \mathrm{H})$ $\leq 7.07 \mathrm{dex}$ ), siendo estos valores esperados para galaxias Hir según indican los trabajos de Hägele et al. (2008); Amorín et al. (2009), aunque podemos ver un leve alejamiento a valores mayores en la componente ancha (7.07 dex). Al igual que en el Nudo A pudimos 
Tabla 4.4. Propiedades físicas (densidades electrónicas $\mathrm{n}_{e} \mathrm{y}$ temperaturas electrónicas $\mathrm{T}_{e}$ ) y abundancias químicas iónicas y totales de II Zw 33 A, derivadas de líneas prohibidas y las líneas de recombinación de helio.

\begin{tabular}{|c|c|c|c|c|}
\hline & \multicolumn{4}{|c|}{ II Zw 33 Nudo A } \\
\hline & Ancha & N1 & $\mathrm{N} 2$ & Global \\
\hline$n_{e}$ & 10: & 107: & $112 \pm 30$ & 31: \\
\hline $\mathrm{t}_{e}[\mathrm{OIII}]$ & $1.00^{b}$ & $1.14 \pm 0.16$ & $1.17 \pm 0.05$ & $1.06 \pm 0.03$ \\
\hline $\mathrm{t}_{e}[\mathrm{SIII}]$ & $0.87^{a}$ & $1.12^{a} \pm 0.24$ & $1.14^{a} \pm 0.04$ & $1.17^{a} \pm 0.02$ \\
\hline $\mathrm{t}_{e}[\mathrm{OII}]$ & $1.09^{a} \pm$ & $1.12^{a} \pm 0.07$ & $0.97^{a} \pm 0.01$ & $1.17^{a} \pm 0.03$ \\
\hline $\mathrm{t}_{e}[\mathrm{SII}]$ & $1.09^{a} \pm$ & $1.12^{a} \pm 0.07$ & $0.97^{a} \pm 0.01$ & $1.17^{a} \pm 0.03$ \\
\hline $\mathrm{t}_{e}[\mathrm{NII}]$ & $1.09^{a} \pm$ & $1.12^{a} \pm 0.07$ & $0.97^{a} \pm 0.01$ & $1.17^{a} \pm 0.03$ \\
\hline $12+\log \left(\mathrm{O}^{+} / \mathrm{H}^{+}\right)$ & $7.84 \pm 0.02$ & $7.97 \pm 0.08$ & $7.87 \pm 0.05$ & $7.71 \pm 0.04$ \\
\hline $12+\log \left(\mathrm{O}^{2+} / \mathrm{H}^{+}\right)$ & $8.04 \pm 0.08$ & $7.99 \pm 0.11$ & $8.07 \pm 0.12$ & $8.14 \pm 0.10$ \\
\hline $12+\log (\mathrm{O} / \mathrm{H})$ & $8.26 \pm 0.05$ & $8.28 \pm 0.10$ & $8.28 \pm 0.09$ & $8.28 \pm 0.08$ \\
\hline $12+\log \left(\mathrm{S}^{+} / \mathrm{H}^{+}\right)$ & 5.94 & 5.60 & 5.67 & 5.72 \\
\hline $12+\log \left(\mathrm{S}^{2+} / \mathrm{H}^{+}\right)$ & 8.58 & 7.29 & 7.88 & 8.18 \\
\hline $\mathrm{ICF}\left(\mathrm{S}^{+}+\mathrm{S}^{2+}\right)$ & 1.15 & 1.09 & 1.15 & 1.27 \\
\hline $12+\log (\mathrm{S} / \mathrm{H})$ & 8.64 & 7.34 & 7.94 & 8.28 \\
\hline $\log (\mathrm{S} / \mathrm{O})$ & 0.38 & -0.94 & -0.34 & 0.00 \\
\hline $12+\log \left(\mathrm{N}^{+} / \mathrm{H}^{+}\right)$ & 6.40 & 6.33 & 6.42 & 6.27 \\
\hline $12+\log (\mathrm{N} / \mathrm{H})$ & 6.81 & 6.33 & 6.42 & 6.84 \\
\hline $\log (\mathrm{N} / \mathrm{O})$ & -1.44 & -1.63 & -1.45 & -1.44 \\
\hline $12+\log \left(\mathrm{Ne}^{2+} / \mathrm{H}^{+}\right)$ & $7.90 \pm 0.04$ & $7.08 \pm 0.33$ & $7.52 \pm 0.08$ & $7.66 \pm 0.07$ \\
\hline $\mathrm{ICF}\left(\mathrm{Ne}^{2+}\right)$ & $1.12 \pm 0.01$ & $1.16 \pm 0.11$ & $1.12 \pm 0.01$ & $1.09 \pm 0.01$ \\
\hline $12+\log (\mathrm{Ne} / \mathrm{H})$ & $7.95 \pm 0.04$ & $7.15 \pm 0.33$ & $7.57 \pm 0.08$ & $7.70 \pm 0.07$ \\
\hline $\log (\mathrm{Ne} / \mathrm{O})$ & $-0.31 \pm 0.07$ & $-1.13 \pm 0.42$ & $-0.71 \pm 0.12$ & $-0.58 \pm 0.11$ \\
\hline $12+\log \left(\mathrm{Ar}^{2+} / \mathrm{H}^{+}\right)$ & 6.19 & 5.84 & 5.71 & 5.98 \\
\hline $12+\log \left(\mathrm{Ar}^{3+} / \mathrm{H}^{+}\right)$ & 9.99 & 9.99 & 9.99 & 9.99 \\
\hline $\mathrm{ICF}\left(\mathrm{Ar}^{2+}\right)$ & 1.11 & 1.13 & 1.11 & 1.12 \\
\hline $\mathrm{ICF}\left(\mathrm{Ar}^{2+}+\mathrm{Ar}^{3+}\right)$ & 9.99 & 9.99 & 9.99 & 9.99 \\
\hline $12+\log (\operatorname{Ar} / H)$ & 6.24 & 5.89 & 5.75 & 6.03 \\
\hline $\log (\operatorname{Ar} / 0)$ & $-2.02 \pm 0.25$ & $-2.39 \pm 0.22$ & $-2.53 \pm 0.19$ & $-2.25 \pm 0.21$ \\
\hline $\mathrm{He}^{+} / \mathrm{H}^{+}$ & & & & \\
\hline 4471 & $0.070 \pm 0.025$ & $0.023 \pm 0.010$ & $0.091 \pm 0.008$ & $0.076 \pm 0.012$ \\
\hline 5876 & - & $0.098 \pm 0.022$ & $0.105 \pm 0.020$ & $0.080 \pm 0.016$ \\
\hline 6678 & - & $0.093 \pm 0.023$ & $0.100 \pm 0.014$ & $0.077 \pm 0.019$ \\
\hline 7065 & - & $0.095 \pm 0.030$ & $0.077 \pm 0.015$ & $0.082 \pm 0.023$ \\
\hline adoptado & $0.070 \pm 0.025$ & $0.075 \pm 0.031$ & $0.092 \pm 0.011$ & $0.078 \pm 0.003$ \\
\hline
\end{tabular}

donde $n_{e}$ corresponde a la densidad en $\mathrm{cm}^{-3}$ y $\mathrm{T}_{e}$ a la temperatura en $10^{4} \mathrm{k}$,

${ }^{a}$ utilizando temperaturas de modelos $\mathrm{y}^{b}$ suponiendo una temperatura inicial de $10000 \mathrm{k}$, 
ajustar las múltiples componentes en las líneas débiles del [ArIII] y del [NeIII], y así estimamos abundancias iónicas para el $\mathrm{Ar}^{2+} / \mathrm{H}^{+}$de $5.52 \pm 0.17 \mathrm{dex}, 5.51 \pm 0.60 \mathrm{dex}$ y $5.71 \pm 0.23$ dex para $\mathrm{N} 1, \mathrm{~N} 2$ y la medida global, respectivamente, estando estos valores en total acuerdo dentro de los errores. Para la componente ancha estimamos valores levemente mayores de $6.13 \pm 0.23 \mathrm{dex}$, que si bien es mayor que el valor medio, al tener en cuenta los errores, estas componentes presentan un excelente acuerdo entre sí. Para el Neón pudimos ajustar las componentes y estimar sus correspondientes abundancias, obteniendo una muy buena relación entre las medidas según los valores esperados para este tipo de objetos $(7.8 \pm 0.1 \mathrm{dex})$, donde la componente $\mathrm{N} 2$ es la que presenta menor abundancia frente a las demás. Todos los valores correspondientes a los parámetros físicos mencionados se presentan en la Tabla 4.12.

Respecto a las abundancias totales de los principales elementos (S, N, Ne y Ar) relativas al oxígeno, determinamos que las componentes ancha se aleja un poco de los posibles esperados en la distribución con respecto a las demás componentes en el S/O y en el $\mathrm{Ar} / \mathrm{O}$. Esto se puede deber a un error en el dato y hay que analizar estos resultados con atención. Para las componentes angostas N1 y N2 los valores se corresponden con los esperados para este tipo de objetos y si tenemos en cuentra los errores para cada una de las medidas, los valores están en acuerdo entre sí. En las Figuras de las abundancias de elementos respecto al oxígeno (ver las Figuras 4.3, 4.4, 4.5 y 4.6) presentamos los cocientes de nuestro análisis y las comparamos con las muestras publicadas por Amorín et al. (2010); Pérez-Montero et al. (2011b); Hägele et al. (2012) donde se puede observar que en general dichos cocientes estimados se hayan dentro de los valores esperados.

Las extinciones determinadas a partir de las líneas más importantes de recombinación del Hidrógeno son bajas, siendo mayor para la componente N1 (0.31 \pm 0.02$)$ y la medida global $(0.18 \pm 0.02)$ y las componente ancha y N2 restantes, son consistente con la baja o nula extinción. 
Tabla 4.5. Intensidad de las líneas corregidas por enrojecimiento para el Nudo B de II Zw 33 $[\mathrm{F}(\mathrm{H} \beta)=\mathrm{I}(\mathrm{H} \beta)=10.000]$.

\begin{tabular}{|c|c|c|c|c|c|c|c|c|c|}
\hline \multirow[b]{2}{*}{$\lambda(\AA)$} & \multicolumn{3}{|c|}{ Ancha } & \multicolumn{2}{|l|}{ N1 } & \multicolumn{2}{|l|}{$\mathrm{N} 2$} & \multicolumn{2}{|c|}{ Global } \\
\hline & $f(\lambda)$ & $I(\lambda)$ & $\begin{array}{l}\text { Error } \\
(\%)\end{array}$ & $I(\lambda)$ & $\begin{array}{c}\text { Error } \\
(\%)\end{array}$ & $I(\lambda)$ & $\begin{array}{c}\text { Error } \\
(\%)\end{array}$ & $\begin{array}{l}I(\lambda) \\
(\%)\end{array}$ & Error \\
\hline 3726 [OII] & 0.272 & $22840 \pm 1463$ & 6.41 & $11852 \pm 571$ & 4.82 & $8120 \pm 938$ & 11.55 & $15183 \pm 587$ & 3.87 \\
\hline $3727[\mathrm{OII}]^{b}$ & 0.271 & $55642 \pm 1201$ & 2.16 & $30353 \pm 652$ & 2.15 & $17210 \pm 1439$ & 8.36 & $37360 \pm 652$ & 1.74 \\
\hline 3729 [OІІ] & 0.271 & $32724 \pm 1485$ & 4.54 & $18570 \pm 616$ & 3.32 & $9055 \pm 1017$ & 11.23 & $22174 \pm 600$ & 2.70 \\
\hline 3868 [NeIII] & 0.238 & $5525 \pm 429$ & 7.77 & $3437 \pm 329$ & 9.57 & $1144 \pm 518$ & 45.33 & $3854 \pm 237$ & 6.15 \\
\hline $4102 \mathrm{H} \delta$ & 0.188 & $3490 \pm 257$ & 7.37 & $3543 \pm 152$ & 4.28 & $2232 \pm 365$ & 16.36 & $3380 \pm 126$ & 3.74 \\
\hline $4340 \mathrm{H} \gamma$ & 0.142 & $5798 \pm 325$ & 5.60 & $6126 \pm 199$ & 3.24 & $3257 \pm 508$ & 15.59 & $5695 \pm 164$ & 2.88 \\
\hline 4363 [ÖнI] & 0.138 & - & - & $333 \pm 66$ & 19.94 & $203 \pm 19$ & 9.30 & $211 \pm 43$ & 20.36 \\
\hline $4471 \mathrm{HeI}$ & 0.106 & $498 \pm 165$ & 33.14 & $351 \pm 85$ & 24.30 & $178 \pm 204$ & 114.66 & $377 \pm 75$ & 19.84 \\
\hline $4861 \mathrm{H} \beta$ & 0.000 & $10000 \pm 366$ & 3.66 & $10002 \pm 197$ & 1.97 & $10000 \pm 556$ & 5.56 & $10001 \pm 171$ & 1.71 \\
\hline 4959 [OіII] & -0.024 & $13852 \pm 1561$ & 11.27 & $11224 \pm 1168$ & 10.40 & $10388 \pm 427$ & 4.11 & $11926 \pm 949$ & 7.96 \\
\hline 5007 [Oin] & -0.035 & $47977 \pm 4788$ & 9.98 & $36004 \pm 2472$ & 6.87 & $27071 \pm 1382$ & 5.10 & $39219 \pm 2503$ & 6.38 \\
\hline $5876 \mathrm{HeI}$ & -0.209 & $1447 \pm 342$ & 23.66 & $985 \pm 172$ & 17.43 & $1337 \pm 579$ & 43.32 & $1159 \pm 155$ & 13.39 \\
\hline $6300[\mathrm{OI}]$ & -0.276 & - & - & $617 \pm 83$ & 13.42 & $204 \pm 197$ & 96.38 & $495 \pm 95$ & 19.14 \\
\hline 6312 [SIII] & -0.278 & $83 \pm 21$ & 25.19 & $220 \pm 53$ & 24.03 & $122 \pm 14$ & 11.33 & $177 \pm 67$ & 37.99 \\
\hline 6548 [NII] & -0.311 & $1062 \pm 226$ & 21.28 & $473 \pm 77$ & 16.19 & $425 \pm 94$ & 22.23 & $633 \pm 81$ & 12.75 \\
\hline $6563 \mathrm{H} \alpha$ & -0.313 & $30817 \pm 372$ & 1.21 & $32633 \pm 181$ & 0.56 & $26645 \pm 663$ & 2.49 & $32069 \pm 167$ & 0.52 \\
\hline $6584[\mathrm{NII}]$ & -0.316 & $3584 \pm 310$ & 8.65 & $1376 \pm 108$ & 7.84 & $1289 \pm 293$ & 22.74 & $1980 \pm 111$ & 5.63 \\
\hline 6678 HeI & -0.329 & $677 \pm 185$ & 27.25 & $250 \pm 80$ & 31.98 & $201 \pm 37$ & 18.48 & $363 \pm 76$ & 21.00 \\
\hline 6717 [SII] & -0.334 & $5798 \pm 377$ & 6.50 & $1961 \pm 121$ & 6.18 & $1156 \pm 312$ & 26.97 & $2933 \pm 128$ & 4.37 \\
\hline 6731 [SII] & -0.336 & $4047 \pm 408$ & 10.07 & $1496 \pm 134$ & 8.95 & $687 \pm 45$ & 6.50 & $2118 \pm 142$ & 6.68 \\
\hline 7065 HeI & -0.377 & - & - & $254 \pm 56$ & 22.10 & $166 \pm 45$ & 27.28 & $197 \pm 57$ & 28.75 \\
\hline 7136 [ArIII] & -0.385 & $599 \pm 201$ & 33.52 & $892 \pm 69$ & 7.71 & $433 \pm 79$ & 18.17 & $790 \pm 72$ & 9.05 \\
\hline 7751 [ArIII] & -0.451 & $498 \pm 161$ & 32.39 & $91 \pm 27$ & 29.48 & $292 \pm 95$ & 32.47 & $214 \pm 50$ & 23.37 \\
\hline 9069 [SIII] & -0.561 & $2685 \pm 508$ & 18.94 & $1298 \pm 164$ & 12.65 & $1328 \pm 509$ & 38.34 & $1678 \pm 174$ & 10.34 \\
\hline $\mathrm{I}(\mathrm{H} \beta)\left(\mathrm{erg} \mathrm{seg}^{-1} \mathrm{~cm}^{-2}\right)$ & & $0.26 \times 10^{-}$ & & $0.50 \times 10^{-}$ & & $0.09 \times 1$ & & $0.85 \times 10^{-}$ & \\
\hline $\mathrm{c}(\mathrm{H} \beta)$ & & 0.00 & & $0.31 \pm 0$ & & $0.05 \pm 0$ & & $0.18 \pm 0$ & \\
\hline$-\mathrm{EW}(\mathrm{H} \beta)$ & & 5.71 & & 14.51 & & 1.76 & & 21.99 & \\
\hline
\end{tabular}




\section{- II Zw 33 Brote C}

En este Nudo hemos medido una importante cantidad de líneas intensas y débiles en emisión de distintos iones pero no hemos podido medir las líneas débiles aurorales de interés para un estudio con la precisión deseada. Los resultados que se presentan fueron estimados a partir de la temperatura inicial de 10.000k, la que corresponde a temperaturas típicas para esta clase de objetos, y utilizando relaciones empíricas y relaciones teóricas se estimaron las temperaturas electrónicas de los distinos iones. La determinación de las densidades y de las abundancias iónicas y totales se realizaron de la misma manera descripta anteriormente.

El mejor ajuste encontrado es de tres componentes en total, dos angostas y una ancha que ajusta de manera correcta las alas del perfil. Las dos componentes angostas se encuentran hacia el azul y rojo respectivamente, respecto al centroide del perfil global de las líneas. Los valores de las abundancias totales del $\mathrm{O} / \mathrm{H}$ estimados son: $8.21 \pm 0.02$ para $\mathrm{N} 1(\approx 3.01$ veces menor que la solar $), 8.15 \pm 0.03$ para $\mathrm{N} 2(\approx 3.46$ veces menor que la solar $), 8.30 \pm 0.05$ para la ancha $(\approx 2.46$ veces menor que la solar) y la medida global de $8.32 \pm 0.03(\approx 2.34$ veces menor que la solar). Estos resultados están en acuerdo con los calculados en la literatura para este tipo de regiones y galaxias. Los ajustes presentan un buen acuerdo y pudimos estimar la densidad con el uso de los cocientes del doblete del [Sir] en: 63, 10, 62 y 25 para las componentes N1, N2, ancha y global, respectivamente, siendo N1 y la componente ancha (Broad) más anchas que la angosta N2. Estas, son densidades bajas tal como es esperable para estas regiones. Hemos determinado la constante $\mathrm{c}(\mathrm{H} \beta)$ para el enrojecimiento a partir de las líneas intensas del hidrógeno medidas, estimando valores de extinciones nulas para la componente ancha y N2 (0.00), y de $0.37 \pm 0.06$ y $0.61 \pm 0.04$ para la componente global y N1 respectivamente. Es la componente angosta más intensa de nuestro ajuste la que presenta mayor apantallamiento del polvo.

Los resultados estimados de nuestros ajustes para los parámetros físicos del gas ionizado del Nudo C de II Zw 33 se indican en la Tabla 4.8. 
Tabla 4.6. Propiedades físicas (densidades electrónicas $\mathrm{n}_{e} \mathrm{y}$ temperaturas electrónicas $\mathrm{T}_{e}$ ) y abundancias químicas iónicas y totales de II Zw 33 B, derivadas de líneas prohibidas y las líneas de recombinación de helio.

\begin{tabular}{|c|c|c|c|c|}
\hline & \multicolumn{4}{|c|}{ II Zw 33 Nudo B } \\
\hline & Ancha & N1 & $\mathrm{N} 2$ & Global \\
\hline$n_{e}$ & $75:$ & 123: & 98: & 69: \\
\hline $\mathrm{t}_{e}[\mathrm{OIII}]$ & $1.00^{b}$ & $1.13 \pm 0.06$ & $1.04 \pm 0.02$ & $0.96 \pm 0.03$ \\
\hline $\mathrm{t}_{e}[\mathrm{SIII}]$ & $0.87^{a}$ & $1.02 \pm 0.16$ & $1.15 \pm 0.25$ & $1.24 \pm 0.28$ \\
\hline $\mathrm{t}_{e}[\mathrm{OII}]$ & $1.05^{a}$ & $1.09^{a}$ & $1.06^{a}$ & $1.03^{a}$ \\
\hline $\mathrm{t}_{e}[\mathrm{SII}]$ & $1.05^{a}$ & $1.09^{a}$ & $1.06^{a}$ & $1.03^{a}$ \\
\hline $\mathrm{t}_{e}[\mathrm{NII}]$ & $1.05^{a}$ & $1.09^{a}$ & $1.06^{a}$ & $1.03^{a}$ \\
\hline $12+\log \left(\mathrm{O}^{+} / \mathrm{H}^{+}\right)$ & $8.24 \pm 0.02$ & $7.91 \pm 0.07$ & $7.72 \pm 0.05$ & $8.10 \pm 0.05$ \\
\hline $12+\log \left(\mathrm{O}^{2+} / \mathrm{H}^{+}\right)$ & $8.19 \pm 0.04$ & $7.90 \pm 0.11$ & $7.91 \pm 0.06$ & $8.16 \pm 0.08$ \\
\hline $12+\log (\mathrm{O} / \mathrm{H})$ & $8.51 \pm 0.03$ & $8.21 \pm 0.09$ & $8.13 \pm 0.06$ & $8.43 \pm 0.06$ \\
\hline $12+\log \left(\mathrm{S}^{+} / \mathrm{H}^{+}\right)$ & 6.30 & 5.81 & 5.57 & 6.03 \\
\hline $12+\log \left(\mathrm{S}^{2+} / \mathrm{H}^{+}\right)$ & $6.97 \pm 0.30$ & $5.93 \pm 0.34$ & $6.25 \pm 0.41$ & $6.28 \pm 0.50$ \\
\hline $\mathrm{ICF}\left(\mathrm{S}^{+}+\mathrm{S}^{2+}\right)$ & $1.07 \pm 0.01$ & $1.08 \pm 0.01$ & $1.15 \pm 0.01$ & $1.10 \pm 0.01$ \\
\hline $12+\log (\mathrm{S} / \mathrm{H})$ & $7.08 \pm 0.27$ & $6.21 \pm 0.24$ & $6.39 \pm 0.37$ & $6.52 \pm 0.39$ \\
\hline $\log (\mathrm{S} / \mathrm{O})$ & $-1.43 \pm 0.27$ & $-1.99 \pm 0.26$ & $-1.74 \pm 0.38$ & $-1.92 \pm 0.39$ \\
\hline $12+\log \left(\mathrm{N}^{+} / \mathrm{H}^{+}\right)$ & 6.78 & 6.34 & 6.34 & 6.55 \\
\hline $12+\log (\mathrm{N} / \mathrm{H})$ & 7.07 & 6.63 & 6.75 & 6.88 \\
\hline $\log (\mathrm{N} / \mathrm{O})$ & -1.46 & -1.57 & -1.38 & -1.55 \\
\hline $12+\log \left(\mathrm{Ne}^{2+} / \mathrm{H}^{+}\right)$ & $7.79 \pm 0.03$ & $7.38 \pm 0.13$ & $7.04 \pm 0.52$ & $7.70 \pm 0.08$ \\
\hline $\mathrm{ICF}\left(\mathrm{Ne}^{2+}\right)$ & $1.18 \pm 0.01$ & $1.17 \pm 0.02$ & $1.12 \pm 0.10$ & $1.15 \pm 0.01$ \\
\hline $12+\log (\mathrm{Ne} / \mathrm{H})$ & $7.86 \pm 0.03$ & $7.45 \pm 0.13$ & $7.08 \pm 0.52$ & $7.76 \pm 0.08$ \\
\hline $\log (\mathrm{Ne} / \mathrm{O})$ & $-0.65 \pm 0.04$ & $-0.76 \pm 0.16$ & $-1.04 \pm 0.65$ & $-0.68 \pm 0.10$ \\
\hline $12+\log \left(\mathrm{Ar}^{2+} / \mathrm{H}^{+}\right)$ & $6.08 \pm 0.23$ & $5.47 \pm 0.17$ & $5.47 \pm 0.60$ & $5.66 \pm 0.23$ \\
\hline $\mathrm{ICF}\left(\mathrm{Ar}^{2+}\right)$ & $1.14 \pm 0.01$ & $1.13 \pm 0.01$ & $1.11 \pm 0.03$ & $1.12 \pm 0.01$ \\
\hline $12+\log (\mathrm{Ar} / \mathrm{H})$ & $6.13 \pm 0.23$ & $5.52 \pm 0.17$ & $5.51 \pm 0.60$ & $5.71 \pm 0.23$ \\
\hline $\log (\mathrm{Ar} / \mathrm{O})$ & $-2.38 \pm 0.23$ & $-2.61 \pm 0.27$ & $-2.68 \pm 0.19$ & $-2.72 \pm 0.24$ \\
\hline $\mathrm{He}^{+} / \mathrm{H}^{+}$ & & & & \\
\hline 4471 & $0.100 \pm 0.033$ & $0.071 \pm 0.017$ & $0.035 \pm 0.012$ & $0.075 \pm 0.015$ \\
\hline 5876 & $0.106 \pm 0.025$ & $0.074 \pm 0.012$ & $0.098 \pm 0.038$ & $0.084 \pm 0.011$ \\
\hline 6678 & $0.175 \pm 0.048$ & $0.066 \pm 0.020$ & $0.052 \pm 0.009$ & $0.093 \pm 0.019$ \\
\hline 7065 & $0.020 \pm 0.003$ & $0.100 \pm 0.025$ & $0.068 \pm 0.019$ & $0.085 \pm 0.025$ \\
\hline adopted & $0.107 \pm 0.045$ & $0.075 \pm 0.013$ & $0.062 \pm 0.022$ & $0.083 \pm 0.006$ \\
\hline
\end{tabular}

donde $n_{e}$ corresponde a la densidad en $\mathrm{cm}^{-3}$ y $\mathrm{T}_{e}$ a la temperatura en $10^{4} \mathrm{k}$,

${ }^{a}$ utilizando temperaturas de modelos $\mathrm{y}^{b}$ suponiendo una temperatura inicial de $10000 \mathrm{k}$, 
Tabla 4.7. Intensidad de las líneas corregidas por enrojecimiento para el Nudo C de II Zw 33 $[\mathrm{F}(\mathrm{H} \beta)=\mathrm{I}(\mathrm{H} \beta)=10.000]$.

\begin{tabular}{|c|c|c|c|c|c|c|c|c|c|}
\hline \multirow[b]{2}{*}{$\lambda(\AA)$} & \multicolumn{3}{|c|}{ Broad } & \multicolumn{2}{|l|}{ N1 } & \multicolumn{2}{|l|}{$\mathrm{N} 2$} & \multicolumn{2}{|l|}{ Global } \\
\hline & $f(\lambda)$ & $I(\lambda)$ & $\begin{array}{c}\text { Error } \\
(\%)\end{array}$ & $I(\lambda)$ & $\begin{array}{c}\text { Error } \\
(\%)\end{array}$ & $I(\lambda)$ & $\begin{array}{c}\text { Error } \\
(\%)\end{array}$ & $\begin{array}{l}I(\lambda) \\
(\%)\end{array}$ & Error \\
\hline $3726[\mathrm{OII}]$ & 0.272 & $8835 \pm 353$ & 4.00 & $10257 \pm 441$ & 4.30 & $6743 \pm 1354$ & 29.68 & $9440 \pm 1038$ & 10.99 \\
\hline $3727\left[^{\mathrm{OII}}\right]^{b}$ & 0.271 & $22329 \pm 3344$ & 14.98 & $24246 \pm 712$ & 2.93 & $19441 \pm 1683$ & 8.66 & $23669 \pm 1569$ & 6.63 \\
\hline 3729 [OII] & 0.271 & $13494 \pm 2554$ & 18.93 & $13984 \pm 836$ & 5.98 & $12697 \pm 1362$ & 10.73 & $14225 \pm 1230$ & 8.65 \\
\hline 3868 [NeIII] & 0.238 & $2410 \pm 170$ & 7.05 & $4939 \pm 651$ & 13.18 & $1513 \pm 422$ & 62.51 & $3490 \pm 687$ & 19.70 \\
\hline $4102 \mathrm{H} \delta$ & 0.188 & $3655 \pm 161$ & 4.41 & $3183 \pm 495$ & 15.54 & $4112 \pm 688$ & 16.72 & $3604 \pm 616$ & 17.10 \\
\hline $4340 \mathrm{H} \gamma$ & 0.142 & $3795 \pm 687$ & 18.10 & $7196 \pm 537$ & 7.46 & $6941 \pm 773$ & 11.14 & $6173 \pm 2278$ & 36.90 \\
\hline $4861 \mathrm{H} \beta$ & 0.000 & $10000 \pm 904$ & 9.04 & $10000 \pm 366$ & 3.66 & $10000 \pm 638$ & 6.38 & $10000 \pm 442$ & 4.42 \\
\hline 4959 [Оіні] & -0.024 & $7671 \pm 334$ & 4.35 & $14933 \pm 1099$ & 7.36 & $10230 \pm 1254$ & 12.26 & $12035 \pm 1170$ & 9.72 \\
\hline 5007 [OIII] & -0.035 & $26305 \pm 264$ & 1.00 & $46192 \pm 842$ & 1.82 & $32599 \pm 1231$ & 3.78 & $37971 \pm 891$ & 2.35 \\
\hline 5876 HeI & -0.209 & $1141 \pm 103$ & 8.99 & $996 \pm 263$ & 26.35 & $1447 \pm 74$ & 5.10 & $1086 \pm 343$ & 31.56 \\
\hline 6548 [NII] & -0.311 & $1371 \pm 384$ & 27.98 & $213 \pm 12$ & 5.60 & $352 \pm 128$ & 36.32 & $489 \pm 150$ & 30.73 \\
\hline $6563 \mathrm{H} \alpha$ & -0.313 & $27510 \pm 653$ & 2.37 & $32355 \pm 246$ & 0.76 & $24868 \pm 697$ & 2.80 & $29849 \pm 297$ & 0.99 \\
\hline $6584[\mathrm{NII}]$ & -0.316 & $1492 \pm 119$ & 8.00 & $1162 \pm 160$ & 13.74 & $1622 \pm 93$ & 5.70 & $1281 \pm 358$ & 27.94 \\
\hline $6678 \mathrm{HeI}$ & -0.329 & $741 \pm 64$ & 8.65 & $363 \pm 113$ & 31.19 & $265 \pm 27$ & 10.37 & $429 \pm 155$ & 36.20 \\
\hline 6717 [SII] & -0.334 & $4618 \pm 155$ & 3.35 & $1396 \pm 171$ & 12.28 & $2339 \pm 471$ & 20.13 & $2216 \pm 156$ & 7.04 \\
\hline 6731 [SII] & -0.336 & $3655 \pm 626$ & 17.12 & $1013 \pm 157$ & 15.49 & $1266 \pm 73$ & 5.75 & $1611 \pm 187$ & 11.61 \\
\hline 7136 [ArIII] & -0.385 & - & - & $842 \pm 107$ & 12.73 & $612 \pm 51$ & 8.31 & $627 \pm 86$ & 13.79 \\
\hline 9069 [SIII] & -0.561 & $2410 \pm 156$ & 6.49 & $1046 \pm 195$ & 18.63 & $2372 \pm 663$ & 27.96 & $1449 \pm 186$ & 12.84 \\
\hline $\mathrm{I}(\mathrm{H} \beta)\left(\mathrm{erg} \mathrm{seg}^{-1} \mathrm{~cm}^{-2}\right)$ & & $0.05 \times 10^{-}$ & & $0.09 \times 10^{-}$ & & $0.03 \times 10^{-}$ & & $0.17 \times 10^{-}$ & \\
\hline $\mathrm{c}(\mathrm{H} \beta)$ & & 0.00 & & $0.61 \pm 0$ & & 0.00 & & $0.37 \pm 0$ & \\
\hline$-\mathrm{EW}(\mathrm{H} \beta)$ & & 0.88 & & 2.97 & & 0.52 & & 4.38 & \\
\hline
\end{tabular}


- Mrk 600 Brote A

Este Nudo es el más complejo de los que hemos analizado. Sus perfiles de líneas con un ajuste global de cuatro componentes cinemáticas, y una alta S/N, nos permitió analizar en detalle los parámetros físicos del gas ionizado. Las cuatro componentes fueron ajustadas en prácticamente todas las líneas intensas de interés con errores menores al $8 \%$ y en los casos de las líneas más débiles, los errores de los ajustes complejos aumentaron hasta un $30 \%$, conduciendo a errores elevados en la propagación para los análisis de las abundancias (en la Tabla 4.9 se pueden ver los errores para cada ajuste). A pesar de esto, los ajustes presentan coherencia entre sí, encontrando soluciones correctas para las líneas más débiles a partir de los ajustes determinados en las líneas de $\mathrm{H} \alpha$ y [OIII] $5007 \AA ̊$.

Las abundancias totales de $\mathrm{O} / \mathrm{H}$ estimadas para nuestras componentes cinemáticas fueron determinadas a partir de las temperaturas electrónicas del [OIII] mediante el método directo. Las múltiples componentes se pudieron ajustar con gran acuerdo en la línea débil auroral a partir de las cuales se estimaron las temperaturas con una muy buena precisión. También se estimaron las $\mathrm{T}_{e}([\mathrm{SIII}])$ a partir las relaciones de las líneas intensas y la línea débil auroral sensible a la temperatura. Las cuatro componentes pudieron ajustarse con incertezas levemente mayores a la de $\mathrm{T}_{e}([\mathrm{OIII}])$. Las temperaturas muestran un comportamiento con valores sensiblemente mayor que los determinados para las regiones de II Zw 33, en un $40 \%$ aproximadamente, con excepción de la componente N3 para los que se derivaron temperaturas del orden de los 20.000k. Esta temperatura es aproximadamente un $30 \%$ mayor que la temperatura estimada para las demás componentes y la medida global, lo que se traduce en una diferencia en las abundancias totales de las especies químicas.

A partir de las estimaciones de las temperaturas se derivaron las abundancias totales del oxígeno, indicando bajas metalicidades respecto a los valores típicos de galaxias Hir: las componentes $\mathrm{N} 1, \mathrm{~N} 2$ y la global presentan valores acordes entre sí $7.68 \pm 0.03(\approx 10.23$ veces menor que la abundancia solar), $7.80 \pm 0.05(\approx 8$ veces menor que la abundancia solar) y $7.80 \pm 0.04(\approx 8$ veces menor que la abundancia solar), respectivamente. La componentes angosta $\mathrm{N} 3$ tiene la abundancia $\mathrm{O} / \mathrm{H}$ más baja de las regiones, con $7.36 \pm 0.06(\approx 22$ veces menor que la abundancia solar $)$ que corresponde a un gas poco enriquecido químicamente, indicando una región menos evoluciona. Por el contrario, la componente ancha con $8.27 \pm 0.06(\approx 3$ veces menor que la abundancia solar) indica que la componente de gas está más evolucionada que todas las demás componentes. La abundancia estimada para N3 es baja con respecto a los valores medios pero sigue estando por encima de valores de metalicidades extremadamente bajos como los determinados por Izotov \& Thuan (1999) en dos de los nudos más intensos de IZw 18 con $7.07 \pm 0.05$ ( $\approx 42$ veces menor que la abundancia solar) y $7.17 \pm 0.06$.

La excelente $\mathrm{S} / \mathrm{N}$ de nuestros espectros nos permitió medir con presición las líneas aurorales del [OIII] y del [SIII], poder estimar las temperaturas de manera directa y luego estimar las abundancias químicas con menor incertidumbre. Las densidades fueron calculadas a partir de los cocientes del [SII], obteniendo valores de densidades bajas esperables para estas regiones. Los valores de referencia para nuestra región son: $10 \mathrm{~cm}^{-3}$ para N1, $91 \mathrm{~cm}^{-3}$ para N2, $127 \mathrm{~cm}^{-3}$ para la $\mathrm{N} 3,10 \mathrm{~cm}^{-3}$ para la ancha y $100 \mathrm{~cm}^{-3}$ para la global. Además, el enrojecimiento medido en nuestras componentes a través de las líneas más intensas de la serie de Balmer del hidrógeno resulta equivalente con que sea nulo $(\mathrm{c}(\mathrm{H} \beta) \approx 0)$, salvo $\mathrm{N} 2$ que presenta un valor de la constante de $0.04 \pm 0.01$, lo que indica que las componentes no están enrojecidas o que tienen un apantallamiento muy bajo. 
Tabla 4.8. Propiedades físicas (densidades electrónicas $\mathrm{n}_{e} \mathrm{y}$ temperaturas electrónicas $\mathrm{T}_{e}$ ) y abundancias químicas iónicas y totales derivadas de líneas prohibidas y las líneas de recombinación de helio, para II Zw $33 \mathrm{C}$.

\begin{tabular}{|c|c|c|c|c|}
\hline & \multicolumn{4}{|c|}{ II Zw 33 Nudo C } \\
\hline & Ancha & N1 & $\mathrm{N} 2$ & Global \\
\hline$n_{e}$ & 62: & 63: & 10: & 25: \\
\hline $\mathrm{t}_{e}[\mathrm{OIII}]$ & $1.00^{b}$ & $1.00^{b}$ & $1.00^{a}$ & $1.00^{b}$ \\
\hline $\mathrm{t}_{e}[\mathrm{SIII}]$ & $0.94^{a}$ & $0.97^{a}$ & $1.21^{a}$ & $1.01^{a}$ \\
\hline $\mathrm{t}_{e}[\mathrm{OII}]$ & $0.94^{a}$ & $0.97^{a}$ & $0.97^{a}$ & $1.01^{a}$ \\
\hline $\mathrm{t}_{e}[\mathrm{SII}]$ & $0.94^{a}$ & $0.97^{a}$ & $0.97^{a}$ & $1.01^{a}$ \\
\hline $\mathrm{t}_{e}[\mathrm{NII}]$ & 0.94 & $0.97^{a}$ & $0.97^{a}$ & $1.01^{a}$ \\
\hline $12+\log \left(\mathrm{O}^{+} / \mathrm{H}^{+}\right)$ & $8.06 \pm 0.08$ & $7.03 \pm 0.08$ & $7.54 \pm 0.04$ & $7.94 \pm 0.04$ \\
\hline $12+\log \left(\mathrm{O}^{2+} / \mathrm{H}^{+}\right)$ & $7.93 \pm 0.01$ & $8.18 \pm 0.01$ & $8.03 \pm 0.02$ & $8.09 \pm 0.02$ \\
\hline $12+\log (\mathrm{O} / \mathrm{H})$ & $8.30 \pm 0.05$ & $8.21 \pm 0.02$ & $8.15 \pm 0.03$ & $8.32 \pm 0.03$ \\
\hline $12+\log \left(\mathrm{S}^{+} / \mathrm{H}^{+}\right)$ & 6.36 & 5.78 & 5.73 & 5.93 \\
\hline $12+\log \left(\mathrm{S}^{2+} / \mathrm{H}^{+}\right)$ & 8.47 & 8.10 & 8.46 & 8.25 \\
\hline $\mathrm{ICF}\left(\mathrm{S}^{+}+\mathrm{S}^{2+}\right)$ & 1.05 & 2.10 & 1.31 & 1.13 \\
\hline $12+\log (\mathrm{S} / \mathrm{H})$ & 8.49 & 8.43 & 8.58 & 8.30 \\
\hline $\log (\mathrm{S} / \mathrm{O})$ & 0.19 & 0.22 & 0.43 & -0.02 \\
\hline $12+\log \left(\mathrm{N}^{+} / \mathrm{H}^{+}\right)$ & 6.69 & 6.34 & 6.26 & 6.40 \\
\hline $12+\log (\mathrm{N} / \mathrm{H})$ & 6.93 & 6.72 & 6.87 & 6.79 \\
\hline $\log (\mathrm{N} / \mathrm{O})$ & -1.37 & -1.69 & -1.28 & -1.54 \\
\hline $12+\log \left(\mathrm{Ne}^{2+} / \mathrm{H}^{+}\right)$ & $7.43 \pm 0.04$ & $7.74 \pm 0.05$ & $7.22 \pm 0.21$ & $7.59 \pm 0.08$ \\
\hline $\mathrm{ICF}\left(\mathrm{Ne}^{2+}\right)$ & $1.22 \pm 0.03$ & $1.07 \pm 0.01$ & $1.09 \pm 0.01$ & $1.13 \pm 0.01$ \\
\hline $12+\log (\mathrm{Ne} / \mathrm{H})$ & $7.51 \pm 0.03$ & $7.77 \pm 0.05$ & $7.26 \pm 0.21$ & $7.64 \pm 0.08$ \\
\hline $\log (\mathrm{Ne} / \mathrm{O})$ & $-0.79 \pm 0.06$ & $-0.44 \pm 0.06$ & $-0.89 \pm 0.21$ & $-0.68 \pm 0.08$ \\
\hline $12+\log \left(A r^{2+} / H^{+}\right)$ & - & 6.04 & 5.90 & 5.91 \\
\hline $\operatorname{ICF}\left(A r^{2+}\right)$ & - & 1.75 & 1.13 & 1.11 \\
\hline $12+\log (\mathrm{Ar} / \mathrm{H})$ & 一 & 6.29 & 5.96 & 5.96 \\
\hline $\log (\mathrm{Ar} / 0)$ & - & $-2.32 \pm 0.22$ & $-2.19 \pm 0.20$ & $-2.36 \pm 0.22$ \\
\hline $\mathrm{He}^{+} / \mathrm{H}^{+}$ & & & & \\
\hline 5876 & $0.083 \pm 0.007$ & $0.073 \pm 0.018$ & $0.106 \pm 0.005$ & $0.079 \pm 0.025$ \\
\hline 6678 & $0.191 \pm 0.016$ & $0.094 \pm 0.026$ & $0.068 \pm 0.007$ & $0.111 \pm 0.040$ \\
\hline Adoptadas & $0.101 \pm 0.035$ & $0.079 \pm 0.008$ & $0.092 \pm 0.020$ & $0.088 \pm 0.017$ \\
\hline
\end{tabular}

donde $n_{e}$ corresponde a la densidad en $\mathrm{cm}^{-3}$ y $\mathrm{T}_{e}$ a la temperatura en $10^{4} \mathrm{k}$,

${ }^{a}$ utilizando temperaturas de modelos $\mathrm{y}^{b}$ suponiendo una temperatura inicial de $10000 \mathrm{k}$, 


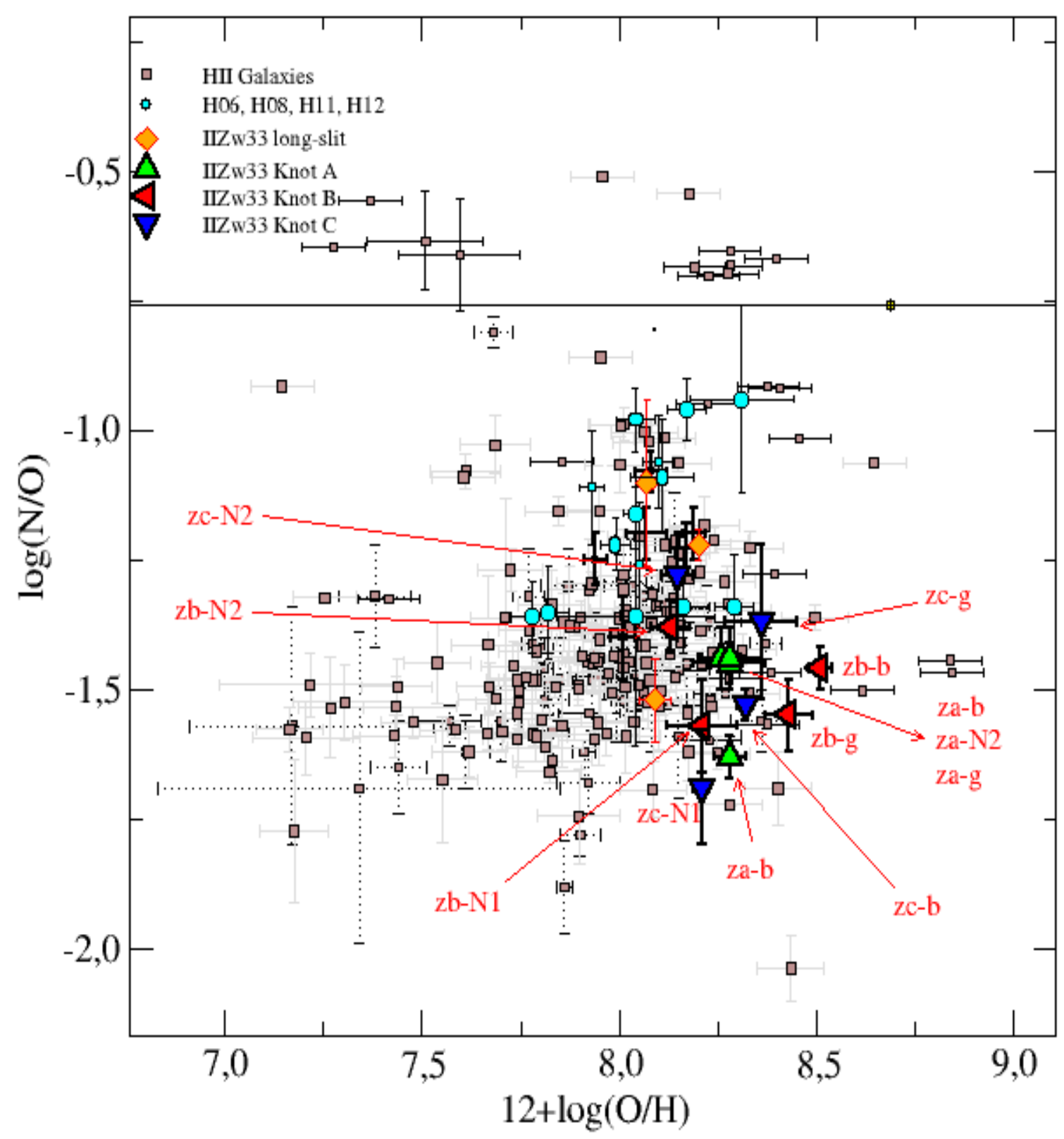

Figura 4.3. $\mathrm{N} / \mathrm{O}$ vs. $12+\log (\mathrm{O} / \mathrm{H})$ para los nudos de la galaxia II Zw 33 (triángulos verdes, rojos y azules). Además se indican las galaxias y RHII de la literatura utilizados en publicaciones previas de nuestro grupo (cuadrados grises) y las regiones estudiadas en los trabajos de Hägele et al. (2006, 2008, 2011) y los brotes intesos de Haro 15 presentados en Hägele et al. (2012) con cículos en celeste. Además se indican los valores de abundancias relativas al oxígeno de II Zw 33 obtenidos con dispersión simple con diamantes rojos (ver CampuzanoCastro et al., 2015). Las regiones estudiadas en este trabajo se indican como za, zb y zc, haciendo referencia a los Nudos A, B y C, respectivamente, y las distintas componentes se indican como: $\mathrm{b}=$ ancha, $\mathrm{N} 1=$ angosta $1, \mathrm{~N} 2=$ angosta 2 y g = medida Global. El valor solar se muestra con el símbolo usual de referencia. 


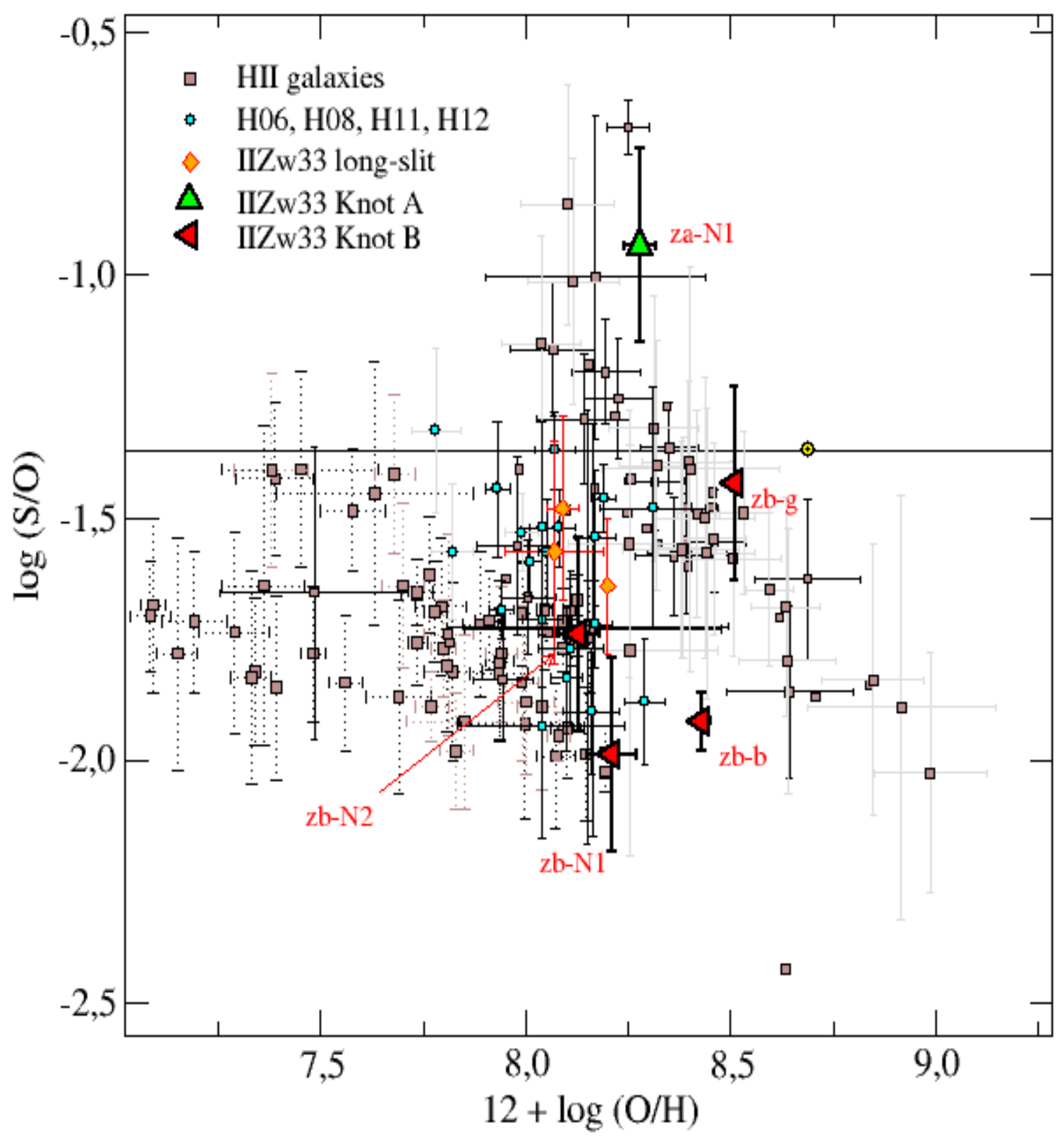

Figura 4.4. $\mathrm{S} / \mathrm{O}$ vs. $12+\log (\mathrm{O} / \mathrm{H})$ para los Nudos A y B de la galaxia II Zw 33 (triángulos verdes y rojos). Además se indican las galaxias y RHı de la literatura utilizados en publicaciones previas de nuestro grupo (cuadrados grises) y las regiones estudiadas en los trabajos de Hägele et al. (2006, 2008, 2011) y los brotes intesos de Haro 15 presentados en Hägele et al. (2012) con cículos en celeste. Además se indican los valores de abundancias relativas al oxígeno de IIZw 33 obtenidos con dispersión simple con diamantes rojos (ver Campuzano-Castro et al., 2015). Las regiones estudiadas en este trabajo se indican como za y zb, haciendo referencia a los Nudos A y B, respectivamente, y las distintas componentes se indican como: $\mathrm{b}=$ ancha, $\mathrm{N} 1=\operatorname{angosta} 1, \mathrm{~N} 2=\operatorname{angosta} 2$ y g = medida Global. El valor solar se muestra con el símbolo usual de referencia. 


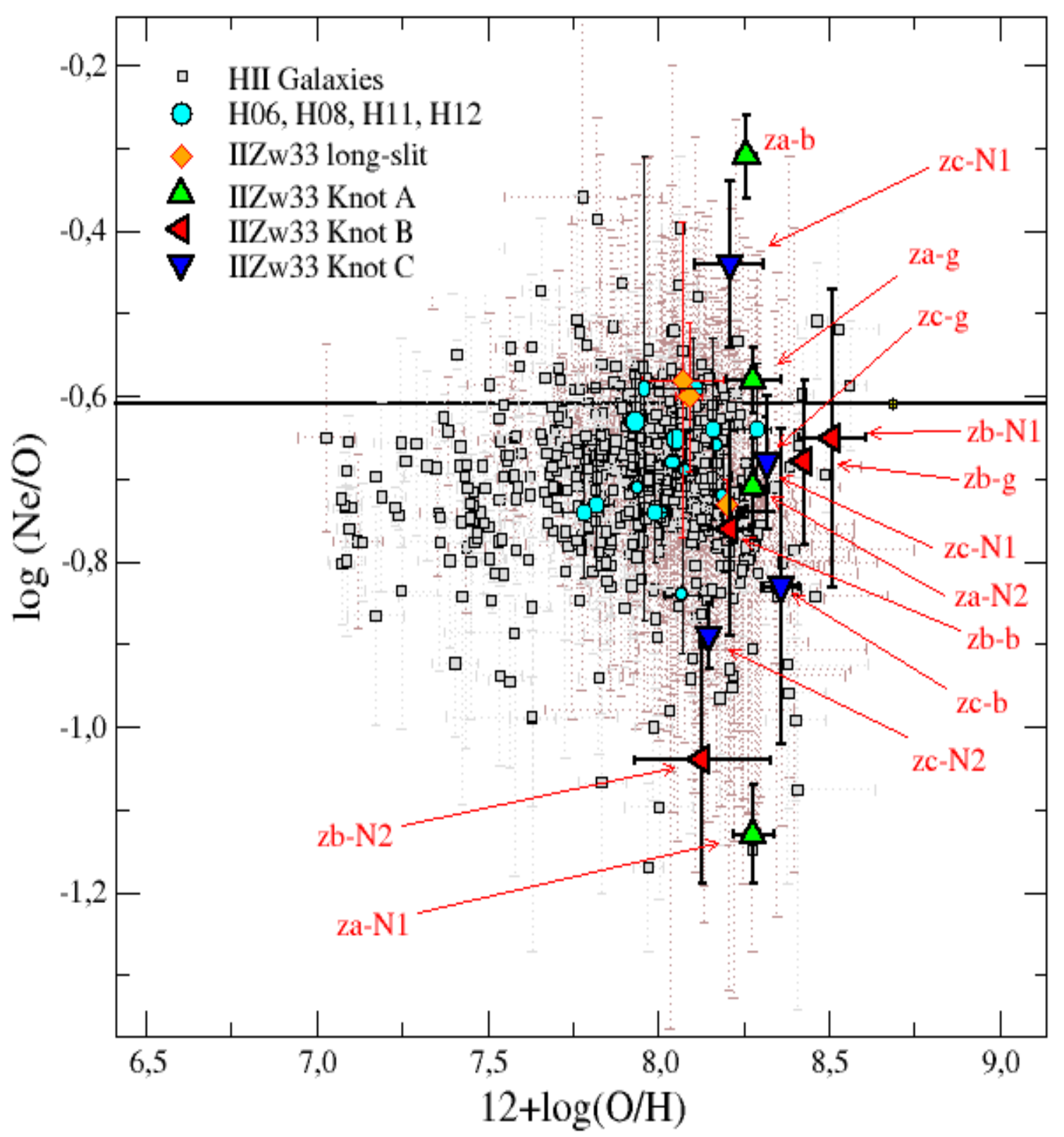

Figura 4.5. $\mathrm{Ne} / \mathrm{O}$ vs. $12+\log (\mathrm{O} / \mathrm{H})$ para los nudos de la galaxia II Zw 33 (triángulos verdes, rojos y azules). Además se indican las galaxias y RHII de la literatura utilizados en publicaciones previas de nuestro grupo (cuadrados grises) y las regiones estudiadas en los trabajos de Hägele et al. (2006, 2008, 2011) y los brotes intesos de Haro 15 presentados en Hägele et al. (2012) con cículos en celeste. Además se indican los valores de abundancias relativas al oxígeno de II Zw 33 obtenidos con dispersión simple con diamantes rojos (ver CampuzanoCastro et al., 2015). Las regiones estudiadas en este trabajo se indican como za, zb y zc, haciendo referencia a los Nudos A, B y C, respectivamente, y las distintas componentes se indican como: $\mathrm{b}=$ ancha, $\mathrm{N} 1=$ angosta $1, \mathrm{~N} 2=$ angosta 2 y g = medida Global. El valor solar se muestra con el símbolo usual de referencia. 


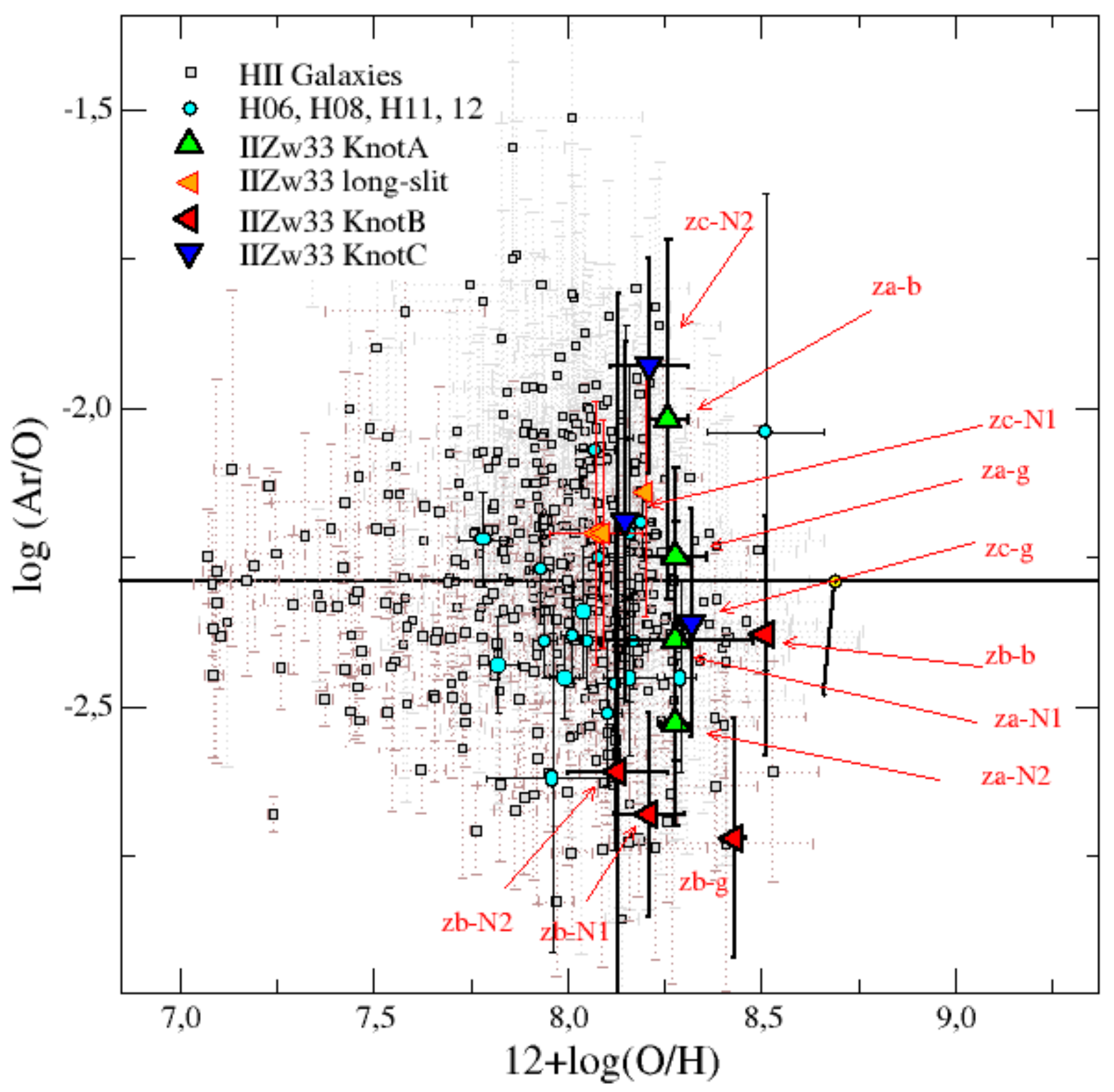

Figura 4.6. Ar/O vs. $12+\log (\mathrm{O} / \mathrm{H})$ para los nudos de la galaxia II Zw 33 (triángulos verdes, rojos y azules). Además se indican las galaxias y RHı de la literatura utilizados en publicaciones previas de nuestro grupo (cuadrados grises) y las regiones estudiadas en los trabajos de Hägele et al. (2006, 2008, 2011) y los brotes intesos de Haro 15 presentados en Hägele et al. (2012) con cículos en celeste. Además se indican los valores de abundancias relativas al oxígeno de II Zw 33 obtenidos con dispersión simple con diamantes rojos (ver CampuzanoCastro et al., 2015). Las regiones estudiadas en este trabajo se indican como za, zb y zc, haciendo referencia a los Nudos A, B y C, respectivamente, y las distintas componentes se indican como: $\mathrm{b}=$ ancha, $\mathrm{N} 1=$ angosta $1, \mathrm{~N} 2=\operatorname{angosta} 2$ y g = medida Global. El valor solar se muestra con el símbolo usual de referencia. 
Hemos determinado abundancias para el Nitrógeno a partir de las líneas de $\lambda \lambda 6548 \AA \mathrm{y}$ $6584 \AA$, encontrando valores acordes a este tipo de regiones y galaxias, muy por debajo del valor de abundancia solar de $\mathrm{N}$ (Holweger, 2001, : $12+\log (\mathrm{N} / \mathrm{H}) \odot=7.93): 5.86$ para $\mathrm{N} 1,6.22$ para N2, 5.65 para N3, 5.9, 7.13 para la ancha y 6.22 para la global (alrededor de 50 veces menor). Para las líneas del azufre nos hemos encontrado con un problema en las intensidades de las líneas que no pudimos solucionar, relacionado con el flujo medido en las líneas de $9069 \AA$. Los valores de los flujos son menores de los esperados, y esto puede deberse a que estas líneas se encuentran en los últimos órdenes del espectro Echelle, y posiblemente la sensibilidad del detector y los flujos se vean perjudicados. Los valores encontrados presentan grandes incertezas, pero al determinar la línea auroral del [SIII] $6312 \AA$, hemos estimado las temperaturas y determinado las abundancias químicas para los casos en los que fue posible.

Por el lado de los elementos Ne y Ar, hemos podido ajustar las componentes cinemáticas fijando los valores de los centros y los $\sigma$ de la solución de $\mathrm{H} \alpha$. En el caso del Ne, hemos podido obtener una buena precisión en el ajuste a pesar de la baja señal de la línea y de ser muy débil intrínsecamente, estimando valores para las abundancias totales en el rango de $6.82 \leq \mathrm{Ne} / \mathrm{H} \leq 7.75$. El límite superior corresponde al valor de la componente ancha, que es considerablemente mayor que las otras componentes (un factor 8.5). Por el lado del Ar, el perfil de la línea y el ajuste cinemático, nos indica que las componentes N2, N3 y la medida global presentan abundancia similares y acordes entre sí dentro de los errores $(4.89 \leq \mathrm{Ne} / \mathrm{H} \leq 5.22)$, mientras que la componente $\mathrm{N} 1$ con $4.66 \pm 0.11$ presenta la abundancia más baja para este elemento y la ancha lo opuesto, con $5.82 \pm 0.15$ representa la componente más abundante. Las componentes parecen ser fases de un gas en distintos estados evolutivos, ya que hay componentes que varían levemente de las otras.

Respecto a las abundancias totales de los principales elementos (S, N, Ne y Ar) relativas al oxígeno, hemos calculado los cocientes para todas las componentes, encontrando valores coherentes y esperables para la gran mayoría de ellas.

Para el N/O (ver Figura 4.7) los valores estimados resultan encontrarse del centro hacia el sector inferior izquierdo de la distribución debido a que las metalicidades estimadas son bajas respecto a los valores medios (con excepción de la componente ancha que presenta una abundancia mayor) y aunque presenta valores bajos, están en la parte inferior de la distribución de objetos compilados de la literarura.

Un comportamiento similar encontramos para el azufre (ver Figura 4.8), pero en estos casos, los valores de los flujos en las líneas del azufre introducen un error extra que hace que nuestros datos tengan una gran incertidumbre. En las Figuras mencionadas presentamos los cocientes para las componentes comparadas con las muestras publicadas en Amorín et al. (2010); Pérez-Montero et al. (2011a). Las discusiones de estos trabajos plantean que estas leves diferencias entre componentes pueden deberse a estados de un mismo gas en distintas fases. Por el lado del Ne (ver Figura 4.9) hemos estimado los cocientes para todas las componentes con un excelente acuerdo, y presentan los valores esperables para estas regiones. En el caso del Ar (ver Figura 4.10), la componente N1 presenta valores levemente mayores en la distribución. Luego, las componentes restantes muestran resultados esperables. 
Tabla 4.9. Intensidad de las líneas corregidas por enrojecimiento para el Nudo A de Mrk 600

$[\mathrm{F}(\mathrm{H} \beta)=\mathrm{I}(\mathrm{H} \beta)=10.000]$.

\begin{tabular}{|c|c|c|c|c|c|c|c|c|c|c|c|}
\hline \multirow[b]{2}{*}{$\lambda(\AA)$} & \multicolumn{3}{|c|}{ Ancha } & \multicolumn{2}{|l|}{ N1 } & \multicolumn{2}{|l|}{ N2 } & \multicolumn{2}{|l|}{ N3 } & \multicolumn{2}{|c|}{ Global } \\
\hline & $\mathrm{f}(\lambda)$ & $I(\lambda)$ & $\begin{array}{c}\text { Error } \\
(\%)\end{array}$ & $I(\lambda)$ & $\begin{array}{c}\text { Error } \\
(\%)\end{array}$ & $I(\lambda)$ & $\begin{array}{c}\text { Error } \\
(\%)\end{array}$ & $\begin{array}{l}I(\lambda) \\
(\%)\end{array}$ & $\begin{array}{c}\text { Error } \\
(\%)\end{array}$ & $I(\lambda)$ & Error \\
\hline 3726 [OII] & 0.272 & $3701 \pm 514$ & 13.88 & $1308 \pm 222$ & 16.98 & $2794 \pm 350$ & 12.52 & $5092 \pm 179$ & 3.52 & $2742 \pm 523$ & 19.07 \\
\hline $3727[\mathrm{OII}]^{b}$ & 0.271 & $8661 \pm 1097$ & 12.67 & $3293 \pm 105$ & 3.19 & $8116 \pm 64$ & 0.79 & $5742 \pm 198$ & 3.45 & $7358 \pm 348$ & 4.74 \\
\hline 3729 [Оіг] & 0.271 & $4953 \pm 659$ & 13.31 & $1982 \pm 96$ & 4.86 & $5322 \pm 297$ & 5.58 & $6503 \pm 216$ & 3.33 & $4629 \pm 436$ & 9.43 \\
\hline 3868 [NeIII] & 0.238 & $23543 \pm 5210$ & 22.13 & $4199 \pm 633$ & 15.07 & $4819 \pm 293$ & 6.09 & $7301 \pm 249$ & 3.41 & $6352 \pm 442$ & 6.96 \\
\hline $4102 \mathrm{H} \delta$ & 0.188 & - & - & $3323 \pm 110$ & 3.31 & $2952 \pm 46$ & 1.54 & $3184 \pm 202$ & 6.36 & $2774 \pm 41$ & 1.49 \\
\hline $4341 \mathrm{H} \gamma$ & 0.142 & $4213 \pm 631$ & 14.97 & $4199 \pm 220$ & 5.23 & $5375 \pm 69$ & 1.29 & $5491 \pm 334$ & 6.09 & $4994 \pm 132$ & 2.65 \\
\hline 4363 [Oіні] & 0.138 & $4724 \pm 392$ & 8.31 & $1347 \pm 14$ & 1.03 & $914 \pm 86$ & 9.44 & $2055 \pm 188$ & 9.16 & $1415 \pm 85$ & 5.98 \\
\hline $4471 \mathrm{HeI}$ & 0.106 & - & - & $631 \pm 67$ & 10.60 & $427 \pm 26$ & 6.18 & $859 \pm 139$ & 16.19 & $476 \pm 25$ & 5.34 \\
\hline $4861 \mathrm{H} \beta$ & 0.000 & $10000 \pm 1047$ & 10.47 & $10000 \pm 176$ & 1.76 & $10000 \pm 55$ & 0.55 & $10000 \pm 262$ & 2.62 & $10000 \pm 101$ & 1.01 \\
\hline 4959 [Оіні] & -0.024 & $59921 \pm 2331$ & 3.89 & $20332 \pm 747$ & 3.67 & $16441 \pm 232$ & 1.41 & $17730 \pm 695$ & 3.92 & $21447 \pm 447$ & 2.08 \\
\hline 5007 [Öні & -0.035 & $222047 \pm 9509$ & 4.28 & $50453 \pm 1285$ & 2.55 & $49376 \pm 566$ & 1.15 & $42025 \pm 1203$ & 2.86 & $63522 \pm 854$ & 1.34 \\
\hline $5876 \mathrm{HeI}$ & -0.209 & - & - & $668 \pm 127$ & 19.02 & $1647 \pm 57$ & 3.45 & $240 \pm 25$ & 10.52 & $1182 \pm 51$ & 4.35 \\
\hline 6312 [SIII] & -0.278 & $657 \pm 111$ & 16.86 & $107 \pm 6$ & 5.48 & $139 \pm 37$ & 26.82 & $304 \pm 18$ & 6.08 & $192 \pm 76$ & 39.56 \\
\hline $6548[\mathrm{NII}]$ & -0.311 & - & - & $65 \pm 2$ & 2.46 & $97 \pm 11$ & 11.21 & $39 \pm 6$ & 14.35 & $78 \pm 10$ & 13.45 \\
\hline $6563 \mathrm{H} \alpha$ & -0.313 & $18898 \pm 302$ & 1.60 & $24804 \pm 143$ & 0.57 & $28500 \pm 46$ & 0.16 & $20859 \pm 215$ & 1.03 & $26604 \pm 77$ & 0.29 \\
\hline $6584[\mathrm{NII}]$ & -0.316 & $613 \pm 125$ & 20.30 & $53 \pm 7$ & 13.00 & $265 \pm 12$ & 4.47 & $148 \pm 14$ & 9.55 & $241 \pm 29$ & 11.90 \\
\hline $6678 \mathrm{HeI}$ & -0.329 & - & - & $205 \pm 46$ & 22.37 & $299 \pm 18$ & 5.91 & $340 \pm 91$ & 26.63 & $264 \pm 18$ & 6.64 \\
\hline 6717 [SII] & -0.334 & $1488 \pm 390$ & 26.21 & $201 \pm 39$ & 19.38 & $673 \pm 30$ & 4.41 & $482 \pm 90$ & 18.74 & $629 \pm 64$ & 10.20 \\
\hline 6731 [SII] & -0.336 & $551 \pm 196$ & 35.54 & $196 \pm 31$ & 15.74 & $532 \pm 4$ & 0.76 & $451 \pm 76$ & 16.80 & $464 \pm 64$ & 13.76 \\
\hline $7065 \mathrm{HeI}$ & -0.377 & - & - & $589 \pm 41$ & 6.96 & $289 \pm 14$ & 4.89 & $718 \pm 78$ & 10.94 & $377 \pm 15$ & 3.99 \\
\hline 7136 [ArIII] & -0.385 & $2417 \pm 413$ & 17.07 & $255 \pm 35$ & 13.60 & $542 \pm 27$ & 5.06 & $540 \pm 101$ & 18.63 & $642 \pm 52$ & 8.10 \\
\hline 7751 [ArIII] & -0.451 & - & - & $97 \pm 7$ & 7.19 & $129 \pm 7$ & 5.22 & $74 \pm 36$ & 48.37 & $109 \pm 7$ & 6.43 \\
\hline 9069 [SIII] & -0.561 & $1205 \pm 362$ & 30.07 & $323 \pm 34$ & 10.42 & $646 \pm 40$ & 6.23 & $773 \pm 280$ & 36.24 & $654 \pm 222$ & 33.93 \\
\hline $\mathrm{I}(\mathrm{H} \beta)\left(\mathrm{erg} \mathrm{seg}^{-1} \mathrm{~cm}^{-2}\right)$ & & $0.13 \times 10^{-1}$ & & $0.33 \times 10^{-}$ & & $0.97 \times 10^{-}$ & & $0.16 \times 10^{-}$ & & $1.59 \times 10$ & \\
\hline $\mathrm{c}(\mathrm{H} \beta)$ & & 0.00 & & 0.00 & & $0.04 \pm 0$. & & 0.00 & & $0.00 \pm 0$ & \\
\hline$-\mathrm{EW}(\mathrm{H} \beta)$ & & 4.97 & & 2.84 & & 8.34 & & 1.60 & & 17.75 & \\
\hline
\end{tabular}


- Mrk600 BroteB

Por último, el Nudo B de Mrk 600 es el segundo de los brotes estudiados en esta galaxia y presenta el espectro con los perfiles cinemáticos más simples analizados. La mejor solución compuesta por una componente angosta y una componente ancha representa el perfil global de las líneas con errores entre el $2 \%$ en los mejores casos y el $\approx 30 \%$ en los casos de líneas con menores relaciones $\mathrm{S} / \mathrm{N}$ (ver Tabla 4.11). Las soluciones cinemáticas calculadas para las líneas de $\mathrm{H} \alpha$ y [OIII] las hemos podido ajustar en las líneas más débiles con un gran acuerdo y los estudios por componente pudieron ser realizados gracias a la gran cantidad de líneas que medimos. Este Nudo es el único de la muestra estudiada en la que pudimos medir y descomponer en múltiples componentes a la línea auroral del $[\mathrm{Sir}] \alpha 4068 \AA$, y junto a las de $[\mathrm{Sin}] 6312 \AA$ y [OIII] $4363 \AA$, hemos podido determinar las temperaturas electrónicas para la nebulosa y para estos iones de una manera directa. Las abundancias totales del $\mathrm{O} / \mathrm{H}$ determinadas para esta región están en acuerdo con las abundancias del Brote A: $7.54 \pm 0.03$ para N1 $(\approx 14$ veces menor que la abundancia solar), $8.19 \pm 0.04$ para la ancha $(\approx 3.2$ veces menor que la abundancia solar) y $7.71 \pm 0.02(\approx 10$ veces menor que la abundancia solar) para la global. El valor de N1 indica una componente de gas menos evolucianado, mientras que la ancha sería un gas más evolucionado.

Las densidades estimadas a partir del doblete del azufre nos indica bajas densidades 10 , $44 \mathrm{~cm}^{-3}$ para la N1 y la ancha, respectivamente, y $52 \mathrm{~cm}^{-3}$ para la componente global, lo que indica componentes con bajas densidades. Los enrojecimientos estimados a partir de $\mathrm{c}(\mathrm{H} \beta)$ son nulos, es decir que componentes no se ven afectadas por la extinción del polvo. En estos casos puntualmente, al realizar la corrección por enrojecimiento de las líneas y así determinar la constante $\mathrm{c}(\mathrm{H} \beta)$, los valores estimados eran negativos. En estos casos asignamos el valor de la constante nulo y suponer así que no hay atenuación por el polvo.

Fue el único Nudo en el que se pudo estimar la abundancia total de He a partir de las líneas débiles de HeI y de la línea débil de alta excitación de HeI en 4868^. Esta línea débil es muy difícil de medir y depende de la estructura de ionización de la nebulosa y se requiere de espectros de buena calidad para poder detectarla (Hägele et al., 2008). Las abundancias totales de $\mathrm{He} / \mathrm{H}$ muestran un excelente acuerdo entre componentes y la medida global, siendo la componente ancha la que presenta mayor abundancia por una diferencia de 0.02 dex.

Las abundancias totales de los elementos más importantes respecto al oxígeno muestran un buen acuerdo entre las componentes cinemáticas, salvo en algunos cosos puntuales en donde alguno de los cocientes presenta valores más altos en relación a las demás componentes.

Para el N/O (ver Figura 4.7) encontramos que los cocientes se encuentran, dentro de los valores esperados, por debajo de los puntos en la distribución, es decir con valores de N/O menores. Es el caso de la componente ancha en el S/O y Ar/O (ver Figuras 4.8 y 4.10, respectivamente), donde los cocientes de la componente ancha son los que se ubican hacia el centro de los diagramas, mientras que las otras componentes al tener metalicidades menores las podemos encontrar hacia la izquierda de las gráficas. Mientras que para el Ne/O (ver Figura 4.9) encontramos un excelente acuerdo para cada una de las componentes con referencia a los valores de la muestra, del orden de la abundancia $\mathrm{Ne} / \mathrm{O} \odot$. La diferencia encontrada entre los distintos elementos en los cocientes $\mathrm{N} / \mathrm{O}$ y $\mathrm{Ne} / \mathrm{O}$, es un poco llamativa. Los valores relativos al nitrógeno son bajos, mientras que los del neón aparecen entorno al valor solar, lo que resulta llamativo debido a que 
Tabla 4.10. Propiedades físicas (densidades electrónicas $\mathrm{n}_{e}$ y temperaturas electrónicas $\mathrm{T}_{e}$ ) y abundancias químicas iónicas y totales de Mrk600 A, derivadas de líneas prohibidas y las líneas de recombinación de helio.

\begin{tabular}{|c|c|c|c|c|c|}
\hline & Ancha & N1 & N2 & N3 & Global \\
\hline$n_{e}$ & 10: & 10: & $91 \pm 44$ & $127 \pm 32$ & 100: \\
\hline $\mathrm{t}_{e}[\mathrm{OIII}]$ & $1.61 \pm 0.07$ & $1.72 \pm 0.03$ & $1.47 \pm 0.06$ & $2.46 \pm 0.16$ & $1.61 \pm 0.05$ \\
\hline $\mathrm{t}_{e}[\mathrm{SIII}]$ & $1.67 \pm 0.33$ & $1.60 \pm 0.24$ & $1.43 \pm 0.17$ & $2.21 \pm 0.13$ & $1.59 \pm 0.18$ \\
\hline $\mathrm{t}_{e}[\mathrm{OHI}]$ & $1.43^{a}$ & $1.47^{a}$ & $1.35^{a}$ & $1.67^{a}$ & $1.39^{a}$ \\
\hline $\mathrm{t}_{e}[\mathrm{SII}]$ & $1.43^{a}$ & $1.47^{a}$ & $1.35^{a}$ & $1.67^{a}$ & $1.39^{a}$ \\
\hline $\mathrm{t}_{e}[\mathrm{NII}]$ & $1.43^{a}$ & $1.47^{a}$ & $1.35^{a}$ & $1.67^{a}$ & $1.39^{a}$ \\
\hline $12+\log \left(\mathrm{O}^{+} / \mathrm{H}^{+}\right)$ & $6.73 \pm 0.07$ & $6.97 \pm 0.05$ & $7.00 \pm 0.04$ & $6.81 \pm 0.04$ & $6.91 \pm 0.05$ \\
\hline $12+\log \left(\mathrm{O}^{2+} / \mathrm{H}^{+}\right)$ & $8.26 \pm 0.06$ & $7.59 \pm 0.03$ & $7.72 \pm 0.05$ & $7.21 \pm 0.06$ & $7.74 \pm 0.04$ \\
\hline $12+\log (\mathrm{O} / \mathrm{H})$ & $8.27 \pm 0.06$ & $7.68 \pm 0.03$ & $7.80 \pm 0.05$ & $7.36 \pm 0.06$ & $7.80 \pm 0.04$ \\
\hline $12+\log \left(\mathrm{S}^{+} / \mathrm{H}^{+}\right)$ & 5.23 & 4.90 & 5.17 & 5.04 & 5.10 \\
\hline $12+\log \left(\mathrm{S}^{2+} / \mathrm{H}^{+}\right)$ & $5.46 \pm 0.29$ & $5.05 \pm 0.12$ & $5.52 \pm 0.34$ & $5.37 \pm 0.29$ & $5.40 \pm 0.55$ \\
\hline $\mathrm{ICF}\left(\mathrm{S}^{+}+\mathrm{S}^{2+}\right)$ & $2.90 \pm 0.63$ & $1.42 \pm 0.02$ & $1.52 \pm 0.02$ & $1.26 \pm 0.01$ & $1.64 \pm 0.01$ \\
\hline $12+\log (\mathrm{S} / \mathrm{H})$ & $6.12 \pm 0.25$ & $5.43 \pm 0.11$ & $5.86 \pm 0.27$ & $5.64 \pm 0.24$ & $5.79 \pm 0.44$ \\
\hline $\log (\mathrm{S} / \mathrm{O})$ & $-2.15 \pm 0.26$ & $-2.25 \pm 0.11$ & $-1.93 \pm 0.27$ & $-1.72 \pm 0.24$ & $-2.01 \pm 0.44$ \\
\hline $12+\log \left(\mathrm{N}^{+} / \mathrm{H}^{+}\right)$ & 5.59 & 5.14 & 5.42 & 5.10 & 5.33 \\
\hline $12+\log (\mathrm{N} / \mathrm{H})$ & 7.13 & 5.86 & 6.22 & 5.65 & 6.22 \\
\hline $\log (\mathrm{N} / \mathrm{O})$ & -1.13 & -1.83 & -1.58 & -1.71 & -1.57 \\
\hline $12+\log \left(\mathrm{Ne}^{2+} / \mathrm{H}^{+}\right)$ & $7.72 \pm 0.14$ & $6.90 \pm 0$ & $7.14 \pm 0.08$ & $6.79 \pm 0.07$ & $7.16 \pm 0.06$ \\
\hline $\mathrm{ICF}\left(\mathrm{Ne}^{2+}\right)$ & $1.07 \pm 0.01$ & $1.08 \pm 0.01$ & $1.08 \pm 0.01$ & $1.09 \pm 0.01$ & $1.07 \pm 0.01$ \\
\hline $12+\log (\mathrm{Ne} / \mathrm{H})$ & $7.75 \pm 0.14$ & $6.93 \pm 0.08$ & $7.18 \pm 0.08$ & $6.82 \pm 0.07$ & $7.19 \pm 0.06$ \\
\hline $\log (\mathrm{Ne} / \mathrm{O})$ & $-0.52 \pm 0.16$ & $-0.76 \pm 0.08$ & $-0.62 \pm 0.09$ & $-0.53 \pm 0.09$ & $-0.61 \pm 0.08$ \\
\hline$\left.{ }^{2+} / \mathrm{H}^{+}\right)$ & $5.34 \pm 0$ & $4.59 \pm 0$ & $5.13 \pm 0.15$ & $4.84 \pm 0.23$ & $5.05 \pm 0.27$ \\
\hline $\mathrm{ICF}\left(\mathrm{Ar}^{2+}\right)$ & $3.00 \pm 0.25$ & $1.18 \pm 0.01$ & $1.23 \pm 0.01$ & $1.12 \pm 0.01$ & $1.31 \pm 0.01$ \\
\hline $12+\log (\mathrm{Ar} / \mathrm{H})$ & $5.82 \pm 0.26$ & $4.66 \pm 0.11$ & $5.22 \pm 0.15$ & $4.89 \pm 0.23$ & $5.17 \pm 0.27$ \\
\hline $\log (\mathrm{Ar} / \mathrm{O})$ & $-2.45 \pm 0.22$ & $-2.72 \pm 0.11$ & $-2.57 \pm 0.16$ & $-2.57 \pm 0.24$ & $-2.63 \pm 0.27$ \\
\hline $\mathrm{He}^{+} / \mathrm{H}^{+}$ & & & & & \\
\hline 4471 & 一 & $0.131 \pm 0.010$ & $0.089 \pm 0.005$ & $0.181 \pm 0.022$ & $0.101 \pm 0.004$ \\
\hline 5876 & - & $0.049 \pm 0.009$ & $0.130 \pm 0.004$ & $0.018 \pm 0.002$ & $0.094 \pm 0.004$ \\
\hline 6678 & - & $0.058 \pm 0.013$ & $0.084 \pm 0.004$ & $0.104 \pm 0.027$ & $0.076 \pm 0.005$ \\
\hline 7065 & - & $0.149 \pm 0.012$ & $0.101 \pm 0.007$ & $0.163 \pm 0.024$ & $0.125 \pm 0.007$ \\
\hline adoptado & - & $0.095 \pm 0.044$ & $0.105 \pm 0.018$ & $0.115 \pm 0.021$ & $0.095 \pm 0.018$ \\
\hline
\end{tabular}

donde $n_{e}$ corresponde a la densidad en $\mathrm{cm}^{-3}$ y $\mathrm{T}_{e}$ a la temperatura en $10^{4} \mathrm{k}$,

${ }^{a}$ utilizando temperaturas de modelos $\mathrm{y}{ }^{b}$ suponiendo una temperatura inicial de $10000 \mathrm{k}$, 
Tabla 4.11. Intensidad de las líneas corregidas por enrojecimiento para el Nudo B de Mrk 600 $[\mathrm{F}(\mathrm{H} \beta)=\mathrm{I}(\mathrm{H} \beta)=10.000]$.

\begin{tabular}{|c|c|c|c|c|c|c|c|}
\hline \multirow[b]{2}{*}{$\lambda(\AA)$} & \multicolumn{3}{|c|}{ Ancha } & \multicolumn{2}{|l|}{ N1 } & \multicolumn{2}{|c|}{ Global } \\
\hline & $f(\lambda)$ & $I(\lambda)$ & $\begin{array}{c}\text { Error } \\
(\%)\end{array}$ & $I(\lambda)$ & $\begin{array}{c}\text { Error } \\
(\%)\end{array}$ & $I(\lambda)$ & $\begin{array}{c}\text { Error } \\
(\%)\end{array}$ \\
\hline 3726 [OII] & 0.272 & $15409 \pm 742$ & 4.82 & $6663 \pm 95$ & 1.42 & $7990 \pm 134$ & 1.68 \\
\hline $3727{\text { [OII }]^{b}}^{b}$ & 0.271 & $36981 \pm 1172$ & 3.17 & $15880 \pm 636$ & 4.01 & $19126 \pm 192$ & 1.00 \\
\hline 3729 [OІІ] & 0.271 & $21572 \pm 884$ & 4.10 & $9183 \pm 111$ & 1.21 & $11068 \pm 156$ & 1.41 \\
\hline 3868 [NeIII] & 0.238 & $18113 \pm 656$ & 3.62 & $3349 \pm 76$ & 2.26 & $5621 \pm 107$ & 1.90 \\
\hline 4068 [SII] & 0.195 & $342 \pm 23$ & 6.81 & $89 \pm 12$ & 13.06 & $128 \pm 30$ & 23.64 \\
\hline $4102 \mathrm{H} \delta$ & 0.188 & $5459 \pm 704$ & 12.90 & $2693 \pm 72$ & 2.66 & $3107 \pm 141$ & 4.53 \\
\hline $4340 \mathrm{H} \gamma$ & 0.142 & $8302 \pm 272$ & 3.27 & $4672 \pm 42$ & 0.90 & $5223 \pm 55$ & 1.05 \\
\hline 4363 [OIII] & 0.138 & $3157 \pm 136$ & 4.32 & $643 \pm 19$ & 2.90 & $1029 \pm 26$ & 2.50 \\
\hline $4471 \mathrm{HeI}$ & 0.106 & $1415 \pm 174$ & 12.26 & $231 \pm 26$ & 11.05 & $414 \pm 34$ & 8.29 \\
\hline 4686 HeII & 0.045 & $101 \pm 19$ & 19.13 & $26 \pm 4$ & 16.42 & $38 \pm 4$ & 10.59 \\
\hline $4861 \mathrm{H} \beta$ & 0.000 & $10000 \pm 887$ & 8.87 & $10000 \pm 140$ & 1.40 & $10000 \pm 182$ & 1.82 \\
\hline 4959 [Oіні] & -0.024 & $38805 \pm 3190$ & 8.22 & $10081 \pm 490$ & 4.86 & $14466 \pm 643$ & 4.44 \\
\hline 5007 [Oıн] & -0.035 & $130189 \pm 2089$ & 1.60 & $28539 \pm 316$ & 1.11 & $44951 \pm 418$ & 0.93 \\
\hline $5876 \mathrm{HeI}$ & -0.209 & $1516 \pm 95$ & 6.29 & $993 \pm 168$ & 16.92 & $1068 \pm 199$ & 18.64 \\
\hline 6312 [SIII] & -0.278 & $564 \pm 118$ & 20.90 & $61 \pm 2$ & 4.03 & $139 \pm 35$ & 24.91 \\
\hline $6548[\mathrm{NII}]$ & -0.311 & $374 \pm 109$ & 29.22 & $61 \pm 9$ & 14.81 & $110 \pm 26$ & 23.55 \\
\hline $6563 \mathrm{H} \alpha$ & -0.313 & $27799 \pm 824$ & 2.96 & $23936 \pm 133$ & 0.56 & $24466 \pm 170$ & 0.69 \\
\hline $6584[\mathrm{NII}]$ & -0.316 & $767 \pm 183$ & 23.88 & $278 \pm 54$ & 19.31 & $353 \pm 25$ & 6.97 \\
\hline $6678 \mathrm{HeI}$ & -0.329 & $367 \pm 113$ & 30.69 & $224 \pm 28$ & 12.64 & $247 \pm 26$ & 10.70 \\
\hline 6717 [SII] & -0.334 & $2585 \pm 168$ & 6.50 & $753 \pm 22$ & 2.95 & $1029 \pm 30$ & 2.95 \\
\hline 6731 [SII] & -0.336 & $2270 \pm 252$ & 11.10 & $499 \pm 36$ & 7.13 & $772 \pm 48$ & 6.25 \\
\hline $7065 \mathrm{HeI}$ & -0.377 & $1050 \pm 246$ & 23.44 & $61 \pm 14$ & 23.02 & $213 \pm 20$ & 9.32 \\
\hline 7136 [ArIII] & -0.385 & $1384 \pm 206$ & 14.90 & $364 \pm 39$ & 10.70 & $520 \pm 33$ & 6.37 \\
\hline 7751 [ArIII] & -0.451 & $496 \pm 70$ & 14.11 & $60 \pm 17$ & 29.11 & $401 \pm 14$ & 3.41 \\
\hline 9069 [SIII] & -0.561 & $2289 \pm 258$ & 11.29 & $285 \pm 50$ & 17.50 & $594 \pm 31$ & 5.28 \\
\hline $\mathrm{I}(\mathrm{H} \beta)\left(\mathrm{erg} \mathrm{seg}^{-1} \mathrm{~cm}^{-2}\right)$ & & $0.16 \times 10^{-}$ & & $0.87 \times 10$ & & $1.03 \times 10$ & \\
\hline $\mathrm{c}(\mathrm{H} \beta)$ & & 0.00 & & 0.00 & & 0.00 & \\
\hline$-\mathrm{EW}(\mathrm{H} \beta)$ & & 4.85 & & 23.66 & & 28.51 & \\
\hline
\end{tabular}

habría una falta de $\mathrm{N}$.

\subsubsection{Comparación de los resultados determinados entre nudos}

\section{- II Zw 33}

Las abundancias totales de oxígeno derivadas para las componentes cinemáticas y para la medida global tienen un muy buen acuerdo con valores típicos de estas regiones. Se determinó la temperatura electrónica a partir de una estimación directa a través de las líneas aurorales del [OIII] en los Nudos A y B, mientras que en el Nudo C no fue posible estimar temperaturas y se utilizó la temperatura típica para esta clase de objetos igual a los 10.000k. Las abundancias $12+\log (\mathrm{O} / \mathrm{H})$ de $8.26 \pm 0.05$ (ancha) y $8.28 \pm 0.10(\mathrm{~N} 1)$, $8.28 \pm 0.09(\mathrm{~N} 2)$ y $8.28 \pm 0.08$ (Global) muestran un excelente acuerdo entre sí para el Nudo A. Estos resultados de abundancias totales encontrados están en acuerdo con los objetos de esta clase. Si comparamos con el Nudo B se determinaron abundancias totales de oxígeno con un mayor rango, $8.19 \leq 12+\log (\mathrm{O} / \mathrm{H}) \leq 8.51$, existiendo acuerdo entre 
Tabla 4.12. Propiedades físicas (densidades electrónicas $\mathrm{n}_{e} \mathrm{y}$ temperaturas electrónicas $\mathrm{T}_{e}$ ) y abundancias químicas iónicas y totales de Mrk 600 B, derivadas de líneas prohibidas y las líneas de recombinación de helio.

\begin{tabular}{|c|c|c|c|}
\hline & \multicolumn{3}{|c|}{ Mrk 600 Nudo B } \\
\hline & Ancha & N1 & Global \\
\hline$n_{e}$ & $44 \pm 32$ & 10: & 52: \\
\hline $\mathrm{t}_{e}[\mathrm{OIII}]$ & $1.70 \pm 0.05$ & $1.61 \pm 0.04$ & $1.64 \pm 0.02$ \\
\hline $\mathrm{t}_{e}[\mathrm{SIII}]$ & $1.71 \pm 0.28$ & $1.59 \pm 0.17$ & $1.63 \pm 0.23$ \\
\hline $\mathrm{t}_{e}[\mathrm{SII}]$ & $0.95 \pm 0.10$ & $1.18 \pm 0.14$ & $1.11 \pm 0.31$ \\
\hline $\mathrm{t}_{e}[\mathrm{OII}]$ & $1.46^{a}$ & $1.42^{a}$ & $1.45^{a}$ \\
\hline $\mathrm{t}_{e}[\mathrm{NII}]$ & $1.46^{a}$ & $1.42^{a}$ & $1.45^{a}$ \\
\hline $12+\log \left(\mathrm{O}^{+} / \mathrm{H}^{+}\right)$ & $7.78 \pm 0.04$ & $6.99 \pm 0.03$ & $7.17 \pm 0.02$ \\
\hline $12+\log \left(\mathrm{O}^{2+} / \mathrm{H}^{+}\right)$ & $7.98 \pm 0.04$ & $7.40 \pm 0.03$ & $7.56 \pm 0.02$ \\
\hline $12+\log (\mathrm{O} / \mathrm{H})$ & $8.19 \pm 0.04$ & $7.54 \pm 0.03$ & $7.71 \pm 0.02$ \\
\hline $12+\log \left(\mathrm{S}^{+} / \mathrm{H}^{+}\right)$ & $5.42 \pm 0.24$ & $5.32 \pm 0.13$ & $5.53 \pm 0.19$ \\
\hline $12+\log \left(\mathrm{S}^{2+} / \mathrm{H}^{+}\right)$ & $6.01 \pm 0.28$ & $5.17 \pm 0.17$ & $5.45 \pm 0.31$ \\
\hline $\mathrm{ICF}\left(\mathrm{S}^{+}+\mathrm{S}^{2+}\right)$ & 1.15 & 1.26 & 1.25 \\
\hline $12+\log (\mathrm{S} / \mathrm{H})$ & $6.44 \pm 0.27$ & $5.65 \pm 0.10$ & $5.89 \pm 0.26$ \\
\hline $\log (\mathrm{S} / \mathrm{O})$ & $-1.75 \pm 0.28$ & $-1.89 \pm 0.10$ & $-1.82 \pm 0.26$ \\
\hline $12+\log \left(\mathrm{N}^{+} / \mathrm{H}^{+}\right)$ & 5.99 & 5.21 & 5.40 \\
\hline $12+\log (\mathrm{N} / \mathrm{H})$ & 6.40 & 5.76 & 5.94 \\
\hline $\log (\mathrm{N} / \mathrm{O})$ & -1.79 & -1.78 & -1.77 \\
\hline $12+\log \left(\mathrm{Ne}^{2+} / \mathrm{H}^{+}\right)$ & $7.54 \pm 0.05$ & $6.88 \pm 0.03$ & $7.08 \pm 0.03$ \\
\hline $\mathrm{ICF}\left(\mathrm{Ne}^{2+}\right)$ & $1.12 \pm 0.01$ & $1.09 \pm 0.01$ & $1.09 \pm 0.01$ \\
\hline $12+\log (\mathrm{Ne} / \mathrm{H})$ & $7.59 \pm 0.05$ & $6.92 \pm 0.03$ & $7.12 \pm 0.03$ \\
\hline $\log (\mathrm{Ne} / \mathrm{O})$ & $-0.60 \pm 0.06$ & $-0.62 \pm 0.04$ & $-0.59 \pm 0.03$ \\
\hline $12+\log \left(\mathrm{Ar}^{2+} / \mathrm{H}^{+}\right)$ & $5.47 \pm 0.17$ & $4.96 \pm 0.13$ & $5.07 \pm 0.15$ \\
\hline $\operatorname{ICF}\left(\mathrm{Ar}^{2+}\right)$ & $1.11 \pm 0.01$ & $1.12 \pm 0.01$ & $1.12 \pm 0.01$ \\
\hline $12+\log (\mathrm{Ar} / \mathbf{H})$ & $5.52 \pm 0.17$ & $5.01 \pm 0.13$ & $5.12 \pm 0.15$ \\
\hline $\log (\mathrm{Ar} / \mathrm{O})$ & $-2.68 \pm 0.11$ & $-2.53 \pm 0.13$ & $-2.59 \pm 0.15$ \\
\hline $\mathrm{He}^{+} / \mathrm{H}^{+}$ & & & \\
\hline 4471 & $0.079 \pm 0.002$ & $0.080 \pm 0.005$ & $0.088 \pm 0.007$ \\
\hline 5876 & $0.117 \pm 0.007$ & $0.081 \pm 0.013$ & $0.086 \pm 0.016$ \\
\hline 6678 & $0.101 \pm 0.006$ & $0.064 \pm 0.008$ & $0.072 \pm 0.007$ \\
\hline 7065 & $0.055 \pm 0.007$ & $0.064 \pm 0.005$ & $0.074 \pm 0.007$ \\
\hline adopted & $0.081 \pm 0.024$ & $0.080 \pm 0.024$ & $0.079 \pm 0.007$ \\
\hline $\mathrm{He}^{2+} / \mathrm{H}^{+}$ & & & \\
\hline 4686 & $0.0009 \pm 0.0001$ & 0.0002: & 0.0003: \\
\hline$(\mathbf{H e} / \mathbf{H})$ & $0.082 \pm 0.024$ & $0.080 \pm 0.011$ & $0.079 \pm 0.007$ \\
\hline
\end{tabular}

donde $n_{e}$ corresponde a la densidad en $\mathrm{cm}^{-3}$ y $\mathrm{T}_{e}$ a la temperatura en $10^{4} \mathrm{k}$,

${ }^{a}$ utilizando temperaturas de modelos $\mathrm{y}^{b}$ suponiendo una temperatura inicial de $10000 \mathrm{k}$, 


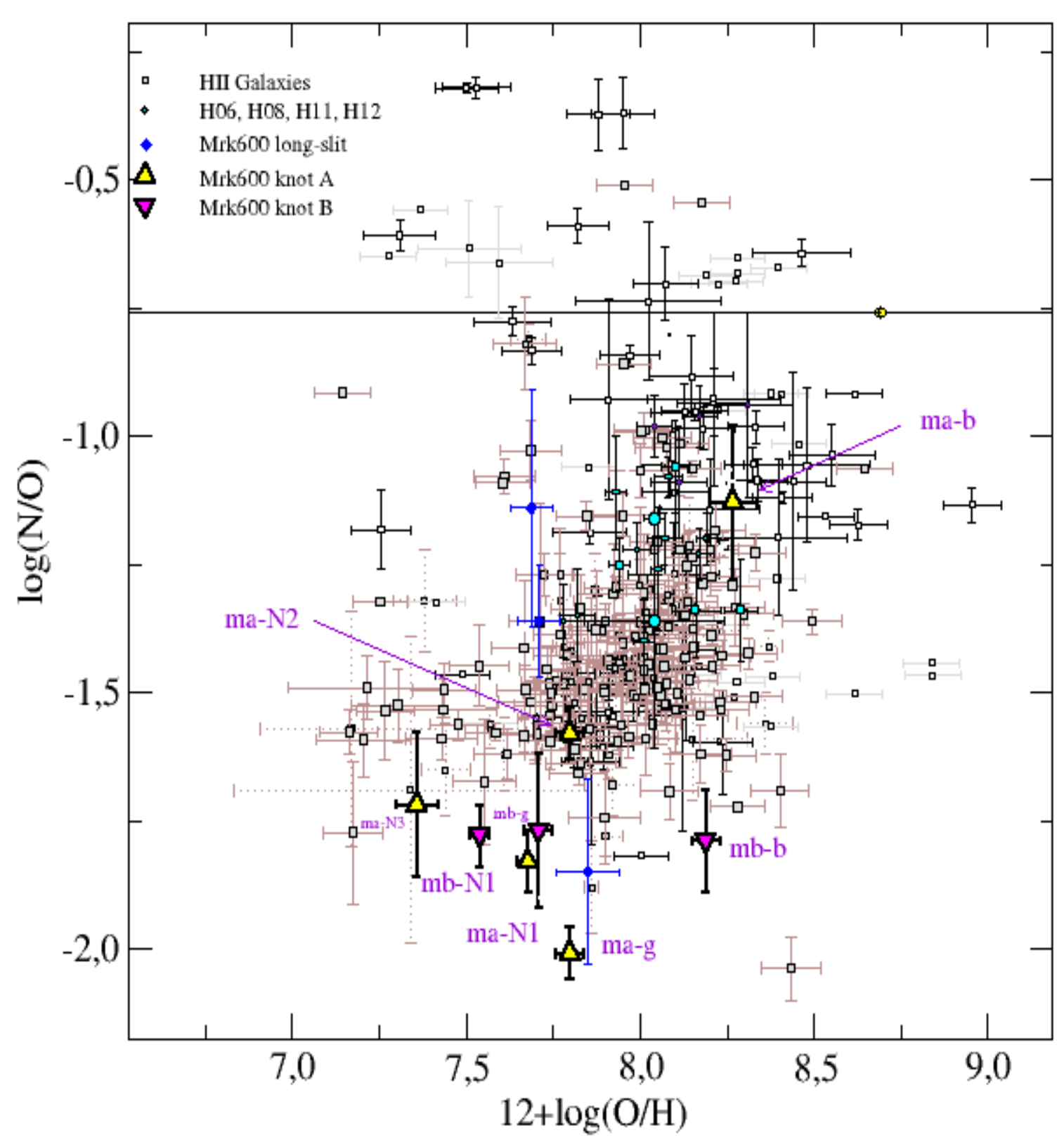

Figura 4.7. $\mathrm{N} / \mathrm{O}$ vs. $12+\log (\mathrm{O} / \mathrm{H})$ para los nudos de Mrk 600 (triángulos rojos y rosas respectivamente), galaxias y RHII de la literatura utilizados en publicaciones previas de nuestro grupo y las regiones estudiadas en los trabajos de Hägele et al. (2006, 2008, 2011, 2012) (círculos grises). Además se indican los valores de abundancias relativas al oxígeno de II Zw 33 obtenidos con dispersión simple con diamantes rojos (ver Campuzano-Castro et al., 2015). Las regiones estudiadas en este trabajo se indican como ma y mb, haciendo referencia a Mrk A y Mrk B respectivamente y las distintas componentes se indican como: $\mathrm{b}=$ ancha, $\mathrm{N} 1=\operatorname{angosta} 1, \mathrm{~N} 2=\operatorname{angosta} 2, \mathrm{~N} 3=\operatorname{angosta} 3 \mathrm{y} \mathrm{g}=$ medida Global. El valor solar se muestra con el símbolo usual de referencia al sol. 


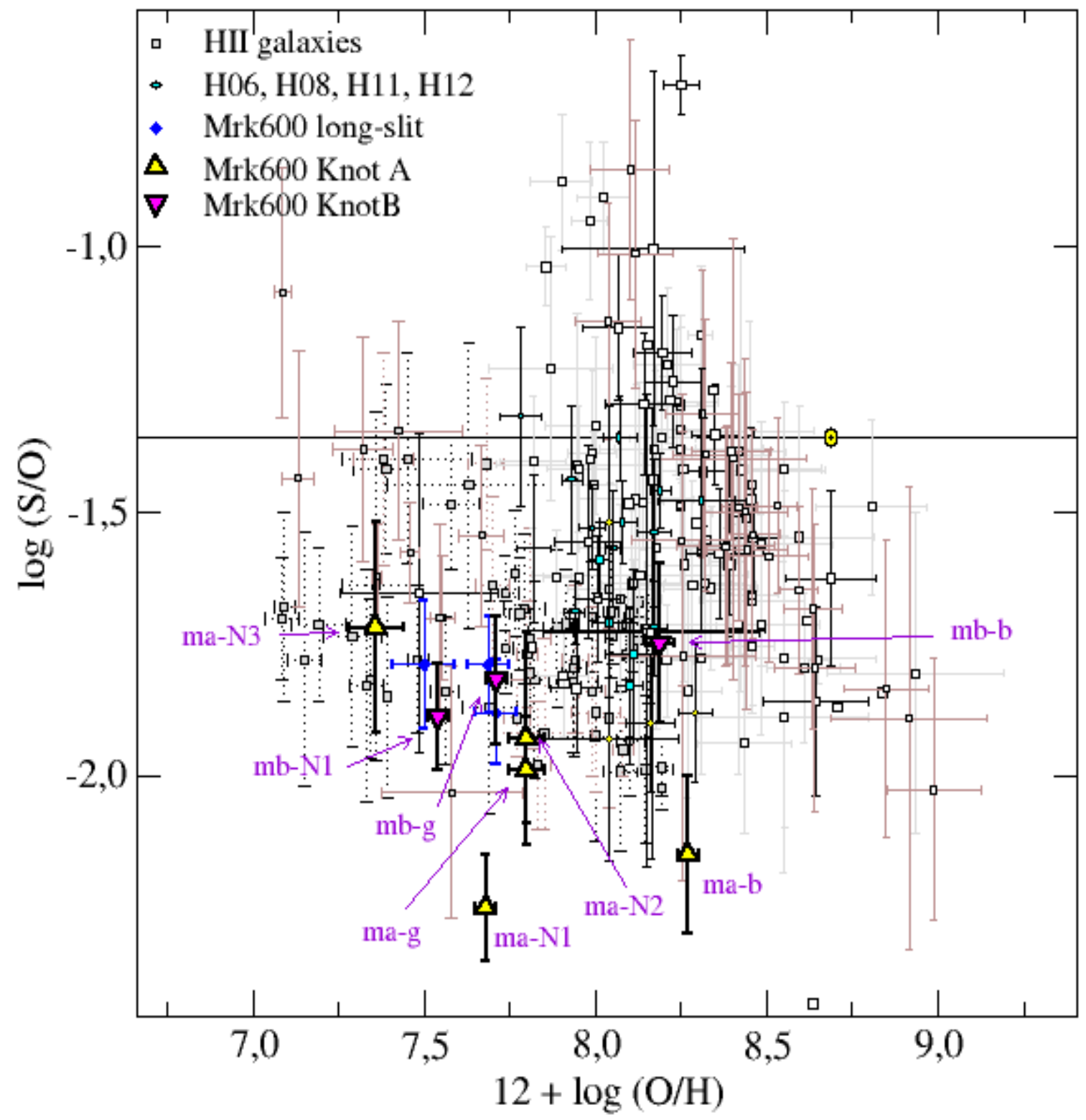

Figura 4.8. $\mathrm{S} / \mathrm{O}$ vs. $12+\log (\mathrm{O} / \mathrm{H})$ para los nudos de la galaxia Mrk 600 (triángulos rosas respectivamente), objetos de la literatura utilizados en publicaciones previas de nuestro grupo (cuadrados grises, Pérez-Montero et al., 2007) y los objetos de los trabajos publicados por Hägele et al. (2006, 2008, 2011, 2012)(círculos celestes). Además agregamos los valores de las abundancias relativas determinadas para los Nudos de esta galaxia observados con rendija larga con diamantes rosas (ver Campuzano-Castro et al., 2015). Las regiones estudiadas en este trabajo se indican como ma y mb, haciendo referencia a Mrk A y Mrk B respectivamente $\mathrm{y}$ las distintas componentes se indican como: $\mathrm{b}=$ ancha, $\mathrm{N} 1=\operatorname{angosta} 1, \mathrm{~N} 2=$ angosta 2, N3 = angosta 3 y g = medida Global. El valor solar se muestra con el símbolo usual de referencia al Sol. 


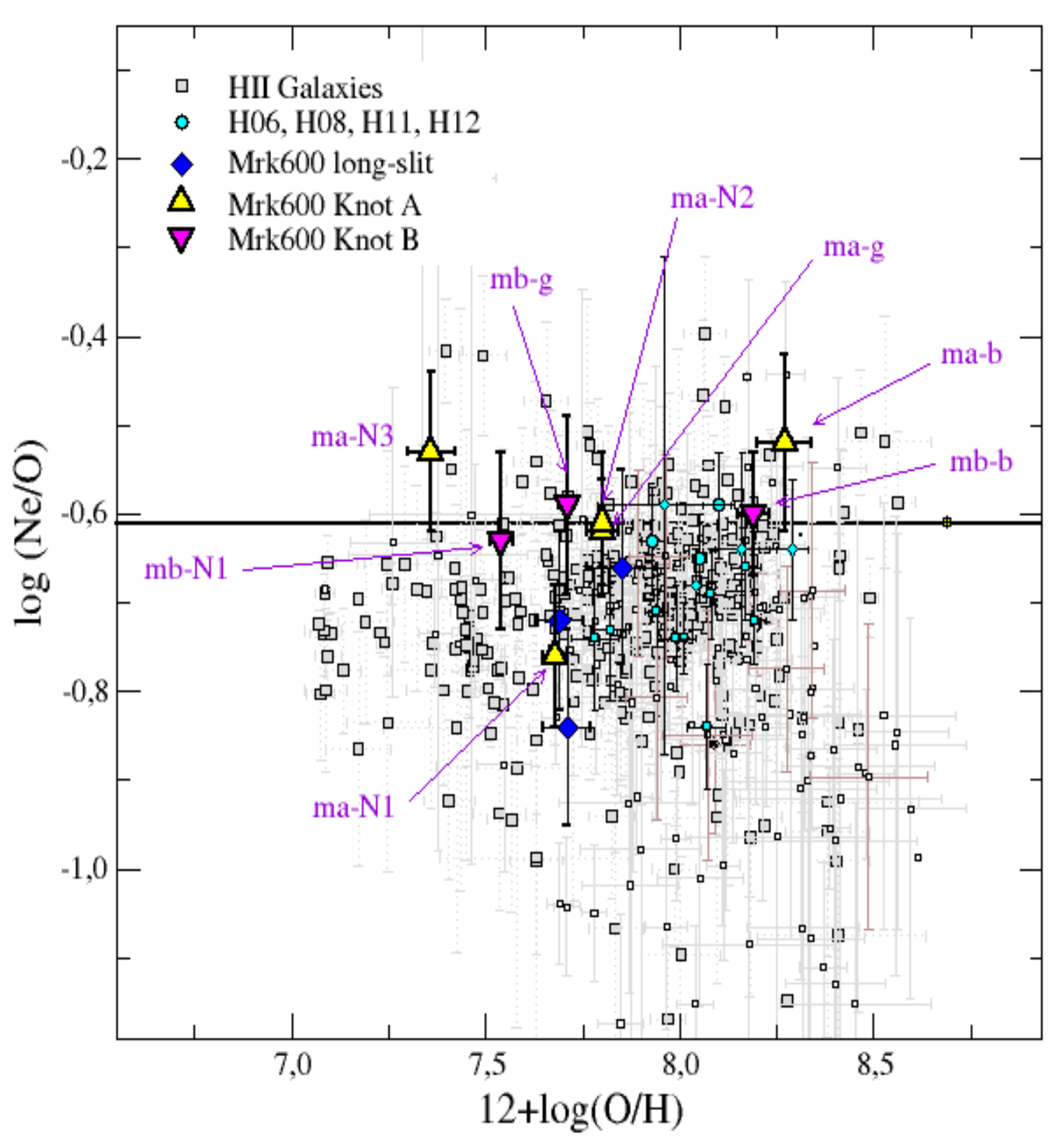

Figura 4.9. $\mathrm{Ne} / \mathrm{O}$ vs. $12+\log (\mathrm{O} / \mathrm{H})$ para los nudos de Mrk600, objetos de la literatura utilizados en publicaciones previas de nuestro grupo (cuadrados grises, Pérez-Montero et al., 2007) y los objetos de los trabajos publicados por Hägele et al. (2006, 2008, 2011, 2012)(círculos celestes). Además agregamos los valores de las abundancias relativas determinadas para los Nudos de esta galaxia observados con rendija larga con diamantes rosas (ver Campuzano-Castro et al., 2015). Las regiones estudiadas en este trabajo se indican como ma y mb, haciendo referencia a Mrk A y Mrk B respectivamente y las distintas componentes se indican como: $\mathrm{b}=$ ancha, $\mathrm{N} 1=$ angosta $1, \mathrm{~N} 2=\operatorname{angosta} 2, \mathrm{~N} 3=\operatorname{angosta} 3 \mathrm{y} \mathrm{g}=$ medida Global. El valor solar se muestra con el símbolo usual de referencia al Sol. 


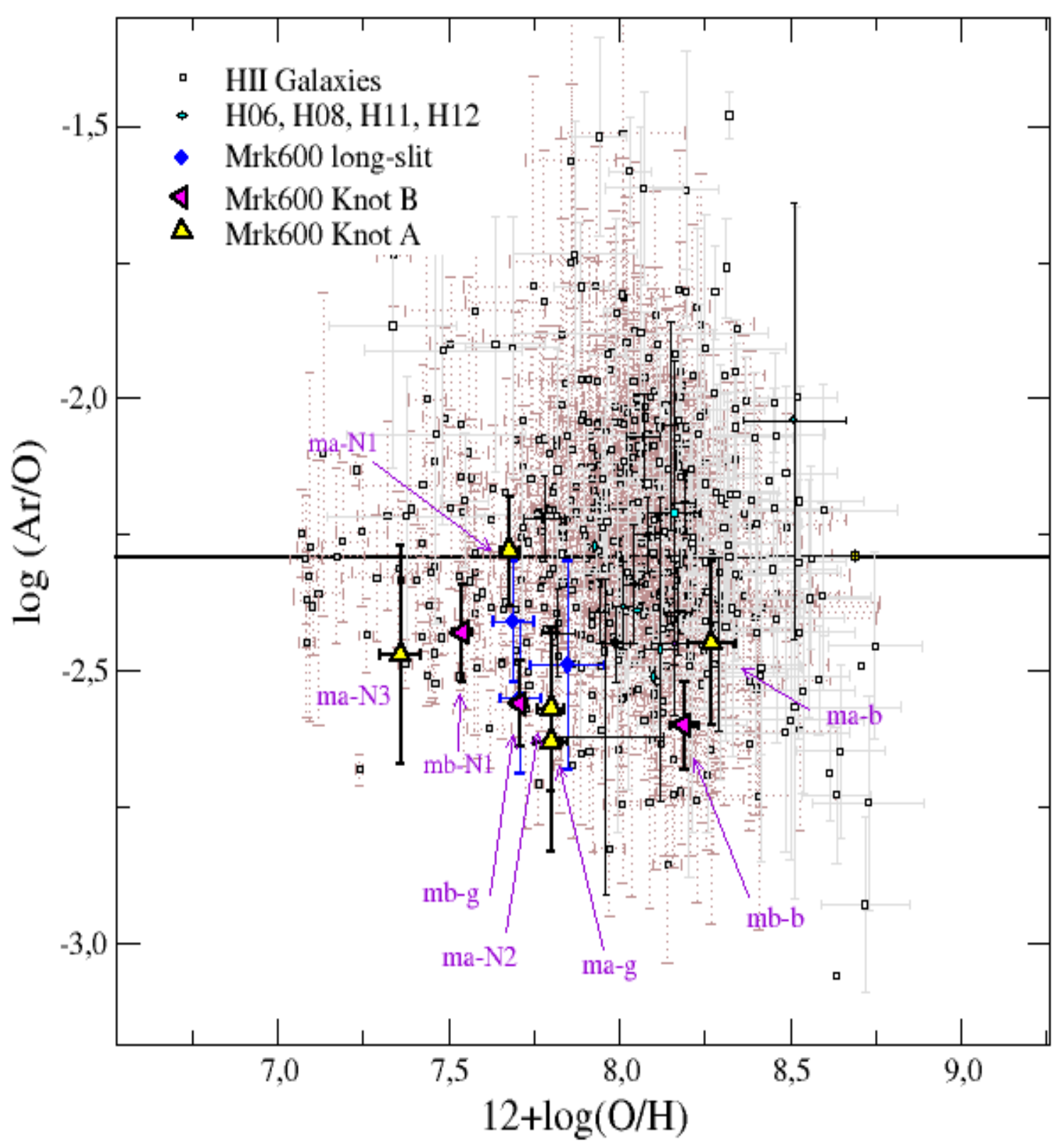

Figura 4.10. $\mathrm{Ar} / \mathrm{O}$ vs. $12+\log (\mathrm{O} / \mathrm{H})$ para los nudos de Mrk 600 (triángulos rojos y rombos rosas respectivamente), objetos de la literatura utilizados en publicaciones previas de nuestro grupo (cuadrados grises, Pérez-Montero et al., 2007) y los objetos de los trabajos publicados por Hägele et al. (2006, 2008, 2011, 2012)(círculos celestes). Además agregamos los valores de las abundancias relativas determinadas para los Nudos de esta galaxia observados con rendija larga con diamantes rosas (ver Campuzano-Castro et al., 2015). Las regiones estudiadas en este trabajo se indican como ma y mb, haciendo referencia a Mrk A y Mrk B respectivamente y las distintas componentes se indican como: $\mathrm{b}=$ ancha, $\mathrm{N} 1=\operatorname{angosta} 1, \mathrm{~N} 2=$ angosta 2, N3 = angosta 3 y g = medida Global. El valor solar se muestra con el símbolo usual de referencia al Sol. 
algunas componentes a $3 \sigma$, pero la componente ancha y la N1 se encuetran 0.11 dex, teniendo en cuentan los errores. Por el lado del Nudo C, las abundancias estimadas de $(\mathrm{O} / \mathrm{H})$ están entre $8.15 \leq 12+\log (\mathrm{O} / \mathrm{H}) \leq 8.32$, y presentan errores bajos porque son estimaciones hechas con temperaturas determinadas a partir de relaciones empíricas y modelos, y los resultados obtenidos son los esperables para este tipo de regiones.

En el Nudo A la temperatura electrónica de [OIII] estimada a través de las relaciones entre líneas intensas y débiles aurorales fue de $\mathrm{T}_{e}([\mathrm{OIII}])=10.600 \mathrm{k} \pm 300 \mathrm{k}$ para la global, $11.400 \mathrm{k} \pm 1.600 \mathrm{k}$ para la $\mathrm{N} 1$ y $11.700 \mathrm{k} \pm 500 \mathrm{k}$ para $\mathrm{N} 2$, estando en acuerdo si tenemos en cuenta los errores a $3 \sigma$. Para el Nudo B la estimación fue de $11.300 \mathrm{k} \pm 600 \mathrm{k}$ y $10.400 \mathrm{k} \pm 200 \mathrm{k}$ y $9.600 \mathrm{k} \pm 300 \mathrm{k}$, para N1, N2 y global respectivamente. Por el lado de la $\mathrm{T}_{e}([\mathrm{SIII}])$ determinada en el Nudo B, estimamos temperaturas levemente mayores para la N2 y para la medida global $(11.500 \mathrm{k} \pm 2.500 \mathrm{k}$ y $12.400 \mathrm{k} \pm 1.600 \mathrm{k}$, respectivamente), pero para la $\mathrm{T}_{e}$ de N1, la medida de $10.200 \pm 1.600 \mathrm{k}$, nos muestra un valor menor, pero dentro de los errores los valores estimados están en acuerdo. Los errores de la estimación de las temperaturas son altos debido a que la línea débil presenta un ajuste con errores entre un $20 \%$ y un $38 \%$.

Estudios realizados en esta galaxia por Méndez et al. (1999) sugieren que el cúmulo de estrellas ionizantes en el Brote B tendría la población más joven de la galaxia $(\approx 100$ Myr). Este nudo presenta un color azul y una alta emisión de fotones UV, lo que indica una actividad reciente de formación estelar, respaldada por la presencia de características Wolf-Rayet en su espectro (Mateo, 1998). Walter et al. (1997) destacan que a través de los espectros de baja resolución con los que ellos contaban no había presencia de múltiples componentes cinemáticas en los perfiles de sus líneas de emisión. En este trabajo utilizando los datos espectroscópicos de Echelle de alta resolución, podemos observar y determinar que efectivamente con resoluciones suficientes pueden estudiarse distintas componentes de gas ionizado y desarrollar el estudio quimiodinámico.

Las abundancias iónicas y totales de O, S, N, Ne y Ar derivadas en los Nudos A y $\mathrm{B}$ presentan valores muy similares, pero con errores grandes en la determinación de las abundancias del azufre debido a los posibles errores en el dato, que posiblemente afectaron las intensidades derivadas de sus medidas. Podemos decir que las diferencias encontradas en los Nudos de esta galaxia son similares a las que se han determinado en trabajos previos que han resuelto espacialmente regiones intensas (para más detalle ver Hägele et al., 2012). Posiblemente, el trabajo llevado adelante en las regiones de esta galaxia sea un primer estimador directo de información confiable de la cinemática y de las propiedades físicas en las regiones más intensas resueltas espacialmente, complementando el análisis de las regiones separadas espacialmente propuestas por Méndez et al. (1999).

\section{- Mrk 600}

Para esta galaxia, podemos comparar los datos estimados de la abundancia de oxígeno de las observaciones de banda ancha obtenidos con VLT/FORS, publicados por Izotov \& Thuan (1999), donde los autores han calculado un promedio de la abundancia total en $12+\log (\mathrm{O} / \mathrm{H})=7.85 \pm 0.09$ para esta galaxia. En una publicación reciente de Lagos et al. (2018), los autores estudiaron los dos Nudos principales: A y B, y determinaron la abundancia de oxígeno total de los perfiles globales de las líneas, a partir de observaciones de VIMOS-IFU en $7.85 \pm 0.09$ y $7.83 \pm 0.01$, respectivamente. Nuestros resultados de las componentes globales estimados en $7.80 \pm 0.04$ y $7.71 \pm 0.02$, para A y $\mathrm{B}$ respectivamente, son similares y para el caso el Nudo A, se encuentran en muy buen acuerdo dentro de los errores. Por el lado del Nudo B, podemos decir que a pesar de 
tener en cuenta los errores a $3 \sigma$ hay una pequeña diferencia de 0.04 dex, lo que indica un buen acuerdo. Lagos y colaboradores estiman que la diferencia entre la abundancia de oxígeno entre ambos brotes es de aproximadamente 0.04 dex, y nosotros obtuvimos a que la diferencia entre ambas regiones es de 0.1 dex. Esto podría deberse a las diferentes hipótesis consideradas para determinar las temperaturas electrónicas y las abundancias químicas, así como también al apuntado y a las regiones observadas en las rendijas con el espectrógrafo.

Para las medidas globales de ambas regiones estimamos las temperatura electrónica del [OIII] $(16100 \pm 500 \mathrm{k}$ y $16200 \pm 300 \mathrm{k}$, respectivamente) usando el método directo. Son coherentes con respecto a los valores estimados por Lagos et al. (2018) de $15.491 \pm 462 \mathrm{k} \mathrm{y}$ $14.156 \pm 438 \mathrm{k}$ ), encontrando en el nudo $\mathrm{B}$ una diferencia de $\approx 2.000 \mathrm{k}$ menor que nuestra determinación. Luego, para cada componente encontramos resultados acordes a lo esperado. Para el caso del Nudo A, N1: $16.100 \pm 300 \mathrm{k}, \mathrm{N} 2: 15.300 \pm 100 \mathrm{k}, \mathrm{N} 3: 25.500 \pm 800 \mathrm{k}$, ancha: $16.100 \pm 900 \mathrm{k}$. La componente N3 presenta una temperatura considerablemente más alta que las otras componentes cinemáticas. A pesar de que la línea auroral $[\mathrm{OIII}] \lambda 4364 \AA$ es $\approx 20$ veces más débil que $[\mathrm{OIII}] \lambda 5007 \AA$, presenta una buena $\mathrm{S} / \mathrm{N}$. Esto nos permitió ajustar las cuatro componentes cinemáticas en el perfil con un excelente acuerdo.

En el Brote B, para la componente ancha la temperatura electrónica de [OIII] es $17.000 \pm 500 \mathrm{k}$, para la componente N1 es de $16.100 \pm 400 \mathrm{k}$, lo que indica hay una diferencia de aproximadamente 1.000k entre ambas, siendo estas muy similares. Además, fue al única región en la cual se midió la línea sensible a la temperatura del [Sir] $\lambda 4068 \AA$ con la que se pudo estimar la temperatura electrónica $\mathrm{T}_{e}[\mathrm{OII}]$ de manera directa. Si a esta le sumamos la temperatura del [SIII], obtuvimos tres de las cinco $\mathrm{T}_{e}$ que podríamos haber medido en el rango óptico (ver Hägele et al., 2006, 2008). Las líneas aurorales del [OII] son débiles, y por ende con bajas relación señal-a-ruido y los ajustes no eran confiables, y decidimos no tenerlas en cuenta.

La c $(\mathrm{H} \beta)$ calculada por Izotov \& Thuan (1999) y Guseva et al. (2011) de 0.24 y 0.225, respectivamente, indica una extinción mayor que nuestras determinaciones valuadas en 0.0 (para ambos Nudos). Estas diferencias afectan a la estimación final de los cocientes y por lo tanto, a la estimación de los parámetros físicos, que lleva a grandes diferencias en las medidas. Si comparamos con la $\mathrm{c}(\mathrm{H} \beta)$ publicada por Lagos et al. (2018) de $0.17 \pm 0.07$ y $0.11 \pm 0.06$ para los Nudos A y B respectivamente, si se tienen en cuenta los errores, son valores bajos y más consistentes con las medidas globales obtenidas en este trabajo. Es posible que los resultados encontrados en nuestro trabajo de extinción 0.0 , estén asociados a los espectros de doble brazo, porque el valor $\mathrm{H} \beta$ se tomó del espectro azul y $\mathrm{H} \alpha$ del espectro rojo. Los valores estimados de $\mathrm{c}(\mathrm{H} \beta)$ para Mrk 600 a partir de espectros de ranura larga dados en Campuzano-Castro et al. (2015) son de $0.49 \pm 0.02$ y $0.58 \pm 0.02$, respectivamente.En ambas regiones fue posible estimar las abundancias iónicas y totales de O, S, N, Ne y Ar. Si bien algunos valores presentaban grandes incertidumbres, los valores estimados están en acuerdo valores con valores de referencia de trabajos previos para este tipo de regiones y galaxias.

\subsection{Abundancias químicas estimadas a partir de calibradores empí- ricos}

En los casos en los que se midieron las líneas débiles aurorales junto a las otras líneas intensas de los distintos elementos, es posible aplicar el método directo para determinar 
las abundancias químicas a partir de una estimación directa de sus temperaturas (Pagel et al., 1992; Hägele et al., 2008, 2012). Pero para los casos en que estas líneas no son lo suficientemente intensas y/o no podemos medirlas, se plantea otro análisis. Comunmente suelen utilizarse diferentes métodos empíricos como estimadores de metalicidades (ver e.g. García-Lorenzo et al., 2008; Cairós et al., 2009; Pérez-Montero et al., 2009; García-Benito et al., 2009, 2010) para hallar la abundancia de oxígeno en los objetos para los cuales la derivación directa de la temperatura electrónica no es factible. Estos métodos empíricos se basan en las propiedades de enfriamiento de la nebulosa ionizada, que se traducen en una relación entre las intensidades de las líneas de emisión y las abundancias de oxígeno. Las intensidades de las líneas están controladas principalmente por la distribución de energía de los fotones ionizantes, de la configuración espacial del gas ionizado y de las propiedades intrínsecas del gas (esencialmente, por la metalicidad y densidad del medio). Es así que, en particular, el alto contenido de oxígeno en la nebulosa actúa como un agente enfriador muy eficiente de la región y es debido a esto que por lo general las líneas prohibidas resultan ser débiles.

Como las líneas intensas de los metales que podemos observar en el rango óptico son de caracter colisional, sus intensidades relativas dependen exponencialmente de la temperatura electrónica, y es necesaria la detección de las líneas aurorales, que son difíciles de medir bajo ciertas condiciones (y que además son intrínsecamente débiles). Para regiones con alto contenido de metales, y por lo tanto, con mayores condiciones para un enfriamiento más eficiente, la relación que estas líneas guardan con la temperatura electrónica las hace muy débiles e indetectables, resultando líneas con baja S/N e imposibles de detectar. Lo ideal sería determinar abundancias a través de las líneas de recombinación de los metales dada su baja dependencia con la temperatura electrónica de la nebulosa, pero desafortunadamente, en los espectros estas son muy débiles, por lo que la única opción es buscar relaciones entre los flujos y las abundancias de las líneas colisionales observadas. Muchas de ellas han sido estudiadas en detalle a través de diferentes métodos empíricos sobre las líneas más fuertes, basados en la calibración de la intensidad relativa de algunas de las líneas de emisión más intensas contra la abundancia de los iones correspondientes presentes en la nebulosa.

Entre los principales estimadores empíricos para la determinación de abundancias, encontramos a: $\mathrm{S}_{23}, \mathrm{O}_{3} \mathrm{~N}_{2}, \mathrm{~S}_{3} \mathrm{O}_{3}, \mathrm{~N}_{2}, \mathrm{Ar}_{3} \mathrm{O}_{3}, \mathrm{O}_{23}$ y $\mathrm{SO}_{23}$. Tales parámetros son determinados a partir de las líneas prohibidas intensas:

\section{- $\mathrm{S}_{23}$}

Este parámetro definido inicialmente por Vilchez \& Esteban (1996) está basado en las líneas intensas de emisión del azufre y permite estimar abundancias de $\mathrm{O}$ cuando es posible medir las líneas del IR cercano en $9069 \AA$ y $9532 \AA$, conjuntamente con las líneas del [SII] en $6717 \AA$ y $6731 \AA$. Esto se basa en el hecho de que tanto O como S son elementos primarios y, por lo tanto, se espera que la relación S/O no varíe. Siguiendo una anología con la expresión mencionada anteriormente, los autores definen el parámetro $\mathrm{S}_{23}$ basado en las líneas [SII] y [SIII] como:

$$
\mathrm{S}_{23}=\frac{\mathrm{I}(6717)+\mathrm{I}(6731)+\mathrm{I}(9069)+\mathrm{I}(9532)}{I(H \beta)} .
$$

Este es un parámetro muy útil porque las líneas utilizadas son intensas hasta en objetos con metalicidades grandes, y como las líneas del [SIII] cumplen la relación teórica de intensidades de flujo $(\mathrm{I}(9532 \AA)=2.44 \times \mathrm{I}(9069 \AA)$, tal que con solo conocer una de las es suficiente para su estimación. 
Pérez-Montero \& Díaz (2005a) proponen un ajuste polinómico para derivar la abundancia total de oxígeno:

$$
12+\log (\mathrm{O} / \mathrm{H})=8.15+0.85 \mathrm{x}+0.58 \mathrm{x}^{2},
$$

donde $\mathrm{x}=\log \left(\mathrm{S}_{23}\right)$. Esta expresión puede ser utilizada hasta la metalicidad solar (es decir, $12+\log (\mathrm{O} / \mathrm{H})=8.69)$ con una dispersión de 0.20 dex como la desviación estándar de los residuos a la $\mathrm{O} / \mathrm{H}$ derivada del método directo, y disminuye a 0.10 dex para las BCDs o galaxias Hiı (ver la Figura 5 dada en Pérez-Montero, 2017, donde se ve la relación de dicho cociente).

Las líneas que definen este parámetro tienen algunas características relevantes, desde un punto de vista observacional, como que es posible detectarlas fácilmente en regiones de gas ionizado (Vilchez \& Pagel, 1988; Díaz \& Pérez-Montero, 2000; Pérez-Montero \& Díaz, 2005a) y las incertidumbres en la calibración en flujo debido al enrojecimiento son mínimas debido a que son línes cercanas en longitud de onda.

Esta relación se mantiene univaluada hasta valores por encima de la solar y a partir de un rango de metalicidades mayores a este valor se espera que las intensidades de las líneas disminuyan. Es decir, que para regiones muy metálicas (mayores que la solar). Como el [SIII] presenta las líneas de emisión en el IR en $18 \mu$ y $33.5 \mu$, el enfriamiento toma un mayor peso a temperaturas menores que el oxígeno, como agente enfriador principal, lo que provoca que la relación entre el parámetro y la metalicidad crezca hacia las metalicidades solares y luego cambie su aspecto a un parámetro bivaluado (para más detalle, ver discusión en Pérez-Montero \& Díaz, 2005a; Hägele et al., 2006).

- $\mathrm{O}_{3} \mathrm{~N}_{2}$

Este calibrador definido por Alloin \& Tenorio-Tagle (1979) fue uno de los primeros estimadores empríicos basado en las líneas más intensas del [OIII] y del [NII], propuesto por la baja dependencia con el enrojecimiento de cada conjuntos de líneas y la relación lineal que mantiene a bajas temperaturas electrónicas, como se observa que sucede en las RHir. Esta relación considera las líneas más intensas de ambos iones y está dada por:

$$
O_{3} N_{2}=\log \left(\frac{\mathrm{I}(5007) / \mathrm{I}(\mathrm{H} \beta)}{\mathrm{I}(6584) / \mathrm{I}(\mathrm{H} \alpha)}\right)
$$

La presencia de las líneas de recombinación de Balmer en la expresión es fundamental para minimizar las incertidumbres introducidas por el enrojecimiento, por su cercanía entre $\mathrm{H} \alpha$ y $\mathrm{H} \beta$ y las líneas intensas colisionales utilizadas. Con esta calibración, para estimar la abundancia de oxígeno, utilizamos $12+\log (\mathrm{O} / \mathrm{H})$ definida por Pettini \& Pagel (2004):

$$
12+\log (\mathrm{O} / \mathrm{H})=8.73-0.32 \times \mathrm{O}_{3} \mathrm{~N}_{2},
$$

con una incerteza de 0.25 dex, es válida para los valores dados por cota: $\mathrm{O}_{3} \mathrm{~N}_{2}<2$.

A partir de este cociente, Pagel (1979) definen al parámetro (actualmente denominado) $\mathrm{O}_{23}$ para evitar la dependencia que el cociente $[\mathrm{OIII}] /[\mathrm{NII}]$ mantiene con el grado de ionización de la nebulosa. A continuación definiremos este parámetro.

\section{- $\mathrm{S}_{3} \mathrm{O}_{3}$}

A este parámetro lo define el cociente entre las líneas más intensas del [SIII] y del [OIII], dado por: 


$$
\mathrm{S}_{3} \mathrm{O}_{3}=\frac{\mathrm{I}(9069)}{\mathrm{I}(5007)}
$$

Cabe mencionar que esta relación puede utilizarse para regiones ionizadas en el universo local, debido a que para objetos lejanos, las líneas del [SIII] se alejan de los límites posibles de observar en el óptico. Aunque no es un impedimento total si se requieren de observaciones con instrumentos y detectores en el IR.

La calibración definida por Stasińska (2006) está dada por:

$$
12+\log (\mathrm{O} / \mathrm{H})=8.70+0.28 x+0.03 x^{2}+0.1 x^{3},
$$

donde $\mathrm{x}=\log \left(\mathrm{S}_{3} \mathrm{O}_{3}\right)$, y el ajuste presenta una desviación de 0.25 dex.

Los autores plantean que debido a que los métodos para derivar abundancias a partir de las líneas más intensas del espectro óptico son relativamente fáciles de aplicar, hay que prestar mucha atención a los errores sistemáticos que estos pueden presentar. Para realizar los ajustes en una base de datos confiable, deberían utilizarse RHiI que tengan las mismas propiedades geométricas y de estructura que las muestras de calibración propuestas en los modelos.

Esto hay que tenerlo en cuenta porque el uso de una misma calibración para diferentes muestras puede producir sesgos importantes, como por ejemplo afirmaciones sobre diferencias en la abundancia de $\mathrm{O}$ entre muestras de galaxias con diferentes historias de evolución química y entre muestras de galaxias con diferentes historias de formación estelar.

Además, vale la pena aclarar que para el extremo de alta metalicidad, dichas calibraciones de $\mathrm{S}_{3} \mathrm{O}_{3}$ se basan en el método-P de Pilyugin (2001). Pilyugin y colaboradores utilizaron una muestra de RHII de galaxias irregulares, a las cuales les estimaron las temperaturas electrónicas y recalibraron el método definido por Pagel (1979) incluyendo el parámetro de excitación $\mathrm{P}$, definido como:

$$
\mathrm{P}=[\mathrm{OIII}] \lambda \lambda 4959 \AA, 5007 \AA /([\mathrm{OII}] \lambda \lambda 3727 \AA, 3729 \AA+[\mathrm{OIII}] \lambda \lambda 4959 \AA, 5007 \AA) .
$$

Esta calibración fue llamada método P y solo es aplicable para RHII de alta metalicidad. Stasińska (2006) indican que cuando se comprendan mejor las RHiı ricas en metales, posiblemente sea necesaria una recalibración de todos los índices de metalicidad de manera consistente.

- $\mathrm{N}_{2}$

Este parámetro fue definido como:

$$
\mathrm{N}_{2}=\log \frac{\mathrm{I}(6584)}{\mathrm{I}(\mathrm{H} \alpha)}
$$

por Storchi-Bergmann et al. (1994) y utilizado posteriormente por Denicoló et al. (2002), Pérez-Montero \& Contini (2009), Firpo (2011) y Hägele et al. (2012), entre otros. Presenta un carácter univaluado en relación con la metalicidad, a diferencia del $\mathrm{S}_{23}$ que tiene un carácter dual. Al igual que el anterior, es independiente del enrojecimiento, dado que $\mathrm{N}_{2}$ depende de dos líneas muy cercanas. Sin embargo, la incerteza es grande debido a la dependencia con el campo de ionización de la nebulosa. Existe además una incerteza debido a la variación de la relación de abundancias entre el $\mathrm{N}$ y el $\mathrm{O}$. Al ser 
el $\mathrm{O}$ un elemento primario y el $\mathrm{N}$ tener un caracter secundario, dicho cociente a metalicidades bajas es constante, pues $\mathrm{N}$ es independiente de la metalicidad. Pero a medida que $12+\log (\mathrm{O} / \mathrm{H})$ aumenta, a partir de $12+\log (\mathrm{O} / \mathrm{H}) \approx 8.0$ (ver panel superior izquierdo de la Figura 8 en Pérez-Montero, 2017), N/O aumenta con la metalicidad debido a la aparición del nitrógeno secundario (para más información ver Denicoló et al., 2002). El rango de valores esperados para el $\mathrm{N}_{2}(=\log (\mathrm{I}(6584 \AA) / \mathrm{I}(\mathrm{H} \alpha)))$ es para $0>\mathrm{N}_{2}>-3$. Luego, para el cálculo de la abundancia de oxígeno utilizamos la expresión de Denicoló et al. (2002) dada por:

$$
12+\log (\mathrm{O} / \mathrm{H})=(9.12 \pm 0.05)+(0.73 \pm 0.10) \mathrm{N}_{2},
$$

la cual tiene asociada una incerteza de 0.23 dex.

- $\mathrm{Ar}_{3} \mathrm{O}_{3}$

Este es el otro parámetro definido también por Stasińska (2006), y utiliza las líneas más intensas del $\mathrm{Ar}^{2+}$ y del $\mathrm{O}^{2+}$, dado por:

$$
\mathrm{Ar}_{3} \mathrm{O}_{3}=\frac{\mathrm{I}(7136)}{\mathrm{I}(5007)}
$$

El comportamiento observado en esta relación se debe principalmente a que la disminución de la temperatura a medida que aumenta la metalicidad

El análisis del parámetro $\mathrm{Ar}_{3} \mathrm{O}_{3}$ presenta un comportamiento que puede explicarse como una consecuencia de que la temperatura electrónica disminuye a medida que aumenta la metalicidad, lo que se traduce en un aumento de la relación de emisividad de $[$ ArIII $] \lambda 7135 \AA$ y $[\mathrm{OIII}] \lambda 5007 \AA$. Izotov et al. (2006b) confirmaron, con las observaciones de RHII pobres en metales, que el Ar y el $\mathrm{O}$ son producidos por las estrellas (como se esperaba de los trabajos de nucleosíntesis estelar), por lo tanto, el parámetro $\mathrm{Ar}_{3} \mathrm{O}_{3}$ no se ve afectado por la evolución química, como sí sucede en el $\mathrm{O}_{3} \mathrm{~N}_{2}$ o el $\mathrm{N}_{2}$. Y por otro lado, el $\mathrm{Ar}_{3} \mathrm{O}_{3}$ se corrije por enrojecimiento (para más detalle ver la discusión en Stasińska, 2006). En nuestros casos, se ha correjido por enrojecimiento y por la determinación de la constante $\mathrm{c}(\mathrm{H} \beta)$ de una manera confiable utilizando las cuatro línea de recombinación de Balmer del $\mathrm{H}$.

A partir de la expresión $\mathrm{Ar}_{3} \mathrm{O}_{3}$, se estima la abundancia del $\mathrm{O}$ dada por:

$$
12+\log (\mathrm{O} / \mathrm{H})=8.91+0.34 x+0.27 x^{2}+0.20 x^{3},
$$

donde $\mathrm{x}=\log \left(\mathrm{Ar}_{3} \mathrm{O}_{3}\right)$, y la interteza de la calibración es de 0.23 dex. Al igual que en el $\mathrm{S}_{3} \mathrm{O}_{3}$, para el extremo de alta metalicidad, dichas calibraciones de $\mathrm{Ar}_{3} \mathrm{O}_{3}$ se basan en el método-P de Pilyugin (2001). Los autores indican que cuando se comprendan mejor las RHII ricas en metales, posiblemente sea necesaria una recalibración de todos los índices de metalicidad de manera consistente (Stasińska, 2006).

\section{- $\mathrm{O}_{23}$}

Uno de los parámetros más utilizado, basado en líneas más intensas del [OII] y [OIII], es el $\mathrm{O}_{23}$, dado por Pagel (1979) y definido como:

$$
\mathrm{O}_{23}=\frac{\mathrm{I}(3727,29)+\mathrm{I}(4959)+\mathrm{I}(5007)}{\mathrm{I}(\mathrm{H} \beta)}
$$


Este parámetro empírico depende exclusivamente de iones del oxígeno que es el elemento dominante de una nebulosa (después del H) y sus líneas no presentan un comportamiento lineal con sus abundancias, por ser este un elemento importante de enfriamiento de la nebulosa. Se observa que al aumentar la metalicidad de una región el enfriamiento se vuelve muy eficaz, es decir que la temperatura electrónica disminuye y en consecuencia lo hacen las intensidades de las líneas. Es por esto que se observa un comportamiento dual en las líneas intensas con respecto a la metalicidad y las abundancias (Searle, 1971; Shields, 1974; Skillman, 1989; Kobulnicky et al., 1999; Pérez-Montero \& Díaz, 2005a, entre otros). Esto se traduce en que a metalicidades bajas las líneas son directamente proporcional a las abundancias y a metalicidades altas se comportan de manera opuesta.

Cuando las temperaturas son mayores producto de una metalicidad baja, las líneas son más intensas hasta que llegan a un máximo. A partir de este pico situado entre $8.15>12+\log (\mathrm{O} / \mathrm{H})>8.3$, se produce un cambio en la pendiente de la relación. A partir de ese cambio, como disminuye la abundancia relativa de oxígeno, se traduce en una disminución en la intensidad de las líneas colisionales, en donde pasamos a tener como la fuente principal de enfriamiento a las líneas de recombinación del $\mathrm{H}$, cuando la temperaturas son más bajas. En la zona del cambio de crecimiento hay una alta dispersión que hace difícil una calibración precisa (ver discusión Hägele et al., 2006; Pérez-Montero \& Contini, 2009; López-Sánchez et al., 2010; Pérez-Montero, 2017).

En este trabajo vamos a utilizar las relaciones obtenidas por Kobulnicky et al. (1999) basadas en un trabajo previo de McGaugh (1991). Este ajuste está basado en el $\mathrm{O}_{23}$ y en la excitación dada por los cocientes [OII] y [OIII].

Para decidir a qué rama pertenece un objeto se utiliza el valor del cociente entre las líneas intensas del $[\mathrm{NII}] \lambda 6584 \AA$ y $[\mathrm{OII}] \lambda 3727 \AA$ y el parámetro $\mathrm{N}_{2}(=[\mathrm{NII}] \lambda 6584 \AA / \mathrm{H} \beta$, que más adelante detallamos). Si el logaritmo del primer cociente es menor que -1.2 entonces consideramos que pertenece a la rama inferior ; si el logaritmo del parámetro $\mathrm{N}_{2}$ es mayor que -1.3 (ver Figura 9 en Kewley \& Ellison, 2008)., pertenece a la rama superior. Las expresiones utilizadas para la determinación de ambas ramas son:

$$
\begin{aligned}
12+\log (\mathrm{O} / \mathrm{H})_{\text {inferior }}= & 12-4.944+0.767 x+0.602 x^{2}-y\left(0.29+0.332 x-0.331 x^{2}\right. \\
12+\log (\mathrm{O} / \mathrm{H})_{\text {superior }} & =12-2.939-0.2 x-0.237 x^{2}-0.305 x^{3}-0.0283 x^{4}- \\
& -y\left(0.0047-0.0221 x-0.102 x^{2}-0.0817 x^{3}-0.00717 x^{4}\right)
\end{aligned}
$$

donde $\mathrm{x}=\log \left(\mathrm{O}_{23}\right), \mathrm{y}=\log ([\mathrm{OH}] 3727 /[\mathrm{OIII}] 4959+5007)$.

Las incertezas que se derivan del ajuste dan para la rama inferior un error cuadrático medio de 0.13 dex y para la rama superior 0.19 dex.

- $\mathrm{SO}_{23}$

Como último parámetro presentamos a $\mathrm{SO}_{23}$ que es una combinación de $\mathrm{S}_{23}$ y $\mathrm{O}_{23}$ :

$$
\mathrm{SO}_{23}=\left(\frac{\mathrm{S}_{23}}{\mathrm{O}_{23}}\right) \text {. }
$$

definido por Díaz \& Pérez-Montero (2000). Como estuvimos describiendo para cada uno de los calibradores empíricos mencionados anteriormente, al estar basados en las líneas brillantes de los espectros de regiones de gas ionizado, las relaciones presentadas 
son válidas en un rango determinado de metalicidades. Si miramos esta relación, el $\mathrm{S}_{23}$ presenta un comportamiento univaluado hasta las metalicidades solares en donde la orientación de la curva cambia. El $\mathrm{O}_{23}$, tiene una incertidumbre mayor debido a que la dependencia bivaluada con la metalicidad ocurre hacia metalicidades menores, en donde se pueden encontrar muchos más objetos (ver panel de la derecha en la Figura 3 de Pérez-Montero \& Díaz, 2005a).

Esto indica que a medida que las líneas intensas del O comienzan a volverse débiles en el espectro por la mayor presencia de metales, las líneas del azufre continúan haciéndose más intensas y hace falta una mayor cantidad de azufre para que este tome un rol protagónico en en enfriamiento de la nebulosa.

Las líneas del azufre y del oxígeno son importantes en el enfriamiento de la nebulosa y tienen un papel muy importante en este mecanismo, pero como lo hacen a diferentes energías, eso quiere decir que son eficientes a distintas temperaturas. A través de este parámetro pueden estudiarse gradientes de metalicidad, es decir objetos que presenten como características de una gran variación interna de la metalicidad (para más información ver Pérez-Montero \& Díaz, 2005a).

La calibración que utilizaremos en nuestro trabajo es la realizada por Pérez-Montero \& Díaz (2005a) dada por:

$$
12+\log (\mathrm{O} / \mathrm{H})=9.09+1.03 x-0.23 x^{2},
$$

donde: $\mathrm{x}=\log \left(\mathrm{S}_{23} / \mathrm{O}_{23}\right)$. Esta calibración presenta una dispersión considerable de 0.27 dex, por la propagación de errores que trae la composición de ambos parámetros (ver panel derecho de la Figura 15 de dicho trabajo).

Quedando definidos los calibradores empíricos, presentaremos los resultados obtenidos. Para la descripción de los métodos, utilicé la bibliografía disponible en la literatura, y principalmente la Tesis de la Dra. Verónica Firpo (para más detalle, ver Firpo, 2011) y los artículos de mi director, G. Hägele (en particular Hägele et al., 2006, 2008, 2012), así como los principales trabajos referenciados en ellos.

A continuación analizamos los resultados obtenidos de la abundancia de oxígeno con los diferentes parámetros empíricos en cada una de las regiones (ver Tabla 4.13)y componentes cinemáticas estudiadas. Como puede verse en las Figuras 4.11 y 4.12 se comparan estas abundancias con las derivadas previamente usando las temperaturas estimadas, donde presentamos las abundancias para cada componente e incertezas correspondientes a $1 \sigma$, así como también los valores de las abundancias totales determinadas para los datos long-slit. En la Figura 4.11, de arriba a abajo presentamos los Nudos A, B y C de II Zw 33, de izquierda a derecha los diferentes parámetros empíricos: $\mathrm{S}_{23}, \mathrm{O}_{3} \mathrm{~N}_{2}, \mathrm{~S}_{3} \mathrm{O}_{3}, \mathrm{~N}_{2}, \mathrm{Ar}_{3} \mathrm{O}_{3}, \mathrm{O}_{23}$ y $\mathrm{SO}_{23}$. Para la Figura 4.12 de arriba a abajo presentamos los Nudos A y B de Mrk600, de izquierda a derecha los diferentes parámetros empíricos. Con triángulo rojo se muestra la medida global; el triángulo azul se refiere a la componente angosta N1; el triángulo naranja la componente angosta N2; el triángulo verde corresponde a la componente ancha y los rombos rojos a las medidas determinadas en el long-slit. Las líneas horizontales continuas representan, según el color de referencia, las abundancias determinadas en el análsis previo a partir de las temperaturas electrónicas. Por un tema de simplicidad del gráfico no se indican los rangos de abundancias determinados previamente, sino que simplemente colocamos con las líneas los valores de referencia y así comparar con lo determinado a partir de los parámetros empíricos. Es importante indicar que debido a que las abundancias determinadas para la componente $\mathrm{N} 1$, N2 y la medida global coinciden en $12+\log (\mathrm{O} / \mathrm{H})=8.28$, hemos graficado con una leve 


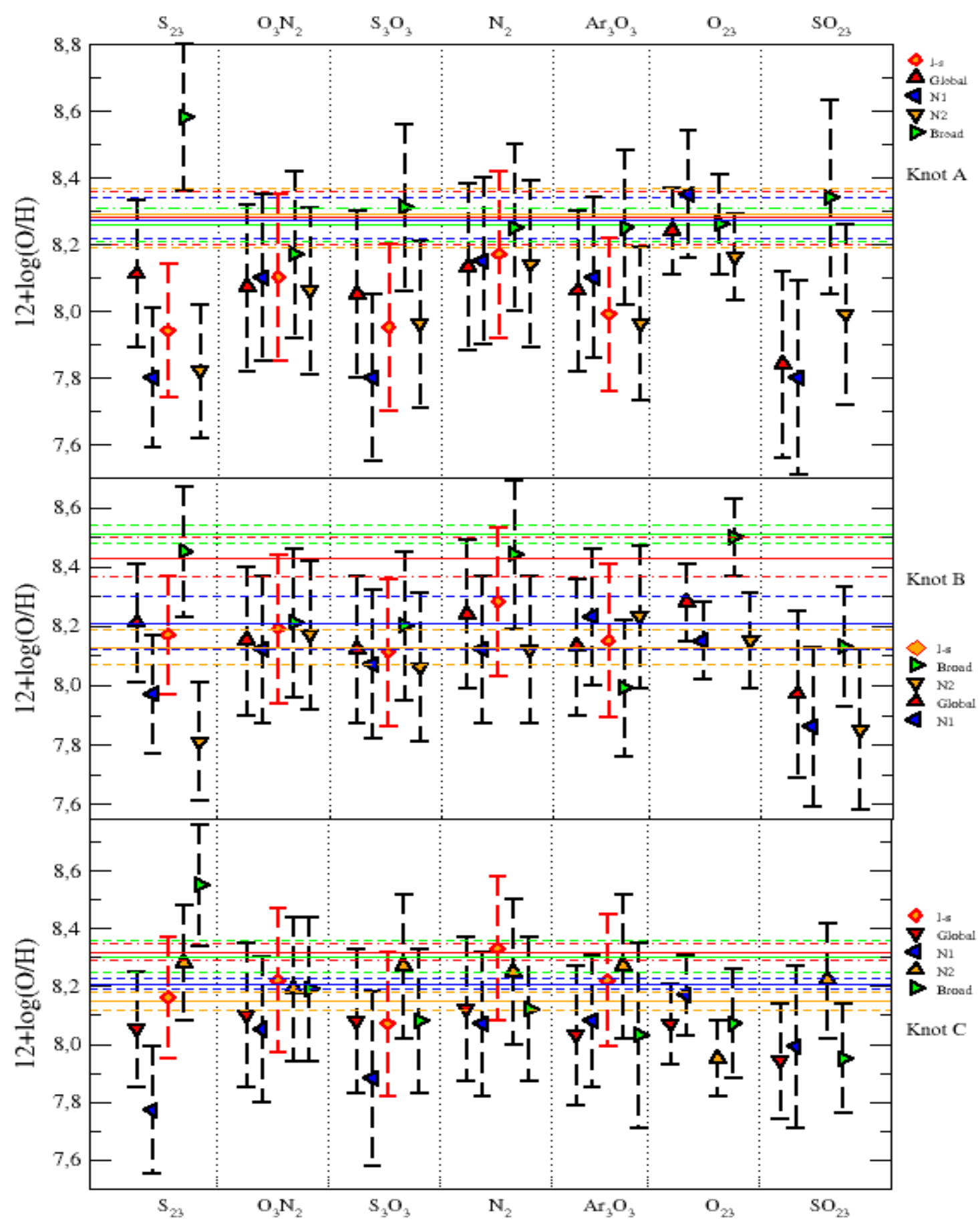

Figura 4.11. Abundancias de oxígeno y sus incertezas para cada brote observado en II Zw 33 calculadas usando los diferentes parámetros empíricos para las diferentes componentes y la medida global. De arriba a abajo: brotes A, B y C. De izquierda a derecha los parámetros empíricos: $\mathrm{S}_{23}, \mathrm{O}_{3} \mathrm{~N}_{2}, \mathrm{~S}_{3} \mathrm{O}_{3}, \mathrm{~N}_{2}, \mathrm{Ar}_{3} \mathrm{O}_{3}, \mathrm{O}_{23}$ y $\mathrm{SO}_{23}$. Con triángulo rojo se muestra la medida global; el triángulo azul se refiere a la componente angosta N1; el triángulo naranja es la componente angosta N2; el triángulo verde es la componente ancha y el rombo son los datos long-slit de la región global. Las líneas horizontales representan el rango de la abundancia total de oxígeno estimadas previamente (Tablas: 4.4, 4.6 y 4.8). 

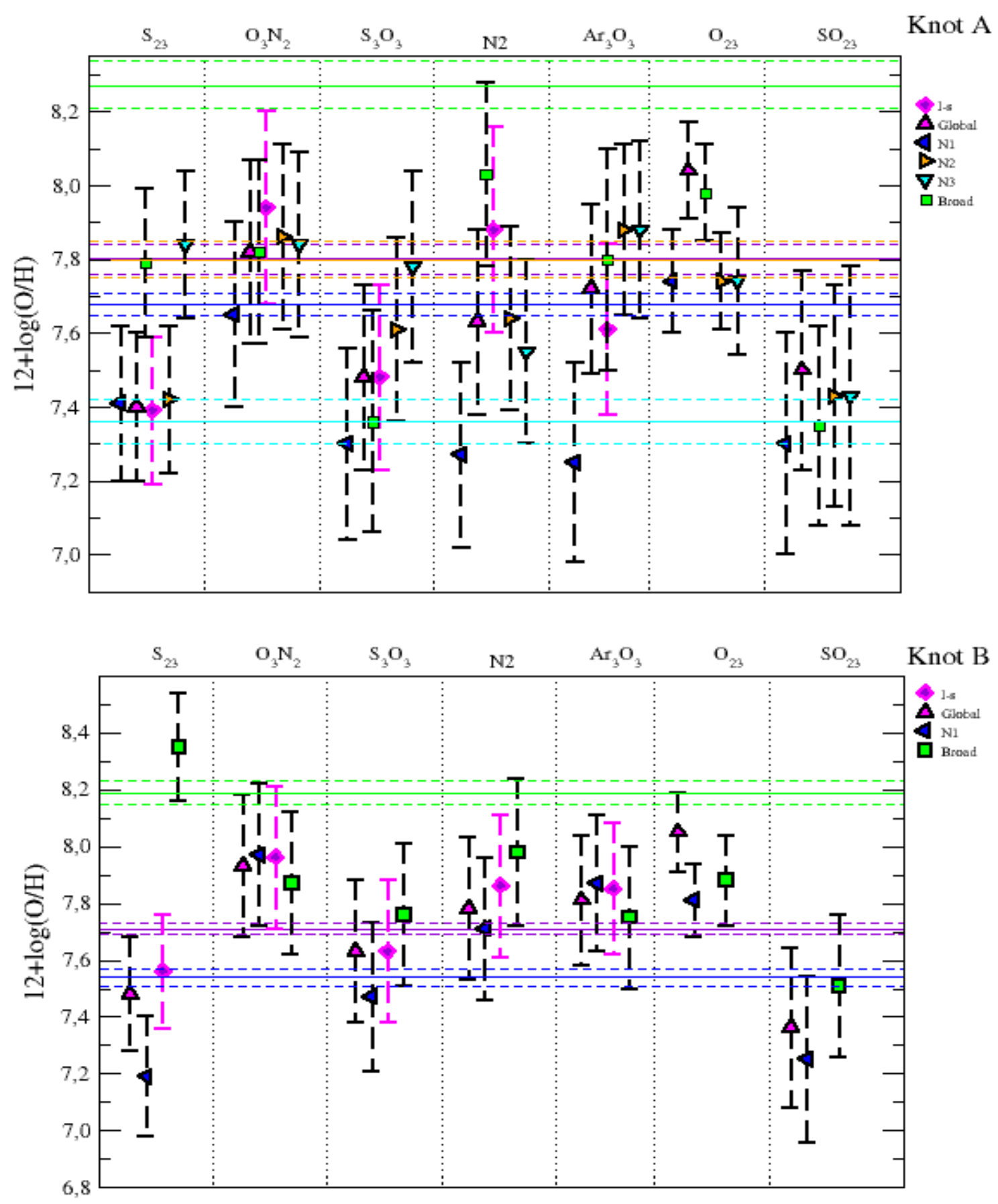

Figura 4.12. Abundancias de oxígeno y sus incertezas para cada brote observado Mrk 600 calculadas usando los diferentes parámetros empíricos para las diferentes componentes y la medida global. De arriba a abajo: brotes A y B. De izquierda a derecha los parámetros empíricos: $\mathrm{S}_{23}, \mathrm{O}_{3} \mathrm{~N}_{2}, \mathrm{~S}_{3} \mathrm{O}_{3}, \mathrm{~N}_{2}, \mathrm{Ar}_{3} \mathrm{O}_{3}, \mathrm{O}_{23}$ y $\mathrm{SO}_{23}$. Con triángulo rojo se muestra la medida global; el triángulo azul se refiere a la componente angosta N1; el triángulo naranja es la componente angosta N2; el triángulo celeste es la componente angosta N3; el cuadrado verde es la componente ancha y el rombo violeta corresponde a los datos long-slit. La componente N2 y N3 las encontramos exclusivamente para el Nudo A. Las líneas horizontales representan el rango de la abundancia total de oxígeno determinadas previamente (Tablas: 4.10 y 4.12). 
diferencia en el valor (únicamente) las líneas horizontales para que sean apreciables en la Figura. En la Tabla 4.13 se indican los valores de los parámetros empíricos para tener como referencia en el las Figuras 4.11 y 4.12. Para el caso de Mrk600, con triángulo rojo presentamos la medida global; el triángulo azul se refiere a la componente angosta N1; el triángulo naranja es la componente angosta N2; el triángulo celeste es la componente angosta N3; el cuadrado verde es la componente ancha y con rombos violetas presentamos a los valores determinados a partir de las temperaturas electrónicas en datos long-slit. La componente N2 y N3 las encontramos exclusivamente para el Nudo A.

Tabla 4.13. Abundancias de oxígeno a partir de los Parámetros Empíricos $\mathrm{S}_{23}, \mathrm{O}_{3} \mathrm{~N}_{2}, \mathrm{~S}_{3} \mathrm{O}_{3}$, $\mathrm{N}_{2}, \mathrm{Ar}_{3} \mathrm{O}_{3}, \mathrm{O}_{23}$ y $\mathrm{SO}_{23}$ para las distintas componentes, las medidas globales y las observaciones long-slit de los Nudos A, B y C de II Zw 33 y los Nudos A y B de Mrk600. Para estos últimos no se han realizado las estimaciones de los parámetros $\mathrm{O}_{23}$ y $\mathrm{SO}_{23}$ por un tema de disponibilidad de líneas.

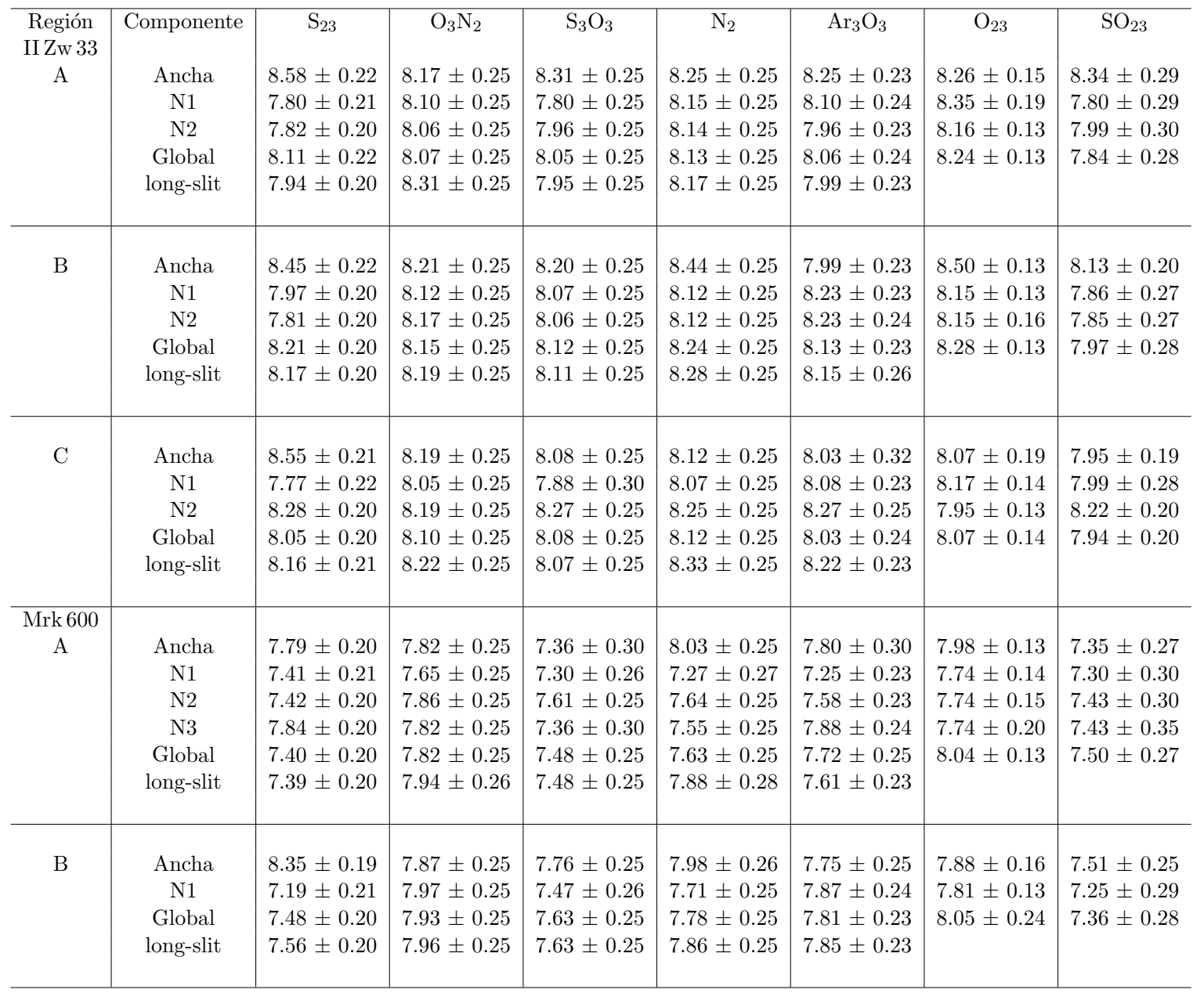

\subsubsection{Discusión de los resultados determinados a partir de los parámetros empíricos para II Zw 33}

En el Nudo A existe una buena concordancia entre la abundancia total de oxígeno de la medida global determinada usando los diferentes parámentro empíricos, dentro de los errores, con la respectiva abundancia derivada usando las temperaturas obtenidas del método directo (línea horizontal continua roja en $12+\log (\mathrm{O} / \mathrm{H})=8.28 \pm 0.08)$. La única que escapa a este 
resultado es la determinación de la abundancia total de oxígeno con el parámetro empírico $\mathrm{SO}_{23}$, que presenta una diferencia de 0.10 dex respecto a la abundancia determinada con el método directo, y teniendo en cuenta el error a tres sigmas serían compatibles, aunque debemos destacar que los errores son grandes. En la componente angosta N1 existe una buena concordancia en la determinación de la abundancia usando los parámetros empíricos: $\mathrm{O}_{3} \mathrm{~N}_{2}$, $\mathrm{N}_{2}, \mathrm{Ar}_{3} \mathrm{O}_{3}$ y $\mathrm{O}_{23}$ con la abundancia determinada usando las temperaturas obtenidas de por el método directo (línea horizontal continua azul en $12+\log (\mathrm{O} / \mathrm{H})=8.28 \pm 0.10$ ). En cambio, la abundancia determinada usando los parámetros empítricos: $\mathrm{S}_{23}, \mathrm{~S}_{3} \mathrm{O}_{3}$ y $\mathrm{SO}_{23}$ resultan menores en 0.20 dex, 0.22 dex y 0.10 dex, respectivamente, pero si tenemos en cuenta las barras de error de las abundancias determinadas por el método directo a $3 \sigma$ estamos en acuerdo con las abundancias estimadas. Por el lado de la componente angosta N2 los parámetros empíricos: $\mathrm{O}_{3} \mathrm{~N}_{2}, \mathrm{~S}_{3} \mathrm{O}_{3}, \mathrm{~N}_{2}, \mathrm{Ar}_{3} \mathrm{O}_{3}, \mathrm{O}_{23}$ y $\mathrm{SO}_{23}$, están en acuerdo con la abundancia determinada usando las temperaturas obtenidas de por el método directo (línea horizontal continua naranja en $12+\log (\mathrm{O} / \mathrm{H})=8.28 \pm 0.09)$. Solo para el parámetro $\mathrm{S}_{23}$ identificamos una diferencia notoria de 0.20 dex, que si tenemos en cuenta los errores a $3 \sigma$, podemos decir que están en acuerdo. Un comportamiento similar a la componente N2 se determinó para la componente ancha, donde $\mathrm{S}_{23}$ es el único parámetro de los determinados que difiere (casi despreciable) con la abundancia calculada usando las temperaturas obtenidas de los modelos y relaciones empíricas en 0.03 dex (línea horizontal continua azul en $12+\log (\mathrm{O} / \mathrm{H})=8.26 \pm 0.05$ ), estando en acuerdo teniendo en cuenta los errores.

Para esta región las estimaciones de abundancias totales de oxígeno usando el parámetro $\mathrm{S}_{23}$ dan valores más bajos de los esperados para todas las componentes salvo para la componente ancha que la diferencia es despreciable. Pero si tenemos en cuenta los errores a $3 \sigma$ las determinaciones están dentro de los rangos de validez. Luego, encontramos en todos los otros estimadores valores coherentes y acordes a lo determinado.

Para el Nudo B encotramos un excelente acuerdo para (prácticamente) todas las componentes. Los valores de las abundancias totales de oxígeno estimadas a partir de los parámetros empíricos es muy buena, obteniendo valores similares, excelente en algunos casos y teniendo en cuenta a los errores en otros, de las abundancias estimadas para todas las componentes cinemáticas y la componente global (en $12+\log (\mathrm{O} / \mathrm{H})=8.43 \pm 0.06)$ en comparación con las abundancias determinadas por el método directo. La componente angosta N2 es la única que al estimar su abundancia con el parámetro $\mathrm{S}_{23}$ presenta una diferencia de $\sim 0.06$, que si teniemos en cuenta los errores de la medida directa en $3 \sigma$, está en acuerdo con lo esperado, determinado por el método directo (línea horizontal continua naranja en $12+\log (\mathrm{O} / \mathrm{H})=8.13 \pm 0.06$ ). Vale indicar y mencionar que para esta región las temperaturas electrónicas del [OIII] y [SIII] fueron estimadas de manera directa a través de las líneas débiles aurorales. En el caso de la componente ancha, es el parámetro $\mathrm{Ar}_{3} \mathrm{O}_{3}$ el que se encuentra por debajo de lo esperado, pero esto puede deber a un problema en la medida de la línea débil del $\mathrm{Ar}^{+2}$, siendo el único parámetro que se aleja de los valores esperados.

Para el Nudo C estimamos las abundancias de todas las componentes a través de los parámetros empíricos y obtuvimos un muy buen acuerdo entre estas y las abundancias calculadas usando las temperaturas a partir de los modelos y relaciones empíricas. En la componente angosta N1 encontramos los parámetros que presentan mayores diferencias con respecto a la abundancias a partir de relaciones empíricas es en el parámetro $\mathrm{S}_{23}$ en donde tenemos que una componente no tiene buen acuerdo, y en este caso para la componente N1 estimamos 0.15 dex menor que la abundancia obtenida a partir de modelos.Y si comparamos los resultados de los parámetros empíricos sobre las observaciones long-slit para los Nudos observados de II Zw 33, encontramos que con respecto a las medidas determinadas previamente en Campuzano-Castro et al. (2015), están en un excelente acuerdo. Las abundancias determinadas a partir de las esti- 
maciones de las temperaturas para cada región fueron: II Zw $33 \mathrm{~A}: 12+\log (\mathrm{O} / \mathrm{H})=8.07 \pm 0.12$, II Zw 33 B: $12+\log (\mathrm{O} / \mathrm{H})=8.09 \pm 0.04$ yII Zw $33 \mathrm{C}: 12+\log (\mathrm{O} / \mathrm{H})=8.20 \pm 0.02$. En absolutamente todos los nudos se encuentra un excelente acuerdo entre lo estimado a partir de los calibradores empíricios y las medidas calculadas previamente.

\subsubsection{Discusión de los resultados determinados a partir de los parámetros empíricos para Mrk 600}

En el Nudo A existe un acuerdo entre algunas de las abundancias totales de oxígeno determinadas a partir de los diferentes parámentro empíricos para las componentes cinemáticas, la medida global y los datos long-slit, con la respectiva abundancia derivada previamente utilizando las temperaturas obtenidas del método directo, teniendo en cuenta los errores (línea horizontal continua violeta en $12+\log (\mathrm{O} / \mathrm{H})=7.80 \pm 0.04)$. Para los parámetros empíricos: $\mathrm{O}_{3} \mathrm{~N}_{2}, \mathrm{~S}_{3} \mathrm{O}_{3}, \mathrm{~N}_{2}, \mathrm{Ar}_{3} \mathrm{O}_{3}$ y $\mathrm{SO}_{23}$ hay un buen acuerdo, tomando un valor similar o diferencias despreciables si tenemos en cuenta los errores $(7.80 \pm 0.04)$. Por el lado del parámetro empírico $\mathrm{O}_{23}$ la abundancia obtenida dentro de los errores está en acuerdo. Pero al igual que en la galaxia anterior, el parámetro $\mathrm{S}_{23}$ es inferior al esperado en 0.20 dex, teniendo en cuenta los errores de las medidas directas.

Para la componente angosta N1 el comportamiento de las abundancias estimadas por medio de los parámetros empíricos es significativamente diferente a la calculada previamente. Puede observarse como los triángulos azules tienden a estar por debajo de todos los valores en prácticamente todos los indicadores, pero debido a las grandes barras de error que estos presentan, podemos encontrar similitudes, dentro de los errores. Los parámetros $\mathrm{O}_{3} \mathrm{~N}_{2}$ y $\mathrm{O}_{23}$ nos devuelven una estimación con una concordancia excelente respecto a la abundancia previamente obtenida. Para los parámetros $\mathrm{S}_{23}$ y $\mathrm{SO}_{23}$ las diferencias están dentro de los $2 \sigma$ de las abundancias determinadas a partir de medidas directas, por lo que encontramos valores en acuerdo. Distinto es el comportamiento de los parámetros empíricos $\mathrm{S}_{3} \mathrm{O}_{3}, \mathrm{~N}_{2}$ y $\mathrm{Ar}_{3} \mathrm{O}_{3}$ donde las estimaciones de las abundancias totales de oxígeno muestran valores muy por debajo del determinado por el método directo con una diferencia de 0.30 dex, que disminuye al tener en cuenta los errores de la medida directa en $3 \sigma$, pero siguen alejados de lo esperado. Además de la componente N1, la componente angosta N3 también muestra una incongruencia entre las abundancias calculadas a partir de los parámetros empíricos y las abundancias estimadas a partir de las temperaturas electrónicas determinadas de manera directa $(12+\log (\mathrm{O} / \mathrm{H})=7.36$, la que representa la componente con menor metalicidad de todas las estudiadas y está representada con la línea continua de color celeste). La mayor inconsistencia en los valores estimados de las abundancias totales de oxígeno frente a las previamente determinadas, las encontramos en los parámetros empíricos: $\mathrm{S}_{23}, \mathrm{O}_{3} \mathrm{~N}_{2}$ y $\mathrm{Ar}_{3} \mathrm{O}_{3}$. Todas las abundancias estimadas a partir de estos parámetros son valores superiores a los 7.36 en hasta 0.50 dex. En los casos de $\mathrm{S}_{3} \mathrm{O}_{3}$ y $\mathrm{O}_{23}$ las diferencias son grandes, pero al tener en cuenta las barras de error, los valores están en acuerdo. Los dos parámetros restantes, $\mathrm{N}_{2}$ y $\mathrm{SO}_{23}$, están en buen acuerdo con las abundancias bajas de 7.36.

Para la componente $\mathrm{N} 2$, las estimaciones dadas por: $\mathrm{O}_{3} \mathrm{~N}_{2}, \mathrm{~S}_{3} \mathrm{O}_{3}, \mathrm{~N}_{2}, \mathrm{Ar}_{3} \mathrm{O}_{3}, \mathrm{O}_{23}$ presentan un muy buen acuerdo en comparación con la abundancia determinada con las temperaturas obtenidas del método directo (línea horizontal continua naranja, con el mismo valor que la medida global de $12+\log (\mathrm{O} / \mathrm{H})=7.36)$. Con el parámetro $\mathrm{SO}_{23}$ encontramos un valor de abundancia total de oxígeno levemente menor, que teniendo en cuenta los errores, encontramos una concordacia con lo previamente determinado. El único de los parámetros que nos indica un valor considerablemente distinto es el parámetro empírico $\mathrm{S}_{23}$ con el que estimamos una abundancia menor de $0.15 \mathrm{dex}$, lo que puede deberse a un problema en la medida del dato y de las líneas que lo componen. 
Por el lado de la componente ancha, para todos los parámetros se han estimado valores de abundancias menores que los determinados por el método directo $(12+\log (\mathrm{O} / \mathrm{H})=8.27 \pm 0.06)$ en unos 0.10 dex y hasta 0.40 dex. El parámetro $\mathrm{N}_{2}$ es el único que con su estimación obtenemos un valor acorde, teniendo en cuenta la barra de error del parámetro empírico, con el esperado. Por el lado del $\mathrm{Ar}_{3} \mathrm{O}_{3}$ y $\mathrm{O}_{23}$, los valores están en acuerdo si tenemos al tener en consideración $3 \sigma$ de la medida directa. Para los demás casos en que las diferencias son mayores, no hay acuerdo y esto puede deberse a que la componente ancha es débil y en líneas con baja señal-a-ruido su determinación ha sido difícil de determinar. De este análisis podemos indicar que el parámetro empírico a partir del cual obtenemos valores estrictamente menores que los calculados previamente es el $\mathrm{S}_{23}$. Esto puede indicar que hay un problema en las abundancias estimadas para estas líneas, lo que tiene coherencia con algunas diferencias en flujo que hemos encontrado para las líneas del [SIII] en 9069 línea de emisión órdenes superiores cercanos al borde del detector, pudiendo esto introducir problemas de borde y como consecuencia un problema en el flujo de las líneas que en estas zonas se encuentren.

La región B de Mrk600, es la última de las regiones que describiremos y es además la que presenta el menor número de componentes cinemáticas que la representan. Existe una buena concordancia entre la abundancia total de oxígeno de la medida global determinada usando los diferentes parámentro empíricos, dentro de los errores, con la respectiva abundancia derivada usando las temperaturas obtenidas del método directo (línea horizontal continua violeta, y su errores en líneas violetas rayadas en $12+\log (\mathrm{O} / \mathrm{H})=7.71 \pm 0.02)$.

La medida global presenta un muy buen acuerdo entre las determinaciones a patir de los parámetros y las medidas previamente estimadas de abundancias totales del oxígeno, salvo en el caso del $\mathrm{O}_{23}$, que presentan una diferencia de 0.15 dex. Si tenemos en cuenta los errores a $3 \sigma$, podríamos decir que la diferencia de 0.09 dex es equivalente a una diferencia de $\sim 1.25$ en abundancias, siendo una diferencia que no es despresiable entre ambas determinaciones, de manera tal que no podemos indicar un buen acuerdo.

En la componente angosta N1 existe una buena concordancia en la determinación de la abundancia usando los parámetros empíricos: $\mathrm{S}_{3} \mathrm{O}_{3}, \mathrm{~N}_{2}$, y $\mathrm{SO}_{23}$ con la abundancia determinada usando las temperaturas obtenidas de por el método directo (línea horizontal continua azul en $12+\log (\mathrm{O} / \mathrm{H})=7.54 \pm 0.03)$. Las estimaciones obtenidas con los parámetros $\mathrm{S}_{23}$ y $\operatorname{Ar}_{3} \mathrm{O}_{3}$ tienen un acuerdo si tenemos en cuenta los errores a $3 \sigma$ de las determinaciones directas de las abundancias. Pero, para los $\mathrm{O}_{3} \mathrm{~N}_{2}$ y $\mathrm{O}_{23}$, los valores estimados resultan mayores que los esperados en $\sim 20$ dex y si tenemos en cuenta los errores en la medida directa a $3 \sigma$, la diferencia es de $0.15 \mathrm{dex}$, equivalente a una diferencia de 1.40 veces mayor en abundancia respecto a la medida determinada directamente. Esto nos está indicando una diferencia considerable en abundancias que no puede ser despreciada.

Por el lado de la componente ancha existe un buen acuerdo en la determinación de la abundancia para los parámetros empíricos: $\mathrm{S}_{23}$ y $\mathrm{N}_{2}$ con la abundancia determinada usando las temperaturas obtenidas de por el método directo (línea horizontal continua verde en $12+\log (\mathrm{O} / \mathrm{H})=8.19 \pm 0.04)$. Para los parámetro $\mathrm{O}_{3} \mathrm{~N}_{2}, \mathrm{~S}_{3} \mathrm{O}_{3}, \mathrm{Ar}_{3} \mathrm{O}_{3}$ y $\mathrm{O}_{23}$ se identifican pequeñas diferencias, dentro de los rangos del error a $3 \sigma$, presentando un acuerdo con los valores esperados. Por el lado del paráemtro $\mathrm{SO}_{23}$ la diferencia es grande, incluso teniendo en cuenta los errores, del $\approx 0.5$ dex. Esto puede ser indicio de que hay algún problema en la medición para la componente ancha en las líneas del azufre, como ya se ha planteado en otras situaciones.

Y por último, las estimaciones de los parámetros empíricos para los datos long-slit en Mrk 600 A y B $(12+\log (\mathrm{O} / \mathrm{H})=7.69 \pm 0.06$ y $12+\log (\mathrm{O} / \mathrm{H})=7.71 \pm 0.08$, respectivamente. Para más detalle ver Campuzano-Castro et al., 2015) muestran un excelente acuerdo con los 
valores esperados de las abundancias derivados de las medidas directas. En las Figuras correspondientes a los Nudos A y B, tomamos como referencia las medidas de la componente angosta N1 (línea continua azul) y de la medida global (línea continua violeta), respectivamente.

Es importante mencionar que todas las relaciones empíricas usadas en este análisis están chequeadas y son utilizadas en la literatura. En particular, en nuestro grupo de trabajo ya han sido testeadas para las componentes cinemáticas en el estudio de múltiples componente en distintos Nudos en Haro 15 en Firpo (2011), Hägele et al. (2011) y Hägele et al. (2012), así como también en los trabajos llevados anteriormente en II Zw 33 y Mrk600 a partir de observaciones long-slit. Es necesario realizar nuevas observaciones y ampliar la muestra de regiones con determinaciones de abundancias por componentes y también, dentro de lo posible, con una determinación directa de la abundancia iónia del $\mathrm{O}^{+}$, lo que daría una medida directa las abundancias de oxígeno para las distintas componentes, para el caso del Nudo C y las componentes anchas de A y B de II Zw 33, que fueron estimadas usando las temperaturas obtenidas de modelos y de relaciones empíricas. Y por el lado de Mrk600, es necesario chequear las líneas del [SIII] y sus relaciones de flujo debido a algunos valores anómalos que se han determinado, que pueden llevar a que sus estimaciones de parámetros que se derivan a través de ellos no sean confiables. Si tenemos en cuenta todos los parámetros empíricos que pudimos utilizar para nuestro análisis, hemos encontrado que a partir del $\mathrm{S}_{23}$ se subestiman los valores de las abundancias totales, lo que nos lleva a pensar en las líneas del $\mathrm{S}_{23}$ pueden presentar un problema.

Para los demás parámetros hemos encontrado un buen acuerdo entre las calibraciones de abundancias a partir de parámetros empíricas y las abundancias determinadas a partir del uso de temperaturas estimadas por el método directo en los casos en que fue posible, y sino a partir de las temperaturas obtenidas de los modelos y relaciones empíricas.

\subsection{Estructura de Ionización}

A través de la medida de las líneas más intensas de los elemento pueden estimarse metalicidades por medio la determinación directa de la temperatura o del uso de parámetros empíricos y de modelos. La geometría de la nebulosa y las líneas de emisión de la nebulosa están vinculadas con el Parámetro de Ionización $U$ que está relacionado directamente con la "dureza" del campo ionizante. Esta relación es fundamental para estimar cómo es el campo de radiación a partir de líneas intensas, que nos ayuda a entender y completar el análisis global sobre las nebulosas y el gas ionizado. Y por lo tanto, resulta importante la determinación que la estructura de ionización depende del tipo de Región Hir que se esté estudiando (Díaz et al., 2007; Hägele et al., 2008).

El oxígeno es un elemento que funciona como enfriador de la nebulosa, y resulta que para metalicidades grandes, el enfriamiento se vuelve más eficaz. Por lo tanto, la temperatura electrónica disminuye, y de la misma manera lo hacen la intensidad de las líneas.

Para estimar el parámetro U, la estimación más razonable sería utilizar los cocientes de las abundancias iónicas de elementos químicos en sucesivos estados de ionización. Esto no es una tarea sencilla pues su determinación resulta difícil dado que son líneas débiles y se requiere espectros de con alta S/N para poder medirlas. Frente a esta limitación, suelen utilizarse los cocientes de las intensidades de los estados consecutivos de ionización de un mismo ion. Vilchez \& Pagel (1988) indican que los cocientes $\mathrm{O}^{+} / \mathrm{O}^{2+}$ y $\mathrm{S}^{+} / \mathrm{S}^{2+}$ están intrínsecamente relacionados con la forma del continuo y son buenos estimadores para clasificar la estructura de ionización.

Los mecanismos de generación de dichos iones dependen de la temperatura efectiva del campo de radiación generado por la fuente ionizante. Para el cociente del oxígeno se observa 
que al ir aumentando la temperatura, los iones de $\mathrm{O}^{+}$se ionizan pasando a $\mathrm{O}^{2+}$, por lo tanto, dicho cociente disminuye. Esto nos permite trabajar con nebulosas con altos grados de ionización.

Por el lado del azufre, a medida que la temperatura efectiva aumenta $\mathrm{S}^{2+}$ pasa a $\mathrm{S}^{3+}$, y por lo tanto, la relación entre $\mathrm{S}^{+} / \mathrm{S}^{2+}$ aumenta ya que el denominador de este cociente se hace más pequeño. Este efecto es pronunciado en grados de ionización altos, por lo que este cociente se usa más en nebulosas con grados de ionización menor $(\log (\mathrm{U}) \leq 2)$. Al cociente entre ambas cantidades se lo conoce como Softness Parameter y comunmente se lo denomina: $\eta=\frac{O^{+}}{O^{+2}} / \frac{S^{+}}{S^{+2}}$. Como esta magnitud depende de la geometría de la nebulosa, se supone una estructura siempre con un factor de llenado constante, lo que permite que este efecto pueda ser representado por el cociente de las líneas del oxígeno mencionadas, que a su vez depende de la $\mathrm{T}_{\text {eff }}$, y por consiguiente de la metalicidad. En la Tabla 4.14 presentamos los cocientes $\log \left(\mathrm{S}^{+} / \mathrm{S}^{2+}\right)$ y $\log \left(\mathrm{O}^{+} / \mathrm{O}^{2+}\right)$ para obtener $\log \eta$ de nuestras regiones. Los valores de este parámetro nos dan una relación para el campo de radiación de las regiones en función de la temperatura efectiva, y esto nos permite comparar dichos campos de radiación según su dureza. Este análisis lo pudimos realizar en los Nudos observados en los que medimos las líneas más débiles aurorales del azufre y del oxígeno, como presentamos en las Figuras 4.13 y 4.14 .

La contraparte puramente observacional del parámetro $\eta$ está dada por el parámetro $\eta$ ' definido también por Vilchez \& Pagel (1988) como el cociente entre las intensidades de las líneas de emisión de $[\mathrm{OII} \lambda](3726 \AA+\lambda 3729 \AA) /[\mathrm{OIII}](\lambda 4959 \AA+\lambda 5007 \AA)$ y $[\operatorname{Sin}](\lambda 6717 \AA+$ $\lambda 6731 \AA) /[\operatorname{SiII}](\lambda 9060 \AA+\lambda 9532 \AA$ ) (ver Figuras 4.15 y 4.16). Se define la relación entre ambos parámetros como:

$$
\log \left(\eta^{\prime}\right)=\log (\eta)-\frac{0.14}{t_{e}}-0.16
$$

donde $t_{e}$ es la temperatura electrónica en unidades de $10^{4} \mathrm{k}$. Dicha relación está dada en función de la temperatura electrónica pero depende de una manera mínima de ella, por lo que un cambio en la temperatura de, por ejemplo, 7000 a $14000 \mathrm{k}$, equivale a un cambio en el logaritmo de 0.1 dex, con lo que se mantiene dentro de los errores observacionales. Además, por como se define al parámetro $\eta$ ' observacional, se tiene que de la relación del $\log \left(\eta^{\prime}\right)$ es siempre inferior a $\log (\eta)$.

En las Figuras 4.13 y 4.14 presentamos los gráficos para II Zw 33 y Mrk 600, respectivamente, con las relaciones estimadas para $\log \left(\mathrm{O}^{+2} / \mathrm{O}^{+}\right)$y $\log \left(\mathrm{S}^{+} / \mathrm{S}^{2+}\right)$. En las Figuras 4.15 y 4.16 presentamos el diagrama con la relación $\log ([\mathrm{OII}] /[\mathrm{OIII}])$ vs. $\log ([\mathrm{SII}] /[\mathrm{SIII}])$ para las componentes cinemáticas y para el perfil golbal para las respectivas galaxias. El parámetro $\eta$ para cada componente cinemática y las medidas globales fue determinado por el método directo únicamente para el Nudo B de Mrk600, mientras que para las demás regiones, al menos una de las abundancias del cociente tuvo que se derivada a partir de una temperatura estimada a partir de relaciones empíricas o por temperaturas obtenidas a partir de relaciones teóricas. Para la determinacón del parámetro $\eta$ ' cada punto corresponde al cociente de las medidas directas tomadas de la observación, y no se requiere del conocimiento de la temperatura electrónicas involucrada en la estimación de los cocientes de abundancias iónicas. Por lo tanto, este parámetro no depende del método para estimar estas temperaturas.

De la Figura 4.13 analizamos las componentes y las regiones de II Zw 33. Para el Nudo B se determinó una estructura de ionización (o valor de $\eta$ ) muy similar entre la medida global y sus distintas componentes cinemáticas. Por el lado de las componentes de los Nudos A y $\mathrm{C}$, hemos determinado valores muy elevados en los cocientes de abundancias relativas S/O imposibles de explicar físicamente, por lo que se lo relaciona a posible problema en las líneas del IR del [SIII]. Es por esto que a la única componente de estas dos regiones que hemos 
Tabla 4.14. Los cocientes $\log \left(\mathrm{S}^{+} / \mathrm{S}^{2+}\right)$ y $\log \left(\mathrm{O}^{+} / \mathrm{O}^{2+}\right)$ para obtener $\log \eta$, y los cocientes $\log ([\mathrm{SII}] /[\mathrm{SIII}])$ y $\log ([\mathrm{OII}] /[\mathrm{OIII}])$ para obtener $\log \eta^{\prime}$, para las distintas componentes y medidas globales de los Nudos A, B y C de II Zw 33 y los Nudos A y B de Mrk 600.

\begin{tabular}{l|c|c|c|c|c}
\hline Galaxias & Componente & $\log \left(\mathrm{S}^{+} / \mathrm{S}^{2+}\right)$ & $\log \left(\mathrm{O}^{+} / \mathrm{O}^{2+}\right)$ & $\log ([\mathrm{SII}] /[\mathrm{SIII}])$ & $\log ([\mathrm{OII}] /[\mathrm{OII}])$ \\
II Zw 33 & & & & & \\
& Ancha & $-1.64 \pm 0.2$ & $-0.2 \pm 0.08$ & $-0.26 \pm 0.10$ & $-0.02 \pm 0.09$ \\
& $\mathrm{~N} 1$ & $-1.00 \pm 0.3$ & $-0.02 \pm 0.083$ & $0.25 \pm 0.03$ & $-0.2 \pm 0.04$ \\
& $\mathrm{~N} 2$ & $-1.21 \pm 0.23$ & $-0.06 \pm 0.01$ & $-0.5 \pm 0.03$ & $-0.68 \pm 0.07$ \\
& Global & $-1.40 \pm 0.2$ & $-0.06 \pm 0.11$ & $-0.28 \pm 0.03$ & $-0.42 \pm 0.06$ \\
\hline B & Ancha & $-0.67 \pm 0.2$ & $0.05 \pm 0.03$ & $0.03 \pm 0.1$ & $-0.05 \pm 0.05$ \\
& N1 & $-0.12 \pm 0.08$ & $0.01 \pm 0.03$ & $-0.11 \pm 0.08$ & $-0.08 \pm 0.04$ \\
& N2 & $-0.68 \pm 0.3$ & $-0.19 \pm 0.08$ & $-0.39 \pm 0.2$ & $-0.34 \pm 0.05$ \\
& Global & $-0.25 \pm 0.2$ & $-0.06 \pm 0.1$ & $-0.06 \pm 0.06$ & $-0.14 \pm 0.04$ \\
& & & & & \\
\hline C & Ancha & $-1.00 \pm 0.24$ & $0.00 \pm 0.08$ & $0 \pm 0.06$ & $-0.18 \pm 0.07$ \\
& N1 & $-1.32 \pm 0.3$ & $-0.06 \pm 0.05$ & $-0.17 \pm 0.12$ & $-0.4 \pm 0.03$ \\
& N2 & $-1.73 \pm 0.05$ & $-0.06 \pm 0.05$ & $-0.35 \pm 0.15$ & $-0.34 \pm 0.06$ \\
& Global & $-1.31 \pm 0.25$ & $-0.06 \pm 0.04$ & $-0.11 \pm 0.09$ & $-0.32 \pm 0.04$ \\
& & & & & \\
\hline Mrk600 & & & & & \\
Ancha & $-0.24 \pm 0.30$ & $-1.5 \pm 0.10$ & $-0.31 \pm 0.20$ & $-1.51 \pm 0.07$ \\
& N1 & $-0.15 \pm 0.10$ & $-0.62 \pm 0.07$ & $-0.45 \pm 0.10$ & $-1.33 \pm 0.03$ \\
& N2 & $-0.35 \pm 0.04$ & $-0.72 \pm 0.04$ & $-0.27 \pm 0.04$ & $-0.91 \pm 0.01$ \\
& N3 & $-0.33 \pm 0.30$ & $-0.41 \pm 0.07$ & $-0.45 \pm 0.19$ & $-1.02 \pm 0.03$ \\
& Global & $-0.3 \pm 0.40$ & $-0.83 \pm 0.06$ & $-0.31 \pm 0.16$ & $-1.06 \pm 0.03$ \\
& & & & & \\
\hline B & Ancha & $0.12 \pm 0.06$ & $-0.2 \pm 0.3$ & $-0.21 \pm 0.08$ & $-0.66 \pm 0.03$ \\
& N1 & $0.15 \pm 0.05$ & $-0.41 \pm 0.2$ & $0.11 \pm 0.09$ & $-0.39 \pm 0.03$ \\
& Global & $0.08 \pm 0.05$ & $-0.39 \pm 0.2$ & $-0.05 \pm 0.04$ & $-0.49 \pm 0.01$ \\
& & & & & \\
\hline
\end{tabular}

incluído en el análisis de $\eta$ es la angosta N1 del Nudo A, la cual se encuentra levemente desplazada hacia la zona de temperaturas efectivas menores con respecto a las del Nudo B, pero si tenemos en cuenta los errores, está en acuerdo con los valores de $\eta$ de N1 y B del Nudo B. Se observa que para los cocientes del $\log \left(\mathrm{O}^{+} / \mathrm{O}^{+2}\right)$ los errores son menores que los obtenidos para los cocientes del azufre $\log \left(\mathrm{S}^{+} / \mathrm{S}^{2+}\right)$, asociado a los errores propagados en la determinación de las temperaturas electrónicas.

Para el Nudo B los resultados están en acuerdo entre las componentes cinemáticas y la medida global, si tenemos en cuenta los errores. Hay una diferencia considerable si comparamos la medidas global estudiada a través del espectro Echelle con el determinado a través del espectro de dispersión simple de dichas galaxias BCDs trabajados previamente. Esta diferencia se observa en los valores de los cocientes de $\log \left(\mathrm{O}^{+} / \mathrm{O}^{+}\right)$para el Nudo B, lo que puede ser resultado de que en este trabajo se determinaron las abundancias de $\mathrm{O}^{+2}$ derivando la temperatura de manera directa pero $\mathrm{O}^{+}$se calculó con la temperatura por modelos y métodos empíricos, y en la dispersión simple las abundancias fueron estimadas por el método directo, siendo este más preciso porque en su determinación se hacen menos suposiciones y hay menor propagación de error. Este análisis nos indica que la temperatura del campo de 
radiación ionizante es muy similar para las componentes cinemáticas N2 y G, de temperaturas mayores por un lado y para las N1 y la global, de temperaturas menores por el otro (triángulos rojos). Los cúmulos estelares masivos responsables de la ionización de cada grupo de componente cinemática sería el mismo. La temperatura estimada para la componente N1 del Nudo A (triángulo verde) es levemente menor que las componentes N1 y la global Nudo B.

En el caso de las regiones A y B de Mrk600 se encuentra un acuerdo en la estructura de ionización de las componentes cinemáticas y de la medida global, dentro de los errores. La única componente que se distancia del resto de las regiones, estando en la zona de alta ionización, es la componente ancha de Mrk600 A. Pero si tenemos en cuenta los errores grandes que presenta en los cocientes del azufre, podemos estimar un $\eta$ muy similar. Esto nos indica que no hay indicios de diferentes estados evolutivos entre las regiones A y B debido a que ambas componentes se ubican hacia regiones con temperaturas de campos de radiaciones ionizantes similares. Para esta galaxia los valores en el diagrama de $\eta$ se encuentran desplazados hacia temperaturas efectivas mayores del campo de radiación ionizante, con respecto a lo encontrado para las regiones de II Zw 33 .

De los comportamientos derivados de las Figuras 4.13 y 4.14 podemos indicar que las regiones de Mrk600 se ven ionizadas por cúmulos más jóvenes que las regiones de II Zw 33.

En los Nudos de II Zw 33 los valores de $\eta$ son mayores, en casi todos los casos, a los valores esperados en las galaxias HiI (Hägele et al., 2008; Firpo, 2011). Solamente las componentes N1 y global se hayan sobre las zonas de temperaturas efectivas esperadas, dadas por líneas paralelas de $\eta$. Por el lado de $\eta$ ', al ser una determinación puramente observacional, no depende de la temperatura de [SIII], por ende, disminuye la dispersión. De igual forma, hay que considerar y ser cuidadosos con la interpretación posible de este parámetro debido al posible problema que presentan las líneas del [SIII] en el IR.

Por el lado de Mrk 600 (ver Figura 4.14) parecería haber una diferencia en la temperatura del campo de radiaciónn, siendo la temperatura del cúmulo vinculado a 1 Nudo A un poco más alta, o sea que sería un cúmulo un poco mś joven el responsable de la ionización. Además, para todos los cúmulos de ambas galaxias, parece también que la temperatura del campo de radiación ionizante sería el mismo para las distintas componentes, lo que sería indicativo que el cúmulo ionizante sería el mismo y no sería un efecto de proyección que veamos las distintas componentes cinemáticas en la misma región del espacio, de manera similar a lo que se encontró en Haro 15 en Hägele et al. (2012).

En cambio para las regiones de Mrk 600 los valores de $\eta$ están en el rango esperado para las galaxias Hir. Finalmente, el análisis del parámetro puramente observacional $\eta$ ' muestra que, salvo la componente ancha de II Zw 33 que es levemente mayor, todas las componentes están en los rangos esperables de las galaxias y regiones HiI.

Por otro lado, considerado el análisis realizado en la Tesis de Guillermo Hägele, a partir de los flujos calculados, $\mathrm{F}(\mathrm{H} \alpha)$, es posible estudiar otras características de las galaxias Hir. La estimación de los flujos para cada una de las componentes cinemáticas nos permite calcular el número de fotones ionizantes de $\mathrm{Q}\left(\mathrm{H}_{0}\right)$ a través de la expresión:

$$
\log Q\left(H_{0}\right)=7.35 \times 10^{11} L(H \alpha)
$$

donde la luminosidad está en unidades de $\operatorname{erg~s}^{-1}$. Con dicho el número de fotones, es posible determinar el parámetro de ionización, $\mathrm{U}$, a partir de:

$$
\mathrm{U}=\frac{\mathrm{Q}\left(\mathrm{H}_{0}\right)}{4 \pi c \mathrm{R}^{2} \mathrm{~N}_{e}},
$$

donde $\mathrm{R}$ corresponde al radio de la región ionizada bajo estudio y c la constante de la velocidad de la luz. Como no necesariamente pueden conocerse las dimensiones de cada una de las 


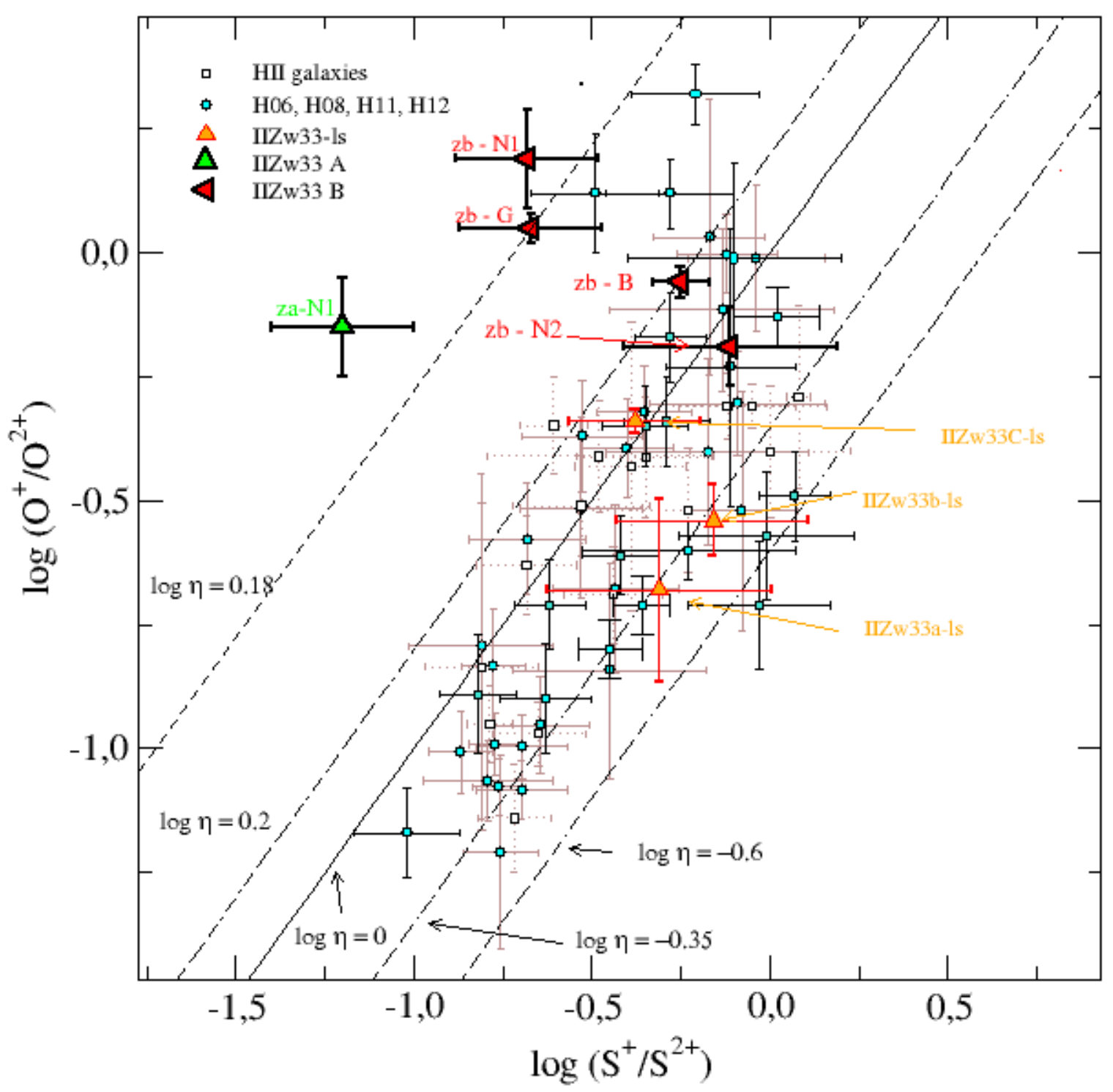

Figura 4.13. Parámetro $\eta$. Las líneas continuas y a trozos que aparecen en el gráfico corresponden a valores constantes del parámetro $\eta$, que nos sirven como indicadores de la temperatura efectiva del campo de radiación ionizante del medio. Podemos ver como las regiones de formación estudiadas en II Zw 33 se encuentran entre la recta correspondiente a $\log (\eta)=0.18$ y $\log (\eta)=-0.35$, si tenemos en cuenta las barras de error. Según Vilchez \& Pagel (1988) las galaxias HiI tienen valores de $\log (\eta)$ entre -0.35 y 0.2 , que corresponden a temperaturas de ionización altas. Para las regiones con $\eta$ mayores, como Za-N1, Zb-G y ZbN1, se cree que son estructuras con grados de ionización más bajos que las correspondientes a Zb-N2 y Zb-B. 
regiones involucradas en el estudio de múltiples componentes, Díaz et al. (2000) definen una serie de ecuaciones que relaciona al parámetro de ionización U con las medidas del [SII]/[SIII] y del $[\mathrm{OII}] /[\mathrm{OIII}]$, dadas como:

$$
\begin{aligned}
& \log \mathrm{U}=-1.68 \log ([\mathrm{SII}] /[\mathrm{SIII}])-2.99, \\
& \log \mathrm{U}=-1.80 \log ([\mathrm{OII}] /[\mathrm{OIII}])-2.99,
\end{aligned}
$$

en donde los cocientes $[\mathrm{SII}] /[\mathrm{SIII}]$ y $[\mathrm{OII}] /[\mathrm{OIII}]$, se determinan a partir de una relación exclusivamente observacional.

En Dors et al. (2011) mejoran los ajustes de estas relaciones y además utilizan la relación entre el $[\mathrm{SII}] / \mathrm{H} \alpha$ para la estimación de U. Luego, las expresiones que utilizamos son:

$$
\begin{gathered}
\log \mathrm{U}=-1.68( \pm 0.07) \log ([\mathrm{SII}] /[\mathrm{SIII}])-3.09( \pm 0.07), \\
\log \mathrm{U}=-1.66( \pm 0.06) \log ([\mathrm{SII}] / \mathrm{HII})-4.13( \pm 0.07), \\
\log \mathrm{U}=-1.22( \pm 0.07) \log ([\mathrm{OII}] /[\mathrm{OIII}])-2.25( \pm 0.05) .
\end{gathered}
$$

Estos parámetros, U y Q, juntos con la densidad, $\mathrm{N}_{e}$, nos permiten estimar el tamaño angular aproximado de la región emisora. Para ello, se utiliza la expresión dada por Castellanos et al. (2002) como:

$$
\phi=0.64\left[\frac{\mathrm{F}(\mathrm{H} \alpha)}{10^{-14}}\right]^{1 / 2}\left(\frac{\mathrm{U}}{10^{-3}}\right)^{-1 / 2}\left(\frac{\mathrm{N}_{e}}{10^{2}}\right)^{-1 / 2} .
$$

En nuestro trabajo hemos realizado una estimación de los diámetros angulares a partir de U para la componente global únicamente, para comparar los valores con los encontrados en la linteratura. En los casos de los nudos de II Zw 33 hemos utilizado los flujos totales dados por la fotometría realizada en Méndez et al. (1999), indicando que las luminosidades y los diámetros de las regiones son los presentados por dicho trabajo. Por el lado de Mrk600, utilizamos las medidas globales de nuestros ajustes, debido a que no hay registro en la literatura de determinación de diámetros de los Nudos A y B para hacer una comparación de las medidas. Como en nuestros datos las líneas del [SIII] en el IR se encuentran sobre los órdenes del extremo del detector y pueden estar afectadas por efectos de borde, no resultan confiables y por eso no se listan esos valores en la Tabla.

También es posible estimar la masa del cúmulo de estrellas ionizantes, capaces de producir los fotones energéticos con $\lambda<912 \AA$, a partir de una relación entre el número de fotones $\mathrm{Q}\left(\mathrm{H}_{0}\right)$ y el ancho equivalente (EW, del inglés: equivalent width) de la línea de H $\beta$. Díaz et al. (2000) afirman que, según los modelos de evolución de cúmulos ionizantes utilizados en la literatura, se puede derivar la siguiente relación:

$$
\mathrm{M}_{\mathrm{ion}}=\frac{Q\left(H_{0}\right)}{10^{44.48+0.86 \cdot \log [E W(H \beta)]}},
$$

en donde $\mathrm{EW}(\mathrm{H} \beta)$ es el ancho equivalente medido de $(\mathrm{H} \beta)$, el cual presentamos para cada región en las tablas de Intensidad de las líneas corregidas por enrojecimiento 4.3, 4.5, 4.7, 4.9 y 4.11 , correspondientes.

El gas ionizado en las regiones observadas puede estimarse con las luminosidades de $\mathrm{H} \alpha$ a partir, por ejemplo, de la relación dada por Macchetto et al. (1990):

$$
\mathrm{M}_{\mathrm{HII}}=3.32 \cdot 10^{-33} L(H \alpha) N e^{-1},
$$

en donde tomamos como un valor mínimo de baja densidad a $\mathrm{Ne}^{-1}=100 \mathrm{~cm}^{-3}$. Los valores de las densidades para las componentes que sean menores a este valor, las redondeamos en 
$100^{-3}$. Hemos tomado este criterio para obtener valores de masas ionizantes que no se vean afectados por los cocientes de números pequeños (en el límite de bajas densidades) y con errores muy grandes de densidades menores a $100 \mathrm{~cm}^{-3}$, lo que genera un valor mayor en las masas ionizantes que no es necesariamente el representativo.

Las luminosidades $\mathrm{L}(\mathrm{H} \alpha)$ para cada componente se definieron según las medidas de emisión correspondintes (ver las Tablas con los parámetros de la cinemática para cada una de las regiones en $3.1,3.2,3.3,3.4$ y 3.5), el parámetro de ionización $\mathrm{U}$, los diámetros de las regiones asociadas al perfil global y a las componentes cinemáticas en pársecs, la cantidad de fotones ionizantes y la masa total de gas ionizado se presentan en la Tabla 4.15.

En la Tabla 4.15 presentamos los valores de U determinados a partir de los cocientes de $[\mathrm{OII}] /[\mathrm{OIII}]$ por ser el más utilizado en la literatura y $\mathrm{U}_{[\mathrm{SII}] / \mathrm{H} \alpha}$ para comparar nuestras estimaciones en las regiones de Mrk 600 con lo realizado en Lagos et al. (2018). Para II Zw 33, como mencionamos anteriormente, las luminosidades y los diámetros presentados corresponden al trabajo de Méndez et al. (1999). Las luminosidades derivadas a partir de los flujos de nuestros ajustes a partir de la calibración de los espectros echelle, son alrededor de 5, 3 y 3 veces menores para los Nudos A, B y C, respectivamente, que las publicadas en Méndez et al. (1999). Para las componentes utilizamos las medidas de emisión determinadas en el análsis de la cinemática y las masas asociadas al cúmulo ionizante y la masa total de HiI son derivados a partir de las correspondientes cantidades. Por el lado de Mrk 600, hemos utilizado las medidas de $\mathrm{F}(\mathrm{H} \alpha)$ totales para las regiones A y B $\left(6.91 \times 10^{-14} \mathrm{erg} \mathrm{cm}^{-2} \mathrm{~s}^{-1}\right.$ y $6.22 \times 10^{-14}$ $\operatorname{erg~} \mathrm{cm}^{-2} \mathrm{~s}^{-1}$, respectivamente) dadas por Lagos et al. (2018).

Además, vale la pena mencionar que el valor de U determinado a partir de la relación del cociente $\log ([\mathrm{SII}]) / \mathrm{H} \alpha$ presentados por Lagos et al. (2018) para los Nudos A y B de Mrk 600 $(-1,76$ y -2.20 , respectivamente) están en acuerdo con los determinados por nosotros de -1.80 y de -2.25 para el Nudo A y B, respectivamente, utilizando las medidas de los cocientes de flujos a partir de nuestras observaciones y ajustes. Los diámetros aproximados del cúmulo calculados a través de la ecuación 4.5, a partir de $\mathrm{U}_{\text {[OIII]/[OII] }}$ y $\mathrm{U}_{[\mathrm{SII}] / \mathrm{H} \alpha}$, respectivamente, resultan de 216pc y de 35pc para Mrk600 A y de $98 \mathrm{pc}$ y $50 \mathrm{pc}$ para Mrk600 B. La diferencia entre los tamaños estimados indica una gran dispersión en la determinación de los tamaños según los distintos indicadores. Las regiones son chicas en comparación a los diámetros de II Zw 33 presentados por Méndez et al. (1999), algunas centenas mayores. Los valores de los diámetros determinados a partir de nuestras medidas de $\mathrm{U}_{\text {[OIII]/[OII] }}$ y $\mathrm{U}_{[\mathrm{SII}] / \mathrm{H} \alpha}$ en comparación con los valores presentados en el trabajo de Méndez et al. (1999) son menores, considerablemente. Los diámetros $\phi_{U_{[\mathrm{OIII}] /[\mathrm{OII}]}}$ resultan de 363pc para el Nudo A, 190pc para el Nudo B y 127pc para el Nudo C. Por el lado de $\phi_{U_{[\mathrm{SII}] / \mathrm{H} \alpha}}$ resultan de 270pc para el Nudo A, 300 pc para el Nudo B y 121pc para el Nudo C.

Los parámetros de ionización, $\mathrm{U}$, determinados a través de los distintos indicadores toman valores dentro de los rangos esperables de -1.14 y -4.09 . Los resultados obtenidos de las masas muestran un excelente acuerdo para las masas de las medidas globales y las de las componentes cinemáticas, y están dentro de los valores esperados para este tipo de objetos, al igual que el número de fotones ionizantes involucrados en estos procesos (ver por ejemplo: Hägele et al., 2007; Guseva et al., 2011; Hägele et al., 2011; James et al., 2015, y ver también los objetos presentados en la Tesis de Guillermo Hägele). 


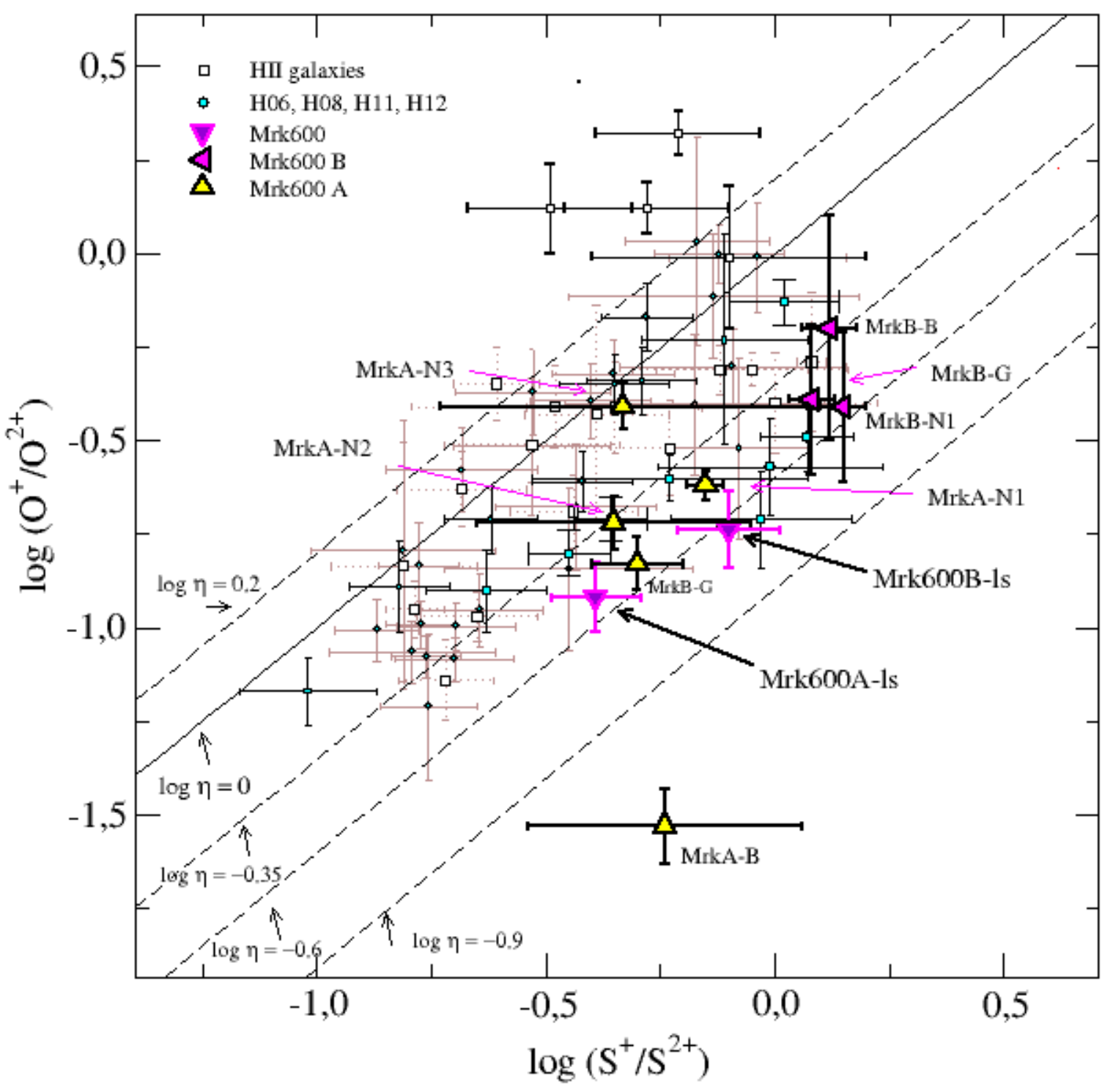

Figura 4.14. Parámetro $\eta$. Al igual que el gráfico de $\eta$ de II Zw 33, las líneas continuas y a trozos indican valores constantes del parámetro $\eta$, que nos sirven como indicadores de la temperatura efectiva del campo de radiación ionizante del medio. Para Mrk600 y las componentes cinemáticas resueltas para las regiones $\mathrm{A}$ y $\mathrm{B}$, vemos que se encuentran entre las rectas $\log (\eta)=0$ y $\log (\eta)=-0.9$. Por el lado del Nudo A, las componentes N1, N2 y la medida global se encuetran en acuerdo entre las rectas $\log (\eta)=-0.35$ y $\log (\eta)=-0.6$, y la componente N3 también lo hace teniendo en cuenta los errores. Además, el valor de $\eta$ determinado a partir de los datos long-slit están en excelente acuerdo con estas medidas. La componente ancha se encuentra levemente desplaza hacia $\eta$, indicando una temperatura efectiva levemente mayor que las demás componentes. Para Mrk 600 B las componentes están en acuerdo entre sí y además en concordancia con las medidas determinadas a partir de los datos long-slit, entre las rectas $\log (\eta)=-0.35$ y $\log (\eta)=-0.6$. 
Figura 4.15. Gráfico del parámetro $\eta$ '. Las líneas continuas y a trozos que aparecen en el gráfico corresponden a valores constantes del parámetro $\eta$ ', que nos sirven como indicadores de la temperatura efectiva del campo de radiación ionizante del medio a través de las cantidades medidas del espectro directamente. En este gráfico, a diferencia del $\eta$, hemos podido incluir las medidas de todas las componentes para los Nudos A y C. Con estas medudas vemos que disminuye la dispersión para las componentes estudiadas de la región B y además podemos indicar un gran acuerd en las medidas de $\eta$ ' para las componentes de las regiones B y C. Por el lado del Nudo A, la componente ancha se ubica hacia temperaturas efectivas menores y la angosta N1 se encuentran levemente desplazadas hacia temperaturas mayores frente a las componente N2 y la medida global, que indican temperaturas del campo de radiación similares.

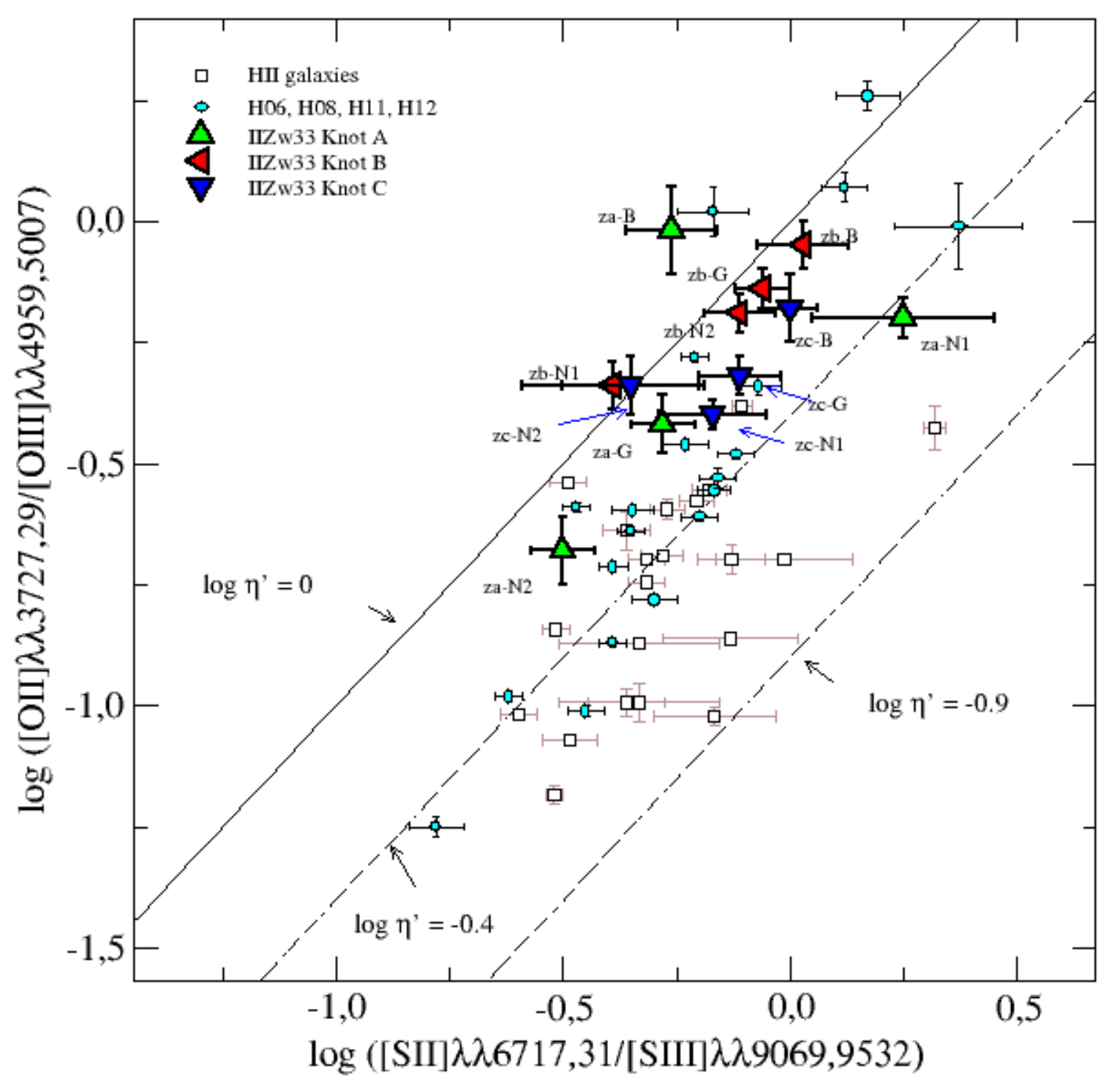


Figura 4.16. Gráfico del parámetro $\eta$ '. Las líneas continuas y a trozos que aparecen en el gráfico corresponden a valores constantes del parámetro $\eta$ ', que nos sirven como indicadores de la temperatura efectiva del campo de radiación ionizante del medio a través de las cantidades medidas del espectro directamente. Por el lado de Mrk600 A encontramos unas temperaturas del campo de radiación similares a las obtenidas a través del parámetro $\eta$, en donde la componente ancha es la que de desplaza hacia abajo en el diagrama, indicando valores de $\eta$ ' menores asociados a temperaturas efectivas mayores. Para Mrk 600 B podemos ver que las componentes estarían siendo ionizadas por un cúmulo con una temperatura efectiva similar.

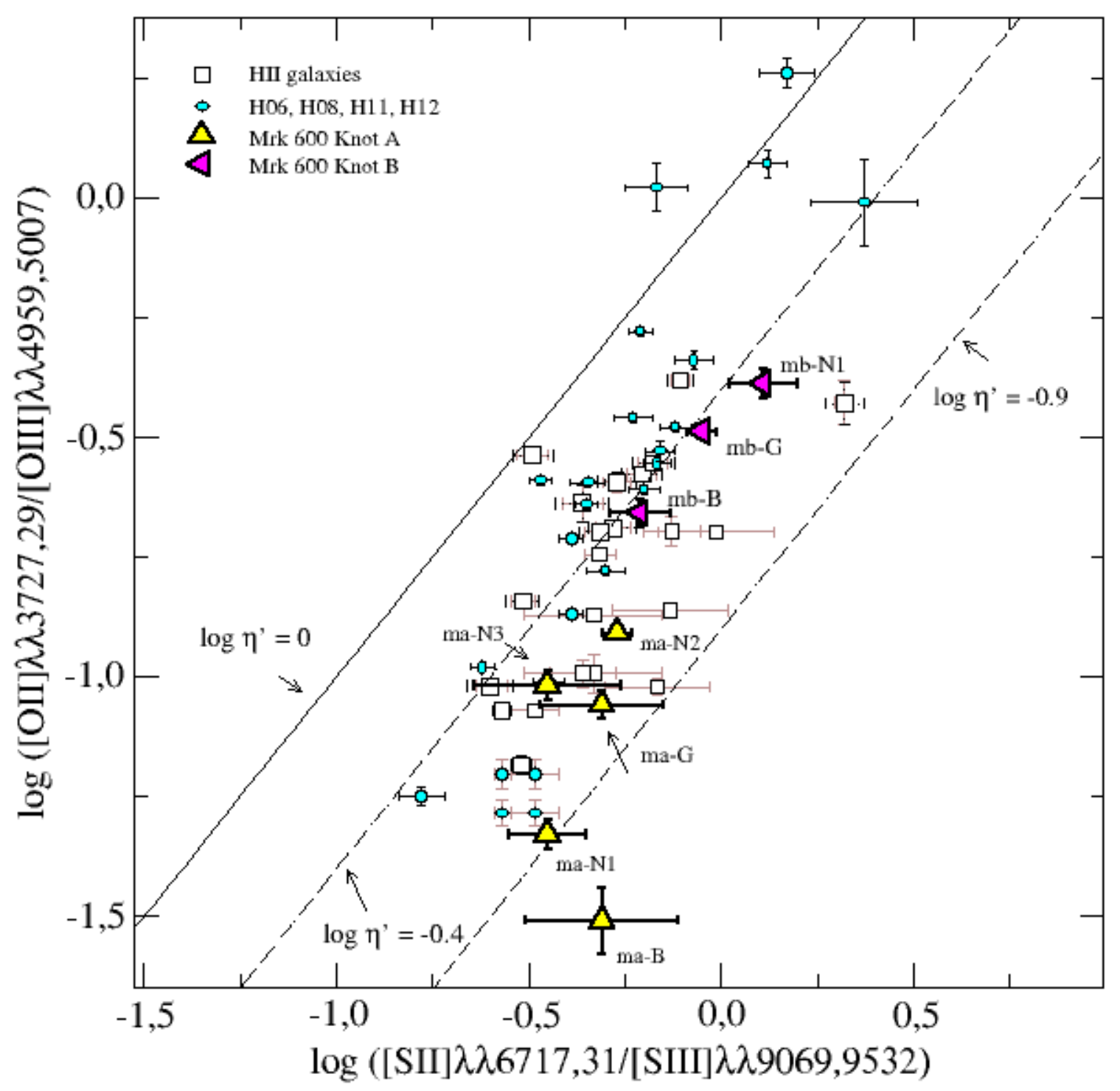


Tabla 4.15. De izquierda a derecha, la identificación de las galaxias y nudos, las componentes cinemáticas, luminosidad en $\mathrm{H} \alpha$ derivada del flujo y de las medidas de emisión para cada componente, el número de fotones ionizantes, $\mathrm{Q}\left(\mathrm{H}_{0}\right)$, el parámetro de ionización, $\mathrm{U}$, estimado con dos indicadores distintos: $\mathrm{U}_{\text {[OII] } /[\mathrm{OII}]}$ y $\mathrm{U}_{[\mathrm{SII}] / \mathrm{H} \alpha}$, el diámetro aproximado $\phi$ para la global [considerando las distancias: II Zw $33=41.76 \pm 2.92 \mathrm{Mpc}=1.154 \times 10^{26} \mathrm{~cm}$ (de Vaucouleurs, 1991) y Mrk $600=11.69 \pm 0.85 \mathrm{Mpc}=4.32 \times 10^{25} \mathrm{~cm}$ (Haynes et al., 1997)], el diámetro aproximado $\phi_{[\mathrm{OII}] /[\mathrm{OIII}]}$ y el diámetro aproximado $\phi_{[\mathrm{SII}] / \mathrm{H} \alpha}$, la masa del cúmulo ionizante medida en $\mathrm{M}_{\odot}$ y la masa de total de $\mathrm{HII}$ en $\mathrm{M}_{\odot}$.

\begin{tabular}{|c|c|c|c|c|c|c|c|c|c|c|}
\hline $\begin{array}{c}\text { Galaxias } \\
\text { Nudo }\end{array}$ & Componente & $\begin{array}{c}\log (\mathrm{L}(\mathrm{H} \alpha)) \\
{\left[10^{39} \mathrm{erg} \mathrm{s}^{-1}\right]}\end{array}$ & $\begin{array}{c}\log \left(\mathrm{Q}\left(\mathrm{H}_{0}\right)\right) \\
{\left[\mathrm{ph} \mathrm{s}^{-1}\right]}\end{array}$ & $\begin{array}{c}\log (\mathrm{U}) \\
{[\mathrm{OII}] /[\mathrm{OIII}]}\end{array}$ & $\begin{array}{c}\log (\mathrm{U}) \\
{[\mathrm{SII}] / \mathrm{H} \alpha}\end{array}$ & $\begin{array}{c}\text { Diámetro }(\phi) \\
{[\mathrm{pc}]}\end{array}$ & $\begin{array}{c}\phi_{[\mathrm{OII}] /[\mathrm{OIII}]} \\
{[\mathrm{pc}]}\end{array}$ & $\begin{array}{c}\phi_{[\mathrm{SII}] / \mathrm{H} \alpha} \\
{[\mathrm{pc}]}\end{array}$ & $\begin{array}{c}\mathrm{M}_{\text {Ionizante }} \\
10^{5} \mathrm{M}_{\odot}\end{array}$ & $\begin{array}{r}\mathrm{M}_{\mathrm{HII}} \\
10^{5} \mathrm{M}_{\odot}\end{array}$ \\
\hline $\begin{array}{c}\text { II Zw } 33 \\
\text { A }\end{array}$ & $\begin{array}{c}\text { Global } \\
\text { Ancha } \\
\text { N1 } \\
\text { N2 }\end{array}$ & $\begin{array}{l}40.37 \\
40.05 \\
39.82 \\
39.75\end{array}$ & $\begin{array}{l}52.24 \\
51.92 \\
51.69 \\
51.61\end{array}$ & $\begin{array}{l}-2.76 \\
-2.27 \\
-2.49 \\
-3.08\end{array}$ & $\begin{array}{l}-2.50 \\
-3.01 \\
-2.20 \\
-2.10\end{array}$ & 735 & 363 & 270 & $\begin{array}{l}48.68 \\
43.37 \\
40.81 \\
38.80\end{array}$ & $\begin{array}{l}7.84 \\
3.75 \\
2.06 \\
1.68\end{array}$ \\
\hline B & $\begin{array}{c}\text { Global } \\
\text { Ancha } \\
\text { N1 } \\
\text { N2 }\end{array}$ & $\begin{array}{l}40.13 \\
39.55 \\
39.95 \\
39.04\end{array}$ & $\begin{array}{l}52.00 \\
51.42 \\
51.82 \\
50.90\end{array}$ & $\begin{array}{l}-2.42 \\
-2.31 \\
-2.35 \\
-2.66\end{array}$ & $\begin{array}{l}-2.80 \\
-3.33 \\
-2.52 \\
-2.19\end{array}$ & 625 & 190 & 300 & $\begin{array}{l}8.82 \\
7.58 \\
3.21 \\
1.25\end{array}$ & $\begin{array}{l}4.54 \\
1.18 \\
2.44 \\
0.37\end{array}$ \\
\hline $\mathrm{C}$ & $\begin{array}{c}\text { Global } \\
\text { Ancha } \\
\text { N1 } \\
\text { N2 }\end{array}$ & $\begin{array}{l}39.52 \\
38.83 \\
39.35 \\
38.59\end{array}$ & $\begin{array}{l}51.40 \\
50.69 \\
51.22 \\
50.46\end{array}$ & $\begin{array}{l}-2.65 \\
-3.27 \\
-2.25 \\
-2.74\end{array}$ & $\begin{array}{l}-2.64 \\
-2.47 \\
-2.74 \\
-2.66\end{array}$ & 420 & 127 & 121 & $\begin{array}{l}23.26 \\
18.07 \\
21.55 \\
16.77\end{array}$ & $\begin{array}{l}1.11 \\
0.22 \\
0.74 \\
0.13\end{array}$ \\
\hline $\begin{array}{c}\text { Mrk } 600 \\
\text { A }\end{array}$ & $\begin{array}{c}\text { Global } \\
\text { Ancha } \\
\text { N1 } \\
\text { N2 } \\
\text { N3 }\end{array}$ & $\begin{array}{l}39.21 \\
38.66 \\
38.41 \\
38.88 \\
38.06\end{array}$ & $\begin{array}{l}51.08 \\
50.52 \\
50.28 \\
50.75 \\
49.92\end{array}$ & $\begin{array}{l}-3.54 \\
-4.09 \\
-3.87 \\
-3.36 \\
-3.49\end{array}$ & $\begin{array}{l}-1.82 \\
-2.52 \\
-1.14 \\
-1.86 \\
-1.89\end{array}$ & - & 216 & 35 & $\begin{array}{l}3.35 \\
2.88 \\
2.57 \\
3.01 \\
1.84\end{array}$ & $\begin{array}{l}0.54 \\
0.15 \\
0.09 \\
0.25 \\
0.04\end{array}$ \\
\hline B & $\begin{array}{c}\text { Global } \\
\text { Ancha } \\
\text { N1 }\end{array}$ & $\begin{array}{l}39.16 \\
38.40 \\
39.08\end{array}$ & $\begin{array}{l}51.03 \\
50.26 \\
50.95\end{array}$ & $\begin{array}{l}-2.85 \\
-3.06 \\
-2.73\end{array}$ & $\begin{array}{l}-2.25 \\
-2.86 \\
-2.01\end{array}$ & - & 98 & 50 & $\begin{array}{l}1.99 \\
1.56 \\
1.94\end{array}$ & $\begin{array}{l}0.49 \\
0.08 \\
0.40\end{array}$ \\
\hline
\end{tabular}




\subsection{Resumen}

En este Capítulo se realizó un análisis detallado de las características físicas y químicas del gas ionizado. Se discriminó por componente cinemática y para el flujo global de las líneas de emisión del gas ionizado en los nudos más brillantes de formación estelar de las galaxias BCD II Zw 33 y Mrk600. Para esto se estimaron temperaturas y densidades electrónicas, y se determinaron las abundancias iónicas y totales de diferentes elementos. Cuando la temperatura no pudo estimarse de manera directa a través de las relaciones entre líneas intensas y débiles aurorales, se utilizaron modelos y relaciones empíricas. En estos caso, en las tablas no se han indicado las estimaciones de los errores en este caso no son realistas y resultan en errores artificialmente bajos. Utilizando los espectros Echelle tomados con el espectrógrafo de alta resolución MIKE del Telescopio Clay, de los Magallanes en el Observatorio Las Campanas, Chile, se obtienen los siguiente resultados:

- II Zw 33

- En el Nudo A, la determinación de las temperaturas electrónicas del [OIII] se realizó a partir de líneas aurorales débiles e intensas para las componentes angostas N1 y N2 y para la medida global, con precisiones de $14 \%, 4 \%$ y $3 \%$, respectivamente. Para la componente ancha se consideró la temperatura de $10.000 \mathrm{k}$ y a través de las relaciones teóricas y de modelos, se obtuvieron las temperaturas $\mathrm{T}_{e}([\mathrm{SIII}])$, $\mathrm{T}_{e}([\mathrm{OII}]), \mathrm{T}_{e}([\mathrm{SII}]) \mathrm{Y}_{e}([\mathrm{NII}])$.

- En el Nudo B pudieron derivarse $\mathrm{T}_{e}([\mathrm{OIII}])$ y $\mathrm{T}_{e}([\mathrm{SIII}])$ con el método directo para las componentes angostas N1 y N2 y para la medida global, con precisiones del $5 \%$ y $15 \% ; 2 \%$ y $20 \% ; 3 \%$ y $20 \%$, respectivamente. Los errores en la estimación de la $\mathrm{T}_{e}([\mathrm{SIII}])$ son mayores que para $\mathrm{T}_{e}([\mathrm{OIII}])$. La temperatura $\mathrm{T}_{e}([\mathrm{OII}])$ se obtuvo a través de las relaciones teóricas, y las temperaturas $\mathrm{T}_{e}([\mathrm{SII}])$ y $\mathrm{T}_{e}([\mathrm{NII}])$ se consideraron iguales a $\mathrm{T}_{e}([\mathrm{OII}])$.

- En el Nudo C, se consideró una temperatura electrónica de [OIII] de 10.000k para la medida global y para las múltiples componentes cinemáticas. La temperatura $\mathrm{T}_{e}([\mathrm{SIII}])$ se calculó usando la relación con la temperatura del [OIII] dada por Hägele et al. (2006). Con un análisis por medio de relaciones teóricas y de modelos, con este valor se estimó la temperatura $\mathrm{T}_{e}([\mathrm{OII}])$ usando la relación dada en PérezMontero et al. (2003). Luego, las temperaturas $\mathrm{T}_{e}([\mathrm{SII}])$ y $\mathrm{T}_{e}([\mathrm{NII}])$ se consideraron iguales a la temperatura de $\mathrm{T}_{e}([\mathrm{OII}])$.

- El análisis de las densidades electrónicas para todas las componentes y medidas globales de las regiones se llevó adelante por medio del cociente del doblete de las líneas del $[\mathrm{SII}] \lambda \lambda 6717,6731 \AA$. Se estimaron valores muy por debajo de la densidad electrónica crítica de desexcitación colisional. En algunos casos los errores y las incertezas en la estimación de la densidad son muy grandes, de manera que se ha indicado el valor medio estimado. Las densidades calculadas en general resultan $\mathrm{n}_{e}<170 \mathrm{~cm}^{-3}$.

- Se estimaron las abundancias iónicas de $\mathrm{O}^{+}, \mathrm{O}^{2+}, \mathrm{S}^{+}, \mathrm{S}^{2+}, \mathrm{N}^{+}, \mathrm{Ne}^{2+}, \mathrm{Ar}^{2+}$ y $\mathrm{He}^{+}$, junto con los ICFs del $\mathrm{S}\left(\mathrm{S}^{+}+\mathrm{S}^{2+}\right)$, del $\mathrm{Ne}\left(\mathrm{Ne}^{2+}\right)$ y $\mathrm{Ar}\left({ }^{2+}\right)$ para las componentes cinemáticas y para las medidas globales también, en las tres regiones estudiadas, encontrando comportamientos diversos para cada componente. A las líneas de emisión de los iones asociados a las zonas de menor excitación $\left(\mathrm{O}^{+}, \mathrm{S}^{+}\right.$, $\mathrm{N}^{+}$) fue posible ajustarle las múltiples componentes en todas las regiones. 
Por el lado de las líneas de $\mathrm{Ne}^{2+}$, asociadas a zonas de alta excitación, en algunas componentes presentan incertidumbres grandes. Para las zonas de una excitación intermedia, para el ión $\mathrm{S}^{2+}$ hemos obtenido valores con mucha incetidumbre que han llevado a obtener resultados dudosos, mientras que el ión de $\mathrm{Ar}^{2+}$ ha sido posible medirlo en todas las componentes.

- Fue posible calcular las abundancias totales de O, N, Ne y Ar para nuestras regiones, que se encuentran en el mismo rango de las abundancias totales medidas para las galaxias Hiı. Los valores determinados para las abundancias totales de oxígeno muestran un buen acuerdo, si tenemos en cuenta los errores. Para el Nudo A hay un acuerdo excelente para los valores estimados de abundancias totales de oxígeno, dentro de los errores. Si se comparan con las abundancias determinadas para los espectros de dispersión simple, se hallan diferencias entre componentes, pero son valores esperables para RHII. Las abundancias totales del S son dudosas para los Nudos A y C, por eso las hemos incluído en las gráficas ni en las discusiones. Para los cocientes $\mathrm{S} / \mathrm{O}$ los valores estimados no son físicamente posibles para los $\mathrm{Nu}$ dos A y C, salvo la componente N1 del Nudo A. El Nudo B está en acuerdo con los valores estimados para este tipo de regiones, pero la componente ancha presenta un exceso respecto a las otras componentes. Las abundancias totales de O estimadas están en general en acuerdo entre las componentes para cada Nudo, pero en los casos en que se observan diferencias, como en N1 y N2 del Nudo B, los valores similares de los cocientes de los elementos químicos respecto al $\mathrm{O}$ calculados, pueden ser indicios de que las componentes cinemáticas corresponden a distintas fases de un mismo gas.

- Para el He se encontró que las abundancias totales presentan un comportamiento similar entre las componente angostas N1 y N2 de los Nudos A y B, en donde la abundancia para la componente N1 es menor. Un comportamiento inverso se identificó en el Nudo B, en donde la abundancia de N1 es mayor. La abundancia del He no pudo ser estimada para todas las componentes debido a que la componente ancha no pudo ser ajustada con errores menores al $40 \%$. Distinta resultó la situación en el Nudo B en donde se pudo ajustar la componente ancha en todas las líneas del $\mathrm{He}$, obteniendo el valor adoptado de $\mathrm{He}^{+} / \mathrm{He}^{+}$más grande entre las componentes de esta región. Los valores estimados de $\mathrm{He}^{+} / \mathrm{H}^{+}$están en acuerdo con los valores espados para estas regiones, teniendo en cuenta los errores. Como las líneas de He observadas en los Nudos tienen perfiles generalmente ruidosos, convendría obtener nuevos datos con mejor $\mathrm{S} / \mathrm{N}$ para tratar de disminuir los errores en las estimaciones.

- Las determinaciones de $\mathrm{c}(\mathrm{H} \beta)$ para el cálculo de la extinción en las regiones presentan valores compatibles con la baja extinción. Aunque hay algunas componentes que presentan $\mathrm{c}(\mathrm{H} \beta) \approx 0.5$, lo que indica una absorción mayor que el resto de los brotes, son extinciones relativamente bajas para esta clase de regiones.

- Las abundancias totales de oxígeno estimadas a través de los diferentes parámetros empíricos en general muestran valores consistentes con las abundancias derivadas usando las temperaturas electrónicas de [OIII] obtenidas con el método directo o a través de relaciones teóricas o empíricas. Se observó que las abundancias determinadas por el parámetro empírico $\mathrm{S}_{23}$ en prácticamente todos los casos subestima la abundancia total de oxígeno obtenida previamente. Los resultados determinados a través de este análisis muestran resultados consistentes con lo determinado previamente a partir del método directo. 
- Las estructuras de ionización estudiadas a partir de los parámetros $\eta$ y $\eta^{\prime}$ nos dan indicios de las caracterísitcas de los campos de radiación para las regiones bajo estudio. Los Nudos A y C presentan mucha incertidumbre debido a incertidumbres en las medidas de las líneas del [SIII] en el IR, por lo que no las hemos analizado en estos diagramas. Para el Nudo B pudimos estimar con un muy buen acuerdo las medidas de $\eta$, revelando una similitud en los valores determinados para sus componentes y lo esperado para estas regiones y galaxias.

- La temperatura efectiva del campo de radiación es similar para las distintas componentes cinemáticas, y por lo tanto, podríamos decir que el cúmulo ionizante de las distintas componentes es el mismo. La presencia de distintas componentes cinemáticas no sería un efecto de proyección o un problema de resolución espacial, lo que se condicen con los resultados obtenidos para los Nudos observados en la galaxia BCD Haro 15 (Hägele et al., 2012).

- Mrk 600

- En el Nudo A se derivaron las temperaturas electrónicas del [OIII] y [Siri] con el método directo para las componentes cinemáticas y para la medida global. Las precisiones fueron del $7 \%$ y $19 \%$ para la componente ancha, $2 \%$ y $15 \%$ para la componente angosta N1, $4 \%$ y $12 \%$ para la componente $\mathrm{N} 2,7 \%$ y $6 \%$ para la componente N3 y $3 \%$ y $11 \%$ para la medida global, para el O y S respectivamente. Las temperaturas $\mathrm{T}_{e}([\mathrm{SII}])$ y $\mathrm{T}_{e}([\mathrm{NII}])$ se consideraron iguales a $\mathrm{T}_{e}([\mathrm{OII}])$.

- En el Nudo B pudieron derivarse $\mathrm{T}_{e}([\mathrm{OIII}])$ y $\mathrm{T}_{e}([\mathrm{SIII}])$ con el método directo para las componentes ancha y angostas N1, y para la medida global con precisiones del $3 \%$ y $16 \% ; 2 \%$ y $11 \% ; 2 \%$ y $14 \%$, respectivamente. Además, esta región fue la única en la que se pudo estimar la $\mathrm{T}_{e}([\mathrm{SII}])$ con el método directo, con precisiones del $10 \%, 12 \%$ y $26 \%$ para las componentes ancha, angostas N1 y para la medida global, respectivamente. $\mathrm{Y}$ por último, las temperaturas $\mathrm{T}_{e}([\mathrm{OII}])$ y $\mathrm{T}_{e}([\mathrm{NII}])$ se consideraron iguales a $\mathrm{T}_{e}([\mathrm{~S} I \mathrm{I}])$.

- Como en el caso de IIZw 33, el análisis de las densidades electrónicas para las componentes y medidas globales de las regiones se determinaron con las líneas del [SII] $\lambda \lambda 6717,6731 \AA$. Se estimaron valores muy por debajo de la densidad electrónica crítica de desexcitación colisional. En el caso de la medida global, de la componente ancha y N1 del Nudo A los errores y las incertezas en la estimación de la densidad son muy grandes y lo hemos indicado con ":".Las densidades estimadas para las componentes $\mathrm{N} 2$ y N3 son de $\mathrm{n}_{e}<91 \mathrm{~cm}^{-3} \mathrm{y} \mathrm{n}_{e}<127 \mathrm{~cm}^{-3}$.

- $\mathrm{Al}$ igual que en las regiones de IIZw 33, se han medido las abundancias iónicas de $\mathrm{O}^{+}, \mathrm{O}^{2+}, \mathrm{S}^{+}, \mathrm{S}^{2+}, \mathrm{N}^{+}, \mathrm{Ne}^{2+}, \mathrm{Ar}^{2+}, \mathrm{He}^{+}$y además se midió $\mathrm{He}^{2+}$ para el Nudo B. Los ICFs del $\mathrm{S}\left(\mathrm{S}^{+}+\mathrm{S}^{2+}\right)$, del $\mathrm{Ne}\left(\mathrm{Ne}^{2+}\right)$ y $\mathrm{Ar}\left({ }^{2+}\right)$ para las componentes cinemáticas y para las medidas globales también, en ambas regiones, determinando abundancias dentro de los rangos esperables.

- Fue posible calcular las abundancias totales de O, S, N, Ne y Ar para todas las componentes analizadas en ambos nudos, encontrando abundancias totales en el rango estimado para galaxias HiI, y donde en particular la componente N3 de la región A muestra abundancias totales de oxígeno bajas, en los extremos de la baja metalicidad. Los valores determinados para las abundancias totales de oxígeno muestran un buen acuerdo entre las componentes de las regiones correspondientes, 
salvo para la componente ancha de los Nudos A y B, presentando un valor levemento mayor a las otras componentes, y para la componente N3 del Nudo A que presenta un valor más bajo del esperado. Para las demás componentes de los $\mathrm{Nu}-$ dos A y B encontramos un buen acuerdo para los valores estimados de abundancias totales de oxígeno, dentro de los errores. Si se comparan con las abundancias determinadas para los espectros de dispersión simple, se hayan diferencias mínimas con respecto a las determinaciones Echelle, pero igualmente son valores acordes y esperables para RHII. La abundancia total de oxígeno derivada en ambas regiones está en muy buen acuerdo con las abundancias obtenidas por Lagos et al. (2018). Hay unas diferencias en las abundancias derivadas para el Nudo B que son menores en nuestro caso ( 0.1 dex aproximadamente) al valor encontrado por Lagos y colaboradores, aunque si se tienen en cuenta los errores a tres $\sigma$ están en acuerdo ambas medidas.

Se encontraron valores de N/O un poco más bajos para las componentes ancha y N1 del Nudo A, en el extremo inferior de la distribución de las galaxias Hiı compiladas de la literatura. Para los demás cocientes, las estimaciones están en acuerdo a los encontradas en la literatura. La similitud de los cocientes (N/O, S/O, Ar/O y Ne/O) de las abundancias relativas determinadas para cada componenete cinemática (dentro de los errores) nos podría indicar una evidencia de que la evolución de la química para las diferentes componentes es muy similar y que pueden estar relacionadas con distintas fases de un mismo gas. Al igual que para II Zw 33, este estudio se realizó para todas las componentes cinemáticas por primera vez en esta galaxia.

- Para el He no fue posible ajustar las múltiples componentes en la región A dado que la componente ancha no fue posible ajustarla con buena precisión. Para las demás componentes se estimó que las abundancias totales presentan un comportamiento similar entre las componente angostas N1, N2, N3 y la medida global en el Nudo A, con diferencias de 0.06 dex, teniendo en cuenta los errores. Para el Nudo B se ajustaron las múltiples componentes y se determinó la abundancia total $(\mathbf{H e} / \mathbf{H})$. Esta fue la única región estudiada en la que fue posible estimar esta abundancia debido a que se pudo medir la línea débil de alta excitación de HeII $\lambda 4686 \AA$ A Las abundancias adoptadas de $\mathrm{He}^{+} / \mathrm{H}^{+}$tienen un muy buen acuerdo entre las componentes y la medida global $(0.081 \pm 0.024,0.080 \pm 0.024$ y $0.079 \pm 0.007)$. Y el mismo comportamiento se determinó para la abundancia total $(\mathrm{He} / \mathrm{H})$ de $0.082 \pm 0.024$, $0.080 \pm 0.011$ y $0.079 \pm 0.007$, mostrando la componente ancha una abundancia de 0.02 dex mayor frente a la N1 y la medida global.

- Las determinaciones del c $(\mathrm{H} \beta)$ para el cálculo de la extinción en las regiones presentan valores compatibles con la baja extinción.

- Las abundancias totales de oxígeno estimadas a través de los diferentes parámetros empíricos difieren en algunas componentes de las abundancias derivadas usando las temperaturas electrónicas de [OIII] obtenidas con el método directo o a través de las relaciones teóricas y empíricas. Si bien en líneas generales hay un buen acuerdo, se observó el mismo comportamiento para las estimaciones realizadas con el parámetro empírico $\mathrm{S}_{23}$ que en prácticamente todos los casos subestima la abundancia total de oxígeno obtenida directamente. Para el Nudo B se encuentra un buen acuerdo dentro de los errores.

- Las estructuras de ionización estudiadas a partir de los parámetros $\eta$ y $\eta^{\prime}$ nos muestran un comportamiento consistente frente a los valores esperados para este 
tipo de galaxias y regiones. Si se consideran los errores estas regiones presentan una estructura de ionización similar entre ellas. La única componente cinemática que está desplazada hacia regiones que indican una temperatura del campo de radiación mayor con respecto a las demás, es la componente ancha del Nudo A. Igualmente, si se tienen en cuenta los errores, esta componente se ubicaría en un $\eta$ que implica una temperatura del campo de radiación similar a las otras componentes. Para el parámetro $\eta$ ' observamos un excelente acuerdo entre todas las componentes determinadas para estas regiones, dentro de los errores. El comportamiento encontrado para estas regiones podría indicar que el gas ionizado de cada componente cinemática recibe la energía ionizante de un mismo supercúmulo jóven y masivo.

- Por último, al igual que en la galaxia anterior, las relaciones empíricas utilizadas para la determinación de $\eta$ como $\eta$ ' y las usadas para los parámetros empíricos fueron derivadas para los brotes de formación estelar violenta de Mrk600 en nuestro trabajo por primera vez en la literatura. Los resultados encontrados nos muestran que aún hay mucho trabajo por hacer y que nuestros resultados pueden ser mejorados con observaciones más profundas. Los parámetros utilizados hay que ver si son válidos en los análisis de múltiples componentes de otras nuevas regiones y así ampliar la muestra. 



\section{Capítulo 5}

\section{Resumen, conclusiones y trabajo a futuro}

Las Regiones Hiı Gigantes que rodean a los cúmulos estelares jóvenes y masivos pueden ser estudiados a partir de las líneas intensas de emisión de sus espectros. En particular, los brotes intensos de formación estelar presentes en las galaxias BCD muestran cinemáticas complejas que pueden ser resueltas con intrumentos lo suficiemente potentes.

En esta Tesis, se han identificado y analizado las características cinemáticas de una muestra de Regiones Hiı Gigantes pertenecientes a dos galaxias observables con telescopios del Hemisferio Sur, las galaxias II Zw 33 y Mrk600. Estas son galaxias BCD que presentan nudos intensos de formación estelar en la escala de unas centenas de pársecs. Dichos nudos son muy luminosos y dominan la emisión de la luz en estas galaxias. Su estudio ha permitido definir la naturaleza y las propiedades físicas de las múltiples componentes de gas ionizado presentes. Esto nos permitió estimar temperaturas y densidades electrónicas, constantes de enrojecimiento y tasas de formación estelar a partir de los flujos de $\mathrm{H} \alpha$. También se analizaron abundancias químicas iónicas y totales de distintas especies químicas.

Para este trabajo se ha utilizado espectroscopía de alta resolución Echelle. Se analizaron, en total, cinco regiones Hir luminosas en BCDs, para las que se obtuvieron los siguientes resultados generales:

- Resultados observacionales

- Las observaciones fueron reducidas y calibradas siguiendo la metodología habitual. Las calibraciones en longitud de onda y flujos de nuestros espectros fue realizada mucho cuidado para poder minimizar los errores e intentando obtener los datos lo mejor calibrado posible, para las que se utilizaron lámparas de calibración y estrellas estándares de flujo tomadas en la misma noche.

- Se eliminaron rayos cósmicos de los espectros en los casos en que fueran posibles para trabajar con espectros limpios.

- Se propuso achicar la ventana de extracción del espectro de líneas para trabajar con la zona de mayor intensidad de las líneas y eliminar posibles efectos espurios de la región observada.

- Resultados de la cinemática

- Hemos desarrollado un código en Python para validar las soluciones cinemática propuestas, a través del indicador y del criterio estadístico de Akaike. Por medio 
de este programa, basado en el LMFit, modelamos soluciones para los perfiles de líneas intensas mediante una composición de gaussianas más una función lineal para modelar el continuo local de las líneas. Se determinaron múltiples componentes cinemáticas para las cinco regiones observadas con espectroscopía Echelle de alta resolución, obtenida en LCO. Para los tres nudos de II Zw 33 se modelaron soluciones con tres componentes, dos angostas y una ancha. Por el lado de Mrk600, para el Nudo A el mejor ajuste indica la presencia de cuatro componentes gaseosas, tres componentes angostas y una ancha. Para el Nudo B la situación es más simple dado que el perfil está compuesto por dos componentes, una angosta y una ancha.

- No se ha realizado la resta del cielo para nuestros objetos debido a que las regiones observadas en estas galaxias son débiles y la resta del cielo podría afectar la información de las líneas. Esta fue la forma en que se ha trabajado en la calibración y reducción de datos para estos objetos difusos por el grupo de trabajo desde el 2006.

- A partir de las soluciones gaussianas propuestas, indicamos si tiene un sentido matemático, penalizando los ajustes que tienen un mayor número de componentes que las estadísticamente válidas. En estos ajustes se encontró que todas las líneas intensas muestran evidencias de un residuo presente en las alas de los perfiles de las líneas de emisión. La solución determinada para estos comportamientos en los residuos de las alas fue la incorporación de una componente ancha. Se determinó que todas las regiones estudiadas presentan componentes cinemáticas supersónicas, salvo la componente angosta N3 en el Nudo A de Mrk600. La alta resolución espectral y la alta relación $\mathrm{S} / \mathrm{N}$ de nuestros imágenes nos permitió resolver los perfiles de las líneas de emisión intensas y débiles. Se ha determinado la dispersión de velocidades, $\sigma$, dada por el ensanchamiento del perfil de las líneas de emisión. Para este análisis, se midieron los ancho observados de los perfiles de las líneas de recombinación de hidrógeno, luego de corregirlo por el perfil instrumental y por la contribución térmica de las líneas. Así hemos confirmado la naturaleza gigante de las regiones candidatas.

- Resultados de las propiedades físicas

- Se realizó un estudio detallado de las propiedades físicas a partir de las componentes cinemáticas y para la medida global de las líneas en las distintas regiones. Esto nos permitió estimar de manera directa la temperaturas electrónicas de los iones, $\mathrm{T}_{e}([\mathrm{OIII}]), \mathrm{T}_{e}([\mathrm{SIII}])$ y $\mathrm{T}_{e}([\mathrm{SII}])$. Esto último fue posible únicamente en el $\mathrm{Nu}-$ do B de Mrk 600. En el resto de los nudos, las temperaturas electrónicas $\mathrm{T}_{e}([\mathrm{NII}])$, $\mathrm{T}_{e}([\mathrm{OII}])$ y la $\mathrm{T}_{e}([\mathrm{SII}])$ fueron estimadas usando relaciones teóricas y empíricas entre las temperaturas y métodos empíricos que relacionan las temperaturas con las intensidades de líneas fuertes. Se han estimado temperaturas electrónicas a través del método directo en un rango de temperaturas esperable para este tipo de objetos, en los casos en que fue posible, para las distintas componentes cinemáticas y para las medida globales con un promedios de error del $5 \%$ para $\mathrm{T}_{e}([\mathrm{OIII}])$, de $15 \%$ para $\mathrm{T}_{e}([\mathrm{SIII}])$ y del $10 \%$ para $\mathrm{T}_{e}([\mathrm{SII}])$. Para las componentes anchas de los Nudos A y C de II Zw 33, donde no fue posible derivar ninguna temperatura, se consideró la temperatura electrónica $\mathrm{T}_{e}([\mathrm{OIII}])$ como $10^{4} \mathrm{k}$ y a partir de ella, usando relaciones entre temperaturas, se obtuvieron las restantes. Las densidades electrónicas, $\mathrm{n}_{e}$, fueron determinadas a partir de los cocientes de las líneas del doblete del $[\mathrm{SII}] \lambda 9069 \AA, 9532 \AA$. En todas las regiones y subcomponentes las densidades determinadas esperables, muy por debajo de los valores críticos para la 
desexcitación colisional. Las líneas del azufre se pudieron determinar en todas las regiones con errores menores al $25 \%$.

- Continuando con la línea de trabajo y la metodología definida por Hägele et al. (2006), Hägele et al. (2008), Hägele et al. (2011), Firpo (2011) y Hägele et al. (2012), se obtuvieron abundancias elementales de O, S, N, Ne y Ar, presentes en el gas ionizado. Se realizó un tratamiento realista de los errores observacionales para estimar las abundancias químicas iónicas y totales de las distintas especies a través del método directo.

Debido a que los errores de los modelos no se pueden cuantificar, no es posible estimar, de forma realista, las relaciones entre las temperaturas electrónicas. Por lo tanto, dichas incertezas no se indican en las abundancias obtenidas. Las abundancias totales determinadas para el O, S, N, Ne y Ar, se encuentran en los rangos esperables y previamente obtenidos en la literatura para las galaxias Hit. Las abundancias total de O estimadas para los Nudos de II Zw 33, están en acuerdo con las obtenidas en el modo de dispersión simple dados por Campuzano-Castro et al. (2015).

Por el lado de Mrk 600, las medidas derivadas para el O resultan levemente menores que las indicadas por Lagos et al. (2018). Si se tienen en cuenta las barras de error, las diferencias resultan de $0.02 \mathrm{dex}$, mostrando un excelente acuerdo con dicho trabajo.

- Al analizar las abundancias de los elementos respecto del oxígeno se obtuvieron cocientes dentro de los rangos esperados para prácticamente todas las regiones. En los casos de las componentes de II Zw 33, se encuentra una gran gran dispersión entre las distintas componentes del gas. Sin embargo, en general están en acuerdo con los valores esperables para los cocientes N/O, Ar/O y Ne/O. En el caso del cociente $\mathrm{S} / \mathrm{O}$, los valores determinados son muy altos respecto a los valores esperados para las galaxias Hiıy RHiı.

Por el lado de Mrk 600, hay una similitud, dentro de los errores, en las abundancias relativas $\mathrm{Ar} / \mathrm{O}$ y Ne/O en las componentes de ambos brotes. Para el N/O y S/O, se estiman valores levemente inferiores dentro de los esperados, pero que están en concordancia con los esperados. En términos generales, los resultados determinados muestran algunas diferencias entre los cocientes de abundancias químicas de las componentes cinemáticas, pero si tenemos en cuenta los errores, se encuentran dentro de los valores esperables. Estas diferencias puede indicar que estas componentes son distintas fases de un mismo gas.

- Para el helio se determinaron abundancias iónicas mediante el cociente $\mathrm{He}^{+} / \mathrm{H}^{+}$ típicas para galaxias Hiı y RHıI (Hägele et al., 2008). Para varias líneas débiles de HeI no fue posible ajustar la componente ancha que ajusta las alas, de manera que no fue posible estimar el $\mathrm{He}^{+} / \mathrm{H}^{+}$, como es el caso del Nudo A de Mrk600, para el cual no pudo ajustarse la componente ancha en ninguna de estas líneas.

Para el Nudo B de Mrk600 fue posible medir la línea intrínsecamente débil de HeII $\lambda 4868 \AA$, por lo que, en este caso, se pudieron estimar las abundancias totales $\mathrm{He} / \mathrm{H}$.

- Las medidas de la extinción c $(\mathrm{H} \beta)$, determinadas para ambas galaxias, son compatibles con bajas extinciones, y se condicen con valores de extinción bajos calculados para estas regiones a partir de observaciones de rendija larga (Campuzano-Castro et al., 2015). Hay diferencias en la constante de enrojecimiento de Mrk 600 determinada entre las observaciones Echelle y dispersión simple, que pueden deberse 
a diferencias en el calado, en las rendijas utilizadas y en las condiciones de observación. Determinar las causas de esta diferencia requiere de un análisis más profundo.

- Las abundancias totales de oxígeno, estimadas a través de los diferentes parámetros empíricos, difieren en algunas componentes de las abundancias derivadas usando las temperaturas electrónicas de [OIII], obtenidas con el método directo o a través de relaciones teóricas y/o relaciones empíricas. Si bien, en líneas generales hay acuerdo, se observó el mismo comportamiento en el parámetro empírico $\mathrm{S}_{23}$. En prácticamente todos los casos, dicho parámetro subestima la abundancia total de oxígeno obtenida previamente. Para el Nudo B se encuentra un buen acuerdo dentro de los errores.

- Para el Nudo B de II Zw 33 se pudo estimar, a partir de la estructura de ionización y del parámetro $\eta$, que, en los rangos de temperaturas bajas, la temperatura del campo de radiación ionizante es muy similar entre las componentes. Los Nudos A y C muestran una dispersión mayor respecto a los valores de $\eta$ típicos para este tipo de regiones. Esto posiblemente se deba a que las temperaturas electrónicas $\mathrm{T}_{e}([\mathrm{SIII}])$ no fueron estimadas a partir de una medida directa de sus líneas, sino que fueron estimadas por relaciones empíricas. Por el lado de los Nudos A y B de Mrk600, se encuentra un excelente acuerdo entre componentes. Los valores de $\eta$ estimados indican temperaturas del campo de radiación ionizante mayores que las encontradas para II Zw 33. La componente ancha del Nudo A se encuentra lévemente desplazada hacia temperaturas mayores, pero si tenemos en cuenta los errores, se estiman valores acordes a los esperados. Para el parámetro $\eta$ ' observamos un excelente acuerdo entre todas las componentes determinadas para estas regiones, dentro de los errores. Como conclusión, el comportamiento encontrado para estas regiones implicaría que la fuente excitatriz de las regiones en cuestión es la mismo.

Los resultados obtenidos en este trabajo confirman y amplían de distintas formas el estudio de las Regiones Hir Gigantes presentes en la literatura. Se continuó con la metodología de trabajo dada por Hägele et al. (2006), Hägele et al. (2008), Firpo (2011) y Hägele et al. (2012), mejorando y desarrollando un programa para el análisis de la cinemática, adaptando los criterios previos para un análisis más óptimo. La combinación del análisis de múltiples componentes cinemáticas con el análisis de sus propiedades físicas, nos permitió completar un estudio quimiodinámico detallado de nuestras regiones. Se midieron una gran cantidad de líneas y, a través de un complejo código en Python desarrollado por el grupo de investigación, pudimos mejorar el estudio de la cinemática de múltiples componentes. Se incorporó el criterio estadístico de Akaike para la validación de los ajustes de múltiples componentes. Los espectros de alta resolución nos permitieron estudiar la compleja cinemática de una gran cantidad de líneas de emisión intensas y débiles aurorales, necesarias para emplear el método directo en nuestro análisis. El estudio fue realizado por primera vez en nuestros candidatos II Zw 33 y Mrk600, aportando información novedosa acerca de la quimiodinámica. Los resultados presentados en esta Tesis son consistentes con los resultados determinados en Firpo (2011), en donde se realizó un estudio detallado de la galaxia BCD Haro 15. Ademas, estos resultados reflejan los distintos estudios y discusiones llevados a cabo durante este proceso. 


\subsection{Trabajo a Futuro}

Como parte de la Tesis, se han realizado propuestas de observación en distintos observatorios con el fin de ampliar la muestra de RHII. Se han observado brotes un total de 13 nudos de formación estelar intensa en las galaxias BCD cercanas $(5 \mathrm{Mpc}<$ distancias $<80 \mathrm{Mpc}$ ): Haro 11, Haro 14, Tol2, Tol 0610-387, SCHG 0341-407 y NGC 1702. Estas observaciones se realizaron utilizando espectroscopía Echelle de alta resolución con el instrumento montado en el telescopio DuPont de 2.5m, en LCO, las noches del 3, 4, 8 y 9 de diciembre del 2015. Las galaxias de la muestra fueron seleccionados por nuestro grupo de trabajo, identificando galaxias BCD que no tengan estudios cinemáticos publicados previamente. Esto garantizará resultados novedosos para dichas regiones, con el afán de aportar información valiosa sobre regiones de formación estelar complejas.

Como se demuestra en este trabajo, a través de la intensidad de las líneas de emisión del gas ionizado es posible analizar la cinemática y las propiedades físicas de las múltiples componentes de dichas regiones. Se espera que, continuando con la metodología presentada en el Capítulo 3 para la determinación, y validación, de las componentes cinemáticas en los perfiles de líneas intensas, se pueda:

- Definir la naturaleza y propiedades físicas para las regiones observadas en las BCDs Haro 11, Haro 14, Tol2, Tol0610-387, SCHG 0341-407 y NGC 1702.

- Dilucidar los posibles mecanismos que retroalimentan la formación de nuevas generaciones de estrellas en estas galaxias evolucionadas, las cuales cuentan con presencia de una población estelar vieja y gas enriquecido previamente.

El análisis de los parámetros físicos como la densidad y temperatura electrónica, la composición química y los estudios de los campos de radiación de las fuentes ionizantes, permitirá caracterizar las regiones de formación estelar más luminosas en galaxias compactas (en las Figuras 5.1, 5.2, 5.3, 5.4, 5.5 y 5.6, se pueden ver algunos resultados preliminares de este estudio). De los ajustes se espera poder detectar las líneas débiles aurorales para determinar temperaturas electrónicas y abundancias químicas de manera directa. En líneas generales, es necesario disminuir las estimaciones a partir de modelos para ampliar los resultados confiables de parámetros físicos, independientes de las soluciones determinadas a partir de líneas más intensas.

Por otro lado, en la propuesta de observación realizada durante el semestre 2018A, se solicitó tiempo de telescopio en GEMINI Norte para observar los dos nudos más intensos de II Zw 18 (en la Figura 5.7, se muestran algunos resultados provisorios), con el espectrógrafo de alta resolución GRACES. Esta galaxia, visible desde el hemisferio Norte, presenta el gas con metelicidad más baja detectado, según Hunt et al. (2003), Papaderos et al. (2003), Izotov et al. (2006b). Resulta de interés analizar esta galaxia de manera detallada para determinar las características cinemáticas, las propiedades físicas y metalicidades para esta galaxia. Por el lado del espectrógrafo y el telescopio solicitados, GRACES es el resultado de la cooperación entre ESPaDOnS (an Echelle SpectroPolarimetric Device for the Observation of Stars at CFHT) y GEMINI. Este combina el gran área colectora del Telescopio Gemini $(8.1 \mathrm{~m})$ con el gran poder resolvente y la alta eficiencia del espectrógrafo ESPaDOnS en CFHT (telescopio Canadiense, Francś y Hawaiano), para obtener una alta resolución espectroscópica a lo largo del espectro óptico. Esto se logra a través de los $270 \mathrm{~m}$ de fibra óptica desde el Telescopio Gemini Norte hasta CFHT, donde se encuentra montado ESPaDOnS. Esto nos permitiría estimar resultados novedosos para estas regiones de formación estelar.

Las observaciones tomadas con el espectrógrafo ESPaDONs cuentan con el código de reducción en Python, llamada OPERA. Este software no hace la calibración en flujo, pero 
Figura 5.1. A la izquierda, en el primer panel, la galaxia BCD Haro 11 observada con el Telescopio Espacial Hubble. Imágen adopatada de https://www.groundai.com/project/ kinematics-of-haro11-the-miniature-antennae/.. A la derecha, en el primer panel, imagen $\mathrm{H} \alpha$ (extraída de $\mathrm{NED}^{(\mathrm{i})}$ ) de Haro 11 con las rendijas superpuestas indicando las regiones de observaciónen y los nudos de formación estelar observados. De arriba hacia abajo, los ajustes gaussianos preliminares desarrollados con el programa Kinematics para el estudio de la cinemática en los tres nudos principales de la galaxia BCD Haro 11. Los paneles derechos muestran la solución cinemática en la línea intensa de $\mathrm{H} \alpha$ y los paneles izquierdos la solución cinemática determinada para [OIII] $\lambda 5007 \AA$. Superpuestas a las líneas, en verde y azul, las múltiples componentes propuestas. En las leyendas se indican las componentes para cada perfil de línea. Las solución fue validada a partir del criterio de Akaike, como se describe en el Capítulo 3. Las calibraciones y ajustes son análisis preliminares que he estado desarrollando y que son la base de los ajustes en los estudios que continuarán en las regiones mencionadas.

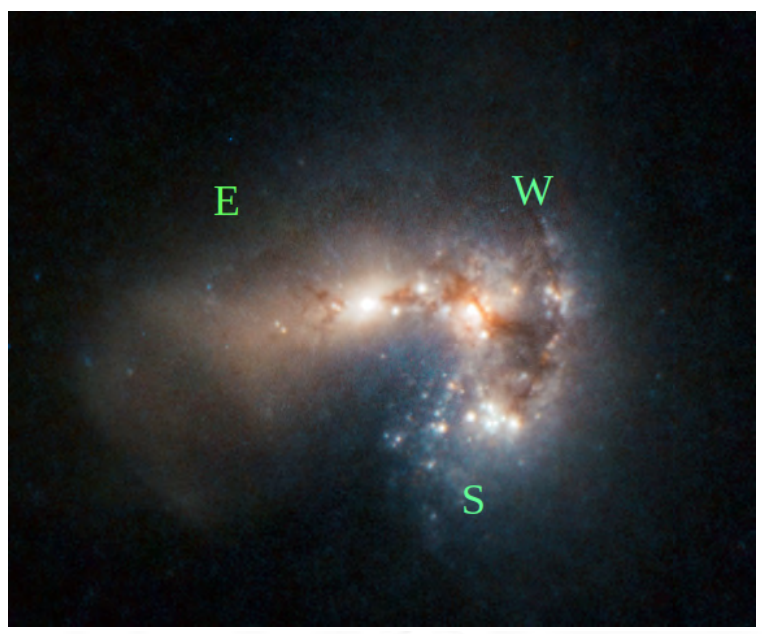

HARO 11

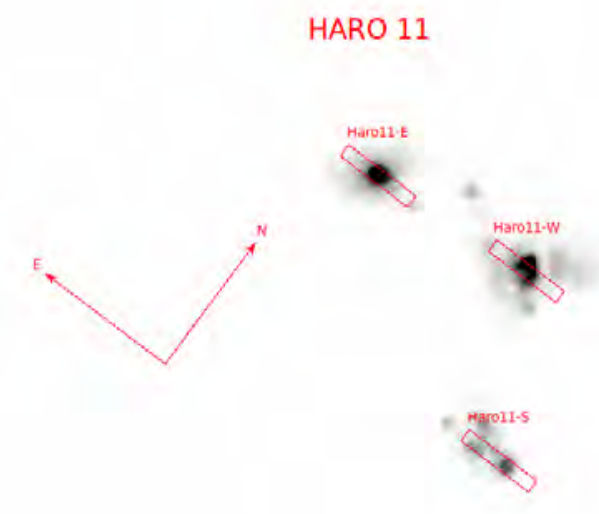

$\mathrm{H} \alpha 6563$

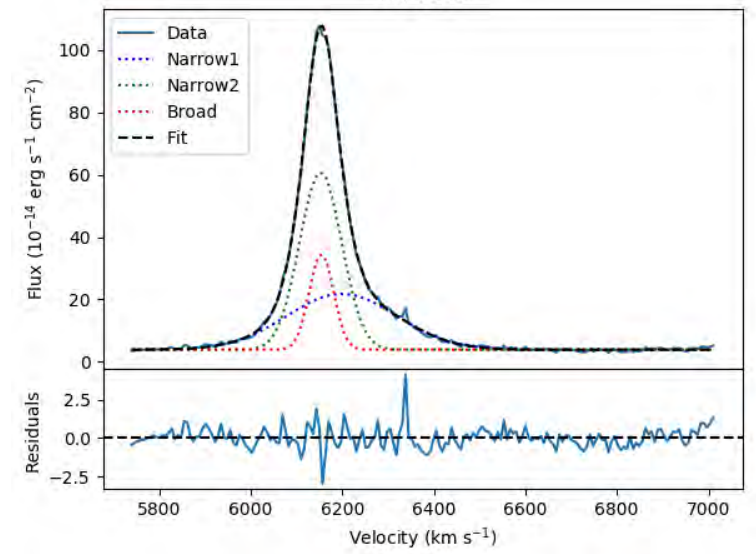

(a) $\mathrm{H} \alpha$ en Haro 11-E

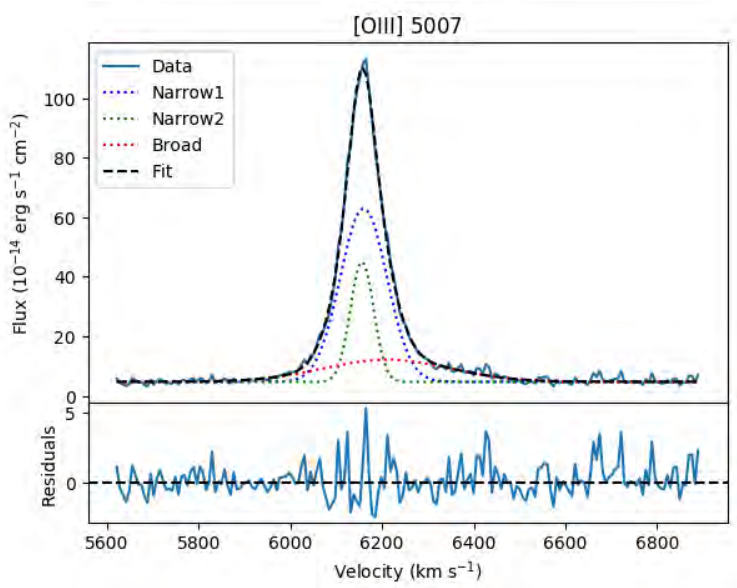

(b) $[\mathrm{OIII}] \lambda 5007 \AA$ en Haro 11-E 


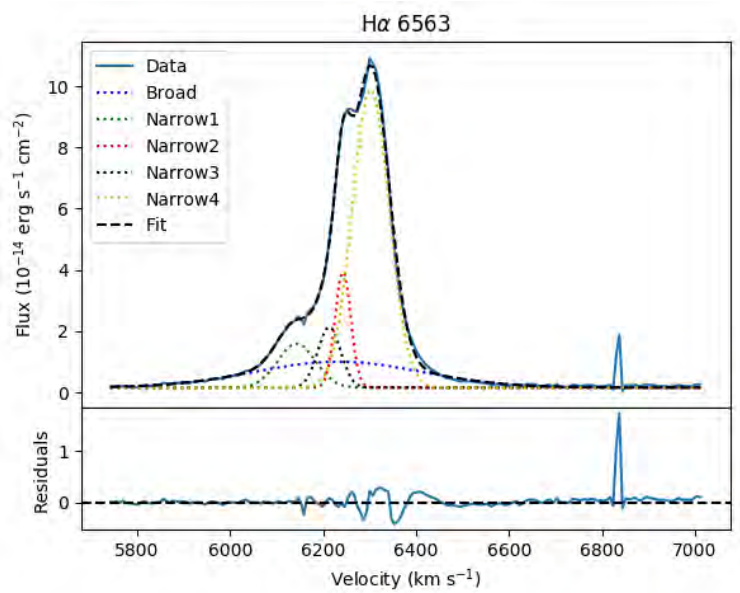

(c) $\mathrm{H} \alpha$ en Haro 11-S



(e) H $\alpha$ en Haro 11-W

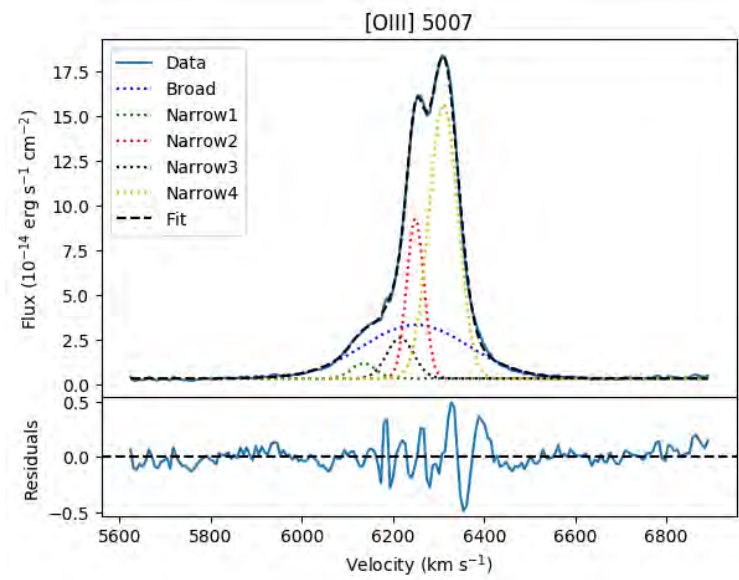

(d) $[\mathrm{OIII}] \lambda 5007 \AA$ en Haro 11-E

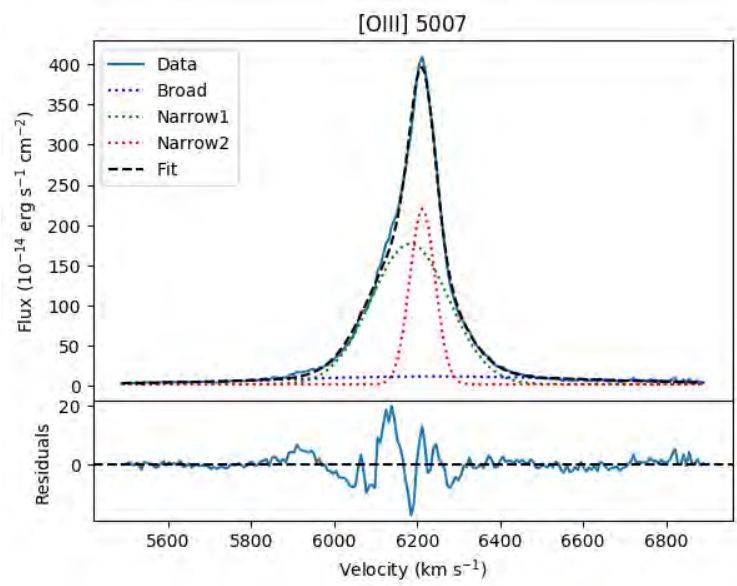

(f) $[\mathrm{OIII}] \lambda 5007 \AA$ en Haro 11-E 
Figura 5.2. De arriba hacia abajo, los ajustes gaussianos desarrollados con el programa $K i$ nematics para el estudio de la cinemática compleja de tres nudos intensos de la galaxia BCD Tol2. Los paneles derechos muestran la solución cinemática preliminar en la línea intensa de $\mathrm{H} \alpha$ y los paneles izquierdos la solución cinemática preliminar determinada para

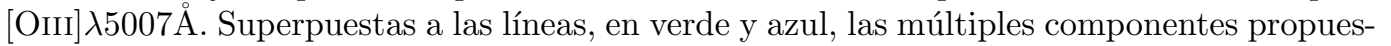
tas. En las leyendas se indican las componentes para cada perfil de línea. Las solución fue validada a partir del criterio de Akaike, como se describe en el Capítulo 3. Las calibraciones y ajustes son análisis preliminares que he estado desarrollando y que son la base de los ajustes en los estudios que continuarán en las regiones seleccionadas.

\section{Tololo 2}

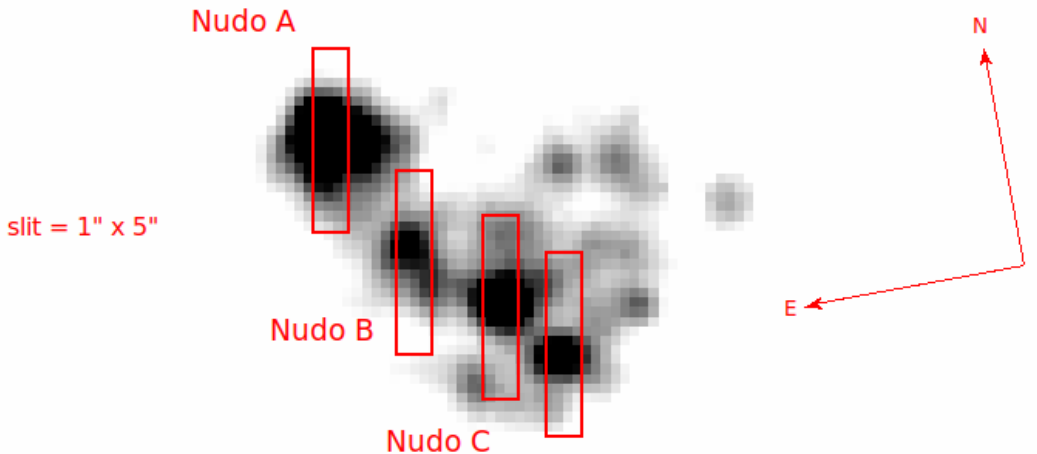

Nudo D

(a) Imagen $\mathrm{H} \alpha$ (extraída de $\mathrm{NED}^{a}$ ) de Tololo 2 con las rendijas superpuestas indicando las regiones de observaciónen y los nudos de formación estelar observados.

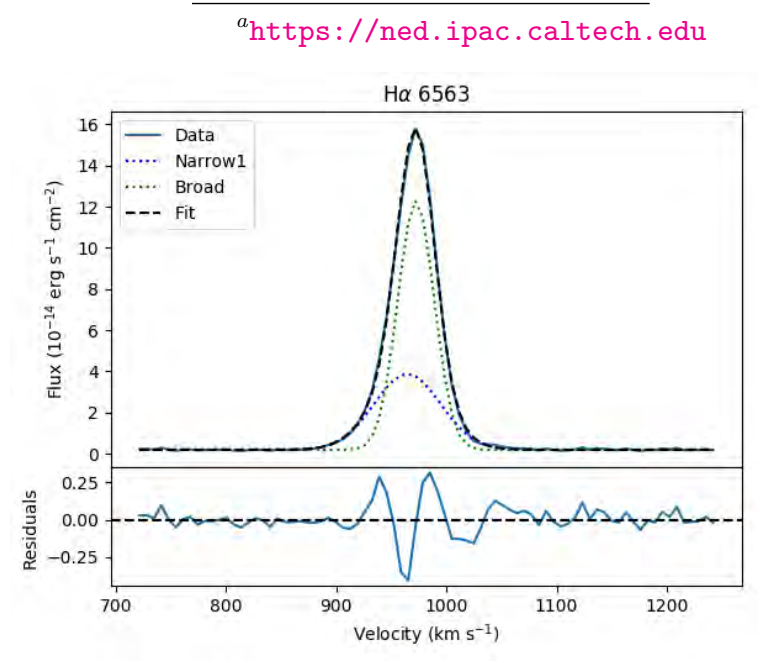

(b) $\mathrm{H} \alpha$ en Tol 2-E

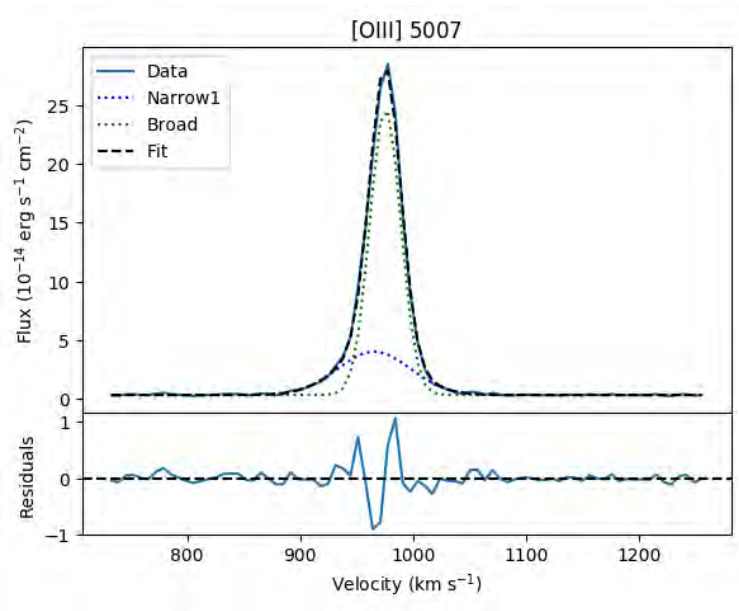

(c) $[\mathrm{OIII}] \lambda 5007 \AA$ en Tol2-E 
$\mathrm{H} \alpha 6563$

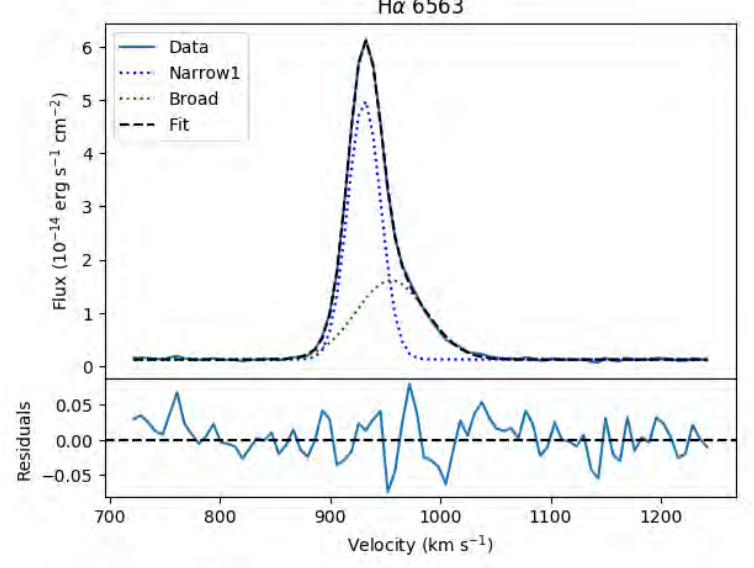

(d) $\mathrm{H} \alpha$ en $\mathrm{Tol} 2-\mathrm{S}$

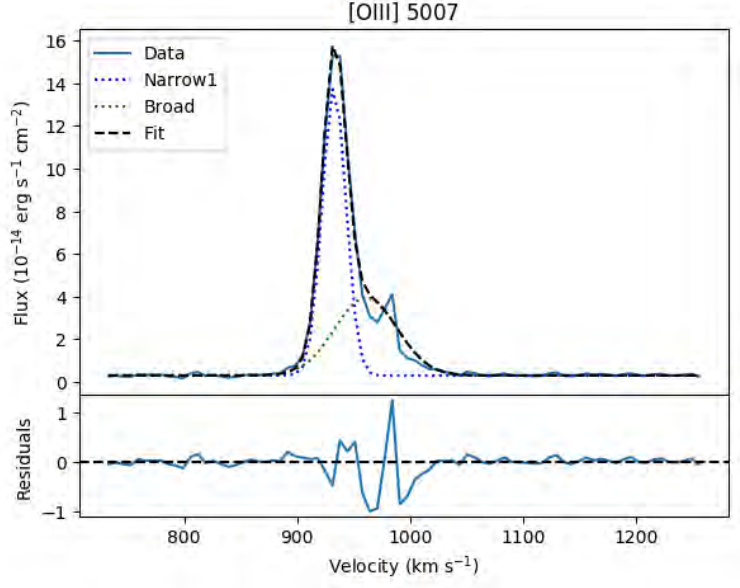

(e) $[\mathrm{OIII}] \lambda 5007 \AA$ en Tol 2-S

[OIII] 4959

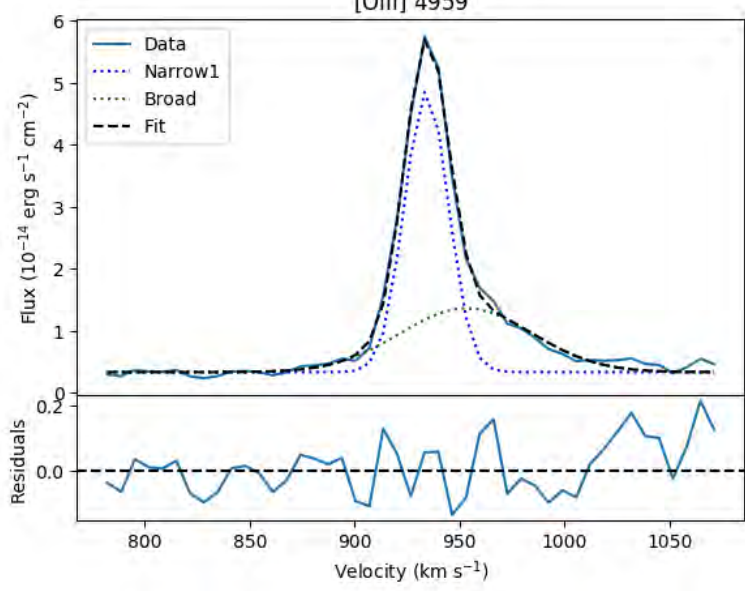

(f) $[\mathrm{OIII}] \lambda 5007 \AA$ en $\mathrm{Tol} 2-\mathrm{S}$ 


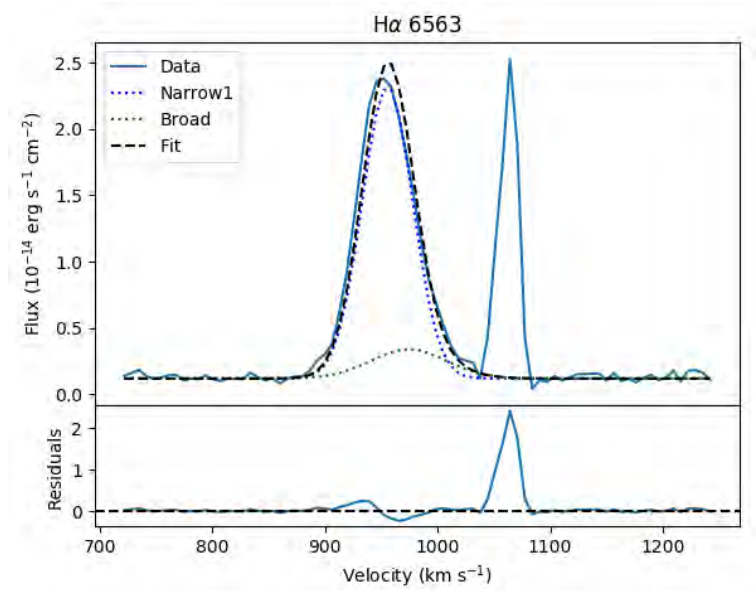

(g) $\mathrm{H} \alpha$ en Tol 2-W

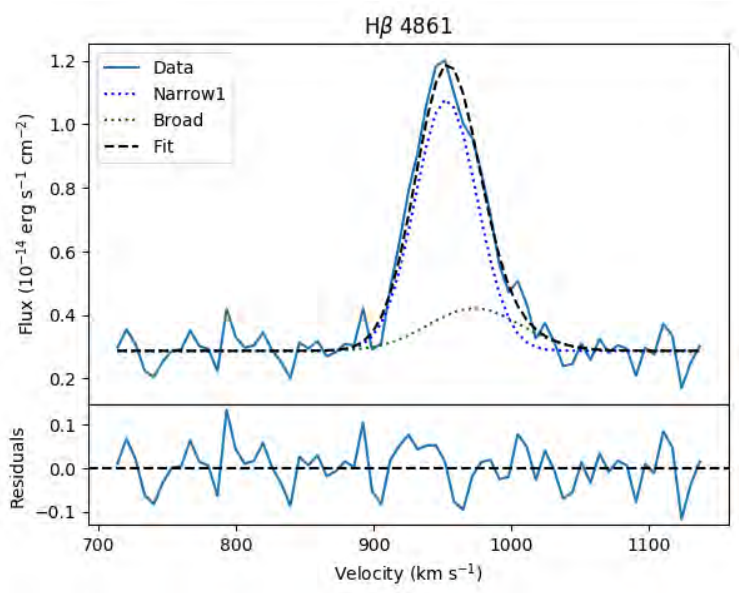

(h) $[$ Oıiı $] \lambda 5007 \AA$ en Tol 2-W

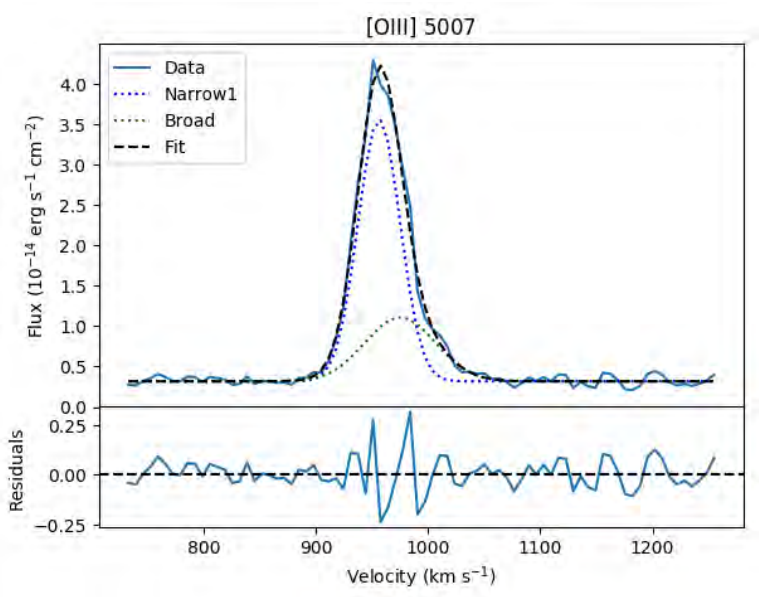

(i) $[\mathrm{OIII}] \lambda 5007 \AA$ en Tol 2-W 
Figura 5.3. Ajustes gaussianos desarrollados con el programa Kinematics para el Nudo Central observado en la galaxia BCD Tol0610-387. El panel derecho muestra la solución cinemática preliminar en la línea intensa de $\mathrm{H} \alpha$ y el panel izquierdo la solución cinemática preliminar determinada para [OIII] $\lambda 5007 \AA$. Superpuestas a las líneas en verde, azul y rojo, las múltiples componentes propuestas. En las leyendas se indican las componentes para cada perfil de línea. Las solución fue validada a partir del criterio de Akaike, como se describe en el Capítulo 3. Las calibraciones y ajustes son análisis preliminares que he estado desarrollando y que son la base de los ajustes en los estudios que continuarán en la región mencionada.

\section{Tololo 0610-387}
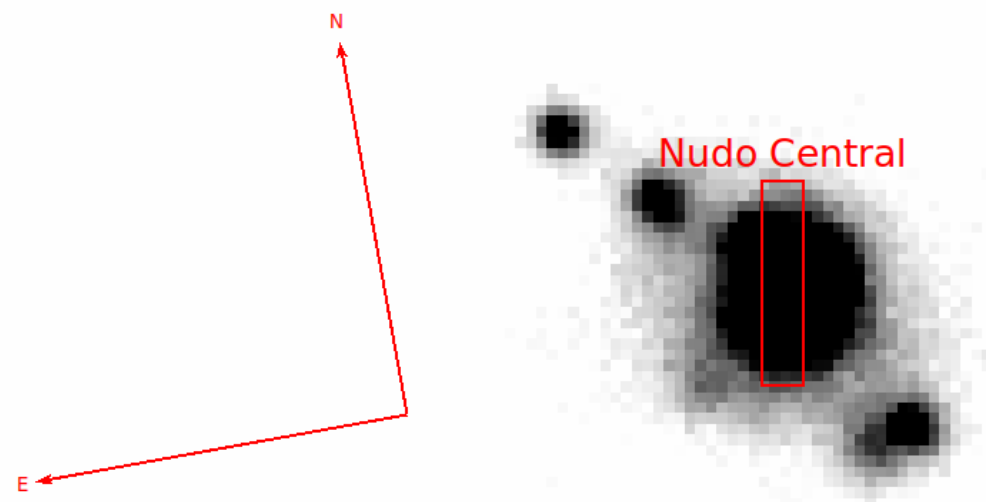

slit $=1^{\prime \prime} \times 5^{\prime \prime}$

(a) Imagen $\mathrm{H} \alpha$ (extraída de $\mathrm{NED}^{a}$ ) de Tol0610-387 con la rendija superpuesta indicando el Nudo central observado.

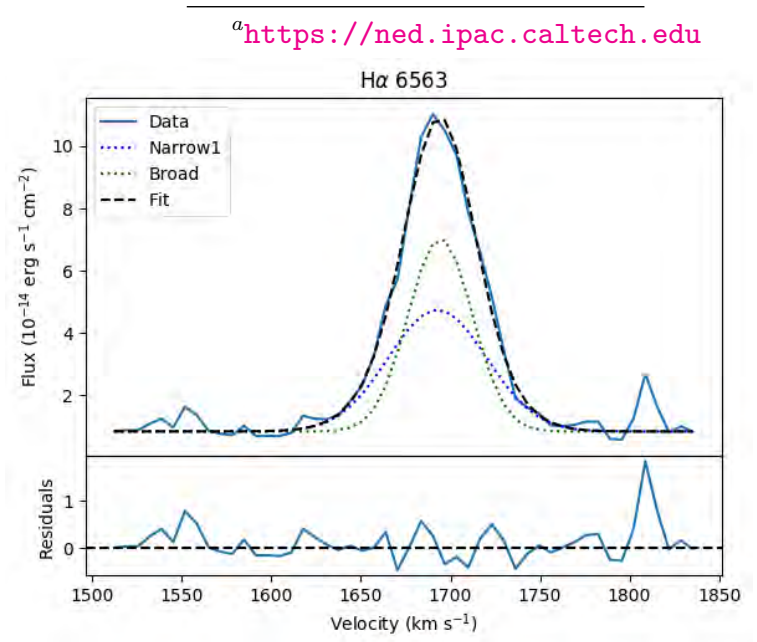

(b) $\mathrm{H} \alpha$ en Tol 0610-387-Central

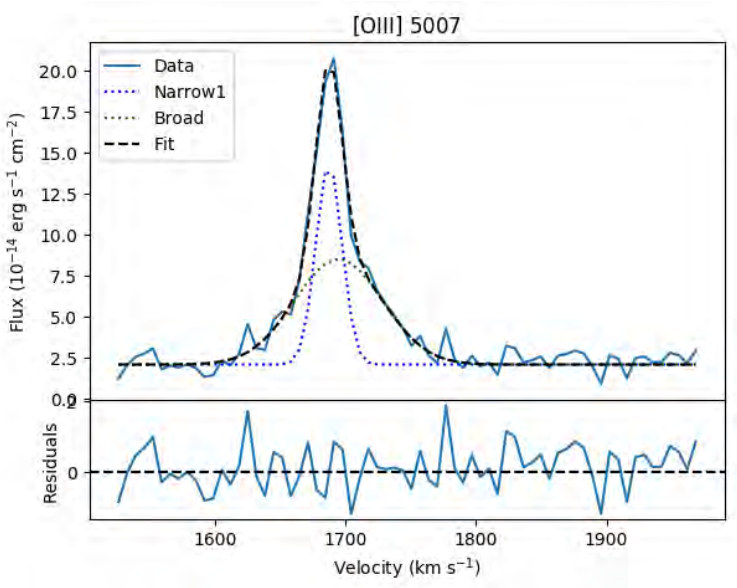

(c) $[\mathrm{OIII}] \lambda 5007 \AA$ en Tol0610-387-Central 
Figura 5.4. De arriba hacia abajo, los ajustes gaussianos desarrollados con el programa Kinematics para las dos regiones de formación estelar observadas en la galaxia BCD SCHG 0341407. Los paneles derechos muestran la solución cinemática preliminar en la línea intensa de $\mathrm{H} \alpha$ y los paneles izquierdos la solución cinemática preliminar determinada para [OIII $] \lambda 5007 \AA$. Superpuestas a las líneas, en verde y azul, las múltiples componentes propuestas. En las leyendas se indican las componentes para cada perfil de línea. Las solución fue validada a partir del criterio de Akaike, como se describe en el Capítulo 3. Las calibraciones y ajustes son análisis preliminares que he estado desarrollando y que son la base de los ajustes en los estudios que continuarán en las regiones mencionadas.

\section{SCHG-0341-407}

Nudo A

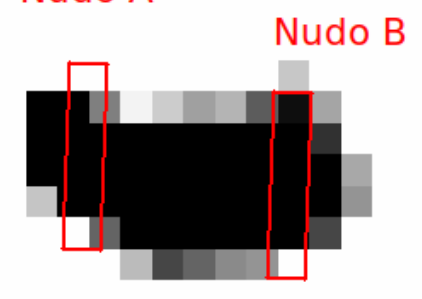

$$
\text { slit }=1^{\prime \prime} \times 5^{\prime \prime}
$$

(a) Imagen $\mathrm{H} \alpha$ (extraída de $\mathrm{NED}^{a}$ ) de SCHG 0341-407 con las rendijas superpuestas indicando las regiones de observaciónen y los nudos de formación estelar observados.

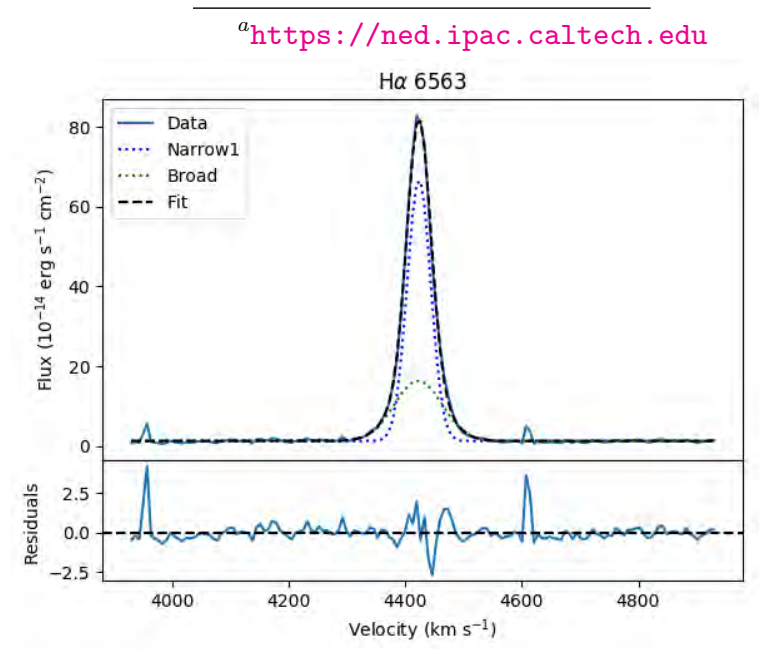

(b) H $\alpha$ en SCHG 0341-407-E

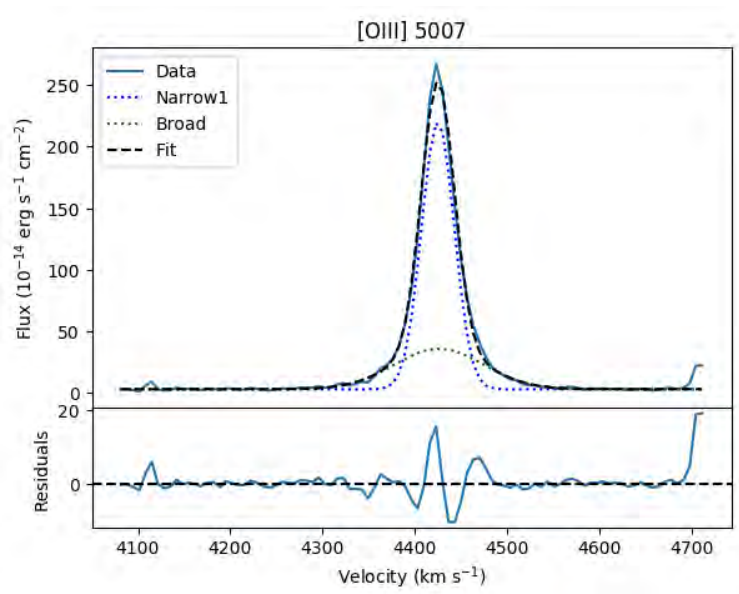

(c) $[\mathrm{OIII}] \lambda 5007 \AA$ en SCHG 0341-407-E 


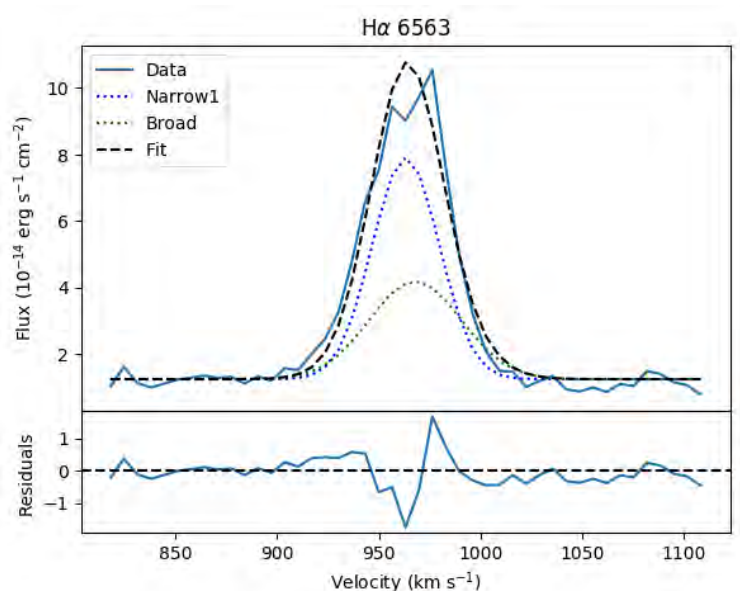

(d) $\mathrm{H} \alpha$ en SCHG 0341-407-C

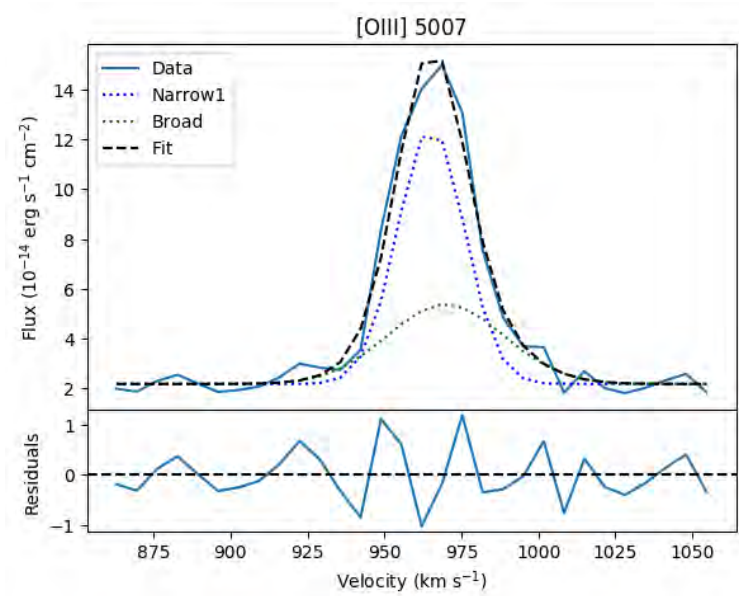

(e) $[\mathrm{OIII}] \lambda 5007 \AA$ en SCHG 0341-407-C

sí hace un pasaje de cuentas del detector a una medida instrumental de flujo. Al utilizar este espectrógrafo con el telescopio Gemini, el programa que procesa y calibra los datos está escrito en IDL, y se define como DraGraces. Este programa tiene el condicionante que se requiere de una licencia paga para poder utilizar IDL, y puede resultar un problema para algunos usuarios del telescopio GEMINI.

Con el objetivo de realizar este estudio sobre dichas galaxias, he estado desarrollando un programa en Python para la calibración en longitud de onda y flujo de los espectros. A través del programa se genera un archivo con las medidas del flujo corregidas por extinción atmosférica y se combinan los órdenes del espectro, al cual llamé PUGLIESE. Luego, se realiza la normalización y la calibración final del flujo con la estrella estándard. Como resultado, el programa devuelve un archivo ascii con las calibraciones finales longitud de onda y el flujo. Además, se generaran los gráficos de los órdenes espectrales de la estrella standard de flujo y de la ciencia (como se presenta en los resultados preliminares de la Figura 5.7), entre otros. En resumen, este programa realizará el proceso completo de calibración el longitud de onda y flujo, y fue testeado y validado por personas del staff de GEMINI. A partir de ello, he estado realizando los ajustes preliminares para el análisis de las múltiples componentes en los brotes principales de formación estelar observados en II Zw 18 (ver Figuras 5.7 con ajustes de las soluciones preliminares).

La incapacidad de resolver el rango completo de temperaturas electrónicas de las galaxias y RHII es una gran limitación actual, que además tiene una implicancia directa en la estimación de metalicidad y parámetros de ionización de dichos objetos.

Completados los estudios quimiodinámicos para las galaxias mencionadas, se estará en condiciones de producir y calibrar nuestras relaciones empíricas entre las diferentes temperaturas electrónicas. A partir de estas, se podrá actulizar y reemplazar a las relaciones que se utilizan actualmente, las cuales están basadas en secuencias de modelos de fotoionización simples. Además, están pobremente contrastados debido a la falta de datos precisos. Por lo tanto, la muestra de galaxias BCD obtenida con espectrógrafos de amplia cobertura y una alta resolución espectral podría aportar para resolver las complejas propiedades físicas y químicas de objetos en los extremos de temperaturas electrónicas bajas y altas. 
Figura 5.5. De arriba hacia abajo, los ajustes gaussianos desarrollados con el programa Kinematics para la galaxia BCD Haro 14. El panel derecho muestra la solución cinemática preliminar en la línea intensa de $\mathrm{H} \alpha$ y el panel izquierdo la solución cinemática preliminar determinada para [OIII] $\lambda 5007 \AA$ A. Superpuestas a las líneas en verde, azul y rojo, las múltiples componentes propuestas. En las leyendas se indican las componentes para cada perfil de línea. Las solución fue validada a partir del criterio de Akaike, como se describe en el Capítulo 3. Las calibraciones y ajustes son análisis preliminares que he estado desarrollando y que son la base de los ajustes en los estudios que continuarán en la región mencionada.

\section{HARO 14}

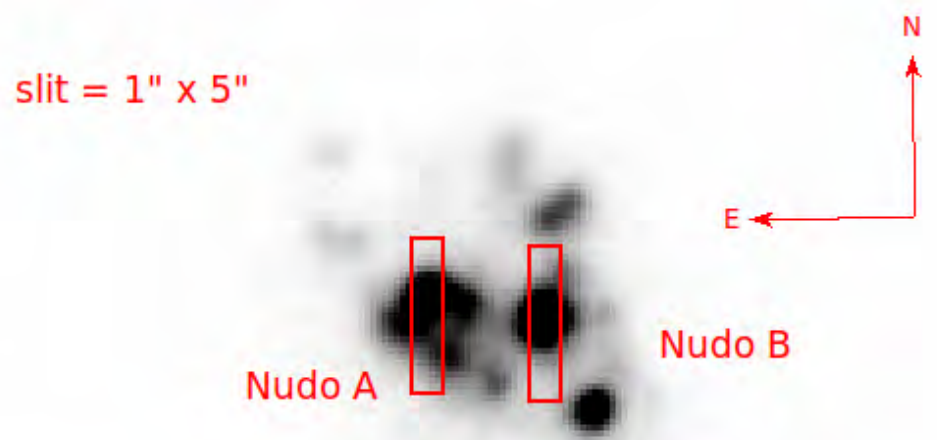

(a) Imagen $\mathrm{H} \alpha$ (extraída de $\mathrm{NED}^{a}$ ) de Haro 14 con las rendijas superpuestas indicando las regiones de observaciónen y los nudos de formación estelar observados.

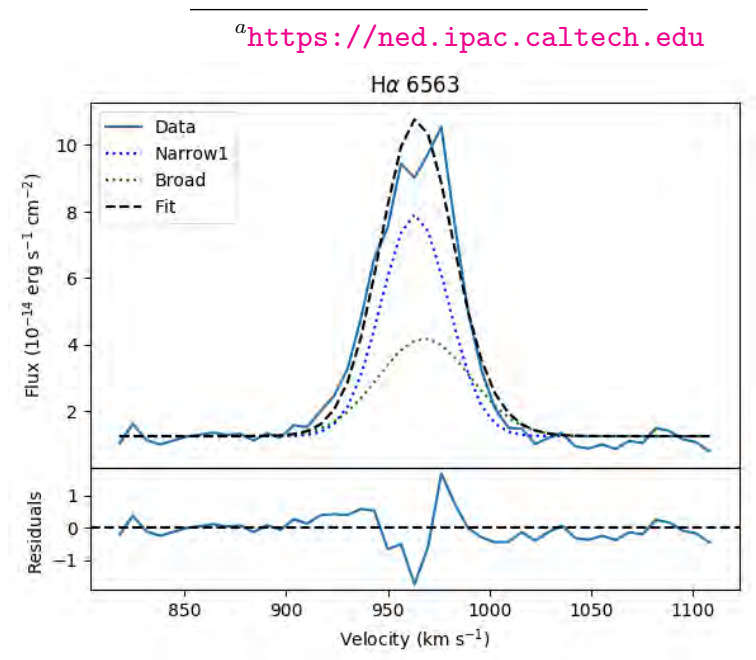

(b) $\mathrm{H} \alpha$ en Haro 14-A

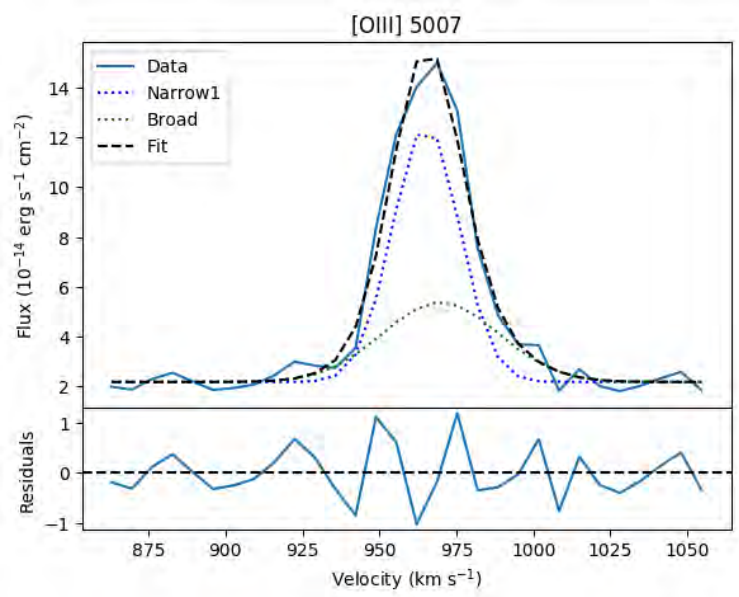

(c) $[\mathrm{OIII}] \lambda 5007 \AA$ en Haro $14-\mathrm{A}$ 
Figura 5.6. De arriba hacia abajo, los ajustes gaussianos desarrollados con el programa Kinematics para la galaxia BCD NGC 1702. El panel derecho muestra la solución cinemática preliminar en la línea intensa de $\mathrm{H} \alpha$ y el panel izquierdo la solución cinemática preliminar determinada para [OIII] $\lambda 5007 \AA$. Superpuestas a las líneas en verde, azul y rojo, las múltiples componentes propuestas. En las leyendas se indican las componentes para cada perfil de línea. Las solución fue validada a partir del criterio de Akaike, como se describe en el Capítulo 3. Las calibraciones y ajustes son análisis preliminares que he estado desarrollando y que son la base de los ajustes en los estudios que continuarán en la región central indicada.

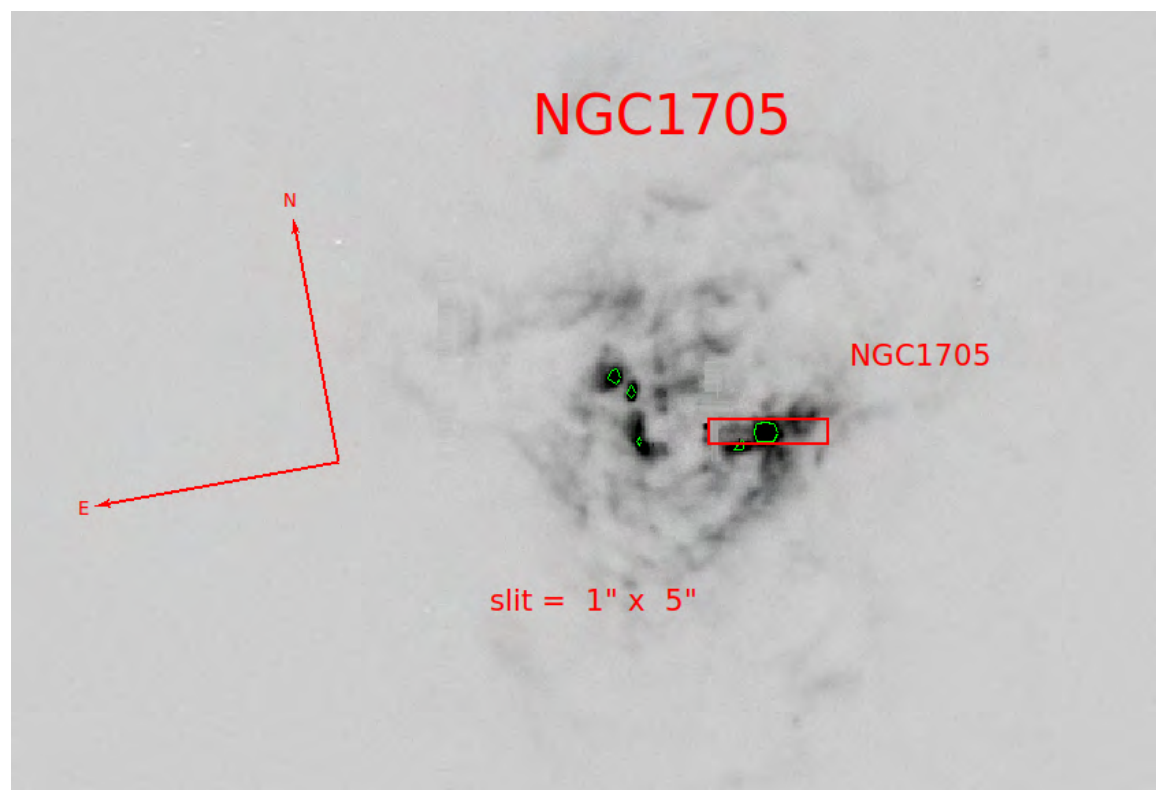

(a) Imagen $\mathrm{H} \alpha$ (extraída de $\mathrm{NED}^{a}$ ) de NGC 1705 con los contornos más intensos y la rendija de observación superpuesta indicando la regiónes central observada.

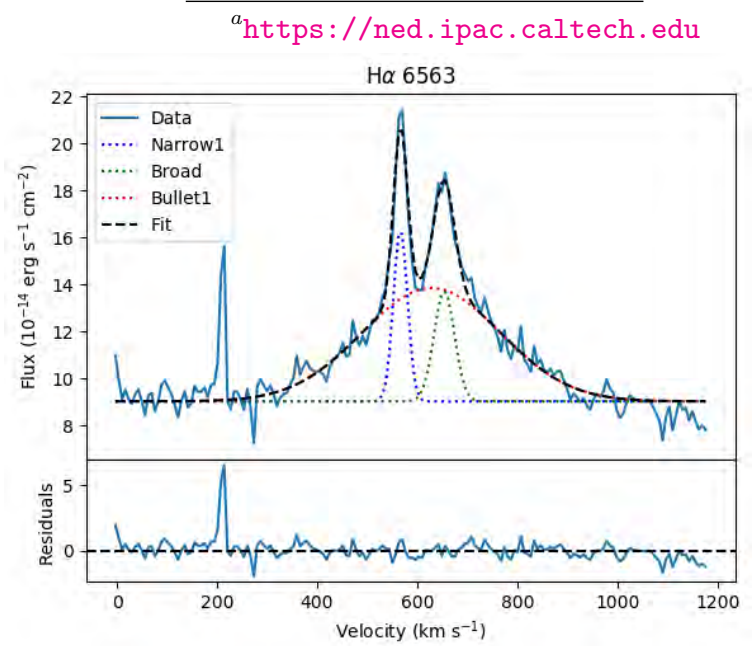

(b) $\mathrm{H} \alpha$ en NGC 1705-Central

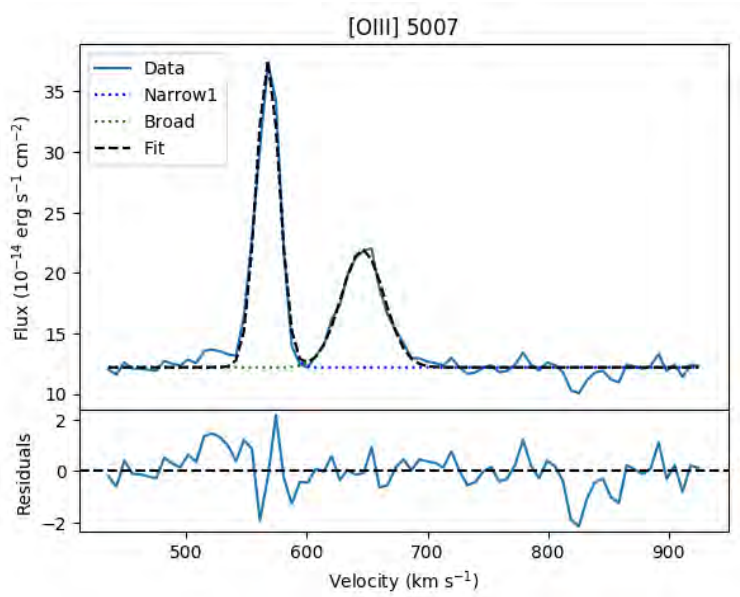

(c) $[\mathrm{OIII}] \lambda 5007 \AA$ en NGC 1705-A 
Figura 5.7. De arriba a abajo, imagen $\mathrm{H} \alpha$ (extraída de $\mathrm{NED}^{(\mathrm{ii})}$ ) de $\mathrm{IZw} 18$ con las fibras de 1.2 arcsec de GRACES (en blanco) superpuestas en los nudos de formación estelar, propuestas para la observación. Las calibraciones y ajustes son análisis preliminares que he estado desarrollando y que son la base de los ajustes en los estudios que continuarán en las regiones observadas. El panel central muestra la calibración preliminar del espectro. En el panel izquierdo, de arriba a abajo, órdenes donde se identifican a las líneas del $\mathrm{H} \beta$ (en el primero y segundo), [OIII] $\lambda \lambda 4959 \AA, 5007 \AA$ (en el 2 do y tercero). En el panel derecho, ye arriba a abajo, órdenes donde podemos identificar $\mathrm{H} \alpha$, el doblete de $[\mathrm{SII}] \lambda \lambda 6717 \AA, 6731 \AA$, y HeI. El panel inferior representa la solución preliminar de las múltiples componentes, ajustadas y superpuestas al perfil de línea de $\mathrm{H} \alpha$.

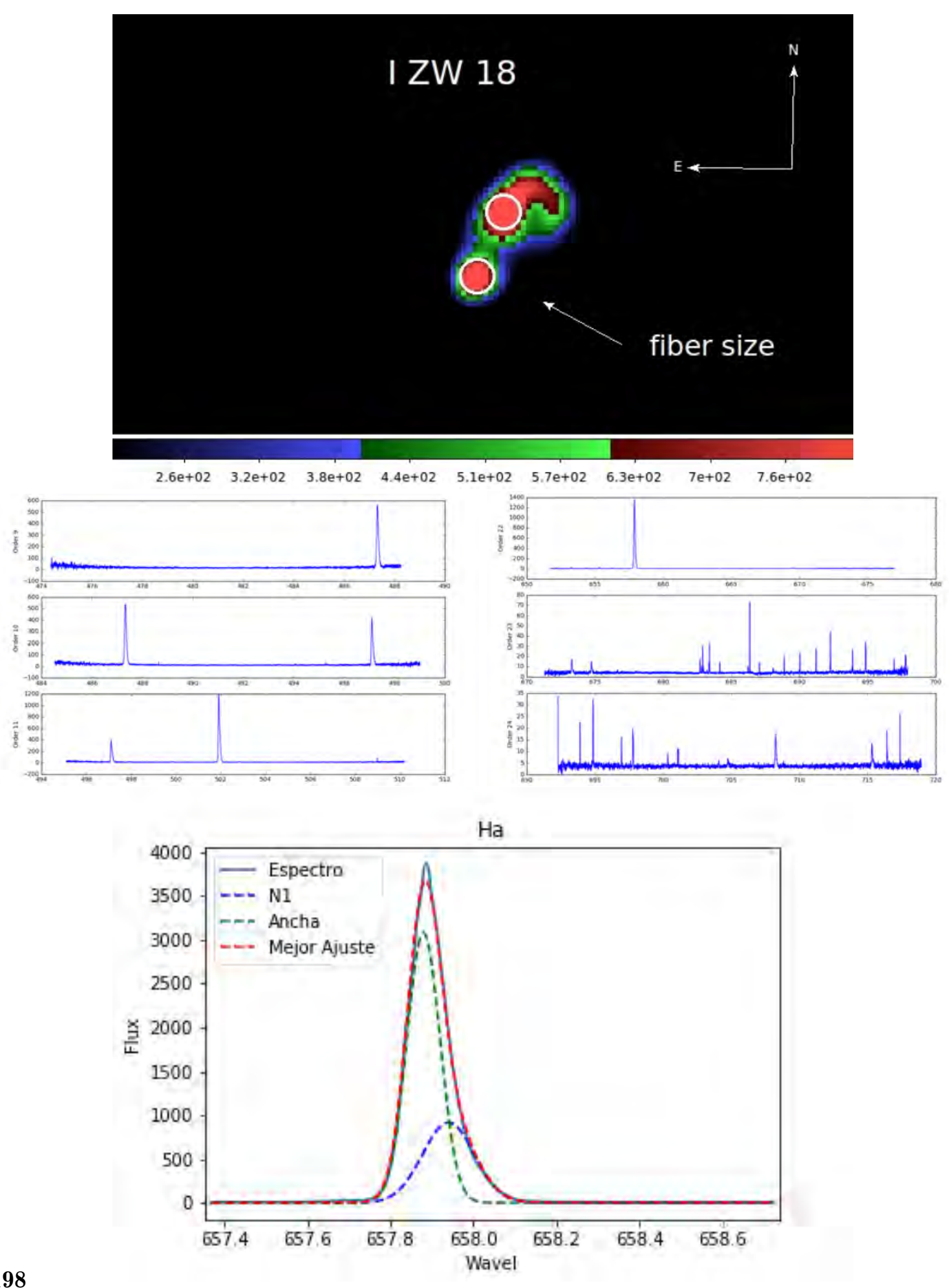




\section{Bibliografía}

2000, Astronomical optics

Abbott D. C., 1982, ApJ, 263, 723

Allende Prieto C., Lambert D. L., Asplund M., 2001, ApJ, 556, L63

Aller L. H., Czyzak S. J., 1983, ApJS, 51, 211

Alloin D., Tenorio-Tagle G., 1979, The Messenger, 18, 12

Amorín R., Aguerri J. A. L., Muñoz-Tuñón C., Cairós L. M., 2009, A\&A, 501, 75

Amorín R. O., Pérez-Montero E., Vílchez J. M., 2010, ApJ, 715, L128

Amorín R., Vílchez J. M., Hägele G. F., Firpo V., Pérez-Montero E., Papaderos P., 2012a, A\&A, 754, L22

Amorín R., Vílchez J. M., Hägele G. F., Firpo V., Pérez-Montero E., Papaderos P., 2012b, ApJ, 754, L22

Amorín R., Fontana A., Pérez-Montero 2017, Nature Astronomy, 1, 0052

Augarde R., Figon P., Vanderriest C., Lemonnier J. P., 1990, A\&A, 233, 348

Baldwin J. A., Phillips M. M., Terlevich R., 1981, A\&A, 93, 5

Barker T., 1980, ApJ, 240, 99

Benjamin R. A., Skillman E. D., Smits D. P., 1999, ApJ, 514, 307

Bergvall N., Östlin G. J., 2002, A\&A, 390, 891

Bernstein R., Shectman S. A., Gunnels S. M., Mochnacki S., Athey A. E., 2003, in Instrument Design and Performance for Optical/Infrared Ground-based Telescopes.

Binette L., Matadamas R., Hägele G. F., Nicholls D. C., Magris C. G., Peña-Guerrero M. Á., Morisset C., Rodríguez-González A., 2012, A\&A, 547, A29

Binggeli B., Popescu C. C., 1995, A\&A, 298, 63

Bosch G., et al., 2019, MNRAS, p. 2153

Bothun G. D., Impey C. D., Malin D. F., 1991, ApJ, 376, 404

Bowen I. S., 1927, Physical Review, 29, 231

Bowen I. S., 1928, ApJ, 67, 1 
Brinchmann J., Ellis R. S., 2000, ApJ, 536, L77

Buckalew B. A., Kobulnicky H. A., 2006, AJ, 132, 1061

Cairós L. M., Méndez D. I., Esteban C., Vílchez J. M., 1998, in Proceedings of the Bonn/Bochum-Graduiertenkolleg Workshop. pp 181-184

Cairós L. M., Caon N., Vílchez J. M., González-Pérez J. N., Muñoz-Tuñón C., 2001, ApJS, 136,393

Cairós L. M., Caon N., Papaderos P., Kehrig C., Weilbacher P., Roth M. M., Zurita C., 2009, ApJ, 707, 1676

Calzetti D., 2000, A UV Imaging Survey of IR-Bright Star- Forming Galaxies, HST Proposal

Calzetti D., Giavalisco M., Ruffini R., 1989, A\&A, 226, 1

Campuzano-Castro F., Hagele G., Cardaci M., Bosch G., Firpo V., 2015, Boletin de la Asociacion Argentina de Astronomia La Plata Argentina, 57, 61

Campuzano-Castro F., Hägele G. F., Bosch G., Firpo V., Morrell N., Cardaci M. V., 2018, in Chemical Abundances in Gaseous Nebulae. p. 189

Campuzano-Castro F., Hägele G. F., Bosch G., Firpo V., Cardaci M., Muthukrishna D., Morrell N., 2019, Boletin de la Asociacion Argentina de Astronomia La Plata Argentina, 61,178

Cardamone C., et al., 2009, MNRAS, 399, 1191

Cardelli J. A., Clayton G. C., Mathis J. S., 1989, in Allamandola L. J., Tielens A. G. G. M., eds, IAU Symposium Vol. 135, Interstellar Dust. pp 5-10

Castellanos M., Díaz A. I., Terlevich E., 2002, MNRAS, 337, 540

Chromey F. R., 2010, To Measure the Sky

Chu Y., Kennicutt R., 1994, ApJ, 425, 720

Clayton C. A., 1990, MNRAS, 246, 712

De Young D. S., Gallagher John S. I., 1990, ApJ, 356, L15

De Young D. S., Heckman T. M., 1994, ApJ, 431, 598

Dekel A., Silk J., 1986, ApJ, 303, 39

Denicoló G., Terlevich R., Terlevich E., 2002, MNRAS, 330, 69

Díaz A. I., 1988, MNRAS, 231, 57

Díaz A. I., Pérez-Montero E., 2000, MNRAS, 312, 130

Díaz A., Terlevich E., Pagel B. E. J., Vílchez J. M., Edmunds M. G., 1987, MNRAS, 226, 19

Díaz A. I., Castellanos M., Terlevich E., Luisa García-Vargas M., 2000, MNRAS, 318, 462

Díaz Á. I., Terlevich E., Castellanos M., Hägele G. F., 2007, MNRAS, 382, 251 
Dors O. L. J., Krabbe A., Hägele G. F., Pérez-Montero E., 2011, MNRAS, 415, 3616

Dors O. L., et al., 2013, MNRAS, 432, 2512

Dors O. L., Pérez-Montero E., Hägele G. F., Cardaci M. V., Krabbe A. C., 2016, MNRAS, 456, 4407

Elmegreen B. G., Burkert A., 2010, ApJ, 712, 294

Esteban C., Vilchez J. M., 1992, ApJ, 390, 536

Fanelli M. N., Marcum P. M., O’Connell R. W., Waller W. H., Cornett R. H., Stecher T. P., UIT Science Team 1998, in American Astronomical Society Meeting Abstracts. p. 08.16

Ferland G. J., Fabian A. C., Hatch N. A., Johnstone R. M., Porter R. L., van Hoof P. A. M., Williams R. J. R., 2008, MNRAS, 386, L72

Figer D. F., Najarro F., Morris M., McLean I. S., Geballe T. R., Ghez A. M., Langer N., 1998, ApJ, 506, 384

Figer D. F., Najarro F., Kudritzki R. P., 2004, ApJ, 610, L109

Firpo V., 2011, PhD thesis, Universidad Nacional de La Plata $<$ EMAIL $>$ vfirpo@fcaglp.unlp.edu.ar $</$ EMAIL $>$

Firpo V., Bosch G., Morrell N., 2005, MNRAS, 356, 1357

Firpo V., Bosch G., Hägele G. F., Morrell N., 2010, MNRAS, 406, 1094

Firpo V., Bosch G., Hägele G. F., Díaz Á. I., Morrell N., 2011, MNRAS, 414, 3288

García-Benito R., Pérez E., Díaz Á. I., Maíz Apellániz J., Cerviño M., 2009, Ap\&SS, 324, 337

García-Benito R., et al., 2010, MNRAS, 408, 2234

García-Lorenzo B., Cairós L. M., Caon N., Monreal-Ibero A., Kehrig C., 2008, ApJ, 677, 201

García-Rojas J., Esteban C., Peimbert M., Rodríguez M., Ruiz M. T., Peimbert A., 2004, ApJS, 153, 501

García-Rojas J., Esteban C., Peimbert A., Peimbert M., Rodriguez M.... Ruiz M. T., 2005, MNRAS, 362, 301

Garnett D. R., 1992a, in Edmunds M. G., Terlevich R., eds, Elements and the Cosmos. p. 188

Garnett D. R., 1992b, AJ, 103, 1330

Gil de Paz A., Madore B. F., 2005, A\&A, 156, 345

Gil de Paz A., Madore B. F., Pevunova O., 2003a, A\&A, 147, 29

Gil de Paz A., Madore B. F., Pevunova O., 2003b, VizieR Online Data Catalog, 214, 70029

Grebel E. K., 2000, in Favata F., Kaas A., Wilson A., eds, ESA Special Publication Vol. 445, Star Formation from the Small to the Large Scale. p. 87 (arXiv:astro-ph/0005296)

Grebel H., Iqbal Z., Lan A., 2001, Chemical Physics Letters, 348, 203 
Grevesse N., Sauval A. J., 1998, A\&A, 85, 161

Guseva N. G., Izotov Y. I., Thuan T. X., 2006, ApJ, 644, 890

Guseva N. G., Izotov Y. I., Stasinska G., Fricke K. J., Henkel C., Papaderos P., 2011, A\&A, 529, A149

Hagele G. F., 2009, PhD thesis, -

Hägele G. F., Pérez-Montero E., Díaz Á. I., Terlevich E., Terlevich R., 2006, MNRAS, 372, 293

Hägele G. F., Díaz Á. I., Cardaci M. V., Terlevich E., Terlevich R., 2007, MNRAS, 378, 163

Hägele G. F., Díaz Á. I., Terlevich E., Terlevich R., Pérez-Montero E., Cardaci M. V., 2008, MNRAS, 383, 209

Hägele G. F., Díaz Á. I., Cardaci M. V., Terlevich E., Terlevich R., 2009, MNRAS, 396, 2295

Hägele G. F., Díaz Á. I., Cardaci M. V., Terlevich E., Terlevich R., 2010, MNRAS, 402, 1005

Hägele G. F., García-Benito R., Pérez-Montero E., Díaz Á. I., Cardaci M. V., Firpo V., Terlevich E., Terlevich R., 2011, MNRAS, 414, 272

Hägele G. F., Firpo V., Bosch G., Díaz Á. I., Morrell N., 2012, MNRAS, 422, 3475

Hägele G. F., Díaz Á. I., Terlevich R., Terlevich E., Bosch G. L., Cardaci M. V., 2013, MNRAS, 432, 810

Haynes M. P., Giovanelli R., Herter T., Vogt N. P., Freudling W., Maia M. A. G., Salzer J. J., Wegner G., 1997, AJ, 113, 1197

Holweger H., 2001, in Wimmer-Schweingruber R. F., ed., American Institute of Physics Conference Series Vol. 598, Joint SOHO/ACE workshop "Solar and Galactic Composition". pp 23-30 (arXiv:astro-ph/0107426), doi:10.1063/1.1433974

Howarth I. D., Wilson B., 1983, MNRAS, 204, 1091

Hubble E. P., 1926, ApJ, 64, 321

Hubble E. P., 1936, Realm of the Nebulae

Hunt L. K., Hirashita H., Thuan T. X., Izotov Y. I., Vanzi L., 2003, arXiv e-prints, pp astro-ph/0310865

Izotov Y. I., Thuan T. X., 1999, ApJ, 511, 639

Izotov Y. I., Thuan T. X., Lipovetsky V. A., 1994, ApJ, 435, 647

Izotov Y. I., Stasińska G., Meynet G., Guseva N. G., Thuan T. X., 2006a, A\&A, 448, 955

Izotov Y. I., Papaderos P., Guseva N. G., Fricke K. J., Thuan T. X., 2006b, A\&A, 454, 137

James B., Koposov S., Stark D., Belokurov V., Pettini M., Olszewski E. W., 2015, in American Astronomical Society Meeting Abstracts \#225. p. 129.03

Jaschek M., Egret D., 1981, Bulletin d'Information du Centre de Donnees Stellaires, 20, 36 
Jun-Jie W., Xue-Feng W., Fulvio M., 2016, A\&AS, 90, 285

Karachentsev I. D., Karachentseva V. E., Huchtmeier W. K., Makarov D. I., 2004, AJ, 127, 2031

Kauffmann G., et al., 2003, MNRAS, 346, 1055

Kewley L. J., Ellison S. L., 2008, ApJ, 681, 1183

Kewley L. J., Dopita M. A., Sutherland R. S., Heisler C. A., Trevena J., 2001, ApJ, 556, 121

Kewley L. J., Groves B., Kauffmann G., Heckman T., 2006, MNRAS, 372, 961

Kingdon J., Ferland G. J., 1995, ApJ, 442, 714

Kniazev A. Y., Pustilnik S. A., Grebel E. K., Lee H., Pramskij A. G., 2004, ApJS, 153, 429

Kobulnicky H. A., Kennicutt Robert C. J., Pizagno J. L., 1999, ApJ, 514, 544

Krabbe A. C., Rosa D. A., Dors O. L., Pastoriza M. G., Winge C., Hägele G. F., Cardaci M. V., Rodrigues I., 2014, MNRAS, 437, 1155

Kunth D., Östlin G., 2000, ApJS, 10, 1

Kunth D., Sargent W. L. W., 1983, ApJ, 273, 81

Lagos P., Scott T. C., Nigoche-Netro A., Demarco R., Humphrey A., Papaderos P., 2018, MNRAS, 477, 392

Lauqué R., 1973, A\&A, 23, 253

Lehnert M. D., Nesvadba N. P. H., Le Tiran L., Di Matteo P., van Driel W., Douglas L. S., Chemin L., Bournaud F., 2009, ApJ, 699, 1660

Liu X. W., Luo S. G., Barlow M. J., Danziger I. J., Storey P. J., 2001, MNRAS, 327, 141

López-Hernández J., Terlevich E., Terlevich R., Rosa-González D., Díaz Á., García-Benito R., Vílchez J., Hägele G., 2013, MNRAS, 430, 472

López-Sánchez A. R., Koribalski B., van Eymeren J., Esteban C., Popping A., Hibbard J., 2010, in Verdes-Montenegro L., Del Olmo A., Sulentic J., eds, Astronomical Society of the Pacific Conference Series Vol. 421, Galaxies in Isolation: Exploring Nature Versus Nurture. p. 65 (arXiv:0909.5500)

López-Sánchez Á. R., Dopita M. A., Kewley L. J., Zahid H. J., Nicholls D. C., Scharwächter J., 2012, MNRAS, 426, 2630

Macchetto F., Colina L., Golombek D., Perryman M. A. C., di Serego Alighieri S., 1990, ApJ, 356,389

Maeder A., 1981, A\&A, 101, 385

Markarian B. E.; Lipovetsky V. A., Stepanian J. A., Erastova L. K., Shapovalova A. I., 1989, A\&A, 62, P.5

Martínez-Delgado D., Pohlen M., Gabany R. J., Majewski S. R., Peñarrubia J., Palma C., 2009, ApJ, 692, 955 
Marzke R. O., da Costa L. N., 1997, AJ, 113, 185

Massey P., Olsen K. A. G., 2003, AJ, 126, 2867

Mateo M., 1998, in Richtler T., Braun J. M., eds, The Magellanic Clouds and Other Dwarf Galaxies. p. 53

McGaugh S. S., 1991, ApJ, 380, 140

Melnick J., Terlevich R., Moles M., 1985a, Rev.Mex.A.A., 11, 91

Melnick J., Terlevich R., Eggleton P. P., 1985b, MNRAS, 216, 255

Melnick J., Tenorio-Tagle G., Terlevich R., 1999, in Franco J., Carraminana A., eds, Interstellar Turbulence. p. 148

Méndez D. I., Cairós L. M., Esteban C., Vílchez J. M., 1999, AJ, 117, 1688

Muñoz-Tunon C., Tenorio-Tagle G., Castaneda H. O., Terlevich R., 1996, AJ, 112, 1636

Newton O., Cautun M., Jenkins A., Frenk C. S., Helly J. C., 2018, MNRAS, 479, 2853

Newville M., Stensitzki T., Allen D. B., Ingargiola A., 2014, LMFIT: Non-Linear LeastSquare Minimization and Curve-Fitting for Python, doi:10.5281/zenodo.11813, https:// doi.org/10.5281/zenodo.11813

Newville M., Stensitzki T., Allen D. B., Rawlik M., Ingargiola A., Nelson A., 2016, Lmfit: Non-Linear Least-Square Minimization and Curve-Fitting for Python (ascl:1606.014)

Nicholson J. W., 1911, MNRAS, 72, 49

Noeske K. G., Papaderos P., Fricke K. J., Thuan T. X., 1998, in Magellanic Clouds and Other Dwarf Galaxies. p. 279

Noeske K. G., Papaderos P., Cairǿs L. M., Fricke K. J., 2005, A\&A, 429, 115

Olive K. A., Skillman E. D., 2001, New Astron., 6, 119

Olive K. A., Skillman E. D., 2004, ApJ, 617, 29

Osterbrock D. E., Ferland G. J., 2006, Astrophysics of gaseous nebulae and active galactic nuclei

Östlin G., Zackrisson E., Bergvall N., Rönnback J., 2003, A\&A, 408, 887

Pagel B. E. J., 1979, in Woolfson M. M., ed., Trends in Physics 1978. pp 247-254

Pagel B. E. J., 1992, in Barbuy B., Renzini A., eds, IAU Symposium Vol. 149, The Stellar Populations of Galaxies. p. 133

Pagel B. E. J., Simonson E. A., Terlevich R. J., Edmunds M. G., 1992, MNRAS, 255, 325

Papaderos P., Fricke K. J., Izotov Y. I., Thuan T. X., 1999, in Astronomische Gesellschaft Abstract Series. p. 46

Papaderos P., Izotov Y. I., Noeske K. G., Cairós L. M., Guseva N. G., Thuan T. X., Fricke K. J., 2003, Ap\&SS, 284, 619 
Papaderos P., Guseva N. G., Izotov Y. I., Fricke K. J., 2008, ApJS, 113, 113

Pauldrach A. W. A., Hoffmann T. L., Lennon M., 2001, A\&A, 375, 161

Peimbert M., 1967, ApJ, 150, 825

Peimbert M., 1978, in Terzian Y., ed., IAU Symposium Vol. 76, Planetary Nebulae. pp 215224

Peimbert M., Costero R., 1969, Boletin de los Observatorios Tonantzintla y Tacubaya, 5, 3

Pérez-Montero E., 2017, PASP, 129, 043001

Pérez-Montero E., Contini T., 2009, MNRAS, 398, 949

Pérez-Montero E., Díaz A. I., 2005a, MNRAS, 361, 1063

Pérez-Montero E., Díaz A. I., 2005b, VizieR Online Data Catalog, 736, 11063

Pérez-Montero E., Díaz A. I., Zamorano J., Gallego J., 2003, in Avila-Reese V., Firmani C., Frenk C. S., Allen C., eds, Revista Mexicana de Astronomia y Astrofisica, vol. 27 Vol. 17, Revista Mexicana de Astronomia y Astrofisica Conference Series. pp 205-205

Pérez-Montero E., Díaz A. I., Vílchez J. M., Kehrig C., 2006, A\&A, 449, 193

Pérez-Montero E., Hägele G. F., Contini T., Díaz Á. I., 2007, MNRAS, 381, 125

Pérez-Montero E., García-Benito R., Díaz A. I., Pérez E., Kehrig C., 2009, A\&A, 497, 53

Pérez-Montero E., García-Benito R., Hägele G. F., Díaz Á. I., 2010, MNRAS, 404, 2037

Pérez-Montero E., et al., 2011a, in Zapatero Osorio M. R., Gorgas J., Maíz Apellániz J., Pardo J. R., Gil de Paz A., eds, Highlights of Spanish Astrophysics VI. pp 395-395

Pérez-Montero E., et al., 2011b, A\&A, 532, A141

Pettini M., Pagel B. E. J., 2004, MNRAS, 348, L59

Pilyugin L. S., 2001, A\&A, 369, 594

Relaño M., Beckman J. E., 2005, A\&A, 430, 911

Robertis M., 1986, IRAS Select Seyferts, IUE Proposal

Rozas M., Richer M. G., Lǿpez J. A., Relaño M., Beckman J. E., 2006, A\&A, 455, 539

Salpeter E. E., 1955, Physical Review, 97, 1237

Sargent W. L. W., Searle L., 1970, ApJ, 162, L155

Schönberner D., Blöcker T., 1996, Ap\&SS, 245, 201

Searle L., 1971, ApJ, 168, 327

Searle L., Sargent W. L. W., 1972, ApJ, 173, p25.

Seaton M. J., 1979, MNRAS, 187, 785

Shaw R. A., Dufour R. J., 1995, PASP, 107, 896 
Shields G. A., 1974, ApJ, 193, 335

Skillman E. D., 1989, ApJ, 347, 883

Slettebak A., 1979, ] 10.1007/BF00212356, 23, 541

Smith M. G., Weedman D. W., 1972, ApJ, 172, 307

Stasińska G., 1978, A\&AS, 32, 429

Stasińska G., 2006, A\&A, 454, L127

Storchi-Bergmann T., Calzetti D., Kinney A. L., 1994, ApJ, 429, 572

Storey P. J., Hummer D. G., 1995, MNRAS, 272, 41

Taylor C. L., Brinks E., Skillman E. D., 1993, A\&A, 25, 1342

Telles E., Melnick J., Terlevich R., 1997, MNRAS, 288, 78

Tenorio-Tagle G., Muñoz-Tuñón C., Pérez E., Melnick J., 1997, ApJ, 490, 179

Terlevich R., Melnick J., 1981, ApJ, 195, 839

Terlevich R., Melnick J., Masegosa J., Moles M., Copetti M. V. F., 1991, ApJ, 91, 285

Terlevich E., Díaz A. I., Terlevich R., González-Delgado R. M., Pérez E., García Vargas M. L., 1996, MNRAS, 279, 1219

Terlevich R., Melnick J., Masegosa J., Moles M., Copetti M. V. F., 1999, VizieR Online Data Catalog, pp J/A+AS/91/285

Thuan T. X., Izotov Y. I., 1999, arXiv e-prints, pp astro-ph/9902369

Thuan T. X., Martin G. E., 1981, A\&A, 247, 823

Tolstoy E., Hill V., Tosi M., 2009, ??jnlARA\&A, 47, 371

Vilchez J. M., Esteban C., 1996, MNRAS, 280, 720

Vilchez J. M., Pagel B. E. J., 1988, MNRAS, 231, 257

Walter F., Brinks E., Duric N., Klein U., 1997, AJ, 113, 2031

Westmoquette M. S. Smith L. J., Gallagher J. S., Exter K. M., 2007, MNRAS, 381, 913

Whitford A. E., 1958, AJ, 63, 201

Yang H., Chu Y.-H., Skillman E. D., Terlevich R., 1996, AJ, 112, 146

Zorec J., Frémat Y., Cidale L., 2004, in Combes F., Barret D., Contini T., Meynadier F., Pagani L., eds, SF2A-2004: Semaine de l'Astrophysique Francaise. p. 327

Zwicky F., 1965, ApJ, 142, 1293

Zwicky F., Rudnicki K., 1966, Zeitschrift für Astrophysik, 64, 246

de Vaucouleurs G., 1991, Science, 254, 1667

van Genderen A. M., The P. S., 1984, A\&A, 39, 317

van der Werf P. P., Snijders L., 2006, arXiv e-prints, pp astro-ph/0601430 